\title{
Manual for Implementing Residual Radioactive Material Guidelines Using RESRAD, Version $\mathbf{5 . 0}$
}

Working Draft for Comment

by C. Yu, A.J. Zielen, J.-J. Cheng, Y.C. Yuan, ${ }^{\star}$ L.G. Jones, D.J. LePoire, Y.Y. Wang, C.O. Loureiro, ${ }^{*}$ E. Gnanapragasam, E. Faillace, A. Wallo III, ${ }^{*}$ W.A. Williams, ${ }^{*}$ and H. Peterson*

Environmental Assessment Division,

Argonne National Laboratory, 9700 South Cass Avenue, Argonne, Illinois 60439

September 1993

Work sponsored by United States Department of Energy,

Assistant Secretary for Environment, Safety and Health, Office of Environmental Guidance, Assistant Secretary for Environmental Restoration and Waste Management, Office of Environmental Restoration

"Yuan is associated with Square Y Consultants, Orchard Park, New York; Loureiro with the School of Engineering of the University of Minas Gerais, Belo Horizonte, Brazil; Wallo with the U.S. Department of Energy, Office of Environmental Guidance, Washington, D.C.; Williams with the U.S. Department of Energy, Office of Environmental Restoration, Washington, D.C.; and Peterson with the U.S. Department of Energy, Office of Environmental Guidance, Washington, D.C. 


\section{Manual for Implementing Residual Radioactive Material Guidelines Using RESRAD, Version $\mathbf{5 . 0}$}

\section{Working Draft for Comment}

by C. Yu, A.J. Zielen, J.-J. Cheng, Y.C. Yuan, ${ }^{*}$ L.G. Jones, D.J. LePoire, Y.Y. Wang, C.O. Loureiro, ${ }^{\star}$ E. Gnanapragasam, E. Faillace, A. Wallo III, ${ }^{*}$ W.A. Williams, ${ }^{*}$ and H. Peterson*

Environmental Assessment Division,

Argonne National Laboratory, 9700 South Cass Avenue, Argonne, Illinois 60439

September 1993

Work sponsored by United States Department of Energy, Assistant Secretary for Environment, Safety and Health, Office of Environmental Guidance, Assistant Secretary for Environmental Restoration and Waste Management, Office of Environmental Restoration

*Yuan is associated with Square Y Consultants, Orchard Park, New York; Loureiro with the School of Engineering of the University of Minas Gerais, Belo Horizonte, Brazil; Wallo with the U.S. Department of Energy, Office of Environmental Guidance, Washington, D.C.; Williams with the U.S. Department of Energy, Office of Environmental Restoration, Washington, D.C.; and Peterson with the U.S. Department of Energy, Office of Environmental Guidance, Washington, D.C. 


\section{DISCLAIMER}

This report was prepared as an account of work sponsored by an agency of the United States Government. Neither the United States Government nor any agency thereof, nor any of their employees, make any warranty, express or implied, or assumes any legal liability or responsibility for the accuracy, completeness, or usefulness of any information, apparatus, product, or process disclosed, or represents that its use would not infringe privately owned rights. Reference herein to any specific commercial product, process, or service by trade name, trademark, manifacturer, or otherwise does not necessarily constitute or imply its endorsement, recommendation, or favoring by the United States Government or any agency thereof. The views and opinions of authors expressed herein do not necessarily state or reflect those of the United States Government or any agency thereof. 


\section{DISCLAIMER}

Portions of this document may be illegible electronic image products. Images are produced from the best available original document. 


\section{CONTENTS}

FOREWORD $\ldots \ldots \ldots \ldots \ldots \ldots \ldots \ldots \ldots \ldots \ldots \ldots \ldots \ldots \ldots \ldots \ldots \ldots \ldots \ldots \ldots$

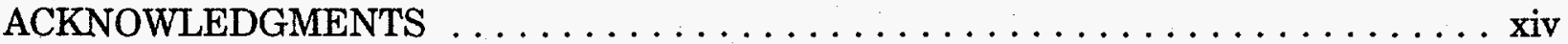

AVAILABILITY OF COMPUTER SOFTWARE $\ldots \ldots \ldots \ldots \ldots \ldots \ldots$

NOTATION $\ldots \ldots \ldots \ldots \ldots \ldots \ldots \ldots \ldots \ldots \ldots \ldots \ldots \ldots \ldots \ldots \ldots \ldots \ldots$

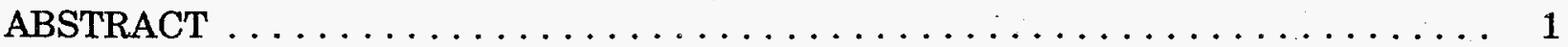

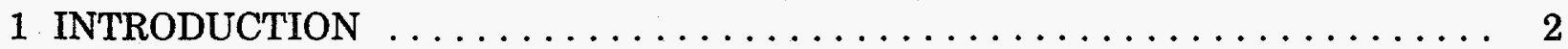

2 PATHWAY ANALYSIS $\ldots \ldots \ldots \ldots \ldots \ldots \ldots \ldots \ldots \ldots \ldots \ldots$

2.1 Source Terms $\ldots \ldots \ldots \ldots \ldots \ldots \ldots \ldots \ldots \ldots \ldots \ldots$

2.1.1 Geometry of the Contaminated Zone ................. 9

2.1.2 Time Dependence .......................... 10

2.1 .3 Redistribution ............................. 10

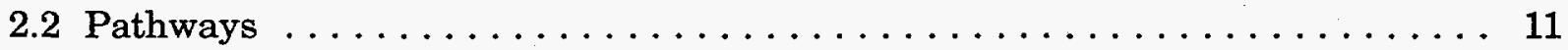

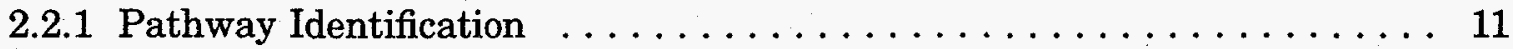

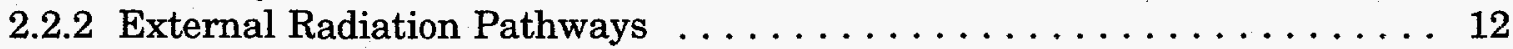

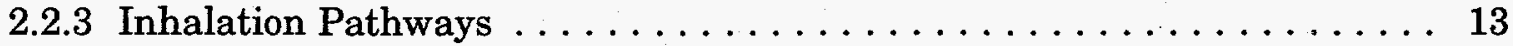

2.2 .4 Ingestion Pathways $\ldots \ldots \ldots \ldots \ldots \ldots \ldots \ldots \ldots \ldots \ldots \ldots \ldots \ldots$

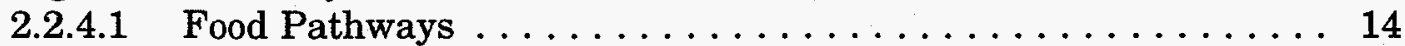

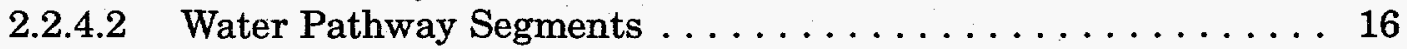

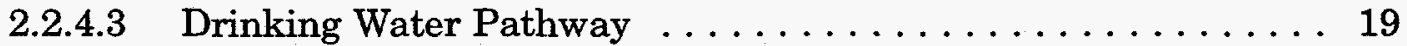

2.2.4.4 Soil Ingestion Pathway . . . . . . . . . . . . . . . . . 19

2.3 Dose Conversion Factors . . . . . . . . . . . . . . . . . . . . 19

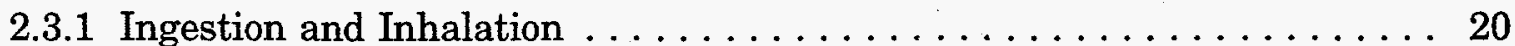

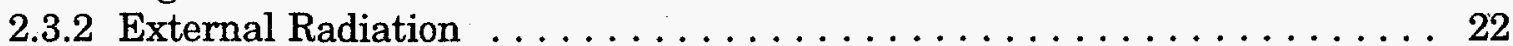

2.4 Exposure Scenarios . . . . . . . . . . . . . . . . . . 23

3 GUIDELINES FOR RADIONUCLIDE CONCENTRATIONS IN SOIL . . . . . . . 28

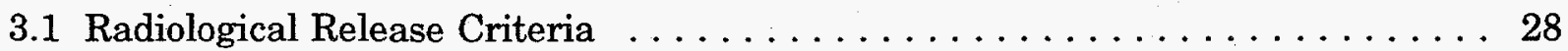

3.2 Dose/Source Concentration Ratios for Uniform Contamination . . . . . . . . 37

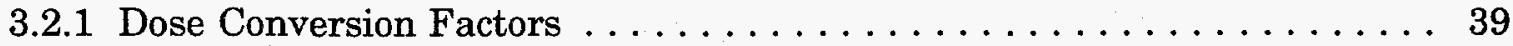

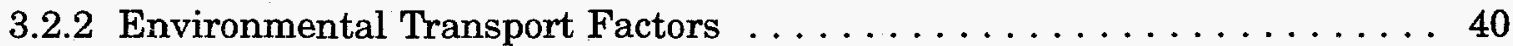

3.2 .3 Source Factors . . . . . . . . . . . . . . . . . . . . . 41

3.3 Guidelines for Inhomogeneous Contamination $\ldots \ldots \ldots \ldots \ldots \ldots \ldots \ldots$

3.3.1 Inhomogeneous Criteria for Project Planning and Review . . . . . . . 42

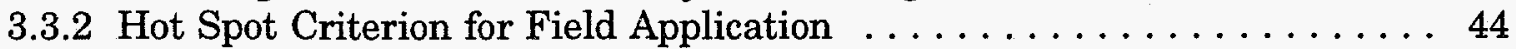


4 USER'S GUIDE FOR $\operatorname{RESRAD} \ldots \ldots \ldots \ldots \ldots \ldots \ldots \ldots \ldots \ldots \ldots$

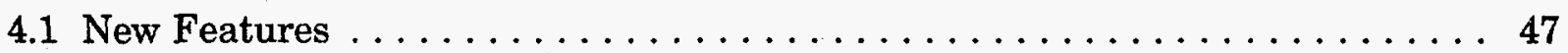

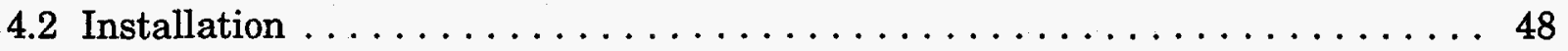

4.3 RESRAD Execution $\ldots \ldots \ldots \ldots \ldots \ldots \ldots \ldots \ldots \ldots \ldots \ldots$

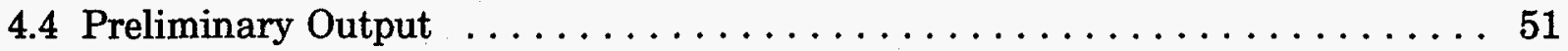

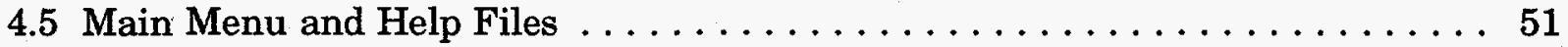

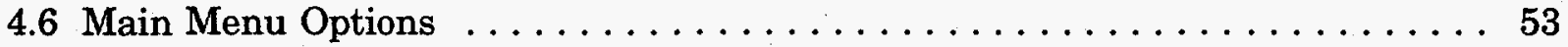

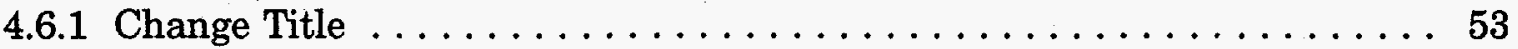

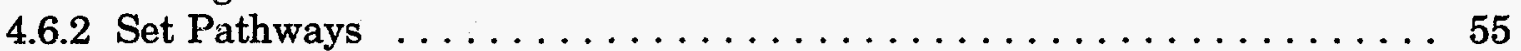

4.6.3 Modify Data ........................ 56

4.6.3.1 Contaminated Zone Parameters . . . . . . . . . . . . 58

4.6.3.2 Initial Concentrations of Principal Radionuclides . . . . . . . 60

4.6.3.3 Cover and Contaminated Zone Hydrological Data . . . . . . . . 63

4.6.3.4 Saturated Zone Hydrological Data . . . . . . . . . . . . . 65

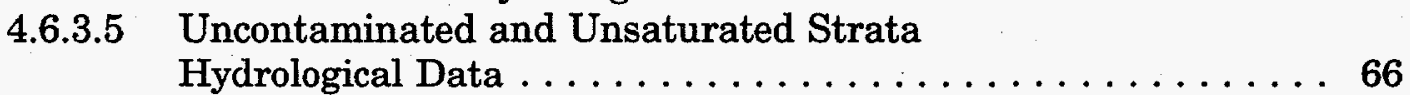

4.6.3.6 Distribution Coefficients and Leach Rate Constants . . . . . . 67

4.6.3.7 Dust Inhalation and External Gamma Parameters . . . . . . . . 69

4.6.3.8 Ingestion Pathway Data, Dietary Parameters . . . . . . . . . 70

4.6.3.9 Ingestion Pathway Data, Nondietary Parameters . . . . . . . 72

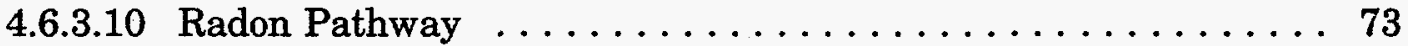

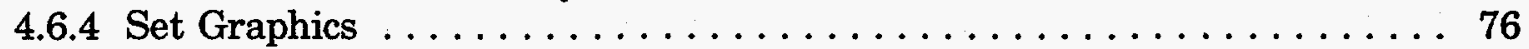

4.6.4.1 Graphics Parameters ................. 77

4.6.4.2 Serial Port Parameters Submenu .................. 80

4.6.4.3 Plot Scaling and Offset Adjustments Submenu . . . . . . . . . 80

4.6.4.4 Dot Matrix Printer Parameters Submenu . . . . . . . . . . . 82

4.6.4.5 Hewlett-Packard Pen Plotter Parameters Submenu ... . . . . 82

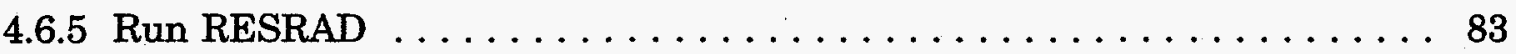

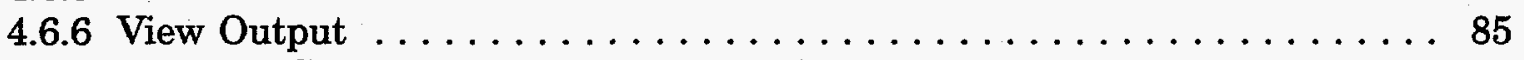

4.6.6.1 Summary Report $\ldots \ldots \ldots \ldots \ldots \ldots \ldots \ldots$

4.6.6.2 Detailed Report $\ldots \ldots \ldots \ldots \ldots \ldots \ldots \ldots \ldots$

4.6.6.3 Health Risk Report . . . . . . . . . . . . . . . . 91

4.6.6.4 Radionuclide Concentration Report ............... 92

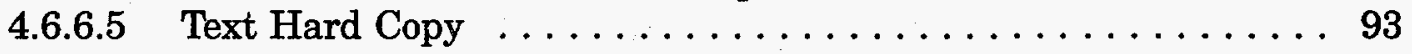

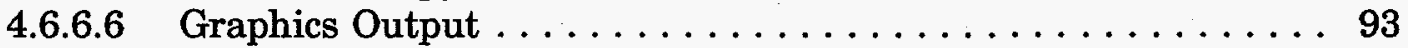

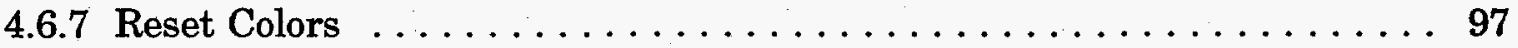

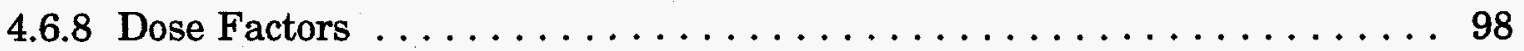

4.6.8.1 Ground External Gamma Effective Dose

Conversion Factors ..................... 100

4.6.8.2 Depth Factors for External Gamma Radiation
from the Ground $\ldots \ldots \ldots \ldots \ldots \ldots \ldots \ldots \ldots 1$

4.6.8.3 Inhalation Effective Dose Conversion Factors . . . . . . . . . . 101

4.6.8.4 Ingestion Effective Dose Conversion Factors . . . . . . . . . . 103

4.6.8.5 Food Transfer Factors . . . . . . . . . . . . . . . . . . . 104 
4.6.8.6 Bioaccumulation Factors for Aquatic Foods . . . . . . . . . 105

4.6.8.7 Slope Factors . . . . . . . . . . . . . . . 106

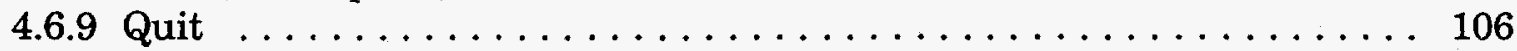

4.7 Technical Summary $\ldots \ldots \ldots \ldots \ldots \ldots \ldots \ldots$

5 APPLICATION OF THE AS LOW AS REASONABLY

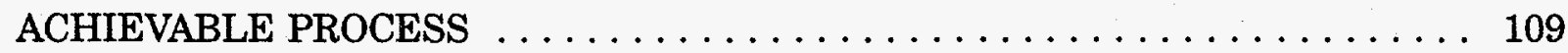

5.1 Determining ALARA $\ldots \ldots \ldots \ldots \ldots \ldots \ldots \ldots \ldots \ldots \ldots \ldots$

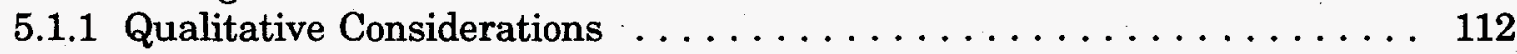

5.1 .2 Cost-Benefit Analysis . . . . . . . . . . . . . . . . 114

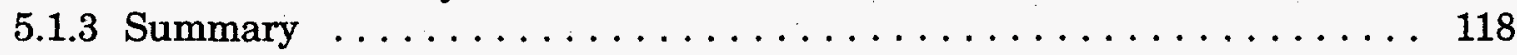

5.2 Documenting ALARA $\ldots \ldots \ldots \ldots \ldots \ldots \ldots \ldots \ldots \ldots \ldots \ldots$

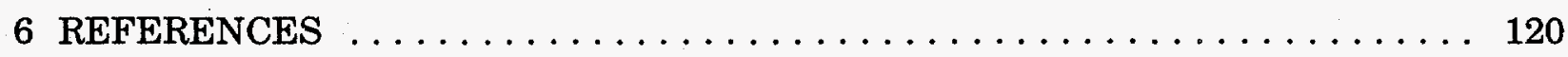

APPENDIX A: External Ground Radiation Pathway Factors $\ldots \ldots \ldots \ldots \ldots \ldots$

APPENDIX B: Inhalation Pathway Factors $\ldots \ldots \ldots \ldots \ldots \ldots \ldots$

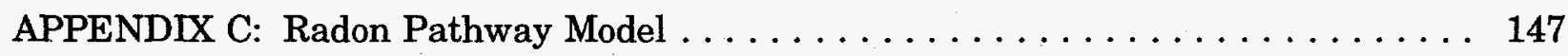

APPENDIX D: Ingestion Pathway Factors $\ldots \ldots \ldots \ldots \ldots \ldots \ldots \ldots$

APPENDIX E: Water Pathway Factors . . . . . . . . . . . . . . . 193

APPENDIX F: Soil Ingestion Pathway Factors . . . . . . . . . . . . . . 219

APPENDIX G: Source Factors . . . . . . . . . . . . . . . . . 225

APPENDIX H: Distribution Coefficients $\ldots \ldots \ldots \ldots \ldots \ldots \ldots \ldots \ldots$

APPENDIX I: Radioactive Decay Product Transfer Function $\ldots \ldots \ldots \ldots \ldots$

APPENDIX J: Estimation of the Distribution Coefficient on the Basis of the Solubility Constant . . . . . . . . . . . . . . . 259

APPENDIX K: Estimation of Individual and Collective Off-Site Doses . . . . . . 283

APPENDIX L: Tritium and Carbon-14 Pathway Models . . . . . . . . . . 303

APPENDIX M: Optimization in Quantitative ALARA Analyses . . . . . . . . 327 


\section{TABLES}

$2.1 \quad$ Potential Pathways $\ldots \ldots \ldots \ldots \ldots \ldots \ldots \ldots \ldots \ldots \ldots \ldots \ldots$

$3.1 \quad$ Principal and Associated Radionuclides $\ldots \ldots \ldots \ldots \ldots \ldots$

$3.2 \quad$ Ranges for Hot Spot Multiplication Factors . . . . . . . . . . . . . 45

$5.1 \quad$ Examples of Expenditures to Limit Cancer Risk . . . . . . . . . . . . 117

A.1 Effective Dose Equivalent Conversion Factors for External Gamma

Radiation from Contaminated Ground . . . . . . . . . . . . . . 128

A.2 Area Factors for External Gamma Radiation from Contaminated Ground . . . . 131

A.3 Depth Factors for External Gamma Radiation from Contaminated

Ground as a Function of Thickness of a Contaminated Layer

Exposed at the Ground Surface and Soil Density for Principal

and Associated Radionuclides

B.1 Committed Effective Dose Equivalent Conversion Factors

for Inhalation

C.1 Ranges of Parameters Affecting Radon Concentrations in Air

from Water Use in the Home

C.2 Summary of $\mathrm{K}$ Factors for Bronchial Dose Calculated for the General Public Relative to Underground Miners

D.1 Committed Effective Dose Equivalent Conversion Factors

for Internal Radiation from Ingestion $\ldots \ldots \ldots \ldots \ldots \ldots \ldots \ldots \ldots$

D.2 Dietary Factors for Human Food Consumption $\ldots \ldots \ldots \ldots \ldots \ldots \ldots$

D.3 Vegetable/Soil Transfer Factors for Root Uptake . . . . . . . . . . . . 183

D.4 Meat and Milk Transfer Factors for Root Uptake $\ldots \ldots \ldots \ldots \ldots \ldots$

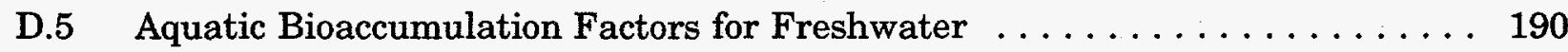

E.1 Runoff Coefficient Values $\ldots \ldots \ldots \ldots \ldots \ldots \ldots \ldots \ldots \ldots \ldots \ldots$

E.2 Representative Values of Saturated Hydraulic Conductivity, Saturated

Water Content, and the Soil-Specific Exponential Parameter . . . . . . . . 200

E.3 Typical Average Distribution Coefficients for Various Elements

in Sand, Soils, and Clays 


\section{TABLES (Cont.)}

E.4 Distribution Coefficients for Strontium and Cesium . . . . . . . . . 203

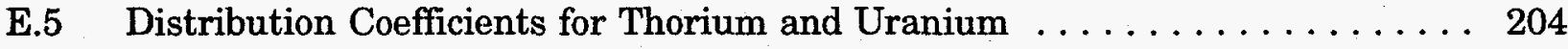

E.6 Distribution Coefficients for Several Radionuclides from Various Sources . . . 205

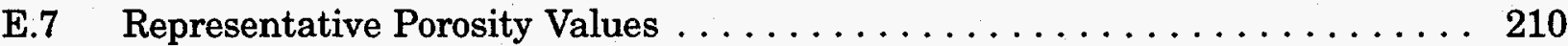

J.1 Constants of Atomic Weight and Specific Activity for Radionuclides in the Current RESRAD Database . . . . . . . . . . . . . . 268

L.1 Water Content of Food Types $\ldots \ldots \ldots \ldots \ldots \ldots \ldots \ldots \ldots \ldots$

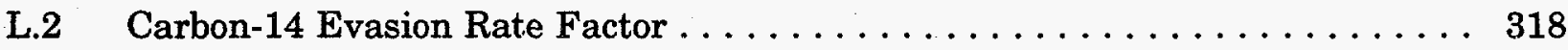

L.3 Carbon Content of Food Types and Water $\ldots \ldots \ldots \ldots \ldots \ldots \ldots \ldots$

\section{FIGURES}

$1.1 \quad$ Exposure Pathways Considered in RESRAD $\ldots \ldots \ldots \ldots \ldots \ldots \ldots \ldots$

2.1 Geometry of Idealized Contaminated Zone $\ldots \ldots \ldots \ldots \ldots \ldots$

2.2 Schematic Representation of RESRAD Pathways $\ldots \ldots \ldots \ldots \ldots$

2.3 Schematic Representation of the Water Pathway Segments . . . . . . . . 17

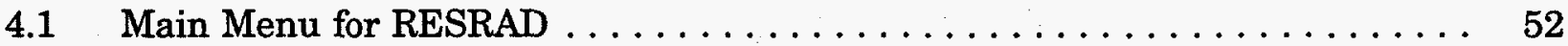

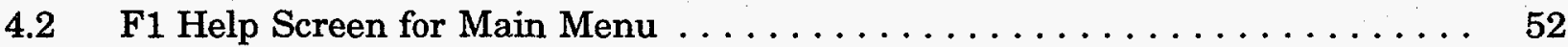

$4.3 \quad$ F1 Help Screen for Data Entry Forms $\ldots \ldots \ldots \ldots \ldots \ldots \ldots \ldots \ldots$

4.4 Input Form R010 for Title and User Data Files . . . . . . . . . . . . . . . 54

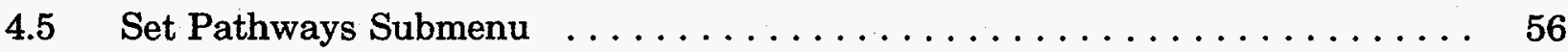

$4.6 \quad$ Modify Data Submenu $\ldots \ldots \ldots \ldots \ldots \ldots \ldots \ldots \ldots \ldots \ldots$

4.7 Input Form R011 for Contaminated Zone Parameters . . . . . . . . . 59

4.8 Input Form R011 with Sensitivity Analysis Pop-Up Menu . . . . . . . . . 59 


\section{FIGURES (Cont.)}

4.9 Input Form R012 for Initial Concentrations of Principal Radionuclides . . . . . 61

4.10 Input Form R012W for Initial Soil and Groundwater Concentrations of Principal Radionuclides . . . . . . . . . . . . . . . . . . 61

4.11 Input Form R013 for Cover and Contaminated Zone Hydrological Data . . . . . 64

4.12 Input Form R014 for Saturated Zone Hydrological Data $\ldots \ldots \ldots \ldots \ldots .65$

4.13 Input Form R015 for Uncontaminated and Unsaturated Strata

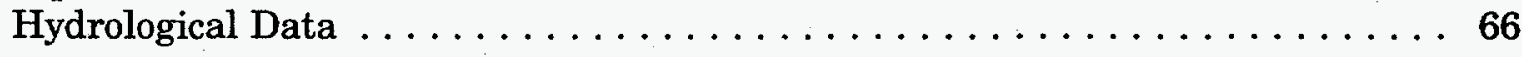

4.14 Input Form R016 for Distribution Coefficients and Leach Rates . . . . . . . 67

4.15 Input Form R017 for Dust Inhalation and External Gamma Parameters . . . . 70

4.16 Input Form R018 for Ingestion Pathway Data, Dietary Parameters . . . . . . 71

4.17 Input Form R019 for Ingestion Pathway Data, Nondietary Parameters . . . . 72

4.18 Input Form $\mathrm{C} 14$ for Carbon-14 Parameters $\ldots \ldots \ldots \ldots \ldots \ldots \ldots \ldots$

4.19 Input Form R021 for Radon Parameters $\ldots \ldots \ldots \ldots \ldots \ldots \ldots \ldots \ldots$

4.20 Input Form R020 for Graphics Parameters $\ldots \ldots \ldots \ldots \ldots \ldots \ldots \ldots$

4.21 Input Form R0205 for Serial Port Parameters $\ldots \ldots \ldots \ldots \ldots \ldots \ldots \ldots$

4.22 Input Form R0206 for Plot Scaling and Offset Adjustments $\ldots \ldots \ldots \ldots$. 81

4.23 Input Form R0207 for Dot Matrix Printer Parameters $\ldots \ldots \ldots \ldots \ldots \ldots 2$

4.24 Input Form R0208 for Hewlett-Packard Pen Plotter Parameters . . . . . . . . 83

4.25 Typical Screen after Run RESRAD Is Selected from Main Menu . . . . . . . 84

4.26 View and Setup Submenu $\ldots \ldots \ldots \ldots \ldots \ldots \ldots \ldots \ldots \ldots \ldots \ldots \ldots \ldots \ldots$

4.27 Setup Printer Submenu $\ldots \ldots \ldots \ldots \ldots \ldots \ldots \ldots \ldots \ldots \ldots \ldots \ldots \ldots \ldots \ldots$

4.28 View Text Output Submenu $\ldots \ldots \ldots \ldots \ldots \ldots \ldots \ldots \ldots \ldots \ldots$

4.29 Typical Table of Contents for Summary Report $\ldots \ldots \ldots \ldots \ldots \ldots \ldots$

4.30 Typical Table of Contents for Detailed Report $\ldots \ldots \ldots \ldots \ldots \ldots \ldots \ldots$ 


\section{FIGURES (Cont.)}

J.4 Relative Stability of Cobalt Solids in an Oxidizing Soil Environment with Phosphate Levels in Equilibrium with Variscite and Gibbsite, Dicalcium Phosphate Dihydrate, and Octacalcium Phosphate ............. 273

J.5 Relative Stability of Various Europium Solids at $\mathrm{pSO}_{4}{ }^{2-}=2.5 \ldots \ldots \ldots \ldots 274$

J.6 Relative Stability of Various Neptunium Solids in an Oxidizing

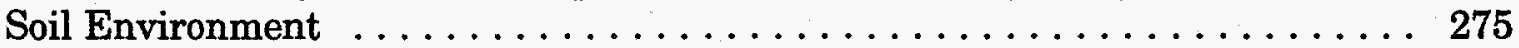

J.7 Relative Stability of Various Plutonium Solids in an Oxidizing

Soil Environment, $\mathrm{pF}^{-}=3.5$, with Phosphate Levels in Equilibrium

with Variscite and Gibbsite, Dicalcium Phosphate Dihydrate,

and Octacalcium Phosphate

J.8 Relative Stability of Various Ruthenium Solids in an Oxidizing Soil

Environment

J.9 Relative Stability of Various Strontium Solids at $\mathrm{pCa}^{2+}=\mathrm{pSO}_{4}{ }^{2-}=2.5$, $\mathrm{pH}_{4} \mathrm{SiO}_{4}=3.1$, and $\mathrm{pCO}_{2(\mathrm{~g})}=1.52$ atm in Equilibrium with Variscite and Gibbsite, Dicalcium Phosphate Dihydrate, and Octacalcium Phosphate

J.10 Relative Stability of Various Thorium Solids in Equilibrium with Variscite and Gibbsite, Dicalcium Phosphate Dihydrate, and Octacalcium Phosphate

J.11 Relative Stability of Various Uranium Solids in an Oxidizing

Soil Environment with Phosphate Levels in Equilibrium

with Variscite and Gibbsite

K.1 Movement of Contaminated Water in the Horizontal Plane . . . . . . . . . 293

L.1 Absolute Humidity by Geographical Region . . . . . . . . . . . . . . . 310 


\section{FIGURES (Cont.)}

4.31 Typical Table of Contents for Health Risk Report $\ldots \ldots \ldots \ldots \ldots \ldots$

4.32 Typical Table of Contents for Radionuclide Concentration Report $\ldots \ldots$. . . 92

4.33 Graphics Selection Menu $\ldots \ldots \ldots \ldots \ldots \ldots \ldots \ldots \ldots \ldots \ldots \ldots \ldots$

4.34 Reset Colors Submenu $\ldots \ldots \ldots \ldots \ldots \ldots \ldots \ldots \ldots$

4.35 Dose Factors Submenu $\ldots \ldots \ldots \ldots \ldots \ldots \ldots \ldots \ldots$

4.36 Input Form A-1 for Ground External Gamma Effective Dose

Conversion Factors $\ldots \ldots \ldots \ldots \ldots \ldots \ldots \ldots \ldots \ldots \ldots \ldots$

4.37 Input Form A-3 for Depth Factors for External Gamma

Radiation from Ground . . . . . . . . . . . . . . . . . . . . . 102

4.38 Input Form B-1 for Inhalation Effective Dose Conversion Factors . . . . . . 102

4.39 Input Form D-1 for Ingestion Effective Dose Conversion Factors . . . . . . . . 104

4.40 Input Form D-34 for Food Transfer Factors $\ldots \ldots \ldots \ldots \ldots \ldots$

4.41 Input Form D-5 for Bioaccumulation Factors for Aquatic Foods $\ldots \ldots$

4.42 Input Form SF for Principal Radionuclide Slope Factors . . . . . . . . . . 107

4.43 Input Form $\mathrm{RN}$ for Radon Slope Factors $\ldots \ldots \ldots \ldots \ldots \ldots$

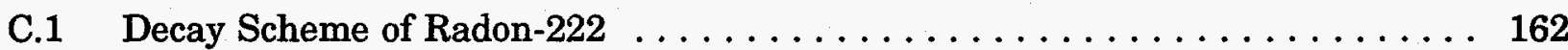

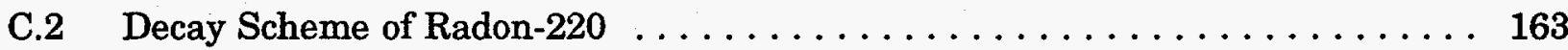

E.1 Dilution of Contaminated Inflow by Uncontaminated Inflow in the Nondispersion Model for a Well Adjacent to the Contaminated Area . . . . . . 212

H.1 Flow Diagram for the Iterative Calculations $\ldots \ldots \ldots \ldots \ldots \ldots$

J.1 Relative Stability of Various Americium Solids in an Oxidizing Soil Environment $\ldots \ldots \ldots \ldots \ldots \ldots \ldots \ldots \ldots \ldots \ldots \ldots \ldots$

J.2 Activity of $\mathrm{SbO}^{+}$in Equilibrium with Various Antimony Solids in an Oxidizing Soil Environment $\ldots \ldots \ldots \ldots \ldots \ldots \ldots \ldots \ldots \ldots$

J.3 Activity of $\mathrm{Ce}^{3+}$ in Equilibrium with Phosphate Levels from Variscite and Gibbsite, Dicalcium Phosphate Dihydrate, and Octacalcium Phosphate 


\section{FOREWORD}

In 1989, a manual for implementing the U.S. Department of Energy's (DOE's) residual radioactive material guidelines ${ }^{*}$ was developed, and the dose assessment methodology recommended for use in deriving site-specific soil guidelines was coded in a microcomputer program called RESRAD. Since then, the manual and the code have been used widely by DOE and its contractors and, to some degree outside DOE, by the U.S. Nuclear Regulatory Commission and licensing states. Comments received from users and new features have been incorporated into the code (Version 5.0). These improvements serve to ease the user's interaction with the code while increasing RESRAD's capability and flexibility.

One of the most substantial improvements to RESRAD has been the addition of graphical output. This option is accessible from within or outside the RESRAD program and can be used to display computational results and any sensitivity analyses that have been requested. This option can generate publication-quality hard copy output from several devices, including dot-matrix printers, laser printers, and plotters.

The user interface to RESRAD has been expanded and redesigned to simplify management of the user's RESRAD work. This improvement has been accomplished by introducing a menu system from which the user can access the data input screens, run the RESRAD calculations, and view the output. The menu system also provides options for suppressing one or more of the nine exposure pathways calculated by RESRAD. Two of the nine exposure pathways are new with Version 5.0: radon inhalation and soil ingestion. Suppressing pathways not only decreases the calculation time but also decreases the number of input parameters that the user must consider. A feature of RESRAD 5.0 blanks out these nonapplicable parameters to simplify data input for the user.

Further improvements to RESRAD include the ability to run sensitivity analyses on most RESRAD parameters, optional input of groundwater radionuclide concentrations, optional user-specified dose factors and transfer factors, an improved groundwater transport model, optional input of a solubility constant, special models for tritium and carbon-14,

\footnotetext{
* The DOE guidelines were incorporated into DOE Order 5400.5 in February 1990 and were included in proposed Title 10, Part 834 of the Code of Federal Regulations (March 1993).
} 
calculation of cancer incidence risks, and context-specific help files. Parameters for sensitivity analysis can be selected directly from the data input forms, and the analyses are performed automatically during RESRAD's calculational phase. The input of the groundwater radionuclide concentration is an option that can be used to derive distribution coefficients for radionuclides on the basis of site specifications. User-specified dose factors (i.e., effective dose conversion, food/soil transfer, and aquatic bioaccumulation factors) can be used to override the RESRAD defaults, which are always the most conservative values if multiple choices exist. An improved groundwater transport model that calculates parent and progeny nuclide concentrations in groundwater and surface water has been developed and incorporated into RESRAD 5.0. Users now have the option of inputting a solubility constant for calculating the leaching of radionuclides from the contaminated zone. Improved models have been formulated for tritium and carbon-14 because of their special transport mechanisms in the environment. In addition to the calculation of dose, slope factors can be used to calculate excess cancer incidence risk. Finally, context-specific help files provide information about every data input parameter accessible to the user. These help files for individual parameters are in addition to the expanded general help files that provide useful keystroke and background information to the user at any time.

The calculational phase of RESRAD Version 5.0 also reflects many improvements made since the last version was released in 1989. These include the addition of 27 new radionuclides: sodium-22, aluminum-26, chlorine-36, potassium-40, calcium-41, manganese-54, cobalt-57, zinc-65, 'germanium-68, ruthenium-106, silver-108m, silver-110m, cadmium-109, antimony-125, cesium-134, cerium-144, promethium-147, samarium-147, gadolinium-152, gadolinium-153, europium-155, gold-195, thallium-204, bismuth-207, plutonium-244, curium-248, and californium-252. The cutoff half-life for associated radionuclides has been reduced to six months. The output reports generated by RESRAD have been redesigned and expanded in Version 5.0 .

Some improvements to RESRAD have not been confined to the actual code. For example, an interactive installation program to simplify and automate the installation procedure has been designed. Also, the FORMPACK library has undergone significant modifications. These modifications include the use of a mouse with menus, the ability to display multiple menus on one screen, the capability of reading imbedded screen files, the 
ability to read data in a Name List format, and the support of far-string memory allocation. This latter modification has resulted in an increase in the number of parameters available for calculations in RESRAD and in the variety of options available to the user. Furthermore, in order to take advantage of the 32 bit chips in 386 and 486 computers, the RESRAD FORTRAN programs are compiled by using the LAHEY F77L/EM32 compiler. The old 16-bit compiler, LAHEY F77L, is still used for 286 and 8088 computers. 


\section{ACKNOWLEDGMENTS}

Since the first release of the RESRAD code in 1989, the authors have received many constructive comments and suggestions from RESRAD users and the scientific community. These comments and suggestions have resulted in a much-improved, more realistic, and more user-friendly computer program - RESRAD, Version 5.0. The authors would like to thank Tom Gerusky, Don Mackenzie, Ken Duval, and Randy Harris of the U.S. Department of Energy and John Russell of Booz-Allen and Hamilton for reviewing this document; Robert E. Baker for providing comments on the ALARA portions of the report; Bill Lahs of the U.S. Nuclear Regulatory Commission for providing comments that resulted in a more realistic groundwater pathway model; Steve M. Dean of the U.S. Environmental Protection Agency for providing a machine-readable copy of the HEAST database; and S.Y. Chen of Argonne National Laboratory for providing the SOLD routine and constant interest and encouragement. Finally, we thank Pat Hollopeter for editorial assistance and the Information and Publishing Division Document Processing Center for document preparation. 


\section{AVAILABILITY OF COMPUTER SOFTWARE}

Electronic copies of the software described in this publication will be distributed with appropriate restrictions by:

Energy Science and Technology Software Center

P.O. Box 1020

Oak Ridge, Tennessee $37831-1020$

For information regarding requests for software, call (615) 576-2606. 


\section{NOTATION}

The following is a list of the acronyms, initialisms, and abbreviations (including units

of measure) used in this document.

\section{ACRONYMS, INITIALISMS, AND ABBREVIATIONS}

$\begin{array}{ll}\text { ALARA } & \text { as low as reasonably achievable } \\ \text { AMAD } & \text { activity median aerodynamic diameter } \\ \text { ASCII } & \text { American Standard Code for Information Interchange } \\ \text { BRC } & \text { below regulatory concern } \\ \text { CFR } & \text { Code of Federal Regulations } \\ \text { CGA } & \text { color graphics adapter } \\ \text { DCF } & \text { dose conversion factor } \\ \text { DCPD } & \text { dicalcium phosphate dihydrate } \\ \text { DE } & \text { dose/exposure-parameter } \\ \text { DF } & \text { dietary factor } \\ \text { DL } & \text { dilution length } \\ \text { DOE } & \text { U.S. Department of Energy } \\ \text { DOS } & \text { disk operating system } \\ \text { DSR } & \text { dose/source ratio } \\ \text { EDE } & \text { effective dose equivalent } \\ \text { EGA } & \text { enhanced graphics adapter } \\ \text { EPA } & \text { U.S. Environmental Protection Agency } \\ \text { ES } & \text { exposure parameter/source concentration } \\ \text { ETF } & \text { environmental transport factor } \\ \text { FS } & \text { shape factor } \\ \text { FUSRAP } & \text { Formerly Utilized Sites Remedial Action Program } \\ \text { G } & \text { gibbsite } \\ \text { GI } & \text { gastrointestinal } \\ \text { GM } & \text { geometric mean } \\ \text { GSD } & \text { geometric standard deviation } \\ \text { GTCR } & \text { General Group Tracer Transfer Concentration Calculation code } \\ \text { ICRP } & \text { International Commission on Radiological Protection } \\ \text { LLD } & \text { lower limit of detection } \\ \text { MB } & \text { mass-balance (model), megabyte } \\ \text { NCRP } & \text { National Council on Radiation Protection and Measurements } \\ \text { ND } & \text { nondispersion (model) } \\ \text { NRC } & \text { U.S. Nuclear Regulatory Commission } \\ \text { OCP } & \text { octacalcium phosphate } \\ \text { ORNL } & \text { Oak Ridge National Laboratory } \\ \text { PC } & \text { personal computer } \\ \text { SF } & \text { source factor } \\ \text { TDS } & \text { total dissolved solids } \\ \text { USLE } & \text { Universal Soil Loss Equation } \\ & \end{array}$



$\mathrm{V}$
variscite
VGA video graphics array

\section{UNITS OF MEASURE}

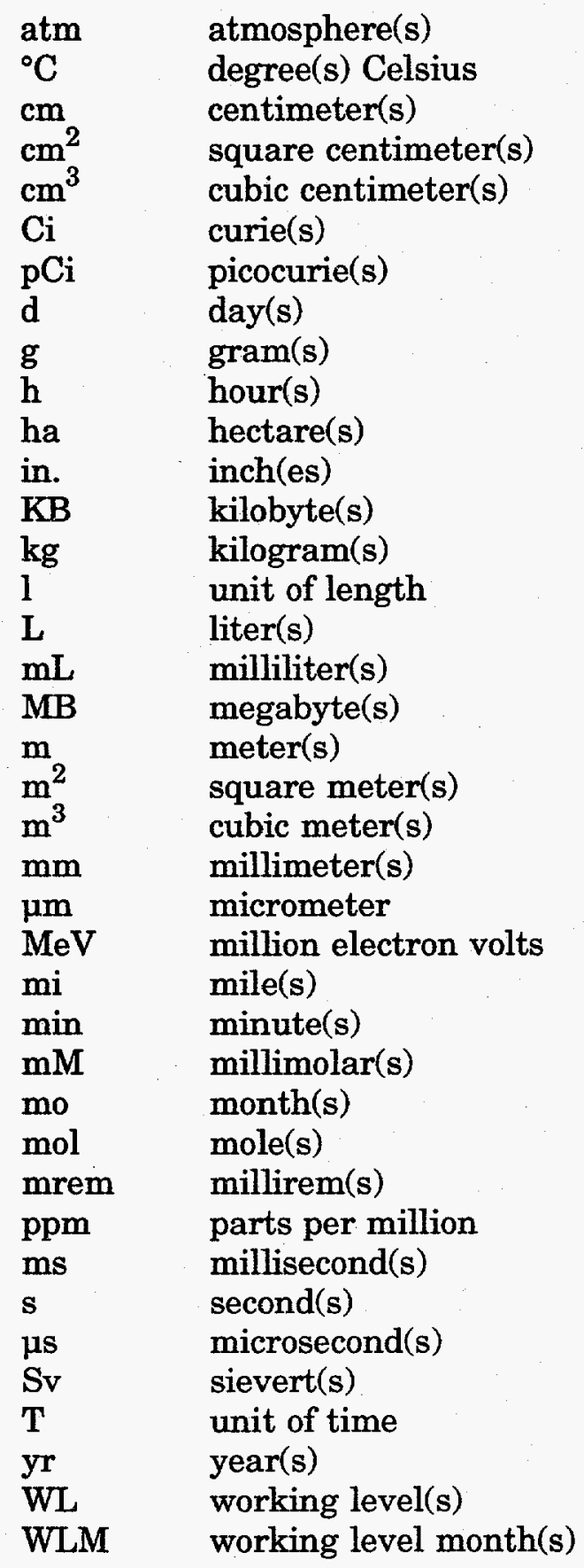




\title{
MANUAL FOR IMPLEMENTING RESIDUAL RADIOACTIVE MATERIAL GUIDELINES USING RESRAD, VERSION 5.0
}

by

C. Yu, A.J. Zielen, J.-J. Cheng, Y.C. Yuan, L.G. Jones, D.J. LePoire, Y.Y. Wang, C.O. Loureiro, E. Gnanapragasam, E. Faillace, A. Wallo III, W.A. Williams, and H. Peterson

\begin{abstract}
This manual presents information for implementing U.S. Department of Energy (DOE) guidelines for residual radioactive material. It describes the analysis and models used to derive site-specific guidelines for allowable residual concentrations of radionuclides in soil and the design and use of the RESRAD computer code for calculating doses, risks, and guideline values. It also describes procedures for implementing DOE policy for reducing residual radioactivity to levels that are as low as reasonably achievable. Two new pathways, radon inhalation and soil ingestion, have been added to RESRAD. Twenty-seven new radionuclides have also been added, and the cutoff half-life for associated radionuclides has been reduced to six months. Other major improvements to the RESRAD code include the ability to run sensitivity analyses, the addition of graphical output, user-specified dose factors, updated databases, an improved groundwater transport model, optional input of a groundwater concentration and a solubility constant, special models for tritium and carbon-14, calculation of cancer incidence risk, and the use of a mouse with menus.
\end{abstract}




\section{INTRODUCTION}

This manual presents information for implementing U.S. Department of Energy (DOE) requirements for residual radioactive material. These requirements are described in Chapter 4 of DOE Order 5400.5 (DOE 1990) and are referred to herein as the "DOE guidelines." This manual describes the analysis and models used to derive site-specific guidelines for allowable residual concentrations of radionuclides in soil and the design and use of the RESRAD computer code for calculating doses, risks, and guideline values. It also describes procedures for implementing DOE policy for reducing residual radioactive material to levels that are as low as reasonably achievable (ALARA) (DOE 1991).

A guideline is defined as a radionuclide concentration or a level of radiation or radioactivity that, given appropriate use scenarios and site parameters, will reasonably ensure that individual dose limits and or constraints will be achieved. Guidelines are expressed as (1) concentrations of residual radionuclides in soil, (2) concentrations of airborne radon decay products, (3) levels of external gamma radiation, (4) levels of radioactivity from surface contamination, and (5) concentrations of residual radionuclides in air and water. Soil is defined as unconsolidated earth material, including rubble and debris that may be present in earth material. Generic guidelines for thorium and radium in soil, airborne radon decay products, external gamma radiation, surface contamination, and residual radionuclides in air and water are specified in the DOE directives.

U.S. Department of Energy Order 5400.5 (DOE 1990) requires that guidelines for other radionuclides be derived on a site-specific basis and that authorized limits for control of residual radioactive material be developed through the ALARA process. Radium and thorium concentrations should also be as far below the generic limits as is practicable. The use of RESRAD is recommended for completing pathway analyses necessary to support guideline development and the ALARA evaluation; Section 4 of the manual is a user's guide for RESRAD.

To complete an ALARA evaluation and select the authorized limits for a site, a number of alternative remedial actions should be evaluated (including no action). U.S. Department of Energy goals are to reduce potential radiation doses due to residual radioactive material to levels that are as near background as is practicable. While it is not 
possible to reduce residual radioactive material levels to background in most cases, remedial actions (including necessary controls) should reduce levels such that potential doses under "actual" or "likely use" conditions are a small fraction of the primary dose limits. While the pathway analyses conducted by using RESRAD should consider and evaluate viable alternative remedial measures that reduce doses to a member of the general public from $100 \mathrm{mrem}$ in a year to a few mrem or less, the $100 \mathrm{mrem}$ in a year limit is for all pathways and sources combined (excluding background and medical exposures). Therefore, in order to comply with DOE Order 5400.5 requirements, potential doses from residual radioactive material must be well below the primary dose limit. Consistent with the recommendations of the International Commission on Radiological Protection (ICRP) regarding the establishment of dose constraints that are lower than the $100 \mathrm{mrem}$ in a year dose limit, DOE recommends that $30 \mathrm{mrem}$ in a year be generally applied as a constraint for dose to any individual under the actual use or likely future use scenarios. That is, remedial measures selected through the ALARA process must be sufficiently protective to ensure that likely potential doses will be less than 30 mrem from a year of exposure.

The radiation dose is defined here as the effective dose equivalent (EDE) from external radiation plus the committed effective dose equivalent from internal radiation (ICRP 1984, Section 2.1). The critical population group is a relatively small, homogeneous group that is representative of those individuals in the population expected to potentially receive the largest radiation dose. An example of the critical population group is a family that establishes residence on a site after the site has been released for use without radiological restrictions. Other examples of the critical population group include industrial workers and recreationists. Section 2.4 provides a detailed discussion of the critical population group.

The controlling principles for all guidelines are (1) the annual radiation dose received by a member of the critical population group from residual radioactive material - predicted by a realistic but reasonably conservative analysis of the actual or likely future use of the site and calculated as the EDE - should not exceed the dose constraint of $30 \mathrm{mrem}$ in a year and (2) doses should be as low as is reasonably achievable giving due consideration to health and environmental impacts, economics, cultural and natural resources, and other appropriate factors. As noted, the final authorized limits should be based on a realistic assessment of 
future use of the subject property, but they should be sufficiently protective to ensure that the other less likely but plausible use scenarios will not cause potential doses to exceed 100 mrem in a year. The worst-case use scenario is typically the family-farm scenario. Where this scenario is not realistic but is plausible, it can generally be assumed to be the most restrictive use and therefore may be used to demonstrate that the potential uses for all plausible scenarios will not exceed the 100 mrem in a year dose limit. In cases where the family-farm scenario is the likely future use, the 30 mrem in a year constraint should be used in developing the guideline values.

All significant exposure pathways for the critical population group must be considered in deriving soil guidelines. These pathways include the following:

- Direct exposure to external radiation from the contaminated soil material;

- Internal dose from inhalation of airborne radionuclides, including radon progeny; and

- Internal dose from ingestion of

- Plant foods grown in the contaminated soil and irrigated with contaminated water,

- Meat and milk from livestock fed with contaminated fodder and water,

- Drinking water from a contaminated well or pond,

- Fish from a contaminated pond, and

- Contaminated soil.

These exposure pathways are illustrated in Figure 1.1. Detailed discussions of the exposure pathways considered in the RESRAD code are presented in Section 2.

The primary purpose of this manual is to provide guidance for use of RESRAD in estimating individual dose resulting from possible reuse of property containing residual radioactive material. Collective dose, however, is an important factor in assessing potential impacts and in selecting the optimum remedial action and authorized limits through the 


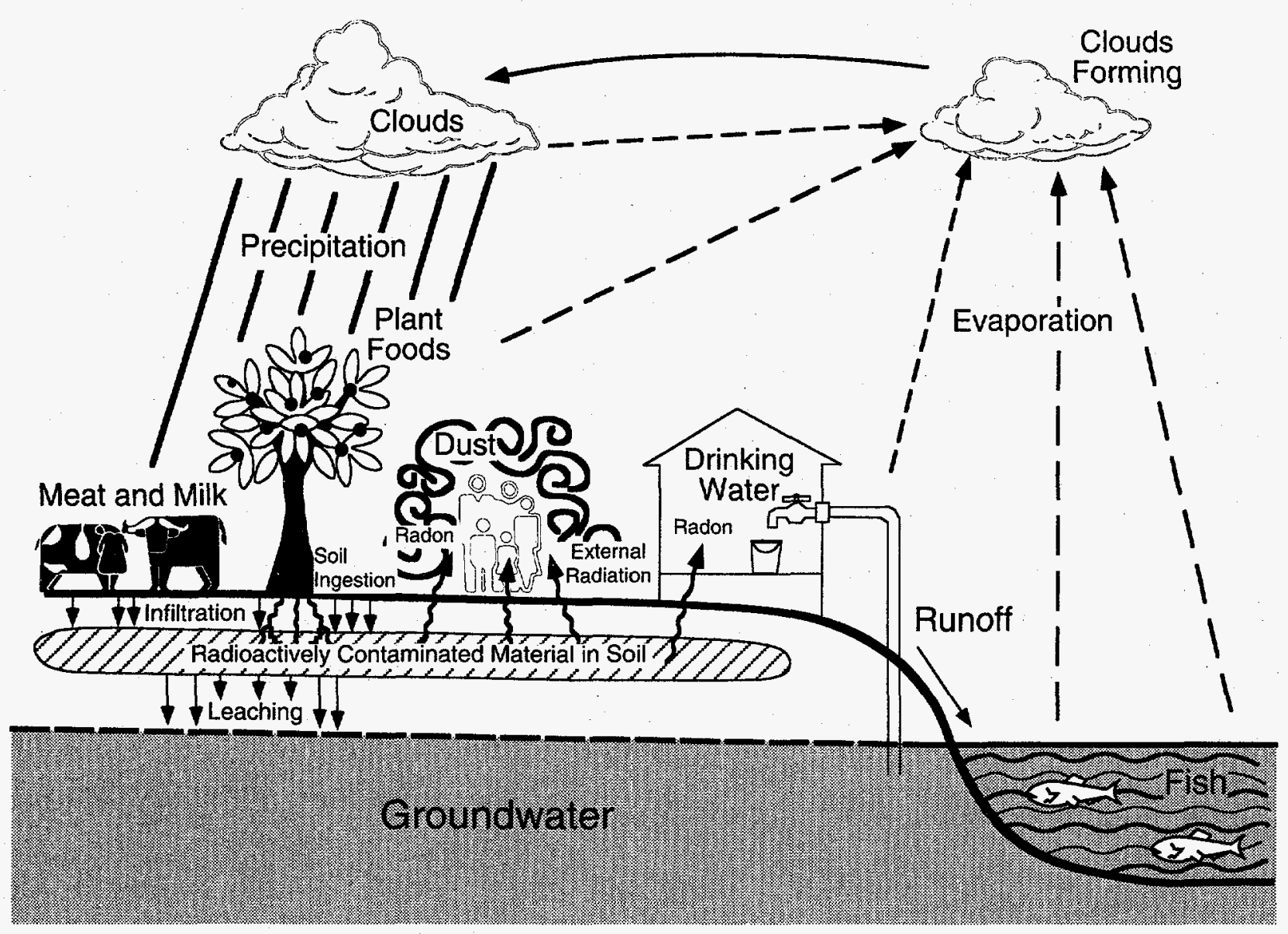

FIGURE 1.1 Exposure Pathways Considered in RESRAD

ALARA process. Estimates of collective dose from current and future use of the site can be made with RESRAD by applying the appropriate site-specific parameters and integrating over the appropriate site occupancy. Calculation of the potential off-site collective dose will require extending the RESRAD methodology and adding calculations. Appendix $\mathrm{K}$ presents alternative methodologies for calculating off-site doses from the air and water pathways.

In addition to requiring that authorized limits be selected such that plausible doses are less than the primary dose limit and estimated doses associated with actual or likely use scenarios be less than the 30 mrem in a year dose constraint, DOE requires as a matter of policy that the ALARA process be applied. Socioeconomic considerations, as well as technical feasibility, are taken into account in implementing this policy. The ALARA requirements apply to the release of properties for use with or without restrictions and to the management 
of sites that cannot be released because it is not practicable to reduce the residual radioactivity below authorized limits.

Models for deriving soil concentration guidelines from dose limits are simplified representations of complex processes. It is not feasible to obtain sufficient data to fully or accurately characterize transport and exposure processes. Similarly, it is not possible to predict future conditions with certainty. Consequently, there will be uncertainties in the derived guideline values. The built-in sensitivity analysis capability in RESRAD 5.0 can be used to study the sensitivity of input parameters and the uncertainty of results. The sensitivity information on input parameters can be used to set priorities for the collection of data for a particular site (Cheng et al. 1991). The models and input parameters described in this manual and incorporated into RESRAD have been chosen so as to be realistic but reasonably conservative, and the calculated doses corresponding to guideline values of the radionuclide concentrations are expected to be reasonably conservative estimates (overestimates) of the actual doses. The methodology for collecting data for input into RESRAD and the ranges and typical values of input parameters are discussed in detail in a separate document (Yu et al. 1993).

The derivation of guideline values for radionuclide concentrations in soil is based on a pathway analysis method known as the concentration factor method (U.S. Nuclear Regulatory Commission [NRC] 1977; ICRP 1979-1982; Till and Meyer 1983; National Council on Radiation Protection and Measurements [NCRP] 1984). With this method, the relation between radionuclide concentrations in soil and the dose to a member of a critical population group is expressed as a sum of the products of "pathway factors." Pathway factors correspond to pathway segments connecting compartments in models of the environment between which radionuclides can be transported or radiation transmitted. Most pathway factors are assumed to be steady-state ratios of concentrations in adjoining compartments. Some are factors for conversion from a radionuclide concentration to a radiation level or radiation dose; others are use and occupancy factors that affect exposure. Each term in the sum corresponds to a pathway of connected segments. A pathway product or pathway factor may be added, deleted, or replaced, in most cases, without affecting the other pathways or pathway factors. This structuring facilitates the use of alternative models for different conditions or transport processes and the incorporation of additional pathways. Thus, in most cases, RESRAD can 
be easily modified or tailored to model any given situation by merely adding or replacing factors or terms in the pathway sum.

This report only covers procedures for deriving site-specific guidelines for radionuclide concentrations in soil and guidelines for implementing DOE's ALARA process. Problems associated with procedures for collecting and interpreting field measurements of residual radioactive material and protocols for determining whether the guidelines have been met are not covered. Guidance on these topics may be found in the data collection handbook for establishing residual radioactive material guidelines ( $Y u$ et al. 1993) and the Environmental Implementation Guide for Radiological Survey Procedures (DOE 1992a). Other sources of supporting information include the summary protocol for identification, characterization, designation, remedial action, and certification of Formerly Utilized Sites Remedial Action Program (FUSRAP) sites (DOE 1984, 1986a, 1986b) and the procedures manual for remedial action survey and certification activities (Oak Ridge National Laboratory [ORNL] 1982).

The information presented in this manual is organized as follows:

- Pathways and scenarios by which a member of the critical population group can be exposed to radiation - Section 2;

- Derivation and application of soil guidelines - Section 3;

- Description of the RESRAD code and instructions for its use Section 4;

- ALARA procedures and general guidelines for implementing the ALARA process - Section 5; and

- Models, formulas, and data used in RESRAD to calculate the pathway factors - Appendixes A through $M$. 


\section{PATHWAY ANALYSIS}

The pathway analysis for deriving soil concentration guidelines from a dose limit has four parts: (1) source analysis, (2) environmental transport analysis, (3) dose/exposure analysis, and (4) scenario analysis.

Source analysis addresses the problem of deriving the source terms that determine the rate at which residual radioactivity is released into the environment. This rate is determined by the geometry of the contaminated zone, the concentrations of the radionuclides present, the ingrowth and decay rates of the radionuclides, and the removal rate by erosion and leaching. Qualitative aspects of these topics are discussed in Section 2.1; quantitative aspects are covered in Section 3 and the appendixes.

Environmental transport analysis addresses the problems of (1) identifying environmental pathways by which radionuclides can migrate from the source to a human exposure location and (2) determining the migration rate along these pathways. The significant environmental pathways are identified and described in Section 2.2. The derivation of environmental transport factors that characterize the rate of migration is covered in Section 3 and the appendixes.

Dose/exposure analysis addresses the problem of the derivation of dose conversion factors for the radiation dose that will be incurred by exposure to ionizing radiation. This problem is discussed in Section 2.3. Appendixes A, B, and D include tabulations of the dose conversion factors used to determine the EDE incurred by exposure to external radiation or to internal radiation from inhalation or ingestion of radionuclides.

The parameters that control the rate of radionuclide release into the environment and the severity and duration of human exposure at a given location are determined by patterns of human activity referred to as exposure scenarios. The credible scenarios that are likely to result in the largest exposure to individuals are discussed in Section 2.4. 


\subsection{SOURCE TERMS}

\subsubsection{Geometry of the Contaminated Zone}

A contaminated zone, defined as a belowground region within which radionuclides are present in above-background concentrations, is the common source term and starting point for all pathways. The derivation of soil guidelines is based on idealized contaminated regions of cylindrical shape, as shown in Figure 2.1, within which radionuclides are assumed to be uniformly distributed. A shape factor, which equals 1 if the actual contaminated zone is a circular cylinder and less than or equal to 1 if it is irregularly shaped, is used to correct for the difference between dose estimates for the actual and idealized areas. The shape factor is used for the external radiation exposure pathway (see Appendix $A$ ). The cover depth $\left(C_{d}\right)$ corresponds to the distance to the uppermost contaminated soil sample; the cover depth plus contaminated zone thickness $\left(\mathrm{C}_{d}+\mathrm{T}\right)$ corresponds to the distance to the lowest contaminated soil sample.

If radionuclide distributions are approximately uniform throughout the contaminated region, the source geometry is a single cylinder that specifies a homogeneous contaminated zone. If the distributions are not approximately uniform, the source geometry consists of two or more cylinders that specify an inhomogeneous contaminated zone. Limiting radionuclide concentrations, referred to as single-radionuclide soil guidelines, are derived for each

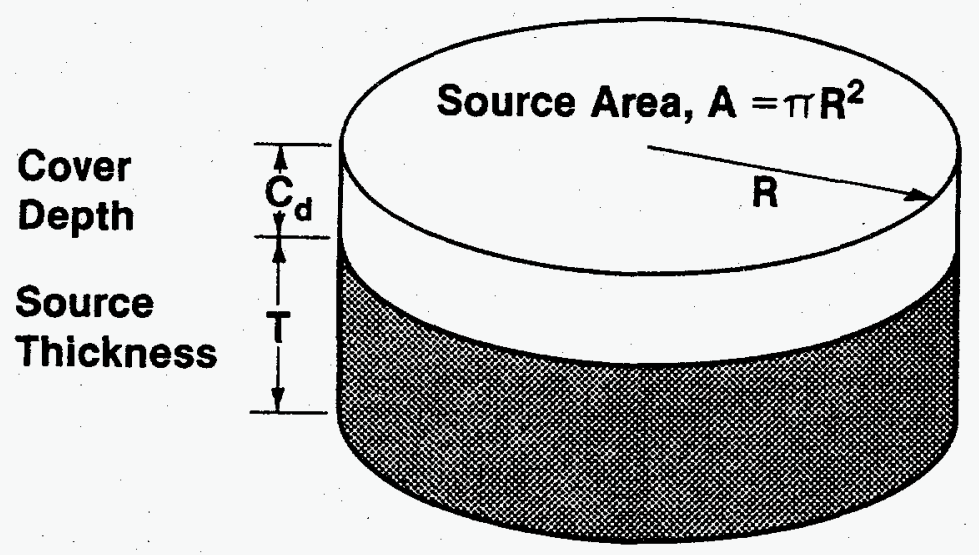

FIGURE 2.1 Geometry of Idealized Contaminated Zone 
cylindrical volume. The manner in which the cylindrical volumes and soil guidelines are determined is described in Sections 3.1 and 3.3.

\subsubsection{Time Dependence}

The time dependence of the annual dose incurred by a member of the critical population group is controlled by (1) the rate at which radionuclides are leached from the contaminated zone, (2) the rate of ingrowth and decay of the radionuclides, (3) the rate of erosion of the cover and contaminated soil material, and (4) the rate of contaminant transport through the environmental pathways. The first and third processes occur within, or primarily within, the contaminated zone; the last process occurs outside the contaminated zone; and the second process occurs in all zones. The models used to account for the time dependence of different transport and erosion processes are described in Appendixes A through $\mathrm{F}$ and $\mathrm{H}$ through $\mathrm{K}$; the models used to account for the rate of ingrowth and decay and leaching are described in Appendixes G and I.

\subsubsection{Redistribution}

A contaminated zone is treated as a single homogeneous or inhomogeneous source of changing thickness and radionuclide concentrations as the result of leaching, erosion, and ingrowth and decay. Erosion or human activities (such as excavation for a building being erected in the contaminated zone) can result in redistribution of the contaminated soil that creates new contaminated zones. Such zones are not explicitly incorporated into RESRAD. If the topography and circumstances are such that redistribution is likely to occur, the new contaminated zone should be taken into account. This can be done by estimating the initial area and thickness of the new contaminated zone and then deriving its contribution to the soil guidelines in the same manner as for the original contaminated zone (see Section 2.3).

Radionuclides can also be redistributed following the use of irrigation water that has been contaminated by radionuclides leached from the original contaminated zone. This contribution is taken into account in the derivation of the contribution from food pathways. 


\subsection{PATHWAYS}

\subsubsection{Pathway Identification}

Potential pathways are identified in Table 2.1. The three major headings correspond to the three exposure pathways by which radionuclides can enter the body. In the first pathway, exposure is by external radiation from radionuclides outside the body. In the second and third pathways, exposure is by internal radiation from radionuclides that are

\section{TABLE 2.1 Potential Pathways}

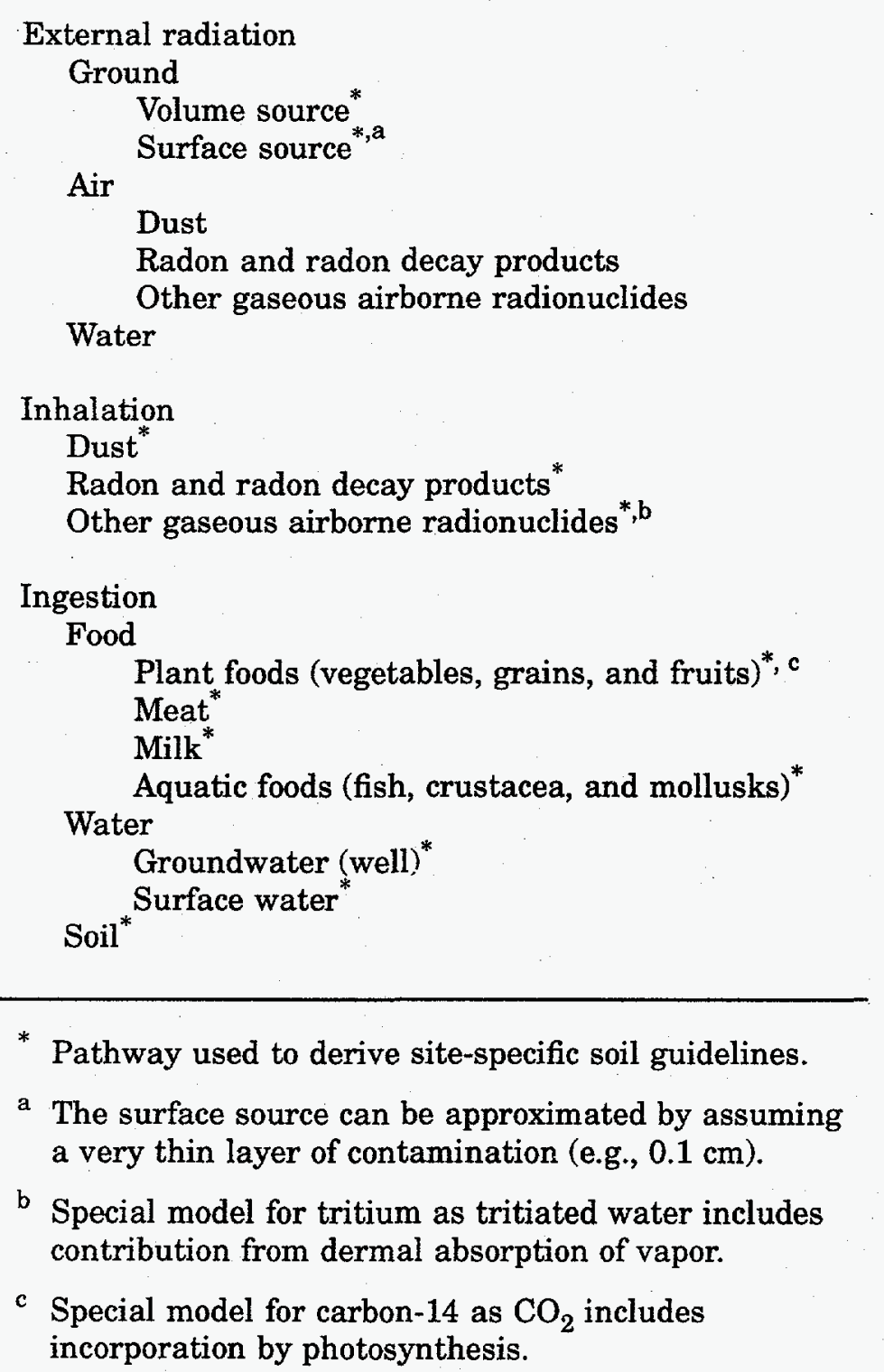


inhaled or ingested. These three types of exposure correspond to the three kinds of dose conversion factors discussed in Section 2.3.

For each exposure pathway, radionuclides can migrate from a source to a human exposure location by many environmental pathways. The major categories of environmental pathways are listed in Table 2.1. The items listed should be regarded as environmental pathway categories rather than individual pathways because many of the items can correspond to more than one pathway and some of the items can occur as segments in more than one pathway. For example, there are many different plant food pathways, and contaminated groundwater can contribute to the human drinking water pathway and also to several food pathways if contaminated water is used to irrigate crops or water livestock.

Major pathways used to derive site-specific soil guidelines in the RESRAD code are identified in Table 2.1 by an asterisk and are illustrated in Figure 2.2. Minor pathways for on-site exposure are not taken into account in deriving soil guidelines because the dose contribution from these pathways is expected to be insignificant. External radiation from a surface layer formed by redeposition of airborne radionuclides carried by the wind from an exposed contaminated zone is expected to be insignificant compared with external radiation from the residual radioactive material in its original location. External radiation from contaminated water is expected to be insignificant compared with internal exposure from radionuclides ingested in drinking water. The external radiation dose from airborne dust is much smaller than the inhalation dose from dust (by a factor of 100 or more for radionuclides in the uranium-238 series [Gilbert et al. 1983, Tables 5.6-5.8]). The external radiation dose from airborne radon decay products is negligible compared with (1) the internal inhalation dose to the lungs, (2) the external radiation dose from the parent radium in the soil, or (3) the internal radiation dose from ingestion of plant foods grown in the radium-contaminated soil (Gilbert et al. 1983). The transport and dosimetry for gaseous airborne radionuclides other than radon decay products (e.g., carbon-14 occurring in carbon dioxide or tritium occurring in tritiated water vapor) require special consideration (see Appendix L).

\subsubsection{External Radiation Pathways}

Gamma radiation from radionuclides distributed throughout the contaminated zone is the dominant external radiation pathway and the only external radiation pathway taken 


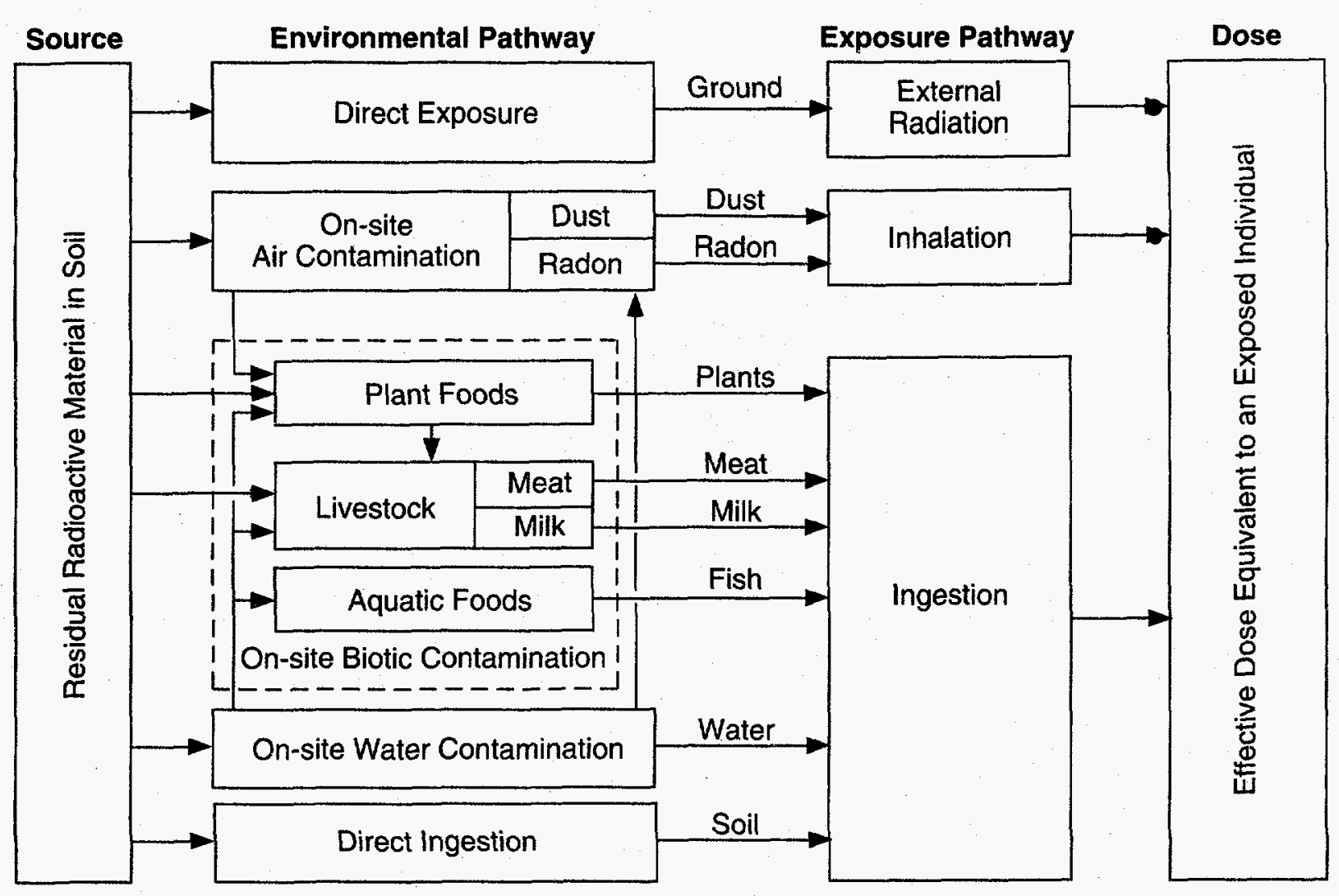

\section{FIGURE 2.2 Schematic Representation of RESRAD Pathways}

into account in calculating soil guidelines. ${ }^{1}$ The dose due to external gamma radiation is first calculated for an individual exposed continuously to radiation from an infinite contaminated zone at a distance of $1 \mathrm{~m}$ from the ground surface. Correction factors are then applied for the finite area and thickness of the contaminated zone, shielding by a cover of uncontaminated soil (if $\mathrm{C}_{\mathrm{d}}>0$; see Figure 2.1), irregular shape, shielding by the floors and walls of a house, and less-than-continuous occupancy. Quantitative details are presented in Section 3 and Appendix A.

\subsubsection{Inhalation Pathways}

Inhalation exposure results primarily from inhalation of radon decay products and contaminated dust. An inhalation pathway consists of two segments: (1) an airborne

1 The dose contribution from electrons (beta particles) is primarily restricted to the skin; the dose contribution from neutron radiation can be significant for some transuranic radionuclides and should be considered if neutron-emitting radionuclides are known to be present. 
exposure segment linking the source (contaminated zone) with the airborne radionuclides at an exposure location and (2) an inhalation segment linking the airborne radionuclides with the exposed individual. The inhalation segment is characterized by an occupancy factor (equivalent fraction of time during which an individual inhales contaminated air) and a factor for the inhalation rate. Numerical values for these factors can be obtained by using well established procedures (ICRP 1975). The airborne exposure pathway segment is the critical segment. It is characterized by the air/soil concentration ratio, which is defined as the ratio of the airborne concentration of a radionuclide at a human exposure location to the concentration in the soil. The air/soil concentration ratio for contaminated dust depends on the complex processes by which soil particles become airborne by resuspension and are transported to an exposure location. This ratio is used in the food chain pathways (for the foliar deposition subpathways) as well as in the inhalation pathways. For the radon pathway, the air/soil concentration ratio is calculated by solving radon diffusion equations for the soil and for the atmosphere.

Modeling the airborne exposure pathway segment consists of two steps: (1) modeling the process by which radionuclides become airborne and (2) modeling the process by which the airborne radionuclides are transported to a human exposure location and diluted before inhalation. The first step gives the ratio of the airborne emission near the source before it is dispersed and diluted to the concentration in the resuspendable layer of dust; the second step gives the ratio of the airborne concentration at the point of exposure to the airborne emission at the source. Quantitative details are presented in Appendix B for dust particles and in Appendix $\mathrm{C}$ for radon and its progeny. A special model for tritium as tritiated water vapor is presented in Appendix L.

\subsubsection{Ingestion Pathways}

\subsubsection{Food Pathways}

Four food pathway categories are considered: plant foods, meat, milk, and aquatic foods. The plant food pathway category is divided into four subcategories corresponding to (1) root uptake from crops grown in the contaminated zone, (2) foliar uptake from contaminated dust deposited on the foliage, (3) root uptake from contaminated irrigation water, and (4) foliar uptake from contaminated irrigation water. A special model for the 
incorporation of carbon-14 in carbon dioxide $\left(\mathrm{CO}_{2}\right)$ as a result of photosynthesis is presented in Appendix L. The plant food pathway subcategories are applicable to livestock fodder; hence, they are applicable to the pathways through which meat and milk become contaminated by ingestion of contaminated fodder. A fifth subcategory for the meat and milk pathways corresponds to ingestion of contaminated water by livestock. A sixth subcategory corresponds to ingestion of contaminated soil by livestock. The aquatic food pathway is for ingestion of fish, crustacea, and mollusks from a nearby pond that has been contaminated by radionuclides that have been leached from the contaminated zone.

The food pathways are activated by scenarios in which crops are grown in or close to the contaminated zone. Plant crops grown in the contaminated zone will be the dominant and most frequent contributor, especially if the crops are irrigated with contaminated water. Contributions from the meat and milk pathways, which involve an additional pathway segment for transfer of radionuclides from fodder or water to the meat or milk, will generally be smaller but not insignificant. Vegetable gardens are common in urban and suburban areas as well as rural areas, whereas raising livestock is generally limited to rural areas. The aquatic food pathway will occur only in areas where the topography and soil characteristics are favorable for building a pond.

The food pathways may be classified as water-independent or water-dependent. The direct root uptake and foliar dust deposition pathways for plant foods, meat, and milk are water-independent, as is the soil intake by livestock, (here water refers to the water that has been contaminated by radionuclides that have been leached from the contaminated zone). Water-dependent pathways are the irrigation water pathway for plant foods, meat, and milk; the livestock water pathway for meat and milk; and the aquatic food pathway. In regions where natural rainfall is the only source of water used in raising crops, the only waterdependent pathways will be the livestock water branch of the meat and milk pathways and the aquatic food pathway. If ditch irrigation is used, the contribution from root uptake of contaminated irrigation water by plant foods and fodder will be added. If overhead irrigation is used, the contribution from the subpathways for foliar and root uptake from irrigation water will be added.

The water-independent pathways are assumed to contribute to the dose as soon as a family establishes a residence and a garden on the site. The time dependence of these 
pathways is determined by the time dependence of the cover, contaminated zone thickness, and the radionuclide concentrations in the contaminated zone. The contribution from water-dependent pathways will be delayed until radionuclides transported by groundwater reach a point of water withdrawal (i.e., well or pond). The time dependence of these pathways is determined by the time dependence of the radionuclide concentrations in the contaminated water, as determined by the hydrological model used for the groundwater pathway segment. A fraction of a radionuclide will have been leached from the root zone before the radionuclide first reaches a point of water withdrawal in above-background concentrations (the breakthrough time); hence, the contributions to the dose from the waterindependent and water-dependent pathways will occur at different times.

After the breakthrough time, the contaminated irrigation water will create a new contaminated zone as it percolates down through the soil. The contribution of this secondary contaminated zone to pathways other than the food pathway is not taken into account because it is assumed to be small compared with the contributions of the food pathway.

Radionuclide transport through the food pathways is determined by the quantities of different foods consumed (dietary factors), the fraction of the diet from foods that are contaminated by radionuclides from the contaminated zone (which is determined by the fraction raised locally and the area of the contaminated zone), the cover depth and contaminated zone thickness relative to the root zone of the plants, the various transfer factors from root or foliage to plants and from fodder or water to meat or milk, and the concentrations of radionuclides in water that has percolated through the contaminated zone. The factors used to take these effects into account are discussed in Section 3 and Appendixes D and E.

\subsubsection{Water Pathway Segments}

A water pathway segment connects the contaminated zone with a point of water withdrawal for drinking or irrigation or with a pond where aquatic foods are raised for human consumption. This segment is characterized by a water/soil concentration ratio for each radionuclide, which is defined as the ratio of the radionuclide concentration in the water at the point of withdrawal or use to the radionuclide concentration in the contaminated zone. Irrigation and drinking water are assumed to be taken from a well or pond. The well is 
assumed to be either in the center of or at the downgradient edge of the contaminated zone (see Appendix E). The pond water is assumed to be contaminated by water that seeps to the surface after percolating through the contaminated zone. Figure 2.3 schematically represents the process by which natural precipitation or irrigation water infiltrates the contaminated zone and transports radionuclides through the unsaturated (vadose) zone and saturated zone (aquifer) to a well or point of seepage into surface water.

\section{Groundwater Pathway Segment}

Two models are used for calculating the water/soil concentration ratio for the groundwater pathway segment: a mass-balance (MB) model and a nondispersion (ND) model. The MB model assumes that all of the radionuclides released annually from the contaminated zone are withdrawn through a well located at the center of the contaminated zone. The ND model assumes that the dispersivity is nil, the vadose zone and aquifer are homogeneous,

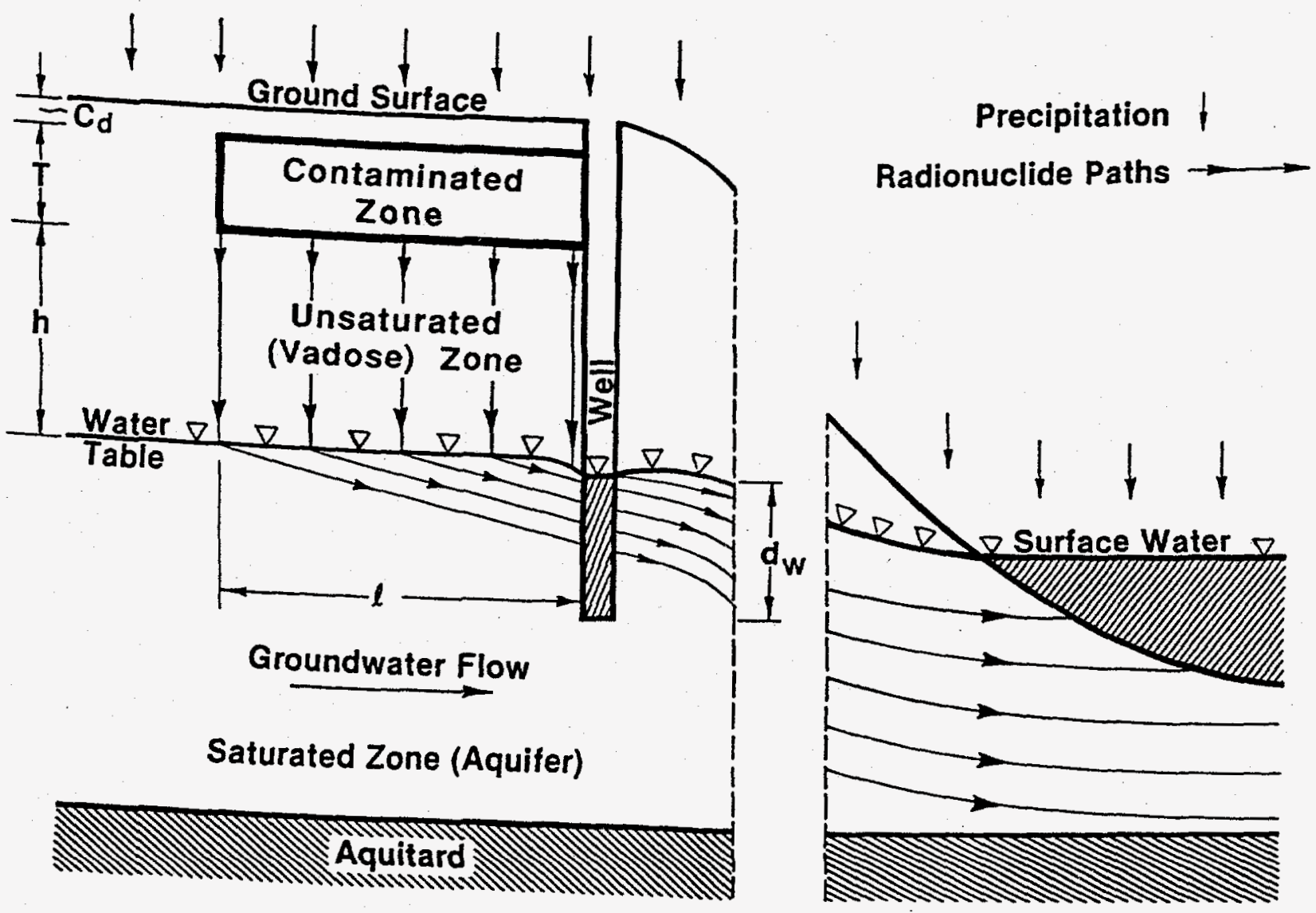

FIGURE 2.3 Schematic Representation of the Water Pathway Segments 
and the well is located at the downgradient edge of the contaminated zone. The MB model is used for smaller contaminated areas (e.g., $1,000 \mathrm{~m}^{2}$ or less), and the ND model can be used for contaminated areas of any size. The MB and ND models are discussed in more detail in Appendix E.

The groundwater pathway segment is analyzed in terms of parameters that are usually used in more sophisticated models. These parameters consist of the breakthrough time, rise time, and dilution factor. The breakthrough time is the time from a radionuclide's release into infiltrating water at the contaminated zone until its detection at the point of withdrawal or use. The rise time is the time from initial detection until the concentration reaches a maximum value. The dilution factor is the steady-state ratio of a radionuclide concentration at the point of withdrawal or use to the concentration of the same radionuclide in infiltrating water as it leaves the contaminated zone. Formulas for calculating the breakthrough times, rise times, and dilution factors for the MB and ND models are given in Section 3 and Appendix E.

The groundwater pathway models implemented in the RESRAD code apply only to situations for which the hydrological strata can reasonably be approximated by a sequence of uniform, horizontal strata. For sites having more complicated strata, such as fracture zones, simple models may be used to provide reasonable estimates if a set of effective hydrogeologic flow parameters are used. However, the accuracy of the results predicted is determined by the accuracy of the effective input parameters used (Yu et al. 1986).

\section{Surface Water Pathway Segment}

The surface water is assumed to be a pond for which (1) the water inflow and outflow are in steady-state equilibrium and (2) the annual inflow of radioactive material into the pond equals the annual quantity of radioactive material leached from the contaminated zone. The dilution factor is assumed to be the ratio of the annual volume of water that infiltrates the contaminated zone to the annual total inflow of water into the pond. If, as also assumed, the infiltrating water flow is vertically downward through the contaminated zone, the surface water dilution factor is given by the ratio of the area of the contaminated zone to the area of the watershed that supplies the pond. Breakthrough time and rise time are assumed to be the same as for an on-site well; no credit is taken for the additional time for radionuclides 
to be transported from the edge of the contaminated zone to the point of seepage. This simplified model will give a conservative estimate of the water/soil concentration ratio for a pond.

\subsubsection{Drinking Water Pathway}

The concentration factor that characterizes the drinking water pathway is obtained by multiplying the water/soil concentration ratio by the annual quantity of contaminated drinking water consumed by an individual. Both well water and surface water can be used for drinking. The fraction of well water blended with or supplemented by surface water is used to calculate the total contribution from groundwater and surface water. The default value for this fraction is unity, that is, $100 \%$ of the drinking water is drawn from a well.

\subsubsection{Soil Ingestion Pathway}

The soil ingestion pathway corresponds to direct ingestion of soil (Healy 1977). This pathway is especially significant for a child afflicted with pica (a compulsive craving for nonfood objects) (U.S. Environmental Protection Agency [EPA] 1984). The dose due to ingestion of soil depends on the amount of soil ingested and the concentration of soil. Quantitative details are presented in Appendix F.

\subsection{DOSE CONVERSION FACTORS}

The distribution of dose within the body depends on the type of radiation involved and the location of the radionuclides emitting the radiation, that is, within or external to the body. The ICRP has provided a useful quantity that, on a risk scale, can be applied to any radiation pattern (ICRP 1977, 1978, 1979-1982, 1991a, 1991b). This quantity, the EDE, is the weighted sum of the dose equivalent in various organs. The weighting factors for the organs are proportional to the potential risk associated with the irradiation of those organs. All dose factors used for deriving soil concentration guidelines are based on the EDE.

Internal radiation doses to organs and tissues of the body are frequently estimated with factors representing the committed dose equivalent ${ }^{2}$ for a unit intake of a radionuclide via inhalation or ingestion. In the case of external radiation, the dose rate in organs depends

2 The committed dose equivalent is the dose equivalent to organs or tissues that will be received by an individual from an intake of radioactive material during the 50-year period following the intake. 
on the concentration of the radionuclide in the environment. These relationships are expressed as dose/exposure ratios called "dose conversion factors (DCFs)." A DCF is the ratio of the committed EDE (for internal exposure) or the EDE rate (for external exposure) to the quantity of a radionuclide inhaled or ingested (for internal exposure) or the concentration of a radionuclide in the air, water, or ground (for external exposure).

\subsubsection{Ingestion and Inhalation}

The radiation dose from inhalation and ingestion of radionuclides has been systematically evaluated by the ICRP (Publication 30; ICRP 1979-1982). This effort was undertaken to compute secondary limits for occupational exposures corresponding to the primary radiation protection guidance of ICRP Publication 26 (ICRP 1977). Dose equivalents in organs or tissues of the body are calculated with models that (1) describe the entrance of materials into the body (respiratory and gastrointestinal [GI] tract) and the deposition and subsequent retention of the radionuclides in body organs (referred to as metabolic models) and (2) estimate the energy deposition in tissues of the body (ICRP 1979-1982). In applying the results of these calculations, some insight into the details of models for the lung and GI tract is required because these models serve as the interface between humans and the environment.

Inhalation of radionuclides attached to airborne particles is a potential route for intake of radionuclides into the body. The ICRP model of the respiratory tract divides the tract into three regions: nasopharyngeal, tracheobronchial, and pulmonary (ICRP 1966). These regions are connected with one another as well as with body fluids and the GI tract. The fraction of inhaled activity deposited in these regions is a function of the size of the airborne particles. The activity median aerodynamic diameter (AMAD) is the measure most widely used to characterize the aerosol. The rate at which the deposited material is removed from the regions is considered to be independent of particle size; the rate is, however, related to the chemical form of the particles. Chemical compounds are assigned to one of three classes to characterize their removal rate from the lung. The inhalation classes are denoted as $\mathrm{D}, \mathrm{W}$, and $\mathrm{Y}$ - corresponding to clearance or removal times from the pulmonary region of the lung on the order of days, weeks, or years, respectively. Details are provided in the ICRP task group's report (ICRP 1966) and subsequent ICRP publications (ICRP 1968, 19791982, 1991a, 1991b). 
A portion of the material initially deposited in the lung enters the GI tract and, of course, ingested radionuclides enter the GI tract directly. The ICRP model of the GI tract divides the tract into four compartments: stomach, small intestine, upper large intestine, and lower large intestine (Eve 1966). Absorption of materials into body fluids is generally considered to occur within the small intestine. The fraction of the ingested material absorbed into body fluids from the tract is denoted as $f_{1}$. The numerical value of $f_{1}$ depends on the chemical form of the ingested material (ICRP 1979-1982).

Radionuclides that enter body fluids from either the lung or the GI tract may be deposited within the various organs of the body. Metabolic processes and radioactive decay reduce the radioactivity in the body. These removal processes are modeled in a rather simple manner in which the organs are represented by a number of mathematical compartments from which the removal rate is directly proportional to the amount of radionuclide present (ICRP 1979-1982). This approach leads to functions involving a sum of exponentials to describe the time course of activity within the body. An exception to this representation occurs for radium, for which the more-detailed metabolic model of ICRP Publication 20 (ICRP 1972) is used.

In calculating the dose from intake of radionuclides into the body, the occurrence of radioactive decay products must be considered. When an atom undergoes radioactive decay, the new atom formed may also be radioactive and thus contribute to the dose. Although these decay products may be treated as independent radionuclides in external exposures, they must be considered in conjunction with the parent in the evaluation of radionuclides inhaled or ingested, because their distribution among the organs of the body depends on the metabolism of the parent (ICRP 1979-1982). Thus, the contributions to dose from decay products formed within the body are included in the DCF for any radionuclide with radioactive decay products.

Factors representing the committed EDE per unit intake of radionuclides are given in Appendix B (Table B.1) for inhalation and Appendix D (Table D.1) for ingestion. These values were taken from a DOE report (DOE 1988a); similar values are given in an EPA report (Eckerman et al. 1988). The DCFs for inhalation of radon and its decay products are discussed in Appendix C. In many instances, several chemical forms have been considered; that is, more than one clearance class (inhalation) or $f_{1}$ (ingestion) value is given for the 
radionuclide. If a user of this manual has information indicating the chemical forms expected in the particular environment being considered, then the appropriate value should be selected after consulting ICRP Publication 30 (ICRP 1979-1982). If no information on the relevant chemical forms is available, then the most conservative value (i.e., the highest estimate of dose) should be used.

Dose conversion factors identified with " $+D$ " in Tables B.1 and D.1 are the sums of DCFs for a principal radionuclide (a radionuclide with a half-life greater than six months) and its associated decay chain (all short-lived decay products of a principal radionuclide down to, but not including, the next principal radionuclide or the final nonradioactive nuclide in the chain). These aggregated DCFs correspond to ingestion or inhalation of the principal radionuclide together with its associated decay product radionuclides, which are assumed to be in secular equilibrium at the time of intake (see Section 3.1 and Table 3.1).

\subsubsection{External Radiation}

Organs of the body may be irradiated by radiation emitted from radionuclides present in the environment. Gamma radiation is the only external radiation taken into account in calculating soil guidelines (see Section 2.2.2). Gamma radiation is of particular concern because this radiation is sufficiently penetrating that the dose at a given location depends on the spatial distribution over considerable distances. In addition, the dose distribution pattern within the body is rather uniform. The actual distribution of dose in the body depends somewhat on the distribution pattern of the radionuclide in the environment. An idealized distribution pattern that is often assumed is a uniform distribution of the radionuclide within an infinite or semi-infinite region. By considering such idealized distributions, DCFs relating the EDE rate to the radionuclide concentration can be tabulated. For other situations, the details of radionuclide distribution must be included in the numerical calculation of dose.

Kocher (1983) has published extensive tabulations of dose-rate factors for radionuclides distributed in air, in water, and on the ground surface. Chen (1991) used the Monte Carlo method to generate dose-rate factors for radionuclides in soil. The methods of Kocher and Sjoreen (1985) and Chen (1991) were used to derive dose-rate factors of radionuclides uniformly distributed in the soil volume. Dose conversion factors for exposure 
from radionuclides in or on the ground are presented in Appendix A (Table A.1). The doserate factors for surface contamination were taken from a DOE report (DOE 1988b).

The DCFs for ground contamination are for exposure at a point $1 \mathrm{~m}$ above the ground. The volume contamination is assumed to be uniformly distributed to an infinite depth and infinite lateral extent. ("Infinite" dimensions reflect consideration of radiation transport and may, in fact, correspond to small physical dimensions. For example, one meter of soil is generally infinite with respect to the dose rate from a photon-emitting radionuclide.) Contributions from all associated radionuclides, which are assumed to be in secular equilibrium with the parent principal radionuclide, are included in the DCFs for principal radionuclides.

Dose conversion factors for radionuclides distributed in the ground depend on the bulk density of the soil $\left(\rho_{b}\right)$. Factors for $\rho_{b}=1.0 \mathrm{~g} / \mathrm{cm}^{3}$ and $\rho_{b}=1.8 \mathrm{~g} / \mathrm{cm}^{3}$ are listed in Table A.1. Values for other soil densities may be obtained by interpolation.

\subsection{EXPOSURE SCENARIOS}

Many parameters that determine the quantity of radionuclides or radiation to which an individual is exposed are determined by exposure scenarios, that is, patterns of human activity that can affect the release of radioactivity from the contaminated zone and the amount of exposure received at the exposure location. In this manual, soil guidelines are based on a family-farm exposure scenario. This scenario includes all environmental pathways for on-site or near-site exposure and is expected to result in the highest predicted lifetime dose. Other scenarios, such as the industrial worker and recreationist, can be taken into account by adjusting the scenario parameters in formulas for calculating the transport of radionuclides through the pathways (see Sections 3 and 4 and Appendixes A through $\mathrm{K}$ ).

In the family-farm scenario, a family is assumed to move onto the site after it has been released for use without radiological restrictions, build a home, and raise crops and livestock for family consumption. Members of the family can incur a radiation dose by (1) direct radiation from radionuclides in the soil, (2) inhalation of resuspended dust (if the contaminated area is exposed at the ground surface), (3) inhalation of radon and its decay products, (4) ingestion of food from crops grown in the contaminated soil, (5) ingestion of milk from livestock raised in the contaminated area, (6) ingestion of meat from livestock raised in 
the contaminated area, (7) ingestion of fish from a nearby pond contaminated by water percolating through the contaminated zone, (8) ingestion of water from a well or pond contaminated by water percolating through the contaminated zone, and (9) ingestion of contaminated soil. The basis for selecting a family-farm scenario for the critical population group is summarized below.

Permanent residents, rather than individuals exposed by activities not associated with residential living, have been chosen as the primary critical population group because the exposure for permanent residents is more likely to be long term and will generally involve exposure by more pathways. The nonresident group most likely to receive significant exposure consists of construction workers. An individual involved in recreational activities (e.g., a baseball field built on the site) will receive a much smaller dose than a permanent resident because the former will spend less time on-site. Scavenging can also occur, although this is less likely considering the lack of economic value of the contaminated material. The exposure of scavengers can reasonably be assumed to be comparable to that of construction workers. The exposure of construction workers or scavengers is unlikely to last longer than a few months and would generally be limited to working hours. The lifetime exposure for construction workers and scavengers is, therefore, unlikely to exceed the lifetime dose for a permanent on-site resident. (The basic dose limit for members of the general public from all sources of radiation [except natural background and radiation received as a patient] is $100 \mathrm{mrem} / \mathrm{yr}$ [DOE 1990, 1992b]. With adequate justification and DOE [EH-1] approval, this limit may be exceeded for a limited period of time [not to exceed a few years], providing that the annual dose is kept below $500 \mathrm{mrem} / \mathrm{yr}$.) Exposure of workers in on-site industrial or commercial buildings can also occur, but this exposure will generally be less than that of residents because the exposure will be limited to working hours and will not include contributions from ingestion of foods grown on-site.

Soil guidelines are based on on-site exposure because on-site residents will receive a radiation dose that is at least as large as the dose to off-site residents and is generally larger. The external radiation dose will decrease rapidly with distance from the site, and secondary off-site sources - such as surface deposits of airborne contaminated soil or water contaminated by radionuclides leached from the soil - will have lower radionuclide concentrations. The contributions from inhalation pathways will decrease with distance from 
the site for the same reasons. The largest contribution from the groundwater pathway will be for drinking water from the unconfined aquifer tapped by a well at the downgradient boundary of the contaminated area. This contribution can be the same for on-site and nearsite residents but will decrease for wells at greater distances from the boundary. The situation is more complicated for food chain pathways because reconcentration can occur along these pathways. However, the predominant contribution is from on-site crops and domestic animals, and this contribution will be greatest for on-site residents who raise food for their own consumption.

Exposure scenarios used for establishing soil guidelines should be bounding in the sense that they correspond to actions, events, and processes that will result in the largest exposure likely to occur to individuals and groups. However, they must also be credible, which implies that the probability of occurrence should be above some threshold value. The basis for specifying a credible bounding scenario is ill-defined because a threshold probability for distinguishing between a credible and a noncredible scenario has not been established, and it is usually not possible to assign a meaningful probability of occurrence for a scenario (unless the scenario is physically impossible, in which case a zero probability can be assigned). A family-farm scenario, in which a family constructs a home on the contaminated site and raises an appreciable fraction of its food on this site, is considered to be a credible bounding scenario for the purpose of this manual. ${ }^{3}$ Even though such a scenario may be unlikely in the foreseeable future for a site located in an industrial or urban area, it cannot be excluded as noncredible at some time several hundred years in the future.

The assignment of appropriate values to the scenario parameters is based on existing patterns of human activity that can be expected to persist for an indefinite time. For most scenario parameters, this criterion enables a straightforward determination of parameter values on the basis of data for current conditions. Scenario parameters and parameter values required for the RESRAD code are given in Appendixes A through $J$. The parameters and models that can be used to estimate the off-site dose are discussed in Appendix $\mathrm{K}$. The basis for selecting some of the key parameter values used in the RESRAD code is discussed below.

3 This scenario is referred to as the intruder-agriculture scenario when used for analyzing the longterm radiological impacts of a low-level radioactive waste disposal site (NRC 1981). This terminology is not used in this manual because a resident cannot be regarded as an intruder after a site has been released for use without radiological restrictions. 
One scenario parameter is the fraction of the family diet that consists of contaminated foodstuffs. This parameter will be determined by the fraction of food that is raised on-site and the fraction of the on-site crops grown in contaminated soil. The fraction of food raised on-site will be determined by the living and farming style and the area of land available to a family for gardening.

Farms or residential gardens that provide a significant fraction of the family diet are fairly common; it is not uncommon for families in rural areas to raise livestock to provide most of their meat and milk. A fully self-sufficient farm is rare. For a "self-sufficient" scenario to apply, the family would have to raise all of its vegetables on-site - that is, grow its own potatoes, get all fruit from an on-site fruit orchard, can or freeze enough of the summer crop to provide all food during the winter, and raise all grain used for bread and cereal. A scenario that is considered to be bounding and credible is for a family to have a garden that provides half of the total plant food diet if the area available for gardens and orchards is 0.1 ha or larger. This value is based on the following estimates for the area required for a year's supply of food for an individual: $50 \mathrm{~m}^{2}$ for a year's supply of leafy and other aboveground vegetables, $200 \mathrm{~m}^{2}$ for root vegetables and grains, and a somewhat larger area for fruit trees. It is inferred from these figures that 0.1 ha would be sufficient for half of the plant food diet for a family of four. It is also assumed, as noted above, that half the diet would be food purchased from a market even if sufficient land were available for a larger garden.

The area required for livestock, including summer pasture and forage for winter use, is somewhat larger. A single pig requires a food supply from an area of about $2,000 \mathrm{~m}^{2}$; a single milk cow needs a forage supply that may require an area of 1 ha or more. It is assumed that an area of 2 ha would be needed to provide sufficient meat and milk, including all forage, for a family of four. Thus, if the contaminated area exceeds 2 ha, the scenario diet factors specify that all of the meat and milk and $50 \%$ of the plant foods in the family diet are potentially contaminated. The fraction of the meat and milk diet that is contaminated is assumed to decrease linearly from 1 to 0 as the area decreases from 2 to 0 ha. The fraction of the plant food diet that is contaminated is assumed to decrease linearly from 0.5 to 0 as the area decreases from 0.1 to 0 ha. 
If the contaminated material is initially underneath a protective cover of uncontaminated material, one should take into account the dose that might be incurred from radioactive material that is redistributed during excavation for the basement of a residence. The excavation may result in the mixing of the uncontaminated cover material with the contaminated material underneath and the redistribution of the mixed material on the surface. However, no credit for concentration reduction as the result of mixing should be taken if the undisturbed contaminated layer is exposed at the surface or if the redistributed layer would result in a predicted potential dose less than the potential dose predicted for the undisturbed layer. 


\section{GUIDELINES FOR RADIONUCLIDE CONCENTRATIONS IN SOIL}

The basic criterion for releasing a site for use without radiological restrictions is the dose limit. The dose limit is converted to soil guidelines - specified as radionuclide concentrations - by means of dose/source ratios (DSRs) that are expressed in terms of three primary factors: dose conversion factors (DCFs), environmental transport factors (ETFs), and source factors (SFs). The definitions and use of these factors for deriving soil guidelines are described in this section. Tables, detailed models, and formulas for calculating the factors are given in Appendixes A through L. The typical range, measurement methodology, and default values used for RESRAD input parameters are discussed in the RESRAD Data Collection Handbook (Yu et al. 1993).

\subsection{RADIOLOGICAL RELEASE CRITERIA}

The basic criterion that must be satisfied before a site can be released for use without radiological restrictions is

$$
H_{E}(t) \leq H_{E L}, \quad t_{r} \leq t \leq t_{h},
$$

where ${ }^{4}$

$$
\begin{aligned}
H_{E}(t)= & \text { average annual EDE received by a member of the critical } \\
& \text { population group at time } t \text { following the radiological survey of the } \\
& \text { site (mrem/yr), } \\
H_{E L}= & \text { basic dose limit ( } 30 \mathrm{mrem} / \mathrm{yr}), \\
t_{r}= & \text { time at which the site is released for use without radiological } \\
& \text { restrictions following the radiological survey ( } 1 \mathrm{yr}) \text {, and } \\
t_{h}= & \text { time horizon }(1,000 \mathrm{yr}) .
\end{aligned}
$$

The time at which a radiological survey is done is the time of origin, or time 0 . The time at which a site is released after the radiological survey is the release time, or time $t_{r}$.

4 Units are specified in parentheses following the variable description. Numerical values are given for input variables to which default values are assigned. Default values are assigned to all input variables except radionuclide concentrations. If the total dose is sensitive to the variable (Cheng et al. 1991), then a site-specific value should be used if its determination is feasible. 
The default value used in the RESRAD code for $t_{r}$ is 1 year; for $t_{h}$, the default value is 1,000 years.

If the radionuclides are uniformly distributed within the contaminated zone, Equation 3.1 may be transformed into the inequality

$$
M(t)=\sum_{i} S_{i}(0) / G_{i}(t) \leq 1, \quad t_{r} \leq t \leq t_{h},
$$

where

$$
\begin{aligned}
M(t) \equiv & H_{E}(t) / H_{E L}=\text { fraction of the basic dose limit received by an average } \\
& \text { member of the critical population group at time } t \text { following the } \\
& \text { radiological survey (dimensionless), } \\
S_{i}(0)= & \text { initial concentration of the } i \text { th principal radionuclide in a uniformly } \\
& \text { contaminated zone at time } 0(\mathrm{pCi} / \mathrm{g}), \text { and } \\
G_{i}(t)= & \text { single-radionuclide soil concentration guideline for the } i \text { th principal } \\
& \text { radionuclide in a uniformly contaminated zone at time } t(\mathrm{pCi} / \mathrm{g}) .
\end{aligned}
$$

The variable $M(t)$ is referred to as the mixture sum. ${ }^{5}$ Principal radionuclides are radionuclides with half-lives greater than six months. The decay products of any principal radionuclide down to, but not including, the next principal radionuclide in its decay chain are called associated radionuclides and consist of radionuclides with half-lives less than six months. It is assumed that a principal radionuclide is in secular equilibrium with its associated radionuclides at the point of exposure. This assumption is reasonable because it usually takes about three years or longer to clean up a site. Principal and associated radionuclides included in the current version of RESRAD are listed in Table 3.1.

A contaminated zone is defined as a belowground volume within which the radionuclide concentrations in soil samples clearly exceed the background concentrations. Background concentrations are determined from measurements in soil samples taken at several nearby off-site locations where contamination is very unlikely. The concentration of a radionuclide is considered to clearly exceed the background concentration if it is larger than

5 The term "sum of fractions" is commonly used in regulatory references. 
TABLE 3.1 Principal and Associated Radionuclides

\begin{tabular}{|c|c|c|c|c|}
\hline \multicolumn{2}{|c|}{$\begin{array}{c}\text { Principal } \\
\text { Radionuclide }^{\mathrm{a}}\end{array}$} & \multirow[b]{2}{*}{ Associated Decay Chain ${ }^{b}$} & \multicolumn{2}{|c|}{$\begin{array}{c}\text { Terminal Nuclide or } \\
\text { Radionuclide }^{c}\end{array}$} \\
\hline Species & Half-life (yr) & & Species & Half-life (yr) \\
\hline $\mathrm{H}-3$ & 12.33 & $-d$ & $\mathrm{He}-3$ & $*$ \\
\hline C-14 & 5,730 & - & $\mathrm{N}-14$ & $*$ \\
\hline $\mathrm{Na}-22$ & 2.602 & - & $\mathrm{Ne}-22$ & $*$ \\
\hline $\mathrm{Al}-26$ & $7.16 \times 10^{5}$ & - & $\mathrm{Mg}-26$ & $*$ \\
\hline \multirow[t]{2}{*}{ Cl-36 } & $3.01 \times 10^{5}$ & - & $\begin{array}{l}\text { S-36 } \\
(98.1 \%)\end{array}$ & $*$ \\
\hline & & & $\begin{array}{l}\text { Ar-36 } \\
(1.9 \%)\end{array}$ & $*$ \\
\hline \multirow[t]{2}{*}{$\mathrm{K}-40$} & $1.28 \times 10^{9}$ & - & $\begin{array}{l}\mathrm{Ca}-40 \\
(89.3 \%)\end{array}$ & * \\
\hline & & & $\begin{array}{l}\text { Ar-40 } \\
(10.7 \%)\end{array}$ & $*$ \\
\hline $\mathrm{Ca}-41$ & $1.03 \times 10^{5}$ & - & $\mathrm{K}-41$ & $*$ \\
\hline $\mathrm{Mn}-54$ & 0.8561 & - & Cr-54 & $*$ \\
\hline $\mathrm{Fe}-55$ & 2.685 & - & $M n-55$ & $*$ \\
\hline $\mathrm{Co}-57$ & 0.7417 & - & $\mathrm{Fe}-57$ & $*$ \\
\hline $\mathrm{Co}-60$ & 5.271 & - & $\mathrm{Ni}-60$ & $*$ \\
\hline $\mathrm{Ni}-59$ & $7.5 \times 10^{4}$ & - & $\mathrm{Co}-59$ & $*$ \\
\hline $\mathrm{Ni}-63$ & 100 & - & $\mathrm{Cu}-63$ & $*$ \\
\hline $\mathrm{Zn}-65$ & 0.6678 & - & $\mathrm{Cu}-65$ & $*$ \\
\hline Ge-68 & 0.789 & - & $\mathrm{Ga}-68$ & $*$ \\
\hline Sr-90 & 28.8 & Y-90 (64.1 h) & $\mathrm{Zr}-90$ & $*$ \\
\hline $\mathrm{Nb}-94$ & $2.0 \times 10^{4}$ & - & Mo-94 & $*$ \\
\hline $\mathrm{Tc}-99$ & $2.14 \times 10^{5}$ & - & Ru-99 & $*$ \\
\hline $\mathrm{Ru}-106$ & 1.0335 & Rh-106 (29.9 s) & Pd-106 & $*$ \\
\hline \multirow[t]{3}{*}{$\mathrm{Ag}-108 \mathrm{~m}$} & 127 & - & $\begin{array}{l}\text { Pd-108 } \\
(91.1 \%)\end{array}$ & $*$ \\
\hline & & $\mathrm{Ag}-108(8.90 \%, 2.37 \mathrm{~min})$ & $\begin{array}{l}\text { Cd-108 } \\
(97.65 \%)\end{array}$ & $*$ \\
\hline & & & $\begin{array}{l}\mathrm{Pd}-108 \\
(2.35 \%)\end{array}$ & $*$ \\
\hline \multirow[t]{3}{*}{ Ag-110m } & 0.6842 & $\because$ & $\begin{array}{l}\text { Cd-110 } \\
(98.67 \%)\end{array}$ & $*$ \\
\hline & & $\mathrm{Ag}-110(1.33 \%, 24.6 \mathrm{~s})$ & $\begin{array}{l}\mathrm{Cd}-110 \\
(99.7 \%)\end{array}$ & $*$ \\
\hline & & & $\begin{array}{l}\mathrm{Pd}-110 \\
(0.3 \%)\end{array}$ & $*$ \\
\hline Cd-109 & 1.27 & - & Ag-109 & $*$ \\
\hline Sb-125 & 2.77 & $\begin{array}{l}\text { Te-125m } \\
(22.8 \%, 58 \mathrm{~d})\end{array}$ & $\mathrm{Te}-125$ & $*$ \\
\hline $\mathrm{I}-129$ & $1.6 \times 10^{7}$ & - & $\mathrm{Xe}-129$ & $*$ \\
\hline Cs-134 & 2.062 & - & $\begin{array}{l}\mathrm{Ba}-134 \\
(99.9997 \%) \\
\mathrm{Xe}-134 \\
(0.0003 \%)\end{array}$ & $\begin{array}{l}* \\
*\end{array}$ \\
\hline
\end{tabular}


TABLE 3.1 (Cont.)

\begin{tabular}{|c|c|c|c|c|}
\hline \multicolumn{2}{|c|}{$\begin{array}{c}\text { Principal } \\
\text { Radionuclide }^{\mathrm{a}}\end{array}$} & \multirow[b]{2}{*}{ Associated Decay Chain ${ }^{b}$} & \multicolumn{2}{|c|}{$\begin{array}{c}\text { Terminal Nuclide or } \\
\text { Radionuclide }^{c}\end{array}$} \\
\hline Species & Half-life (yr) & & Species & Half-life (yr) \\
\hline Cs-135 & $3 \times 10^{6}$ & - & Ba-135 & * \\
\hline Cs-137 & 30.17 & Ba-137m $(94.6 \%, 2.551 \mathrm{~min})$ & $\mathrm{Ba}-137$ & $*$ \\
\hline $\mathrm{Ce}-144$ & 0.7784 & $\begin{array}{l}{[\operatorname{Pr}-144(98.22 \%, 17.3 \mathrm{~min})} \\
\operatorname{Pr}-144 \mathrm{~m}(1.78 \%, 7.2 \mathrm{~min})]\end{array}$ & Nd-144 & $*$ \\
\hline Pm-147 & 2.6234 & - & $\mathrm{Sm}-147$ & $1.06 \times 10^{11}$ \\
\hline Sm-147 & $1.06 \times 10^{11}$ & - & Nd-143 & $*$ \\
\hline $\mathrm{Sm}-151$ & 90 & - & Eu-151 & $*$ \\
\hline Eu-152 & 13.3 & - & $\begin{array}{l}\mathrm{Sm}-152 \\
(72.1 \%)\end{array}$ & $*$ \\
\hline & & & $\begin{array}{l}\text { Gd-152 } \\
(27.9 \%)\end{array}$ & $1.08 \times 10^{14}$ \\
\hline Eu-154 & 8.5 & - & $\begin{array}{l}\text { Gd-154 } \\
(99.98 \%)\end{array}$ & \\
\hline & & & $\begin{array}{l}\mathrm{Sm}-154 \\
(0.02 \%)\end{array}$ & $*$ \\
\hline Eu-155 & 4.96 & - & Gd-155 & $*$ \\
\hline $\mathrm{Gd}-152$ & $1.08 \times 10^{14}$ & - & $\mathrm{Sm}-148$ & $*$ \\
\hline $\mathrm{Gd}-153$ & 0.663 & - & $\mathrm{Eu}-153$ & $*$ \\
\hline Au-195 & 0.501 & - & Pt-195 & $*$ \\
\hline $\mathrm{Tl}-204$ & 3.779 & - & $\begin{array}{l}\mathrm{Pb}-204 \\
(97.42 \%)\end{array}$ & $*$ \\
\hline & & $\therefore$ & $\begin{array}{l}\mathrm{Hg}-204 \\
(2.58 \%)\end{array}$ & * \\
\hline $\mathrm{Pb}-210$ & 22.3 & $\begin{array}{l}\operatorname{Bi}-210(5.01 \mathrm{~d}) \\
\operatorname{Po}-210(138.38 \mathrm{~d})\end{array}$ & $\mathrm{Pb}-206$ & * \\
\hline $\mathrm{Bi}-207$ & 38 & - & $\mathrm{Pb}-207$ & * \\
\hline $\mathrm{Ra}-226$ & $1.60 \times 10^{3}$ & $\begin{array}{l}\text { Rn-222 }(3.8235 \mathrm{~d}) \\
\mathrm{Po-218}(3.05 \mathrm{~min}) \\
{[\mathrm{Pb}-214(99.98 \%, 26.8 \mathrm{~min})} \\
\text { At-218 }(0.02 \%, 2 \mathrm{~s})] \\
\mathrm{Bi}-214(19.7 \mathrm{~min}) \\
{\left[\mathrm{Po}-214\left(99.98 \%, 1.64 \times 10^{-4} \mathrm{~s}\right)\right.} \\
\mathrm{T} 1-210(0.02 \%, 1.3 \mathrm{~min})]\end{array}$ & $\mathrm{Pb}-210$ & 22.3 \\
\hline $\mathrm{Ra}-228$ & 5.76 & $\operatorname{Ac}-228(6.13 \mathrm{~h})$ & Th-228 & 1.9131 \\
\hline Ac-227 & 21.733 & $\begin{array}{l}{[\mathrm{Th}-227(98.6 \%, 18.71 \mathrm{~d})} \\
\text { Fr-223 }(1.4 \%, 21.8 \mathrm{~min})] \\
\operatorname{Ra}-223(11.435 \mathrm{~d}) \\
\operatorname{Rn}-219(3.96 \mathrm{~s}) \\
\text { Po-215 }(1.78 \mathrm{~ms}) \\
\text { Pb-211 }(36.1 \mathrm{~min}) \\
\text { Bi-211 }(2.15 \mathrm{~min}) \\
{[\mathrm{T}-207(99.72 \%, 4.77 \mathrm{~min})} \\
\text { Po-211 }(0.28 \%, 0.516 \mathrm{~s})]\end{array}$ & $\mathrm{Pb}-207$ & $*$ \\
\hline
\end{tabular}


TABLE 3.1 (Cont.)

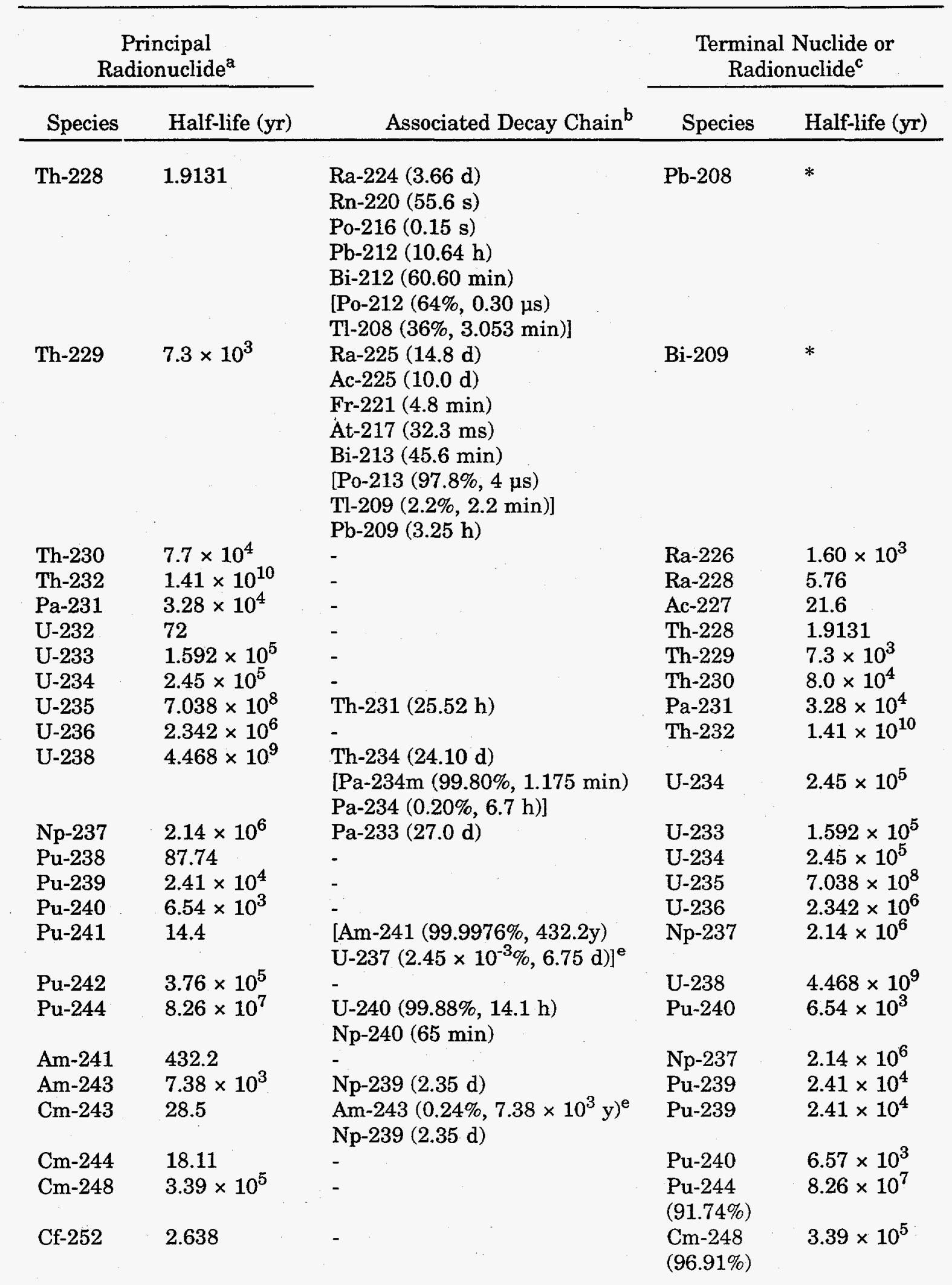


TABLE 3.1 (Cont.)

a Radionuclides with half-lives greater than six months.

b The chain of decay products of a principal radionuclide extending to (but not including) the next principal radionuclide or a stable nuclide. Half-lives are given in parentheses. Branches are indicated by square brackets with branching ratios in parentheses.

c The principal radionuclide or stable nuclide that terminates an associated decay chain. Stable nuclides are indicated by an asterisk (*) in place of the half-life.

d A hyphen indicates that there are no associated decay products.

e The branching decay for $\mathrm{Pu}-241$ and $\mathrm{Cm}-243$ involves multiple principal radionuclides and associated radionuclides. For a detailed description of the decay chain, see ICRP Publication 38 (ICRP 1983b) and Appendix G.

the mean background concentration plus twice the standard deviation of the background measurements. If the concentrations in the samples used for determining the background concentration are below the lower limit of detection (LLD) of the instrument used, the concentration of that radionuclide is considered to exceed background if it exceeds the LLD of the instrument.

A distinction should be made between a uniformly contaminated zone and a homogeneously contaminated zone. In a uniformly contaminated zone, radionuclide concentrations are exactly the same at every point. A uniformly contaminated zone is a mathematical construct used to calculate soil guidelines. A homogeneously contaminated zone is a volume within which deviations from uniformity of the actual radionuclide concentrations are considered small enough to allow the volume to be treated as if it were uniformly contaminated.

Actual radionuclide distributions are nonuniform. The potential annual individual dose received through a particular pathway is an areal average of the nonuniform residual radioactivity over an area determined by the scenario activities; for example, the area of daily activities for external radiation or the size of the garden for plant food pathways. For the purpose of deriving soil guidelines, it is assumed that this area is $100 \mathrm{~m}^{2}$ for all pathways. The effect of vertical nonuniformities is taken into account by averaging over the radionuclide concentrations in a $0.15-\mathrm{m}$-thick layer. Thus, the criterion for releasing a site for use without 
radiological restrictions is as follows: for any $100-\mathrm{m}^{2}$ area and $0.15-\mathrm{m}$-thick layer within the contaminated zone,

$$
\bar{M}(t) \equiv \sum_{i} \bar{S}_{i}(0) / G_{i}(t) \leq 1, \quad t_{r} \leq t \leq t_{h}
$$

where

$$
\begin{aligned}
\bar{M}(t)= & \text { average mixture sum at time } t \text { (dimensionless), } \\
\bar{S}_{i}(0)= & \text { initial concentration of the } i \text { th principal radionuclide averaged over } \\
& \text { a } 100-\mathrm{m}^{2} \text { area and } 0.15-\mathrm{m} \text {-thick layer }(\mathrm{pCi} / \mathrm{g}), \text { and } \\
G_{i}(t)= & \text { single-radionuclide soil concentration guideline for the } i \text { th principal } \\
& \text { radionuclide in a uniformly contaminated zone at time } t(\mathrm{pCi} / \mathrm{g}) .
\end{aligned}
$$

Equation 3.3 is the homogeneous release criterion. It is directly applicable to homogeneous contamination, which is defined as contamination in a volume within which radionuclide concentrations in individual soil samples do not exceed the average concentrations by a factor greater than 3. For a contaminated site, if the number and distribution of soil samples required by characterization and certification protocols result in only one soil sample from a contaminated region that is $100 \mathrm{~m}^{2}$ in area and $0.15 \mathrm{~m}$ thick, then the contamination within the volume is assumed to be homogeneous and the average radionuclide concentrations are assumed to equal the concentrations in that sample. If no soil samples are taken from the volume, the radionuclide concentrations are assumed to be averages of concentrations in soil samples from the nearest neighboring volumes from which samples are taken. (See ORNL 1982, DOE 1984, and Ahrends 1987 for sampling protocols.)

For field applications, the homogeneous criterion as specified by Equation 3.3 may be replaced by the criterion

$$
\bar{M}=\sum_{i} \bar{S}_{i}(0) / G_{i}\left(t_{m}\right) \leq 1
$$

where

$$
\bar{S}_{i}(0)=\text { as defined for Equation } 3.3, \text { and }
$$


$G_{i}\left(t_{m}\right)=$ single-radionuclide soil guideline for the $i$ th principal radionuclide in a homogeneous contaminated zone at time $t=t_{m}$ within the interval $t_{r} \leq t \leq t_{h}$, at which the time-dependent value $G_{i}(t)$ is minimal (pCi/g).

The variable $G_{i}\left(t_{m}\right)$ should be obtained from RESRAD prior to the field actions. Equations 3.3 and 3.4 are equivalent when the minima in $G_{i}(t)$ for different principal radionuclides all occur at the same time. Equation 3.4 is more restrictive than Equation 3.3 because the minima in $G_{i}(t)$ usually occur at different times for different principal radionuclides.

For inhomogeneous contamination, defined as contamination in a volume within which the above-background concentration of a radionuclide at one or more sampling locations exceeds three times the average concentration, the average concentration $\bar{S}_{i}(0)$ in Equation 3.3 may be conservatively replaced by $\hat{S}_{i}(0) / 3$, where $\hat{S}_{i}(0)$ is the maximum concentration in a soil sample taken from within the area.

Alternatively, when sufficient data are available and conditions warrant, one may apply less conservative, inhomogeneous contamination criteria in place of homogeneous contamination criteria. The purpose of the inhomogeneous contamination criteria is to provide a more realistic guideline for cleanups in cases in which the conservatism inherent in the homogeneous assumptions would cause excessive expenditures in comparison to the benefits associated with the more conservative approach. The inhomogeneous contamination criteria, which are discussed in Section 3.3.1, should not be confused with the hot spot criterion for field application, which is described in Section 3.3.2.

The purpose of the hot spot criteria is to ensure that applying the homogeneous criteria, in which the concentrations of residual radioactive material are averaged over a $100-\mathrm{m}^{2}$ area, does not result in the release of small areas that, due to averaging, contain unacceptably high concentrations of residual radioactive material. Although the inhomogeneous criteria may, under appropriate conditions, be used in place of the general guidelines, the hot spot criteria must be used along with the general guidelines or authorized limits resulting from the homogeneous criteria. 
A single-radionuclide soil concentration guideline for a uniformly contaminated zone is defined as

$$
G_{i}(t) \equiv H_{E L} / D S R_{i}(t)
$$

where

$$
\begin{aligned}
& H_{E L}=\text { basic dose limit }(30 \mathrm{mrem} / \mathrm{yr}) \text {, } \\
& D S R_{i}(t)=\sum_{p} D S R_{i p}(t)=\text { dose/soil-concentration ratio for the } i \text { th } \\
& D S R_{i p}(t)=\text { dose/soil-concentration ratio for the } i \text { th principal radionuclide }
\end{aligned}
$$

The dose/soil-concentration ratios for individual principal radionuclides and pathways are defined as

$$
D S R_{i p}(t) \equiv H_{E, i p}(t) / S_{i}(0)
$$

where

$$
\begin{aligned}
H_{E, i p}(t)= & \text { average annual EDE received at time } t \text { by a member of the } \\
& \text { critical population group from the } i \text { th principal radionuclide } \\
& \text { transported through the pth environmental pathway together } \\
& \text { with its associated decay products (mrem/yr), and } \\
S_{i}(0)= & \text { initial concentration of the } i \text { th principal radionuclide in a } \\
& \text { uniformly contaminated zone }(\mathrm{pCi} / \mathrm{g}) .
\end{aligned}
$$

The total annual effective dose equivalent to an individual is

$$
H_{E^{(}}(t)=\sum_{i} \sum_{p} H_{E, i p}(t)
$$

By substituting Equations 3.5 through 3.7 into Equation 3.1 and rearranging terms, Equation 3.2 is obtained. 


\subsection{DOSE/SOURCE CONCENTRATION RATIOS FOR UNIFORM CONTAMINATION}

For the radon pathway $(p=9)$, the indoor and outdoor air concentrations for radon and its decay products are calculated by RESRAD, then the concentrations are converted to the WL (working level) values, which in turn are converted to the average annual EDE. Therefore, the dose/source $(D / S)$ concentration ratios for the radon pathway are calculated by using a different method from the other pathways. In short, the dose/source concentration ratios can be written as

$$
D S R_{i 9}(t)=H_{E, 9}(t) / S_{i}(0)
$$

where

$$
\begin{aligned}
H_{E, 9}= & \text { the average annual EDE received at time } t \text { by a member of the } \\
& \text { critical population group from the radon pathway (mrem/yr); and } \\
S_{i}(0)= & \text { initial concentration of the principal radionuclide } i \text {, which is a } \\
& \text { radon source, at time } 0(\mathrm{pCi} / \mathrm{g}) .
\end{aligned}
$$

The dose/source $(D / S)$ concentration ratios for the other pathways are calculated by first expressing them as the products of dose conversion factors [dose/exposure-parameter (D/E) ratios], environmental transport factors [exposure-parameter/source-concentration (E/S) ratios], and modified source factors (ratios of the concentration of a radionuclide to the initial concentration). The factored expression for a $D / S$ ratio is

$$
D S R_{i p}(t)=\sum_{j} D C F_{j, x(p)} \times B R F_{i, j} \times \sum_{q} E T F_{i j, p q}(t) \times S F_{i j, p q}^{\prime}(t),
$$

where

$$
\begin{aligned}
D C F_{j, x(p)=} & \text { dose conversion factor for the } j \text { th principal radionuclide and } \\
& x(p) \text { th exposure pathway }\left[(\mathrm{mrem} / \mathrm{yr}) /\left(\mathrm{pCi} / \mathrm{cm}^{3}\right)\right. \text { for external } \\
& \text { radiation from the contaminated zone; } \mathrm{mrem} / \mathrm{pCi} \text { for internal } \\
& \text { radiation from ingestion or inhalation of radionuclides }] . \\
B R F_{i, j}= & \text { a branching factor (dimensionless) that is the fraction of the } \\
& \text { total decay of radionuclide } i \text { that results in the ingrowth of } \\
& \text { radionuclide } j .
\end{aligned}
$$




$$
\begin{aligned}
E T F_{i j, p q}(t)= & E T F_{j p}(t) \text {, environmental transport factor for the } j \text { th principal } \\
& \text { radionuclide at time } t \text {, when } p=1,2 \text {, and } 8\left(\mathrm{~g} / \mathrm{cm}^{3}\right. \text { for } \\
& \text { external radiation from the contaminated zone, } p=1 ; \mathrm{g} / \mathrm{yr} \\
& \text { for internal radiation from dust inhalation, } p=2 \text {; and soil } \\
& \text { ingestion, } p=8) \text {. When } p=3,4 \text {, and } 5 \text { (ingestion of plant, } \\
& \text { meat, and milk pathways), it is the environmental transport } \\
& \text { factor for the jth principal radionuclide originating from the } \\
& \text { decay of the } i \text { th principal radionuclide for the } q \text { th component } \\
& \text { of the } p \text { th environmental pathway at time } t \text { (g/yr). When } \\
& p=6 \text { and } 7 \text { (ingestion of fish and drinking water), it is } \\
& \text { equivalent to } E T F_{i j p}(t) \text {, the environmental transport factor } \\
& \text { for the } j \text { th principal radionuclide originating from the decay } \\
& \text { of the } i \text { th principal radionuclide for the } p \text { th environmental } \\
& \text { pathway at time } t \text { (g/yr) (see Appendixes A through D for } \\
& \text { detailed derivations). }
\end{aligned}
$$

$S F^{\prime}{ }_{i j, p q}(t)=S F_{i j}(t)$, factor for ingrowth and decay and leaching of the $j$ th principal radionuclide at time $t$ from the $i$ th principal radionuclide present initially (dimensionless), when $p=1,2$, and 8. For $p=3, S F^{\prime} i j, p q(t)$ is equivalent to $S F_{i j}(t)$ when $q=1$ and 2 ( $q=1$ for root uptake component, $q=2$ for foliar deposition component), and it is 1 when $q=3$ and 4 ( $q=3$ for ditch irrigation component, and $q=4$ for overhead irrigation component). For $p=4$ and $5, S F_{i j, p q}(t)$ is equivalent to $S F_{i j}(t)$ when $q=1,2$, and 6 ( $q=6$ for livestock intake of soil component), and it is 1 when $q=3,4$, and 5 ( $q=5$ for livestock water). For $p=6$ and $7, S F^{\prime}{ }_{i j p q}(t)$ is 1 . $x(p)=$ index label for exposure pathways, which is a function of the environmental pathway $p$ (for $p=1$ lexternal radiation from the ground], $x(p)=1$; for $p=2$ and 9 [inhalation of dust and radon], $x(p)=2$; and for $p=3,4,5,6,7$, and 8 [ingestion of 
plants, meat, milk, fish, water, and soil, respectively], $x(p)=3)$.

$p=$ index label for environmental pathways.

$q=$ index label for pathway components. For $p=1,2,6,7$, and $8, q=1$; that is, there is no further division of the pathway p. For $p=3, q$ ranges from 1 to 4 . For $p=4$ and $5, q$ ranges from 1 to 6 . Indexes are $q=1$ for root uptake, $q=2$ for foliar deposition, $q=3$ for ditch irrigation, $q=4$ for overhead irrigation, $q=5$ for livestock water, and $q=6$ for livestock intake of soil.

$i_{\nu} j=$ index labels for principal radionuclides, where $i$ refers to radionuclides that exist initially at time $t$, and $j$ refers to radionuclides in the decay chain of radionuclide $i$.

\subsubsection{Dose Conversion Factors}

A dose conversion factor is the ratio

$$
D C F_{i x}=H_{E, i x} / E_{i x}
$$

where

$H_{E, i x}=$ annual effective dose equivalent resulting from exposure to external radiation $(x=1)$ from the $i$ th principal radionuclide and its associated radionuclides ( $\mathrm{mrem} / \mathrm{yr}$ ) or the annual committed EDE resulting from exposure for 50 years to internal radiation from the amount of the ith principal radionuclide and its associated radionuclides inhaled $(x=2)$ or ingested $(x=3)$ in 1 year (mrem/yr); and

$E_{i x}=$ exposure parameter for the $i$ th principal radionuclide (concentration of the $i$ th principal radionuclide in a standard source [for external radiation pathways] or the annual quantity of the $i$ th principal radionuclide inhaled or ingested [for internal radiation 
pathways]) ( $\mathrm{pCi} / \mathrm{cm}^{3}$ for external radiation $[x=1]$ from the contaminated zone; $\mathrm{pCi} / \mathrm{yr}$ for internal radiation from inhalation $[x=2]$ or ingestion $[x=3])$.

An internal DCF for any radionuclide includes the contribution from ingrowth following ingestion or inhalation. The internal DCF for a principal radionuclide includes, in addition, the contribution from inhalation or ingestion of associated radionuclides along with the principal radionuclide. The additional contribution is significant only for associated radionuclides with half-lives that are not small compared to the biological half-life. The DCF for external radiation for a principal radionuclide is defined here as including the dose from all of its associated radionuclides, which are assumed to be in secular equilibrium.

Dose conversion factors for external radiation from the contaminated zone, internal radiation from inhalation of airborne radionuclides, and internal radiation from ingestion are given in Tables A.1, B.1, and D.1, respectively, of Appendixes A, B, and D. The dose conversion factors for internal radiation from inhalation of radon and its decay products are discussed in Appendix C.

\subsubsection{Environmental Transport Factors}

An environmental transport factor is the time-dependent ratio

$$
E T F_{i j, p q}(t)=E_{i j, p q}(t) / S_{i}(0) \times S F_{i j, p q}^{\prime}(t)
$$

where

$E_{i j, p q}(t)=$ exposure parameter value at time $t$ for the $j$ th principal radionuclide (or radiation therefrom) transported through the $p q$ th environmental pathway as a result of the decay of the initially existent radionuclide $i$ in the soil $\left(\mathrm{pCi} / \mathrm{cm}^{3}\right.$ for external radiation from the contaminated zone; $\mathrm{pCi} / \mathrm{yr}$ for internal radiation). For $p=1,2$, and 8 (external radiation, dust inhalation, and soil ingestion pathways), $E_{i j p q}=E_{j p}$; for $p=3,4$, and 5 (ingestion of plant food, meat, and milk pathways), when $q=1$ and 2 (root uptake and foliar deposition), $E_{i j, p q}=E_{j p q}$; for $p=4$ and 5 , when $q=6$ (livestock 
ingestion of soil), $E_{i j, p q}=E_{j p q}$; and for $p=6$ and 7 (ingestion

of seafood and drinking water pathways), $E_{i j, p q}=E_{i j p}$.

$p=$ index label for environmental pathways.

$q=$ index label for the component of the environmental pathway $p$.

$S_{i}(0)=$ average concentration of the $i$ th principal radionuclide in a uniformly contaminated zone at time $0(\mathrm{pCi} / \mathrm{g})$.

$S F_{i j, p q}^{\prime}(t)=$ an adjusting factor to modify the soil concentration. For $p=1,2$, and $8, S F_{i j, p q}^{\prime}(t)=S F_{i j}(t) ; S F_{i j}(t)$ is the source factor, which when multiplied by $S_{i}(0)$ will give the soil concentration of radionuclide $j$ at time $t, S_{j}(t)$. For $p=3,4$, and 5 , when $q=1$ and $2, S F_{i j, p q}^{\prime}(t)=S F_{i j}(t) ;$ when $q=3$ and 4 , $S F^{\prime}{ }_{i j, p q}(t)=1$. For $p=4$ and 5 , when $q=5, S F_{i j, p q}^{\prime}(t)=1$, and $S F_{i j, p q}^{\prime}(t)=S F_{i j}(t)$, when $q=6$. For $p=6$ and $7, S F_{i j, p q}(t)=1$.

The exposure parameter for external radiation from the contaminated zone is the concentration of the $j$ th principal radionuclide in the ground, adjusted for occupancy and the size and depth of the contaminated zone by means of multiplying factors. The exposure parameter for internal radiation pathways is the annual quantity of the $j$ th principal radionuclide that is inhaled or ingested after migrating through the pqth environmental pathway. There are only two internal exposure pathways (inhalation and ingestion); several environmental pathways can contribute to each.

Models and formulas for calculating environmental transport factors are given in Appendixes A through $\mathrm{F}$ and $\mathrm{H}$ through $\mathrm{L}$.

\subsubsection{Source Factors}

A source factor is the time-dependent ratio

$$
S F_{i j}(t)=S_{i j}(t) / S_{i}(0)
$$


where

$$
\begin{aligned}
& S_{i j}(t)= \text { concentration at time } t \text { of the } j \text { th principal radionuclide remaining } \\
& \text { in the contaminated zone after leaching and ingrowth from the } i \text { th } \\
& \text { principal radionuclide, if } j \neq i(\mathrm{pCi} / \mathrm{g}) \text {; or the concentration at time } t \\
& \text { of the } i \text { th principal radionuclide remaining in the contaminated } \\
& \text { zone after leaching and decaying (or transforming), excluding } \\
& \text { contributions from ingrowth from other radionuclides, if } j=i \\
&(\text { pCi } / g) \text {; and } \\
& S_{i}(0)= \text { initial concentration of the } i \text { th principal radionuclide in the } \\
& \text { contaminated zone (pCi/g). }
\end{aligned}
$$

Formulas for calculating source factors are given in Appendix G.

\subsection{GUIDELINES FOR INHOMOGENEOUS CONTAMINATION}

Two separate formulations of inhomogeneous release criteria have been developed: (1) the inhomogeneous criteria intended for use during project planning and review as an alternative to the homogeneous criteria and (2) the hot spot criterion, intended for field use in conjunction with the homogeneous criteria or for use when determination of compliance must be made immediately.

\subsubsection{Inhomogeneous Criteria for Project Planning and Review}

A contaminated zone is inhomogeneous if it contains a contaminated region within which the concentration of a radionuclide exceeds three times the average for the contaminated zone. The inhomogeneous release criteria, described below, are generally more realistic and hence less restrictive than the homogeneous release criteria. In general, inhomogeneous contamination should be remediated in order to meet homogeneous contamination limits. However, the inhomogeneous contamination criteria may be used in cases in which it is inappropriate to use the homogeneous contamination criteria. For example, homogeneous criteria might be inappropriate if the contamination exists under a roadway, railroad right-of-way, or building. Inhomogeneous criteria can also be used if an area of elevated contamination is found during the verification survey after equipment and crews have left the site. An area of elevated contamination must always be remediated 
before a site can be released for use without radiological restrictions, if that area does not comply with inhomogeneous criteria.

The mixture-sum release criterion for any zone of inhomogeneous contamination is

$$
M^{*}(t)+\bar{M}(t) \leq 1, \quad t_{r} \leq t \leq t_{h}
$$

where

$$
\begin{aligned}
M^{*}(t)= & \text { mixture sum for area of elevated contamination (dimensionless); } \\
& \text { and } \\
\bar{M}(t)= & \text { mixture sum for averaging zone (dimensionless), and } t_{r} \text { and } t_{h} \text { are } \\
& \text { as defined for Equation } 3.1 .
\end{aligned}
$$

The averaging zone for inhomogeneous contamination is an area of $100 \mathrm{~m}^{2}$ that encloses the elevated contamination.

The two mixture sums are defined as follows:

$$
M^{*}(t)=\sum_{i}\left[S_{i}^{*}(0)-\bar{S}_{i}(0)\right] / G_{i}^{*}(t)
$$

and

$$
\bar{M}(t)=\sum_{i} \bar{S}_{i}(0) / G_{i}(t)
$$

where

$S_{i}^{*}(0)=$ initial concentration of the $i$ th principal radionuclide in the area of elevated contamination ( $\mathrm{pCi} / \mathrm{g}$ );

$G_{i}^{*}(t)=$ single-radionuclide, time-dependent soil guideline for the $i$ th principal radionuclide in the area of elevated contamination (pCi/g);

$\vec{S}_{i}(0)=$ average initial concentration of the $i$ th principal radionuclide in the averaging zone over a $100-\mathrm{m}^{2}$ area and a $0.15-\mathrm{m}$-thick layer, excluding the area of elevated contamination ( $\mathrm{pCi} / \mathrm{g})$; and 


$$
\begin{aligned}
G_{i}(t)= & \text { single-radionuclide, time-dependent soil guideline for the } i \text { th } \\
& \text { principal radionuclide in a uniformly contaminated zone }(\mathrm{pCi} / \mathrm{g}) .
\end{aligned}
$$

If the area of elevated contamination is inhomogeneous, one of two approaches may be used: (1) the area may be treated as if it were homogeneous with a concentration $\hat{S}_{i}(0) / 3$, where $S_{i}(0)$ is the peak concentration of the $i$ th principal radionuclide in a sample from within the area of elevated contamination, or (2) the area may be divided into subzones by using the same procedure that is used for an inhomogeneous contaminated zone.

When inhomogeneous release criteria are used, Equation 3.12 must be satisfied for every area of inhomogeneous contamination; in addition, Equation 3.3 must be satisfied for any region within the homogeneous portion of the contaminated zone.

\subsubsection{Hot Spot Criterion for Field Application}

Hot spots are small areas that have levels of residual radioactive material that are considerably above the levels in the surrounding area. The derivation of remedial action criteria generally assumes homogeneous contamination of large areas (several hundred square meters or more), and the derived concentration guide is stated in terms of concentrations averaged over an area of $100 \mathrm{~m}^{2}$. Because of this averaging process, hot spots can exist within these $100-\mathrm{m}^{2}$ areas that contain concentrations of radionuclides that are significantly higher than the authorized limit. Therefore, the presence of hot spots could potentially pose a greater risk of exposure to individuals using the site than the risk associated with homogeneous contamination. To ensure that individuals are adequately protected and to ensure that the ALARA process is satisfied, the following hot spot criterion must be applied, along with the general criterion for homogeneous contamination. The hot spot criterion for field application is

$$
M^{* *} \equiv \sum_{i} S_{i}^{*} / G_{i}^{* *} \leq 1
$$

where

$$
\begin{aligned}
M^{* *}= & \text { hot spot mixture sum for field use (dimensionless), } \\
S_{i}^{*}= & \text { measured concentration of the } i \text { th principal radionuclide in the hot } \\
& \text { spot (pCi/g), and }
\end{aligned}
$$




$$
\begin{aligned}
G_{i}^{* *}= & \text { single-radionuclide soil guideline for the } i \text { th principal radionuclide } \\
& \text { in the hot spot ( } \mathrm{pCi} / \mathrm{g}) .
\end{aligned}
$$

The measured hot spot concentrations $S_{i}{ }^{*}$ are the peak concentrations if the hot spot area is $1 \mathrm{~m}^{2}$ or less, or the average concentrations if the hot spot area is larger than $1 \mathrm{~m}^{2}$.

The formula for single-radionuclide, hot spot soil guidelines is

$$
G_{i}^{* *}=G_{i}\left(t_{m}\right) \times(100 / A)^{1 / 2}
$$

where

$$
\begin{aligned}
G_{i}\left(t_{m}\right) & =\text { as defined for Equation } 3.4 \\
A & =\text { area of hot spot }\left(\mathrm{m}^{2}\right), \text { and } \\
(100 / A)^{1 / 2} & =\text { hot spot multiplication factor. }
\end{aligned}
$$

Equations 3.15 and 3.16 apply to hot spots with areas of $25 \mathrm{~m}^{2}$ or less. For larger hot spot areas, the homogeneous release criterion is sufficient. An area of $A=1 \mathrm{~m}^{2}$ is used in Equation 3.16 if the actual hot spot area is less than $1 \mathrm{~m}^{2}$. The average radionuclide concentrations for any $100-\mathrm{m}^{2}$ area must always comply with the homogeneous release criterion, irrespective of hot spot criteria.

For general field applications, it is recommended that the ranges of hot spot multiplication factors provided in Table 3.2 be used. The hot spot guideline for radionuclide $i$ is calculated for each specific site by Equation 3.16. The term $G_{i}\left(t_{m}\right)$ in Equation 3.16 can be substituted by $G_{i}$, the authorized limit at a specific site for the $i$ th principal radionuclide.

The authorized limit is considered adequate to protect the public for areas larger than $25 \mathrm{~m}^{2}$; hence, no special hot spot limits are required for areas larger than $25 \mathrm{~m}^{2}$.
TABLE 3.2 Ranges for Hot Spot Multiplication Factors

\begin{tabular}{cc}
\hline Range & $\begin{array}{c}\text { Factor } \\
\text { (multiple of } \\
\text { authorized limit) }\end{array}$ \\
\hline$<1 \mathrm{~m}^{2}$ & $10^{\mathrm{a}}$ \\
$1-<3 \mathrm{~m}^{2}$ & 6 \\
$3-<10 \mathrm{~m}^{2}$ & 3 \\
$10-25 \mathrm{~m}^{2}$ & 2 \\
\hline
\end{tabular}

a Areas less than $1 \mathrm{~m}^{2}$ are to be averaged over a $1-\mathrm{m}^{2}$ area, and that average shall not exceed 10 times the authorized limit. 
Averaging of hot spots less than or equal to $25 \mathrm{~m}^{2}$ should be done only over the local hot spot area.

Every reasonable effort should be made to identify and remove any source that has a radionuclide concentration exceeding 30 times the authorized limit, irrespective of area. 


\section{USER'S GUIDE FOR RESRAD}

The analytical method for deriving guidelines for allowable concentrations of residual radioactive material in soil is described in Sections 2 and 3 and Appendixes A through M. This method has been implemented in a computer code named RESRAD. RESRAD is designed for use on an IBM or IBM-compatible personal computer (PC), with internal help files for information on input and output data. The code consists of three modules:

- RESPC, an interactive module for data entry and display via forms;

- RESMAIN, the main calculational and report-generating module; and

- RESPLOT, an interactive graphics module that displays the latest RESMAIN results.

All of the modules are integrated into a single package and can be automatically executed, as needed, by the user. Instructions for installing and using the code are presented in Sections 4.2 through 4.7 .

\subsection{NEW FEATURES}

RESRAD, Version 5.0 (i.e., RESRAD5) has several improvements compared to Version 3.121A (i.e., RESRAD3), which was the last release of the code (Gilbert et al. 1989). These improvements are as follows:

- Calculation of potential health effects (excess risk of cancer incidence);

- Interactive graphics with publication-quality printouts;

- Performance of sensitivity analysis on most RESRAD parameters;

- New pathways: (1) radon inhalation and (2) soil ingestion;

- Optional external ground radiation pathway model;

- Groundwater radionuclide concentrations as optional input data for deriving water/soil distribution coefficients;

- Optional input of a solubility constant for leaching of radionuclides; 
- Optional user selection for deriving distribution coefficients from plant/soil concentration ratios;

- Optional user selection of all dose conversion and related factors, including cancer incidence slope factors;

- Twenty-seven additional radionuclides;

- Expanded and redesigned main menu;

- Context-sensitive help files for data input;

- Improved groundwater transport model; and

- User selection of accuracy criterion for groundwater pathway computations.

RESRAD5 reads and processes data files from previous versions and assigns default values to all new parameters.

\subsection{INSTALLATION}

An IBM or IBM-compatible microcomputer with a 3.1 or higher disk operating system (DOS), a hard disk drive, and approximately 500 kilobytes (KB) of free memory are required. A printer capable of 132-column output is desirable. Most PC printers have a condensed print option (17 characters per inch) that is satisfactory. A mathematics coprocessor, a color monitor, and a mouse are optional but recommended. A mouse may not work, however, with all monitor/graphics cards used in IBM or clone configurations. No problems have occurred with the Microsoft Bus Mouse, the Logitech Bus Mouse, or the Mouse Systems PS/2 Mouse. Any hardware problems encountered should be reported to the contact address on the final RESRAD screen.

The RESRAD code is available on a single 1.44 megabyte (MB) 3.5-in. or $1.2 \mathrm{MB}$ 5.25-in. diskette, two $720 \mathrm{~KB} 3.5$-in. diskettes, or four $360 \mathrm{~KB} 5.25$-in. diskettes. In all cases, installation diskette no. 1 should be inserted first, the default should be set to the appropriate floppy disk drive, and the command INSTALL should be entered. The INSTALL.BAT file will issue a few simple prompts to determine where the RESRAD5 files are to be stored. Approximately 2.4 MB of disk space are needed for a complete installation. The installation program will create new directories, as needed, or use specified old 
directories. The diskette files will overwrite existing ones of the same name, only if the diskette files have been created at a later date.

The installation batch file will prompt for the insertion of new diskettes, as they are needed, and will announce when the installation has been completed. The installation procedure can be repeated any number of times. All RESRAD5 files on the installation diskettes are in a compressed format and should only be added to the hard disk via the INSTALL.BAT program.

In addition to successful completion of INSTALL.BAT, RESRAD5 requires a CONFIG.SYS file (root directory of C: disk) that contains at least the following statements:

FILES $=30$

BUFFERS $=16$

SHELL $=\mathrm{C}: \backslash$ DOS $\backslash$ COMMAND. COM $/ \mathrm{P} / \mathrm{E}: 512$

A CONFIG.SYS consisting of the above lines will be found in the RESRAD5 execution files. This CONFIG.SYS may be copied directly to the $\mathrm{C}$ drive, or the existing CONFIG.SYS may be modified with a suitable editor such as EDLIN. The number 512 in the SHELL command sets the size of the DOS environment-string table. If an out-of-memory environment error occurs, the size of the $\mathbf{E}$ parameter should be increased. A system reboot (Ctrl-Alt-Del) is needed whenever changes are made to the CONFIG.SYS file.

\subsection{RESRAD EXECUTION}

If the RESRAD5 execution files are located in C:\RESRAD5, the prompts (in boldface) and responses for starting RESRAD5 are as follows:

C: $\backslash\rangle$ cd $\backslash$ resrad5

C: IRESRAD5> resrad

Up to three optional command line arguments may also be included. The same information can be supplied (or superseded) later via the regular RESRAD menu input; however, command line input for argument \#1, other than RESRAD.DEF, is more efficient. The optional arguments are as follows:

Argument 1: The initial (template) site-specific data file. The default file for this purpose is RESRAD.DEF. It is supplied on the distribution diskette and contains the default values for all RESRAD parameters. It also serves as a sample test 
problem. In general, users are advised to specify an initial data file that more closely matches their current site.

Argument 2: The final site-specific data file. This is the file that actually serves as input data for RESMAIN, and it is part of the header record for each page of printed output. In the data entry phase of RESRAD (see Sections 4.6.1 and 4.6.3), any of the parameters supplied by the initial site-specific data file (argument \#1) may be modified. All changes are saved in the final site-specific data file (argument \#2). If there are no command line arguments, the default for the \#2 argument is SAMPLE.DAT. If only one argument is supplied, argument \#2= argument \#1 is the default (except if RESRAD.DEF is used, in which case the default for argument \#2 is again SAMPLE.DAT). WARNING: if the initial site-specific data file is to serve as a template for additional runs, a different name must be supplied for the final site-specific data file.

Argument 3: The destination of RESRAD hard copy output. The default is LPT1 for normal printer output, or the user may specify a disk file name to save all or selected portions of RESRAD output. In the case of an existing disk file, the user can select to overwrite or to append to the end of the file.

Each of the command line arguments that can be used to start RESRAD may include disk drive and path names up to a maximum of 36 characters each. As a special feature, the DOS wild card characters "*" or "?" may be included in the file name(s). RESRAD will produce an appropriate directory listing(s) before starting normal output, and the user can then input the desired file name or try another wild card directory listing. In the following example, all three arguments are used:

C: \RESRAD5> resrad mysite.dat mysite1.dat a:mysite1.prn

In the above example, MYSITE.DAT must exist in the default C: $\backslash$ RESRAD5 directory, MYSITE1.DAT will be produced (or overwritten) in the C: RRESRAD5 directory, and all hard copy output will be "printed" in MYSITE1.PRN on the A drive.

A final feature that is available for starting RESRAD is the standard IBM-PC font. This font uses American Standard Code for Information Interchange (ASCII) characters 
128-255, and it is assumed that it is a print option. ${ }^{6}$ If strange characters appear on the printed output, RESRAD1 should be used instead of RESRAD to start the code, that is,

C:IRESRAD5> resrad1 [optional arguments \#1 \#2 \#3]

\subsection{PRELIMINARY OUTPUT}

The initial RESRAD output is a banner page with a brief code definition. If a mouse is available, the banner screen is followed by RESRAD-specific instructions for using the mouse. Although optional, a mouse provides three unique data entry advantages: direct access to any menu item, instant review of a parameter's default value, and automatic restoration of a parameter's default value. Also, a mouse is very useful for repositioning plot legends in the graphics phase of the code.

\subsection{MAIN MENU AND HELP FILES}

The true starting point for using RESRAD is the main menu, reproduced in Figure 4.1. This appears as soon as the preliminary output described in Section 4.4 is completed. The main menu is also the return point after RESRAD's calculational and/or graphics phase has been completed.

Instructions for interactive use of the code are provided in help files that can be displayed on the screen by pressing the F1 or F2 function keys. ${ }^{7}$ The F1 help screen contains information for positioning the cursor and selecting options. Descriptions of data to be input and general information are given in the F2 help files. The F1 screen for the main menu is shown in Figure 4.2.

The main menu provides the primary options for running RESRAD. Many of the options have associated submenus that define the user's options more explicitly. A menu

6 For a Hewlett-Packard LaserJet II or III, the internal font 01 or symbol set PC- 8 should be selected, respectively. For an Epson dot matrix printer, DIP switch 1-3 (CG table) should be on.

7 The F1 and F2 function key help files are used throughout all interactive phases of RESRAD. However, the exact screen information will vary. The F1 help screen always contains cursor and display information that remains constant until a major phase change occurs. The F2 screens concern data input, and usually the information is keyed to the current active menu field (cursor position). In addition, the last line of every menu is a brief help message for the current field. 


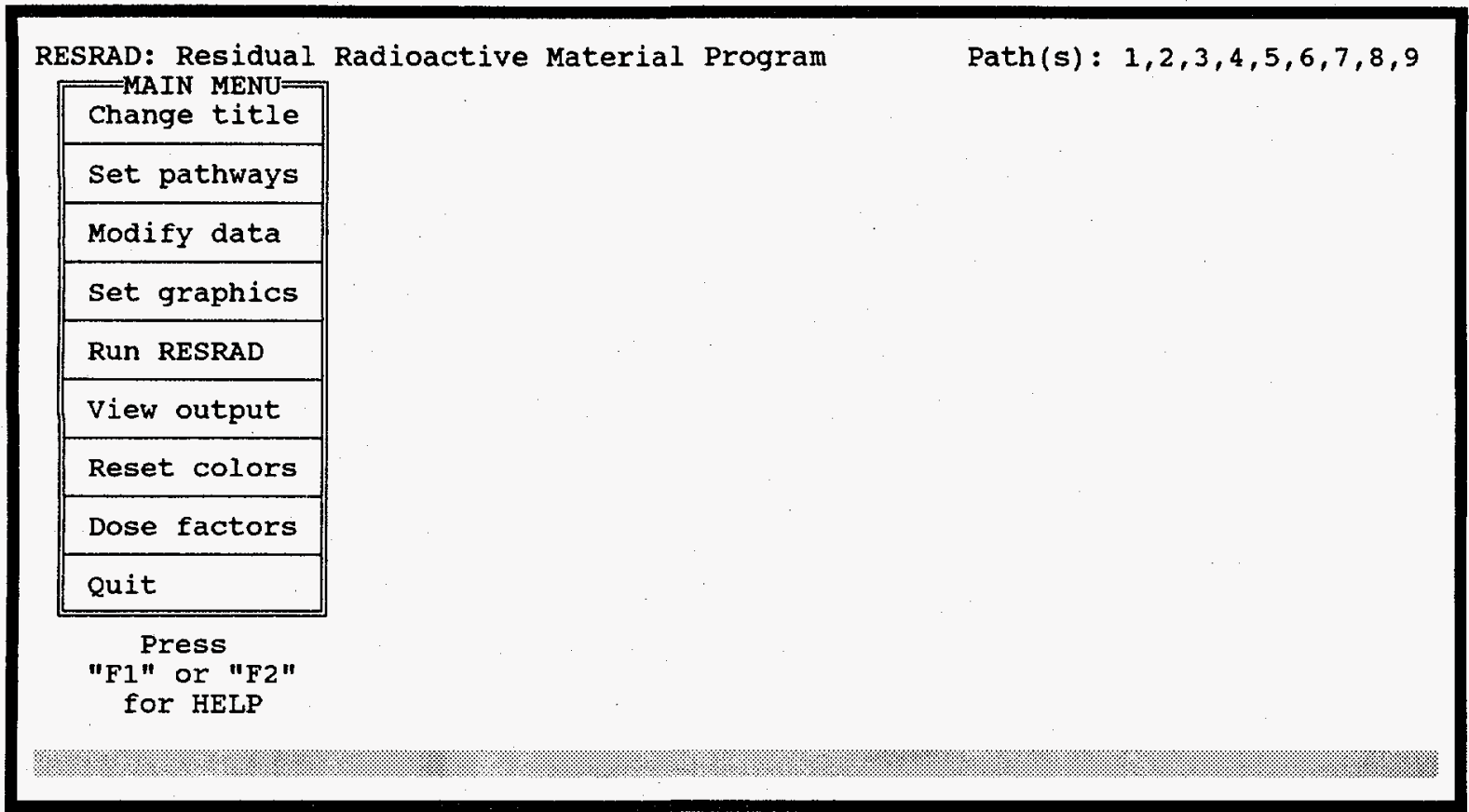

\section{FIGURE 4.1 Main Menu for RESRAD}

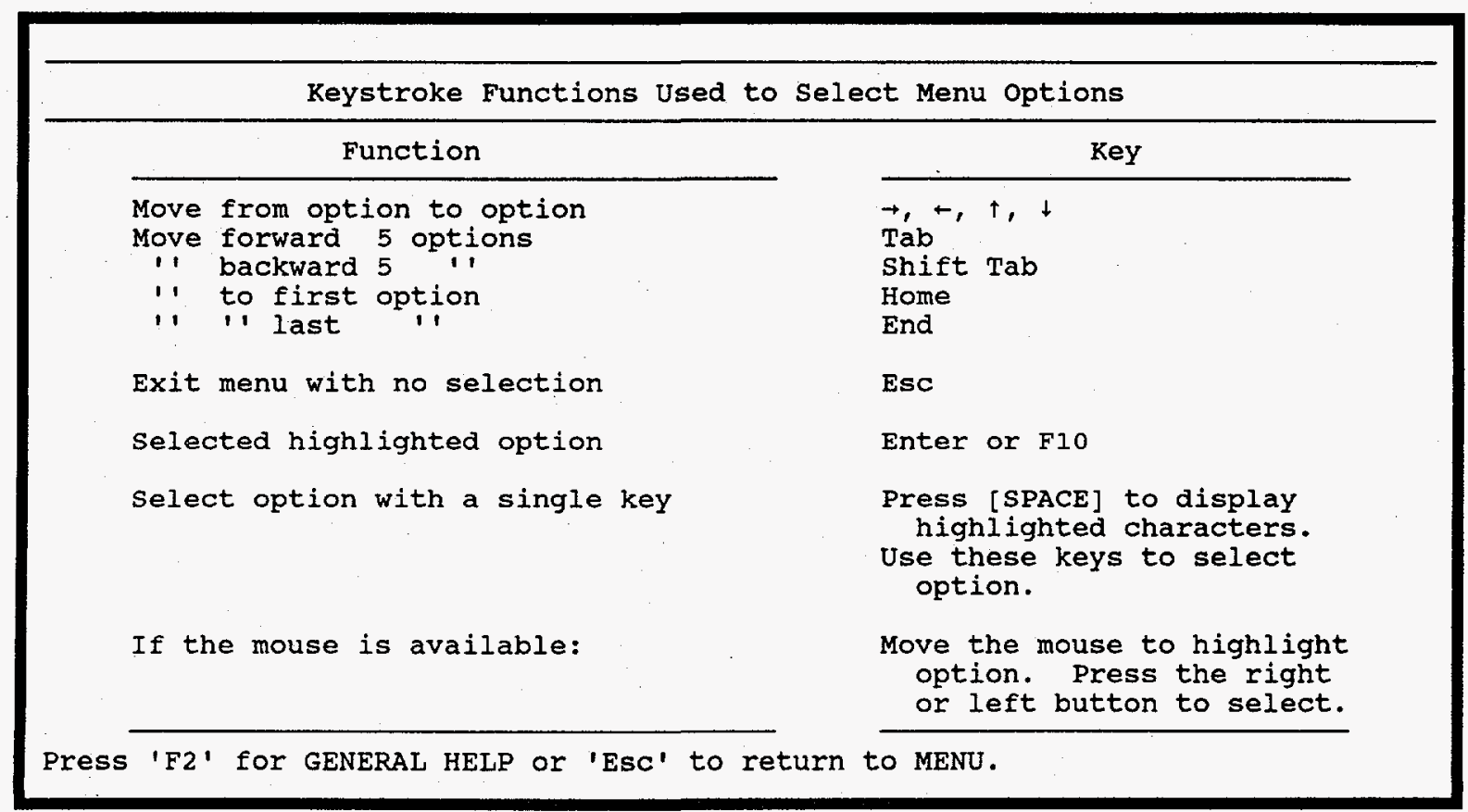

\section{FIGURE 4.2 F1 Help Screen for Main Menu}


option is selected by highlighting the field (see F1 above) and pressing Enter, F10, or the mouse button. An alternative selection method is to press the space bar and then to press the highlighted letter key in the desired option field. The nine main menu options are discussed in Sections 4.6.1 through 4.6.9.

If a submenu appears, additional help information is always available via the F2 function key. The main menu is active when it is the only menu displayed; if a submenu shares the screen, the submenu is active. The Esc key can always be used to return to the main menu; other methods, depending on the submenu, may be available. To exit RESRAD (back to DOS), a two-key combination is used as a safety feature. The main menu must appear alone on the screen with the Quit option highlighted (Esc does this), and then Enter should be pressed. Another method is to press the space bar followed by the highlighted Q key.

\subsection{MAIN MENU OPTIONS}

Change Title, Modify Data, and Set Graphics are the primary main menu data entry options. These full-screen submenus have identical keystrokes for data entry; the keystrokes differ somewhat, however, from those used in the main menu and the other submenus. The F1 help file, reproduced in Figure 4.3, provides a complete keystroke list. Pressing the F10 key records all data fields and can be used to exit the menu; the Esc key can also be used to exit (as always) but cancels all the changes made on the form.

\subsubsection{Change Title}

Change Title is a single, full-screen, data entry submenu (Form R010). The screen contains fields for the title of the current RESRAD run, the initial (template) and final site-specific data files for RESMAIN input, and the destination for RESRAD printer output. The RESRAD.DEF default form is shown in Figure 4.4; the blank gray line at the bottom will contain the help message associated with the current cursor position.

The Title field, which is used to identify the run, can consist of up to 70 alphabetical or numerical characters. It is part of the header for every page of RESRAD output.

The other three fields correspond exactly to the three optional command line arguments that can be used to start RESRAD. Input to these fields overrides the command 


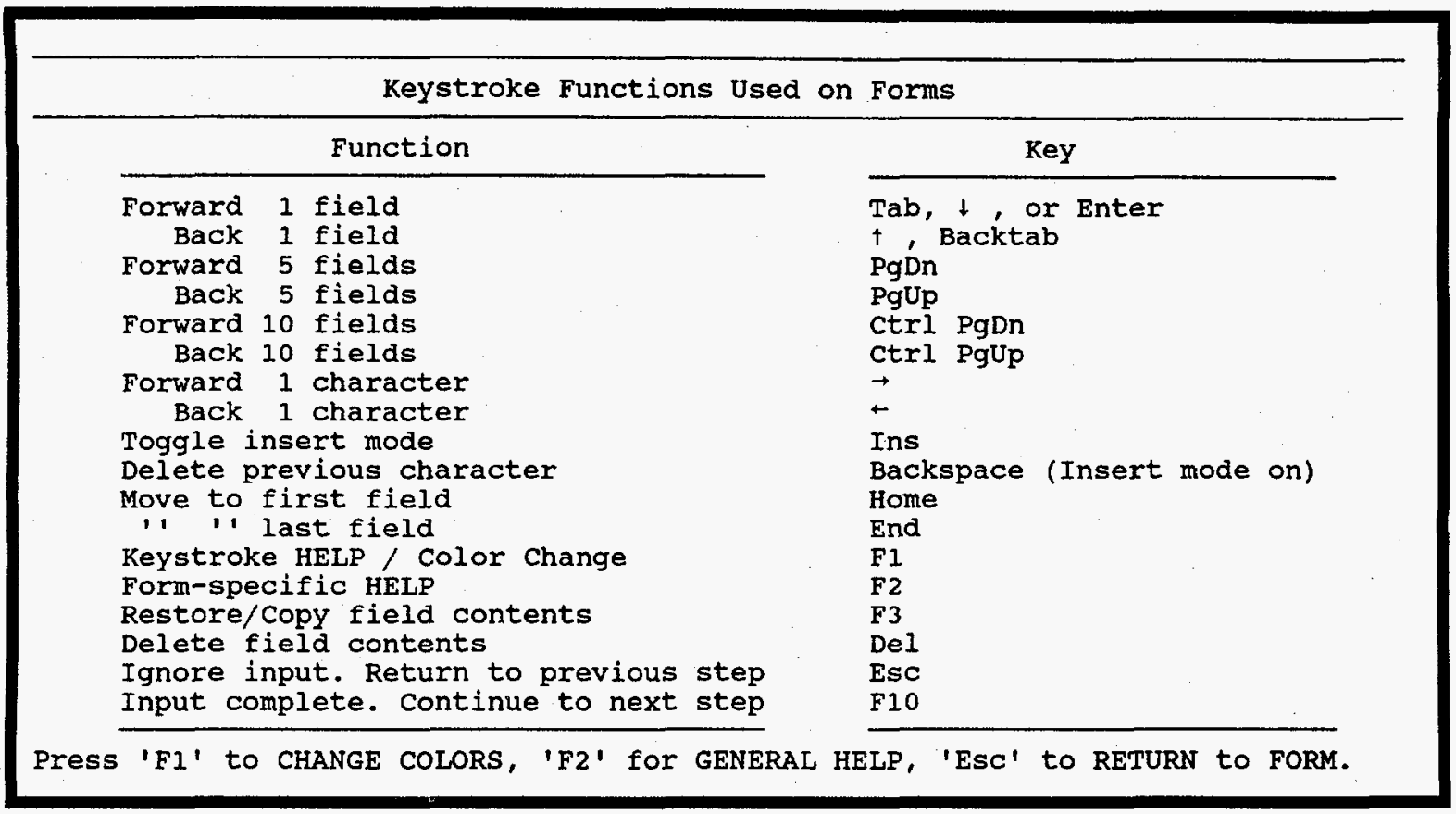

\section{FIGURE 4.3 F1 Help Screen for Data Entry Forms}

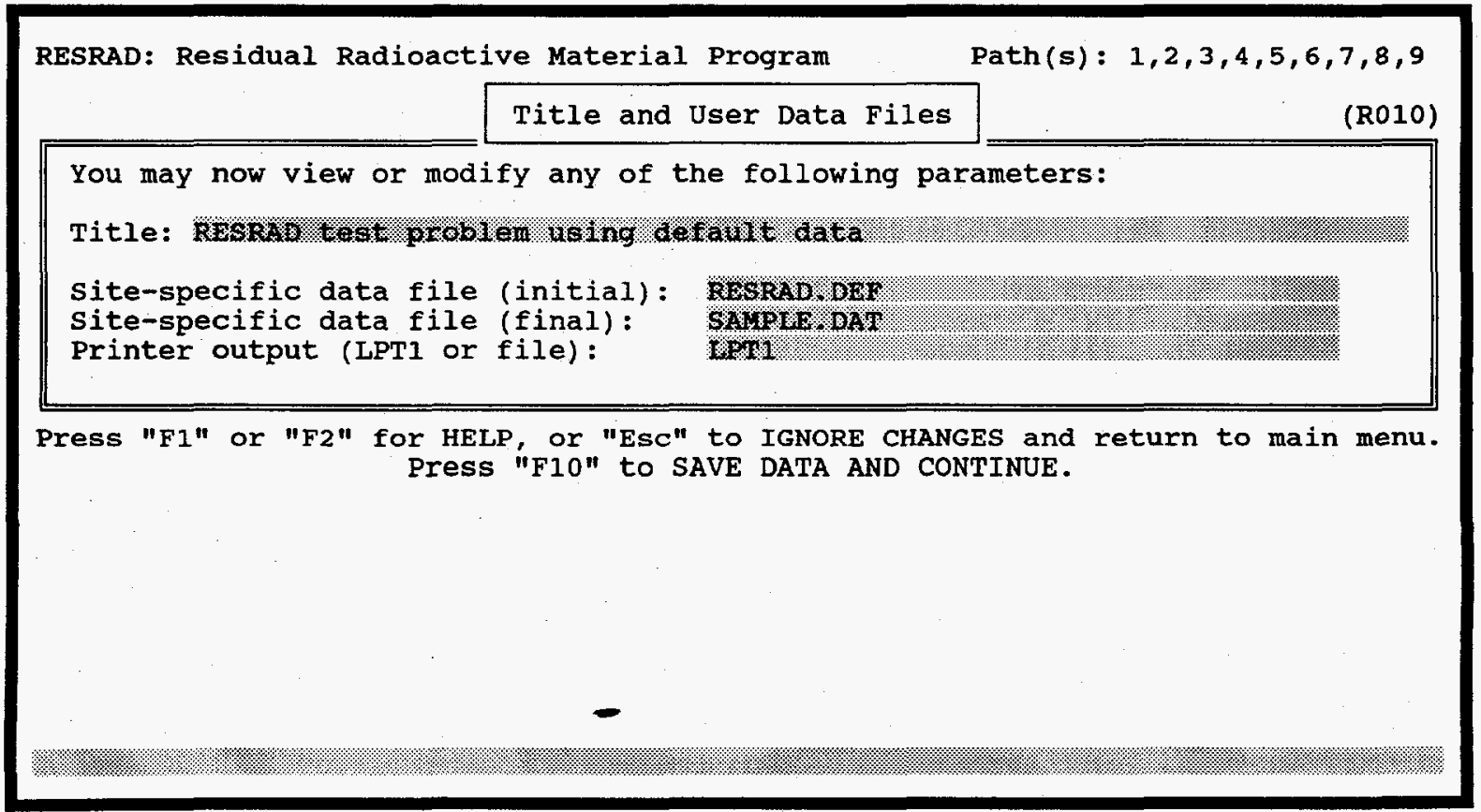

FIGURE 4.4 Input Form R010 for Title and User Data Files 
line arguments. If the initial site-specific data file is to be changed, this should be done first and then the F10 key should be pressed before any other changes are made. This procedure includes all the Modify Data forms. The new data file is input and overwrites all previous values as soon as the F10 key is pressed.

Before entering text-string data, it is usually best to clear the field with the Del key. The "*" and "?" DOS wild card characters can be used in the file names just as for the command line arguments in Section 4.3.

After each RESMAIN execution and return to the main menu, the initial site-specific data file is replaced with the final site-specific data file of the run just completed. The final site-specific data file and the printer output file remain unchanged, which allows the parameter input of the previous run to serve as the template for the next execution. The user, of course, can alter these automatic selections at any time.

\subsubsection{Set Pathways}

This submenu, shown in Figure 4.5, gives the user control over the nine potential pathways RESRAD uses to compute the radiation dose. After highlighting the desired pathway, pressing Enter, F10, or the mouse button will toggle the pathway between active and inactive. A pathway can also be selected by pressing the space bar and highlighting the letter in the pathway. Any combination of the pathways can be turned on or off. The last two fields provide the appropriate return to the main menu. The current list of active pathways is always given at the top of all RESPC menus. The pathways are described in more detail below:

- Direct exposure to external radiation from the contaminated soil,

- Internal radiation from inhalation of contaminated dust,

- Internal radiation from ingestion of plant foods grown on-site and irrigated with water drawn from an on-site well or pond,

- Internal radiation from ingestion of meat from livestock fed with fodder grown on-site and water drawn from an on-site well or pond, 


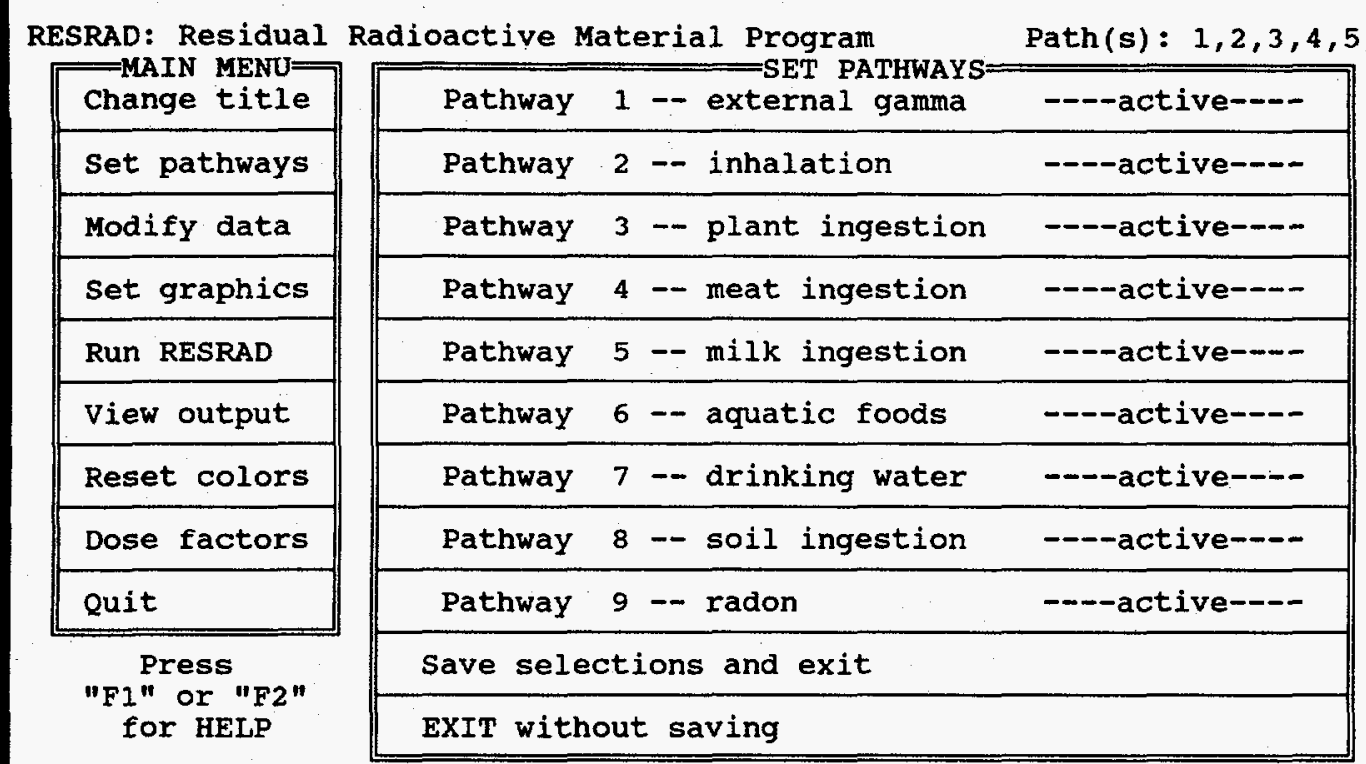

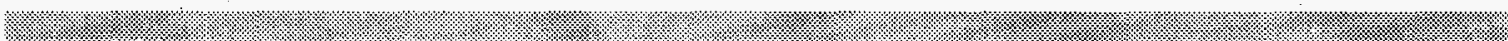

FIGURE 4.5 Set Pathways Submenu (with Main Menu)

- Internal radiation from ingestion of milk from livestock fed with fodder grown on-site and water drawn from an on-site well or pond,

- Internal radiation from ingestion of aquatic foods from an on-site pond,

- Internal radiation from ingestion of drinking water drawn from an on-site well or pond,

- Internal radiation from ingestion of contaminated soil, and

- Internal radiation from inhalation of radon-222 and/or radon-220.

\subsubsection{Modify Data}

The first portion of this main menu option is the submenu shown in Figure 4.6. This is the starting and return point for a series of 10 full-screen menus that are the primary data entry forms for RESMAIN. The menus can be selected repeatedly in any order by highlighting the appropriate field and pressing Enter, F10, or the mouse button. Pressing the space bar and highlighting the letters that follow can also be used to select a menu. Initial values for all data on the forms were obtained from the initial site-specific data file 


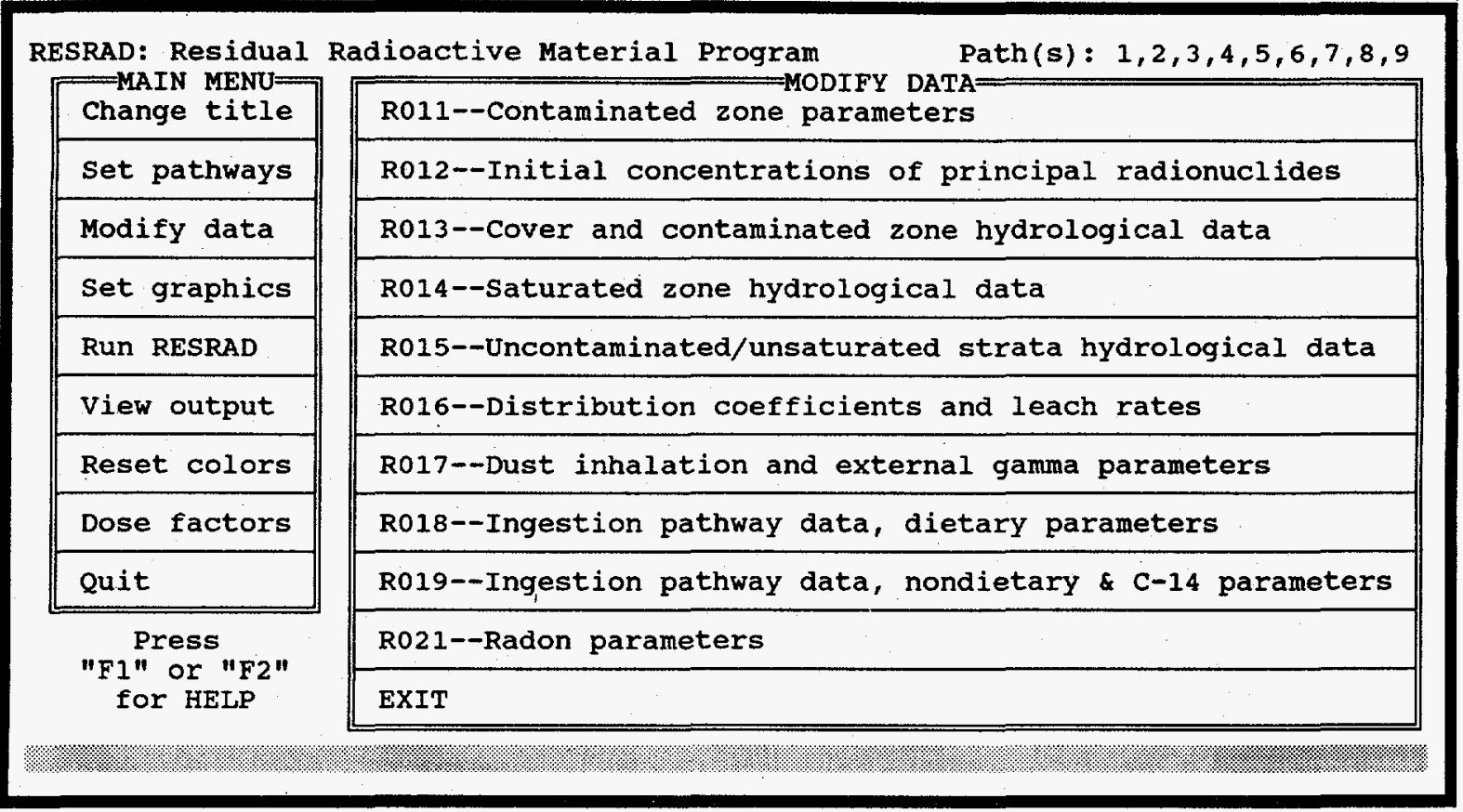

FIGURE 4.6 Modify Data Submenu (with Main Menu)

(Sections 4.3 and 4.6.1). The current values on any form can be reviewed and changed as desired.

Parameters pertaining to suppressed pathways are blanked out in the data entry screens because these parameters are not used in the RESMAIN calculations. This action eases data entry and ensures that only variables pertinent to the calculations are subject to modification. If all of the variables are blanked out in a particular data screen, the message "NO APPLICABLE PARAMETERS" will be displayed in the Modify Data menu and access to this data form will be denied.

Pressing the F2 key will give specific help information for the current active field (cursor position) for all forms in this group. If a mouse is available, pressing the left button while within a numeric data field will display the field's default value; pressing the right button will replace the data item with the default value. Exiting from a form is accomplished by either pressing F10, which saves all data on the screen, or by pressing Esc, which results in all changes being ignored.

RESRAD input parameters are briefly discussed in the following 10 subsections (4.6.3.1 through 4.6.3.10). Detailed discussions on the range of parameter values, 
measurement methodology, and default parameter values are presented in a separate data collection handbook (Yu et al. 1993). The sensitivities of RESRAD input parameters are presented in a report entitled RESRAD Parameter Sensitivity Analysis (Cheng et al. 1991).

\subsubsection{Contaminated Zone Parameters}

The first input form (Form R011) in the Modify Data series is reproduced in Figure 4.7; the result of selecting the Sensitivity Analysis ${ }^{8}$ pop-up menu feature is shown in Figure 4.8. The data fields are general parameters concerning the contaminated zone and are described in more detail below.

The Area of Contaminated Zone parameter is a compact area specified in square meters $\left(\mathrm{m}^{2}\right)$. This area contains the locations of all soil samples with radionuclide concentrations that are clearly above background; these locations are separated from the locations of other above-background soil samples by a distance of at least $100 \mathrm{~m}$. The concentration of a radionuclide is clearly above background if it exceeds background level measurements by at least two standard deviations (see Section 3.1). To justify the use of two or more contaminated zones, credible evidence must be provided on the basis of radiological survey data that the intervening distance between any two contaminated zones is uncontaminated; otherwise, the contaminated zone should be characterized by a single compact area that contains the locations of all soil samples with above-background radionuclide concentrations.

8 Most RESRAD parameters can be subjected to a sensitivity analysis by pressing the F9 function key with the cursor positioned on the parameter of interest. A pop-up submenu will appear that allows specification of a \pm percent range of parameter values. Any of five preset values or a userspecified range can be selected. The Esc key can be used to exit with no change in the sensitivity analysis settings; however, F10 must be used to exit and to save any new settings. Parameter values selected for sensitivity analysis are enclosed by parentheses on the screen. The sensitivity analysis is carried out after the regular RESRAD calculations have been completed by taking each parameter selected and repeating the entire RESRAD calculation, with the parameter being tested set at the two extremes specified. Only a single test parameter is varied at a time. The factor $F$, which is used to vary the parameter, is obtained from the percent range by using $F=1+\% / 100$. Thus a $400 \%$ range converts to a factor of 5 . The base parameter value is first multiplied by $\mathrm{F}$ and then divided by $\mathrm{F}$. The results of the analysis are presented graphically with separate curves for the base case; both ranges are on the same plot. The maximum number of sensitivity analyses per RESRAD run is 5 . 
You may now view or modify any of the following parameters.

Area of contaminated zone: Thickness of contaminated zone: Length parallel to aquifer flow: meters Radiation dose limit:

Elapsed time of waste placement:

Times for calculations (years since initial time)

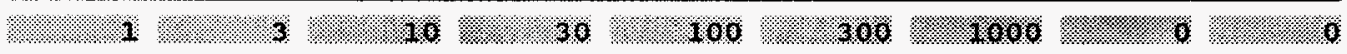

Press "FI" or "F2" for HELP, or "Esc" to IGNORE CHANGES and return to main menu. Press "Fg" for sensitivity Analysis. Press "F10" to SAVE DATA AND CONTINUE.

FIGURE 4.7 Input Form R011 for Contaminated Zone Parameters

Contaminated zone Parameters

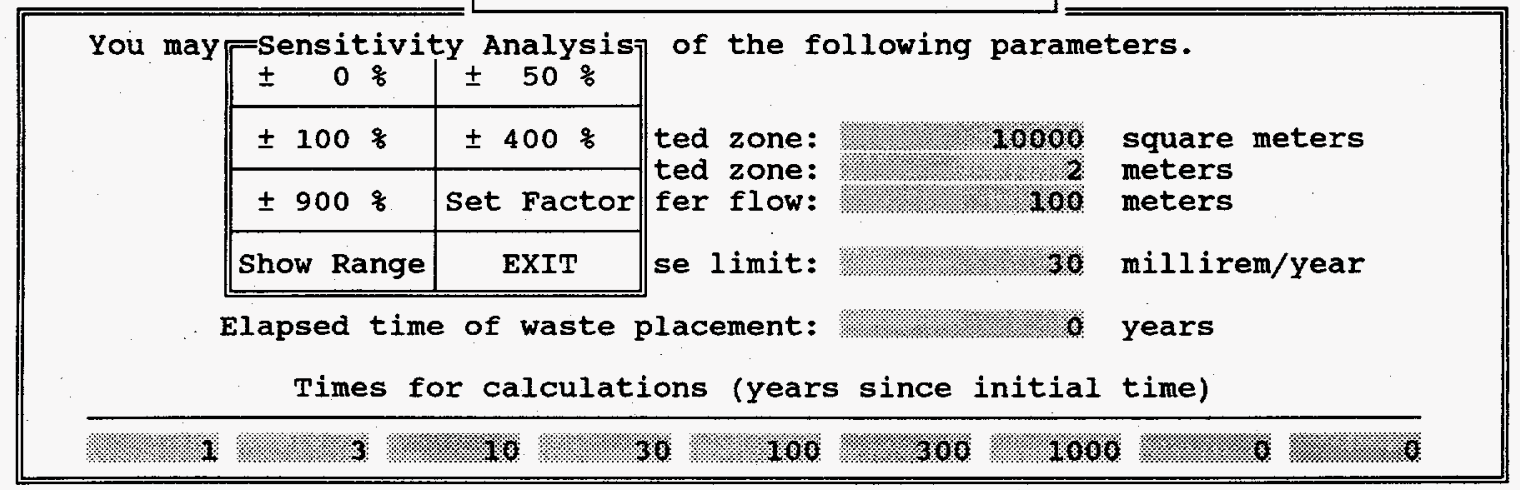

Press "FI" or "F2" for HELP, or "Esc" to IGNORE CHANGES and return to main menu. Press "F9" for Sensitivity Analysis. Press "F10" to SAVE DATA AND CONTINUE.

FIGURE 4.8 Input Form R011 with Sensitivity Analysis Pop-Up Menu 
The Thickness of Contaminated Zone parameter is the distance, in meters (m), between the uppermost and lowermost soil samples with radionuclide concentrations that are clearly above background.

The Length Parallel to Aquifer Flow parameter is the distance, in meters (m), between two parallel lines perpendicular to the direction of aquifer flow, one at the upgradient edge of the contaminated zone and the other at the downgradient edge.

The Basic Radiation Dose Limit parameter is the annual radiation dose limit, in millirems per year (mrem/yr) used to derive all site-specific soil guidelines.

The Time Since Material Placement parameter is the time, in years, that has elapsed since the disposal of the radioactive materials. If the radioactive materials might have been disposed of on-site at different times, then an appropriate representative value should be input for all of the waste materials. A value greater than zero must be specified if groundwater radionuclide concentrations are to be input (Menu R012).

The Times for Calculations parameter consists of the times, in years (yr), following the radiological survey at which tabular single radionuclide soil guidelines and mixture sums will be calculated. Graphical output will supply intermediate values between the first and last user-specified times. The default values are 1, 3, 10,30,100, 300, and 1,000 years. The guidelines require that the total dose be less than the basic radiation dose limit at all times out to the time horizon. A time horizon of 1,000 years is commonly used; however, calculations can be carried out to longer periods to identify potential problems from delayed contributions from the groundwater or other pathways. RESRAD always calculates values for year 0 . All years must be input in ascending order. If fewer than 10 time periods are selected, a zero must be input for the time period immediately following the last one desired. Because logarithmic time scales are used for all graphics output, integer powers of 10 are recommended for the first and last specified years.

\subsubsection{Initial Concentrations of Principal Radionuclides}

There are two possible forms (R012 and R012W) for this option, and they are illustrated in Figures 4.9 and 4.10. The R012W form with radionuclide groundwater concentrations [Wi(0)] will only appear if the Time Since Material Placement parameter of Form R011 is set greater than zero. All principal radionuclides for which guidelines can be 


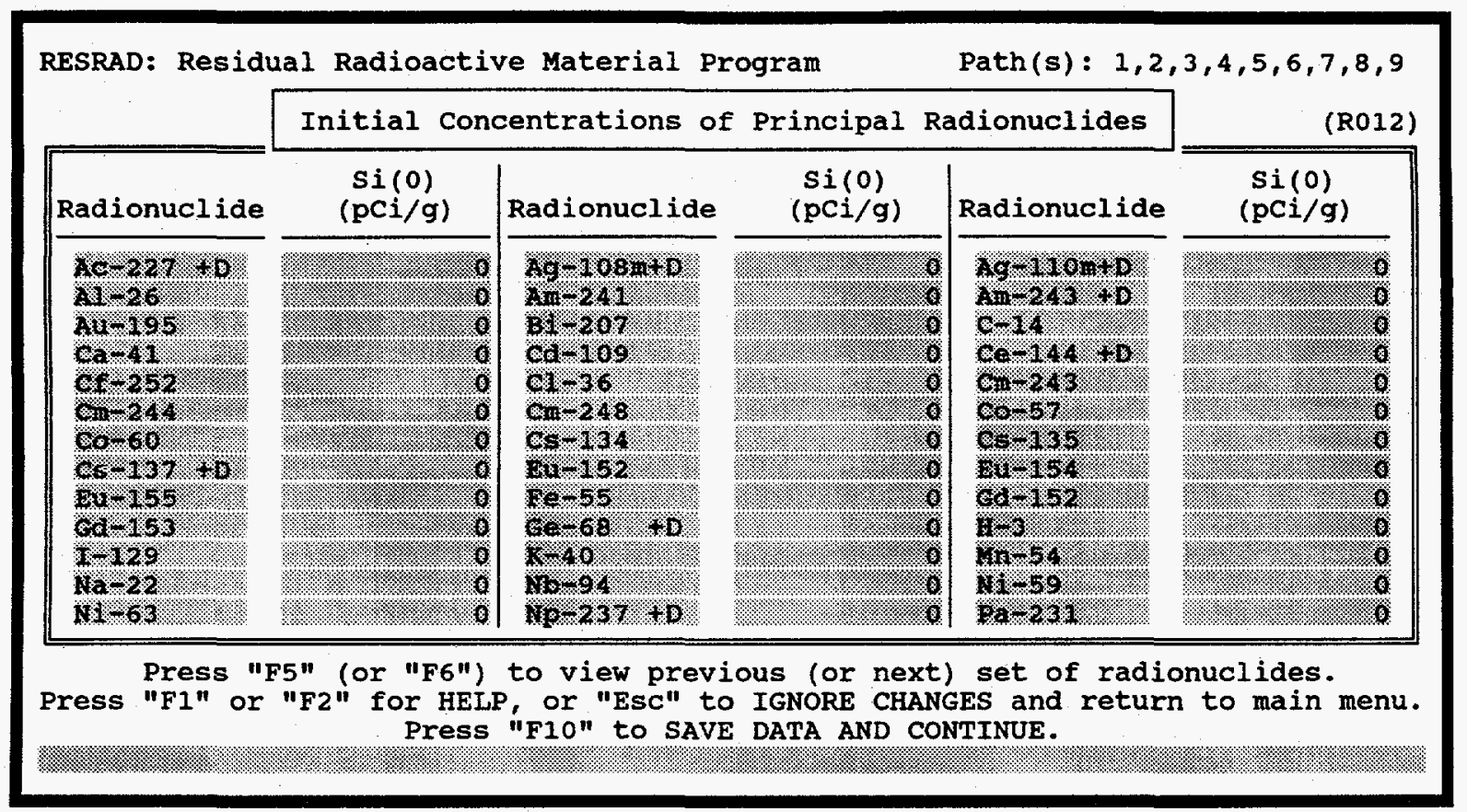

FIGURE 4.9 Input Form R012 for Initial Concentrations of Principal Radionuclides

\begin{tabular}{|c|c|c|c|c|c|c|}
\hline \multirow[t]{2}{*}{ RESRAD: Re } & \multicolumn{6}{|c|}{$\operatorname{Path}(s): 1,2,3,4,5,6,7,8,9$} \\
\hline & \multicolumn{5}{|c|}{ Initial Concentrations of Principal Radionuclides } & (R012W) \\
\hline Radionuclide & $\begin{array}{l}\operatorname{si}(0) \\
(\mathrm{pCi} / \mathrm{g})\end{array}$ & $\begin{array}{l}\text { wi }(0) \\
(p c i / L)\end{array}$ & & Radionuclide & $\begin{array}{l}\operatorname{si}(0) \\
(p C i / g)\end{array}$ & $\begin{array}{l}\text { Wi (0) } \\
(\mathrm{pCi} / \mathrm{L})\end{array}$ \\
\hline $20-227 / 10$ & 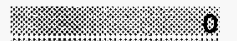 & & 8 & 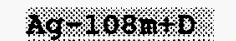 & $7^{2}$ & (6) \\
\hline 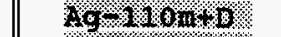 & \% & 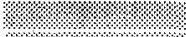 & 8 & $\mathrm{~A}+\mathrm{l}=\mathrm{6}$ & i & ঋ: \\
\hline An 2848 & $1 \%$. & (. & 0 & 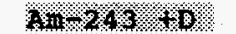 & / & 0 \\
\hline $282=199.5$ & $1 \% .2 \%$ & \%. & 0 & 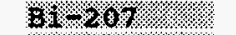 & 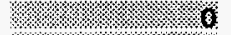 & 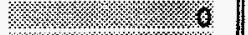 \\
\hline c. $11 \%$ & 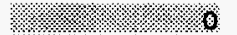 & & 0 & ca $-4:$ & 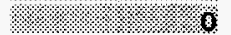 & \%: \\
\hline Ka $1009:=2:$ & ?/: & & 0 & een $144: 40$. & / & 3.2. \\
\hline $8+252$. & 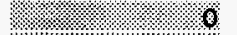 & 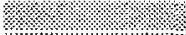 & 0 & el. .36 . & $1 / .8 .8$. & 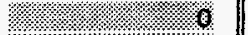 \\
\hline $\operatorname{lom}=243.3$. & ?.8. & Y: & 0 & Cut $-2 / 44: 4$. & 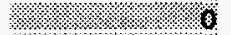 & ? \\
\hline cur 248. & 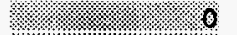 & 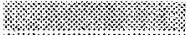 & 0 & 60.57. & 8 & l: \\
\hline \%. $60.0 .=1 .:$ & \%.: & স / & 0 & $C_{s}-13_{3} x_{1}=$ & \% & 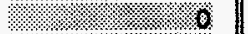 \\
\hline es-135. & 3.9. & //. & 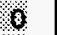 & $0-1.37 \%=0$ & 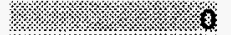 & 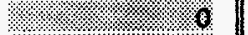 \\
\hline 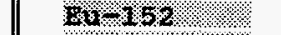 & 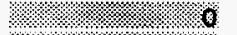 & ‥ & 0 & Eut $15 \mathrm{su}$. & 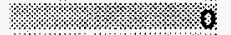 & (1.: \\
\hline 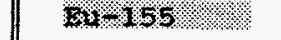 & 10.0 .0 & 2: & 0 & 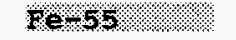 & / & $\sqrt[3]{3}=1 / 8$ \\
\hline $\begin{array}{l}\text { Press "F5 } \\
\text { Press "Fl" or }\end{array}$ & $\begin{array}{r}\text { " (or "F6") } \\
\text { F2" for HEL } \\
\text { Press }\end{array}$ & $\begin{array}{l}\text { view pre } \\
\text { or "Esc" } \\
10 \text { " to SA }\end{array}$ & $\begin{array}{l}\text { to } \\
\text { AVE }\end{array}$ & $\begin{array}{l}\text { Jus (or next) } \\
\text { IGNORE CHANC } \\
\text { DATA AND COI }\end{array}$ & $\begin{array}{l}\text { set of radi } \\
\text { IS and retur } \\
\text { [INUE. }\end{array}$ & $\begin{array}{l}\text { luclides. } \\
\text { to main }\end{array}$ \\
\hline
\end{tabular}

FIGURE 4.10 Input Form R012W for Initial Soil and Groundwater Concentrations of Principal Radionuclides 
derived by using RESRAD5 are listed alphabetically on the forms. More isotopes exist than can be accommodated in a single screen, however. The F5 and F6 function keys are used to go forward or backward in the list of radionuclides. Soil guidelines are calculated only for those isotopes with nonzero initial (zero survey time) soil concentrations. Nonzero groundwater concentrations are allowed only for radionuclides with nonzero initial soil concentrations. (These input groundwater concentrations should be measured at the same time as the soil concentrations, that is, during a radiological survey.) If a nonzero groundwater concentration is input for a radionuclide, iterations are performed to find values for the corresponding water/soil distribution coefficients. Water/soil distribution coefficients are discussed in Appendix $\mathrm{H}$.

The term principal radionuclide is an important concept to remember when using RESRAD. A principal radionuclide is a radionuclide with a half-life longer than one-half year. ${ }^{9}$ An associated radionuclide is a decay product with a half-life of one-half year or less. The radionuclides "associated" with a principal radionuclide consist of all decay products down to, but not including, the next principal radionuclide in the chain. It is assumed that all associated radionuclides are in secular equilibrium with their principal radionuclide in the contaminated zone and also at the location of human exposure.

All radionuclides in a decay chain are taken into account in calculating ingrowth and decay; however, the associated radionuclide concentrations are set equal to their principal radionuclide concentrations for the purpose of calculating the mixture sums. $A$ " $+D$ " suffix on a principal radionuclide in Forms R012 or R012W indicates that all associated progeny in the decay chain will be included in the dose calculations.

The single radionuclide soil guidelines do not depend on the soil concentrations; even if the concentrations are not known, values for these quantities can be obtained by entering any nonzero concentration. ${ }^{10}$ The mixture sums, however, depend on the soil concentrations; thus, calculated mixture sum values are valid only if the concentrations are known.

9 This differs from the one-year definition used in RESRAD3.

${ }^{10}$ If a nonzero groundwater concentration is input to derive the water/soil distribution coefficient, the actual soil concentration should be used (see Appendix $H$ ). 
If the contaminated zone is treated as a single homogeneous contaminated zone, the mixture sum should be calculated by using the radionuclide concentrations averaged over a compact $100-\mathrm{m}^{2}$ area or one-third of the peak radionuclide concentrations within the same area, whichever gives the largest mixture sum. A $100-\mathrm{m}^{2}$ area that gives the largest mixture sum should be used if the area of the contaminated zone is larger than $100 \mathrm{~m}^{2}$. The entire contaminated zone area should be used if this area is less than $100 \mathrm{~m}^{2}$ but greater than $1 \mathrm{~m}^{2}$. If the contaminated zone area is less than $1 \mathrm{~m}^{2}$, peak radionuclide concentrations should be used.

If the contaminated zone is treated as an inhomogeneous contaminated zone, then it may be divided into subzones; within each subzone, the peak concentration does not exceed the average concentration by a factor larger than three. The mixture sums for these subzones are then calculated as if each subzone were an isolated, homogeneous contaminated zone. The total of the mixture sums for subzones within a $100-\mathrm{m}^{2}$ area plus the average contribution from the ambient zone (that part of the contaminated zone lying outside the $100-\mathrm{m}^{2}$ area) must be less than 1 for any $100-\mathrm{m}^{2}$ area within the contaminated zone.

The current version of RESRAD calculates the mixture sum only for a single homogeneous zone or subzone. For an inhomogeneous contaminated zone, the subzone and ambient zone contributions must be summed separately according to the procedures described in Section 3.3.

The number of principal radionuclides that can be run simultaneously by RESRAD is limited only by the amount of random access memory available in the user's computer. The code will issue an error message during execution if this memory limit has been exceeded.

\subsubsection{Cover and Contaminated Zone Hydrological Data}

Input Form R013, as shown in Figure 4.11, is used to access cover and contaminated zone hydrological data. Data definitions and information for determining appropriate site-specific values are given in Appendix E. The Density of Cover Material and Cover Erosion Rate appear on the form only if the Cover Depth is not zero. 




\section{FIGURE 4.11 Input Form R013 for Cover and Contaminated Zone Hydrological Data}

The Cover Depth is the distance, in meters (m), from the ground surface to the location of the uppermost soil sample with radionuclide concentrations that are clearly above background.

The bulk density of dry soil, specified in grams per cubic centimeter $\left(\mathrm{g} / \mathrm{cm}^{3}\right)$, should be used for the Density of Cover Material and the Density of Contaminated Zone. A default value of $1.5 \mathrm{~g} / \mathrm{cm}^{3}$ can be used for preliminary estimates.

The Cover Erosion Rate and Contaminated Zone Erosion Rate are the rates, in meters per year ( $\mathrm{m} / \mathrm{yr})$, at which soil is removed by erosion. They can be estimated by means of the universal soil loss equation described in Appendix A. A default value of $0.001 \mathrm{~m} / \mathrm{yr}$ is used for screening estimates. The contaminated zone erosion rate is only significant if and when the cover depth becomes zero.

The Humidity in Air entry field will appear only if a nonzero tritium concentration was entered. The special model for tritium is discussed in Appendix L, which includes a map of average U.S. humidity values. 
All other parameters on Form R013 are discussed in Appendix E. Typical values for various soil materials are presented in Tables E.1 through E.7.

\subsubsection{Saturated Zone Hydrological Data}

Input Form R014, shown in Figure 4.12, is used to access saturated zone hydrological data. The definitions and information for determining appropriate values are given in Appendix E. Parameters with special consideration are listed below.

The Water Table Drop Rate parameter is the rate, in meters per year ( $\mathrm{m} / \mathrm{yr})$, that the depth of the water table decreases. If the water table drop rate is not zero, the unsaturated zone thickness will be created or increased. The saturation of this newly created unsaturated zone is estimated by the hydrological parameters of the saturated zone. The Saturated Zone b Parameter appears on the form only if the water table drop rate is greater than zero.

The NonDispersion or Mass Balance parameter selects which of two models will be used for water/soil concentration ratio calculations. A " 0 " (the default) selects the ND model, and " 1 " selects the MB model. The MB model is not recommended for contaminated zones with an area greater than $1,000 \mathrm{~m}^{2}$. The ND model can be used for an area of any size.

RESRAD: Residual Radioactive Material Program

Saturated Zone Hydrological Data

Path(s): $1,2,3,4,5,6,7,8,9$ (R014)

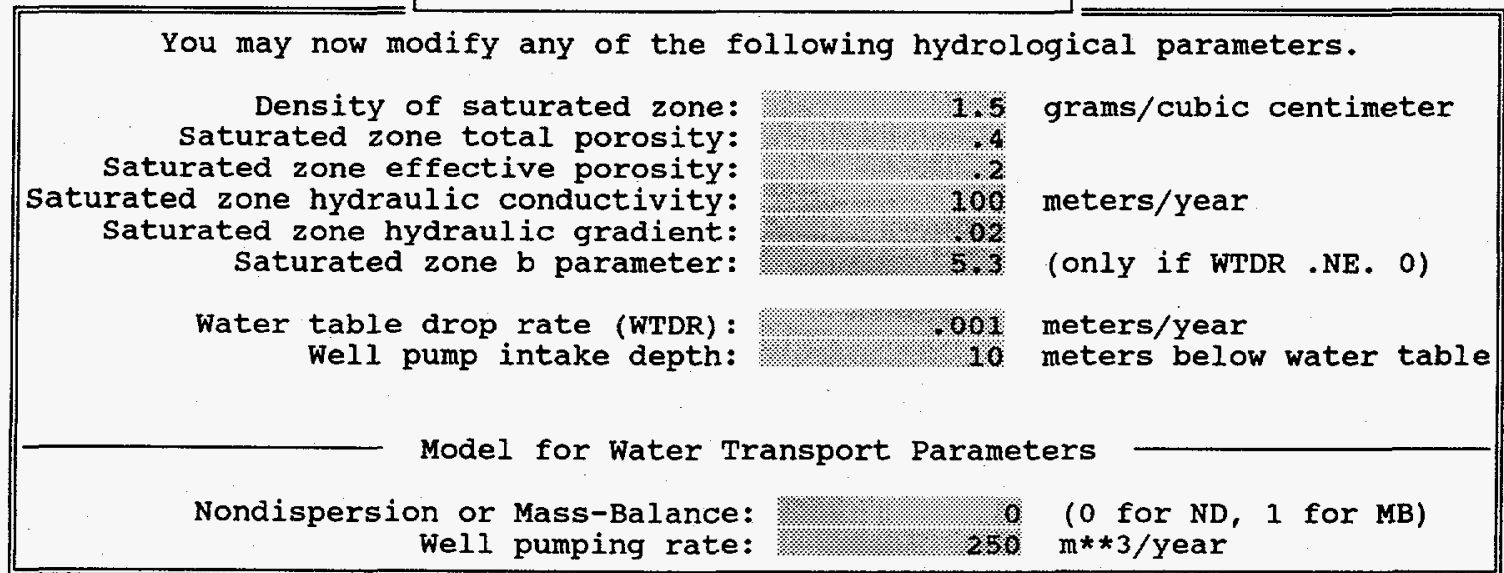

Press "F1" or "F2" for HELP, or "Esc" to IGNORE CHANGES and return to main menu. Press "F9" for Sensitivity Analysis. Press "F10" to SAVE DATA AND CONTINUE. \%.

FIGURE 4.12 Input Form R014 for Saturated Zone Hydrological Data 


\subsubsection{Uncontaminated and Unsaturated Strata Hydrological Data}

The uncontaminated and unsaturated zone is the portion of the uncontaminated zone that lies below the bottom of the contaminated zone and above the groundwater table. The code has provisions for up to five different horizontal strata within this zone. Each stratum is characterized by six radionuclide-independent parameters: thickness, soil density, total porosity, effective porosity, soil-specific b parameter, and hydraulic conductivity. Input Form R015 for these data is shown in Figure 4.13.

The Well Pumping Rate parameter is the total volume of water obtained annually from the well for use by humans and livestock and for agricultural and other purposes. The default value is $250 \mathrm{~m}^{3} / \mathrm{yr}$.

Data must be entered for each stratum used in the calculation. Entering a nonzero thickness for a stratum activates that stratum and, similarly, changing the thickness to zero deletes the stratum. Default values are supplied by the code for all parameters of an active stratum; however, the use of true site-specific data is strongly recommended. Data for estimating hydrological parameters for different soil types are given in Appendix E.

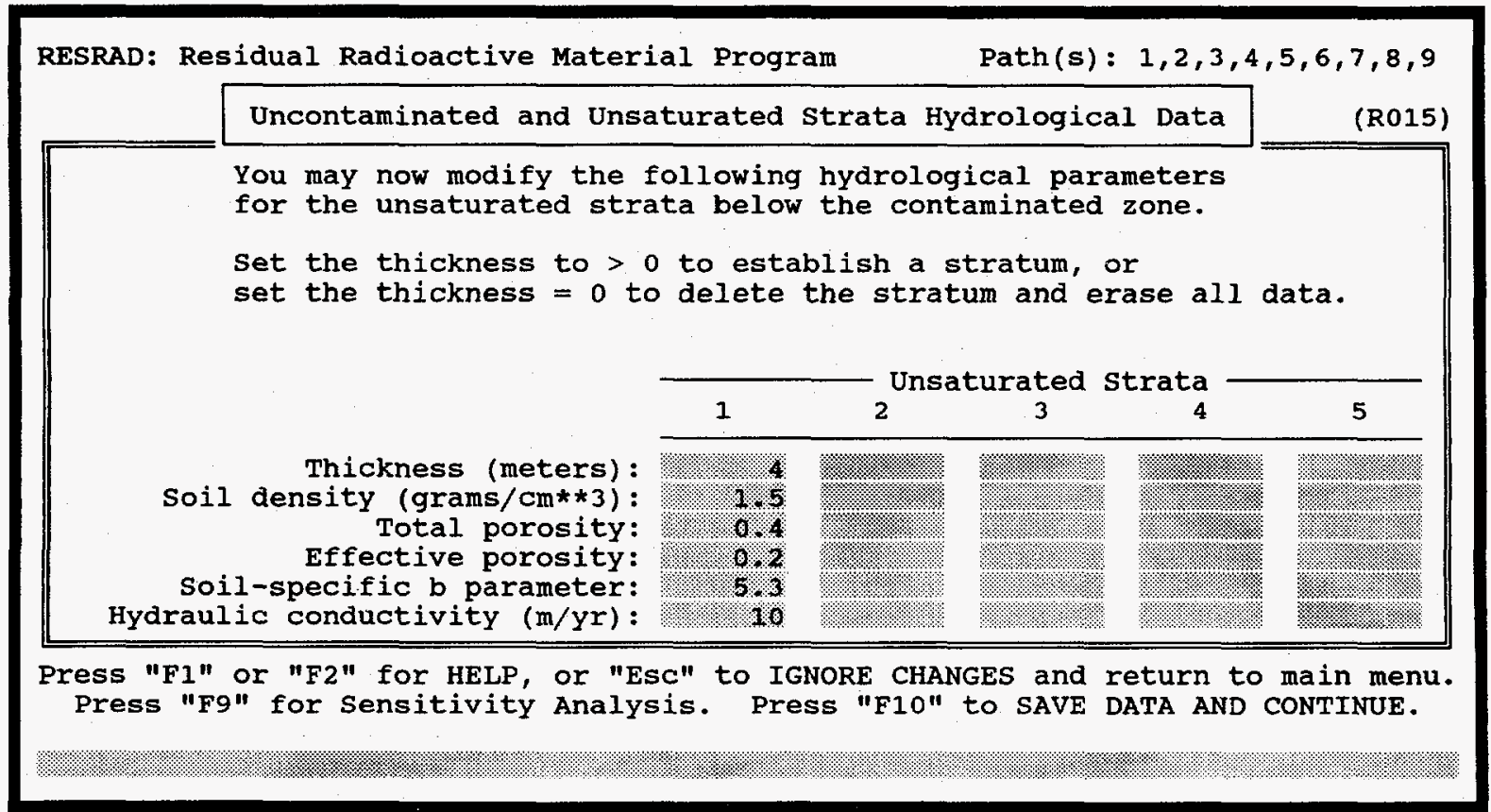

FIGURE 4.13 Input Form R015 for Uncontaminated and Unsaturated Strata Hydrological Data 


\subsubsection{Distribution Coefficients and Leach Rate Constants}

Input Form R016, shown in Figure 4.14, is used to access distribution coefficients and leach rate constants. If the radionuclide leach rate constants in the contaminated zone are known, these leach rate constants should be entered in the Leach Rate fields. The leach rate constant is defined as the fraction of available radionuclide leached out per year (Appendix E). If a Leach Rate is entered (i.e., $>0$ ), it will be used to calculate the leaching of radionuclides from the contaminated zone; the distribution coefficient will be calculated according to Equation E.3. Input values of distribution coefficients from the same screen will be replaced with the calculated values as long as they are within a reasonable range, that is, $>0$. However, if the calculated distribution coefficients are negative, which may result from entering an inappropriate number for the input leach rate, then the nonzero input leach rate will be neglected during RESRAD calculations; this leads to the same result as if the input value were 0 . If a Leach Rate is not entered (i.e., $=0$ ), the program will calculate the leach rate by using the distribution coefficient for the contaminated zone. In addition to the on-site nuclides, Form R016 allows parameters to be entered for decay product principal radionuclides. A "*" prefix is added to the symbol of all principal radionuclides present as decay products only.

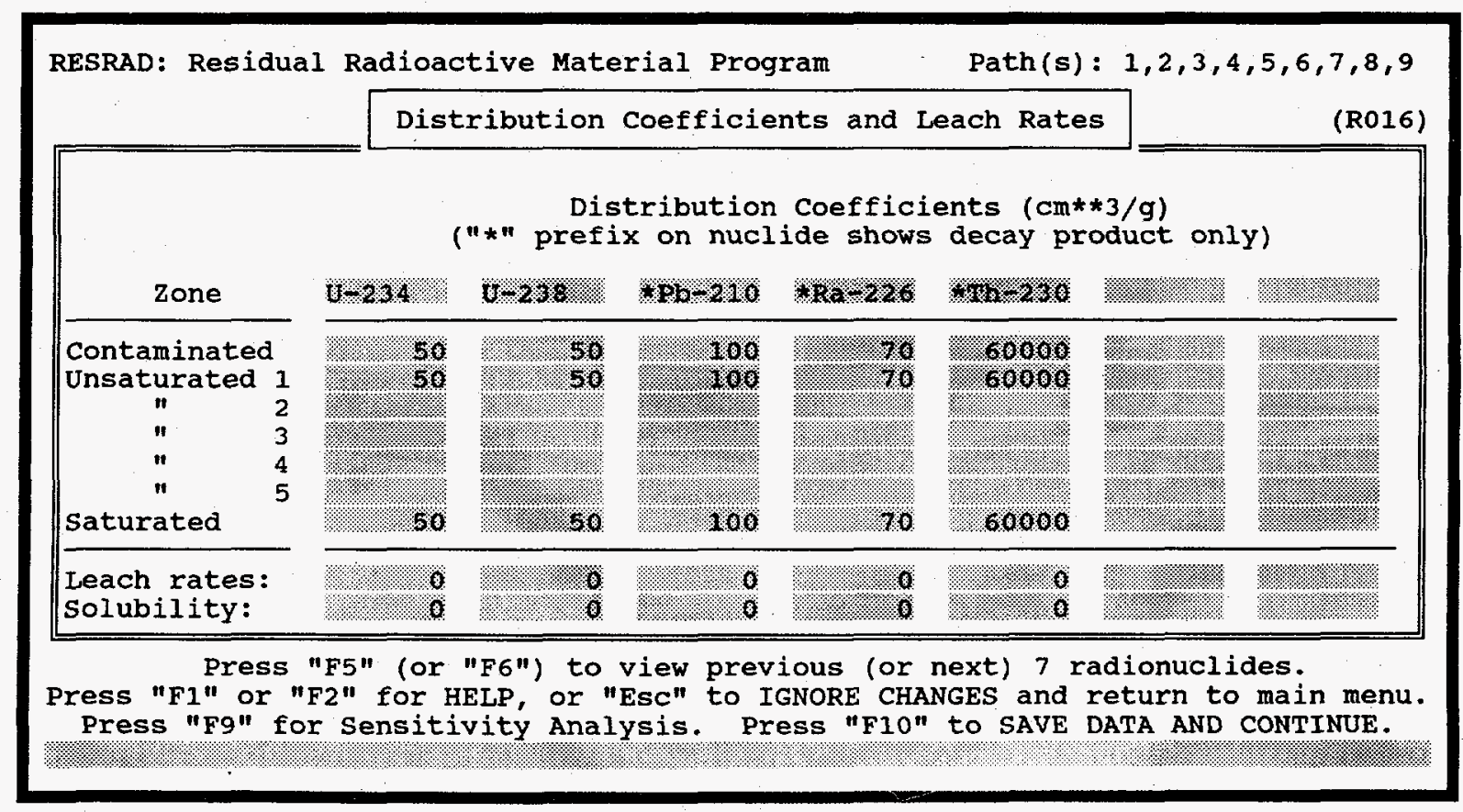

FIGURE 4.14 Input Form R016 for Distribution Coefficients and Leach Rates 
The solubility limit - the aqueous equilibrium solubility concentration - of the nuclide in the pore solution of the contaminated zone can also be used to calculate the distribution coefficient (Appendix $\mathrm{J}$ ). If the solubility limits are known or have been estimated, these values $(\mathrm{mol} / \mathrm{L})$ can be entered in the solubility field. A nonzero value of the solubility limit will be used to compute the distribution coefficient. The computed distribution coefficients will override the input distribution coefficients if they are positive. The input solubility values will be ignored if they result in negative distribution coefficients. In this case, the distribution coefficients entered in the first seven rows of Form R016 will be used.

The input form displays lines for the contaminated and saturated zones and for each unsaturated stratum below the contaminated zone with a nonzero thickness. Default distribution coefficients are provided automatically by the code for most nuclides. These values should be used with care, however, because site-specific distribution coefficients can vary over many orders of magnitude depending on the soil type, $\mathrm{pH}$, redox potential, and the presence of other ions. Replacement of the default values with site-specific data is always recommended. In addition to the default values for distribution coefficients, generic values of these parameters can be calculated by using the plant/soil concentration ratios. Whenever a value of -1 is chosen by the user, this option will be invoked, and either the default plant/soil concentration ratio or a user input value will be used for the calculation. The default plant/soil concentration ratio can be changed by first choosing "DOSE FACTORS" from the main menu and then choosing "Food Transfer Factors" from the submenu. Tables of generic distribution coefficients for various nuclides are given in Appendix E. A detailed discussion on the distribution coefficients is provided in Appendix $H$ and the RESRAD Data Collection Handbook (Yu et al. 1993).

As discussed in Appendix $\mathrm{H}$, it is anticipated that the user will utilize only one of the four optional methods available for computing distribution coefficients. However, if the user provides input values for more than one option, the following order of preference will be followed by the code in selecting the input to be used: groundwater concentration measured at the same time as the soil concentration first, solubility limit in the contaminated zone pore solution second, leach rate constant third, and plant/soil concentration correlation fourth. In this case, if the input with the highest preference fails to produce a nonzero value for the 
partition coefficient, the code will directly use the distribution coefficients in the first seven rows of the input screen.

Seven radionuclides are displayed on the screen simultaneously, but the user can page through all the radionuclides, seven at a time, by pressing the F5 (previous set) or F6 (next set) key. If fewer than seven principal radionuclides are active, the F5 and F6 keys will have no effect. Also, the instruction line for F5 and F6 near the bottom of the screen will be blanked out.

The on-site principal radionuclides can be changed by using Form R012, which is obtained as an option on the Modify Data submenu. The associated decay product principal radionuclides are automatically added to Form R016. The number of unsaturated strata can be changed by using Form R015, which is obtained by an option on the Modify Data submenu.

\subsubsection{Dust Inhalation and External Gamma Parameters}

Input Form R017 for external gamma and dust inhalation parameters is shown in Figure 4.15. With one exception, the values of these parameters, which are applicable in the long term, are not strongly site dependent; thus, in most cases, the generic default values can be used. The F2 help file should be used for additional information.

The one parameter that is site specific is the Shape Factor (External Gamma). This parameter, FS1, plays a quadruple role. Setting it to zero will turn off the external ground pathway entirely. If FS1 is greater than zero, it serves as a flag to use the dose conversion factors and environmental transport factors from Appendix A (Table A.1 and Equation A.1). In addition, if FS1 does not equal 1.0, it is the shape factor for an irregularly shaped contaminated zone. (See Appendix A and the data collection handbook [Yu et al. 1993] for instructions on calculating the shape factor for an irregularly shaped contaminated zone.)

If FS1 is less than zero, the parameter is not used; instead, it serves as a flag to use the new optional SOILD routine (Chen 1991) for the external ground pathway. If FS1 is less than zero but not equal to -1.0 , the flag also shows an irregularly shaped contaminated zone. The shape factor used by SOILD, in this case, is calculated from the 12 annular area fractions that appear on Form R017 only when FS1 is less than 0 but not equal to -1 . 


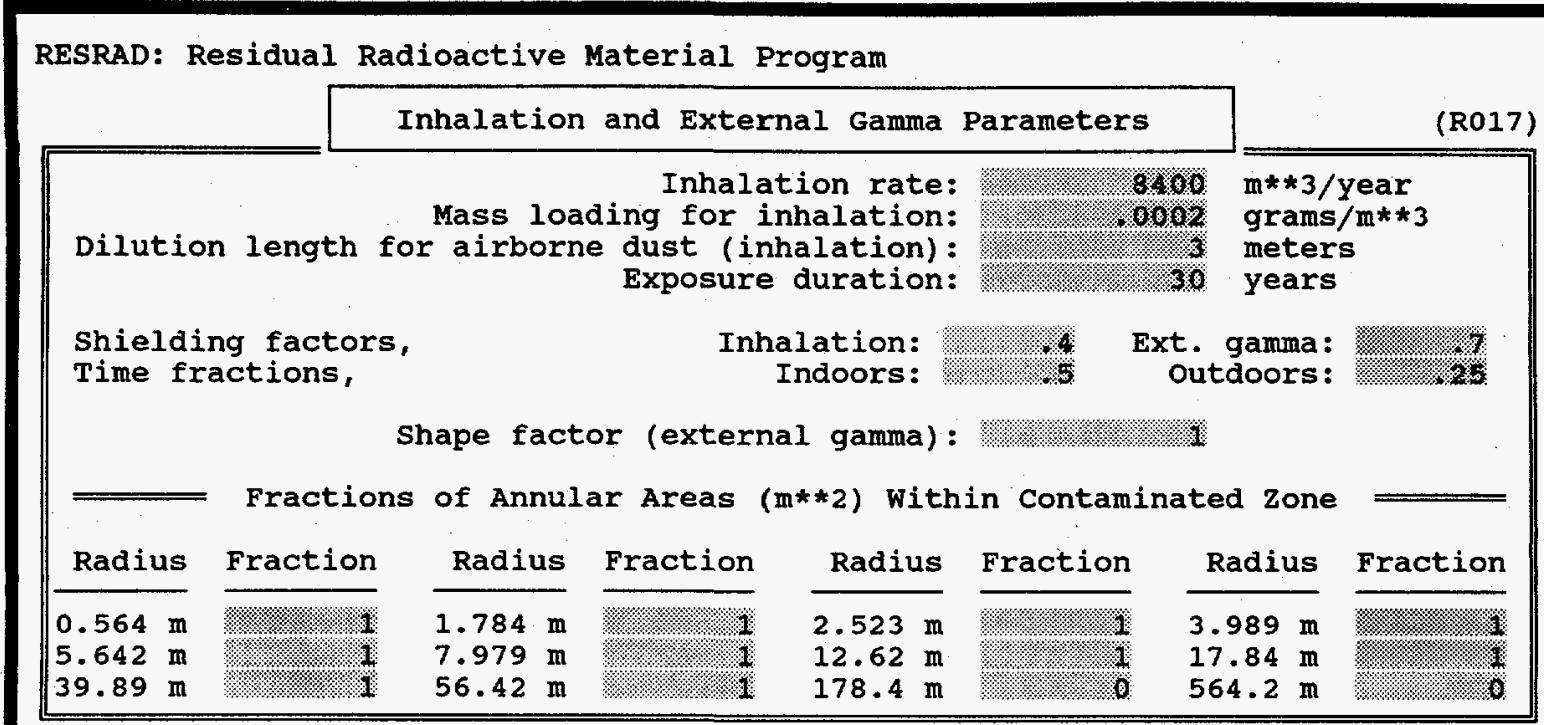

Press "F1" or "F2" for HELP, or "Esc" to IGNORE CHANGES and return to main menu. Press "F9" for sensitivity Analysis. Press "F10" to SAVE DATA AND CONTINUE.

FIGURE 4.15 Input Form R017 for Dust Inhalation and External Gamma Parameters

The Fractions of Annular Areas within Contaminated Zone parameter consists of the 12 area fraction fields that will appear on this form only if the Shape Factor (External Gamma) parameter has been set to a negative value (to use SOILD) but not equal to -1.0 (to show an irregularly shaped contaminated zone). To estimate the fraction of each annular area contained within the irregularly shaped contaminated zone, a circle should be drawn to enclose the contaminated zone. The user should then envision 12 concentric circles emanating from the contaminated zone with radii as listed on Form R017. The fractions of each annular area contained within the contaminated zone are the values to be entered. The values displayed in Figure 4.15 correspond to the proper values for the RESRAD.DEF case, that is, a circular contaminated zone of $10,000 \mathrm{~m}^{2}$.

\subsubsection{Ingestion Pathway Data, Dietary Parameters}

Input Form R018 for the ingestion pathway is shown in Figure 4.16. The default parameter values have been chosen to correspond to national averages. The parameters in the top half of the screen are not strongly site dependent; thus, use of the generic default 


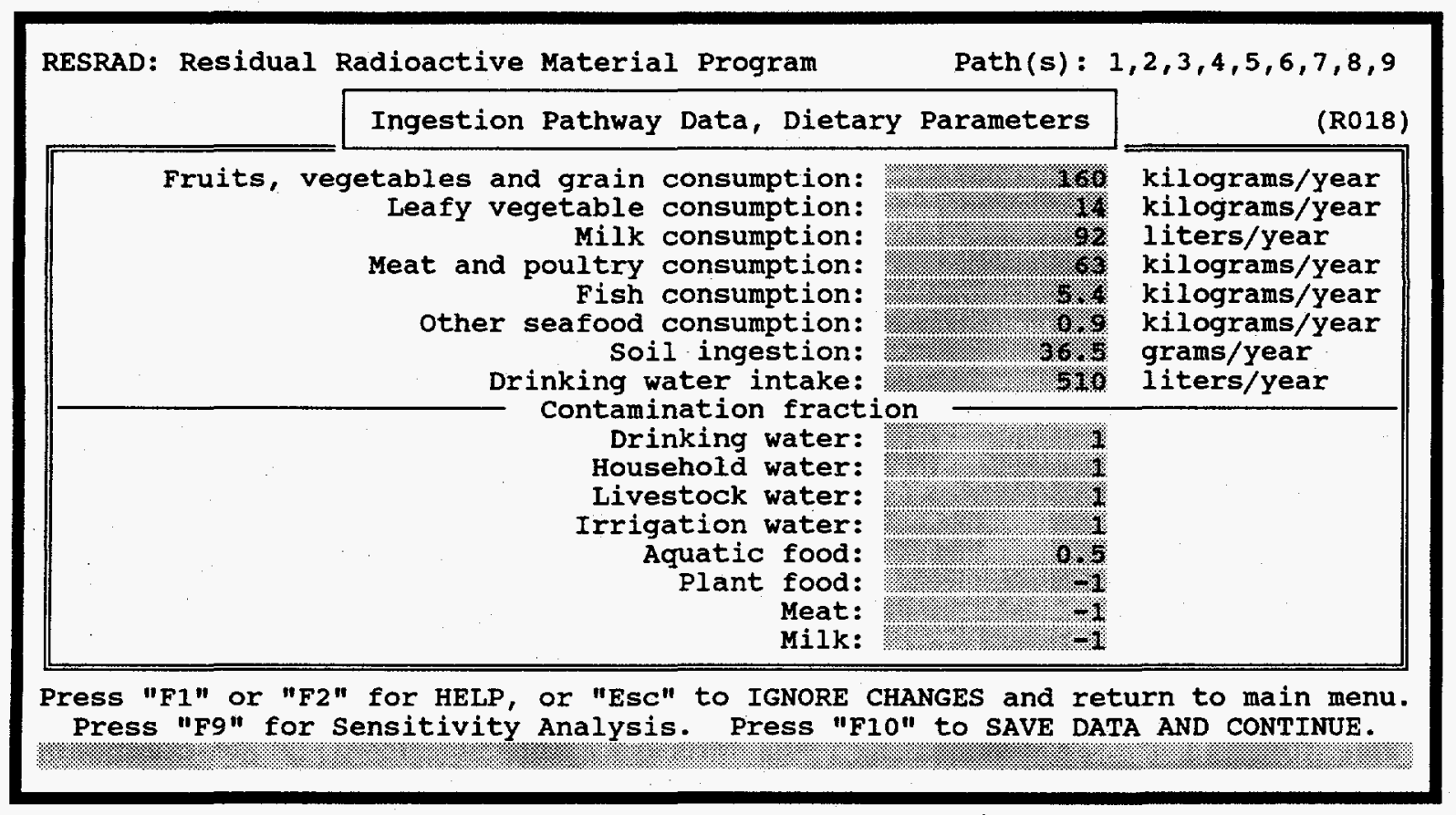

\section{FIGURE 4.16 Input Form R018 for Ingestion Pathway Data, Dietary Parameters}

values is always acceptable. Adjustments for regional differences in diet may sometimes be appropriate.

The Contamination Fraction of Drinking, Household, Livestock, and Irrigation Water parameters allow specification of the fraction of water used that originates from the contaminated site. Off-site water is assumed to be uncontaminated. Defaults for the fractions are 1.0 (100\% of water is contaminated).

The default Contamination Fraction for aquatic food is 0.5 , assuming that half of the fish and other seafood are imported. For the last three pathways (plant, meat, and milk), the default Contamination Fraction is -1 , a flag indicating that RESRAD will automatically calculate the contaminated fraction of these food products using the area factor method discussed in Section D.2.1.2 of Appendix D. The user may override this calculation by entering a number between 0 and 1 that represents the fraction of produce, meat, or milk that is contaminated. 


\subsubsection{Ingestion Pathway Data, Nondietary Parameters}

Input Form R019 for the ingestion pathway is shown in Figure 4.17. The default parameter values have been chosen to correspond to the national averages. The parameters, other than the final four, are not strongly site dependent; thus, use of the generic default values is usually acceptable. Adjustments for regional differences in farming practices may sometimes be appropriate.

The four Groundwater Fractional Usage parameters (Drinking Water, Household Usage, Livestock Water, and Irrigation), are included primarily to allow for groundwater (well or spring) and surface water (pond or river) scenarios. Thus, the fractions will usually be set at 1 or 0 . A value of 1 selects $100 \%$ groundwater usage; 0 selects $100 \%$ surface water. The default for all four parameters is 1 . The fractions of contaminated water, that is, from the site, can be varied via the parameters in Form R018.

Special parameters that are available only if carbon-14 is selected as a contaminant may be accessed by pressing the "F5" key. Figure 4.18 shows the carbon-14 submenu parameters. A detailed discussion of these parameters is included in Appendix L.

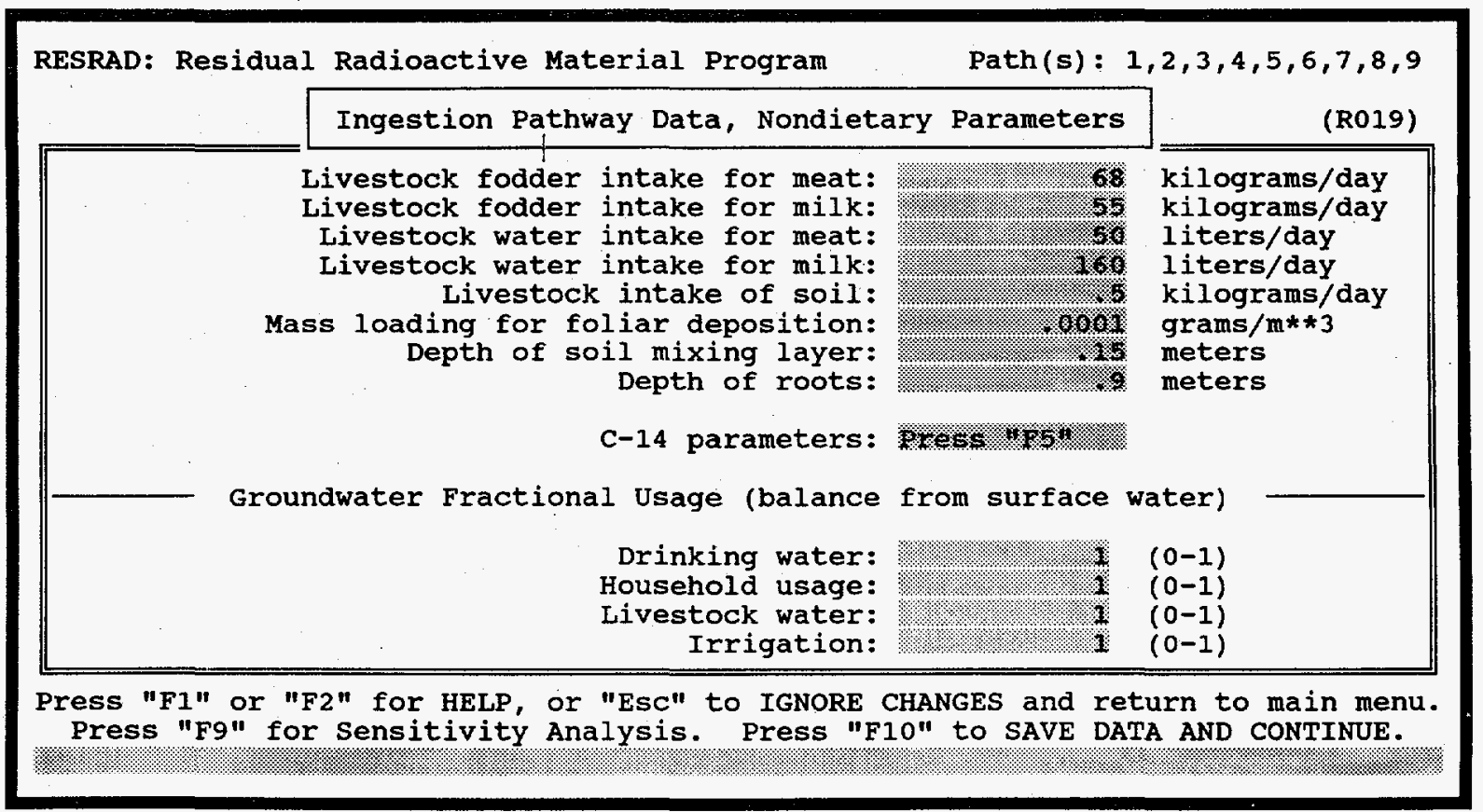

FIGURE 4.17 Input Form R019 for Ingestion Pathway Data, Nondietary Parameters 


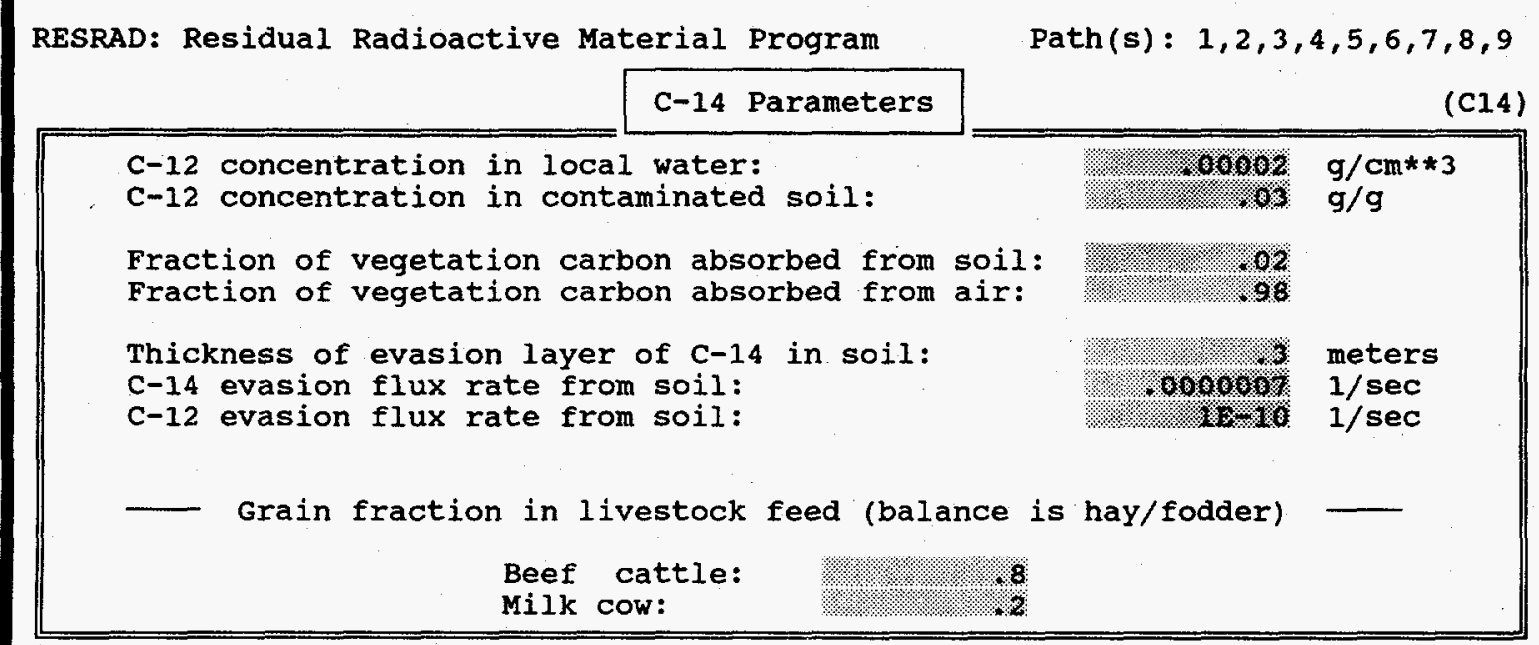

Press "F1" or "F2" for HELP, or "ESC" to IGNORE CHANGES and return to main menu. Press "F9" for sensitivity Analysis. Press "F10" to SAVE DATA AND CONTINUE.

\section{FIGURE 4.18 Input Form C14 for Carbon-14 Parameters}

\subsubsection{Radon Pathway}

The radon pathway is discussed in detail in Appendix C. Inhalation dose contributions from both radon-222 (parent principal radionuclides uranium-238, uranium-234, thorium-230, and radium-226) and radon-220 (parent principal radionuclides thorium-232, radium-228, and thorium-228) are included. The pathway model considers both outdoor radon exhalation from the ground surface and indoor radon exposure from a house built on-site. The indoor dose calculations are made on the assumption that the house is built in the current year. Input Form R021 for the radon pathway is shown in Figure 4.19. All cover material fields will be blank and inaccessible if the contaminated zone does not have a clean cover zone. The data fields are described below. The thickness and density of the cover material are displayed on Form R021 solely for user convenience. Form R013 must be used to change these parameters.

Thickness is the average thickness of the cover material and the building shell structure in the subsurface of the soil. A typical value for the building foundation thickness is about $0.15 \mathrm{~m}$, which is the RESRAD default value. 


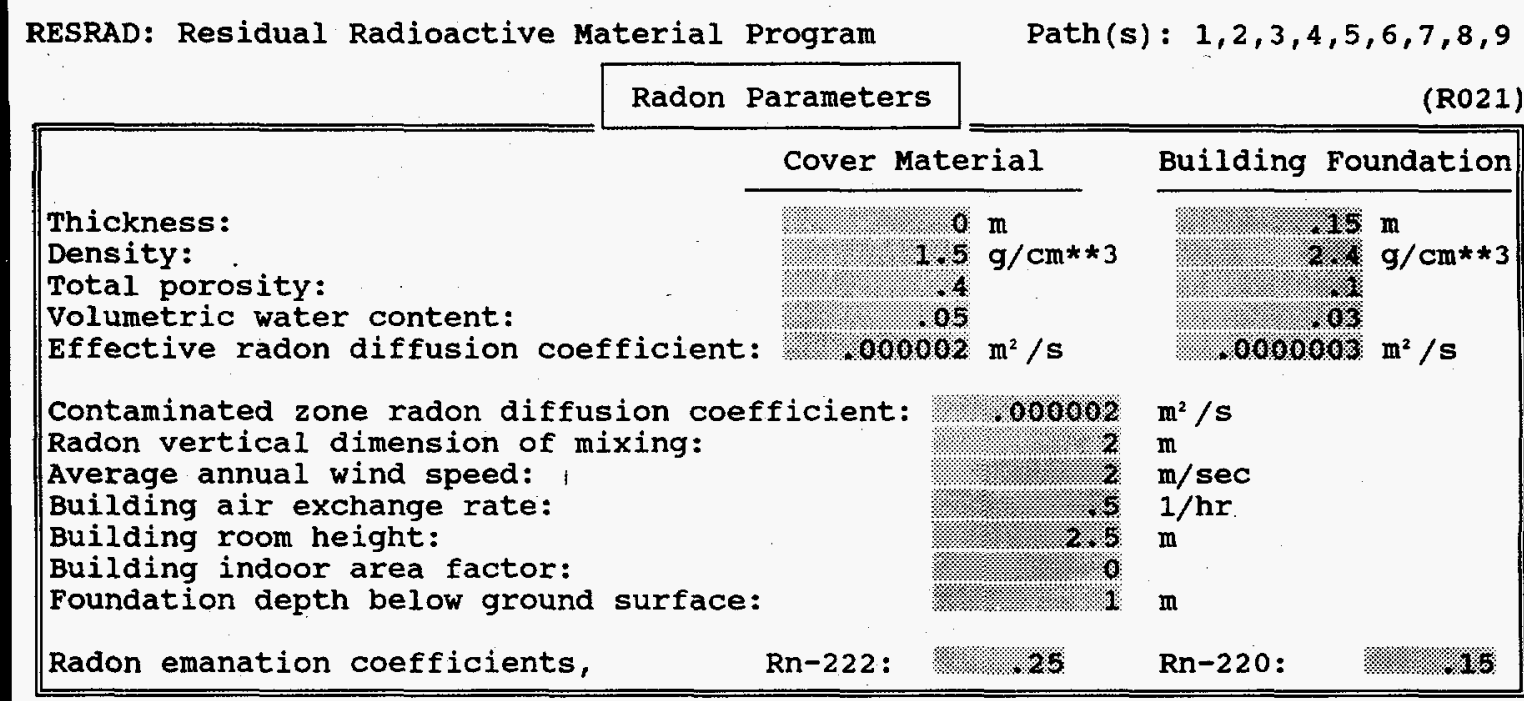

Press "F1" or "F2" for HELP, or "Esc" to IGNORE CHANGES and return to main menu. Press "Fg" for sensitivity Analysis. Press "F10" to SAVE DATA AND CONTINUE.

(1.

\section{FIGURE 4.19 Input Form R021 for Radon Parameters}

Bulk Density is the ratio of the solid phase mass to the total volume and is given in units of $\mathrm{g} / \mathrm{cm}^{3}$. These data fields should be differentiated from particle density, which is defined as the ratio of the solid phase mass to the volume of the solid phase. The bulk density is equal to the product of the particle density and $\left(1-p_{t}\right)$, where $p_{t}$ is the total porosity. Default values of 1.5 and $2.4 \mathrm{~g} / \mathrm{cm}^{3}$ are used in RESRAD for the cover material and building foundation, respectively.

Total Porosity is the ratio of the void space volume to the total volume of a porous medium. Typical ranges for the porosity values in unconsolidated deposits are 0.25 to 0.40 for gravel, 0.25 to 0.40 for sand, 0.35 to 0.50 for silt, and 0.40 to 0.70 for clay. For concrete, a typical total porosity is in the range of 0.10 to 0.15 . The default values are 0.4 for cover material and 0.1 for building foundation.

Volumetric Water Content in a porous medium is the ratio of the total volume of water present in the pore space to the total volume of the medium. For a water-saturated medium, the volumetric water content is equal to the total porosity. Values of volumetric water content depend on the degree of saturation and can range from very low to the value of the total porosity. The default values are 0.05 for cover material and 0.03 for building foundation. 
Effective Radon Diffusion Coefficient D (all zones) is the ratio of the radon flux across the pore area to the gradient of the radon concentration in the pore space. The dimensions of the coefficient are in units of length squared over unit of time $\left(1^{2} / T\right)$. In porous media, the radon diffusion coefficient is a property of the pore structure and the fluid present in the pores. Typical values of $D$ for radon in low moisture, undisturbed soils are in the range of $(3.0 \pm 1.5) \times 10^{-6} \mathrm{~m}^{2} / \mathrm{s}$. In RESRAD, a D default value of $2.0 \times 10^{-6} \mathrm{~m}^{2} / \mathrm{s}$ is adopted in both the contaminated zone and in the cover material. A default value of $3.0 \times 10^{-7} \mathrm{~m}^{2} / \mathrm{s}$ is used for the building foundation. If -1 is entered for a diffusion coefficient, RESRAD will generate a value of $D$ on the basis of the porosity and water content of the medium.

Radon Vertical Dimension of Mixing is the assumed height to which the radon emission from the ground surface is uniformly mixed in the outdoor air. The default value used in RESRAD is $2 \mathrm{~m}$.

Average Annual Wind Speed is the inverse of the overall average of the inverse wind speeds measured in a one-year period (i.e., the harmonic mean of the wind speeds in a year). The wind speed is given in units of length over time (l/T). A default value of $2 \mathrm{~m} / \mathrm{s}$ is used in RESRAD.

Building Air Exchange Rate (or ventilation rate) is the number of total volumes of air contained in the building that is being exchanged with outside air per unit of time. The rate is measured in units of $1 / \mathrm{T}$. For example, a building with a ventilation rate of $1(1 / \mathrm{h})$ has its volume of air replaced once each hour on the average. The exchange rate depends on the construction and operating features of the building as well as on environmental conditions. Typically in the United States, the average ventilation rate during seasons when houses are kept closed is in the range of 0.1 to 1.0 . A default value of $0.5(1 / \mathrm{h})$ is used in RESRAD.

Building Room Height is the average height of the living area of the building. Typical values are in the range of 2.2 to $3.0 \mathrm{~m}$. A default value of $2.5 \mathrm{~m}$ is used in RESRAD.

Building Indoor Area Factor or FAI is the fraction of the floor area built on the contaminated area. Values greater than 1.0 indicate a contribution from walls penetrating the contaminated zone. A default value of 0 is used in the code, which means the code will automatically calculate a time-dependent area factor on the basis of an assumed floor area of $100 \mathrm{~m}^{2}$ and the amount of wall area extending into the contaminated zone. For 
example, if the walls extend to a depth of $0.5 \mathrm{~m}$ into the contaminated zone, $\mathrm{FAI}=1+0.5^{*} 4 / \mathrm{SQRT}(100)$ or 1.2 . If the user inputs a value greater than zero, this input value will be used in the RESRAD calculation. The FAI is time dependent because of erosion and the assumption that the house is built in the current year.

Foundation Depth Below Ground Surface is the vertical distance in the soil from the very bottom of the basement floor slab to the ground surface. Typical values are in the range of 0.0 to $3.0 \mathrm{~m}$. A default value of $1 \mathrm{~m}$ is used in RESRAD. If a negative value is entered, the absolute value will be adjusted (if needed) so that the foundation depth will not extend into the contaminated zone. Thus, due to erosion of the cover and contaminated zones, the foundation depth could be time dependent and less than the (absolute) specified value. The indoor radon flux is always calculated on the assumption that the house is built in the current year.

The Radon Emanation Coefficient is the fraction of the total amount of radon gas generated by radium decay within the solid phase of the porous medium (the soil) that escapes from the solid particles and is ultimately entrapped within the pores. It is a dimensionless parameter and is represented as a decimal fraction. The radon emanation coefficient depends on the radon isotope being considered (either radon-222 or radon-220) and on many other soil-related factors such as the mineral composition of the soil, total porosity, particle size distribution, and moisture content of the medium. Observed values range from about 0.01 to 0.80 . For soils with a low moisture content, a typical value for radon-222 is about 0.25. In RESRAD5, a radon-222 default value of 0.25 is used for all mediums; similarly, a value of 0.15 is used for radon-220. The radon-222 or radon-220 field will be blank if there are no source principal radionuclides (i.e., uranium-238, uranium-234, thorium-230, and radium-226 for radon-222, and thorium-232, radium-228, and thorium-228 for radon-220). The radon pathway is automatically suppressed if there are no sources for radon isotopes.

\subsubsection{Set Graphics}

Graphic display of RESRAD output is dependent on many factors, most of them having to do with hardware. A series of five forms, R020, R0205, R0206, R0207, and R0208, is used to enter the graphics parameters. Form R020 is the most important and is the one 
that is first displayed when the Set Graphics option is selected from the main menu. After Form R020 has been completed, depending on the parameters entered, some of the other forms may be needed to complete the graphics setup. After a satisfactory set of parameters has been selected to match the hardware configuration, the Set Graphics option will seldom be used again.

All the graphics parameters are saved in a file named GRAPHICS.DAT; a complete working set is also supplied on the distribution diskettes called GRAPHICS.DEF. If RESRAD does not find GRAPHICS.DAT, a file of that name is created and GRAPHICS.DEF is copied into it. This is a good starting point, but the user's graphics hardware must be matched with a tailored file created from the forms described in Sections 4.6.4.1 through 4.6.4.5.

With two exceptions, all of the graphics parameters can be changed, and the results can be checked without rerunning RESMAIN. An initial Run RESRAD (see Section 4.6.5) must be made. After the return to RESPC, Set Graphics must be selected, the desired parameters must be changed, and the output viewed (see Sections 4.6.5 and 4.6.5.4). The two parameters that cannot be changed without rerunning RESMAIN are the graphics points per curve and the RAM disk drive, both of which are in Form R020. New values for these two parameters can be input, but they will not be used until the next execution of RESMAIN.

\subsubsection{Graphics Parameters}

Form R020 is shown in Figure 4.20. The "Press 'F5', 'F7', and 'F8'" lines will only appear if the appropriate parameters are selected in Form R020. The six parameters on the form are discussed below.

Graphics Points Per Curve determines the smoothness of the calculated RESRAD curves. If zero is selected, the graphics output is entirely disabled. This speeds up program execution, but it also precludes parameter sensitivity analysis. Normal choices are limited to $32,64,128$, or 256 points per curve. All plots with a 1.0 scale factor have an abscissa ( $x$-axis) $8.0 \mathrm{in}$. long. Thus the two extremes available translate to $8 / 31=0.258 \mathrm{in}$. and $8 / 255=0.031 \mathrm{in}$. between generated data points. Straight line segments connect the points. For most work, 32 or 64 points per curve is an adequate choice. The time required to generate the computer graphics is directly proportional to the number of points per curve. 


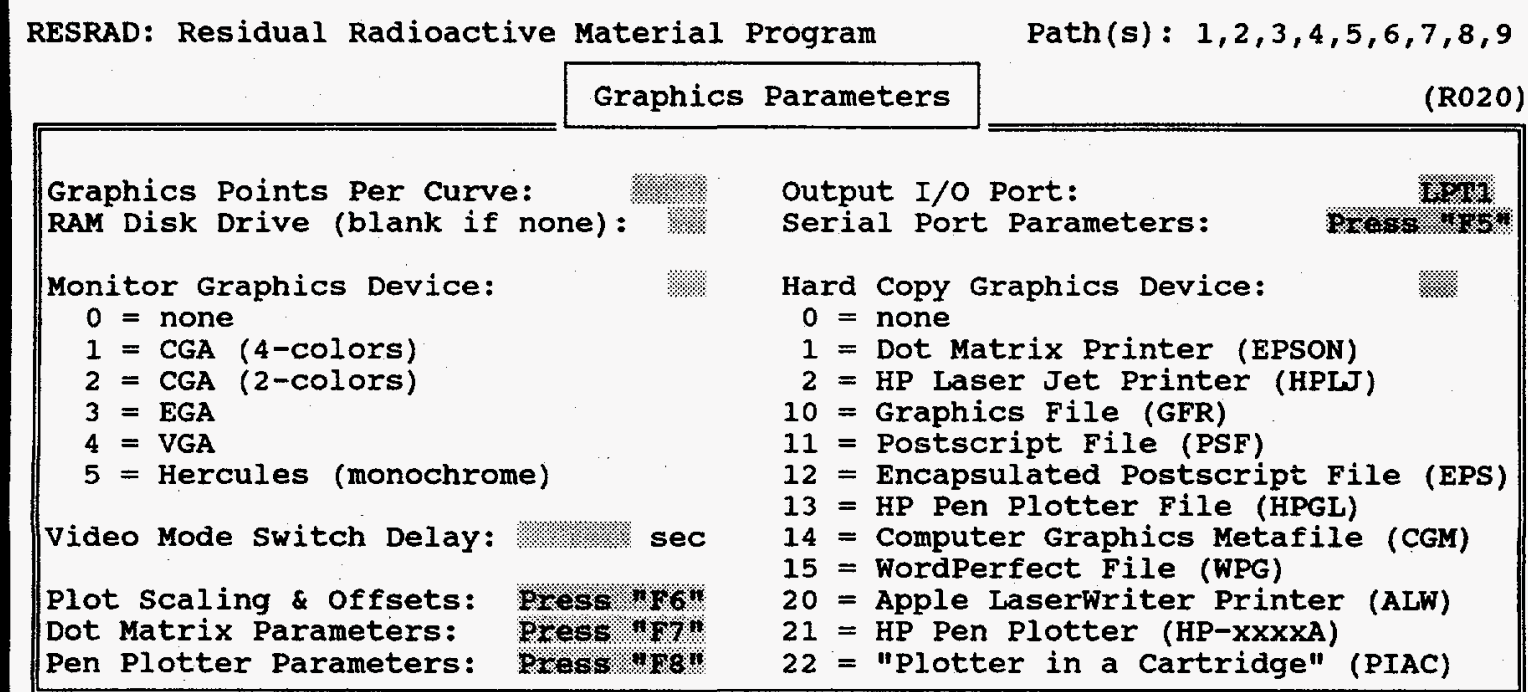

Press "F1" or "F2" for HELP, or "Esc" to IGNORE CHANGES and return to main menu. Press "F10" to SAVE DATA AND CONTINUE.

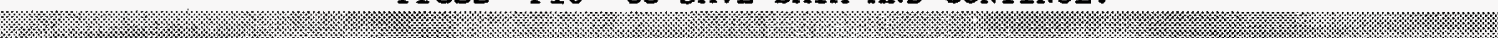

\section{FIGURE 4.20 Input Form R020 for Graphics Parameters}

Graphics I/O Port selects the port to be used for directly attached hard copy devices such as printers and pen plotters. The choices are limited to the following:

- COM1 - Primary serial port,

- COM2 - Secondary serial port,

- LPT1 - Primary parallel port, and

- LPT2 - Secondary parallel port.

If a COM port is selected, the "Serial Port Parameters: Press 'F5"' line will become visible. It is essential to check the settings on this form before exiting Form R020.

RAM Disk Drive selects the disk drive to be used for all RESRAD temporary files, including SUMMARY.REP, DETAILED.REP, INTRISK.REP, and all graphics files. If an extended memory RAM disk with about $1 \mathrm{MB}$ of free space is available, it can be selected by merely entering D:, E:, and so forth. If the field is left blank, all files will be written to the current directory. Use of a RAM disk will speed up all RESRAD operations. The disadvantage is that all such files will disappear if the power is turned off or the computer 
is rebooted. Even if a RAM disk is not used, a hard disk drive letter can be entered. In this case, all files will be written in the root directory of the designated drive.

MONITOR GRAPHICS DEVICE is the code number (0-5) for the computer monitor and graphics card combination. The choices are limited to the following:

0 - No graphics capability;

1 - IBM CGA, $320 \times 200$ pixels, 4 colors;

2 - IBM CGA, $640 \times 200$ pixels, 2 colors;

3 - IBM EGA, $640 \times 350$ pixels, 16 colors;

4 - IBM VGA, $640 \times 480$ pixels, 16 colors; and

5 - Hercules monochrome, $720 \times 348$ pixels.

Hard Copy Graphics Device is the code number (0-2, 10-15, or 20-22) for graphics hard copy. The devices supported are as follows:

0 - No graphics capability (or turned off),

1 - Attached dot matrix printer (Epson or IBM-compatible),

2 - Attached Hewlett-Packard (HP) LaserJet printer,

10 - Graphics File (RESRAD.GRF) for use with VECTOR program,

11 - Postscript File (RESRAD.PSF) for Apple Laser Writer or Postscript printer,

12 - Encapsulated Postscript File (RESRAD.EPS) for word processors,

13 - HP HPGL File (RESRAD.HPG) for HP pen plotters,

14 - Computer Graphics Metafile (RESRAD.CGM) for suitable graphics devices,

15 - WordPerfect Graphics File (RESRAD.WPG) for WordPerfect word processor,

20 - Attached Apple Laser Writer printer,

21 - Attached HP pen plotter (or compatible equivalent), and

22 - Plotter in a Cartridge (Pacific Data Products) in an HP LaserJet. 
If 1 or 21 is selected, the "F7 Dot Matrix Parameters" or "F8 Pen Plotter Parameter" line will become visible. The parameters in these submenus (R0207 and R0208) should be checked before exiting the Graphics Parameters Form (R020).

True immediate hard copy is only obtained with devices 20 through 22 . Delayed hard copy is obtained automatically with devices 1 through 2 via the VECTOR program. If a HP LaserJet is available, the addition of a Plotter in a Cartridge (code 22) is highly recommended. Code 22 plots are produced at least an order of magnitude faster than code 2 and with no loss in plot quality.

The file hard copy devices 10 through 15 may be useful in special situations. Devices 12, 14, and 15, however, are limited to only one plot frame per file. Additional information on devices 10 through 15 can be found in the "RESPLOT Data Files" section of RESRAD5.DOC, which is one of the supplementary files on the distribution diskettes.

Video Mode Switch Delay is the delay time between the switch from the graphics to the text mode. Most multiscanning monitors require a short time delay to adjust when switched between modes. The result is a brief but annoying period of screen jitters. If video jitter does not occur or if it is not troublesome, leave this parameter at the default value of zero. Otherwise, a delay of 0.75 seconds is recommended. The delay time merely blanks the screen for the specified period, which helps to conceal the jitters.

\subsubsection{Serial Port Parameters Submenu}

Form R0205 is shown in Figure 4.21. It is only accessible if a COM port is selected in the Graphics Parameters menu, Form R020, which in turn activates the F5 function key and the Serial Port Parameters display line. The hardware manual for the attached graphics device should be consulted for the proper parameter values.

\subsubsection{Plot Scaling and Offset Adjustments Submenu}

Form R0206 is shown in Figure 4.22. It is always accessible from the Graphics Parameters menu, Form R020, by pressing the F6 function key. The display varies depending on the current Hard Copy Graphics Device selection in Form R020. Changes to 
RESRAD: Residual Radioactive Material Program $\operatorname{Path}(\mathrm{s}): 1,2,3,4,5,6,7,8,9$

Serial Port Parameters Submenu (R0205)

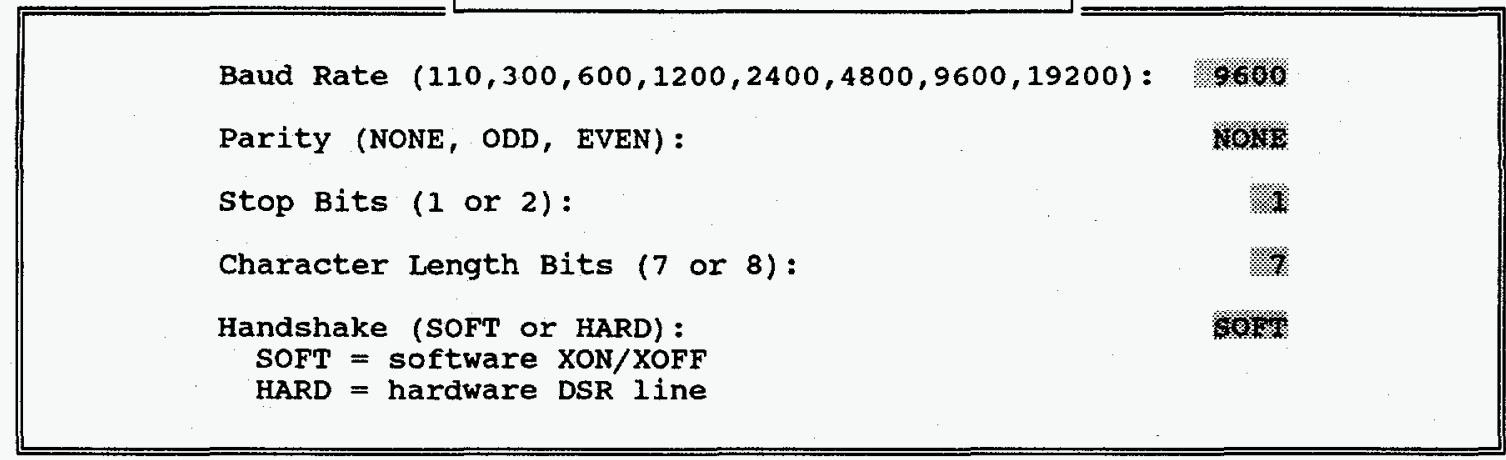

Press "F1" or "F2" for HELP, or "Esc" to IGNORE CHANGES and return to main menu. Press "F10" to SAVE DATA AND CONTINUE.

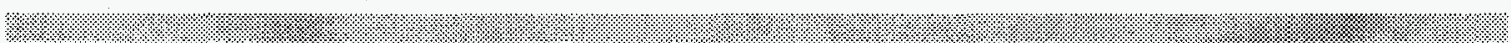

FIGURE 4.21 Input Form R0205 for Serial Port Parameters (Submenu for R020)

RESRAD: Residual Radioactive Material Program $\operatorname{Path}(s): 1,2,3,4,5,6,7,8,9$

Plot scaling and offset Adjustments Submenu (R0206)

Adust the following parameters only if needed to properly position the plot. Monitor Plots

$\mathrm{X}$-axis scaling: $\quad \mathrm{Y}$-axis scaling:

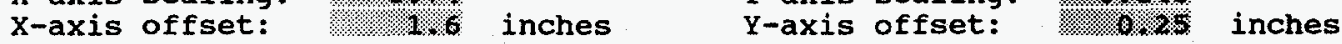

Hard Copy Plots

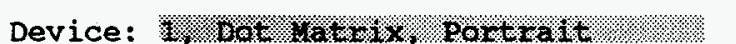

$x$-axis scaling: $0.60 \%$

$\mathrm{X}$-axis offset:

Angle:

$\mathrm{Y}$-axis scaling: $00.68 \%$

degrees

$Y$-axis offset: 2 inches

Press "F1" or "F2" for HELP, or "EsC" to IGNORE CHANGES and return to main menu. Press "FI0" to SAVE DATA AND CONTINUE.

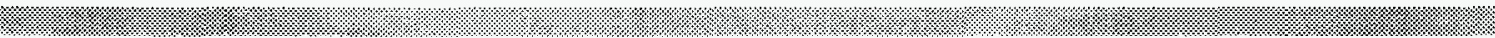

FIGURE 4.22 Input Form R0206 for Plot Scaling and Offset Adjustments (Submenu for R020) 
the parameter settings should be made with caution and only after checking the F2 help files for each parameter. The original values can always be restored from the GRAPHICS.DEF file.

\subsubsection{Dot Matrix Printer Parameters Submenu}

Form R0207 is shown in Figure 4.23. It is only accessible if the Hard Copy Graphics Device parameter is set at 1 in the Graphics Parameters menu, Form R020. This activates the F7 function key and the corresponding Dot Matrix Parameters display line. The printer hardware manual should be consulted for suggested parameter settings. If available, high resolution plots will undoubtedly look better; the user must decide, however, whether the extra time to produce them is worthwhile.

\subsubsection{Hewlett-Packard Pen Plotter Parameters Submenu}

Form R0208 is shown in Figure 4.24. It is only accessible if the Hard Copy Graphics Device parameter is set at 21 in the Graphics Parameters menu, Form R020. This activates the F8 function key and the corresponding Pen Plotter Parameters display line. The plotter

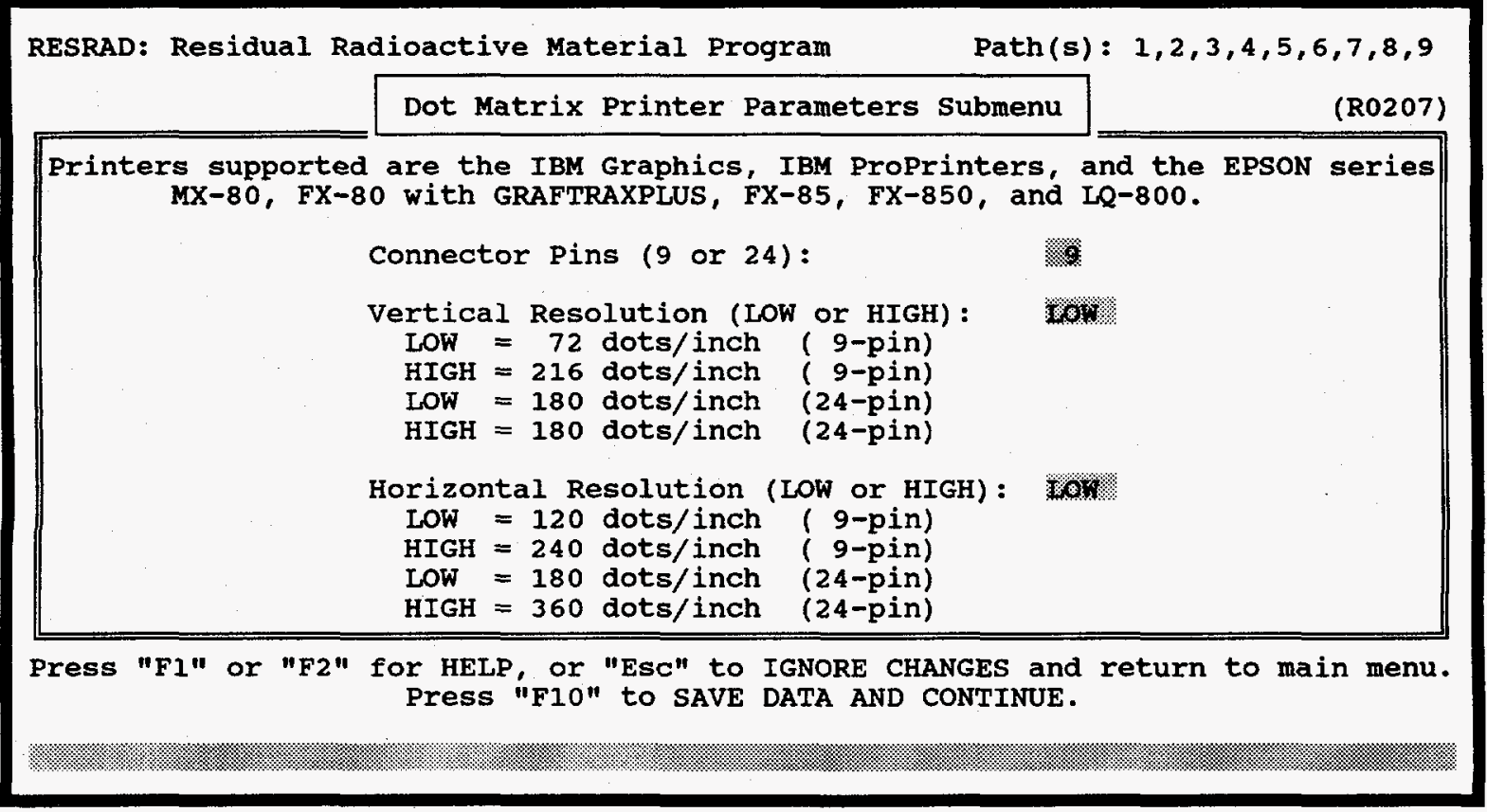

FIGURE 4.23 Input Form R0207 for Dot Matrix Printer Parameters (Submenu for R020) 


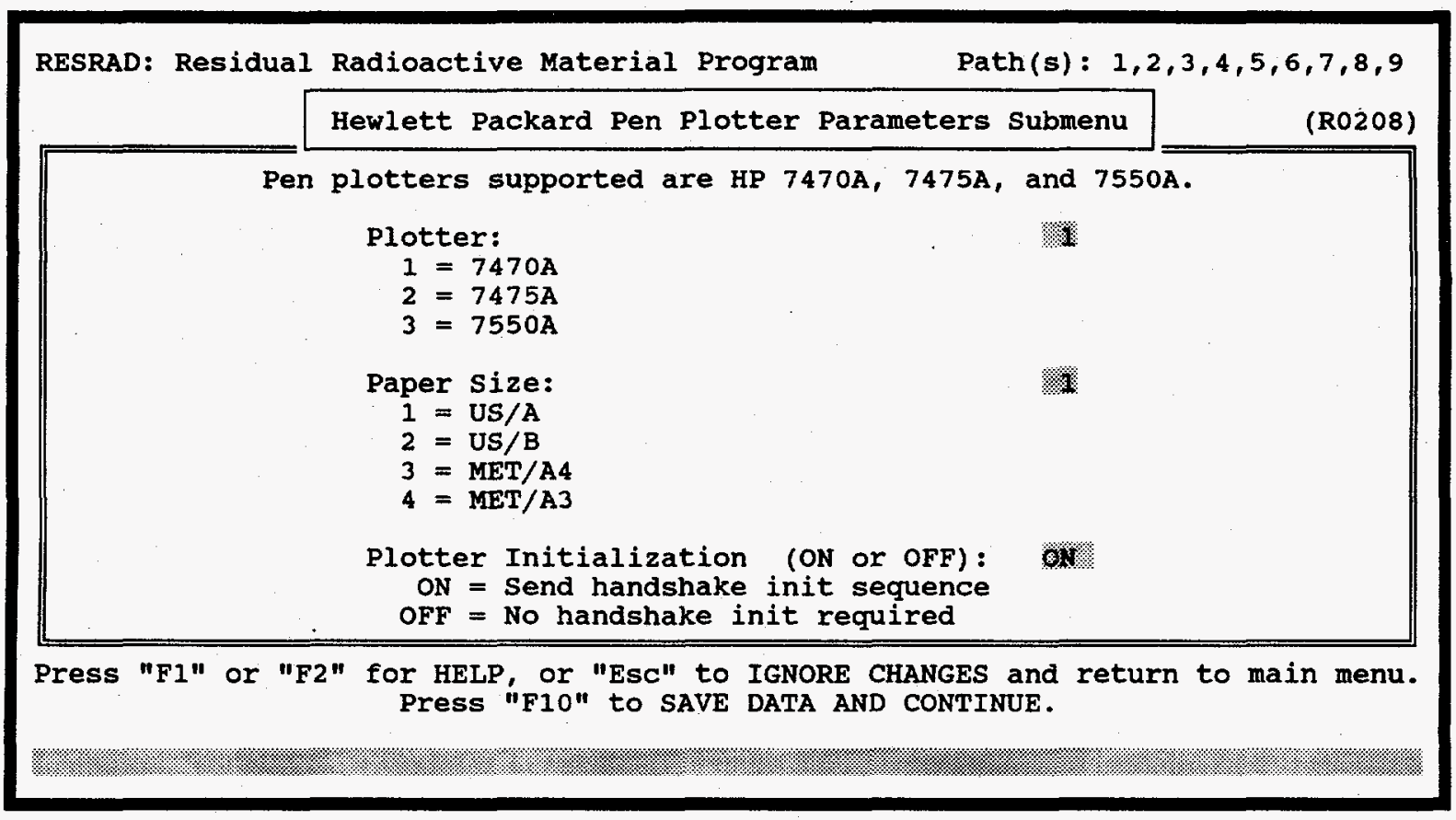

\section{FIGURE 4.24 Input Form R0208 for Hewlett-Packard Pen Plotter Parameters (Submenu for R020)}

hardware manual should be consulted for suggested parameter settings. The Plot Scaling and Offset Adjustments Submenu, Form R0206, may be needed to properly position the plots.

\subsubsection{Run RESRAD}

This main menu option starts RESMAIN, the computational and report-generating phase of RESRAD. Before exiting RESPC, however, the code first displays a summary report on the activated sensitivity analysis settings (if any). This report provides a final $\mathrm{Y} / \mathrm{N}$ validity check for the user before starting the calculations. If an $\mathbf{N}$ or $\mathbf{n}$ response is received (any other key is a default $\mathrm{Y}$ ), RESMAIN is canceled, and an immediate return is made to the RESPC main menu. This procedure can be used at any time for a quick status check on the sensitivity analysis settings.

Before the actual start of RESMAIN, the user must input one final item, the dose factor file to be used in the calculations. ${ }^{11}$ The input options are $R$ (or $r$ ) for the default

11 This option appears only if the user has modified any of the dose factors in the current or previous runs (see Section 4.6.8 on modifying dose factors). 
RESRAD file (RESRAD.BIN) and $U$ (or $u$ ) for user-selected dose factors (USER.BIN). User-selected dose factors are discussed in Section 4.6.8. Any other input cancels the pending start of RESMAIN and returns the RESPC Main Menu to the screen. A typical monitor display is shown in Figure 4.25.

If RESMAIN starts, a series of brief status reports will appear on the display monitor. The reports are primarily intended to reassure the user that the calculations are proceeding normally. The continuously updated lap and iteration counters, which trace the progress of the calculations, require clarification. In brief, there are four main period or lap values, and each iteration is a complete evaluation of all variables at the current time value. The four lap values correspond to the following activities:

1) All variables are computed at user-specified times as specified on Form R011.

2) Variables are computed and saved for graphical output. The time periods are equally spaced increments (log scale) between the first and last user-specified times on Form R011.

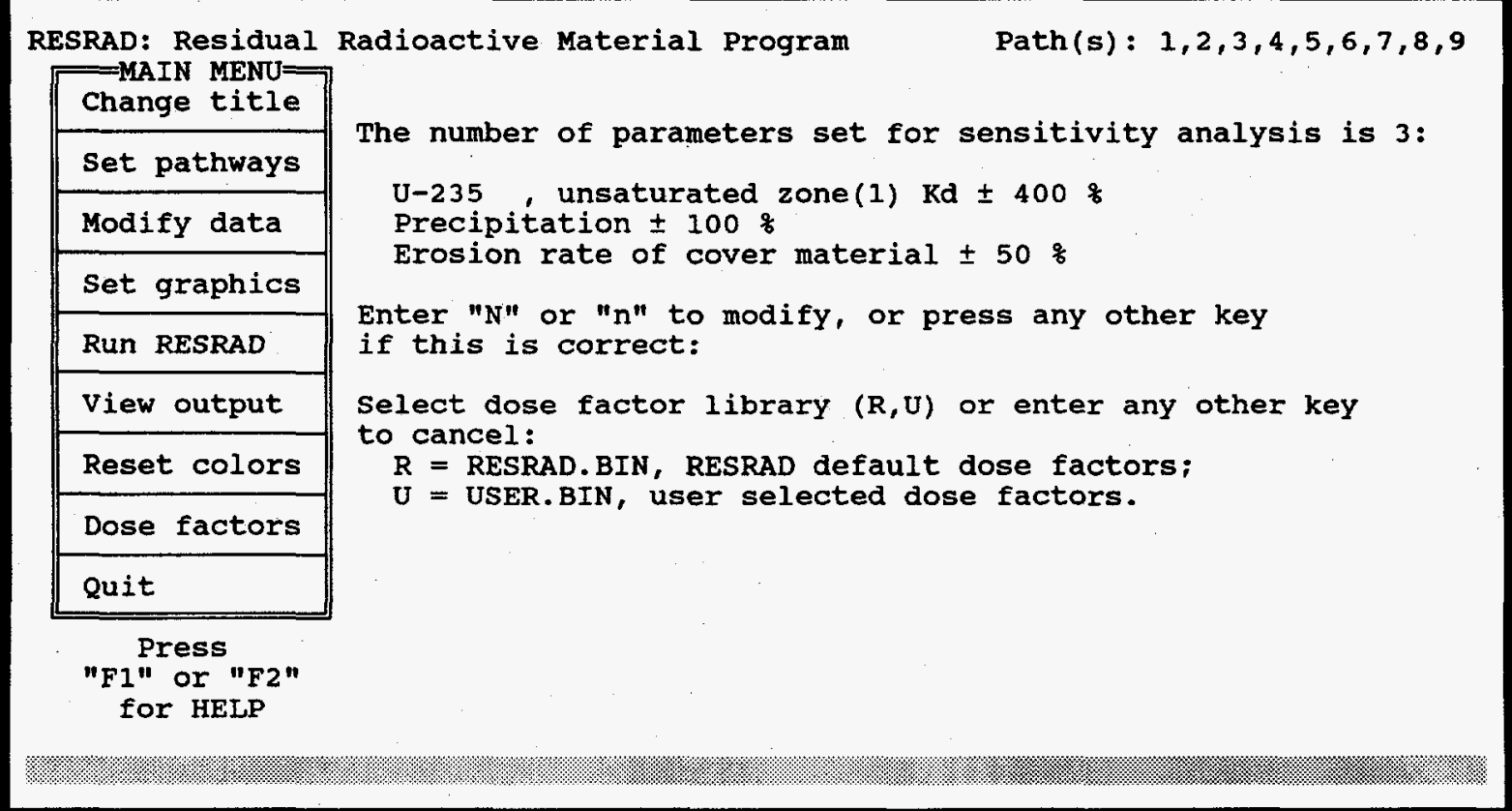

FIGURE 4.25 Typical Screen after Run RESRAD Is Selected from Main Menu 
3) Variables are computed in a standard iterative procedure (Press et al. 1986) to find the time of the maximum dose/source ratio for each initially present principal radionuclide.

4) Variables are computed by the same iterative procedure to find the time of maximum total dose, that is, the dose summed over all radionuclides and pathways.

If sensitivity analysis has been requested, the lap and iteration reports are repeated for the two extremes of each parameter specified. However, lap $=3$ and lap $=4$ are not used during the sensitivity analysis portion of the calculations. More information on the RESMAIN calculations can be obtained in the RESMAIN Major Cycles section of RESRAD5.DOC.

The successful completion of RESMAIN is marked by the following display line: RESMAIN regular output complete, elapsed time $=x x x x \cdot x x x x$ seconds

The RESMAIN output consists of three tabular report files, SUMMARY.REP, DETAILED.REP, and INTRISK.REP, plus a series of (binary) graphics data files. The user can view and/or obtain a hard copy of the text and graphics by using the View Output option of the main menu discussed in Section 4.6.6.

\subsubsection{View Output}

This main menu option is the customary choice after the completion of RESMAIN (see Section 4.6.5). However, a prior execution of RESMAIN is not necessary if the output files from the last run are still available. The View Output option consists of three submenus:

- View and Setup, shown in Figure 4.26, is the starting point and allows basic selecting of a printer setup, viewing of text output, or viewing of graphics output. If graphics output is selected, RESPC terminates and RESPLOT starts automatically (see Section 4.6.6.5).

- Setup Printer, shown in Figure 4.27, allows easy setup for 80- or 132-column output. 


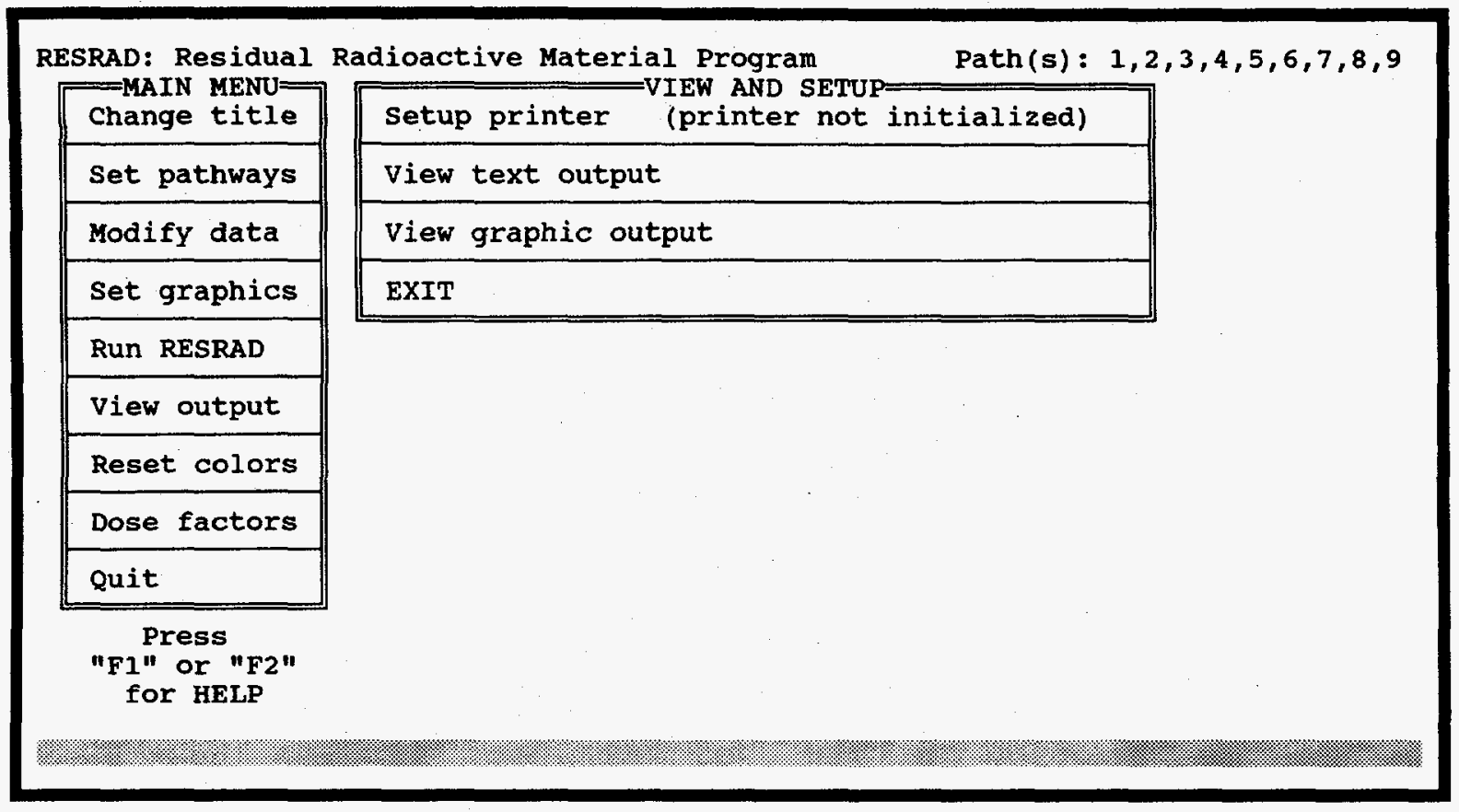

FIGURE 4.26 View and Setup Submenu (with Main Menu)

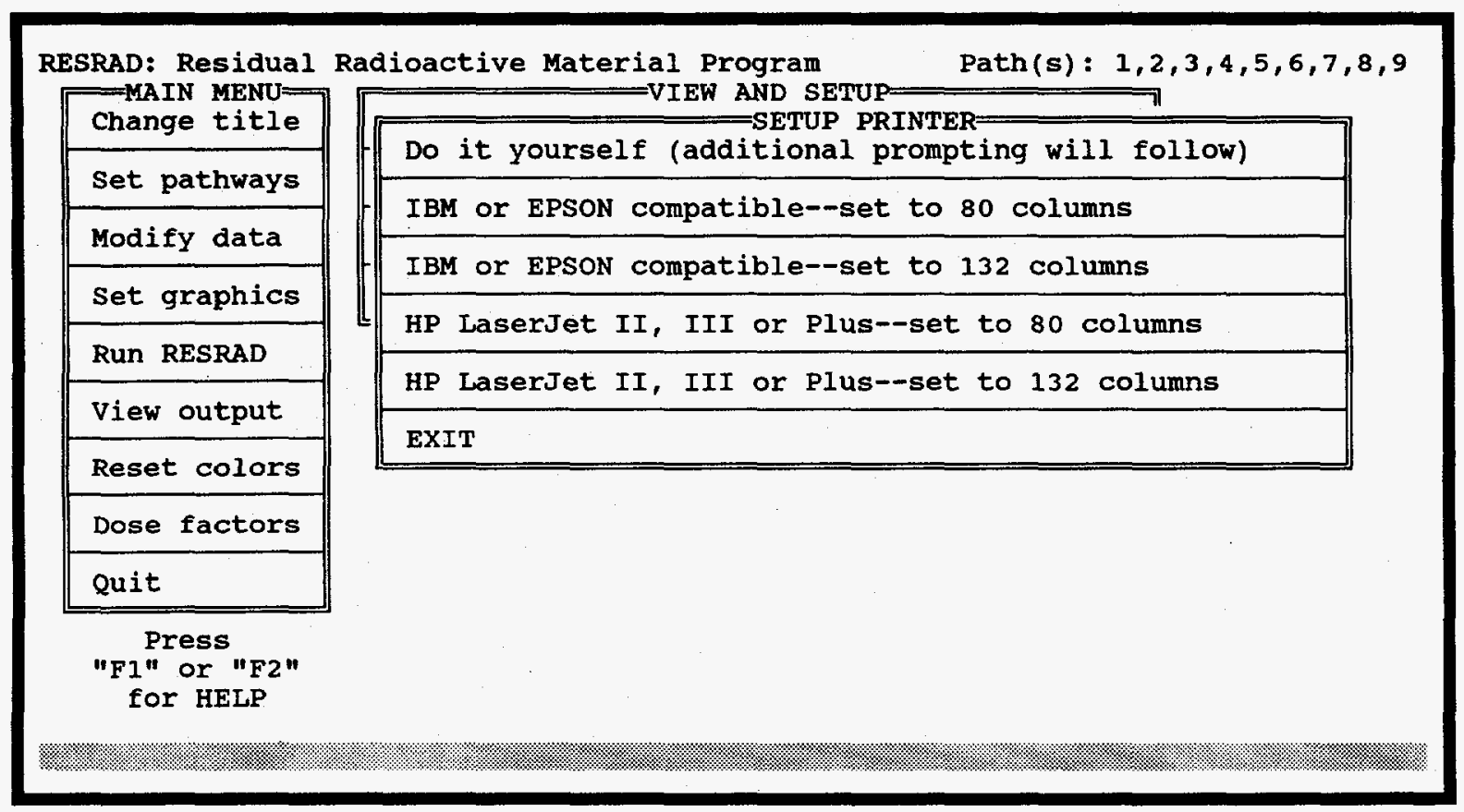

FIGURE 4.27 Setup Printer Submenu (with Main Menu) 
- View Text Output, shown in Figure 4.28, selects among the RESMAIN output text files, SUMMARY.REP, DETAILED.REP, or INTRISK.REP, or any text (ASCII) file the user selects. The last option is particularly useful for examining saved output from a prior RESRAD run.

\subsubsection{Summary Report (SUMMARY.REP FILE)}

When the summary report option is selected, the first page (table of contents) of a multiple page summary report is displayed on the screen. The table of contents is shown in Figure 4.29. Subsequent report pages can be displayed by entering the page number and pressing either the Enter or F10 function key. Pressing Esc executes a return to the main menu. Online help, primarily key strokes to aid in the page display, is available via the F1 function key. Function keys F7 and F8 can be used to produce hard copy output (see Section 4.6.6.4).

The summary report starts with lists of all DCFs; site-specific parameter values, used and default; and pathways selected for the current run. The two parameter lists are followed by a brief summary of the contaminated zone parameters. Summaries of the estimated total dose, $T D O S E(t)$, and total mixture sum, $M(t)$, for the user-selected time

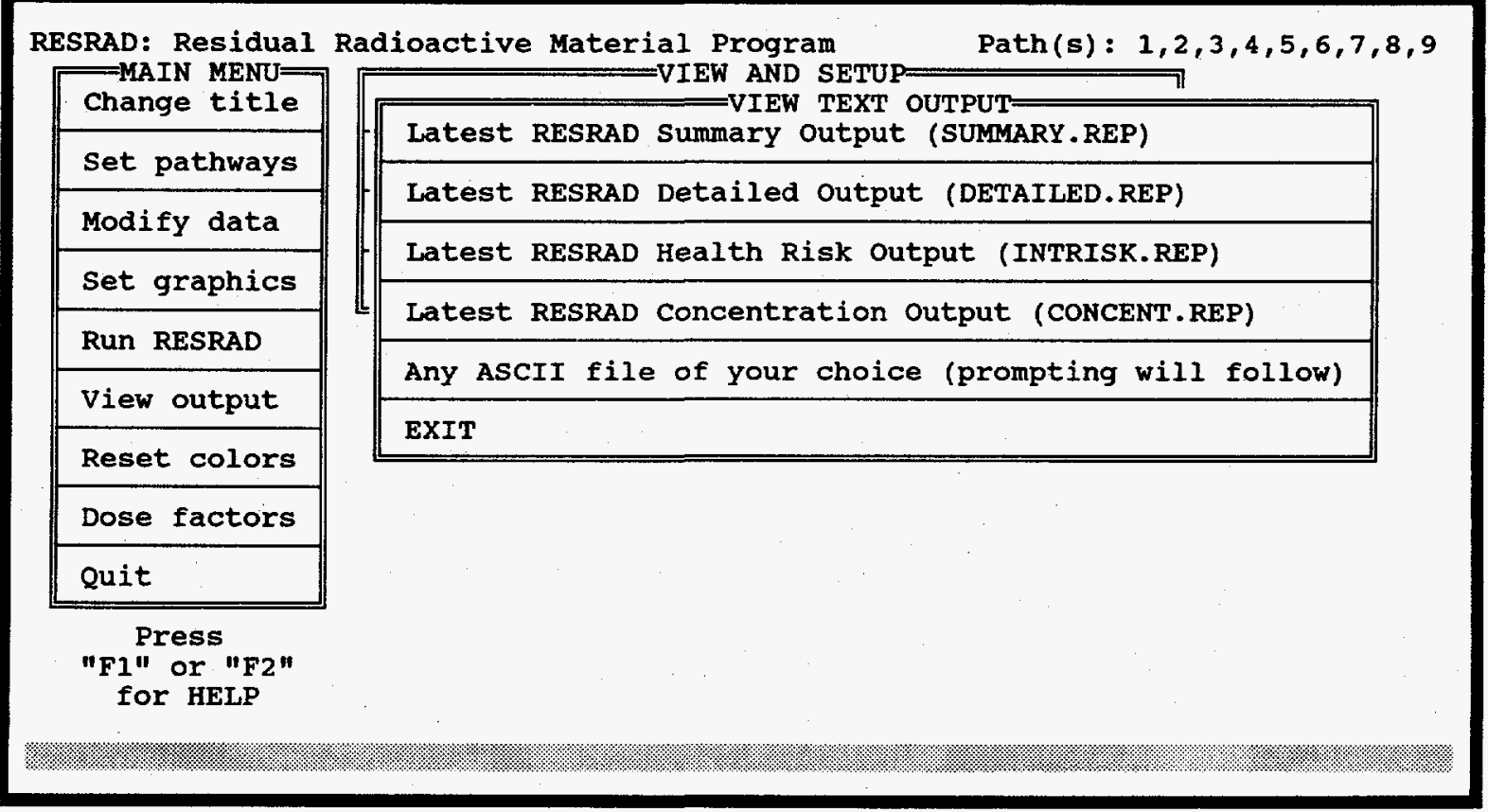

FIGURE 4.28 View Text Output Submenu (with Main Menu) 
Residual Radioactivity Program, Version $\mathbf{5 . 0 0}$ Summary : RESRAD Demonstration Data
$09 / 21 / 93$ 10:42 Page 1
File: OEMO1. DAT

Table of Contents

Part I: Mixture Sums and Single Radionuclide Guidelines

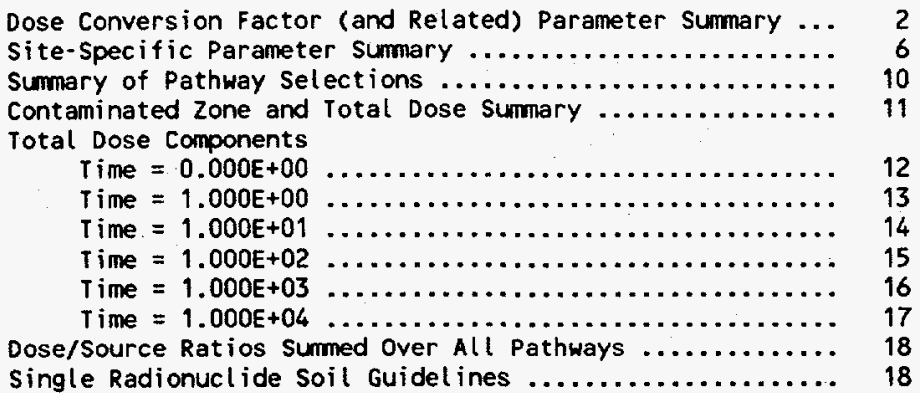

FIGURE 4.29 Typical Table of Contents for Summary Report

periods, $t$, are listed next (TDOSE $[t]$ in mrem/yr and $t$ in $\mathrm{yr}$ ). If a total dose maximum has been bracketed within the time frame selected, ${ }^{12}$ this maximum and its time of occurrence is determined by a standard iterative procedure (Press et al. 1986, p. 283).

The total mixture sum, $M(t)$, is the estimated effective dose equivalent, expressed as a multiple of the basic dose limit, that a member of the critical population group might receive at time $t$ following the radiological survey as a consequence of the residual radioactivity. A site may be certified in compliance with guidelines only if the mixture sum does not exceed the value of 1 at any time within the time horizon. The summary report continues with the total dose components for all individual pathways at different times. The results are presented both as actual dose (mrem/yr) and as the fractional contribution to the total. In addition, the pathways are grouped into water-independent and water-dependent sets. Four pathways (radon, plant, meat, and milk) have components in both sets.

The final table series of the summary report presents the following:

- Total dose/source ratios, $D S R(i, t)$ in (mrem/yr)/(pCi/g), summed over all pathways for principal radionuclide $i$ and time $t$;

12 Only the user-specified time frame (i.e., $t=0$ years excluded) is used to bracket a maximum. If multiple peaks are found to occur, separate runs with appropriate time frames can be used to bracket each peak. 
- Single-radionuclide soil guidelines, $G(i, t)$ in $\mathrm{pCi} / \mathrm{g}$, for principal radionuclide $i$ and time $t$; and

- Values of $D S R(i, t)$ and $G(i, t)$ for $t$ equals the time of minimum $G(i, t)$ for radionuclide $i$ and for $t$ equals the time of maximum total dose.

The single radionuclide soil guidelines are the concentration guidelines that would apply if only one radionuclide were present. A single radionuclide soil guideline, $G(i, t)$, is the magnitude of the initial concentration of the $i$ th principal radionuclide that would result in a potential radiation dose equal to the basic radiation limit to a member of the critical population group at time $t$. Thus, if only the $i$ th radionuclide were present, the initial concentration $S(i, 0)$ would have to satisfy the inequality $S(i, 0) / G(i, t)<1$ for all times up to the time horizon. The value of $G(i, t)$ is readily obtained by dividing the basic radiation dose limit by the total dose/source ratio, $D S R(i, t)$. The minimum $G(i, t)$ values, or more correctly, the corresponding maximum $D S R(i, t)$, are obtained for each $i$ with the same iterative procedure used for the maximum total dose.

The total mixture sum enables the user to determine whether the concentrations of residual radioactive material are in compliance with guidelines. The total dose components and single radionuclide soil guidelines enable the user to identify the critical pathways and radionuclides.

\subsubsection{Detailed Report (DETAILED.REP FILE)}

When the detailed report option is selected, the first page (table of contents) of a multiple-page detailed report is displayed on the screen. A typical table of contents is shown in Figure 4.30. Individual report pages can be displayed and printed (or saved) in the same manner as for the summary output displays. The detailed displays present all of the intermediate results used to evaluate the formulas in Appendixes $A$ through $L$ for different pathway contributions. Users will not normally make use of these tables. The detailed data are used for verifying the code and can be useful for gaining insight into the transport mechanisms. The latter is accomplished by enabling a more detailed investigation of the effect of parameter changes on pathway factors, environmental transport factors, and dose/source ratios for the different pathways. 
Residual Radioactivity Program, Version 5.00

09/21/93 13:10 Page 1 Detailed: RESRAD Demonstration Data

file: DEMO1.DAT

Table of Contents

Part 11: Source Terms, Factors, and Parameters for Individual Pathways

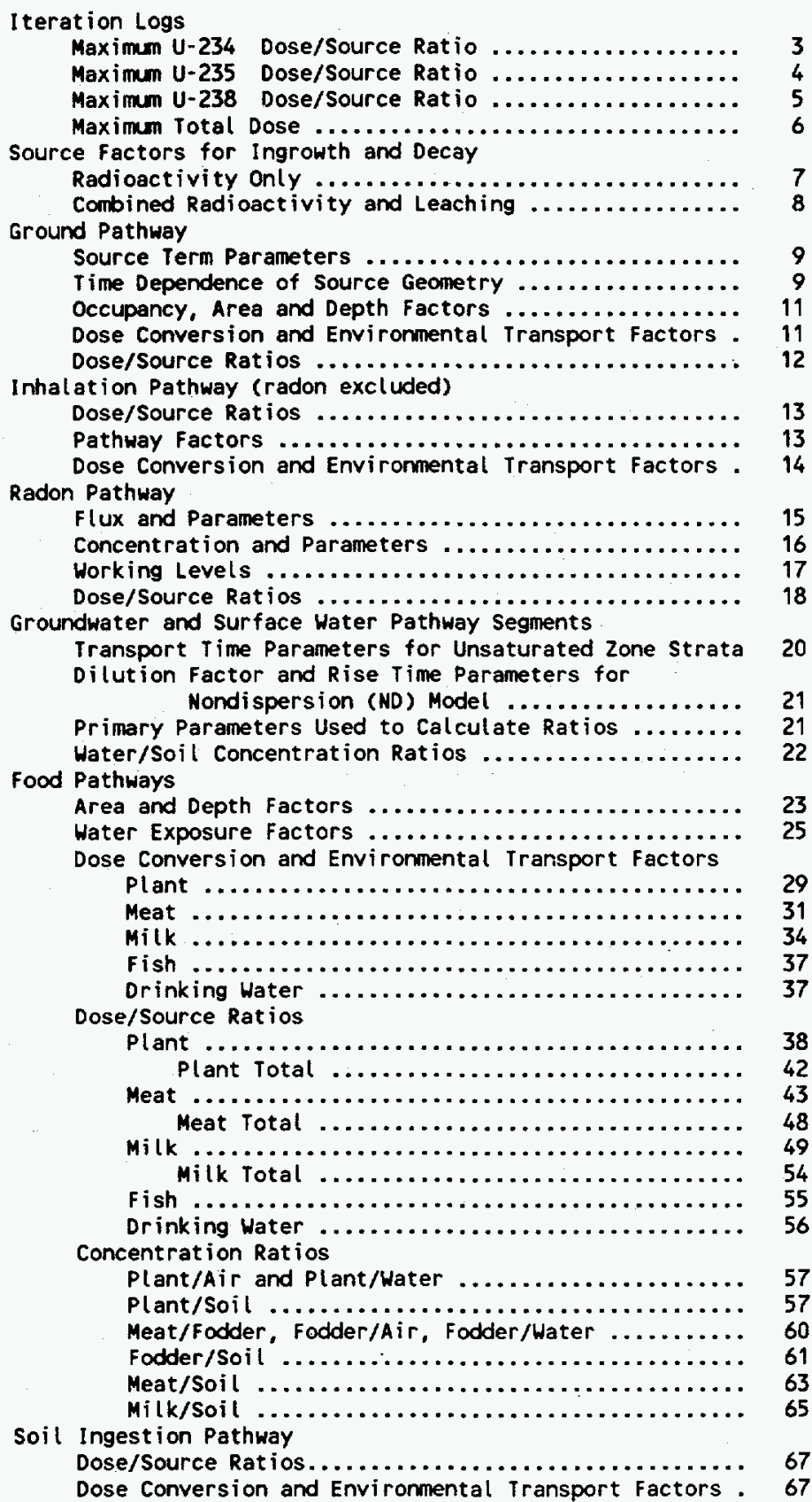

FIGURE 4.30 Typical Table of Contents for Detailed Report 


\subsubsection{Health Risk Report (INTRISK.REP FILE)}

The calculation of radionuclide intake quantities for use in estimating excess cancer incidence risks is a new feature of the RESRAD code. Excess cancer risk calculations are based on the EPA's slope factor methodology (EPA 1992).

When the health risk report option is selected, the table of contents for this report is displayed on the screen. A typical table of contents is shown in Figure 4.31. This report contains the slope factors and intake quantities used to calculate excess cancer risks. Individual report pages can be displayed and printed (or saved) in the same manner as for the summary and detailed report displays.

The health risk report starts with a list of the cancer risk slope factors, both userselected and default values, for the external exposure, inhalation, and ingestion pathways. Then, for each of the user-selected time periods, a series of intake and risk tables is displayed.

The first set of tables contains the intake quantities; the radionuclide intakes from inhalation of dust and ingestion of plants, meat, milk, water, and soil are given in $\mathrm{pCi} / \mathrm{yr}$. A separate table is provided for the inhalation of radon-222, radon-220, and their respective progeny. The intake quantities from each pathway are broken down by water-independent and water-dependent contributions.

Following the tables of intake quantities are the excess cancer risk tables for the principal radionuclides and associated principal radionuclides. Excess cancer risks and the fractional contribution from each pathway and radionuclide (parent and principal progeny

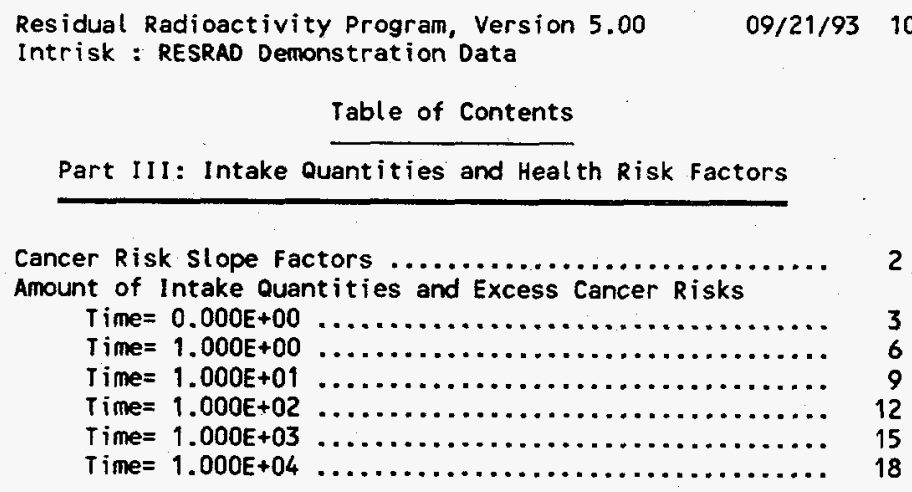


listed separately) are divided into water-independent and water-dependent components. The contribution from the ground pathway is included, along with the risks from radionuclide intakes presented in previous tables. Again, the water-independent and water-dependent risks from inhalation of radon and associated progeny are tabulated separately.

The last series of tables presents the total risks from each principal radionuclide (including the contributions from principal progeny in the decay chain) and each pathway. Once again, pathways are divided into water-independent and water-dependent contributions. However, these two tables also incorporate the radon contribution to the total risk. The individual contributions are then summed to provide the total risks for all pathways and radionuclides.

\subsubsection{Radionuclide Concentration Report (CONCENT.REP)}

The fourth output report generated by RESRAD tabulates the radionuclide concentrations in various environmental media. As with the other three reports, a table of contents is displayed on the screen when the concentration report option is selected. A typical table of contents is shown in Figure 4.32. Printing of the entire report or individual pages is accomplished in the same manner as with the first three reports.

Separate tables are generated for each user-selected time. In each table, the concentrations of both initially present and ingrown radionuclides (excluding radon) are displayed for the following media: contaminated and surface soil layers (in $\mathrm{pCi} / \mathrm{g}$ ); air (in $\mathrm{pCi} / \mathrm{m}^{3}$, based on the mass loading for inhalation); well water, surface water, and milk (in

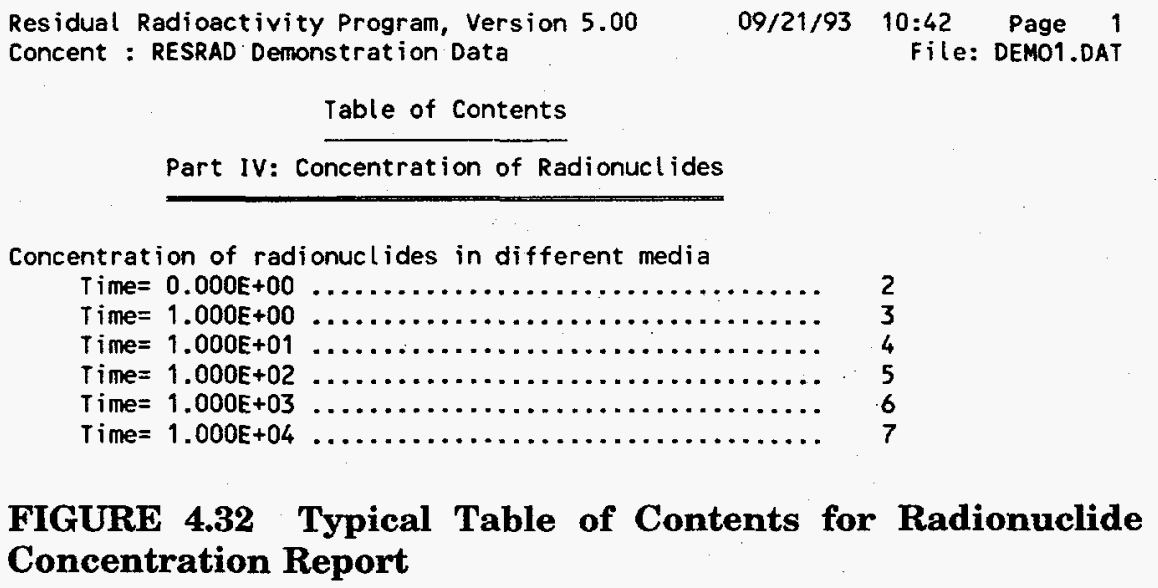

FIGURE 4.32 Typical Table of Contents for Radionuclide Concentration Report 
pCi/L); and leafy vegetables, nonleafy vegetables (including fruits and grain), fodder, meat, fish, and other aquatic organisms (in pCi/kg).

\subsubsection{Text Hard Copy}

At any time RESRAD text output (or any other ASCII file) is being viewed, hard copy can be produced (if the printer is turned on) by pressing the F7 or F8 function keys. The F8 key produces an immediate copy of the current screen page only.

The F7 function key is a more versatile but complicated option. ${ }^{13}$ The user is first informed of the current hard copy destination (printer or disk file name) and allowed to make a change, if desired. ${ }^{14}$ If an existing disk file is selected the user can specify that it be overwritten or appended. The following series of options can then be exercised repeatedly (until Esc):

- Enter the first and last page numbers, separated by a space or comma, to print (or save);

- Enter a single page number to print (or save) that page only;

- Press the Enter key only to print the current page (the current page number will be displayed on the screen);

- Press D or d to print entire document (file);

- Press P or $p$ to pass ASCII control characters to the printer; and

- Press Esc to resume normal RESRAD viewing of the file.

\subsubsection{Graphics Output}

All graphics output is obtained via the interactive RESPLOT program. RESPLOT can be executed in any of three ways:

- Immediately at the conclusion of RESMAIN by entering $G$ at the concluding prompt;

\footnotetext{
${ }^{13}$ To preserve an option of an earlier RESRAD version, the * is identical to the F7 function key.

14 This is usually more convenient than command line or Form R010 input.
} 
- From the main menu via the View Output option, provided that the graphics files from the last RESMAIN run still exist; and

- From the DOS prompt by entering DORP, an acronym for DO ResPlot. Again, the graphics files from RESMAIN must still exist.

RESPLOT is based on a series of random access files generated by RESMAIN. These files contain the computed dose/source ratios for each time value, pathway, and principal radionuclide. Data for any isotope and pathway component can be retrieved and manipulated to produce a variety of plots. RESPLOT enables the user to quickly review the graphs on the display monitor; after each plot is displayed, the user is given the option of producing a high quality hard copy.

The principal Graphics Selection Menu is shown in Figure 4.33. The following parameters can be specified to select a plot:

- IDOSE - Selects the plot ordinate units; all abscissas are the same (log scale years):

1) dose/source ratio (mrem/yr)/(pCi/g)

2) soil guideline $(\mathrm{pCi} / \mathrm{g})$

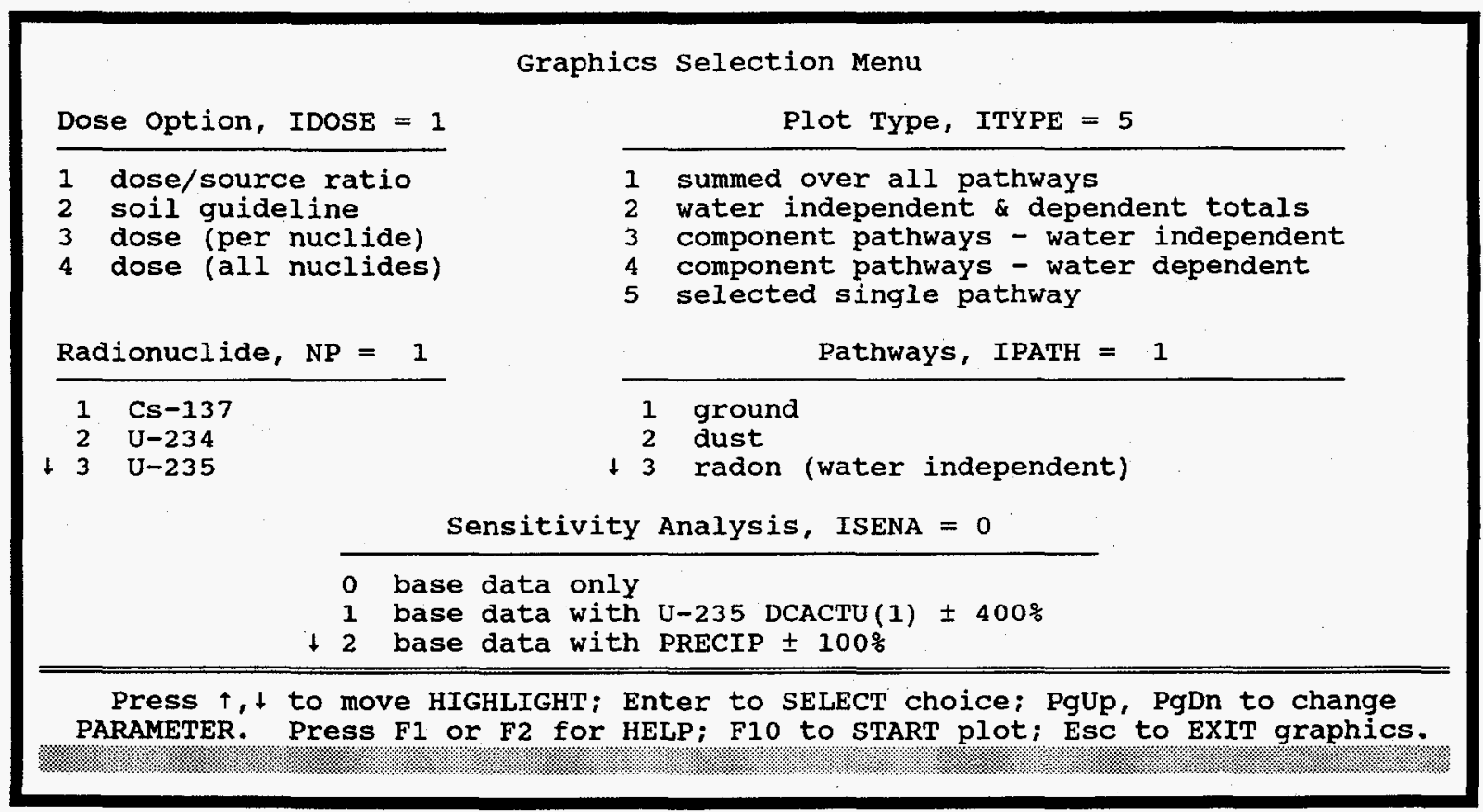

FIGURE 4.33 Graphics Selection Menu 
3) dose per nuclide (mrem/yr)

4) dose, summed over all nuclides (mrem/yr)

- ITYPE - Selects the ordinate data sets (if IDOSE $=2$, ITYPE $=1$ is automatic):

1) summed over all pathways

2) subtotals for water-independent and -dependent pathways

3) component pathways, water-independent

4) component pathways, water-dependent

5) selected single pathway

- NP - Selects the principal radionuclide (if IDOSE $\neq 4$ )

- IPATH - Selects the pathway, if a single pathway was selected (ITYPE = 5):

1) ground

2) dust inhalation

3) radon (water-independent)

4) plant (water-independent)

5) meat (water-independent)

6) milk (water-independent)

7) soil ingestion
8) drinking water

9) fish

10) radon (water-dependent)

11) plant (water-dependent)

12) meat (water-dependent)

13) milk (water-dependent)

14) all pathways, in sequence

- ISENA - Selects the sensitivity analysis case, if any (only if ITYPE = 1 or 5 ).

Each of the five parameters is displayed in subwindows with a header line showing the current value (in bright numerals) followed by the available options. For some IDOSE and ITYPE values, entire subwindows will be blank, indicating that no option is available. For the last three subwindows, there are usually too many options to fit within the allotted space. If this is the case, a bar will appear to the left with direction arrows to indicate a scrolling options menu.

The current active subwindow is indicated by a highlighted option line. The up and down arrow keys are used to move among the options, and Enter is pressed to select the desired highlighted choice. To move among the subwindows without changing parameter values, the PgUp and PgDn keys are used. Other editing keystrokes are possible. A complete keystroke list is displayed with the F1 function key, and a general help screen is displayed with the F2 key. When all parameters are properly set, pressing F10 starts the plot, or the Esc key can be used to exit graphics and to return to either the RESPC Main Menu or the DOS prompt. 
The current plot will remain on the screen until the Enter key is pressed (note the speaker beep and "Cr:" prompt in the upper right corner). However, if ITYPE = 2, 3, or 4, a movable legend is part of the plot, and the user must first answer a $\mathrm{Y} / \mathrm{N}$ prompt to indicate whether the legend position is satisfactory. If the response is $\mathrm{N}$ or $\mathrm{n}$ (any other key is a $\mathrm{Y}$ default), the lower left corner of the legend can be moved repeatedly with the mouse (if present) or by inputting X,Y displacements (if no mouse is available). The code will not allow the user to position any portion of the legend outside of the limits of the X,Y axes. The Cr: prompt and beep, which indicate the end of plotting, will not appear until the user indicates that the legend is in a satisfactory location. The legend position will remain fixed on all subsequent plots until the user moves it again.

After Enter is pressed, the monitor screen is erased and returned to text mode in order to query whether a hard copy is to be produced. ${ }^{15}$ If hard copy is selected, it is produced immediately if the attached device is an Apple Laser Writer printer, an HP Pen Plotter, or an HP LaserJet with Plotter in a Cartridge. Otherwise, the hard copy is a disk file, $d$ :RESRAD. $x x x$, where $d:$ is the optional RAM disk drive and $x x x$ indicates the file type (see Section 4.6.4.1). The $d$ :RESRAD.GRF file, which serves as input to the VECTOR plotting program, is produced automatically if the attached hard copy device is a dot matrix printer or an HP LaserJet printer.

When RESPLOT terminates (i.e., the Esc key is pressed while the Graphics Selection Menu is on screen) and if output via VECTOR is pending, the user is given the option of an automatic, immediate start of VECTOR or a return to the RESPC main menu. If VECTOR execution is delayed, the program must be started at the DOS prompt by entering "VECTOR"; this must be done before rerunning RESPLOT, otherwise, $d$ :RESRAD.GRF will be overwritten. The automatic start option of VECTOR does not occur if RESPLOT was started at the DOS prompt via DORP; in this case, VECTOR must always be started at the DOS prompt.

Because graphics hard copy is usually a slow process, the user may wish to delay the actual output to a more opportune time. Such a delay is easily executed in RESRAD. For dot matrix or laserjet printers, RESRAD automatically saves the plot data in

15 Software print spoolers may cause problems with graphics hard copy output and, if so, should be turned off. Such problems often occur with PrintCache from Laser Tools Corporation. 
$d:$ RESRAD.GRF. In a simple case, such as when RESMAIN is not run again and the RAM disk files are still intact, all that is necessary for a delayed plot is to type VECTOR at the DOS prompt. In a more typical situation, the user may elect to rename and/or transfer the graphics file(s) to a floppy disk. If a delayed hard copy is selected in RESPLOT, the renaming and/or copying of $d$ :RESRAD.GRF is an automatic user option.

For example, if the user wants graphics hard copy after completing two RESMAIN runs and saving the RESPLOT $d:$ RESRAD.GRF files as A:RUN1.GRF and A:RUN2.GRF, either floppy disk file should be copied to $d$ :RESRAD.GRF, and VECTOR should be entered at the DOS prompt. A more sophisticated example would require moving to another personal computer, possibly with different graphics hardware and without RESRAD5. In this case, the files VECTOR.EXE, VECTOR.CFG., and PROCESS.FIL should also be copied to the floppy disk, and the floppy drive should be made the default. To plot, an editor must be used to change the single record in PROCESS.FIL from $d:$ RESRAD.GRF to RUN1.GRF or RUN2.GRF. If the graphics hardware is different, the VECTOR.CFG file must be edited, and the DEVICE $=X X X$ record changed to DOT (dot matrix printer), PEN (HP pen plotter), JET (HP LaserJet printer), or WRITER (Apple Laser Writer). Scaling and offset changes in VECTOR.CFG may also be needed if the device is changed. To start the plotting, VECTOR must be entered.

If the hard copy device is a HP pen plotter, Plotter in Cartridge, or Apple Laser Writer printer, delayed graphics hard copy is even simpler. For the first two cases, the HP HPGL File (code number 13 on the RESPC Set Graphics Form R020) should be selected; for

the third case, the Postscript File (code number 11 on Form R020) should be selected. A direct copy to the printer port of the $d:$ RESRAD. $x x x$ file ( $x x x$ is HPG or PSF) that is produced by RESPLOT will generate the graphics (e.g., COPY G:RESRAD.HPG LPTI) . Renaming and/or copying of $d$ :RESRAD. $x x x$ is an automatic RESPLOT option.

\subsubsection{Reset Colors}

This submenu, shown in Figure 4.34, allows the user to experiment with the monitor display and, if desired, to save the new color selections as the permanent RESRAD default. The submenu is also a convenient method of resetting the default for a monochrome monitor. 


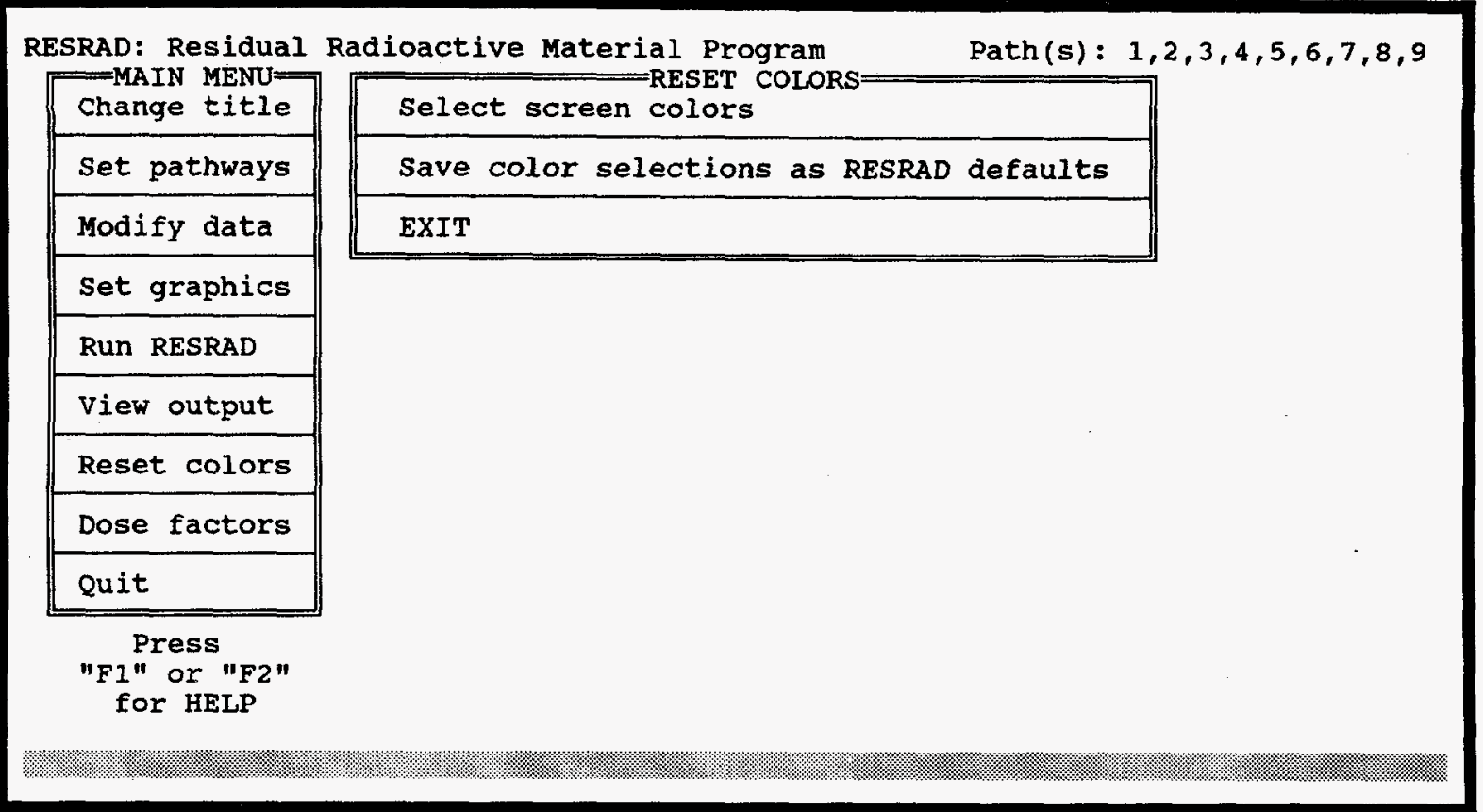

FIGURE 4.34 Reset Colors Submenu (with Main Menu)

\subsubsection{Dose Factors}

This option allows the user to review and modify the effective dose equivalent conversion factors (and related others) as well as slope factors for the RESRAD pathways. Seven submenu choices, shown in Figure 4.35, display parameters corresponding to Tables A.1, A.3, B.1, D.1, D.3, D.4, and D.5 in the appendixes of this report. Tables D.3 and D.4 are combined into a single submenu and data entry Form D-34. The slope factors (last submenu option) are taken from EPA reports (EPA 1992).

A submenu is selected by the standard RESRAD5 method: highlighting the field and pressing Enter, F10, or the mouse button, or pressing the space bar and then pressing the desired highlighted letter. The data form that appears will be quite similar to the corresponding appendix table but with two main differences:

1) The principal radionuclides displayed will be limited to the current subset with an initial concentration greater than zero plus principal radionuclides present as decay products only. 


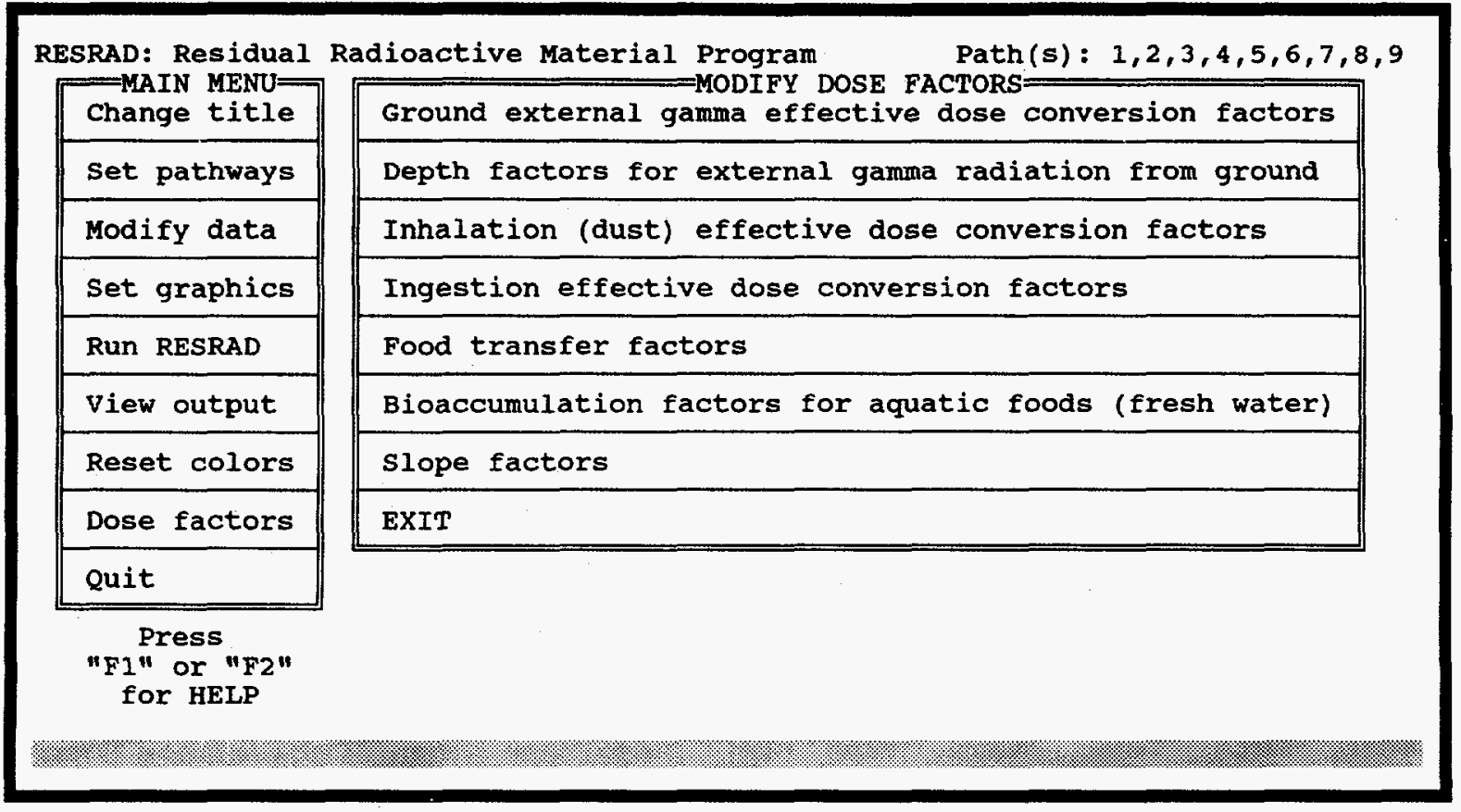

\section{FIGURE 4.35 Dose Factors Submenu (with Main Menu)}

2) The parameter values will be the current user-specified values. In addition, for inhalation and ingestion dose conversion factors, the user selection among multiple lung clearance classes or gastrointestinal (GI) tract fractions will be marked with an "!".

Other than the fact that there is no parameter sensitivity analysis, operation of the data entry forms in this series is very similar to the Modify Data series of Section 4.6.3. One important difference is that when the F10 key is pressed to save the screen data, the permanent user-defined dose factor data bank, USER.BIN, is updated. For the Modify Data series, only the current site-specific data file is rewritten. Particular care should be taken with radionuclides marked with the " $+D^{\prime \prime}$ suffix, which indicates that the dose factor is an aggregate value that includes all associated radionuclides in the decay chain. (See Table 3.1 for the decay chains.) Details on the maintenance of USER.BIN and the corresponding RESRAD defaults in RESRAD.BIN are not important for the average user. The following summary may be helpful for interested users.

Eight ASCII files exist with the general name TAB-*.DAT (e.g., TAB-B1.DAT or TAB-SF.DAT); these files contain the user-selected parameters for the dose factors series. Every time RESRAD is loaded, a check is made to ensure that all eight files of the 
TAB-*.DAT series exist. If any file is missing, it is generated by making a copy of the corresponding TAB-*.DEF file, which is a matching ASCII file that contains the actual data of Table A.1, Table A.3, and so forth. Thus initially, the user-specified data bank is identical to the RESRAD defaults. Thereafter, whenever the F10 key is pressed for any data form in this series, the screen values replace the parameters in the corresponding TAB-*.DAT file; if USER.BIN exists, it is deleted. RESMAIN starts by attempting, per the user's selection, to read either RESRAD.BIN or USER.BIN. If the selected binary file does not exist, or fails other internal checks, a new RESRAD.BIN or USER.BIN file is generated from the respective TAB-*.DEF or TAB-*.DA'T files. ${ }^{16}$

RESRAD also performs an automatic update on the TAB-*.DAT files by checking whether any new radionuclides have been added to the default TAB-*.DEF file. If so, records containing the new data are merged into TAB-*.DAT from the corresponding TAB-*.DEF files.

\subsubsection{Ground External Gamma Effective Dose Conversion Factors}

This submenu selection brings up Form A-1, which contains data corresponding to Table A.1. A sample display is shown in Figure 4.36. The DCF for the external ground radiation pathway is the annual effective dose equivalent received from exposure to radiation from the listed principal radionuclide present at a unit concentration in a uniformly contaminated zone of infinite depth and lateral extent. The radiation field is assumed to be equal to the radiation level at a distance of $1 \mathrm{~m}$ above the ground surface. The DCFs for surface contamination (infinite thinness and lateral extent) are presented in Form A-1 (and Table A.1) for information only. Volume DCFs are used in RESRAD, and factors with soil densities of 1.0 and $1.8 \mathrm{~g} / \mathrm{cm}^{3}$ are listed in the form. Linear interpolation of $\log$ (DCF) is used to obtain values at other densities. Adjustments for shape and finite area and finite depth must also be made. See Appendix A for details.

${ }^{16}$ Other ASCII files are included and common to both binary files. NUCLIDES.DAT contains the data of Table 3.1 plus default distribution coefficients and isotope mass values. ROTCOEF.DAT, ISOCOEF.DAT, APCOEF.DAT, AIRSHLD.DAT, COVER.DAT, and PHOTON.DAT are all used by the SOILD routine (Chen 1991). 


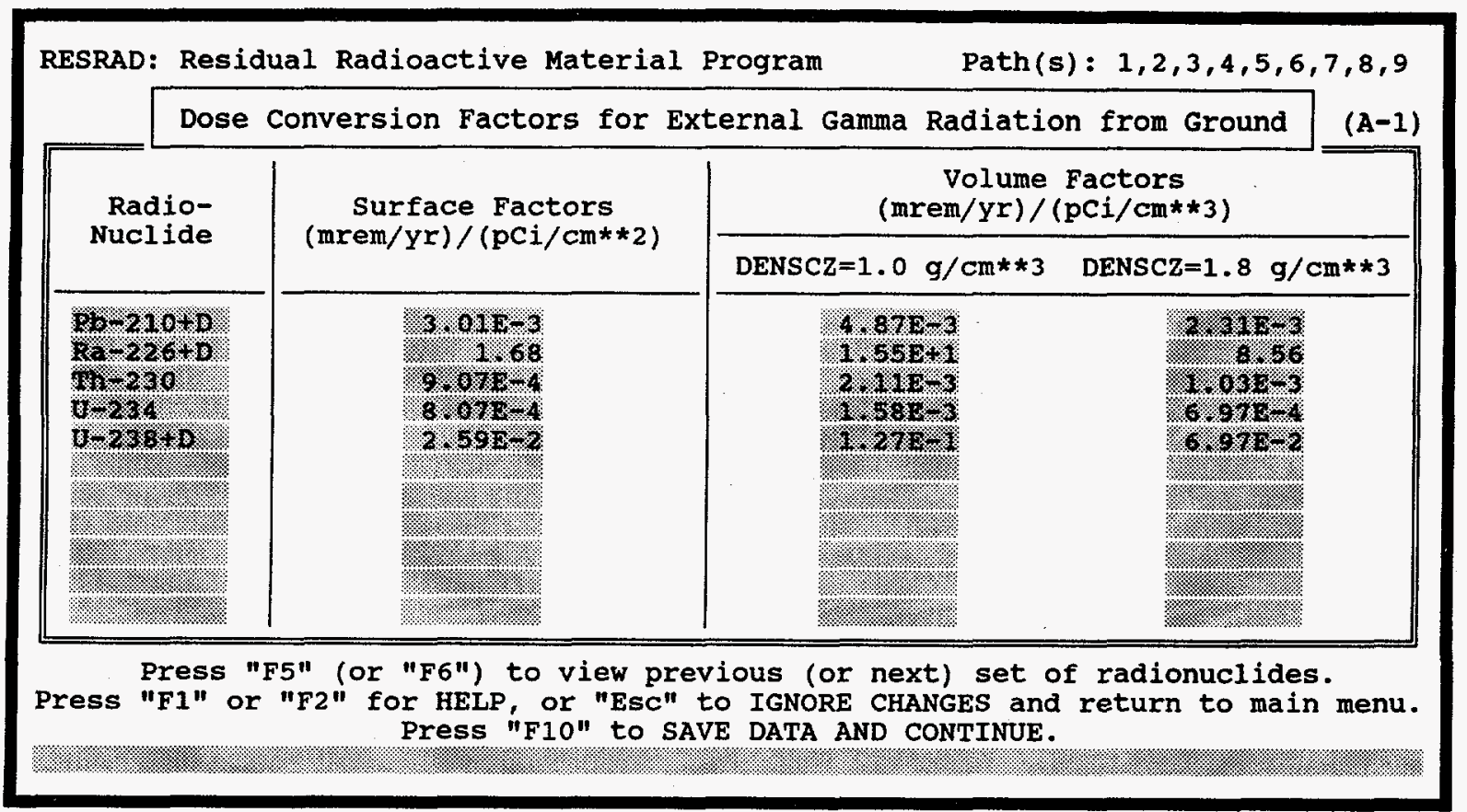

FIGURE 4.36 Input Form A-1 for Ground External Gamma Effective Dose Conversion Factors

\subsubsection{Depth Factors for External Gamma Radiation from the Ground}

Input Form A-3, corresponding to Table A.3, is the result of this submenu choice. A typical screen is shown in Figure 4.37. For each radionuclide, the form lists two data sets containing three depth factors per set. The two sets are for soil densities of 1.0 and $1.8 \mathrm{~g} / \mathrm{cm}^{3}$ (matching the densities of Form A-1), and the three subentries per set are for contaminated zone thickness values of $0.15,0.5$, and $1.0 \mathrm{~m}$. The depth factor for a finite contaminated zone thickness is obtained by interpolation or extrapolation of Form A-3 values. The calculation is sophisticated, however; see Section A.2.1 in Appendix A for the method used.

\subsubsection{Inhalation (Dust) Effective Dose Conversion Factors}

Input Form B-1, corresponding to Table B.1, is the result of this submenu choice. A typical screen is presented in Figure 4.38. A DCF for inhalation is the dose/exposure ratio, $D C F(i)=H_{E} / E(i)$, for the committed effective dose equivalent $H_{E}$ that an individual incurs from exposure by inhalation of a quantity $E(i)$ of the $i$ th principal radionuclide in the 


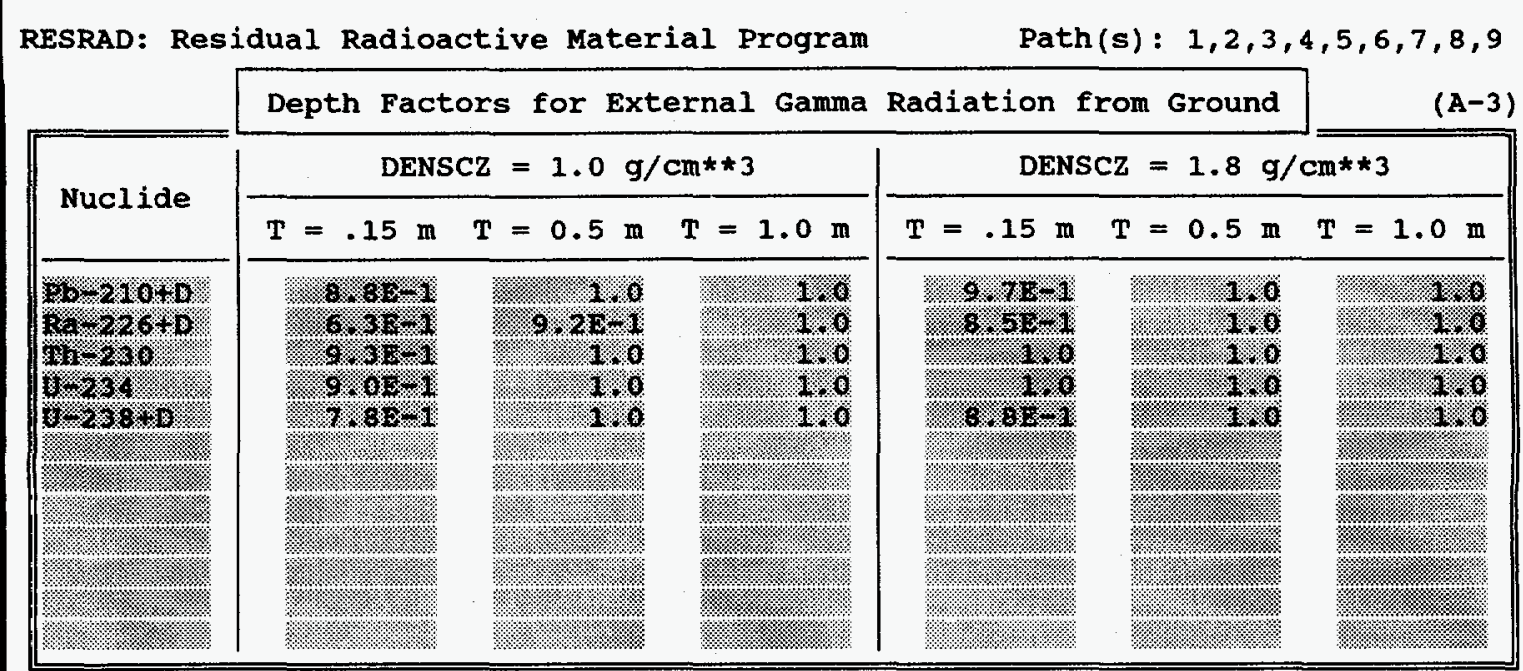

Press "F5" (or "F6") to view previous (or next) set of radionuclides.

Press "F1" or "F2" for HELP, or "Esc" to IGNORE CHANGES and return to main menu. Press "F1O" to SAVE DATA AND CONTINUE.

\%.॥.:.

FIGURE 4.37 Input Form A-3 for Depth Factors for External Gamma Radiation from Ground

\begin{tabular}{|c|c|c|c|c|c|c|c|c|}
\hline \multirow[t]{2}{*}{ RESRAD: } & \multicolumn{3}{|c|}{ Residual Radioactive } & \multicolumn{2}{|c|}{ Material Program } & \multicolumn{3}{|c|}{$\operatorname{Path}(s): 1,2,3,4,5,6,7,8,9$} \\
\hline & Effective & Dose & Equivalent & Conversion & Factors for & Dust Inh & halation & $(B-1)$ \\
\hline Ra & dionuclide & & Inhalation & Class & Dose Convers & ion Facto & or (mrem/ & Ci) \\
\hline & 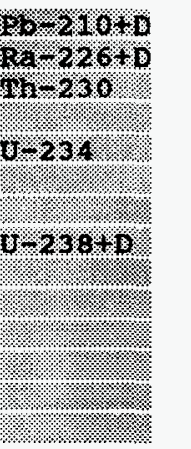 & & 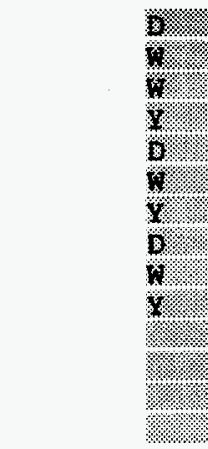 & 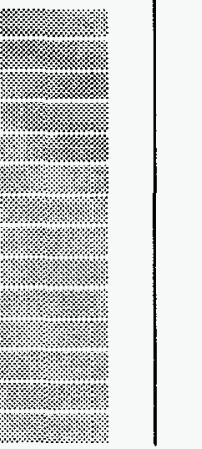 & 8 & 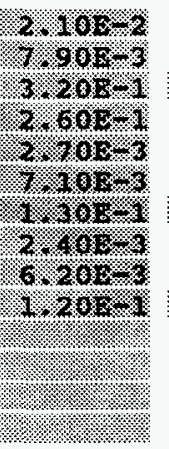 & $\begin{array}{l}\text { W } \\
\text { XY }\end{array}$ & \\
\hline
\end{tabular}

Press "F5" (or "F6") to view previous (or next) set of radionuclides.

Press "F1" or "F2" for HELP, or "Esc" to IGNORE CHANGES and return to main menu. Press "FIO" to SAVE DATA AND CONTINUE.

\%: .

FIGURE 4.38 Input Form B-1 for Inhalation (Dust) Effective Dose Conversion Factors 
contaminated dust. The $D C F(i)$ is measured in mrem/pCi. The values are for dust particles with an AMAD of 1 pn.

The inhalation class or rate of clearance from the lung is also listed. The three classes $\mathrm{D}, \mathrm{W}$, and $\mathrm{Y}$ correspond to retention half-times of less than 10 days, 10 to 100 days, and greater than 100 days, respectively. Usually more than one inhalation class is listed per radionuclide. If so, the DCF that will be used in the RESRAD calculations is marked by an "!" suffix. Also, the other inhalation classes for the same radionuclide appear in subdued print. To change the selected DCF, the cursor must be positioned in the desired field and the F4 key pressed. The RESRAD default inhalation class in TAB-B1.DEF and RESRAD.BIN is always the most conservative (highest dose) DCF.

For a few radionuclides, inhalation classes other than $\mathrm{D}, \mathrm{W}$, and $\mathrm{Y}$ are listed, indicating a gaseous airborne radionuclide. Currently the only such cases are hydrogen-3, carbon-14, nickel-59, and nickel-63. Radon is excluded because it involves a separate RESRAD pathway (see Appendix C).

\subsubsection{Ingestion Effective Dose Conversion Factors}

Input Form D-1, corresponding to Table D.1, is the result of this submenu choice. A typical screen is presented in Figure 4.39. A DCF for ingestion is the dose/exposure ratio, $D C F(i)=H_{E} / E(i)$, for the committed effective dose equivalent $H_{E}$ that an individual incurs from exposure by intake of a quantity $E(i)$ of the $i$ th principal radionuclide. The units of measure for $D C F(i)$ are $\mathrm{mrem} / \mathrm{pCi}$.

Ingestion DCFs depend on the chemical form of the radionuclide; the chemical form determines the fraction, $f_{1}$, of a radionuclide that enters the GI tract and reaches body fluids. The $f_{1}$ values are also shown in Form D-1. If more than one $f_{1}$ value is listed for a radionuclide, the corresponding DCF that will be used in the RESRAD calculations is marked by an "!" suffix, and the other $f_{1}$ value is shown in subdued print. To change the DCF selection, the cursor must be positioned in the desired DCF field and the F4 key pressed. The RESRAD default $f_{1}$ fraction in TAB-D1.DEF and RESRAD.BIN is always the most conservative (highest dose) DCF. 


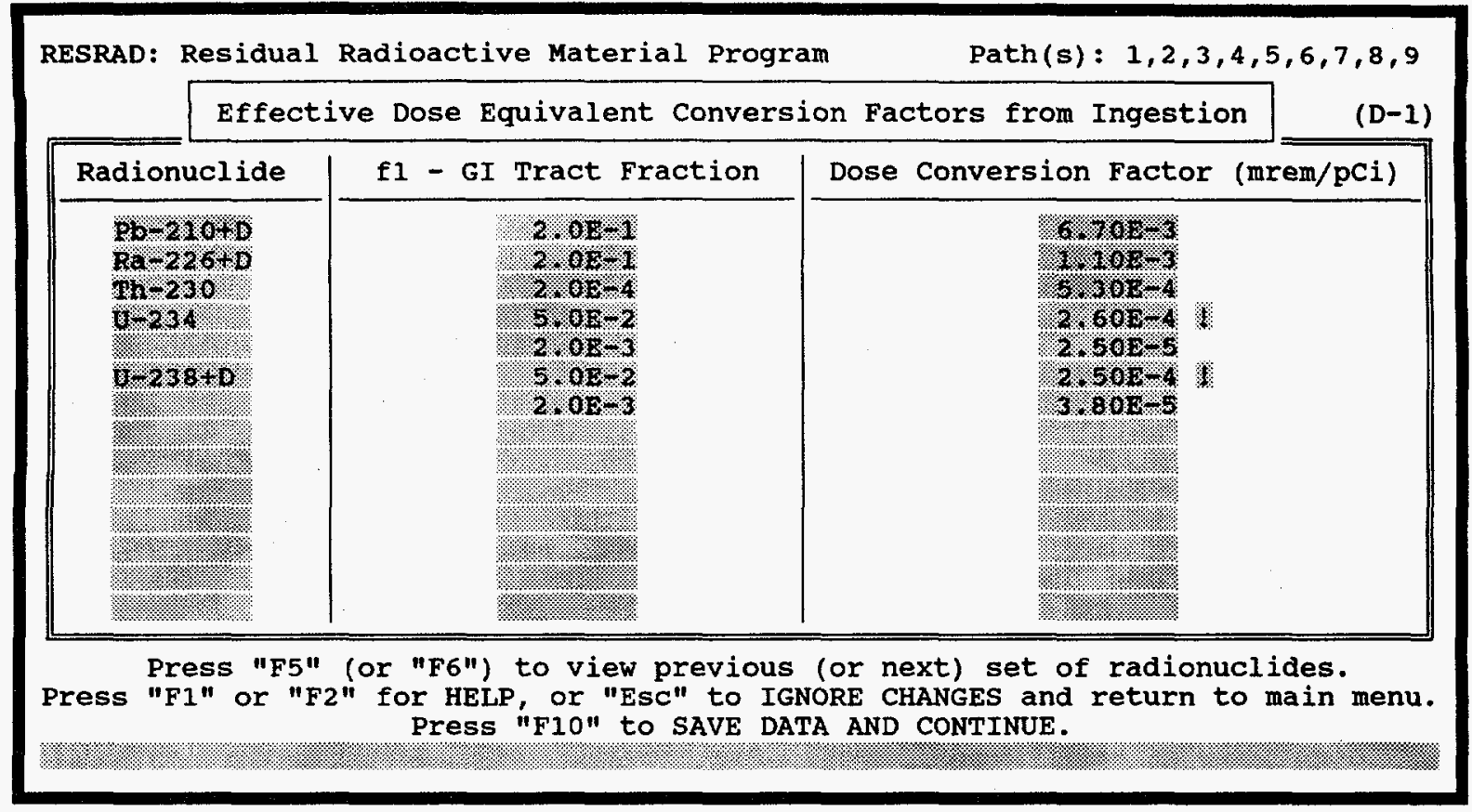

\section{FIGURE 4.39 Input Form D-1 for Ingestion Effective Dose Conversion Factors}

The $f_{1}$ values listed in Form D-1 cannot be changed. This is of no consequence, however, in the calculations to follow because RESRAD only uses the corresponding DCFs.

\subsubsection{Food Transfer Factors}

The result of selecting this submenu is Form D-34, which corresponds to a combination of Tables D.3 and D.4. A typical screen is shown in Figure 4.40. The transfer factors are used to compute environmental transport factors for the plant, meat, and milk pathways. See Section D.2.1 in Appendix D for details. The three transfer factors per element are summarized below.

Plant is the plant/soil transfer factor for root uptake. It is the ratio of radionuclide concentration in the edible portions of the plant at harvest time to the dry soil radionuclide concentration. The factor is dimensionless. It is assumed that the same transfer factor can be used for leafy and nonleafy vegetables.

Beef is the meat/livestock-intake transfer factor. It is the ratio of radionuclide concentration in beef to the daily intake of the same radionuclide in livestock feed, water, or soil. The units of measure are $(\mathrm{pCi} / \mathrm{kg}) /(\mathrm{pCi} / \mathrm{d})$ or $\mathrm{d} / \mathrm{kg}$. 


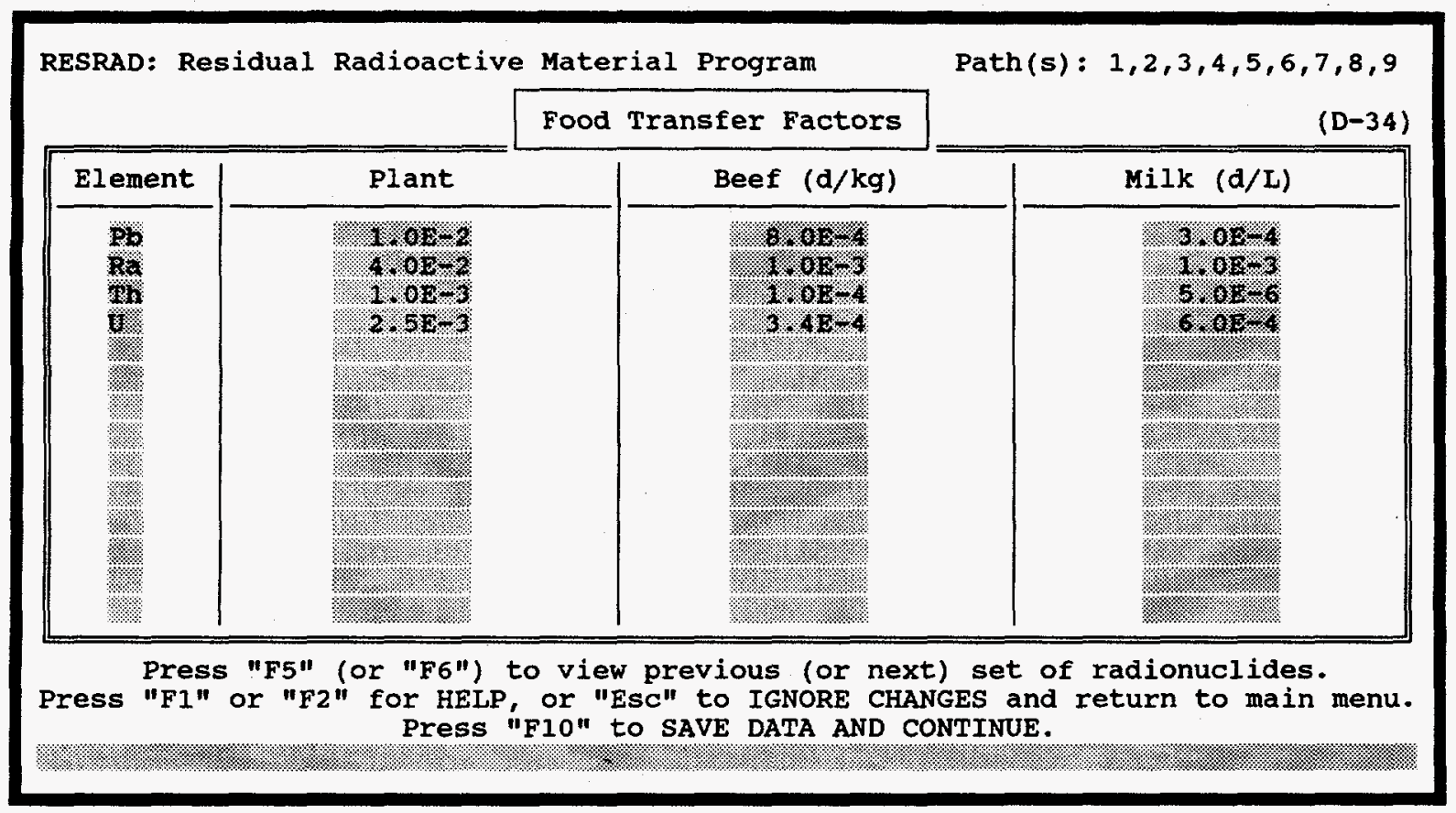

FIGURE 4.40 Input Form D-34 for Food Transfer Factors

Milk is the milk/livestock-intake transfer factor. It is the ratio of radionuclide concentration in milk to the daily intake of the same radionuclide in livestock feed, water, or soil. The units of measure are $(\mathrm{pCi} / \mathrm{L}) /(\mathrm{pCi} / \mathrm{d})$ or $\mathrm{d} / \mathrm{L}$.

\subsubsection{Bioaccumulation Factors for Aquatic Foods}

Input Form D-5, corresponding to Table D.5, is the result of this submenu choice. A typical screen is presented in Figure 4.41. The bioaccumulation factors are used to calculate environmental transport factors for the aquatic food pathway. See Section D.2.2 in Appendix $\mathrm{D}$ for details. The bioaccumulation factor is the ratio of radionuclide concentration in the aquatic food to the concentration of the same radionuclide in water. The units of measure are $(\mathrm{pCi} / \mathrm{kg}) /(\mathrm{pCi} / \mathrm{L})$ or $\mathrm{L} / \mathrm{kg}$.

The two factors presented per element are for fish and crustacea and mollusks. The RESRAD factors are for freshwater only. 


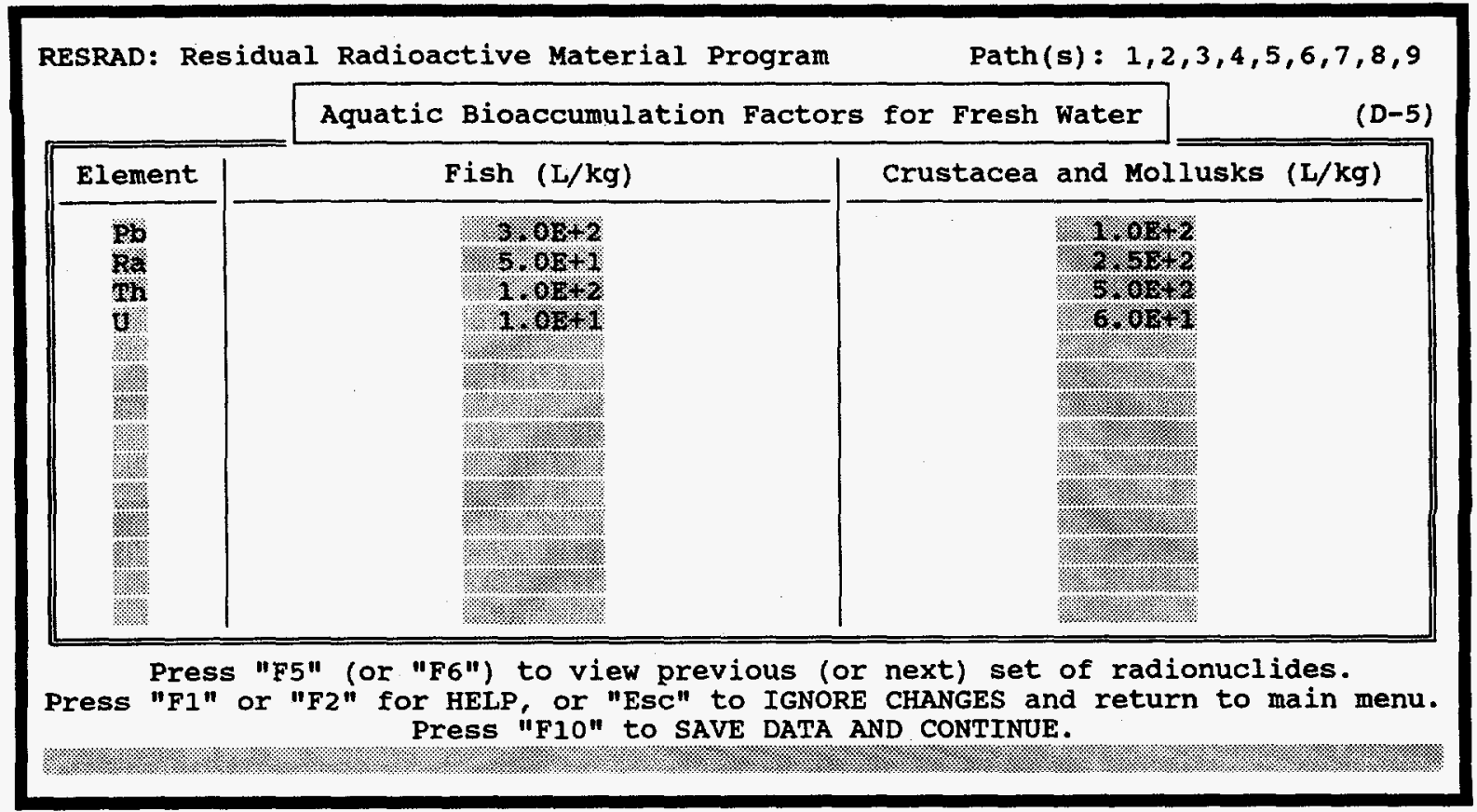

FIGURE 4.41 Input Form D-5 for Bioaccumulation Factors for Aquatic Foods

\subsubsection{Slope Factors}

The selection of this submenu choice enables the user to view and/or modify the slope factors used to calculate excess cancer risks. Two further options are presented: slope factors for principal radionuclides and slope factors for radon (and radon progeny).

A typical screen showing slope factors for principal radionuclides and associated principal progeny is presented in Figure 4.42 (Form SF). This table displays the slope factors for external radiation (in risk/yr per $\mathrm{pCi} / \mathrm{g}$ ), inhalation (in risk/pCi), and ingestion (in risk/pCi).

A typical screen showing the radon slope factors is displayed in Figure 4.43 (Form RN). In this case, only one set of slope factors is provided (in risk/pCi) since inhalation is the only major pathway contributing to the radon dose.

\subsubsection{Quit}

The final submenu option terminates RESRAD and returns the screen to the DOS prompt. The Enter key must be pressed with the Quit field highlighted and the main menu 
RESRAD: Residual Radioactive Material Program

$\operatorname{Path}(s): 1,2,3,4,5,6,7,8,9$

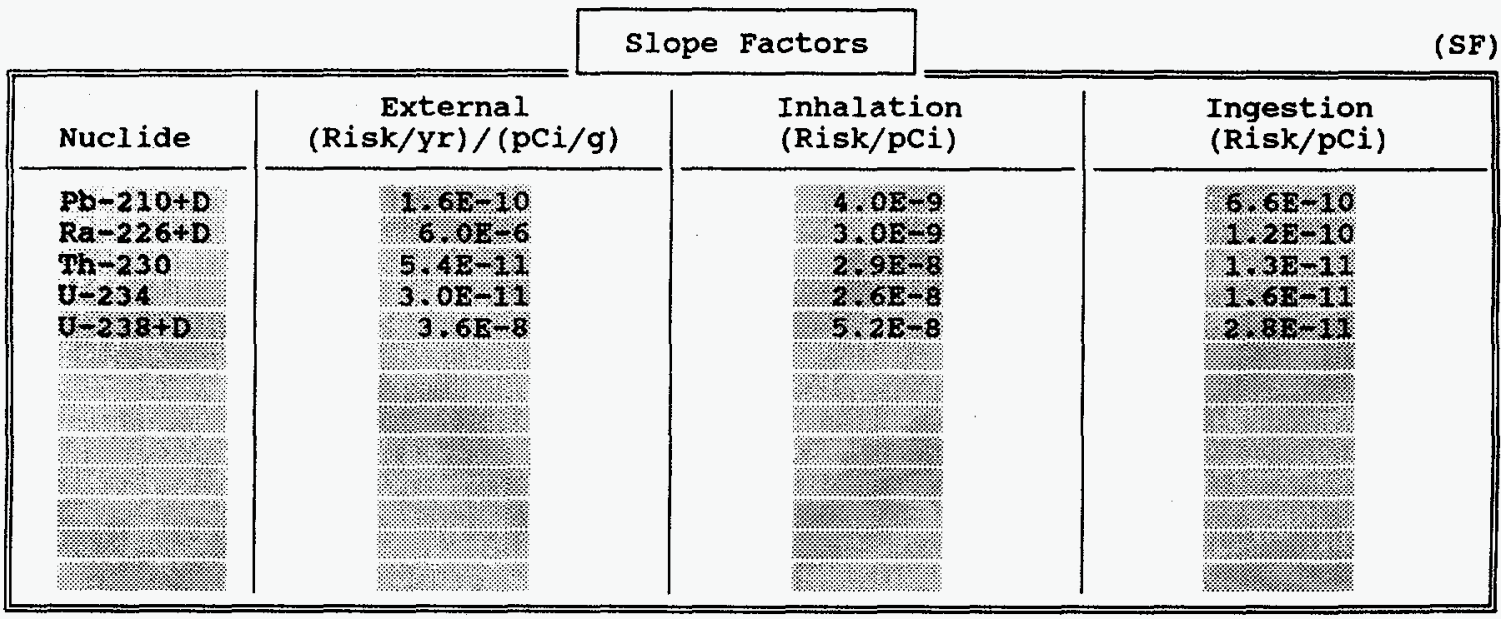

Press "F5" (or "F6") to view previous (or next) set of radionuclides.

Press "F1" or "F2" for HELP, or "Esc" to IGNORE CHANGES and return to main menu. press "F1O" to SAVE DATA AND CONTINUE.

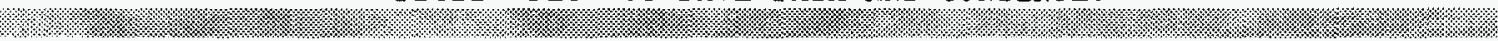

FIGURE 4.42 Input Form SF for Principal Radionuclide Slope Factors

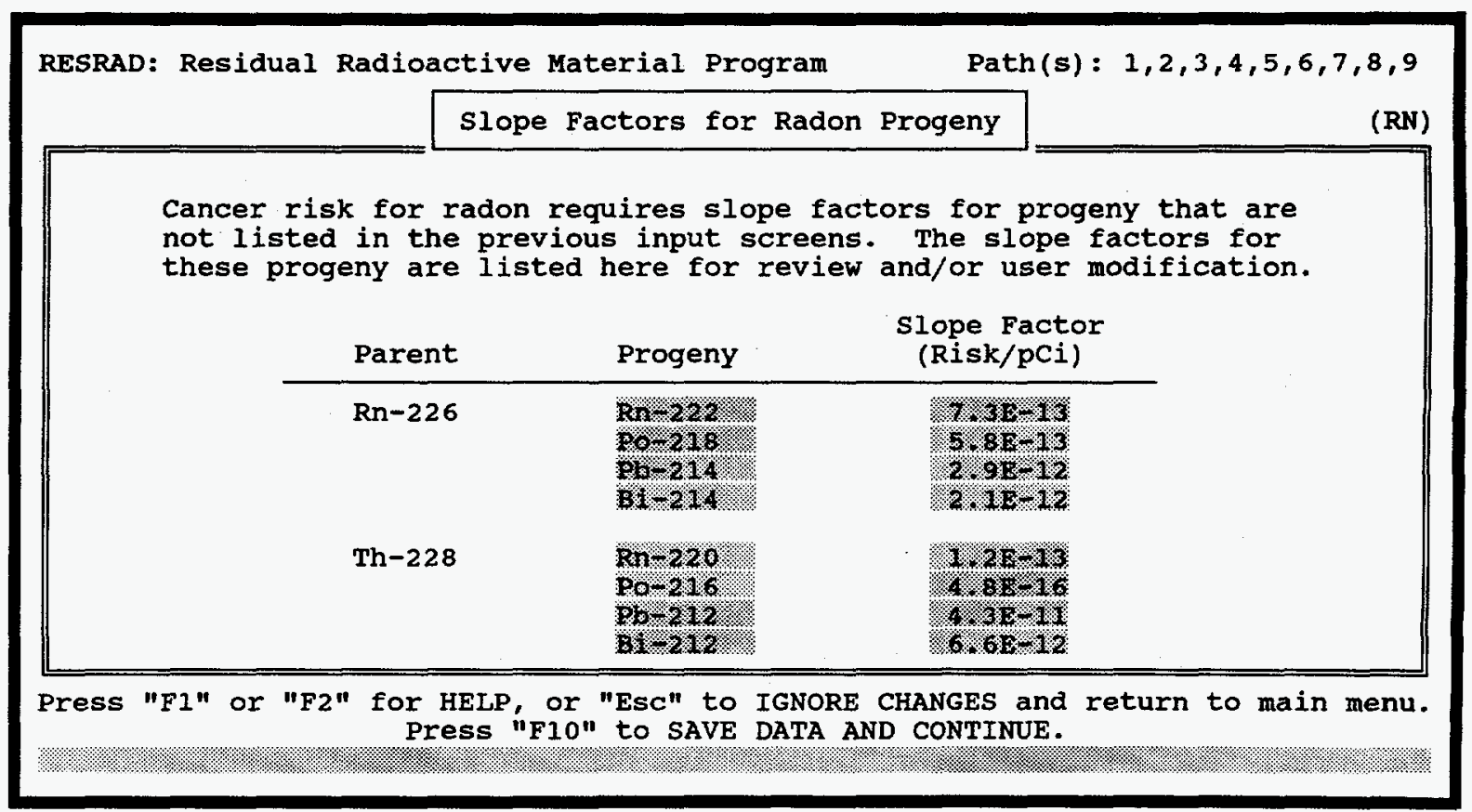

FIGURE 4.43 Input Form RN for Radon Slope Factors 
alone on the screen. Repeatedly pressing the Esc key is not sufficient; Enter must also be pressed. An alternative is to press the space bar followed by the $Q$ key. This multikey sequence guards against accidental termination of a RESRAD session.

\subsection{TECHNICAL SUMMARY}

It is not the intent of this manual to include a programmer's guide, but a brief technical summary should be useful.

RESRAD5 consists of three main programs: RESPC, RESMAIN, and RESPLOT. RESPC is a set of compiled BASIC programs (Microsoft BASIC 7.1) that handles most user interaction with RESRAD. RESMAIN is the FORTRAN code (Lahey F77L 5.0) that does the actual computations and generates the output tables and graphics data files. RESPLOT is a stand-alone FORTRAN system (Lahey F77L 5.0 and MicroGlyph SCIPLOT 4.1) that executes the computer graphics. The user can select whether RESPLOT is loaded automatically when RESMAIN is completed or whether it is called from within RESPC.

RESPC is loaded first (via RESRAD.BAT, NEXT.ORI, and NEXT.BAT). Within RESPC, the user may input selected site-specific data files, review and/or modify the parameters, select parameters for sensitivity analysis, specify graphics hardware parameters and options, save the data in a selected file, view and/or print selected results (including graphics) from the last RESMAIN computation, or run RESMAIN for a new computation. If RESMAIN or RESPLOT is called, RESPC is reloaded automatically when RESMAIN or RESPLOT is completed. The user may then continue with any of the RESPC options. 


\section{APPLICATION OF THE AS LOW AS REASONABLY ACHIEVABLE PROCESS}

Application of the ALARA process is required in DOE environmental protection and health and safety orders, for example, DOE Order 5400.5 (DOE 1990), and in the DOE residual radioactive material guidelines. The objective of the ALARA process is the attainment of dose levels that are as far below applicable limits as is practicable and reasonably achievable, taking into account many factors, including technical, economic, safety, and social considerations. The recommendations contained in this manual do not address "below regulatory concern" (BRC) or "de minimis" concepts. ${ }^{17}$ The ALARA process should be applied throughout the project, from planning to field work. Application of the ALARA process through planning activities occurs both in the development of guidelines and in the site-specific application of the guidelines. The ALARA process should be implemented in a manner consistent with DOE guidance (DOE 1991).

Application of the ALARA process to any radiation protection activity or project is necessary but somewhat subjective. The success of the process depends on the good judgment of the health physicists and managers who are responsible for its application. Applying the ALARA process to remedial actions is, in many cases, more complex than applying it to facility operating situations. For operating facilities, one can identify the sources of radiation, waste streams, pathways, potentially exposed individuals, and likely operating times. The dose reductions from various control actions, which have specific costs over the expected time period, can be estimated and compared. In the case of contaminated facilities requiring remedial action and other environmental protection activities, it may be more difficult to define all of these elements, particularly if potential impacts must be projected for many years into the future and if such impacts could extend for considerable distances from the site of the activity. The assessments may be simplified and conducted in an implementable manner if it is recognized that the primary purpose of the ALARA analysis is to support an environmental and public protection management decision. In this situation, time periods

17 These are concepts wherein authorities would select dose levels at which (1) the presumed probability of the recipient(s) experiencing a radiation-induced health effect would be so low that the resources required for any technical or administrative effort to further reduce the potential dose could not be justified (BRC) or (2) the associated incremental risk to any exposed individual or collectively to the exposed population would be so low as to be deemed "trivial" and should be acceptable without further consideration of benefit, cost, or any other factor (de minimis). 
and distances need not extend beyond those necessary to make a reasonable comparison of competing alternatives.

For sites to be certified in accordance with the guidelines, the intent is to reduce the concentrations of residual radioactive materials to levels (authorized limits) selected through the ALARA process, with consideration given to technical, economic, and social factors. Examples of specific factors include public and worker health, the ecology, and cultural and natural resources. At sites where the residual materials can be reduced only to levels that require that restrictions be placed on the use of the site, the ALARA process includes consideration of the establishment of institutional controls to reduce exposure.

In remedial action or decontamination and decommissioning work under DOE jurisdiction, the concentration limit, or "guideline," for a particular radionuclide in soil or on surfaces is derived from the basic dose limit by using site-specific pathway analysis as a starting point, or "base case," for applying the ALARA process. Each remedial action can differ, in some respect, from those at other sites. In one situation, the contamination might be reasonably uniform over the surface; in others, "hot spots" of radioactive material might exist at various depths. A portion of the radioactive material might have eroded and been transported to another area by wind or water. In buildings, the contamination might be on the surface or buried beneath paint or other wall coverings. A nonuniform distribution of the contamination is the rule rather than the exception. In applying the ALARA process, the first task is to ensure that the area being remediated is at or below the authorized limit or dose constraint; the second is to determine that the residual radioactive material is reduced to levels that are as low as reasonably achievable below the dose constraint.

\subsection{DETERMINING ALARA}

DOE guidance (DOE 1991) indicates that the level of detail in an ALARA analysis should be commensurate with the potential dose or risks associated with the residual radioactive material and the costs or benefits associated with remedial alternatives. Approaches used for supporting the ALARA process range from qualitative, or judgmental, procedures to quantitative, or optimization, procedures. Both types of procedures involve consideration of economic, social, and technical factors to ultimately determine a limit for the site being studied. The level of detail and documentation involved in a qualitative 
comparison can vary significantly. If overall risks are very low and the cost of further reducing potential doses is very high, the decision is obvious, and minimal analysis and documentation are required. However, if potential doses approach levels that are significant in comparison to the dose limit, or the collective dose is significant, more detail is warranted (DOE 1991). The quantitative procedures involve a cost-benefit analysis, as proposed by the ICRP $(1973,1977,1983 a)$, to quantify the costs of improved radiological protection, the costs associated with health detriment from radiation, and the cost of other factors such as ecological detriment and damages to natural resources.

The ALARA process generally involves a multiattribute type of decision-making process. A significant part of the process consists of a cost-benefit analysis; however, frequently, nonquantifiable factors must also be considered.

Many times social factors are the most difficult to address in the ALARA process; when the value of such factors is assessed, it is essential that input be obtained from concerned, affected individuals or concerned groups or organizations on many of the factors considered in the ALARA process. Some social factors can be quantified and addressed in the cost-benefit analysis (e.g., costs of temporary displacement, reduced or increased property values, and reduced access). For others, however, it is difficult, if not impossible, to assign a monetary equivalent (e.g., damage to or reduced access to cultural resources, or confiscation or modification to heirlooms of personal value) except in retrospect. In some cases, the importance of social factors may totally override other considerations. Even in the case of the quantitative risk assessment, these factors generally must be considered qualitatively.

As a result of the need to compare such diverse factors in the ALARA analysis, a key element in high quality (i.e., detailed) ALARA analysis consists of the judgments of the responsible individuals (analysts and decision-makers). These judgments are based on each person's understanding of the relevant economic, social, and technical factors. Therefore, it is important that responsible individuals be appropriately trained in the process and methods of obtaining necessary external input, especially on nontechnical factors, and that they use such input in weighing factors in the decision-making process. Because of the subjective nature associated with weighing many of the factors being considered, documentation of the assumptions and thought processes (rationale) used in qualitative assessments is extremely important. 


\subsubsection{Qualitative Considerations}

The difference between the qualitative and quantitative ALARA analyses (or a semiquantitative analysis) is the level of data associated with value/impact analyses or cost-benefit assessment. In both cases, the same factors should be considered. However, as the analysis becomes more quantitative, more details regarding the economics of each option and the equivalent monetary values for impacts are required. Qualitative assessments may involve the observation that the ecological impacts are low for several options and high for others, and that likely collective doses are significantly reduced (e.g., by a factor of 10) or not significantly different. Rough estimates of costs and doses associated with alternatives are typically provided, but no formal optimization analysis is conducted. Similarly, social factors (e.g., concerns regarding cultural resources) are considered and identified, if they exist, but are not quantified. Such an analysis may list the alternatives evaluated and provide a qualitative value (e.g., no impact, minor concern, or significant impact). The appropriate action would be selected on the basis of the data provided. Qualitative assessments would not be expected to have as many alternatives as quantitative analyses. In the extreme case, where alternatives are very limited and the choice is obvious, the qualitative ALARA assessment may be no more than a memorandum documenting the fact that key factors were considered and that the selected option provided the only reasonable and practicable solution. The discussion below describes factors that should be considered in an ALARA analysis.

One important element in the economic factor is the cost of doing the work. The cost of remedial action typically includes the initial planning cost as well as the cost of operation and associated labor, materials, equipment, energy, and services over the period of remediation. The initial planning cost includes all costs relative to planning and engineering tasks such as performance of radiation surveys; calculation of contamination inventories; performance of engineering studies; preparation of a remedial action plan, with descriptions and specifications; and preparation of detailed cleanup and removal procedures. The operating cost includes all costs associated with physical tasks such as removal of contamination; removal of equipment and structures; storage, packaging, shipping, and burial of wastes; and final site and facility restoration or preservation. In addition to the cost of doing the work, economic factors should also include the potential impact on current facilities, operations, and the environment. The impact and associated cost of either the action or no 
action may have a positive or negative effect on current operations or the environment, and such cost may be significant in comparison with the cost of performing the remedial action. The cost of waste management (disposal, storage, and transport) must also be considered in the ALARA assessment. While qualitative or semiquantitative ALARA analyses do not normally require the detailed economic analysis required for the optimization process conducted in a quantitative ALARA assessment, cost estimates of each alternative should be provided in a qualitative assessment.

Social factors to be considered can cover a wide range of issues including protection of natural and cultural resources, public perception and concerns, environmental equity, impacts on property values, and future industrial development. In the qualitative assessment, the magnitude or the existence of such impacts should be identified for specific alternatives considered. In quantitative assessments, attempts to assign equivalent monetary values to some of these factors may be useful. However, in many cases, prospectively quantifying those costs is likely to be impossible. Hence, the weighing of these factors is expected to be based on subjective decisions, even in the quantitative ALARA process. The societal costs can be determined retrospectively. The societal cost is the cost beyond that which can be justified for the health detriment.

Routinely, consideration is given to the potential exposure scenarios and the risk to those persons who might occupy the area immediately following cleanup; however, many of the sites are contaminated with long-lived radioactive materials, and cleanup will affect the potential risks received over many years and perhaps over areas in the vicinity of the remediated site. Therefore, in evaluating risks, costs, and benefits, these temporal and spatial factors must be taken into account. In carrying out remedial actions on contaminated areas and vicinity properties, occupational exposures will occur. Controlling the radiation exposure of the work force should be based on DOE guidance for occupational exposure, and all unnecessary doses should be avoided. In applying the ALARA process, the reductions in risks (collective dose) to the population of the site and in the area surrounding the disposal site following the remedial action should be compared with the incremental risks (collective dose) to the population in the same areas and to workers during remedial action activities. It is most desirable that such a comparison result in a beneficial balance in overall risk. 
Technical factors are primarily related to technological alternatives for treatment and disposal of the contaminated materials. Also, the technique used to determine the level and extent of contamination is important. The number of soil samples that can be taken might be limited, and the measurement methods might have limited sensitivity. Increasing the number of samples taken and using state-of-the-art instruments or methods for all samples in order to accurately determine the above-background distribution of all radionuclides might be prohibitively expensive. Therefore, a decision must be made regarding the number and location of samples and the sensitivity of the instruments used for establishing compliance with concentration guidelines and for implementing the ALARA process.

A traditional method for determining ALARA consists of making a series of individual decisions for each of the situations encountered. This process typically involves qualitative assessments and is regularly applied in the field. A number of such decisions may be made for different parts of the overall area as work progresses; that is, it is not necessary to make ALARA decisions only after all of the work has been completed or before it is begun.

Frequently, there are subareas with difficult problems and before moving to the next subarea, the work can be concentrated on these difficult areas until the ALARA process is satisfied. This ALARA process activity falls under the category of "good management practices." The temptation to set an "ALARA limit" for this portion of the process should be avoided. Similarly, while the benefits of the "field ALARA" can be used qualitatively in the establishment of authorized limits for a specific action, it should not necessarily be assumed that because previous remedial actions have resulted in actual cleanups achieving levels that were less than derived limits, it is cost-effective to lower (make more restrictive) authorized limits to account for field application of ALARA. ALARA is a process rather than a limit; the lowering of authorized limits based on anticipated outcomes of ALARA being applied in the field may result in greatly increased costs, unnecessary risks, or even in the inability to meet the limit because of unexpected developments.

\subsubsection{Cost-Benefit Analysis}

The ICRP has recommended the use of cost-benefit analysis to determine the levels of population exposure that are reasonably achievable. This quantitative technique compares the cost of health detriment and other factors relative to radiation with the cost of radiation dose reduction (see Appendix $M$ ). As stated previously, even when a rigorous cost-benefit 
analysis is conducted, the decision-makers frequently must integrate nonquantifiable factors in the process.

To apply cost-benefit analysis to radiation protection, a policy decision is often made that the action is already justified (a net positive benefit); this decision may be based on a policy or on broad political, economic, or social considerations. A form of differential costbenefit analysis can be used. The objective is to ensure that the resultant individual doses are kept as low as reasonably achievable below the dose limit. In this differential analysis, consideration shifts from the total cost and benefit to the change in net cost and benefit that might result from requiring the action to be performed at one level of remedial action rather than another. The benefit of a reduction in collective dose is compared with the cost of achieving the reduction. The optimum radiological protection activity or system is that which is defined by a marginal increase in the cost of protection, which is exactly balanced by a marginal decrease in the cost of detriment; that is,

$$
\frac{d X}{d S}=-\frac{d Y}{d S}
$$

where

$$
\begin{aligned}
& X=\text { the cost of achieving a given level of protection, } \\
& Y=\text { the cost of the radiological detriment at the level of protection, }{ }^{18} \text { and } \\
& S=\text { the collective effective dose equivalent. }
\end{aligned}
$$

This is described in more detail in the DOE guidance on meeting ALARA requirements for compliance with DOE Order 5400.5 (DOE 1990).

Cost-benefit analysis is a helpful procedure for quantifying both the costs of improved protection and the costs associated with radiological detriment from alternative courses of

18 The classical expression for the total cost of detriment, $Y$, is given by

$$
Y=\alpha S+\beta \mathrm{f}(S)
$$

where $\alpha$ is the selected monetary value for (avoiding) a unit of collective dose; $S$ is the collective dose; $\alpha S$ is the cost of the health-effect-related detriment; and $\beta$ is the selected monetary value for the nonhealth-effect related detriment that generally is applied to collective dose in a nonlinear manner to yield the cost of the nonhealth detriment (see Appendix M). 
action. This technique is particularly useful when extensive remedial action activities for radiological protection are being considered, when individuals are being exposed to levels close to the dose limit, or when potential collective doses are large. For small-scale problems, simplified analyses are sufficient. However, because a cost-benefit analysis does not encompass all judgments that might be involved in a specific decision concerning radiological protection, such an analysis is only one procedure for quantitatively determining some of the input to that decision.

To implement the ALARA process by using cost-benefit analysis, the costs of radiological protection and radiological detriment must be assessed. The degree of sophistication and effort given to a cost-benefit assessment should reflect the scale of the radiological problem being considered. For a large-scale problem, the assessment might be an evaluation combining engineering designs, control technologies, accounting techniques, and the use of mathematical models for pathway analyses and dose calculations. For a smallscale problem, the assessment might require only simple, intuitive estimates. The estimation of protection costs is generally straightforward.

The assignment of costs to health detriment requires both scientific and other judgments. For example, the NRC established quantitative ALARA guidelines for radionuclide emissions for light-water reactors after several years of intensive study of the emission control technology and operating costs (NRC 1974). The monetary worth of avoiding a unit of collective dose used in the cost-benefit analysis for deriving ALARA guidelines was assumed to be $\$ 1,000$ per person-rem. This same value for $\alpha$ was also used by the NRC in its proposed safety goals for nuclear power plants (NRC 1982); these safety goals were intended to set design objectives for the reliability of safety systems. The EPA has also used an ALARA approach when deriving many of its regulations. Table 5.1 summarizes some examples of expenditures to limit cancer risk that were derived from criteria and guidelines issued by the EPA and NRC. The costs range from $\$ 1$ million to $\$ 200$ million per cancer averted. Assuming a lifetime cancer fatality risk estimate of $5 \times 10^{-4}$ per person-rem, the cost/risk ratios in Table 5.1 are equivalent to economic values of about $\$ 20$ to $\$ 4,000$ per person-rem of radiological detriment. The range resulting from this limited analysis is typical of that in the open literature (i.e., less than $\$ 100 /$ person-rem to more than 
TABLE 5.1 Examples of Expenditures to Limit Cancer Risk

\begin{tabular}{|c|c|c|c|c|}
\hline Example & Agency & $\begin{array}{l}\text { Cost per } \\
\text { Cancer } \\
\text { Averted }^{\mathrm{a}} \\
\left(10^{6} \$\right) \\
\end{array}$ & $\begin{array}{c}\text { Lifetime } \\
\text { Risk Level } \\
\end{array}$ & Comments \\
\hline $\begin{array}{l}\text { Routine radioactive releases } \\
\text { from light-water reactor } \\
\text { effluents }\end{array}$ & NRC & $2^{b}$ & $6 \times 10^{-5}$ & $\begin{array}{l}1975 \text { dollars; no correction } \\
\text { for inflation; only fatal } \\
\text { cancers }\end{array}$ \\
\hline $\begin{array}{l}\text { Dioxin contamination at } \\
\text { Times Beach, Missouri }\end{array}$ & EPA & $2-160$ & $1 \times 10^{-2}$ & Includes nonfatal cancers \\
\hline Drinking water criteria & EPA & $20-200$ & $1 \times 10^{-5}$ & Includes nonfatal cancers \\
\hline $\begin{array}{l}\text { Safety goals for nuclear } \\
\text { power plants }\end{array}$ & NRC & $2^{b}$ & $1 \times 10^{-4}$ & $\begin{array}{l}1982 \text { dollars; inflation } \\
\text { correction included; only } \\
\text { fatal cancers }\end{array}$ \\
\hline Proposed asbestos ban & EPA & 1 & $4 \times 10^{-5}$ & Fatal cancers \\
\hline
\end{tabular}

a Estimated cancers, not actual incidence.

b Revised estimates in the original source are based on a risk factor of $5 \times 10^{-4}$ fatal cancers/person-rem ( $1 / 5$ th of original estimate).

Source: McKone (1986).

$\$ 10,000 /$ person-rem). Herrington et al. (1988) reviewed the literature to identify a monetary equivalent for collective dose and concluded that a monetary equivalent value for worker dose based only on health effects would be on the order of $\$ 100 /$ person-rem. This is the classical ALARA "alpha" term. They noted"that other factors (normally classified as the "beta" portion of the ALARA equation) could exceed the value of the alpha term such that the combined monetary equivalent value would be on the order of $\$ 1,000 /$ person-rem or more depending on site-specific considerations. Similarly, the beta term in estimating the monetary equivalence of public doses can vary by site and alternative. Factors such as duration of the dose, age of the affected population, willingness of the population to accept risks, and even the magnitude of the dose relative to dose limits can affect the beta term of the monetary equivalence. 


\subsubsection{Summary}

In applying the ALARA process on the basis of either qualitative or quantitative analysis, the risks/cost incurred from implementing remedial action must first be justified by the reduction in risk (increasing the benefit) that will result. The ALARA process requires that the responsible persons use judgment with respect to what is "reasonably achievable." The economic, social, and technical factors influencing this judgment are highly variable and site specific. At a minimum, the following parameters should be evaluated to the extent practicable before making a decision:

- Maximum dose to an individual member of the public;

- Collective dose to the population;

- Time and duration of exposure of the affected individual or population;

- Likelihood of a specific use scenario occurring at a site;

- Technological alternatives, such as alternative methods for treatment and disposal of the contaminated materials, alternative operating methods for conducting cleanup activities, or alternative institutional controls for reducing exposure;

- Demographic data;

- Occupational doses associated with each technological alternative;

- Costs of each technological alternative;

- Variations in social and cultural impacts associated with the various alternatives; and

- Ecological and resource impacts.

In allocating resources relative to ALARA considerations, the total value should depend on the size of the affected population, the level of risk already experienced from background radiation sources, and the magnitude of risk for each individual. Because the individual dose must be in compliance with the dose limit regardless of costs, greater cost to reduce collective doses composed of individual doses approaching the dose limit can be more easily justified than costs to reduce doses composed of individual doses far below the limit. 
Because radionuclides considered for DOE sites have long half-lives, the potential radiation risk must be calculated for populations that might exist hundreds and thousands of years from now. This calculation becomes increasingly uncertain as the time period of radiological dose assessment increases. To realistically account for these uncertainties, it is reasonable to attach more weight to doses predicted for the present or the near future than to doses predicted for the distant future, especially when the use scenarios appear unlikely.

\subsection{DOCUMENTING ALARA}

The documentation of any decision regarding the ALARA process in remedial action provides an invaluable record to show that the process was applied. Because DOE is responsible for the work being done, any judgment regarding the ALARA process is ultimately the responsibility of DOE, and DOE personnel should be involved in the decisions and plans that document how the process will be applied to the project and in the field. Therefore, it is essential (and required by DOE directives) that ALARA decisions be well documented, including those made in the field and those made prior to or following field work. 


\section{REFERENCES}

Ahrends, S.W., 1987, Revised Post Remedial Action Survey Protocol, attachment to letter from Ahrends (U.S. Department of Energy, Oak Ridge Operations, Oak Ridge, Tenn.) to G. Hovey (Bechtel National, Inc., Oak Ridge, Tenn.), May 26.

Chen, S.Y., 1991, "Calculation of Effective Dose Equivalent Responses for External Exposure from Residual Photon Emitters in Soil," Health Physics 60:411-426.

Cheng, J.J., C. Yu, and A. Zielen, 1991, RESRAD Parameter Sensitivity Analysis, ANL/EAIS-3, Argonne National Laboratory, Argonne, Ill.

Eckerman, K.F., et al., 1988, Limiting Values of Radionuclide Intake and Air Concentration and Dose Conversion Factors for Inhalation, Submersion, and Ingestion, EPA-520/1-88-020, Federal Guidance Report No. 11, prepared by Oak Ridge National Laboratory, Oak Ridge, Tenn., for U.S. Environmental Protection Agency, Office of Radiation Programs, Washington, D.C.

Eve, I.S., 1966, "A Review of the Physiology of the Gastrointestinal Tract in Relation to Radiation Doses from Radioactive Material," Health Physics 12:131.

Gilbert, T.L., et al., 1983, Pathways Analysis and Radiation Dose Estimates for Radioactive Residues at Formerly Utilized MED/AEC Sites, ORO-832 (Rev.), prepared by Division of Environmental Impact Studies, Argonne National Laboratory, Argonne, Ill., for U.S. Department of Energy, Oak Ridge Operations, Oak Ridge, Tenn., March 1983 (reprinted with corrections January 1984).

Gilbert, T.L., et al., 1989, A Manual for Implementing Residual Radioactive Material Guidelines, ANL/ES-160, DOE/CH/8901, prepared by Argonne National Laboratory, Argonne, III., for U.S. Department of Energy.

Healy, J.W., 1977, An Examination of the Pathways from Soil to Man for Plutonium, LA-6741-MS, Los Alamos Scientific Laboratory, Los Alamos, N.M.

Herrington, W.N., et al., 1988, Health Physics Manual of Good Practices for Reducing Radiation Exposures to Levels That Are as Low as Reasonably Achieveable (ALARA), PNL6577, U.S. Department of Energy, June.

International Commission on Radiological Protection, 1966, "Deposition and Retention Models for Internal Dosimetry of the Human Respiratory Tract," Health Physics 12:173.

International Commission on Radiological Protection, 1968, The Metabolism of Compounds of Plutonium and Other Activities, ICRP Publication 19, Pergamon Press, Oxford, United Kingdom. 
International Commission on Radiological Protection, 1972, Alkaline Earth Metabolism in Adult Man, ICRP Publication 20, Pergamon Press, Oxford, United Kingdom.

International Commission on Radiological Protection, 1973, Implications of Commission Recommendations That Doses Be Kept as Low as Readily Achievable, ICRP Publication 22, Pergamon Press, New York, N.Y.

International Commission on Radiological Protection, 1975, Report of the Task Group on Reference Man, prepared by a Task Group of Committee 2, adopted by the Commission in October 1974, ICRP Publication 23, Pergamon Press, New York, N.Y.

International Commission on Radiological Protection, 1977, Recommendations of the International Commission on Radiological Protection, ICRP Publication 26, Annals of the ICRP, 1(2), Pergamon Press, New York, N.Y.

International Commission on Radiological Protection, 1978, Radionuclide Release into the Environment: Assessment of Doses to Man, a report of Committee 4 of the International Commission on Radiological Protection, adopted by the Commission in October 1978, ICRP Publication 29, Annals of the ICRP, 2(2), Pergamon Press, New York, N.Y.

International Commission on Radiological Protection, 1979-1982, Limits for Intakes of Radionuclides by Workers, a report of Committee 2 of the International Commission on Radiological Protection, adopted by the Commission in July 1978, ICRP Publication 30, Part 1 (and Supplement), Part 2 (and Supplement), Part 3 (and Supplements A and B), and Index, Annals of the ICRP, Pergamon Press, New York, N.Y.

International Commission on Radiological Protection, 1983a, Cost-Benefit Analysis on the Optimization of Radiation Protection, ICRP Publication 37, Annals of the ICRP, 10(2/3), Pergamon Press, New York, N.Y.

International Commission on Radiological Protection, 1983b, Radionuclide Transformations: Energy and Intensity of Emissions, ICRP Publication 38, Annals of the ICRP, Vols. 11-13, Pergamon Press, New York, N.Y.

International Commission on Radiological Protection, 1984, A Compilation of the Major Concepts and Quantities in Use by ICRP, ICRP Publication 42, Annals of the ICRP, 14(4), Pergamon Press, New York, N.Y.

International Commission on Radiological Protection, 1991a, 1990 Recommendations of the International Commission on Radiological Protection, ICRP Publication 60, Annals of the ICRP, 21(1-3), Pergamon Press, New York, N.Y.

International Commission on Radiological Protection, 1991b, Annual Limits on Intake of Radionuclides by Workers Based on the 1990 Recommendations, ICRP Publication 61, Annals of the ICRP, 21(4), Pergamon Press, New York, N.Y. 
Kocher, D.C., 1983, "Dose-Rate Conversion Factors for External Exposure to Photons and Electrons," Health Physics 45(3):665.

Kocher, D.C., and A.L. Sjoreen, 1985, "Dose-Rate Conversion Factors for External Exposure to Photon Emitters in Soil," Health Physics 28:193-205.

McKone, T.E., 1986, The Implicit Valuation of Environmental Cancer by U.S. Regulatory Agencies, UCRL-93355, Lawrence Livermore National Laboratory, Livermore, Calif.

National Council on Radiation Protection and Measurements, 1984, Radiological Assessment: Predicting the Transport, Bioaccumulation, and Uptake by Man of Radionuclides Released to the Environment, NCRP Report No. 76, Bethesda, Md.

Oak Ridge National Laboratory, 1982, Procedures Manual: Remedial Action Survey and Certification Activities, ORNL-6000, Oak Ridge National Laboratory, Oak Ridge, Tenn.

Press, W.H., et al., 1986, Numerical Recipes, The Art of Scientific Computing, Cambridge University Press, New York, N.Y.

Till, J.E., and H.R. Meyer (editors), 1983, Radiological Assessment: A Textbook on Environmental Dose Analysis, NUREG/CR-3332, ORNL-5968, prepared by Oak Ridge National Laboratory, Oak Ridge, Tenn., for Division of Systems Integration, U.S. Nuclear Regulatory Commission, Washington, D.C.

U.S. Department of Energy, 1984, Formerly Utilized Sites Remedial Action Program, Summary Protocol: Identification - Characterization - Designation - Remedial Action Certification, Division of Remedial Action Projects, Washington, D.C.

U.S. Department of Energy, 1986a, Formerly Utilized Sites Remedial Action Program, Summary Protocol: Identification - Characterization - Designation - Remedial Action Certification, Office of Nuclear Energy, Division of Facility and Site Decommissioning Projects, Washington, D.C.

U.S. Department of Energy, 1986b, Formerly Utilized Sites Remedial Action Program, Designation/Elimination Protocol - Supplement No. 1 to the FUSRAP Summary Protocol, Office of Nuclear Energy, Division of Facility and Site Decommissioning Projects, Washington, D.C.

U.S. Department of Energy, 1988a, Internal Dose Conversion Factors for Calculation of Dose to the Public, DOE/EH-0071, Assistant Secretary for Environment, Safety and Health, Washington, D.C.

U.S. Department of Energy, 1988b, External Dose-Rate Conversion Factors for Calculation of Dose to the Public, DOE/EH-0070, Assistant Secretary for Environment, Safety and Health, Washington, D.C. 
U.S. Department of Energy, 1990, Radiation Protection of the Public and the Environment, DOE Order 5400.5, Feb.

U.S. Department of Energy, 1991, DOE Guidance on the Procedures in Applying the ALARA Process for Compliance with DOE 5400.5, March 8, 1991.

U.S. Department of Energy, 1992a, Environmental Implementation Guide for Radiological Survey Procedures, Draft Report for Comment, Assistant Secretary for Environment, Safety and Health, Washington, D.C.

U.S. Department of Energy, 1992b, Radiological Control Manual, Assistant Secretary for Environment, Safety and Health, Washington, D.C.

U.S. Environmental Protection Agency, 1984, Risk Analysis of TCDD Contaminated Soil, EPA 600/8-84-03, Office of Health and Environmental Assessment, Washington, D.C.

U.S. Environmental Protection Agency, 1992, Health Effects Assessment Summary Tables, Annual FY 1992, OERR 9200.6-303(92-1), Washington, D.C.

U.S. Nuclear Regulatory Commission, 1974, "Numerical Guides for Design Objectives and Limiting Conditions for Operation to Meet the Criterion As Low As Is Reasonably Achievable for Radioactive Material in Light Water Cooled Nuclear Power Reactors Effluents," Code of Federal Regulations, title 10, part 50, Appendix I.

U.S. Nuclear Regulatory Commission, 1977, Calculation of Annual Doses to Man from Routine Releases of Reactor Effluents for the Purpose of Evaluating Compliance with 10 CFR Part 50, Appendix I, Regulatory Guide 1.109, Rev. 1, Washington, D.C.

U.S. Nuclear Regulatory Commission, 1981, Draft Environmental Impact Statement on 10 CFR Part 61, "Licensing Requirements for Land Disposal of Radioactive Waste," NUREG-0782, 4 Vol., Office of Nuclear Material Safety and Safeguards, Washington, D.C.

U.S. Nuclear Regulatory Commission, 1982, Safety Goals for Nuclear Power Plants: A Discussion Paper, NUREG-0880, Office of Policy Evaluation, Washington, D.C.

Yu, C., et al., 1986, "Hydrogeologic Parameter Identification from Nuclear Tracer Breakthrough Curve Data for Waste Disposal in Porous Media," Radioactive Waste Management and the Nuclear Fuel Cycle 7(1):63-82.

Yu, C., et al., 1993, Data Collection Handbook to Support Modeling the Impacts of Radioactive Material in Soil, ANL/EAIS-8, Argonne National Laboratory, Argonne, $\mathrm{Il}$. 
124 
APPENDIX A:

EXTERNAL GROUND RADIATION PATHWAY FACTORS 


\section{APPENDIX A:}

\section{EXTERNAL GROUND RADIATION PATHWAY FACTORS}

Models, formulas, and data for calculating pathway factors for the external ground radiation pathway are presented in this appendix. Exposure to external radiation occurs primarily as a result of radiation emanating from radionuclides in a contaminated zone. Exposure can also occur from radiation emanating from radionuclides in the air, water, or ground surface that have been transported from their original location in the contaminated zone. In general, the radiation dose resulting from these secondary sources is negligibly small compared with the dose resulting from direct exposure to the primary source. Therefore, these secondary sources are not taken into account in deriving soil concentration guidelines.

The contribution to the effective dose equivalent from the external ground radiation pathway for the $i$ th principal radionuclide at time $t$ following the radiological survey is given by the dose/source ratio $D S R_{i 1}(t)$. This ratio may be expressed as the sum of the product of the dose conversion factor $\left(D C F_{j 1}\right)$, the environmental transport factor $\left(E T F_{j 1}\right)$, and the source factor $\left(S F_{i j}\right.$ ) (see Equation 3.9). Tables of DCFs for the external ground radiation pathway are given in Section A.1. Models and formulas for calculating the ETFs are given in Section A.2. Formulas for calculating the SFs are given in Section 3.2.3 and Appendix G.

\section{A.1 DOSE CONVERSION FACTORS}

The dose conversion factor $D C F_{i 1}$ for the external ground radiation pathway is the annual effective dose equivalent received from exposure to radiation from the $i$ th principal radionuclide present at the unit concentration in a uniformly contaminated zone of infinite depth and lateral extent. The radiation field is assumed to be equal to the radiation level at a distance of $1 \mathrm{~m}$ above the ground surface. The DCFs for surface contamination (infinite thinness and lateral extent) were taken from a DOE report (DOE 1988). The DCFs for volume contamination (infinite depth and lateral extent) were calculated by using the methods of Kocher and Sjoreen (1985) and Chen (1991). The results are given in Table A.1. In the RESRAD code, the volume contamination DCFs are used. Values for densities other than 1.0 and $1.8 \mathrm{~g} / \mathrm{cm}^{3}$ were obtained by linear interpolation or extrapolation of $\log$ (DCF). 
TABLE A.1 Effective Dose Equivalent Conversion Factors (DCF $_{\text {i1 }}$ ) for External Gamma Radiation from Contaminated Ground

\begin{tabular}{|c|c|c|c|}
\hline \multirow[b]{2}{*}{ Radionuclide } & \multirow[b]{2}{*}{$\begin{array}{c}\text { Surface Factors }^{\mathrm{a}} \\
(\mathrm{mrem} / \mathrm{yr}) /\left(\mathrm{pCi} / \mathrm{cm}^{2}\right)\end{array}$} & \multicolumn{2}{|c|}{$\begin{array}{c}\text { Volume Factors }{ }^{b} \\
(\mathrm{mrem} / \mathrm{yr}) /\left(\mathrm{pCi} / \mathrm{cm}^{3}\right)\end{array}$} \\
\hline & & $\begin{array}{c}\rho_{b}=1.0 \\
\mathrm{~g} / \mathrm{cm}^{3}\end{array}$ & $\begin{array}{c}\rho_{\mathrm{b}}=1.8 \\
\mathrm{~g} / \mathrm{cm}^{3}\end{array}$ \\
\hline $\mathrm{H}-3$ & 0 & 0 & 0 \\
\hline C-14 & 0 & 0 & 0 \\
\hline $\mathrm{Na}-22$ & 2.10 & $1.54 \times 10^{1}$ & 8.54 \\
\hline Al-26 & 2.50 & $2.00 \times 10^{1}$ & $1.11 \times 10^{1}$ \\
\hline Cl-36 & $4.66 \times 10^{-8}$ & $1.02 \times 10^{-3}$ & $5.69 \times 10^{-4}$ \\
\hline $\mathrm{K}-40$ & $1.37 \times 10^{-1}$ & 1.17 & $6.52 \times 10^{-1}$ \\
\hline $\mathrm{Ca}-41$ & $2.06 \times 10^{-5}$ & $1.94 \times 10^{-6}$ & $1.08 \times 10^{-6}$ \\
\hline Mn-54 & $8.41 \times 10^{-1}$ & 5.88 & 3.27 \\
\hline $\mathrm{Fe}-55$ & $2.21 \times 10^{-4}$ & $4.44 \times 10^{-6}$ & $2.47 \times 10^{-6}$ \\
\hline $\mathrm{Co}-57$ & $1.43 \times 10^{-1}$ & $5.03 \times 10^{-1}$ & $2.80 \times 10^{-1}$ \\
\hline Co-60 & 2.27 & $2.27 \times 10^{1}$ & $1.25 \times 10^{1}$ \\
\hline Ni-59 & $4.16 \times 10^{-4}$ & $5.38 \times 10^{-6}$ & $2.99 \times 10^{-6}$ \\
\hline $\mathrm{Ni}-63$ & 0 & 0 & 0 \\
\hline $\mathrm{Zn}-65$ & $5.48 \times 10^{-1}$ & 3.71 & 2.06 \\
\hline $\mathrm{Ge}-68+\mathrm{D}$ & $9.77 \times 10^{-1}$ & 6.39 & 3.55 \\
\hline Sr-90+D & 0 & 0 & 0 \\
\hline $\mathrm{Nb}-94$ & 1.59 & $1.42 \times 10^{1}$ & 7.83 \\
\hline Tc-99 & $6.26 \times 10^{-7}$ & $1.68 \times 10^{-6}$ & $9.32 \times 10^{-7}$ \\
\hline$R u-106+D$ & $2.10 \times 10^{-1}$ & 1.35 & $7.53 \times 10^{-1}$ \\
\hline Ag-108m+D & 1.66 & $1.11 \times 10^{1}$ & 6.15 \\
\hline $\mathrm{Ag}-110 \mathrm{~m}+\mathrm{D}$ & 2.68 & $1.92 \times 10^{1}$ & $1.07 \times 10^{1}$ \\
\hline Cd-109 & $9.45 \times 10^{-3}$ & $1.17 \times 10^{-2}$ & $6.49 \times 10^{-3}$ \\
\hline $\mathrm{Sb}-125+\mathrm{D}$ & $4.48 \times 10^{-1}$ & 2.81 & 1.56 \\
\hline I-129 & $2.20 \times 10^{-2}$ & $3.24 \times 10^{-2}$ & $1.75 \times 10^{-2}$ \\
\hline Cs-134 & 1.58 & $1.08 \times 10^{1}$ & 5.98 \\
\hline Cs-135 & 0 & 0 & 0 \\
\hline Cs-137+D & $6.11 \times 10^{-1}$ & 5.03 & 2.77 \\
\hline $\mathrm{Ce}-144+\mathrm{D}$ & $5.11 \times 10^{-2}$ & $3.12 \times 10^{-1}$ & $1.74 \times 10^{-1}$ \\
\hline Pm-147 & $4.10 \times 10^{-6}$ & $1.47 \times 10^{-5}$ & $8.18 \times 10^{-6}$ \\
\hline $\mathrm{Sm}-147$ & 0 & 0 & 0 \\
\hline $\mathrm{Sm}-151$ & $5.20 \times 10^{-6}$ & $5.45 \times 10^{-7}$ & $3.03 \times 10^{-7}$ \\
\hline Eu-152 & 1.11 & 9.91 & 5.47 \\
\hline Eu-154 & 1.21 & $1.10 \times 10^{1}$ & 6.06 \\
\hline Eu-155 & $7.15 \times 10^{-2}$ & $1.65 \times 10^{-1}$ & $9.19 \times 10^{-2}$ \\
\hline Gd-152 & 0 & 0 & 0 \\
\hline Gd-153 & $1.27 \times 10^{-1}$ & $2.12 \times 10^{-1}$ & $1.18 \times 10^{-1}$ \\
\hline $\mathrm{Au}-195$ & $9.86 \times 10^{-2}$ & $1.67 \times 10^{-1}$ & $9.52 \times 10^{-2}$ \\
\hline Tl-204 & $1.30 \times 10^{-3}$ & $2.20 \times 10^{-3}$ & $1.22 \times 10^{-3}$ \\
\hline $\mathrm{Pb}-210+\mathrm{D}$ & $3.01 \times 10^{-3}$ & $4.87 \times 10^{-3}$ & $2.31 \times 10^{-3}$ \\
\hline
\end{tabular}


TABLE A.1 (Cont.)

\begin{tabular}{|c|c|c|c|}
\hline \multirow[b]{2}{*}{ Radionuclide } & \multirow[b]{2}{*}{$\begin{array}{c}\text { Surface Factors }{ }^{a} \\
(\mathrm{mrem} / \mathrm{yr}) /\left(\mathrm{pCi} / \mathrm{cm}^{2}\right)\end{array}$} & \multicolumn{2}{|c|}{$\begin{array}{c}\text { Volume Factors }{ }^{\mathrm{b}} \\
(\mathrm{mrem} / \mathrm{yr}) /\left(\mathrm{pCi} / \mathrm{cm}^{3}\right)\end{array}$} \\
\hline & & $\begin{array}{c}\rho_{\mathrm{b}}=1.0 \\
\mathrm{~g} / \mathrm{cm}^{3}\end{array}$ & $\begin{array}{c}\rho_{\mathrm{b}}=1.8 \\
\mathrm{~g} / \mathrm{cm}^{3}\end{array}$ \\
\hline $\mathrm{Bi}-207$ & 1.51 & 9.72 & 5.40 \\
\hline $\mathrm{Ra}-226+\mathrm{D}$ & 1.68 & $1.55 \times 10^{1}$ & 8.56 \\
\hline $\mathrm{Ra}-228+\mathrm{D}$ & $9.10 \times 10^{-1}$ & 8.18 & 4.51 \\
\hline $\mathrm{Ac}-227+\mathrm{D}$ & $4.37 \times 10^{-1}$ & 2.76 & 1.52 \\
\hline Th-228+D & 1.45 & $1.33 \times 10^{1}$ & 7.36 \\
\hline Th-229+D & $3.60 \times 10^{-1}$ & 2.20 & 1.21 \\
\hline Th-230 & $9.07 \times 10^{-4}$ & $2.11 \times 10^{-3}$ & $1.03 \times 10^{-3}$ \\
\hline Th-232 & $6.66 \times 10^{-4}$ & $1.35 \times 10^{-3}$ & $6.04 \times 10^{-4}$ \\
\hline $\mathrm{Pa}-231$ & $3.58 \times 10^{-2}$ & $2.21 \times 10^{-1}$ & $1.21 \times 10^{-1}$ \\
\hline U-232 & $1.03 \times 10^{-3}$ & $2.19 \times 10^{-3}$ & $1.01 \times 10^{-3}$ \\
\hline U-233 & $5.00 \times 10^{-4}$ & $1.40 \times 10^{-3}$ & $7.12 \times 10^{-4}$ \\
\hline U-234 & $8.07 \times 10^{-4}$ & $1.58 \times 10^{-3}$ & $6.97 \times 10^{-4}$ \\
\hline $\mathrm{U}-235+\mathrm{D}$ & $1.90 \times 10^{-1}$ & $8.94 \times 10^{-1}$ & $4.90 \times 10^{-1}$ \\
\hline U-236 & $7.33 \times 10^{-4}$ & $1.35 \times 10^{-3}$ & $5.80 \times 10^{-4}$ \\
\hline $\mathrm{U}-238+\mathrm{D}$ & $2.59 \times 10^{-2}$ & $1.27 \times 10^{-1}$ & $6.97 \times 10^{-2}$ \\
\hline $\mathrm{Np}-237+\mathrm{D}$ & $2.68 \times 10^{-1}$ & 1.61 & $8.90 \times 10^{-1}$ \\
\hline $\mathrm{Pu}-238$ & $8.58 \times 10^{-4}$ & $1.56 \times 10^{-3}$ & $6.65 \times 10^{-4}$ \\
\hline Pu-239 & $3.78 \times 10^{-4}$ & $8.14 \times 10^{-4}$ & $3.76 \times 10^{-4}$ \\
\hline Pu-240 & $8.20 \times 10^{-4}$ & $1.48 \times 10^{-3}$ & $6.35 \times 10^{-4}$ \\
\hline $\mathrm{Pu}-241+\mathrm{D}$ & $3.94 \times 10^{-6}$ & $1.88 \times 10^{-5}$ & $1.05 \times 10^{-5}$ \\
\hline $\mathrm{Pu}-242$ & $6.82 \times 10^{-4}$ & $1.24 \times 10^{-3}$ & $5.29 \times 10^{-4}$ \\
\hline $\mathrm{Pu}-244+\mathrm{D}$ & $3.37 \times 10^{-1}$ & 2.23 & 1.24 \\
\hline Am-241 & $2.99 \times 10^{-2}$ & $4.79 \times 10^{-2}$ & $2.58 \times 10^{-2}$ \\
\hline $\mathrm{Am}-243+\mathrm{D}$ & $2.58 \times 10^{-1}$ & 1.08 & $5.95 \times 10^{-1}$ \\
\hline $\mathrm{Cm}-243$ & $1.46 \times 10^{-1}$ & $7.26 \times 10^{-1}$ & $4.01 \times 10^{-1}$ \\
\hline $\mathrm{Cm}-244$ & $8.29 \times 10^{-4}$ & $1.51 \times 10^{-3}$ & $6.67 \times 10^{-4}$ \\
\hline $\mathrm{Cm}-248$ & $5.88 \times 10^{-4}$ & $6.10 \times 10^{-6}$ & $3.39 \times 10^{-6}$ \\
\hline Cf- 252 & $6.34 \times 10^{-4}$ & $6.32 \times 10^{-5}$ & $3.51 \times 10^{-5}$ \\
\hline
\end{tabular}

a Surface factors represent infinite thinness.

b Volume factors represent infinite depth.

\section{A.2 ENVIRONMENTAL TRANSPORT FACTORS}

The environmental transport factor $E T F_{i 1}$ for the external ground radiation pathway is the ratio of the effective dose equivalent for the actual source to the effective dose equivalent for the standard source, multiplied by an occupancy and shielding factor. The standard source is a contaminated zone of infinite depth and lateral extent with no cover. 
The actual source is approximated by a cylindrical contaminated zone of radius $R$ and depth $T$ located at distance $C_{d}$ below the ground surface (see Figure 2.1).

Calculations by Napier et al. (1984) have indicated that the dependence on source size can be approximated reasonably well by an area factor. The ETF for the external ground radiation pathway is expressed as the product

$$
E T F_{i 1}(t)=\rho_{b}^{(c z)} \times F O_{1} \times F S_{1} \times F A_{1} \times F D_{i 1}(t) \times F C_{i 1}(t)
$$

where $^{1}$

$$
\begin{aligned}
\rho_{b}{ }^{(c z)}= & \text { bulk density of soil material in the contaminated zone } \\
& \left(1.5 \mathrm{~g} / \mathrm{cm}^{3}\right), \\
F O_{1}= & \text { occupancy and shielding factor }(0.6, \text { dimensionless), } \\
F S_{1}= & \text { shape factor (1.0, dimensionless), } \\
F A_{1}= & \text { area factor (dimensionless), } \\
F D_{i 1}\left[\rho_{b}{ }^{(c z)}, T(t)\right]= & \text { depth factor for radionuclide } i \text { at time } t \text { (dimension- } \\
& \text { less), } \\
F C_{i 1}\left[\rho_{b}{ }^{(c v)}, C_{d}{ }^{(t)]=} \text { cover factor for radionuclide } i \text { at time } t\right. \text { (dimension- } & \text { less), } \\
T(t)= & \text { thickness of contaminated zone at time } t(\mathrm{~m}), \\
\rho_{b}{ }^{(c v)}= & \text { bulk density of cover material }\left(1.5 \mathrm{~g} / \mathrm{cm}^{3}\right), \text { and } \\
C_{d}(t)= & \text { thickness of cover at time } t(\mathrm{~m}) .
\end{aligned}
$$

The default occupancy and shielding factor of $F O_{1}=0.6$ used in the RESRAD code is based on the assumption that $50 \%$ of a person's time is spent indoors (where the radiation exposure level is $70 \%$ of the outdoor exposure level), $25 \%$ is spent outdoors in the contaminated area, and $25 \%$ is spent in uncontaminated areas. The area factor is calculated in the RESRAD code by linear interpolation using the contaminated zone area (as input parameter) and the values listed in Table A.2. The area factor calculated is for a circular-area-equivalent

1 Numbers preceding the units in parentheses are default values and are given only for parameters for which site-specific input values can be provided by the user. If the units are not preceded by a number, the parameter is a derived quantity. 
contaminated zone. A shape factor (FS) (input parameter) is used to correct for the noncircular-shape area factor. The FS for a circular contaminated area is 1.0. For an irregularly shaped contaminated area, the FS may be obtained by enclosing the irregularly shaped contaminated area in a circle, multiplying the area factor of each annulus by the fraction of the annulus area that is contaminated, summing the products, and dividing by the area factor of a circular-areaequivalent contaminated zone. The area factor of an annulus is the area factor from an annular zone bounded by the radii tabulated in Table A.2. For example, the area factor is 0.2 for the annulus with inner and outer radii of $13 \mathrm{~m}$ and $20 \mathrm{~m}$, respectively; 0.25 for the annulus with radii of 5.6 and $13 \mathrm{~m}$; and so forth. An example of calculating the shape factor for an irregularly shaped contaminated area can be found in the RESRAD Data Collection Handbook (Yu et al. 1993).

\section{A.2.1 Depth Factor}

The depth factor $F D_{i 1}$ for a specified contaminated zone thickness is obtained by interpolation or extrapolation from Table A.3. It is assumed that the depth factor can be approximated by the equation

$$
F D_{i 1}\left[\rho_{b}^{(c z)}, T(t)\right]=1-\exp \left[-k_{i} \rho_{b}^{(c z)} T(t)\right],
$$

where

$$
\begin{aligned}
k_{i}= & \text { an empirical constant }\left(\mathrm{m}^{2} / \mathrm{kg}\right) \text {, and } \\
\rho_{b}{ }^{(c z)}= & \text { bulk density of soil material in the contaminated zone } \\
& \left(1,500 \mathrm{~kg} / \mathrm{m}^{3}\right),
\end{aligned}
$$

and the other parameters are as defined for Equation A.1. The empirical constant $k_{i}$ is a function of $\rho_{b}{ }^{(c z)}$, that is, $k_{i}=k_{i}\left[\rho_{b}{ }^{(c z)}\right]$. The units for $\rho_{b}{ }^{(c z)}$ in Equations A.1 and A.2 are 
TABLE A.3 Depth Factors $\left(F D_{i 1}\right.$ ) for External Gamma Radiation from Contaminated Ground as a Function of Thickness $(T)$ of a Contaminated Layer Exposed at the Ground Surface and Soil Density $\left(p_{b}\right)$ for Principal and Associated Radionuclides

\begin{tabular}{|c|c|c|c|c|c|c|}
\hline \multirow[b]{2}{*}{ Radionuclide $^{\mathrm{a}}$} & \multicolumn{3}{|c|}{$\rho_{b}=1.0 \mathrm{~g} / \mathrm{cm}^{3}$} & \multicolumn{3}{|c|}{$\rho_{b}=1.8 \mathrm{~g} / \mathrm{cm}^{3}$} \\
\hline & $\mathrm{T}=0.15 \mathrm{~m}$ & $\mathrm{~T}=0.5 \mathrm{~m}$ & $\mathrm{~T}=1.0 \mathrm{~m}$ & $\mathrm{~T}=0.15 \mathrm{~m}$ & $\mathrm{~T}=0.5 \mathrm{~m}$ & $\mathrm{~T}=1.0 \mathrm{~m}$ \\
\hline $\mathrm{H}-3$ & 1.0 & 1.0 & 1.0 & 1.0 & 1.0 & 1.0 \\
\hline C-14 & 1.0 & 1.0 & 1.0 & 1.0 & 1.0 & 1.0 \\
\hline $\mathrm{Na}-22$ & $7.01 \times 10^{-1}$ & $9.71 \times 10^{-1}$ & 1.0 & $8.69 \times 10^{-1}$ & 1.0 & 1.0 \\
\hline Al- 26 & $6.57 \times 10^{-1}$ & $9.53 \times 10^{-1}$ & $9.98 \times 10^{-1}$ & $8.36 \times 10^{-1}$ & $9.95 \times 10^{-1}$ & 1.0 \\
\hline $\mathrm{Cl}-36$ & $7.80 \times 10^{-1}$ & $9.96 \times 10^{-1}$ & 1.0 & $9.33 \times 10^{-1}$ & 1.0 & 1.0 \\
\hline $\mathrm{K}-40$ & $6.48 \times 10^{-1}$ & $9.48 \times 10^{-1}$ & 1.0 & $8.25 \times 10^{-1}$ & $9.97 \times 10^{-1}$ & 1.0 \\
\hline $\mathrm{Ca}-41$ & 1.0 & 1.0 & 1.0 & 1.0 & 1.0 & 1.0 \\
\hline Mn-54 & $7.03 \times 10^{-1}$ & $9.72 \times 10^{-1}$ & 1.0 & $8.67 \times 10^{-1}$ & 1.0 & 1.0 \\
\hline $\mathrm{Fe}-55$ & 1.0 & 1.0 & 1.0 & 1.0 & 1.0 & 1.0 \\
\hline $\mathrm{Co}-57$ & $9.07 \times 10^{-1}$ & 1.0 & 1.0 & $9.85 \times 10^{-1}$ & 1.0 & 1.0 \\
\hline $\mathrm{Co}-60$ & $6.80 \times 10^{-1}$ & 1.0 & 1.0 & $8.60 \times 10^{-1}$ & 1.0 & 1.0 \\
\hline $\mathrm{Ni}-59$ & 1.0 & 1.0 & 1.0 & 1.0 & 1.0 & 1.0 \\
\hline $\mathrm{Ni}-63$ & 1.0 & 1.0 & 1.0 & 1.0 & 1.0 & 1.0 \\
\hline $\mathrm{Zn}-65$ & $7.06 \times 10^{-1}$ & 1.0 & 1.0 & $8.93 \times 10^{-1}$ & 1.0 & 1.0 \\
\hline $\mathrm{Ge}-68+\mathrm{D}$ & $7.78 \times 10^{-1}$ & $9.97 \times 10^{-1}$ & 1.0 & $9.32 \times 10^{-1}$ & 1.0 & 1.0 \\
\hline $\mathrm{Sr}-90+\mathrm{D}$ & 1.0 & 1.0 & 1.0 & 1.0 & 1.0 & 1.0 \\
\hline $\mathrm{Nb}-94$ & $7.20 \times 10^{-1}$ & 1.0 & 1.0 & $8.90 \times 10^{-1}$ & 1.0 & 1.0 \\
\hline Tc-99 & $9.60 \times 10^{-1}$ & 1.0 & 1.0 & 1.0 & 1.0 & 1.0 \\
\hline $\mathrm{Ru}-106+\mathrm{D}$ & $7.63 \times 10^{-1}$ & $9.96 \times 10^{-1}$ & 1.0 & $9.21 \times 10^{-1}$ & 1.0 & 1.0 \\
\hline $\mathrm{Ag}-108 \mathrm{~m}+\mathrm{D}$ & $7.56 \times 10^{-1}$ & $9.89 \times 10^{-1}$ & 1.0 & $9.11 \times 10^{-1}$ & 1.0 & 1.0 \\
\hline $\mathrm{Ag}-110 \mathrm{~m}+\mathrm{D}$ & $7.04 \times 10^{-1}$ & $9.74 \times 10^{-1}$ & 1.0 & $8.71 \times 10^{-1}$ & $9.99 \times 10^{-1}$ & 1.0 \\
\hline Cd-109 & $9.60 \times 10^{-1}$ & 1.0 & 1.0 & 1.0 & 1.0 & 1.0 \\
\hline Sb-125+D & $7.71 \times 10^{-1}$ & $9.93 \times 10^{-1}$ & 1.0 & $9.23 \times 10^{-1}$ & 1.0 & 1.0 \\
\hline $\mathrm{I}-129$ & 1.0 & 1.0 & 1.0 & 1.0 & 1.0 & 1.0 \\
\hline Cs-134 & $7.39 \times 10^{-1}$ & $9.83 \times 10^{-1}$ & 1.0 & $8.97 \times 10^{-1}$ & 1.0 & 1.0 \\
\hline Cs-135 & $9.60 \times 10^{-1}$ & 1.0 & 1.0 & 1.0 & 1.0 & 1.0 \\
\hline $\mathrm{Cs}-137+\mathrm{D}$ & $7.20 \times 10^{-1}$ & $9.80 \times 10^{-1}$ & 1.0 & $9.10 \times 10^{-1}$ & 1.0 & 1.0 \\
\hline $\mathrm{Ce}-144+\mathrm{D}$ & $6.95 \times 10^{-1}$ & $9.54 \times 10^{-1}$ & $9.97 \times 10^{-1}$ & $8.51 \times 10^{-1}$ & $9.99 \times 10^{-1}$ & 1.0 \\
\hline Pm-147 & $9.15 \times 10^{-1}$ & 1.0 & 1.0 & $9.88 \times 10^{-1}$ & 1.0 & 1.0 \\
\hline $\mathrm{Sm}-147$ & 1.0 & 1.0 & 1.0 & 1.0 & 1.0 & 1.0 \\
\hline Sm-151 & 1.0 & 1.0 & 1.0 & 1.0 & 1.0 & 1.0 \\
\hline Eu-152 & $6.80 \times 10^{-1}$ & $9.60 \times 10^{-1}$ & 1.0 & $8.50 \times 10^{-1}$ & 1.0 & 1.0 \\
\hline Eu-154 & $6.90 \times 10^{-1}$ & $9.80 \times 10^{-1}$ & 1.0 & $8.60 \times 10^{-1}$ & 1.0 & 1.0 \\
\hline Eu-155 & $9.42 \times 10^{-1}$ & 1.0 & 1.0 & $9.97 \times 10^{-1}$ & 1.0 & 1.0 \\
\hline Gd-152 & 1.0 & 1.0 & 1.0 & 1.0 & 1.0 & 1.0 \\
\hline Gd-153 & $9.44 \times 10^{-1}$ & 1.0 & 1.0 & $9.96 \times 10^{-1}$ & 1.0 & 1.0 \\
\hline $\mathrm{Au}-195$ & $9.75 \times 10^{-1}$ & 1.0 & 1.0 & $9.99 \times 10^{-1}$ & 1.0 & 1.0 \\
\hline Tl-204 & $9.83 \times 10^{-1}$ & 1.0 & 1.0 & 1.0 & 1.0 & 1.0 \\
\hline $\mathrm{Pb}-210+\mathrm{D}$ & $8.80 \times 10^{-1}$ & 1.0 & 1.0 & $9.70 \times 10^{-1}$ & 1.0 & 1.0 \\
\hline $\mathrm{Bi}-207$ & $7.39 \times 10^{-1}$ & 1.0 & 1.0 & $9.14 \times 10^{-1}$ & 1.0 & 1.0 \\
\hline $\mathrm{Ra}-226+\mathrm{D}$ & $6.30 \times 10^{-1}$ & $9.20 \times 10^{-1}$ & 1.0 & $8.50 \times 10^{-1}$ & 1.0 & 1.0 \\
\hline $\mathrm{Ra}-228+\mathrm{D}$ & $6.80 \times 10^{-1}$ & $9.70 \times 10^{-1}$ & 1.0 & $8.50 \times 10^{-1}$ & 1.0 & 1.0 \\
\hline $\mathrm{Ac}-227+\mathrm{D}$ & $7.90 \times 10^{-1}$ & $9.70 \times 10^{-1}$ & 1.0 & $9.10 \times 10^{-1}$ & 1.0 & 1.0 \\
\hline Th-228+D & $6.10 \times 10^{-1}$ & $9.40 \times 10^{-1}$ & 1.0 & $7.50 \times 10^{-1}$ & 1.0 & 1.0 \\
\hline
\end{tabular}


TABLE A.3 (Cont.)

\begin{tabular}{|c|c|c|c|c|c|c|}
\hline \multirow[b]{2}{*}{ Radionuclide $^{\mathrm{a}}$} & \multicolumn{3}{|c|}{$\rho_{\mathrm{b}}=1.0 \mathrm{~g} / \mathrm{cm}^{3}$} & \multicolumn{3}{|c|}{$\rho_{\mathrm{b}}=1.8 \mathrm{~g} / \mathrm{cm}^{3}$} \\
\hline & $\mathrm{T}=0.15 \mathrm{~m}$ & $\mathrm{~T}=0.5 \mathrm{~m}$ & $\mathrm{~T}=1.0 \mathrm{~m}$ & $\mathrm{~T}=0.15 \mathrm{~m}$ & $\mathrm{~T}=0.5 \mathrm{~m}$ & $\mathrm{~T}=1.0 \mathrm{~m}$ \\
\hline Th-229+D & $6.50 \times 10^{-1}$ & $9.50 \times 10^{-1}$ & 1.0 & $8.50 \times 10^{-1}$ & $9.90 \times 10^{-1}$ & 1.0 \\
\hline Th-230 & $9.30 \times 10^{-1}$ & 1.0 & 1.0 & 1.0 & 1.0 & 1.0 \\
\hline $\mathrm{Th}-232+\mathrm{D}$ & $9.50 \times 10^{-1}$ & 1.0 & 1.0 & 1.0 & 1.0 & 1.0 \\
\hline $\mathrm{Pa}-231$ & $7.90 \times 10^{-1}$ & 1.0 & 1.0 & $9.20 \times 10^{-1}$ & 1.0 & 1.0 \\
\hline $\mathrm{U}-232$ & $8.80 \times 10^{-1}$ & 1.0 & 1.0 & 1.0 & 1.0 & 1.0 \\
\hline U-233 & $9.60 \times 10^{-1}$ & 1.0 & 1.0 & 1.0 & 1.0 & 1.0 \\
\hline U-234 & $9.00 \times 10^{-1}$ & 1.0 & 1.0 & 1.0 & 1.0 & 1.0 \\
\hline $\mathrm{U}-235+\mathrm{D}$ & $8.70 \times 10^{-1}$ & 1.0 & 1.0 & 1.0 & 1.0 & 1.0 \\
\hline U-236 & $9.40 \times 10^{-1}$ & 1.0 & 1.0 & 1.0 & 1.0 & 1.0 \\
\hline $\mathrm{U}-238+\mathrm{D}$ & $7.80 \times 10^{-1}$ & 1.0 & 1.0 & $8.80 \times 10^{-1}$ & 1.0 & 1.0 \\
\hline $\mathrm{Np}-237+\mathrm{D}$ & $8.20 \times 10^{-1}$ & 1.0 & 1.0 & $9.30 \times 10^{-1}$ & 1.0 & 1.0 \\
\hline Pu-238 & $9.30 \times 10^{-1}$ & 1.0 & 1.0 & 1.0 & 1.0 & 1.0 \\
\hline $\mathrm{Pu}-239$ & $9.20 \times 10^{-1}$ & 1.0 & 1.0 & 1.0 & 1.0 & 1.0 \\
\hline $\mathrm{Pu}-240$ & $9.20 \times 10^{-1}$ & 1.0 & 1.0 & 1.0 & 1.0 & 1.0 \\
\hline $\mathrm{Pu}-241+\mathrm{D}$ & $9.01 \times 10^{-1}$ & 1.0 & 1.0 & $9.83 \times 10^{-1}$ & 1.0 & 1.0 \\
\hline $\mathrm{Pu}-242$ & $9.60 \times 10^{-1}$ & 1.0 & 1.0 & 1.0 & 1.0 & 1.0 \\
\hline $\mathrm{Pu}-244+\mathrm{D}$ & $7.43 \times 10^{-1}$ & $9.85 \times 10^{-1}$ & 1.0 & $9.03 \times 10^{-1}$ & 1.0 & 1.0 \\
\hline Am-241 & $9.40 \times 10^{-1}$ & 1.0 & 1.0 & 1.0 & 1.0 & 1.0 \\
\hline $\mathrm{Am}-243+\mathrm{D}$ & $9.00 \times 10^{-1}$ & 1.0 & 1.0 & $9.70 \times 10^{-1}$ & 1.0 & 1.0 \\
\hline $\mathrm{Cm}-243$ & $8.70 \times 10^{-1}$ & 1.0 & 1.0 & $9.60 \times 10^{-1}$ & 1.0 & 1.0 \\
\hline $\mathrm{Cm}-244$ & $9.60 \times 10^{-1}$ & 1.0 & 1.0 & 1.0 & 1.0 & 1.0 \\
\hline $\mathrm{Cm}-248$ & 1.0 & 1.0 & 1.0 & 1.0 & 1.0 & 1.0 \\
\hline Cf-252 & $9.26 \times 10^{-1}$ & 1.0 & 1.0 & $9.91 \times 10^{-1}$ & 1.0 & 1.0 \\
\hline
\end{tabular}

a $\mathrm{A}$ " $+\mathrm{D}$ " signifies that the contributions from the associated decay chain are included; radionuclides in the associated decay chain are identified in Table 3.1.

different. The empirical constant $k_{i}$ for radionuclide $i$ is a function of soil material density; it is determined from the tabulated values of the quantity $F D_{i 1}\left[\rho_{b}{ }^{(c z)}, T(t)\right]$ in Table A.3. The $0.15 \mathrm{~cm}$ depth factor, $F D_{i 1}\left[\rho_{b}{ }^{(c z)}, T(t) 0.15\right]$, is obtained by linearly interpolating between $F D_{i 1}$ $[1,000 ; 0.15]$ and $F D_{i 1}[1,800 ; 0.15]$. If the interpolated value of $F D_{i 1}\left[\rho_{b}{ }^{(c z)}, T(t) 0.15\right]$ is greater than 0.995 , then it is set equal to 0.995 . The parameter $k_{i}\left[\rho_{b}{ }^{(c z)}\right]$ is calculated by using

$$
k_{i}\left[\rho_{b}^{(c z)}\right]=-\log _{e}\left\{1-F D_{i 1}\left[\rho_{b}^{(c z)}, 0.15\right]\right\} /\left[0.15 \rho_{b}^{(c z)}\right] .
$$


The time dependence of the contaminated zone thickness is given by

$$
\begin{aligned}
T(t) & =T(0), & & 0 \leq t \leq t_{c} \\
& =T(0)-v^{(c z)}\left(t-t_{c}\right), & & t_{c}<t
\end{aligned}
$$

where

$$
\begin{aligned}
T(t) & =\text { thickness of the contaminated zone at time } t(\mathrm{~m}), \\
T(0) & =\text { initial thickness of the contaminated zone }(2 \mathrm{~m}), \\
t_{c} & =C_{d}(0) / v^{(c v)}=\text { time for the cover to be removed by erosion }(\mathrm{yr}), \\
v^{(c v)} & =\text { erosion rate of cover material }(0.001 \mathrm{~m} / \mathrm{yr}), \text { and } \\
v^{(c z)} & =\text { erosion rate of contaminated zone }(0.001 \mathrm{~m} / \mathrm{yr}) .
\end{aligned}
$$

Erosion rates for both the cover and the contaminated zone may be estimated by means of the Universal Soil Loss Equation (USLE), an empirical model that has been developed for predicting the rate of soil loss by sheet and rill erosion. If sufficient site-specific data are available, a site-specific erosion rate can be calculated. Details are discussed in Wischmeier and Smith (1978) and Foster (1979). Estimates based on the range of erosion rates for typical sites in humid areas east of the Mississippi River (based on model site calculations for locations in New York, New Jersey, Ohio, and Missouri) may also be used (Knight 1983). For a site with a $2 \%$ slope, these model calculations predict a range of $8 \times 10^{-7}$ to $3 \times 10^{-6} \mathrm{~m} / \mathrm{yr}$ for natural succession vegetation, $1 \times 10^{-5}$ to $6 \times 10^{-5} \mathrm{~m} / \mathrm{yr}$ for permanent pasture, and $9 \times 10^{-5}$ to $6 \times 10^{-4} \mathrm{~m} / \mathrm{yr}$ for row-crop agriculture. The rate increases by a factor of about 3 for a $5 \%$ slope, 7 for a $10 \%$ slope, and 15 for a $15 \%$ slope. If these generic values are used for a farm-garden scenario, in which the dose contribution from food ingestion pathways is expected to be significant, an erosion rate of $0.06 \mathrm{~cm} / \mathrm{yr}$ should be assumed for a site with a $2 \%$ slope. This would lead to erosion of $0.6 \mathrm{~m}$ of soil in 1,000 years. A proportionately higher erosion rate must be used if the slope exceeds $2 \%$. An erosion rate of $6 \times 10^{-5} \mathrm{~m} / \mathrm{yr}$, leading to erosion of $0.06 \mathrm{~m}$ of soil in 1,000 years, may be used for a site with a $2 \%$ slope if it can be reasonably shown that the farm-garden scenario is unreasonable; for example, because the site is, and will likely continue to be, unsuitable for agricultural use.

The erosion rates are more difficult to estimate for arid sites in the West than for humid sites in the East. Although water erosion is generally more important than wind 
erosion, the latter can also be significant. Water erosion in the West is more difficult to estimate because it is likely to be due to infrequent heavy rainfalls for which the empirical constants used in the USLE may not be applicable. Long-term erosion rates are generally lower for sites in arid locations than for sites in humid locations. More information on the erosion rate may be found in the RESRAD Data Collection Handbook (Yu et al. 1993). The cover factors and the formula for time dependence of the cover depth are described in Section A.2.2.

\section{A.2.2 Cover Factor}

The cover factor for radionuclide $i$ in the contaminated zone with a cover thickness of $C_{d}(t)$ and a cover density of $\rho_{b}{ }^{(c v)}$ is approximated by the formula

$$
F C_{i 1}\left[\rho_{b}^{(c v)}, C_{d}(t)\right]=\exp \left[-k_{i} \rho_{b}^{(c v)} C_{d}(t)\right]
$$

where

$$
\begin{aligned}
k_{i} & =\text { empirical constant }\left(\mathrm{m}^{2} / \mathrm{kg}\right), \\
\rho_{b}{ }^{(c v)} & =\text { bulk density of the cover material }\left(1,500 \mathrm{~kg} / \mathrm{m}^{3}\right), \text { and } \\
C_{d}(t) & =\text { cover depth at time } t(\mathrm{~m}) .
\end{aligned}
$$

The empirical constant $k_{i}$ for radionuclide $i$ is a function of soil material density. Linear interpolation is used to obtain $k_{i}\left(\rho_{b}{ }^{(c v)}\right)$ for the actual cover density (see Section A.2.1).

The time dependence of the cover depth is given by

$$
\begin{aligned}
C_{d}(t) & =C_{d}(0)-v^{(c v)} t, & & 0 \leq t<t_{c} \\
& =0, & & t_{c} \leq t
\end{aligned}
$$

where

$$
\begin{aligned}
C_{d}(t) & =\text { cover depth at time } t(\mathrm{~m}), \\
C_{d}(0) & =\text { initial cover depth }(0 \mathrm{~m}), \\
v^{(c v)} & =\text { erosion rate of the cover material }(0.001 \mathrm{~m} / \mathrm{yr}), \text { and } \\
t_{c} & =\text { time for the cover to be removed by erosion }(\mathrm{yr}) .
\end{aligned}
$$




\section{A.3 REFERENCES}

Chen, S.Y., 1991, "Calculation of Effective Dose Equivalent Responses for External Exposure from Residual Photon Emitters in Soil," Health Physics 60:411-426.

Foster, G.R., 1979, "Sediment Yield from Farm Fields: The Universal Soil Loss Equation and Onfarm 208 Plan Implementation," in Universal Soil Loss Equation: Past, Present, and Future, Soil Science Society of America Special Publication No. 8, Soil Science Society of America, Inc., Madison, Wis., pp. 17-24.

Knight, M.J., 1983, The Effect of Soil Erosion on the Long-Term Stability of FUSRAP NearSurface Waste-Burial Sites, ANL/EIS-18, revised, prepared by Division of Environmental Impact Studies, Argonne National Laboratory, Argonne, Ill., for U.S. Department of Energy, Oak Ridge Operations Office.

Kocher, D.C., and A.L. Sjoreen, 1985, "Dose-Rate Conversion Factors for External Exposure to Photon Emitters in Soil," Health Physics 28:193-205.

Napier, B.A., et al., 1984, Intruder Dose Pathway Analysis for the Onsite Disposal of Radioactive Wastes: The ONSITE/MAXI1 Computer Program, NUREG/CR-3620, PNL-4054, prepared by Pacific Northwest Laboratory, Richland, Wash., for Division of Waste Management, U.S. Nuclear Regulatory Commission, Washington, D.C.

U.S. Department of Energy, 1988, External Dose-Rate Conversion Factors for Calculation of Dose to the Public, DOE/EH-0070, Assistant Secretary for Environment, Safety and Health, Washington, D.C.

Wischmeier, W.H., and D.D. Smith, 1978, Predicting Rainfall Erosion Losses - A Guide to Conservation Planning, Agricultural Handbook No. 537, prepared by Science and Education Administration, Washington, D.C., for U.S. Department of Agriculture, Washington, D.C.

Yu, C., et al., 1993, Data Collection Handbook to Support Modeling the Impacts of Radioactive Material in Soil, ANL/EAIS-8, prepared by Argonne National Laboratory, Argonne, Ill., for U.S. Department of Energy, Washington, D.C. 
APPENDIX B:

INHALATION PATHWAY FACTORS 


\section{APPENDIX B:}

\section{INHALATION PATHWAY FACTORS}

Models, formulas, and data for calculating pathway factors for the inhalation pathway are presented in this appendix. Inhalation exposure can occur from inhalation of dust, radon and radon decay products, and other gaseous airborne radionuclides. The radon pathway is discussed in Appendix C. The other gaseous airborne decay products are primarily tritium (in tritiated water vapor) and carbon-14 (in $\mathrm{CO}_{2}$ ); the transport and dosimetry for these radionuclides require special consideration. The models for these special radionuclides are discussed in Appendix L.

The contribution to the committed effective dose equivalent from the dust inhalation pathway for the $i$ th principal radionuclide at time $t$ following the radiological survey is given by the dose/source ratio $D S R_{i 2}(t)$. This ratio may be expressed as the sum of the product of the dose conversion factor $\left(D C F_{j 2}\right)$, the environmental transport factor $\left(E T F_{j 2}\right)$, and the source factor $\left(S F_{i j}\right.$ ) (see Equation 3.9). A tabulation of DCFs for dust inhalation is presented in Section B.1. Models and formulas for calculating the ETFs are given in Section B.2. Formulas for calculating the source factors are given in Section 3.2.3 and Appendix G.

\section{B.1 DOSE CONVERSION FACTORS}

A DCF for inhalation is the dose/exposure ratio $D C F_{i 2}=H_{E, i 2} / E_{i 2}$ for the committed effective dose equivalent $H_{E, i 2}$ that is incurred by an individual from exposure by inhalation of a quantity $E_{i 2}$ of the ith principal radionuclide in contaminated dust. Values of dose conversion factors for inhalation were taken from a DOE report (DOE 1988) and are listed in Table B.1. Similar values are given in an EPA report (Eckerman et al. 1988). The values listed in Table B.1 are for dust particles with an activity median aerodynamic diameter (AMAD) of $1 \mu \mathrm{m}$. Values for different inhalation classes are also listed in Table B.1. The inhalation class for inhaled radioactive material is defined according to its rate of clearance from the lung. The three inhalation classes D, W, and $\mathrm{Y}$ correspond to retention half-times of less than 10 days, 10 to 100 days, and greater than 100 days, respectively. If the inhalation class for a radionuclide is not known, the largest DCF for that radionuclide should 
TABLE B.1 Committed Effective Dose Equivalent Conversion Factors $\left(\mathrm{DCF}_{\mathbf{i} 2}\right)$ for Inhalation

\begin{tabular}{|c|c|c|c|c|c|}
\hline Radionuclide $^{b}$ & $\begin{array}{c}\text { Inhalation } \\
\text { Class } \\
\end{array}$ & $\begin{array}{c}\mathrm{DCF}_{\mathrm{i} 2} \\
(\mathrm{mrem} / \mathrm{pCi})\end{array}$ & Radionuclide $^{b}$ & $\begin{array}{c}\text { Inhalation } \\
\text { Class }\end{array}$ & $\begin{array}{c}\mathrm{DCF}_{\mathrm{i} 2} \\
(\mathrm{mrem} / \mathrm{pCi})\end{array}$ \\
\hline $\mathrm{H}-3$ & $\left(\mathrm{H}_{2} \mathrm{O}\right)^{c}$ & $6.3 \times 10^{-8}$ & Cs- $137+D$ & D & $3.2 \times 10^{-5}$ \\
\hline \multirow[t]{3}{*}{ C-14. } & (organic) $^{c}$ & $2.1 \times 10^{-6}$ & $\mathrm{Ce}-144+\mathrm{D}$ & $\mathrm{W}$ & $1.9 \times 10^{-4}$ \\
\hline & $(\mathrm{CO})^{\mathrm{c}}$ & $2.9 \times 10^{-9}$ & & $\mathrm{Y}$ & $3.5 \times 10^{-4}$ \\
\hline & $\left(\mathrm{CO}_{2}\right)^{\mathrm{c}}$ & $2.4 \times 10^{-8}$ & Pm-147 & $\mathrm{W}$ & $2.5 \times 10^{-5}$ \\
\hline $\mathrm{Na}-22$ & $\mathrm{D}^{\mathrm{d}^{2}}$ & $8.0 \times 10^{-6}$ & & $\mathrm{Y}$ & $3.4 \times 10^{-5}$ \\
\hline \multirow[t]{2}{*}{$\mathrm{Al}-26$} & $\mathrm{D}$ & $7.9 \times 10^{-5}$ & Sm-147 & $\mathrm{W}$ & $7.1 \times 10^{-2}$ \\
\hline & W. & $5.9 \times 10^{-5}$ & Sm-151 & W & $2.9 \times 10^{-5}$ \\
\hline \multirow[t]{2}{*}{ Cl-36 } & D & $2.1 \times 10^{-6}$ & Eu-152 & $\mathrm{W}$ & $2.2 \times 10^{-4}$ \\
\hline & W & $2.0 \times 10^{-5}$ & Eu-154 & W & $2.6 \times 10^{-4}$ \\
\hline $\mathrm{K}-40$ & $\mathrm{D}$ & $1.2 \times 10^{-5}$ & Eu-155 & $\mathrm{W}$ & $3.9 \times 10^{-5}$ \\
\hline $\mathrm{Ca}-41$ & W & $1.3 \times 10^{-6}$ & Gd-152 & D & $2.4 \times 10^{-1}$ \\
\hline \multirow[t]{2}{*}{$M n-54$} & D & $5.4 \times 10^{-6}$ & Gd-152 & W & $61 . \times 10^{-2}$ \\
\hline & $\mathbf{W}$ & $6.4 \times 10^{-6}$ & Gd-153 & D & $2.1 \times 10^{-5}$ \\
\hline \multirow[t]{2}{*}{$\mathrm{Fe}-55$} & D & $2.6 \times 10^{-6}$ & & W & $8.4 \times 10^{-6}$ \\
\hline & W & $1.2 \times 10^{-6}$ & $\mathrm{Au}-195$ & D & $1.1 \times 10^{-6}$ \\
\hline \multirow{2}{*}{ Co-57 } & $\mathrm{W}$ & $1.8 \times 10^{-6}$ & & $\mathbf{W}$ & $3.5 \times 10^{-6}$ \\
\hline & $\mathrm{Y}$ & $7.5 \times 10^{-6}$ & & $\mathrm{Y}$ & $1.2 \times 10^{-5}$ \\
\hline \multirow{2}{*}{ Co-60 } & $\mathrm{W}$ & $3.0 \times 10^{-5}$ & Tl-204 & D & $2.3 \times 10^{-6}$ \\
\hline & $\mathrm{Y}$ & $1.5 \times 10^{-4}$ & $\mathrm{~Pb}-210$ & D & $2.1 \times 10^{-2}$ \\
\hline \multirow{3}{*}{$\mathrm{Ni}-59$} & D & $1.3 \times 10^{-6}$ & Bi-207 & D & $2.9 \times 10^{-6}$ \\
\hline & $\mathrm{W}$ & $7.0 \times 10^{-7}$ & & $\mathrm{~W}$ & $1.4 \times 10^{-5}$ \\
\hline & (vapor) $^{c}$ & $2.7 \times 10^{-6}$ & $\mathrm{Ra}-226+\mathrm{D}$ & W & $7.9 \times 10^{-3}$ \\
\hline \multirow[t]{3}{*}{$\mathrm{Ni}-63$} & $\mathrm{D}$ & $3.0 \times 10^{-6}$ & $\mathrm{Ra}-228+\mathrm{D}$ & W & $4.5 \times 10^{-3}$ \\
\hline & W & $1.9 \times 10^{-6}$ & Ac-227+D & D & 6.7 \\
\hline & $(\text { vapor })^{c}$ & $6.3 \times 10^{-6}$ & & $\mathbf{W}$ & 1.7 \\
\hline $\mathrm{Zn}-65$ & $\mathrm{Y}$ & $1.8 \times 10^{-5}$ & & $\mathrm{Y}$ & 1.2 \\
\hline \multirow[t]{2}{*}{$\mathrm{Ge}-68+\mathrm{D}$} & D & $3.1 \times 10^{-7}$ & Th-228+D & W & $2.5 \times 10^{-1}$ \\
\hline & W & $3.4 \times 10^{-7}$ & & $\mathrm{Y}$ & $3.1 \times 10^{-1}$ \\
\hline \multirow[t]{2}{*}{$\mathrm{Sr}-90+\mathrm{D}$} & D & $2.3 \times 10^{-4}$ & Th-229+D & W & 2.0 \\
\hline & $\mathrm{Y}$ & $1.3 \times 10^{-3}$ & & $\mathrm{Y}$ & 1.7 \\
\hline \multirow{2}{*}{$\mathrm{Nb}-94$} & W & $2.6 \times 10^{-5}$ & Th-230 & $\mathrm{W}$ & $3.2 \times 10^{-1}$ \\
\hline & $\mathrm{Y}$ & $3.3 \times 10^{-4}$ & & $\mathbf{Y}$ & $2.6 \times 10^{-1}$ \\
\hline \multirow{2}{*}{ Tc-99 } & D & $8.4 \times 10^{-7}$ & Th-232 & W & 1.6 \\
\hline & $\bar{W}$ & $7.5 \times 10^{-6}$ & & $\mathrm{Y}$ & 1.1 \\
\hline \multirow[t]{3}{*}{ Ru-106+D } & D & $5.7 \times 10^{-5}$ & $\mathrm{~Pa}-231$ & W & 1.3 \\
\hline & $\mathrm{W}$ & $9.3 \times 10^{-5}$ & & $\mathbf{Y}$ & $8.6 \times 10^{-1}$ \\
\hline & $\mathrm{Y}$ & $4.4 \times 10^{-4}$ & U-232 & $\mathrm{D}$ & $1.2 \times 10^{-2}$ \\
\hline \multirow[t]{3}{*}{$\mathrm{Ag}-108 \mathrm{~m}+\mathrm{D}$} & D & $2.8 \times 10^{-5}$ & & $\mathrm{~W}$ & $1.3 \times 10^{-2}$ \\
\hline & $w$ & $1.9 \times 10^{-5}$ & & $\mathrm{Y}$ & $6.7 \times 10^{-1}$ \\
\hline & $\mathrm{Y}$ & $2.0 \times 10^{-4}$ & U-233 & D & $2.7 \times 10^{-3}$ \\
\hline \multirow[t]{3}{*}{$\mathrm{Ag}-110 \mathrm{~m}+\mathrm{D}$} & $\mathrm{D}$ & $3.8 \times 10^{-5}$ & & W & $7.1 \times 10^{-3}$ \\
\hline & $\mathrm{W}$ & $2.7 \times 10^{-5}$ & & $\mathrm{Y}$ & $1.3 \times 10^{-1}$ \\
\hline & $\mathrm{Y}$ & $5.3 \times 10^{-5}$ & U-234 & $\mathrm{D}$ & $2.7 \times 10^{-3}$ \\
\hline \multirow{3}{*}{ Cd-109 } & $\mathrm{D}$ & $1.0 \times 10^{-4}$ & & $\mathrm{~W}$ & $7.1 \times 10^{-3}$ \\
\hline & $\mathrm{W}$ & $3.6 \times 10^{-5}$ & & $\mathrm{Y}$ & $1.3 \times 10^{-1}$ \\
\hline & $\mathrm{Y}$ & $4.2 \times 10^{-5}$ & $\mathrm{U}-235+\mathrm{D}$ & $\mathrm{D}$ & $2.5 \times 10^{-3}$ \\
\hline \multirow{2}{*}{$\mathrm{Sb}-125$} & D & $3.2 \times 10^{-6}$ & & W & $6.7 \times 10^{-3}$ \\
\hline & $\mathrm{W}$ & $1.1 \times 10^{-5}$ & & $Y$ & $1.2 \times 10^{-1}$ \\
\hline I-129 & D & $1.8 \times 10^{-4}$ & U-236 & $\mathrm{D}$ & $2.5 \times 10^{-3}$ \\
\hline Cs-134 & D & $4.7 \times 10^{-5}$ & & $w$ & $6.7 \times 10^{-3}$ \\
\hline Cs-135 & $\mathrm{D}$ & $4.5 \times 10^{-6}$ & & $Y$ & $1.2 \times 10^{-1}$ \\
\hline
\end{tabular}


TABLE B.1 (Cont.)

\begin{tabular}{|c|c|c|c|c|c|}
\hline Radionuclide $^{b}$ & $\begin{array}{l}\text { Inhalation } \\
\text { Class }\end{array}$ & $\begin{array}{c}\mathrm{DCF}_{\mathrm{i} 2} \\
(\mathrm{mrem} / \mathrm{pCi})\end{array}$ & Radionuclide $^{\mathrm{b}}$ & $\begin{array}{l}\text { Inhalation } \\
\text { Class }\end{array}$ & $\begin{array}{c}\mathrm{DCF}_{\mathrm{i} 2} \\
(\mathrm{mrem} / \mathrm{pCi})\end{array}$ \\
\hline \multirow[t]{3}{*}{$\mathrm{U}-238+\mathrm{D}$} & D & $2.4 \times 10^{-3}$ & $\mathrm{Pu}-242$ & $\mathrm{~W}$ & $4.8 \times 10^{-1}$ \\
\hline & $\mathrm{W}$ & $6.2 \times 10^{-3}$ & & $\mathrm{Y}$ & $3.1 \times 10^{-1}$ \\
\hline & $\mathrm{Y}$ & $1.2 \times 10^{-1}$ & $\mathrm{Pu}-244+\mathrm{D}$ & $\mathrm{W}$ & $4.8 \times 10^{-1}$ \\
\hline $\mathrm{Np}-237+\mathrm{D}$ & W & $4.9 \times 10^{-1}$ & & $\mathrm{Y}$ & $3.1 \times 10^{-1}$ \\
\hline \multirow[t]{2}{*}{$\mathrm{Pu}-238$} & W & $4.6 \times 10^{-1}$ & Am-241 & W & $5.2 \times 10^{-1}$ \\
\hline & $\mathrm{Y}$ & $3.0 \times 10^{-1}$ & $\mathrm{Am}-243+\mathrm{D}$ & W & $5.2 \times 10^{-1}$ \\
\hline \multirow[t]{2}{*}{$\mathrm{Pu}-239$} & W & $5.1 \times 10^{-1}$ & $\mathrm{Cm}-243$ & $\mathrm{~W}$ & $3.5 \times 10^{-1}$ \\
\hline & $\mathrm{Y}$ & $3.3 \times 10^{-1}$ & $\mathrm{Cm}-244$ & W & $2.7 \times 10^{-1}$ \\
\hline \multirow[t]{2}{*}{ Pu-240 } & W & $5.1 \times 10^{-1}$ & $\mathrm{Cm}-248$ & $\mathrm{~W}$ & 1.9 \\
\hline & $\mathrm{Y}$ & $3.3 \times 10^{-1}$ & Cf- -252 & $\mathrm{~W}$ & $1.2 \times 10^{-1}$ \\
\hline $\mathrm{Pu}-241+\mathrm{D}$ & W & $1.0 \times 10^{-2}$ & & $Y$ & $1.3 \times 10^{-1}$ \\
\hline $\mathrm{Pu}-241+\mathrm{D}$ & $\mathrm{Y}$ & $5.7 \times 10^{-3}$ & & & \\
\hline
\end{tabular}

a Inhalation factors are for an AMAD of $1 \mu \mathrm{m}$.

b Dose conversion factors for entries labeled by " $+\mathrm{D}$ " are aggregated dose conversion factors for intake of a principal radionuclide together with radionuclides of the associated decay chain in secular equilibrium (see Section 3.1 and Table 3.1).

c Indicates a gaseous material.

d The three inhalation classes $D, W$, and $Y$ correspond to retention half-times of less than 10 days, 10 to 100 days, and greater than 100 days, respectively.

be used. The default value for a radionuclide used in the RESRAD code is the largest DCF for that radionuclide.

\section{B.2 ENVIRONMENTAL TRANSPORT FACTORS}

An ETF for dust inhalation is the ratio $E T F_{i 2}(t)=E_{i 2}(t) / S_{i}(t)$ of the annual intake $E_{i 2}(t)$ of the $i$ th principal radionuclide by dust inhalation to the concentration $S_{i}(t)$ of that radionuclide in the soil for the $i$ th year following the radiological survey. It can be expressed as the product

$$
E T F_{i 2}(t)=A S R_{2} \times F A_{2} \times F C D_{2}(t) \times F O_{2} \times F I_{2}
$$

where

$$
\begin{aligned}
E T F_{i 2}(t)= & \text { environmental transport factor at time } t \text { for dust inhalation for } \\
& \text { the } i \text { th principal radionuclide }(\mathrm{g} / \mathrm{yr}),
\end{aligned}
$$




$$
\begin{aligned}
A S R_{2}= & \text { air/soil concentration ratio }=\text { average mass loading of airborne } \\
& \text { contaminated soil particles }\left(2 \times 10^{-4} \mathrm{~g} / \mathrm{m}^{3}\right), \\
F A_{2}= & \text { area factor (dimensionless), } \\
F C D_{2}(t)= & \text { cover and depth factor (dimensionless), } \\
F O_{2}= & \text { occupancy factor (0.45, dimensionless), and } \\
F I_{2}= & \text { annual intake of air }\left(8,400 \mathrm{~m}^{3} / \mathrm{yr}\right) .
\end{aligned}
$$

The mass loading is a conservative estimate that takes into account short periods of high mass loading and sustained periods of normal activity on a typical farm (Gilbert et al. 1983, Appendix A).

Three models are commonly used for the process by which dust becomes airborne (Healy and Rodgers 1979, Appendix E; Oztunali et al. 1981, Appendix A; Gilbert et al. 1983, Appendix A). One is a resuspension factor model in which the airborne dust concentration $\left(C_{d u s t}\right)$ is given as a function of an empirically determined resuspension factor $\left(R_{f}\right)$, the effective depth of the layer of dust from which resuspension occurs $\left(d_{r}\right)$, and the bulk soil density $\left(\rho_{b}\right)$. The formula relating these variables is

$$
C_{d u s t}=R_{f} d_{r} \rho_{b}
$$

The second is a resuspension rate model in which the airborne dust concentration is given as a function of an empirically determined resuspension rate $\left(R_{r}\right)$, surface dust concentration $\left(\sigma_{s}=\rho_{b} d_{r}\right)$, and average deposition velocity $\left(v_{d}\right)$. The formula is

$$
C_{d u s t}=R_{r} \sigma_{s} / v_{d}
$$

The third is a mass loading model in which an average value of the airborne dust concentration is specified on the basis of empirical data.

The resuspension factor and resuspension rate are related by the equality $R_{r}=v_{d} R_{f} ;$ hence, the resuspension factor and resuspension rate models are not independent. Both resuspension models require two parameters that must be determined empirically and can vary over a wide range: (1) a resuspension factor or rate and (2) the thickness of the resuspendable layer. (The deposition velocity can also vary because of dependence on particle size, but the uncertainty in assigning a value is somewhat smaller.) The mass loading model 
uses a single parameter that is more directly measurable and for which empirical data are more readily available. The mass loading model has, therefore, been used in RESRAD for estimating the airborne dust concentration near the source.

For on-site exposure, the transport process may be regarded as a dilution process in which the resuspended contaminated dust is mixed with uncontaminated dust blown in from off-site. This dilution can be modeled by a Gaussian plume model (using an area distribution of point sources with a zero release height) or by a simple mixing model that assumes perfect mixing of resuspended on-site contaminated dust with off-site uncontaminated dust within a volume defined by a mixing height and the area of the exposed contaminated zone. The Gaussian plume model, as applied to annual average meteorological data, using sector averages, is generally regarded as applicable over distances from one hundred meters to several thousand meters; it has not been validated for on-site exposure in which the exposure occurs immediately above the source. There are uncertainties regarding exposure in the immediate area, primarily because of possible air turbulence or downdrafts created by buildings and structures. Considering these uncertainties regarding the Gaussian plume model for on-site exposure and the large increase in the amount of code that incorporation of an area-source Gaussian plume model would entail, a simple mixing model has been used in the current version of RESRAD.

The area factor represents the fraction of airborne dust that is contaminated. It is calculated by using a mixing model for estimating the dilution of contaminated dust that is resuspended on-site by uncontaminated dust blown in from off-site and is given by the formula

$$
F A_{2}=\frac{\sqrt{A}}{\sqrt{A}+D L}
$$

where

$$
\begin{aligned}
A & =\text { area of contaminated zone }\left(10,000 \mathrm{~m}^{2}\right), \text { and } \\
D L & =\text { dilution length }(3 \mathrm{~m}) .
\end{aligned}
$$

The dilution length $(D L)$ depends on the wind speed, mixing height, resuspension rate, and thickness of the resuspendable dust layer (Gilbert et al. 1983, Appendix A). Estimates of the lower and upper bounds of $D L$ for bounding values of the independent variables are $0.03 \mathrm{~m}$ 
and $250 \mathrm{~m}$, respectively. The geometric mean of the bounds, $D L=3 \mathrm{~m}$, is used as the default value.

The cover and depth factor is the fraction of resuspendable soil particles at the ground surface that are contaminated. It is calculated by assuming that mixing of the soil will occur within a layer of thickness $d_{m}$ at the surface. The cover and depth factor $F C D_{2}(t)$ is calculated by the formula

$$
\begin{aligned}
F C D_{2}(t) & =1 & & C_{d}(t)=0, T(t) \geq d_{m} \\
& =T(t) / d_{m} & & C_{d}(t)+T(t)<d_{m} \\
& =1-C_{d}(t) / d_{m} & & C_{d}(t)<d_{m}, C_{d}(t)+T(t) \geq d_{m} \\
& =0 & & C_{d}(t) \geq d_{m}
\end{aligned}
$$

where

$$
\begin{aligned}
d_{m} & =\text { depth of soil mixing layer }(0.15 \mathrm{~m}), \\
C_{d}(t) & =\text { cover depth at time } t(\mathrm{~m}), \text { and } \\
T(t) & =\text { thickness of contaminated zone at time } t(\mathrm{~m}) .
\end{aligned}
$$

The default occupancy factor of $\mathrm{FO}_{2}=0.45$ used in the RESRAD code is based on the assumption that $50 \%$ of a person's time is spent indoors (where the dust level is $40 \%$ of the outdoor level [Alzona et al. 1979]), 25\% is spent outdoors in the contaminated area, and 25\% is spent in uncontaminated areas.

The annual air intake of $8,400 \mathrm{~m}^{3} / \mathrm{yr}$ used in the RESRAD code is the value recommended by the International Commission on Radiological Protection (1975).

\section{B.3 REFERENCES}

Alzona, J., et al., 1979, "Indoor-Outdoor Relationships for Airborne Particulate Matter of Outdoor Origin," Atmospheric Environment 13:55-60.

Eckerman, K.F., et al., 1988, Limiting Values of Radionuclide Intake and Air Concentration and Dose Conversion Factors for Inhalation, Submersion, and Ingestion, EPA-520/1-88-020, Federal Guidance Report No. 11, prepared by Oak Ridge National Laboratory, Oak Ridge, Tenn., for U.S. Environmental Protection Agency, Office of Radiation Programs, Washington, D.C. 
Gilbert, T.L., et al., 1983, Pathways Analysis and Radiation Dose Estimates for Radioactive Residues at Formerly Utilized MED/AEC Sites, ORO-832 (Rev.), prepared by Division of Environmental Impact Studies, Argonne National Laboratory, Argonne, Ill., for U.S. Department of Energy, Oak Ridge Operations, Oak Ridge, Tenn., March 1983 (reprinted with corrections January 1984).

Healy, J.W., and J.C. Rodgers, 1979, Limits for the Burial of the Department of Energy Transuranic Wastes, LA-UR-79-100, Los Alamos Scientific Laboratory, Los Alamos, N.M.

International Commission on Radiological Protection, 1975, Report of the Task Group on Reference Man, prepared by a Task Group of Committee 2, adopted by the Commission in October 1974, ICRP Publication 23, Pergamon Press, New York, N.Y.

Oztunali, O.I., et al., 1981, Data Base for Radioactive Waste Management, Impact Analyses Methodology Report, NUREG/CR-1759, Vol. 3, prepared by Dames \& Moore, White Plains, N.Y., for Office of Nuclear Material Safety and Safeguards, U.S. Nuclear Regulatory Commission, Washington, D.C.

U.S. Department of Energy, 1988, Internal Dose Conversion Factors for Calculation of Dose to the Public, DOE/EH-0071, Assistant Secretary for Environment, Safety and Health, Washington, D.C. 


\section{6}


APPENDIX C:

RADON PATHWAY MODEL 


\section{APPENDIX C:}

\section{RADON PATHWAY MODEL}

\section{C.1 INTRODUCTION}

The inhalation of radon decay products is a major, and in many cases, the dominant dose contributor of internal radiation when radium isotopes are present in the soil. This is especially true in many sites identified by the Formerly Utilized Sites Remedial Action Program, the Surplus Facilities Management Program, and the Uranium Mill Tailings Remedial Action Program. The radon exposure pathway is included in RESRAD because radon and its decay products can accumulate to high concentrations in homes located on radium-contaminated sites. In the BEIR IV report (National Research Council 1988), radon-222 and its decay products are singled out as the most important sources of radiation exposure to the general public from naturally occurring radioactivity. The estimated annual dose equivalent to the bronchial epithelium from inhaled alpha-emitting radionuclides approaches $2,500 \mathrm{mrem} / \mathrm{yr}$, due almost entirely to the short-lived radon progeny. The Environmental Protection Agency's rule (Code of Federal Regulations, Title 40, Part 61) also identifies radon as one of the important radiation sources from airborne emissions in the United States. Although generic guidelines for radium have been established (see U.S. Department of Energy [DOE] Order 5400.5 [DOE 1990]), the addition of the radon pathway is necessary for deriving guidelines in a more consistent manner, especially for hot spots and mixtures in which radium or radium precursors, such as uranium and thorium isotopes, occur.

Radon-222 is the most common of the radon isotopes. Other radioisotopes of radon, such as radon-219 (actinon) and radon-220 (thoron), also occur naturally and have alphaemitting decay products. Actinon has an extremely short half-life (3.9 s). Accordingly, concentrations of radon-219 and its progeny in the air are extremely low, and the decay of actinon contributes little to human exposure. Radon-220 has a decay half-life of $55.6 \mathrm{~s}$. The concentration of thoron in the air is also usually low. Dosimetric considerations suggest that the dose to the lung from thoron progeny is, for an equal concentration of inhaled alpha energy, less by a factor of 3 than that due to the progeny of radon-222. However, in some DOE remedial action sites, high concentrations of thorium-232 and radium-228 are present 
and, in these circumstances, exposure to radon-220 and its decay products may be predominant. The radon-220 exposure pathway therefore is also included in RESRAD.

Calculation of the effective dose equivalents (EDEs) from airborne radon and radon decay products requires an estimate of (1) the radon exhalation from the ground surface, (2) the radon concentration in the air that results from this flux, and (3) the airborne concentration of radon decay products associated with this concentration. At a radiumcontaminated site, the radon release rate varies with the local distribution of radium, soil type, moisture content, and meteorological factors. Covering the contaminated area with soil and clay during remedial action or with a concrete floor when a house is being constructed can substantially reduce the emission rate. The actual reduction would depend on the physical properties and thickness of the covering materials. Inside a house, the concrete floor pad in the foundation would further reduce the radon emanation because the diffusion of radon through concrete is much smaller than through soil.

Radon and radium are soluble in water. When groundwater moves through radiumbearing soil, radon and radium are dissolved and transported with the water. When radoncontaining groundwater is used for domestic purposes, the radon can be released into the living space. The amount of radon available for release depends on the type and degree of processing that the water undergoes before use.

A generalized radon pathway model is included in RESRAD for estimating the amount of radon released, its concentrations in both indoor and outdoor air, and the resulting radiation dose as a function of time from the initial time of consideration. The effects of cover materials and other major parameters such as geological and meteorological conditions, decay and ingrowth, and leaching and erosion are considered with respect to near-term radon releases and long-term releases extending for thousands of years. All time-dependent parameters that would affect the radon release rate are considered by the radon pathway model. The indoor air concentration is calculated by a model in which radon enters the room through the floor and through ventilation inflow from the outdoor air. The radon mixed with the air in the room is then removed by radioactive decay and ventilation. In the current version of RESRAD, a single-compartment model that assumes uniform mixing is used to estimate the radon concentration distribution in a house. Even though the pathway involving radon in groundwater is usually not the dominant route of exposure for radium-contaminated 
sites, it is included for completeness. The EDE from inhalation of radon and its decay products is calculated on the basis of the recommendations of the International Commission on Radiological Protection's (ICRP's) Publications 32 and 47 (ICRP 1981, 1986), the BEIR IV report (National Research Council 1988), and a recent study by the National Research Council (1991).

\section{C.2 RADON EXHALATION}

Radon exhales continuously in the ground and migrates through the soil from radium-contaminated soil. Radon in the soil gas near buildings can move into houses by diffusion or through cracks or holes in the foundation by convection. Radon progeny, however, are chemically active and attach to soil particles and consequently do not move significantly in the ground. The movement of radon atoms through the pores may be caused by diffusion or convection. The size distribution and configuration of the pore spaces as well as their moisture content and spatial distribution are key parameters in determining the radon diffusion rate. Fractures or holes in building foundations as small as $0.5 \mathrm{~mm}$ are enough to allow convective migration. Convective movement of soil gas and the respective transport of radon within the soil and near the subsurface structure of the building can be induced by pressure differences created by meteorological factors and operational conditions of the house. These factors and conditions are highly time dependent and practically unpredictable. Consequently, the rate of radon exhalation to the outdoor environment and the infiltration into indoor air usually vary with time and cannot be readily quantified. However, the effect of the diurnal atmospheric pressure variation on the radon exhalation rate has been shown to be insignificant when averaged over long periods (Yuan and Roberts 1981).

Assuming a horizontally infinite and homogeneous soil, and neglecting the convective flow of the soil gas in the porous matrix of the soil, the radon flux $J$, (the amount of radon activity crossing a unit area of soil surface per unit of time, $\mathrm{pCi} / \mathrm{m}^{2} \cdot \mathrm{s}$ ), can be mathematically related to the gradient of the radon concentration in the pore space, $C$, by the Fickian diffusion equation 


$$
J=p_{t} D \frac{d C}{d z}
$$

where

$$
\begin{aligned}
& p_{t}=\text { total porosity } \\
& D=\text { diffusion coefficient of radon in soil }\left(\mathrm{m}^{2} / \mathrm{s}\right) \\
& C=\text { radon concentration in the pore space }\left(\mathrm{pCi} / \mathrm{m}^{3}\right), \text { and } \\
& z=\text { axial distance in the direction of diffusion }(\mathrm{m}) .
\end{aligned}
$$

The radon concentration and flux along a one-dimensional direction within multiple layers of radium-contaminated soil, the cover material, and the foundation of a building are calculated by using the one-dimensional radon diffusion equation

$$
\frac{d\left(p_{t} C\right)}{d t}=-\frac{d}{d z}(J)-p_{t} \lambda C+p_{t} Q
$$

or

$$
\frac{d C}{d t}=\frac{d}{d z}\left(D \frac{d C}{d z}\right)-\lambda C+Q
$$

where

$$
\begin{aligned}
& t=\text { time }(\mathrm{s}) \\
& \lambda=\operatorname{radon} \text { decay constant }(1 / \mathrm{s}), \text { and } \\
& Q=\text { radon source term into the pore space }\left(\mathrm{pCi} / \mathrm{m}^{3} \cdot \mathrm{s}\right)
\end{aligned}
$$

Assuming steady-state conditions, Equation C.2 can be written as

$$
-\frac{d}{d z}\left(D \frac{d C}{d z}\right)+\lambda C=Q
$$


The rate of radon generation, $Q$, or the source of radon released into the pore volume of the porous medium, is dependent on the concentration of radium in the contaminated zone. It can be evaluated by the following expression:

$$
Q=\frac{\varepsilon \rho_{b} S_{R a} \lambda}{p_{t}},
$$

where

$$
\begin{aligned}
\varepsilon & =\text { radon emanation coefficient (dimensionless), } \\
\rho_{b} & =\text { bulk density of the soil material }\left(\mathrm{kg} / \mathrm{m}^{3}\right), \\
S_{R a} & =\text { radium concentration in soil }(\mathrm{pCi} / \mathrm{kg}), \text { and } \\
\lambda & =\text { radon decay constant }(1 / \mathrm{s}) .
\end{aligned}
$$

The emanation coefficient $\varepsilon$ represents the fraction of radon generated by radium decay that escapes from the soil particles. Radon enters air-filled pores in the soil primarily from the recoil of radon atoms during radium decay. The contribution from diffusion through the solid mineral grains is less important because most of the radon atoms decay before escaping. Observed values of $\varepsilon$ range from about 0.01 to 0.80 (Mueller Associates, Inc. 1986). The observed emanation coefficient depends on many factors such as mineral composition of the soil, porosity, particle size distribution, and moisture content.

The radium concentration in soil, $S_{R a}$, is the radon precursor principal radionuclide concentration in the contaminated soil. Its value depends on the specific radon isotope being considered. For radon-222 or radon-220, respectively, $S_{R a}$ represents the radium-226 or thorium-228 concentration in soil. (The immediate parent of radon-220 is radium-224, which is an associated radionuclide assumed to be in equilibrium with its principal parent radionuclide thorium-228).

The total porosity, or void fraction $p_{t}$, is the fraction of the total volume that is not occupied by solid soil particles. Values of total porosity are given in Appendix E.

The boundary conditions used in RESRAD for solving Equation C.3 are as follows:

$$
\begin{aligned}
C\left(Z_{a}\right)= & 0, \text { the radon concentration at either the air-ground or the floor- } \\
& \text { indoor air interfaces, } Z_{a} \text {, is zero; }
\end{aligned}
$$


$J(0)=0$, the radon flux is zero at the bottom of the boundary; and

$C(z)$ and $J(z)$, that is, the radon concentration and flux, respectively, are continuous across medium interfaces in the ground.

In RESRAD, Equations C.1 and C.4, together with the above boundary conditions, are solved numerically for the vertical profiles of $C$ and $J$ by using a finite-difference method. The radon flux, $J$, is then evaluated (from Equation C.1) at two distinct locations: (1) $J_{o}$, the outdoor flux at the interface between the ground surface and the atmosphere and (2) $J_{i}$, the indoor flux at the interface between the floor and the indoor air.

In estimating the radon flux, the choice of a value for the diffusion coefficient, $D$, is critical. Values of $D$ have been measured experimentally for a variety of materials (Nazaroff and Nero 1988; Rogers and Nielson 1991). Some of the typical values of $D$ for different soils and building materials are summarized in the RESRAD Data Collection Handbook (Yu et al. 1993). Because the moisture content has such a dominant effect on $D$ (i.e., much smaller effects on $D$ are from other soil properties), $D$ has been correlated with moisture content as (Rogers and Nielson 1991)

$$
D=\left(1.1 \times 10^{-5}\right) p_{t} \exp \left(-6 R_{s} p_{t}-6 R_{s}^{14 p_{t}}\right),
$$

where $1.1 \times 10^{-5}$ is the radon diffusion coefficient in $\operatorname{air}\left(\mathrm{m}^{2} / \mathrm{s}\right)$, and $R_{s}$ is the saturation ratio, defined as the ratio of water content over the total porosity (see Appendix E). This correlation is based primarily on laboratory data for earthen materials and is used in RESRAD as a default approach for the diffusion coefficient if no measured data are available for the site of interest. Nevertheless, whenever possible, site-specific diffusion coefficients should be obtained, especially for materials other than soil.

\section{C.3 RADON CONCENTRATIONS}

\section{C.3.1 Outdoors}

The radon concentration in the outdoor air above a site contaminated with radium isotopes is influenced by the radon flux from the ground surface, environmental factors, and location and time. 
The estimation of the radon flux from a contaminated site has been described in Section C.2. The primary environmental parameters that can influence the dispersion of radon in outdoor air are meteorological conditions such as wind speed and the stability class. All of these site-specific environmental factors are time dependent and difficult to obtain. The calculation of outdoor radon concentrations by using all these parameters requires a comprehensive atmospheric dispersion code such as the MILDOS-AREA code (Yuan et al. 1989). These extensive computations are not practical for the purposes of the RESRAD code because the average radon concentration outdoors on top of a radium-contaminated area would most likely be dependent on the size of the contaminated area and the average wind speed and would not be very sensitive to other meteorological parameters. Also, the concentration outdoors would be relatively small compared to that indoors. The most feasible approach, thereafter, is to use a simplified model with some conservative assumptions. The following assumptions are used in the current model:

1. The annual average estimates are appropriate,

2. An average wind speed is used (a default wind speed of $2 \mathrm{~m} / \mathrm{s}$ is assumed) to conservatively represent annual average wind speed,

3. The wind direction flow is uniform around the compass and the frequency of flow toward the affected receptor is evenly distributed,

4. The receptor location and the house are at the geometric center of the contaminated area,

5. The vertical dimension of the plume is conservatively bounded at $2 \mathrm{~m}$ and a uniform concentration exists along the vertical plane for the downwind distance evaluated,

6. The radon-222 concentration to flux ratio is limited by a value of $500 \mathrm{~s} / \mathrm{m}$ for a very large area of contamination - a value corresponding to the ratio of the radon concentration to the flux level generally observed in the natural environment,

7. The radon-220 concentration to flux ratio is limited by a value of $10 \mathrm{~s} / \mathrm{m}$ for sites with a very large area of contamination - a value also based on the natural environment, and 
8. All emissions from ground surfaces are uniform.

According to this model, the annual average radon concentration outdoors is calculated as

$$
C_{o}=\frac{J_{o} F_{a o}}{\lambda H_{o}}\left[1-e^{-\left(\frac{\lambda X}{2 U}\right)}\right],
$$

where

$$
\begin{aligned}
C_{o} & =\text { annual average concentration of radon outdoors }\left(\mathrm{pCi} / \mathrm{m}^{3}\right), \\
J_{o} & =\text { radon flux at the soil surface outdoors }\left(\mathrm{pCi} / \mathrm{m}^{2} / \mathrm{s}\right), \\
H_{o} & =\text { height into which plume is uniformly mixed }(2 \mathrm{~m}), \\
F_{a o} & =\text { outdoor area factor (dimensionless), } \\
A & =\text { area of the contaminated zone }\left(\mathrm{m}^{2}\right), \\
X & =\sqrt{A}=\text { effective length of the contaminated area }(\mathrm{m}), \text { and } \\
U & =\text { annual average wind speed }(2 \mathrm{~m} / \mathrm{s})
\end{aligned}
$$

The outdoor area factor, $F_{a o}$, is a correction factor to adjust for the lateral dispersion effect for a small contaminated area and is given by the formula

$$
\begin{aligned}
F_{a o} & =\frac{A}{100}, 0 \leq A<100 \mathrm{~m}^{2} \\
& =1.0, A \geq 100 \mathrm{~m}^{2} .
\end{aligned}
$$

As a constraint for the ratio $C_{o}$ to $J_{o}$, it is assumed in RESRAD that

$$
\left(\frac{C_{o}}{J_{o}}\right)_{R n-222} \leq 500 \mathrm{~s} / \mathrm{m}
$$

and

$$
\left(\frac{C_{o}}{J_{o}}\right)_{R n-220} \leq 10 \mathrm{~s} / \mathrm{m}
$$




\section{C.3.2 Indoor}

Indoor radon levels depend on many parameters, including building characteristics, geographic area, and meteorological conditions (Nazaroff and Nero 1988; Cohen 1991). In RESRAD, the radon concentration indoors is determined by assuming a balance between the rate of radon entry from sources and its rate of removal. Radon enters a house by exhalation from the ground through the foundation floor and below-grade walls, by the inflow of outdoor air brought in by ventilation, and by the use of water. The rate of radon removal in a house is affected by processes such as air exchange and radioactive decay. Under steady-state conditions, the radon gain from exhalation and ventilation inflow equals the radon loss by decay and ventilation outflow. In the current version of RESRAD, a steady-state onecompartment model is used to determine the radon concentration indoors. A multicompartment and dynamic model can be used for a more realistic analysis if specific information regarding the design of a house is available.

Under the steady-state, single-compartment model, the mass balance of radon inside a home can be expressed as

$$
\frac{d C_{i}}{d t}=\frac{J_{i} A_{i} F_{a i}}{V}-(\lambda+v) C_{i}+v C_{o}=0
$$

where

$$
\begin{aligned}
C_{i} & =\text { radon concentration indoors }\left(\mathrm{pCi} / \mathrm{m}^{3}\right), \\
J_{i} & =\text { radon flux from the floor built on the contaminated area }\left(\mathrm{pCi} / \mathrm{m}^{2} \cdot \mathrm{s}\right), \\
A_{i} & =\text { interior surface area of the house floor }\left(100 \mathrm{~m}^{2}\right), \\
F_{a i} & =\text { indoor area factor (dimensionless), } \\
V & =\text { interior volume of the house }\left(\mathrm{m}^{3}\right), \\
\lambda & =\text { decay constant of radon }(1 / \mathrm{s}), \text { and } \\
v & =\text { ventilation rate of the house }(1 / \mathrm{s}) .
\end{aligned}
$$


The indoor area factor, $F_{a i}$, is the fraction of the foundation floor area that is built on the contaminated area and is given by the following formula:

$$
\begin{aligned}
F_{a i} & =1.0, & & A=A_{i} \\
& =A / A_{i}, & & A<A_{i} \\
& =1+4 D_{h} / \sqrt{A_{i}}, & & A>A_{i},
\end{aligned}
$$

where

$$
\begin{aligned}
A= & \text { area of contaminated zone }\left(\mathrm{m}^{2}\right) \\
A_{i}= & \text { interior surface area of the house floor }\left(100 \mathrm{~m}^{2}\right), \text { and } \\
D_{h}= & \text { depth of the foundation wall below and within the contaminated zone } \\
& (\mathrm{m}) .
\end{aligned}
$$

The radon flux, $J_{i}$, from the floor built on the contaminated area is calculated by solving Equations C.1 and C.4 and by assuming that the floor is a 15-cm (default) thick concrete slab with a default radon diffusion coefficient of $3.0 \times 10^{-7} \mathrm{~m}^{2} / \mathrm{s}$. The $3.0 \times 10^{-7} \mathrm{~m}^{2} / \mathrm{s}$ default radon diffusion coefficient for the concrete slab is a conservative value compared with the value of $6.0 \times 10^{-9} \mathrm{~m}^{2} / \mathrm{s}$ used by the U.S. Nuclear Regulatory Commission (1980). It is used to account for possible cracks and other penetrations that may develop in the foundation as the house ages. The variation of radon-222 levels in homes with the age of the house has been investigated by Cohen (1991). Of more than 30,000 homes investigated, the variations have been found to be within $36 \%$ of the mean values.

The solution for Equation C.10 for the indoor radon concentration, $C_{i}$, can be written as

$$
C_{i}=\frac{\left(\frac{J_{i} F_{a i}}{H}+v C_{o}\right)}{(\lambda+v)},
$$

where $H=V / A_{i}(\mathrm{~m})$ is the ratio of the interior volume of the house to the floor area of the house. For a single-story house, $H$ is the ceiling height. Because the ventilation rate, $v$, is much greater than the radon-222 decay rate, the indoor radon-222 concentration would be very sensitive to the air exchange rate of a house. For radon-220, because of its high decay rate, $v$ is not an important factor. The air exchange rate is usually expressed as air changes 
per hour and expresses the average number of times, in an hour, that the interior volume of the house is replaced by the inflow volume of outside air. Three basic mechanisms cause the exchange between indoor and outdoor air: (1) infiltration due to pressure gradient - air leakage inward through cracks, ceilings, floors, and walls of a house; (2) natural ventilation - the movement of air into and out of a house through intentionally provided openings such as windows and doors; and (3) mechanical ventilation - the forced movement of air between the indoors and outdoors by fans or air conditioners.

Typically, the air exchange rates for U.S. residences without weatherizing measures are within the following ranges (Mueller Associates, Inc. 1986):

Single-family detached house -0.5 to $1.5 \mathrm{~h}^{-1}$,

Single-family attached house -0.35 to $1.0 \mathrm{~h}^{-1}$,

Mobile homes - 0.3 to $1.5 \mathrm{~h}^{-1}$, and

Apartments -0.3 to $0.9 \mathrm{~h}^{-1}$.

In RESRAD, the radon concentration in the indoor air, $C_{i}$, is first calculated by Equation C.12. Subsequently, depending on the usage of groundwater in the house, its contribution to the final value of $C_{i}$ is added to that calculated from Equation C.12 as described in Section C.3.2.1.

\section{C.3.2.1 Indoor Radon from Water Use}

Groundwater containing radium can add to the amount of radon in the air indoors. Radon is partitioned between air and water in about a ratio of 4 to 1 , respectively, at $20^{\circ} \mathrm{C}$ in the environment under equilibrium conditions (Nazaroff and Nero 1988). When water is used, a large portion of the dissolved radon can escape. In the natural environment, the concentration of radon-222 in groundwater depends on the concentration of its parent, radium-226, in the underlying soil. The rate of migration of groundwater is usually slow enough to allow the radon-222 in water to be in approximate secular equilibrium with radium-226 in the local soil rock. Various studies (Mueller Associates, Inc., 1986; Bodansky et al. 1987) on correlating the radon-222 concentrations in the air of typical homes $\left(C_{a}\right)$ with the concentrations in water supplies $\left(C_{w}\right)$ have indicated that the ratios of $C_{a} / C_{w}$ are on the general order of $10^{-4}$. This value implies that water containing $10,000 \mathrm{pCi} / \mathrm{L}$ of radon would 
typically increase the indoor radon-222 concentration by $1 \mathrm{pCi} / \mathrm{L}$. The ratio of radon concentrations in indoor air to the radon concentrations in potable water supplies derived by the EPA has the following form (EPA 1984):

$$
\frac{C_{a}}{C_{w}}=\frac{f_{w a} U_{w}}{(\lambda+v) V}
$$

where

$$
\begin{aligned}
C_{a} & =\text { radon concentration increment in the indoor air }(\mathrm{pCi} / \mathrm{L}), \\
C_{w} & =\text { concentration of radon in water }(\mathrm{pCi} / \mathrm{L}), \\
f_{w a} & =\text { transfer efficiency of radon from water to air (dimensionless), } \\
U_{w} & =\text { household water use }(\mathrm{L} / \mathrm{h}), \\
v & =\text { ventilation rate }(1 / \mathrm{h}), \text { and } \\
V & =\text { volume of house }(\mathrm{L}) .
\end{aligned}
$$

Table C.1 presents typical ranges of these parameters. This table indicates that under typical conditions, the ratio $C_{a} / C_{w}$ should approximate the $10^{-4}$ empirical value previously determined for radon-222. A more recent study of the contribution of radon-222 from domestic water wells to indoor air in Colorado houses (Lawrence et al. 1992) has shown values approaching $10^{-3}$ in homes with a high radon-222 concentration in their water supplies. This value is an order of magnitude greater than the $10^{-4} \quad C_{a} / C_{w}$ ratio as calculated by Equation C.13. One of the reasons that Equation C.13 or its equivalent form underestimates the contribution of radon-222 from water can be attributed to the assumption that Equation C.13 uses the entire volume of air in a house. The equation implicitly assumes that the indoor air is

TABLE C.1 Ranges of Parameters Affecting Radon Concentrations in Air from Water Use in the Home

\begin{tabular}{ccc}
\hline Parameter & Ranges & Typical Values \\
\hline $\mathrm{f}_{\mathrm{wa}}$ & $0.25-1.0$ & 0.55 \\
$\mathrm{U}_{\mathrm{w}}(\mathrm{L} / \mathrm{h})$ & $4.75-19$ & 9.5 \\
$\mathrm{~V}(\mathrm{~L})$ & $37,500-150,000$ & 75,000 \\
$\mathrm{v}(1 / \mathrm{h})$ & $0.2-2.0$ & 1.0 \\
\hline
\end{tabular}

Note: $f_{w a}=$ transfer efficiency of radon from water to air, $U_{w}=$ individual water use, $\mathrm{V}=$ volume of house, and $v=$ ventilation rate per hour.

Source: Mueller Associates, Inc. (1986). 
perfectly mixed. In reality, the distribution of radon-222 in homes from water usage is highly uneven and dependent on the location of the water outlets. Higher radon-222 concentrations are expected in rooms (e.g., bathroom and kitchen) where water is accessible and frequently used than in rooms where no water outlets exist. Because the half-life of radon-220 is only $55.6 \mathrm{~s}$, the concentration of radon-220 from water usage would be much lower than that for an equal concentration of radon in water.

In estimating the radon concentration in the air from radon-contaminated water in homes, it is assumed in RESRAD that the air to water concentration of radon-222 is $1.0 \times 10^{-3}$ times the fraction of household water from the site. For radon-220, $C_{a} / C_{w}$ is calculated by the following equation:

$$
\left(\frac{C_{a}}{C_{w}}\right)_{R n-220}=1.0 \times 10^{-3}\left(\frac{v+\lambda_{R n-222}}{v+\lambda_{R n-220}}\right) \times F,
$$

where

$$
\begin{aligned}
\lambda_{R n-222} & =\text { radon-222 decay constant, } \\
\lambda_{R n-220} & =\text { radon-220 decay constant, and } \\
F & =\text { fraction of household water from the site (1.0). }
\end{aligned}
$$

The radon concentration in water, $C_{w}$, is calculated by using the groundwater and surface water concentrations, which are adjusted by the fraction of household water from these sources.

\section{C.4 DECAY AND INGROWTH OF RADON AND ITS PROGENY}

The radon that is breathed into the lungs as a noble gas is mostly breathed out before decay. The hazard from radon arises from its progeny, which are not gaseous; when they are breathed in they deposit on the interior surfaces of the lung. The decay schemes of radon-222 and radon-220 are shown in Figures C.1 and C.2, respectively. The physical properties of radon-220 and its progeny, and consequently their behavior in the atmosphere, are almost the same as those of radon-222 and its progeny. The first four decay progeny of 


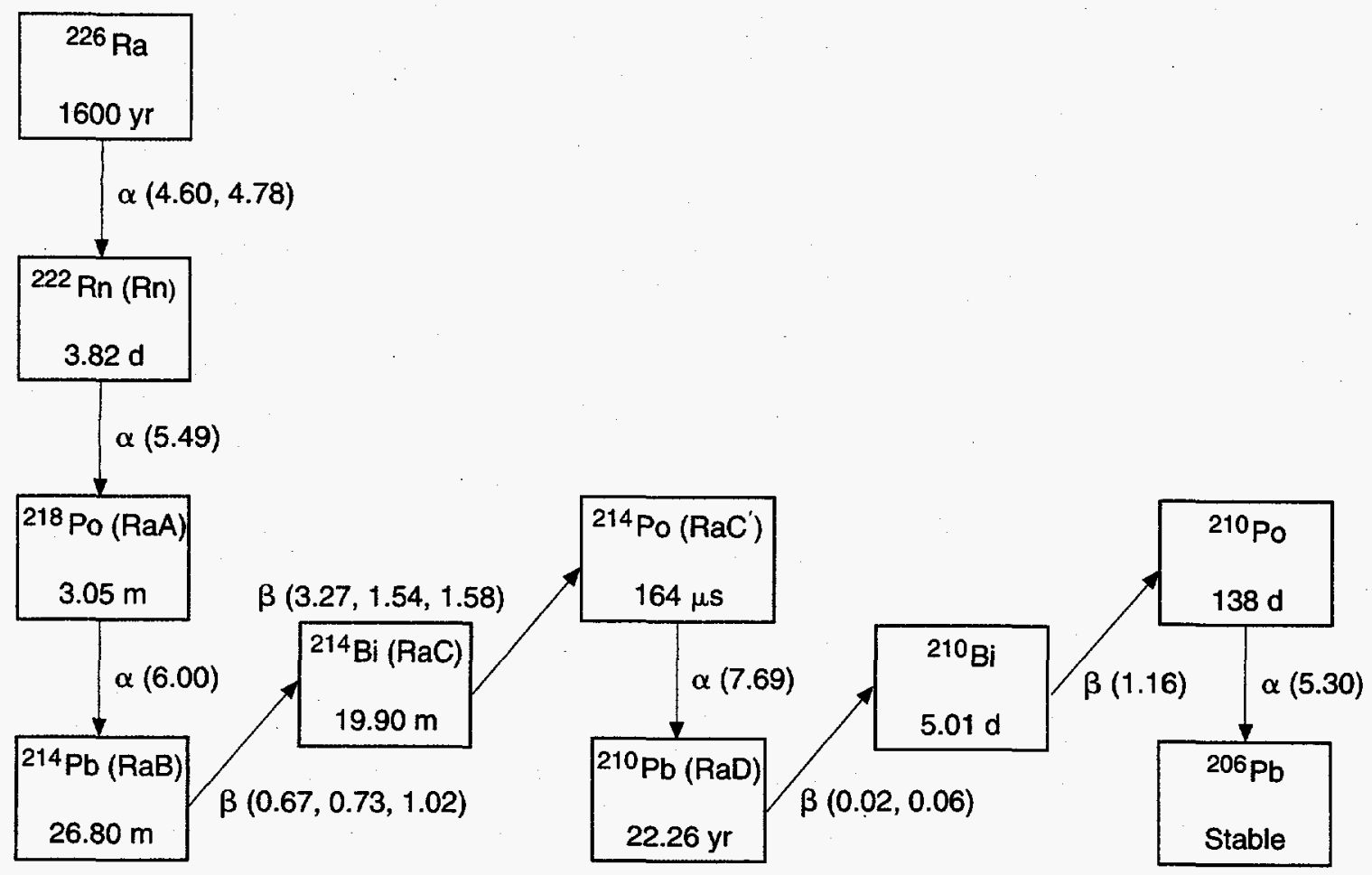

FIGURE C.1 Decay Scheme of Radon-222 (including its long-lived parent radium-226) (Source: Kocher 1981)

radon-222 - polonium-218, lead-214, bismuth-214, and polonium-214 (or $\mathrm{RaA}, \mathrm{RaB}, \mathrm{RaC}$, and $\mathrm{RaC}^{\prime}$, respectively) - have half-lives that are very short compared to the 22 years of lead-210 (RaD). Under most circumstances, only these short-lived alpha-emitting nuclides have health consequences. The half-life of radon-220 is $55.6 \mathrm{~s}$; it decays via polonium-216 with a half-life of $0.15 \mathrm{~s}$, to lead-212 with a half-life of $10.64 \mathrm{~h}$. Thus, levels of lead-212 will, in general, reflect concentrations of radon-220 in air. Once radon decays, the decay products become solids with electrical charges as a result of the decay process. In the air, most of the charged atoms rapidly become attached to aerosol particles. Because the fraction of ions that do not become attached is particularly important to the radon dosimetry, it has been given a special designation, that is, the unattached fraction $f$.

\section{C.4.1 Working Levels}

The working level (WL) was first introduced in 1957 (Holaday et al. 1957) as a convenient one-parameter measure of the concentration of radon decay products in uranium 


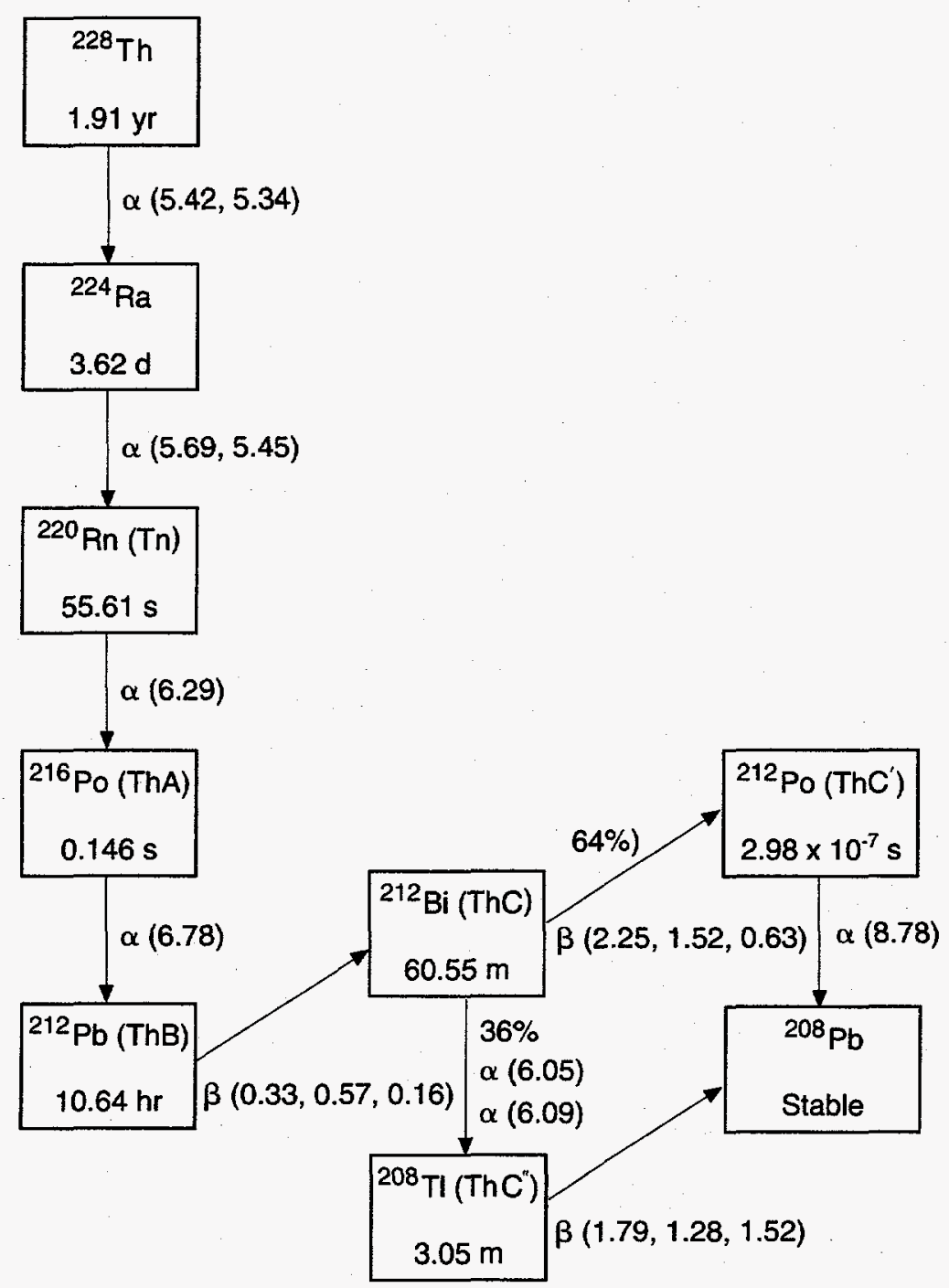

FIGURE C.2 Decay Scheme of Radon-220 (including its long-lived parent thorium-228) (Source: Kocher 1981)

mine air that can be used as a measure of exposure. Since then, WL has become a convention for measuring the concentration of radon progeny. The WL is defined as any combination of the short-lived radon progeny in $1 \mathrm{~L}$ of air that results in the ultimate release of $1.3 \times 10^{5} \mathrm{MeV}$ of potential alpha energy. Only the short-lived progeny are included in the definition of the WL because they contribute most of the dose to the lungs. The dose due to radon itself is minimal. For the same amount of activity inhaled, the radon-222 progeny mixture would result in a factor of about 40 to 100 higher than the dose from radon-222 alone, and the radon-220 progeny mixture (lead-212 and bismuth-212) would result in a factor of about 60 to 400 higher than radon-220 alone (ICRP 1981). Based on the definition 
of WL and using the alpha energy for the different radon-222 progeny, the WL value for an atmosphere containing a mixture of radon progeny can be evaluated as

$$
W L=1.03 \times 10^{-6} A+5.07 \times 10^{-6} B+3.73 \times 10^{-6} C,
$$

where $\mathrm{A}, \mathrm{B}$, and $\mathrm{C}$ represent the individual concentrations of, respectively, polonium-218, lead-214, and bismuth-214 in units of $\left(\mathrm{pCi} / \mathrm{m}^{3}\right)$. Similarly, for radon-220 progeny, the WL value can be evaluated as

$$
W L=9.48 \times 10^{-10} A^{\prime}+1.23 \times 10^{-4} B^{\prime}+1.17 \times 10^{-5} C^{\prime},
$$

where $A^{\prime}, B^{\prime}$, and $C^{\prime}$ represent the individual concentrations of, respectively, polonium-216, lead-212, and bismuth-212 in units of $\mathrm{pCi} / \mathrm{m}^{3}$.

Equations C.15 and C.16 are used in RESRAD for calculating the WL for radon-222 and radon-220, respectively. To calculate WLs by using the above equations, the radon progeny concentrations in the air must be determined first.

\section{C.4.1.1 Radon Progeny Concentrations Outdoors}

The decay and ingrowth of the short-lived radon progeny in the outdoor air are calculated on the basis of an average transit time from the source to the receptor. For outdoor radon, the most important removal processes are dilution by wind and radioactive decay. Other processes, such as deposition of radon progeny on the ground surfaces, are insignificant because the transport distances from the radon sources to the receptors considered are relatively short, that is, in the range of up to a few hundred meters. As discussed in Section C.3.1, the MILDOS-AREA code can be used for a detailed site-specific analysis if meteorological data are available. In RESRAD, the transit time $t$ during which radioactive decay occurs is approximated by dividing the average distance of the contaminated area by the annual average wind speed $U$, that is, $t=X /(2 U)$, where $X$ is the effective length of the contaminated area as defined in Equation C.7. 
The outdoor concentration of the radon progeny in the air is calculated by

$$
C_{o_{(n)}}=\frac{J_{o} F_{a o}}{H_{o}} \sum_{i=1}^{n} A_{(i, n)}\left[\frac{1-e^{-\left(\frac{\lambda_{i} X}{2 U}\right)}}{\lambda_{i}}\right],
$$

where

$$
\begin{aligned}
C_{o_{(n)}} & =n \text {th radon progeny concentration outdoors }\left(\mathrm{pCi} / \mathrm{m}^{3}\right), \\
\lambda_{\mathrm{i}} & =i \text { th radon progeny decay constant, } \\
A_{(i, n)} & =\frac{\lambda_{2} \ldots \lambda_{n}}{\left(\lambda_{1}-\lambda_{i}\right) \ldots\left(\lambda_{n}-\lambda_{i}\right)}, n \neq i
\end{aligned}
$$

and

$$
A_{(1,1)}=1 \text {. }
$$

The index $n$ is for radon and its progeny. For the radon-222 decay chain, $n=1,2,3$, and 4 represent, respectively, radon-222, polonium-218, lead-214, and bismuth-214; for the radon-220 decay chain, $n=1,2,3$, and 4 represent, respectively, radon-220, polonium-216, lead-212, and bismuth-212. In RESRAD, the outdoor concentrations of the radon-220 progeny, lead-212 and bismuth-212, are limited by the following constraint:

and

$$
\frac{C_{o_{(P b-212)}}}{C_{o_{(R n-220)}}} \leq 0.015,
$$

$$
\frac{C_{o_{(B i-212)}}}{C_{o_{(R n-220)}}} \leq 0.015 .
$$

This limit is used to correct the approximation made by Equation C.17 for a very large area of contamination. The concentration ratio of 0.015 for lead-212 and bismuth-212 to radon-220 is the value generally expected in the natural environment where an infinite area of contamination is almost reached. 


\section{C.4.1.2 Indoor Radon Progeny Concentration}

The removal processes by ventilation and plateout of radon progeny on surfaces within a house become important for indoor radon because the time for decay and ingrowth is much longer than that for outdoor radon. Inside a house, the decay products may plate out on any available surface. For an enclosed space with complex surfaces, such as a room with walls and furniture, plateout and detachment from them are complicated, and data are not readily available. The rate of plateout can be specified in terms of the ratio of the amount deposited on a surface from the air (in atoms removed per unit area per unit time) to the concentration in the air (in atoms per unit volume). Such a ratio is usually called the deposition velocity.

The plateout process has been reviewed recently (Knutson et al. 1983; Bodansky et al. 1987). The suggested values of the plateout deposition velocity range from $0.05 \mathrm{~cm} / \mathrm{s}$ for the highly reactive unattached fraction to $0.00075 \mathrm{~cm} / \mathrm{s}$ for the fraction that has already attached to the aerosol in a house. For a room with a floor area of $3 \mathrm{~m} \times 4 \mathrm{~m}$ and a ceiling height of $2 \mathrm{~m}$, these deposition velocities correspond to removal rates of 0.065 per minute for the unattached fraction and 0.00090 per minute for the attached fraction. These removal rates compared with typical air changes range between 0.3 to 1.0 interior volumes per hour, which corresponds to removal rates of 0.005 to 0.0167 per minute. The unattached fraction $f$ in the home has a range of about $1 \%$ to $10 \%$ (National Research Council 1991). In the current version of RESRAD, plateout is not considered in calculating radon progeny in a home. This constitutes a conservative approach because by neglecting plateout, the indoor air concentrations of radon progeny are overestimated.

The indoor air concentrations of the radon progeny are calculated by a mass balance equation. In this equation, the time variation of the concentration of any element of the decay series is equal to the sum of the ingrowth rate from the decay of its immediate predecessor plus the inflow rate from the outdoor concentration due to the ventilation, minus its decay rate and the outflow rate due to the ventilation. The mass balance equation of radon progeny can be written as

$$
\frac{d C_{i_{(n)}}}{d t}=\left[\lambda_{n} C_{i_{(n-1)}}+v C_{o_{(n)}}\right]-\left(\lambda_{n}+v\right) C_{i_{(n)}}
$$


where $n$ is equal to 1 for radon and $C_{i_{(1)}}$ is calculated by using Equation C.10 for the radon indoor concentration; $n$ is equal to $2,3,4$ for the radon progeny (i.e., polonium-218, lead-214, and bismuth-214 for the radon-222 decay chain, and polonium-216, lead-212, and bismuth-212 for the radon-220 decay chain, respectively); and, $C_{i_{(n)}}$ and $C_{o(n)}$ are the $n$th radon progeny concentration indoors and outdoors, respectively, given in units of $\mathrm{pCi} / \mathrm{m}^{3}$. Under steady-state conditions, the mass balance equation above can be solved for $C_{i_{(n)}}$ as

$$
C_{i_{(n)}}=\frac{\lambda_{n} C_{i_{(n-1)}}+v C_{o_{(n)}}}{\left(\lambda_{n}+v\right)}, \quad n=2,3,4
$$

\section{C.5 RADON DOSIMETRY}

The radiation dose from inhalation of radon and its progeny depends on the emitted alpha energies and radioactivities, on the various lung tissues in which they are retained, and the period of time retained. Inhalation of radon, or more specifically radon progeny, leads to the retention of radioactive atoms on the mucus layer covering the respiratory system, especially in the bronchial region of the lung. The short-lived radon progeny, consisting of various isotopes of polonium, lead, and bismuth emit high energy alpha particles and irradiate the cells of the lung tissue. These irradiated cells may become cancerous. The rate of lung cancer induction depends on the rate of retention of the radon progeny atoms in various parts of the lungs. This in turn depends on many factors, including the concentrations of radon and its progeny in the air, the fraction of the progeny that are attached to dust particles, the sizes of the dust particles, and the breathing rate. Given data for these factors and an adequate model of the lungs' dynamics, it is possible to calculate the radiation dose. A number of complicated lung models for calculating the dose from inhalation of radon and its progeny have been developed (ICRP 1979-1982; Jacobi and Eisfeld 1980; James et al. 1980; Harley and Pasternack 1982). Even though the calculated results from using these models are not in complete agreement, they are close enough to permit reasonably good estimates of the radiation dose to the lung from radon and its progeny (National Research Council 1988).

In RESRAD, the radiation dose from radon and its progeny is calculated by using the accumulated exposure in terms of working level month (WLM). Working level month is a cumulative exposure unit historically applied to uranium miners and is now defined as the 
product of WL and the duration of exposure, normalized to a 170-hour working month exposure (ICRP 1981, 1986). This unit was introduced so that both the duration and level of exposure could be taken into account. To convert WLM, or the total cumulative potential alpha energy of radon progeny deposited in the lungs to the effective dose equivalent, as required by RESRAD, doses to the other organs have to be determined. Retention studies of inhaled radon-222 progeny in the human respiratory systems indicate that the dose from radon-222 progeny is primarily delivered to the lung within a few hours after intake. The dose to other tissues is relatively small compared to the lung dose (ICRP 1981). For radon-220, with the exception of lead-212, the dose from its progeny is also primarily delivered to the lungs within a few hours of intake because of the progeny's short decay halflives. Because the radioactive half-life of lead-212 is comparable to its biological half-life in the lung, the ratio of the bronchial to pulmonary dose is lower than that for radon-222 progeny, and a considerable fraction of the deposited lead-212 is transferred to the blood and other organs and tissues. Dosimetric considerations indicate that for an equal concentration of inhaled alpha energy, the dose to the tracheobronchial epithelium from radon-220 progeny is less by a factor of about 3 than that due to the progeny of radon-222. The numerical values recommended by ICRP Publications 32 and 47 for workers in uranium and other mines are used as a basis in RESRAD for converting WLM to the EDE. The ICRP. recommended dosimetric conversion factors are 1,000 $\mathrm{mrem} / \mathrm{WLM}$ for radon-222 progeny and $350 \mathrm{mrem} / \mathrm{WLM}$ for radon-220 progeny. Because the exposure for the general population is continuous and the breathing rate is lower and shallower than for miners, the use of WLM has to be adjusted for the general population. In RESRAD, the application of WLM to the general population follows the approach of a proportional factor $K$, which was first introduced qualitatively in the BEIR IV report (National Research Council 1988) and later analyzed quantitatively by a companion study to BEIR IV (National Research Council 1991).

\section{C.5.1 K Factor: Extrapolation of Doses from Mines to Homes}

Because the environmental conditions in a mine and the physiological characteristics of miners are, respectively, significantly different from those in a home and members of the general public, the dose conversion factors (DCFs) derived for miners have to be extrapolated to the general population in homes. The methodology used in RESRAD is based on the $K$ factor introduced in the BEIR IV report. The $K$ factor is a dimensionless proportionality 
factor that converts the risk or dose to the lung to miners per WLM to the risk or dose to an individual in a home per WLM. In deriving the $K$ factor, the National Research Council uses a dosimetric model recently developed (National Research Council 1991) to estimate the uncertainty introduced by differences in exposure-dose relations for miners and the general population. The model incorporates a wide range of physical and biological parameters that differ in these two environments. The parameters analyzed include aerosol size distribution, unattached fraction, and breathing rate and route. The $K$ factors derived by the National Research Council are presented in Table C.2. For the range of exposure scenarios as well as sex and age groups considered, most values of $K$ were less than unity. Thus, it can be concluded that the delivered bronchial dose per WLM exposure is less in a home than in a mine. In RESRAD, internal DCFs from inhalation and ingestion pathways are based on the ICRP dosimetric model for the reference man. For consistency with the reference man approach, a $K$ factor of 0.76 (see Table C.2 for the $K$ factor for target cells: secretory) is used for the adult male in RESRAD, even though the $K$ factors for children and infants were somewhat greater than those for adults. This $K$ factor translates to an effective dose equivalent dose conversion factor of $760 \mathrm{mrem} / \mathrm{WLM}$ for radon-222 progeny indoors. As discussed earlier, for exposure to radon-222 progeny, the doses to tissues other than the lungs are relatively small. Thus, the $K$ factor derived to convert the lung doses is assumed to be applicable for converting the EDEs for the general population and miners.

TABLE C.2 Summary of K Factors for Bronchial Dose Calculated for the General Public Relative to Underground Miners

For exposure to radon-222 progeny outdoors, the $K$ factor has to be reevaluated because of the higher ventilation (i.e., the effect of wind), the higher unattached fraction, the larger particle size, and the higher breathing rate than in the indoor environment. Assuming unattached fractions of $8 \%$ indoors and $15 \%$ outdoors, particle sizes of $0.15 \mu \mathrm{m}$ activity median aerodynamics diameter (AMAD) indoors and $0.25 \mu \mathrm{m}$ AMAD outdoors and minute volumes of $12.5 \mathrm{~L} / \mathrm{min}$

\begin{tabular}{lcc}
\hline & \multicolumn{2}{c}{ K Factor } \\
\cline { 2 - 3 } Subject Category & $\begin{array}{c}\text { Basal } \\
\text { Cells }\end{array}$ & $\begin{array}{c}\text { Secretory } \\
\text { Cells }\end{array}$ \\
\hline Infant, age 1 mo & 0.64 & 0.74 \\
Child, age 1 yr & 0.87 & 1.00 \\
Child, age 5-10 yr & 0.72 & 0.83 \\
Female & 0.62 & 0.72 \\
Male & 0.69 & 0.76 \\
\hline
\end{tabular}

Source: National Research Council (1991). 
indoors and $21 \mathrm{~L} / \mathrm{min}$ outdoors, a $K$ factor of 0.57 can be derived for exposure to radon-222 progeny outdoors (by using Figures 2B-2, 2B-3, and 2B-4 provided in the BEIR IV report). This $K$ factor corresponds to an EDE conversion factor of $570 \mathrm{mrem} / \mathrm{WLM}$ for radon-222 outdoors. The minute volume of $21 \mathrm{~L} / \mathrm{min}$ outdoors is estimated on the basis of the assumption that an average individual would do light work $75 \%$ of the time and rest $25 \%$ of the time outdoors.

For exposure to radon-220 progeny indoors, the National Research Council study (National Research Council 1991) introduced a $K^{\prime}$ factor that extrapolates the lung dose for exposure to radon-220 progeny in the home relative to radon-222 in mines. Because the dose from exposure to radon-220 progeny is determined principally by the behavior of lead-212, which has a relatively long radioactive half-life $(10.6 \mathrm{~h})$, a considerable fraction of the deposited lead-212 is transferred to blood and other organs or tissues (e.g., bone surface [critical tissue], liver, and red marrow). The $K^{\prime}$ factor thus derived solely for the lung dose may not be appropriate for estimating the EDE, which is the sum of the products of the dose equivalent to the organ or tissue and the weighting factors applicable to each of the organs or tissues that are irradiated. Among the factors that would affect lung dose from exposure to lead-212, only a minute volume would possibly be of any significance when the EDE is the dose of concern. Using the assumptions of a minute volume of $30 \mathrm{~L} / \mathrm{min}$ for the miners and $12.5 \mathrm{~L} / \mathrm{min}$ for an average person breathing in a home, the ratio of the corresponding EDE proportional factor for these two minute volumes was estimated to be about 0.42 . This value corresponds to a dose conversion of about $150 \mathrm{mrem} / \mathrm{WLM}$ indoors and is used in RESRAD for calculating doses from exposure to radon-220 progeny indoors. For exposure to radon-220 progeny outdoors, the minute volume of $21 \mathrm{~L} / \mathrm{min}$ assumed for exposure to outdoor radon-222

progeny can be applied. The conversion factor of 0.42 for indoor exposure therefore increases to 0.70 for outdoor exposure. This increased conversion factor corresponds to an EDE factor of about $250 \mathrm{mrem} / \mathrm{WLM}$ for radon-220 outdoors.

\section{C.6 REFERENCES}

Bodansky, D., et al., 1987, Indoor Radon and Its Hazards, University of Washington Press, Seattle, Washington.

Cohen, B.L., 1991, "Variation of Radon Levels in U.S. Homes Correlated with House Characteristics, Location, and Socioeconomic Factors," Health Physics 60:631-642. 
Harley, N.H., and B.S. Pasternack, 1982, "Environmental Radon Daughter Alpha Factors in Five-Lobed Human Lung," Health Physics 42:789-799.

Holaday, D.A., et al., 1957, Control of Radon and Daughters in Uranium Mines and Calculations of Biological Effects, U.S. Public Health Service Publication No. 494, U.S. Government Printing Office, Washington, D.C.

International Commission on Radiological Protection, 1979-1982, Limits for Intakes of Radionuclides by Workers, a report of Committee 2 of the International Commission on Radiological Protection, adopted by the Commission in July 1978, ICRP Publication 30, Part 1 (and Supplement), Part 2 (and Supplement), Part 3 (and Supplements A and B), and Index, Annals of the ICRP, Pergamon Press, New York, N.Y.

International Commission on Radiological Protection, 1981, Limits for Inhalation of Radon Daughters by Workers, Annals of the ICRP, ICRP Publication 32, Vol. 6, No. 1, Pergamon Press, Oxford, United Kingdom.

International Commission on Radiological Protection, 1986, Radiation Protection of Workers in Mines, Annals of the ICRP, ICRP Publication 47, Vol. 16, No. 1, Pergamon Press, Oxford, United Kingdom.

Jacobi, W., and K. Eisfeld, 1980, Dose to Tissue and Effective Dose Equivalent by Inhalations of Radon-222 and Their Short-Lived Daughters, GSF Report S-626, Geschelscheft Fur Strahlen and Umwelfforschung, Neurherberg, Germany.

James, A.C., et al., 1980, "A Domestic Model for Tissues of the Human Respiratory Tract at Risk from Inhaled Radon and Thoron Daughters," in Radiation Protection. A Systematic Approach to Safety, proceedings of the 5th Congress of the International Radiation Protection Association (IRPA), Vol. 2, Jerusalem, Pergamon Press, New York, N.Y., pp. 1045-1048.

Kocher, D.C., 1981, Radioactive Decay Data Tables: A Handbook of Decay Data for Application to Radiation Dosimetry and Radiological Assessments, DOE/TIC-11026, U.S. Department of Energy, Washington, D.C.

Knutson, E.O., et al., 1983, "Radon Daughter Plateout-II, Prediction Model," Health Physics 45:445.

Lawrence, E.P., et al., 1992, "Contribution of ${ }^{222} \mathrm{Rn}$ in Domestic Water Supplies to ${ }^{222} \mathrm{Rn}$ in Indoor Air in Colorado Homes," Health Physics 62:171-177.

Mueller Associates, Inc., 1986, Indoor Air Quality Environmental Information Handbook: Radon, DOE/PE/72013-2, prepared by Mueller Associates, Inc., Baltimore, Md., for U.S. Department of Energy, Washington, D.C.

National Research Council, 1988, Health Risks of Radon and other Internally Deposited Alpha-Emitters, BEIR IV, Committee on the Biological Effects of Ionizing Radiations, National Academy Press, Washington, D.C. 
National Research Council, 1991, Comparative Dosimetry of Radon in Mines and Homes, National Academy Press, Washington, D.C.

Nazaroff, W.W., and A.V. Nero (editors), 1988, Radon and Its Decay Products in Indoor Air, John Wiley \& Sons, New York, N.Y.

Rogers, V.C., and K.K. Nielson, 1991, "Correlations for Predicting Air Permeabilities and Rn-222 Diffusion Coefficients of Soils," Health Physics 61:225-2300.

U.S. Department of Energy, 1990, Radiation Protection of the Public and the Environment, DOE Order 5400.5, Feb.

U.S. Environmental Protection Agency, 1984, Risk Analysis of TCDD Contaminated Soil, EPA 60018-84-03, Office of Health and the Environmental Assessment, Washington, D.C.

U.S. Nuclear Regulatory Commission, 1980, Final Generic Environmental Impact Statement on Uranium Milling, NUREG-0706, Washington, D.C.

Yu, C., et al., 1993, Data Collection Handbook to Support Modeling the Impacts of Radioactive Material in Soil, ANL/EAIS-8, prepared by Argonne National Laboratory, Argonne, Ill., for U.S. Department of Energy, Washington, D.C.

Yuan, Y.C., and C.J. Roberts, 1981, "Numerical Investigation of Radon Transport through a Porous Medium," Transactions of the American Nuclear Society 38:108-110.

Yuan, Y.C., et al., 1989, MILDOS-AREA: An Enhanced Version of MILDOS for Large-Area Sources, ANL/ES-161, Argonne National Laboratory, Argonne, Ill. 
APPENDIX D:

INGESTION PATHWAY FACTORS 


\section{APPENDIX D:}

\section{INGESTION PATHWAY FACTORS}

Ingestion pathways consist of six environmental pathways and the common exposure pathway to which they contribute (see Table 2.1). The doses from these pathways are specified by the dose/source ratios described in Section 3. A dose/source ratio (DSR) may be defined as the sum of the product of the dose conversion factor (DCF) (which characterizes the exposure pathway), the environmental transport factor (ETF) (which characterizes the environmental pathways), and the source factor (SF) (which characterizes ingrowth and decay and leaching of the radionuclides) (see Equation 3.9). Dose conversion factors for ingestion are described in Section D.1. Environmental transport factors and the models and formulas used to calculate them are presented in Sections D.2 through D.4. The models used for the ingestion of contaminated soil pathway are discussed in Appendix F. Formulas for calculating the source factors are given in Section 3.2.3 and Appendix G.

\section{D.1 DOSE CONVERSION FACTORS}

A DCF for ingestion is the dose/exposure ratio $D C F_{i 3}=H_{E, i 3} / E_{i}$, where $H_{E, i 3}$ is the committed effective dose equivalent (EDE) incurred by an individual from intake by ingestion of a quantity $E_{i}$ of the $i$ th principal radionuclide. Values of DCFs for ingestion were taken from a DOE report (DOE 1988) and are tabulated in Table D.1. Similar values are given in an EPA report (Eckerman et al. 1988). Dose conversion factors depend on the chemical form, which determines the fraction $f_{1}$ of a radionuclide entering the gastrointestinal (GI) tract that reaches body fluids. Data on the appropriate fractions for different chemical forms are given in Publication 30 of the International Commission on Radiological Protection (ICRP) (ICRP 1979-1982). The DCFs used in RESRAD are the values corresponding to the largest values

of $f_{1}$ in Table D.1. If the chemical form of a radionuclide is known and a different value of $D C F_{i 3}$ is needed, it can be modified by choosing the "Dose Factors" option from the RESRAD main menu and selecting the "Ingestion Effective Dose Conversion Factors" from the submenu and then entering the appropriate value. 
TABLE D.1 Committed Effective Dose Equivalent Conversion Factors $\left(\mathrm{DCF}_{\mathrm{i} 3}\right)$ for Internal Radiation from Ingestion

\begin{tabular}{|c|c|c|c|c|c|}
\hline Radionuclide $^{a}$ & $f_{1}^{b}$ & $\begin{array}{c}\mathrm{DCF}_{\mathrm{i} 3} \\
(\mathrm{mrem} / \mathrm{pCi}) \\
\end{array}$ & Radionuclide $^{\mathbf{a}}$ & $\mathrm{f}_{1}^{\mathrm{b}}$ & $\begin{array}{c}\mathrm{DCF}_{\mathrm{i} 3} \\
(\mathrm{mrem} / \mathrm{pCi})\end{array}$ \\
\hline $\mathrm{H}-3$ & 1.0 & $6.3 \times 10^{-8}$ & $\mathrm{~Pb}-210+\mathrm{D}$ & $2 \times 10^{-1}$ & $6.7 \times 10^{-3}$ \\
\hline C-14 & 1.0 & $2.1 \times 10^{-6}$ & $\mathrm{Bi}-207$ & $5 \times 10^{-2}$ & $4.9 \times 10^{-6}$ \\
\hline $\mathrm{Na}-22$ & 1.0 & $1.2 \times 10^{-5}$ & $\mathrm{Ra}-226+\mathrm{D}$ & $2 \times 10^{-1}$ & $1.1 \times 10^{-3}$ \\
\hline $\mathrm{Al}-26$ & $1 \times 10^{-2}$ & $1.3 \times 10^{-5}$ & $\mathrm{Ra}-228+\mathrm{D}$ & $2 \times 10^{-1}$ & $1.2 \times 10^{-3}$ \\
\hline Cl-36 & 1.0 & $3.0 \times 10^{-6}$ & $\mathrm{Ac}-227+\mathrm{D}$ & $1 \times 10^{-3}$ & $1.5 \times 10^{-2}$ \\
\hline $\mathrm{K}-40$ & 1.0 & $1.9 \times 10^{-5}$ & Th $-228+\mathrm{D}$ & $2 \times 10^{-4}$ & $7.5 \times 10^{-4}$ \\
\hline $\mathrm{Ca}-41$ & $3 \times 10^{-1}$ & $1.2 \times 10^{-6}$ & Th-229+D & $2 \times 10^{-4}$ & $4.3 \times 10^{-3}$ \\
\hline Mn-54 & $1 \times 10^{-1}$ & $2.7 \times 10^{-6}$ & Th-230 & $2 \times 10^{-4}$ & $5.3 \times 10^{-4}$ \\
\hline $\mathrm{Fe}-55$ & $1 \times 10^{-1}$ & $5.8 \times 10^{-7}$ & Th-232 & $2 \times 10^{-4}$ & $2.8 \times 10^{-3}$ \\
\hline \multirow[t]{2}{*}{ Co-57 } & $3 \times 10^{-1}$ & $1.1 \times 10^{-6}$ & $\mathrm{~Pa}-231$ & $1 \times 10^{-3}$ & $1.1 \times 10^{-2}$ \\
\hline & $5 \times 10^{-2}$ & $6.8 \times 10^{-7}$ & U-232 & $5 \times 10^{-2}$ & $1.3 \times 10^{-3}$ \\
\hline \multirow[t]{2}{*}{ Co-60 } & $3 \times 10^{-1}$ & $2.6 \times 10^{-5}$ & & $2 \times 10^{-3}$ & $6.8 \times 10^{-5}$ \\
\hline & $5 \times 10^{-2}$ & $1.0 \times 10^{-5}$ & U-233 & $5 \times 10^{-2}$ & $2.7 \times 10^{-4}$ \\
\hline $\mathrm{Ni}-59$ & $5 \times 10^{-2}$ & $2.0 \times 10^{-7}$ & & $2 \times 10^{-3}$ & $2.5 \times 10^{-5}$ \\
\hline $\mathrm{Ni}-63$ & $5 \times 10^{-2}$ & $5.4 \times 10^{-7}$ & U-234 & $5 \times 10^{-2}$ & $2.6 \times 10^{-4}$ \\
\hline $\mathrm{Zn}-65$ & $5 \times 10^{-1}$ & $1.4 \times 10^{-5}$ & & $2 \times 10^{-3}$ & $2.5 \times 10^{-5}$ \\
\hline $\mathrm{Ge}-68+\mathrm{D}$ & $1 \times 10^{-1}$ & $1.4 \times 10^{-6}$ & $\mathrm{U}-235+\mathrm{D}$ & $5 \times 10^{-2}$ & $2.5 \times 10^{-4}$ \\
\hline \multirow[t]{2}{*}{ Sr-90+D } & $3 \times 10^{-1}$ & $1.4 \times 10^{-4}$ & & $2 \times 10^{-3}$ & $2.6 \times 10^{-5}$ \\
\hline & $1 \times 10^{-2}$ & $2.2 \times 10^{-5}$ & U-236 & $5 \times 10^{-2}$ & $2.5 \times 10^{-4}$ \\
\hline $\mathrm{Nb}-94$ & $1 \times 10^{-2}$ & $5.1 \times 10^{-6}$ & & $2 \times 10^{-3}$ & $2.4 \times 10^{-5}$ \\
\hline Tc-99 & $8 \times 10^{-1}$ & $1.3 \times 10^{-6}$ & $\mathrm{U}-238+\mathrm{D}$ & $5 \times 10^{-2}$ & $2.5 \times 10^{-4}$ \\
\hline Ru-106+D & $5 \times 10^{-2}$ & $2.2 \times 10^{-5}$ & & $2 \times 10^{-3}$ & $3.8 \times 10^{-5}$ \\
\hline $\mathrm{Ag}-108 \mathrm{~m}+\mathrm{D}$ & $5 \times 10^{-2}$ & $7.5 \times 10^{-6}$ & $\mathrm{~Np}-237+\mathrm{D}$ & $1 \times 10^{-3}$ & $3.9 \times 10^{-3}$ \\
\hline $\mathrm{Ag}-110 \mathrm{~m}+\mathrm{D}$ & $5 \times 10^{-2}$ & $1.1 \times 10-^{5}$ & $\mathrm{Pu}-238$ & $1 \times 10^{-3}$ & $3.8 \times 10^{-3}$ \\
\hline Cd-109 & $5 \times 10^{-2}$ & $1.2 \times 10^{-5}$ & & $1 \times 10^{-5}$ & $5.4 \times 10^{-5}$ \\
\hline \multirow[t]{2}{*}{ Sb-125+D } & $1 \times 10^{-1}$ & $3.2 \times 10^{-6}$ & Pu-239 & $1 \times 10^{-3}$ & $4.3 \times 10^{-3}$ \\
\hline & $1 \times 10^{-2}$ & $3.4 \times 10^{-6}$ & & $1 \times 10^{-5}$ & $5.8 \times 10^{-5}$ \\
\hline I-129 & 1.0 & $2.8 \times 10^{-4}$ & $\mathrm{Pu}-240$ & $1 \times 10^{-3}$ & $4.3 \times 10^{-3}$ \\
\hline Cs-134 & 1.0 & $7.4 \times 10^{-5}$ & & $1 \times 10^{-5}$ & $5.8 \times 10^{-5}$ \\
\hline Cs-135 & 1.0 & $7.1 \times 10^{-6}$ & $\mathrm{Pu}-241+\mathrm{D}$ & $1 \times 10^{-3}$ & $8.6 \times 10^{-5}$ \\
\hline $\mathrm{Cs}-137+\mathrm{D}$ & 1.0 & $5.0 \times 10^{-5}$ & & $1 \times 10^{-5}$ & $9.2 \times 10^{-7}$ \\
\hline $\mathrm{Ce}-144+\mathrm{D}$ & $3 \times 10^{-4}$ & $2.0 \times 10^{-5}$ & $\mathrm{Pu}-242$ & $1 \times 10^{-3}$ & $4.1 \times 10^{-3}$ \\
\hline Pm-147 & $3 \times 10^{-4}$ & $9.5 \times 10^{-7}$ & & $1 \times 10^{-5}$ & $5.6 \times 10^{-5}$ \\
\hline Sm-147 & $3 \times 10^{-4}$ & $1.8 \times 10^{-4}$ & $\mathrm{Pu}-244+\mathrm{D}$ & $1 \times 10^{-3}$ & $4.0 \times 10^{-3}$ \\
\hline Sm-151 & $3 \times 10^{-4}$ & $3.4 \times 10^{-7}$ & & $1 \times 10^{-5}$ & $3.8 \times 10^{-5}$ \\
\hline Eu-152 & $1 \times 10^{-3}$ & $6.0 \times 10^{-6}$ & Am-241 & $1 \times 10^{-3}$ & $4.5 \times 10^{-3}$ \\
\hline Eu-154 & $1 \times 10^{-3}$ & $9.1 \times 10^{-6}$ & $\mathrm{Am}-243+\mathrm{D}$ & $1 \times 10^{-3}$ & $4.5 \times 10^{-3}$ \\
\hline Eu-155 & $1 \times 10^{-3}$ & $1.3 \times 10^{-6}$ & $\mathrm{Cm}-243$ & $1 \times 10^{-3}$ & $2.9 \times 10^{-3}$ \\
\hline Gd-152 & $3 \times 10^{-4}$ & $1.5 \times 10^{-4}$ & $\mathrm{Cm}-244$ & $1 \times 10^{-3}$ & $2.3 \times 10^{-3}$ \\
\hline Gd-153 & $3 \times 10^{-4}$ & $1.1 \times 10^{-6}$ & $\mathrm{Cm}-248$ & $1 \times 10^{-3}$ & $1.6 \times 10^{-2}$ \\
\hline $\mathrm{Au}-195$ & $1 \times 10^{-1}$ & $1.1 \times 10^{-6}$ & Cf-252 & $1 \times 10^{-3}$ & $9.4 \times 10^{-4}$ \\
\hline Tl-204 & 1.0 & $3.2 \times 10^{-6}$ & & & \\
\hline
\end{tabular}

a Dose conversion factors for entries labeled by " $+\mathrm{D}^{\prime \prime}$ are aggregated dose conversion factors for intake of a principal radionuclide together with radionuclides of the associated decay chain in secular equilibrium (see Section 3.1).

b Fraction of a stable element entering the GI tract that reaches body fluids. 


\section{D.2 ENVIRONMENTAL TRANSPORT FACTORS}

\section{D.2.1 Plant, Meat, and Milk Pathways}

\section{D.2.1.1 Environmental Transport Factor Components}

Environmental transport factors for the plant, meat, and milk pathways can be factored into the product

$$
E T F_{i j, p q}(t)=F A_{p} \times F C D_{p q}(t) \times \sum_{k} D F_{p k} \times F S R_{i j, p q k}(t),
$$

where

$$
\begin{aligned}
E T F_{i j, p q}(t)= & \text { environmental transport factor for the } j \text { th principal radio- } \\
& \text { nuclide and } p q \text { th environmental pathway at time } t(\mathrm{~g} / \mathrm{yr}) ; \\
i j= & \text { subscript index of a variable whose quantity is assigned to } \\
& \text { radionuclide } j \text { as a result of the radioactive decay of } \\
& \text { radionuclide } i, \text { which exists in the contaminated zone from } \\
& \text { the beginning }(t=0){ }^{1} \\
p= & \text { primary pathway index for the plant }(p=3), \text { meat }(p=4), \\
& \text { and milk }(p=5) \text { pathways; } \\
q= & \text { secondary index for root uptake ( } q=1), \text { foliar deposition } \\
& (q=2), \text { ditch irrigation ( } q=3), \text { overhead irrigation }(q=4) \\
& \text { livestock water ( } q=5), \text { and livestock intake of soil }(q=6) ; \\
F A_{p}= & \text { area factor for } p \text { th primary pathway (dimensionless); } \\
F C D_{p q}(t)= & \text { cover and depth factor for the } p q \text { th ingestion pathway at } \\
& \text { time } t \text { (dimensionless); } \\
k= & \text { food class index; }
\end{aligned}
$$

1 In the remainder of this appendix, a variable with an $i j$ subscript index refers directly to radionuclide $j$. However, the quantity of the variable is actually associated with the ingrowth and decay relationship of radionuclide $j$ and $i$. 


$$
\begin{aligned}
D F_{p k}= & \text { dietary factor }=\text { annual consumption of the } k \text { th food class for } \\
& \text { the } p \text { th food pathway (g/yr); and } \\
F S R_{i j, p q k}(t)= & \text { food/soil concentration ratio at time } t \text { for the } j \text { th principal } \\
& \text { radionuclide, } p q \text { th ingestion pathway, and } k \text { th food class at } \\
& \text { time } t \text { (dimensionless), when } q=1,2 \text {, and } 6 . \text { When } q=3,4, \\
& \text { and } 5, \text { it is the ratio of the food concentration of radionuclide } \\
& j \text { at time } t \text { to the soil concentration of radionuclide } i \text { at time } \\
& \text { o, for the } p q \text { th ingestion pathway and the } k \text { th food class } \\
& \text { (dimensionless). }
\end{aligned}
$$

The sum is over the applicable food classes. The plant pathway consists of two food classes: (1) fruit, nonleafy vegetables, and grain $(k=1)$ and (2) leafy vegetables $(k=2)$. For the meat and milk pathways, only a single food class is used in the current version of RESRAD. Dietary factors are commonly given in $\mathrm{kg} / \mathrm{yr}$; they must be converted to $\mathrm{g} / \mathrm{yr}$ before substitution into Equation D.1 in order for $F S R=C / S$ to be a dimensionless quantity when the soil concentration $S$ is specified in $\mathrm{pCi} / \mathrm{g}$.

For water-dependent pathways (pathways with $q=3,4$, or 5 , which include a groundwater or surface water pathway segment), the food/soil concentration ratios can be factored into the products:

$$
F S R_{i j, p q k}(t)=F W R_{j p q k} \times W S R_{i j, r}(t),
$$

where

$$
\begin{aligned}
F W R_{j p q k}= & \text { food/water concentration ratio for the } j \text { th principal radio- } \\
& \text { nuclide, } p q \text { th water-dependent pathway, and } k \text { th food class } \\
& (\mathrm{L} / \mathrm{g}) ; \text { and } \\
W S R_{i j, r}(t)= & \text { ratio of the water concentration of radionuclide } j \text { at time } t \text { to } \\
& \text { the soil concentration of radionuclide } i \text { at time } 0,(\mathrm{~g} / \mathrm{L}) \text {, and } \\
& r=\text { water pathway segment index for groundwater }(r=1) \text { or } \\
& \text { surface water }(r=2) .
\end{aligned}
$$

By substituting Equation D.2 into Equation D.1, the following is obtained: 


$$
E T F_{i j, p q}(t)=W E F_{i j, p q}(t) \times W S R_{i j, r}(t)
$$

where

$$
W E F_{i j, p q}(t)=F A_{p} \times F C D_{p q}(t) \times \sum_{k} D F_{p k} \times F W R_{j p q k}
$$

is the water exposure factor for the pqth pathway in units of $\mathrm{L} / \mathrm{yr}$, and the quantities on the right are as defined for Equations D.1 and D.2. A water exposure factor is equal to the ratio of the annual intake of the $j$ th principal radionuclide in food contaminated through the $p q$ th water-dependent pathway to the concentration of the radionuclide in the contaminated water. Water/soil concentration ratios are defined and discussed in Appendixes $\mathrm{E}$ and $\mathrm{I}$.

\section{D.2.1.2 Area Factors}

The area factor (plant, $\mathrm{FA}_{3}$; meat, $\mathrm{FA}_{4}$; and milk, $\mathrm{FA}_{5}$ ) is used to account for the fraction of consumption that is obtained from the contaminated site. If the input value of the "contamination fraction" is - 1 , then the area factors will be calculated by using Equation D.5. Otherwise, the input value will be assigned to the area factor.

$$
\begin{aligned}
F A_{3} & =A / 2,000, & 0 \leq A & \leq 1,000 \mathrm{~m}^{2} \\
& =0.5, & A & >1,000 \mathrm{~m}^{2} \\
F A_{4}=F A_{5} & =A / 20,000, & 0 \leq A & \leq 20,000 \mathrm{~m}^{2} \\
& =1, & A & >20,000 \mathrm{~m}^{2}
\end{aligned},
$$

where $A=$ area of contaminated zone $\left(10,000 \mathrm{~m}^{2}\right) .^{2}$ An area of $10,000 \mathrm{~m}^{2}=1$ hectare (ha).

\section{D.2.1.3 Cover and Depth Factors}

The cover and depth factors $F C D_{p q}$ for $q=1$ through $q=4$ are applicable to the plant $(p=3)$, meat $(p=4)$, and milk $(p=5)$ pathways. The cover and depth factors $F C D_{p 5}$ and $F C D_{p 6}$ for the livestock water and soil pathways are applicable only to the meat and milk pathways.

2 If a number is included with the unit identification, the variable is an input variable and the number is the default value. 


\section{D.2.1.3.1 Root Uptake}

The cover and depth factor for root uptake $(q=1)$ is

$$
\begin{aligned}
F C D_{p 1}(t) & =0, & & d_{r}=0 \text { or } C_{d}(t) \geq d_{r} \\
& =1, & & C_{d}(t)=0, T(t) \geq d_{r} \\
& =T(t) / d_{r}, & & C_{d}(t)+T(t)<d_{r} \\
& =1-C_{d}(t) / d_{r}, & & C_{d}(t)<d_{r}, C_{d}(t)+T(t) \geq d_{r}
\end{aligned}
$$

where

$$
\begin{aligned}
C_{d}(t) & =\text { cover depth at time } t(\mathrm{~m}), \\
T(t) & =\text { thickness of contaminated zone at time } t(\mathrm{~m}), \text { and } \\
d_{r} & =\text { maximum root depth }(0.9 \mathrm{~m}) .
\end{aligned}
$$

The cover depth is given by Equation A.6. Equation D.6 is based on the assumption of a sharp boundary between the bottom of any uncontaminated cover and the top of the contaminated zone. The effect of mixing uncontaminated and contaminated soil in a surface layer by plowing or other disturbance of the soil close to the ground surface is not taken into account.

\section{D.2.1.3.2 Foliar Deposition and Livestock Soil Intake}

The cover and depth factors for foliar deposition, $F C D_{p 2}(t)$, and livestock soil intake, $F C D_{p 6}(t)$, for the ingestion pathways are the same as the cover and depth factor $F C D_{2}(t)$ for the inhalation pathway (see Equation B.5).

\section{D.2.1.3.3 Ditch Irrigation, Overhead Irrigation, and Livestock Water}

The contributions from ditch irrigation $(q=3)$, overhead irrigation $(q=4)$, and livestock water $(q=5)$ are independent of the depth of the contaminated zone in the models used because the infiltrating water will carry the contamination to the aquifer and contaminate the water regardless of the depth and the subsequent contamination mechanisms do not depend on depth. Hence, the cover and depth factors for these subpathways are 


$$
F C D_{p 3}(t)=F C D_{p 4}(t)=F C D_{p 5}(t)=1
$$

\section{D.2.1.4 Dietary Factors}

The dietary factor for human food consumption is a tabulated quantity. The default values used are given in Table D.2.

\section{D.2.1.5 Food/Soil Concentration Ratios for Plant Foods}

\section{D.2.1.5.1 Root Uptake}

The plant-food/soil concentration ratios for root uptake are given by

$$
F S R_{i j, 31 k}=F S R_{j 31 k}=B_{j v}
$$

TABLE D.2 Dietary Factors $\left(\mathrm{DF}_{\mathrm{pk}}\right)$ for Human Food Consumption

\begin{tabular}{clcll}
\hline $\begin{array}{c}\text { Primary } \\
\text { Pathway } \\
\text { Index, } \\
\mathrm{p}\end{array}$ & $\begin{array}{c}\text { Ingestion } \\
\text { Pathway }\end{array}$ & $\begin{array}{c}\text { Food } \\
\text { Class } \\
\text { Index, } \\
\mathrm{k}\end{array}$ & \multicolumn{1}{c}{ Class Description } & $\begin{array}{c}\text { Fietary } \\
\text { Factor, } \\
\text { DF }_{\mathrm{pk}}\end{array}$ \\
\hline 3 & Plant foods & 1 & Fruits, vegetables, and grain & $160 \mathrm{~kg} / \mathrm{yr}$ \\
3 & Plant foods & 2 & Leafy vegetables & $14 \mathrm{~kg} / \mathrm{yr}$ \\
4 & Meat & 1 & Meat and poultry & $63 \mathrm{~kg} / \mathrm{yr}$ \\
5 & Milk & 1 & Milk & $92 \mathrm{~L} / \mathrm{yr}$ \\
6 & $\begin{array}{l}\text { Aquatic } \\
\text { foods }\end{array}$ & 1 & Fish & $5.4 \mathrm{~kg} / \mathrm{yr}$ \\
6 & $\begin{array}{l}\text { Aquatic } \\
\text { foods }\end{array}$ & 2 & Crustacea and mollusks & $0.9 \mathrm{~kg} / \mathrm{yr}$ \\
7 & $\begin{array}{l}\text { Drinking } \\
\text { water }\end{array}$ & 1 & Drinking water & $510 \mathrm{~L} / \mathrm{yr}$ \\
\hline
\end{tabular}


where $B_{j v}$ represents the vegetable/soil transfer factors listed in Table D.3. It is assumed that the same root uptake transfer factors can be used for nonleafy vegetables $(k=1)$ and leafy vegetables $(k=2)$.

\section{D.2.1.5.2 Foliar Deposition}

The plant-food/soil concentration ratio for uptake from airborne contaminants that deposit on foliage is given by

$$
F S R_{i j, 32 k}=F S R_{j 32 k}=F A_{2} \times F A R_{j 32 k} \times A S R_{3}
$$

where

$$
\begin{aligned}
F S R_{j 32 k}= & \text { plant-food/soil concentration ratio for foliar deposition for the } \\
& j \text { th principal radionuclide and } k \text { th food class (dimensionless), } \\
F A_{2}= & \text { area factor for dilution of resuspended contaminated dust } \\
& \text { (dimensionless), } \\
F A R_{j 32 k}= & \text { plant-food/air concentration ratio for radionuclide transfer by } \\
& \text { airborne foliar deposition for the } j \text { th principal radionuclide and } \\
& \left.k \text { th food class ( } \mathrm{m}^{3} / \mathrm{g}\right), \text { and } \\
A S R_{3}= & \text { air/soil concentration ratio, } \mathrm{specified} \mathrm{as} \mathrm{the} \mathrm{average} \mathrm{mass} \\
& \text { loading of airborne contaminated } \mathrm{soil} \mathrm{particles} \mathrm{in} \mathrm{a} \mathrm{garden} \\
& \text { during the growing season }\left(1 \times 10^{-4} \mathrm{~g} / \mathrm{m}^{3}\right) .
\end{aligned}
$$

The area factor for dilution of resuspended contaminated dust by mixing with uncontaminated dust blown in from off-site is given by Equation B.4. The formula for the plant-food/air concentration ratio is

$$
F A R_{j 32 k}=3.16 \times 10^{4} \frac{\left(V_{d j} \times f_{r} \times T_{j v k}\right)\left[1-\exp \left(-\lambda_{w} t_{e k}\right)\right]}{Y_{v k} \times \lambda_{w}},
$$

where

$$
\begin{aligned}
V_{d j}= & \text { deposition velocity of contaminated dust }(0 \mathrm{~m} / \mathrm{s} \text { for hydrogen, } \\
& \text { carbon, argon, krypton, and xenon; } 1 \times 10^{-2} \mathrm{~m} / \mathrm{s} \text { for fluorine, }
\end{aligned}
$$


TABLE D.3 Vegetable/Soil Transfer Factors $\left(B_{j v}\right)$ for Root Uptake

\begin{tabular}{lllllllll}
\hline Element & \multicolumn{1}{c}{$\mathrm{B}_{\mathrm{jv}}$} & Element & \multicolumn{1}{c}{$\mathrm{B}_{\mathrm{jv}}$} & Element & $\mathrm{B}_{\mathrm{jv}}$ & Element & $\mathrm{B}_{\mathrm{jv}}$ \\
\hline $\mathrm{H}$ & 4.8 & $\mathrm{In}$ & $3.0 \times 10^{-3}$ & $\mathrm{Cu}$ & $1.3 \times 10^{-1}$ & $\mathrm{~W}$ & $1.8 \times 10^{-2}$ \\
$\mathrm{Be}$ & $4.0 \times 10^{-3}$ & $\mathrm{Sn}$ & $2.5 \times 10^{-3}$ & $\mathrm{Zn}$ & $4.0 \times 10^{-1}$ & $\mathrm{Ir}$ & $3.0 \times 10^{-2}$ \\
$\mathrm{C}$ & 5.5 & $\mathrm{Sb}$ & $1.0 \times 10^{-2}$ & $\mathrm{Ge}$ & $4.0 \times 10^{-1}$ & $\mathrm{Au}$ & $1.0 \times 10^{-1}$ \\
$\mathrm{~N}$ & 7.5 & $\mathrm{Te}$ & $6.0 \times 10^{-1}$ & $\mathrm{As}$ & $8.0 \times 10^{-2}$ & $\mathrm{Hg}$ & $3.8 \times 10^{-1}$ \\
$\mathrm{~F}$ & $2.0 \times 10^{-2}$ & $\mathrm{I}$ & $2.0 \times 10^{-2}$ & $\mathrm{Se}$ & $1.0 \times 10^{-1}$ & $\mathrm{~T}$ & $2.0 \times 10^{-1}$ \\
$\mathrm{Na}$ & $5.0 \times 10^{-2}$ & $\mathrm{Xe}$ & 0 & $\mathrm{Br}$ & $7.6 \times 10^{-1}$ & $\mathrm{~Pb}$ & $1.0 \times 10^{-2}$ \\
$\mathrm{Al}$ & $4.0 \times 10^{-3}$ & $\mathrm{Cs}$ & $4.0 \times 10^{-2}$ & $\mathrm{Kr}$ & 0 & $\mathrm{Bi}$ & $1.0 \times 10^{-1}$ \\
$\mathrm{P}$ & 1.0 & $\mathrm{Ba}$ & $5.0 \times 10^{-3}$ & $\mathrm{Rb}$ & $1.3 \times 10^{-1}$ & $\mathrm{Po}$ & $1.0 \times 10^{-3}$ \\
$\mathrm{~S}$ & $6.0 \times 10^{-1}$ & $\mathrm{La}$ & $2.5 \times 10^{-3}$ & $\mathrm{Sr}$ & $3.0 \times 10^{-1}$ & $\mathrm{Rn}$ & 0 \\
$\mathrm{Cl}$ & 20.0 & $\mathrm{Ce}$ & $2.0 \times 10^{-3}$ & $\mathrm{Y}$ & $2.5 \times 10^{-3}$ & $\mathrm{Ra}$ & $4.0 \times 10^{-2}$ \\
$\mathrm{~K}$ & $3.0 \times 10^{-1}$ & $\mathrm{Pr}$ & $2.5 \times 10^{-3}$ & $\mathrm{Zr}$ & $1.0 \times 10^{-3}$ & $\mathrm{Ac}$ & $2.5 \times 10^{-3}$ \\
$\mathrm{Ar}$ & 0 & $\mathrm{Nd}$ & $2.4 \times 10^{-3}$ & $\mathrm{Nb}$ & $1.0 \times 10^{-2}$ & $\mathrm{Th}$ & $1.0 \times 10^{-3}$ \\
$\mathrm{Ca}$ & $5.0 \times 10^{-1}$ & $\mathrm{Pm}$ & $2.5 \times 10^{-3}$ & $\mathrm{Mo}$ & $1.3 \times 10^{-1}$ & $\mathrm{~Pa}$ & $1.0 \times 10^{-2}$ \\
$\mathrm{Sc}$ & $2.0 \times 10^{-3}$ & $\mathrm{Sm}$ & $2.5 \times 10^{-3}$ & $\mathrm{Tc}$ & 5.0 & $\mathrm{U}$ & $2.5 \times 10^{-3}$ \\
$\mathrm{Cr}$ & $2.5 \times 10^{-4}$ & $\mathrm{Eu}$ & $2.5 \times 10^{-3}$ & $\mathrm{Ru}$ & $3.0 \times 10^{-2}$ & $\mathrm{~Np}$ & $2.0 \times 10^{-2}$ \\
$\mathrm{Mn}$ & $3.0 \times 10^{-1}$ & $\mathrm{Gd}$ & $2.5 \times 10^{-3}$ & $\mathrm{Rh}$ & $1.3 \times 10^{-1}$ & $\mathrm{Pu}$ & $1.0 \times 10^{-3}$ \\
$\mathrm{Fe}$ & $1.0 \times 10^{-3}$ & $\mathrm{~Tb}$ & $2.6 \times 10^{-3}$ & $\mathrm{Pd}$ & $1.0 \times 10^{-1}$ & $\mathrm{Am}$ & $1.0 \times 10^{-3}$ \\
$\mathrm{Co}$ & $8.0 \times 10^{-2}$ & $\mathrm{Ho}$ & $2.6 \times 10^{-3}$ & $\mathrm{Ag}$ & $1.5 \times 10^{-1}$ & $\mathrm{Cm}$ & $1.0 \times 10^{-3}$ \\
$\mathrm{Ni}$ & $5.0 \times 10^{-2}$ & $\mathrm{Ta}$ & $2.0 \times 10^{-2}$ & $\mathrm{Cd}$ & $3.0 \times 10^{-1}$ & $\mathrm{Cf}$ & $1.0 \times 10^{-3}$ \\
\hline
\end{tabular}

bromine, iodine, and chlorine; and $1 \times 10^{-3} \mathrm{~m} / \mathrm{s}$ for all remaining elements listed in Table D.3),

$f_{r}=$ fraction of deposited radionuclides retained on the vegetation $(0.25$, dimensionless),

$T_{j v k}=$ foliage-to-food radionuclide transfer coefficient for the $j$ th principal radionuclide and $k$ th food class $\left(T_{j v 1}=0.1, T_{j v 2}=1.0\right.$, and $T_{j v 3}=1.0$, dimensionless),

$\lambda_{w}=$ weathering removal constant for vegetation $\left(20 \mathrm{yr}^{-1}\right)$,

$t_{e k}=$ time of exposure of the $k$ th food class to contamination during the growing season $\left(t_{e 1}=0.17 \mathrm{yr}, t_{e 2}=0.25 \mathrm{yr}\right.$, and $\left.t_{e 3}=0.08 \mathrm{yr}\right)$, and

$Y_{v k}=$ wet-weight crop yield for the $k$ th food class $\left(Y_{v 1}=0.7 \mathrm{~kg} / \mathrm{m}^{2}\right.$, $Y_{v 2}=1.5 \mathrm{~kg} / \mathrm{m}^{2}$, and $Y_{v 3}=1.1 \mathrm{~kg} / \mathrm{m}^{2}$ ). 
The numerical factor $3.16 \times 10^{4}(\mathrm{~kg} / \mathrm{g})(\mathrm{s} / \mathrm{yr})$ is used to convert $F A R_{j 32 k}$ to units of

$\mathrm{m}^{3} / \mathrm{g}$. The foliage-to-food transfer coefficient $T_{j v k}$ is assumed to be radionuclide independent in the current version of the code. The weathering removal constant corresponds to a contaminant removal half-time of two weeks. The values for $k=3$ are for fodder; they are not included in the sum over $k$ in Equation D.1.

\section{D.2.1.5.3 Ditch Irrigation}

The plant-food/soil concentration ratio for ditch irrigation is given by

$$
F S R_{i j, 33 k}(t)=F W R_{j 33 k} \times\left[W S R_{i j, 1}(t) \times F I 1+W S R_{i j, 2}(t) \times(1-F I 1)\right],
$$

where

$$
\begin{aligned}
F S R_{i j, 33 k}(t)= & \text { ratio of the plant concentration of radionuclide } j \text { at time } t \\
& \text { to the soil concentration of radionuclide } i \text { at time } 0 \text { for the } \\
& k \text { th food class (dimensionless), } \\
F W R_{j 33 k}= & \text { plant-food/water concentration ratio for ditch irrigation for } \\
& \text { the } j \text { th principal radionuclide and the } k \text { th food class }(\mathrm{L} / \mathrm{g}), \\
W S R_{i j, 1}(t)= & \text { ratio of well-water concentration of radionuclide } j \text { at time } t \\
& \text { to the soil concentration of radionuclide } i \text { at time } 0 \text { (g/L), } \\
F I 1= & \text { fraction of well water used for irrigation (balance from } \\
& \text { surface water; 1.0, dimensionless), and } \\
W S R_{i j, 2}(t)= & \text { ratio of surface-water concentration of radionuclide } j \text { at } \\
& \text { time } t \text { to the soil concentration of radionuclide } i \text { at time } 0 \\
& (\mathrm{~g} / \mathrm{L}) .
\end{aligned}
$$

The formula for the plant-food/water concentration ratio, $F W R_{j 33 k}$, for ditch irrigation is

$$
F W R_{j 33 k}=\frac{I_{r r} B_{j v}\left[1-\exp \left(-L_{j} t_{e k}\right)\right]}{\rho_{e} \times L_{j}}
$$

where

$$
I_{r r}=\text { irrigation rate }(0.2 \mathrm{~m} / \mathrm{yr}) \text {, }
$$




$$
\begin{aligned}
& L_{j}=\text { leach rate constant for radionuclide } j\left(\mathrm{yr}^{-1}\right) \text {, and } \\
& \rho_{e}=\text { effective surface depsity of soil }\left(240 \mathrm{~kg} / \mathrm{m}^{2}\right) .
\end{aligned}
$$

The effective surface density is expressed in dry weight, and the default value is based on a plow layer of $15 \mathrm{~cm}$. The plant-food/water concentration ratio $F W R_{j 33 k}$ is in dimensions of $\mathrm{L} / \mathrm{g}\left(\mathrm{m}^{3} / \mathrm{kg}\right), B_{j v}$ is as defined for Equation D.8, and $t_{e k}$ and $Y_{v k}$ are as defined for Equation D.10. The leach rate constant $L_{j}$ is discussed in Appendix E. The default value used in the RESRAD code for fraction of well water used for irrigation is 1.0, that is, $100 \%$ of irrigation water is well water. A value of 0.5 means $50 \%$ of irrigation water is well water and $50 \%$ is surface (pond) water. The water/soil concentration ratios are discussed in Appendixes E and I.

\section{D.2.1.5.4 Overhead Irrigation}

The factoring of the plant-food/soil concentration ratio for overhead irrigation, $F S R_{i j, 34 k}$, is the same as the factoring for ditch irrigation in Equation D.11. That is,

$$
F S R_{i j, 34 k}(t)=F W R_{j 34 k} \times\left[W S R_{i j, 1}(t) \times F I 1+W S R_{i j, 2}(t) \times(1-F I 1)\right] .
$$

The only difference is that, for overhead irrigation, the plant-food/water concentration ratio is given by

$$
F W R_{j 34 k}=\frac{\left(I_{r r} \times f_{r} \times T_{j v k}\right)\left[1-\exp \left(-\lambda_{w} t_{e k}\right)\right]}{Y_{v k} \times \lambda_{w}}+\left[F W R_{j 33 k} \times\left(1-f_{r}\right)\right],
$$

where $F W R_{j 34 k}$ is in units of $\mathrm{L} / \mathrm{g}\left(\mathrm{m}^{3} / \mathrm{kg}\right), I_{r r}$ is as defined for Equation D.12, and the remaining quantities are as defined for Equation D.10.

\section{D.2.1.6 Food/Soil Concentration Ratios for Meat and Milk}

The food/soil concentration ratios $F S R_{i j p q}(t)$ for meat and milk can be factored into the product

$$
F S R_{i j, p q}(t)=F Q R_{j p} \times F I_{p q} \times Q S R_{i j, p q}(t)
$$


where

$$
\begin{aligned}
F Q R_{j p}= & \text { radionuclide transfer factor for meat }(p=4) \text { or milk }(p=5) \\
& =\text { ratio of the concentration of the jth principal radionuclide } \\
& \text { in meat or milk in pCi/kg to the rate of intake in fodder, soil, } \\
& \text { or water by livestock of the } j \text { th principal radionuclide in } \\
& \text { pCi/d ( } / \mathrm{kg}) ; \\
F I_{p q}= & \text { daily intake of fodder ( } q=1,2,3 \text {, and } 4) \text {, water }(q=5) \text { or } \\
& \text { soil ( } q=6) \text { by livestock (kg/d }- \text { see below for default values); } \\
& \text { and } \\
Q S R_{i j, p q}(t)= & \text { fodder/soil concentration ratio at time } t \text { for meat ( } p=4) \text { or } \\
& \text { milk }(p=5) \text { for the } j \text { th principal radionuclide and } q \text { th } \\
& \text { subpathway (dimensionless), when } q=1 \text { and } 2 . \text { When } q=3, \\
& 4, \text { and } 5, \text { it is the ratio of the fodder or livestock-water } \\
& \text { concentration of radionuclide } j \text { to the initial soil } \\
& \text { concentration of radionuclide } i \text { (dimensionless). For the soil } \\
& \text { intake by livestock ( } q=6), Q S R_{i j, p 6}=1 \text {. }
\end{aligned}
$$

The radionuclide transfer factors $F Q R_{j p}$ are listed in Table D.4. The livestock fodder intake rates are $F I_{4 q}=68 \mathrm{~kg} / \mathrm{d}$ and $F I_{5 q}=55 \mathrm{~kg} / \mathrm{d}$ for $q=1,2,3$, and 4 for meat and milk, respectively, and the livestock water intake rates are $F I_{45}=50 \mathrm{~L} / \mathrm{d}$ and $F I_{55}=160 \mathrm{~L} / \mathrm{d}$ for $q=5$. ( $1 \mathrm{~L}$ of water weighs $1 \mathrm{~kg}$; hence, units of $\mathrm{kg} / \mathrm{d}$ may be used for all intakes.) The livestock soil intake rates are the same for both meat and milk pathways: $F I_{46}=F I_{56}=0.5 \mathrm{~kg} / \mathrm{d}$.

The expressions for the fodder/soil concentration ratios are the same as the expressions for the food/soil concentration ratios given previously; the only difference is in a few parameter values. The parameter $Q S R_{i j, p q}$ is time independent for the waterindependent pathways $(q=1,2)$ and depends on time only through the water/soil concentration ratio $W S R_{i j, 1}(t)$ or $W S R_{i j, 2}(t)$ for the water-dependent pathways $(q=3,4$, and 5). The formulas for the fodder/soil concentration ratios are as follows:

$$
Q S R_{i j, 41}=Q S R_{i j, 51}=Q S R_{j 41}=Q S R_{j 51}=B_{j v}
$$


TABLE D.4 Meat $\left(\mathrm{FQR}_{\mathrm{i} 4}\right)$ and Milk $\left(\mathrm{FQR}_{\mathrm{i} 5}\right)$ Transfer Factors for Root Uptake

\begin{tabular}{|c|c|c|c|c|c|}
\hline Element & $\begin{array}{c}\text { Beef } \\
\left(\mathrm{FQR}_{\mathrm{i4}}\right) \\
(\mathrm{d} / \mathbf{k g}) \\
\end{array}$ & $\begin{array}{c}\text { Milk } \\
\left(\mathrm{FQR}_{\mathrm{i} 5}\right) \\
(\mathrm{d} / \mathrm{L})\end{array}$ & Element & $\begin{array}{c}\text { Beef } \\
\left(\mathrm{FQR}_{\mathrm{i} 4}\right) \\
(\mathrm{d} / \mathrm{kg}) \\
\end{array}$ & $\begin{array}{c}\text { Milk } \\
\left(F_{Q} R_{i 5}\right) \\
(\mathrm{d} / \mathrm{L})\end{array}$ \\
\hline $\mathrm{H}$ & $1.2 \times 10^{-2}$ & $1.0 \times 10^{-2}$ & In & $4.0 \times 10^{-3}$ & $2.0 \times 10^{-4}$ \\
\hline $\mathrm{Be}$ & $1.0 \times 10^{-3}$ & $2.0 \times 10^{-6}$ & $\mathrm{Sn}$ & $1.0 \times 10^{-2}$ & $1.0 \times 10^{-3}$ \\
\hline $\mathrm{C}$ & $3.1 \times 10^{-2}$ & $1.2 \times 10^{-2}$ & $\mathrm{Sb}$ & $1.0 \times 10^{-3}$ & $1.0 \times 10^{-4}$ \\
\hline $\mathbf{N}$ & $1.0 \times 10^{-2}$ & $1.0 \times 10^{-2}$ & $\mathrm{Te}$ & $7.0 \times 10^{-3}$ & $5.0 \times 10^{-4}$ \\
\hline F & $2.0 \times 10^{-2}$ & $7.0 \times 10^{-3}$ & I & $7.0 \times 10^{-3}$ & $1.0 \times 10^{-2}$ \\
\hline $\mathrm{Na}$ & $8.0 \times 10^{-2}$ & $4.0 \times 10^{-2}$ & $\mathrm{Xe}$ & 0 & 0 \\
\hline $\mathrm{Al}$ & $5.0 \times 10^{-4}$ & $2.0 \times 10^{-4}$ & Cs & $3.0 \times 10^{-2}$ & $8.0 \times 10^{-3}$ \\
\hline$P$ & $5.0 \times 10^{-2}$ & $1.6 \times 10^{-2}$ & $\mathrm{Ba}$ & $2.0 \times 10^{-4}$ & $5.0 \times 10^{-4}$ \\
\hline S & $2.0 \times 10^{-1}$ & $2.0 \times 10^{-2}$ & $\mathrm{La}$ & $2.0 \times 10^{-3}$ & $2.0 \times 10^{-5}$ \\
\hline $\mathrm{Cl}$ & $6.0 \times 10^{-2}$ & $2.0 \times 10^{-2}$ & $\mathrm{Ce}$ & $2.0 \times 10^{-5}$ & $3.0 \times 10^{-5}$ \\
\hline $\mathrm{Ar}$ & 0 & 0 & Pr & $2.0 \times 10^{-3}$ & $2.0 \times 10^{-5}$ \\
\hline $\mathbf{K}$ & $2.0 \times 10^{-2}$ & $7.0 \times 10^{-3}$ & $\mathrm{Nd}$ & $2.0 \times 10^{-3}$ & $2.0 \times 10^{-5}$ \\
\hline $\mathrm{Ca}$ & $1.6 \times 10^{-3}$ & $3.0 \times 10^{-3}$ & $\mathrm{Pm}$ & $2.0 \times 10^{-3}$ & $2.0 \times 10^{-5}$ \\
\hline $\mathrm{Sc}$ & $1.5 \times 10^{-2}$ & $5.0 \times 10^{-6}$ & $\mathrm{Sm}$ & $2.0 \times 10^{-3}$ & $2.0 \times 10^{-5}$ \\
\hline $\mathrm{Cr}$ & $9.0 \times 10^{-3}$ & $2.0 \times 10^{-3}$ & $\mathrm{Eu}$ & $2.0 \times 10^{-3}$ & $2.0 \times 10^{-5}$ \\
\hline $\mathrm{Mn}$ & $5.0 \times 10^{-4}$ & $3.0 \times 10^{-4}$ & $\overline{G d}$ & $2.0 \times 10^{-3}$ & $2.0 \times 10^{-5}$ \\
\hline $\mathrm{Fe}$ & $2.0 \times 10^{-2}$ & $3.0 \times 10^{-4}$ & $\mathrm{~Tb}$ & $2.0 \times 10^{-3}$ & $2.0 \times 10^{-5}$ \\
\hline Co & $2.0 \times 10^{-2}$ & $2.0 \times 10^{-3}$ & Ho & $2.0 \times 10^{-3}$ & $2.0 \times 10^{-5}$ \\
\hline $\mathrm{Ni}$ & $5.0 \times 10^{-3}$ & $2.0 \times 10^{-2}$ & $\mathrm{Ta}$ & $5.0 \times 10^{-6}$ & $5.0 \times 10^{-6}$ \\
\hline $\mathrm{Cu}$ & $1.0 \times 10^{-2}$ & $2.0 \times 10^{-3}$ & W & $4.0 \times 10^{-2}$ & $3.0 \times 10^{-4}$ \\
\hline $\mathrm{Zn}$ & $1.0 \times 10^{-1}$ & $1.0 \times 10^{-2}$ & Ir & $2.0 \times 10^{-3}$ & $2.0 \times 10^{-6}$ \\
\hline $\mathrm{Ge}$ & $2.0 \times 10^{-1}$ & $1.0 \times 10^{-2}$ & $\mathrm{Au}$ & $5.0 \times 10^{-3}$ & $1.0 \times 10^{-5}$ \\
\hline As & $1.5 \times 10^{-3}$ & $1.0 \times 10^{-4}$ & $\mathrm{Hg}$ & $1.0 \times 10^{-1}$ & $5.0 \times 10^{-4}$ \\
\hline $\mathrm{Se}$ & $1.0 \times 10^{-1}$ & $1.0 \times 10^{-2}$ & $\mathrm{TI}$ & $2.0 \times 10^{-3}$ & $3.0 \times 10^{-3}$ \\
\hline $\mathrm{Br}$ & $2.0 \times 10^{-2}$ & $2.0 \times 10^{-2}$ & $\mathrm{~Pb}$ & $8.0 \times 10^{-4}$ & $3.0 \times 10^{-4}$ \\
\hline $\mathrm{Kr}$ & 0 & 0 & $\mathrm{Bi}$ & $2.0 \times 10^{-3}$ & $5.0 \times 10^{-4}$ \\
\hline $\mathrm{Rb}$ & $1.5 \times 10^{-2}$ & $1.0 \times 10^{-2}$ & Po & $5.0 \times 10^{-3}$ & $3.4 \times 10^{-4}$ \\
\hline $\mathrm{Sr}$ & $8.0 \times 10^{-3}$ & $2.0 \times 10^{-3}$ & $\mathrm{Rn}$ & 0 & 0 \\
\hline $\mathrm{Y}$ & $2.0 \times 10^{-3}$ & $2.0 \times 10^{-5}$ & $\mathrm{Ra}$ & $1.0 \times 10^{-3}$ & $1.0 \times 10^{-3}$ \\
\hline $\mathbf{Z r}$ & $1.0 \times 10^{-6}$ & $6.0 \times 10^{-7}$ & Ac & $2.0 \times 10^{-5}$ & $2.0 \times 10^{-5}$ \\
\hline $\mathrm{Nb}$ & $3.0 \times 10^{-7}$ & $2.0 \times 10^{-6}$ & Th & $1.0 \times 10^{-4}$ & $5.0 \times 10^{-6}$ \\
\hline Mo & $1.0 \times 10^{-3}$ & $1.7 \times 10^{-3}$ & $\mathrm{~Pa}$ & $5.0 \times 10^{-3}$ & $5.0 \times 10^{-6}$ \\
\hline $\mathrm{Tc}$ & $1.0 \times 10^{-4}$ & $1.0 \times 10^{-3}$ & $\mathbf{U}$ & $3.4 \times 10^{-4}$ & $6.0 \times 10^{-4}$ \\
\hline $\mathrm{Ru}$ & $2.0 \times 10^{-3}$ & $3.3 \times 10^{-6}$ & $\mathrm{~Np}$ & $1.0 \times 10^{-3}$ & $5.0 \times 10^{-6}$ \\
\hline $\mathrm{Rh}$ & $1.0 \times 10^{-3}$ & $5.0 \times 10^{-3}$ & $\mathrm{Pu}$ & $1.0 \times 10^{-4}$ & $1.0 \times 10^{-6}$ \\
\hline $\mathrm{Pd}$ & $1.0 \times 10^{-3}$ & $5.0 \times 10^{-3}$ & $\mathrm{Am}$ & $5.0 \times 10^{-5}$ & $2.0 \times 10^{-6}$ \\
\hline $\mathrm{Ag}$ & $3.0 \times 10^{-3}$ & $2.5 \times 10^{-2}$ & $\mathrm{Cm}$ & $2.0 \times 10^{-5}$ & $2.0 \times 10^{-6}$ \\
\hline $\mathrm{Cd}$ & $4.0 \times 10^{-4}$ & $1.0 \times 10^{-3}$ & $\mathrm{Cf}$ & $6.0 \times 10^{-5}$ & $7.5 \times 10^{-7}$ \\
\hline
\end{tabular}


is the ratio for root uptake by fodder, where $B_{j v}$ is the vegetable/soil transfer factor for root uptake, tabulated in Table D.3.

$$
Q S R_{i j, 42}=Q S R_{i j, 52}=Q S R_{j 42}=Q S R_{j 52}=F A_{2} \times F A R_{j 323} \times A S R_{3}
$$

is the ratio for foliar deposition on fodder, where $F A_{2}$ is an area factor and $A S R_{3}$ is the air/soil concentration ratio, both defined for Equation D.9, and FAR $R_{j 323}$ is the plant-food/air concentration ratio defined by Equation D.10.

$$
\begin{aligned}
\operatorname{QSR}_{i j, 43}(t) & =\mathrm{QSR}_{i j, 53}(t) \\
& =F W R_{j 333} \times\left[W S R_{i j, 1}(t) \times F I 1+W S R_{i j, 2}(t) \times(1-F I 1)\right]
\end{aligned}
$$

is the ratio for ditch irrigation of fodder, where $F W R_{j 333}$ is the plant-food/water concentration ratio given by Equation D.12; WSR $R_{i j, 1}(t)$ and $W S R_{i j, 2}$ are the water/soil concentration ratios for well water and surface water, respectively, as derived in Appendix E; and FII is as defined for Equation D.11.

$$
\begin{aligned}
\operatorname{QSR}_{i j, 44}(t) & =Q S R_{i j, 54}(t) \\
& =F W R_{j 343} \times\left[W S R_{i j, 1}(t) \times F I 1+W S R_{i j, 2}(t) \times(1-F I 1)\right]
\end{aligned}
$$

is the ratio for overhead irrigation of fodder, where $F W R_{j 343}$ is the fodder/ water concentration ratio for overhead irrigation given by Equation D.14.

$$
\begin{aligned}
\mathrm{QSR}_{i j, 45}(t) & =Q S R_{i j, 55}(t) \\
& =W S R_{i j, 1}(t) \times F L 1+W S R_{i j, 2}(t) \times(1-F L 1) \times 10^{-3}
\end{aligned}
$$

is the ratio for intake of contaminated livestock water, where FL1 is the fraction of well water used for feeding livestock (balance from surface water; 1.0, dimensionless). A factor of $10^{-3}$ is used to convert $\mathrm{g}$ to $\mathrm{L}$.

\section{D.2.2 Aquatic Food Pathway}

The environmental transport factor for the aquatic food pathway $(p=6)$ can be factored into the product

$$
E T F_{i j, 6}(t)=F R_{6} \times\left(\sum_{k} D F_{6 k} \times F W R_{j 6 k}\right) \times W S R_{i j, 2}(t),
$$


where the summation is over the aquatic food classes (see Table D.2) and

$$
\begin{aligned}
E T F_{i j, 6}(t)= & \text { environmental transport factor for the aquatic food pathway } \\
& \text { (fish, crustacea, and mollusks) (g/yr), } \\
D F_{6 k}= & \text { dietary factors for annual consumption of fish }(k=1) \text { and } \\
& \text { crustacea and mollusks }(k=2)(\mathrm{kg} / \mathrm{yr}), \\
F R_{6}= & \text { fraction of aquatic food consumed that is contaminated }(0.5, \\
& \text { dimensionless), } \\
F W R_{j 6 k}= & \text { fish/water }(k=1) \text { and crustacea-mollusk/water }(k=2) \text { concen- } \\
& \text { tration ratios (bioaccumulation factors) (L/kg), and } \\
W S R_{i j, 2}(t)= & \text { ratio of surface water concentration of radionuclide } j \text { at time } t \\
& \text { to the soil concentration ratio of radionuclide } i \text { at time } 0(\mathrm{~g} / \mathrm{L}) .
\end{aligned}
$$

Dietary factors are given in Table D.2. Bioaccumulation factors are given in Table D.5. The water/soil concentration ratio for surface water is calculated by using the surface water model described in Appendixes $\mathrm{E}$ and $\mathrm{I}$.

The ETFs may also be written as products of a water exposure factor and a water/soil concentration ratio

$$
E T F_{i j, 6}(t)=W E F_{j 6} \times W S R_{i j, 2}(t)
$$

\section{D.2.3 Drinking Water Pathway}

The formula for the environmental transport factors for the drinking water pathway is

$$
E T F_{i j, 7}(t)=D F_{7} \times F D W \times\left[W S R_{i j, 1}(t) \times F D 1+W S R_{i j, 2}(t) \times(1-F D 1)\right]
$$

where

$$
\begin{aligned}
E T F_{i j, 7}(t)= & \text { environmental transport factors for the drinking water } \\
& \text { pathway }(\mathrm{g} / \mathrm{yr}) \\
D F_{7}= & \text { annual intake of drinking water }(510 \mathrm{~L} / \mathrm{yr})
\end{aligned}
$$


TABLE D.5 Aquatic Bioaccumulation Factors $\left(\right.$ FWR $_{\mathbf{i 6 k}}$ ) for Freshwater

\begin{tabular}{|c|c|c|c|c|c|}
\hline Element & $\begin{array}{c}\text { Fish }(\mathbf{k}=1) \\
(\mathrm{L} / \mathrm{kg})\end{array}$ & $\begin{array}{c}\text { Crustacea } \\
\text { and Mollusks } \\
(\mathrm{k}=2)(\mathrm{L} / \mathrm{kg})\end{array}$ & Element & $\begin{array}{c}\text { Fish }(\mathrm{k}=1) \\
(\mathrm{L} / \mathrm{kg})\end{array}$ & $\begin{array}{c}\text { Crustacea } \\
\text { and Mollusks } \\
(\mathrm{k}=2)(\mathrm{L} / \mathrm{kg})\end{array}$ \\
\hline $\mathrm{H}$ & 1.0 & 1.0 & In & $1.0 \times 10^{4}$ & $1.5 \times 10^{4}$ \\
\hline $\mathrm{Be}$ & $1.0 \times 10^{2}$ & $1.0 \times 10^{1}$ & Sn & $3.0 \times 10^{3}$ & $1.0 \times 10^{3}$ \\
\hline $\mathrm{C}$ & $5.0 \times 10^{4}$ & $9.1 \times 10^{3}$ & $\mathrm{Sb}$ & $1.0 \times 10^{2}$ & $1.0 \times 10^{1}$ \\
\hline $\mathbf{N}$ & $1.5 \times 10^{5}$ & 0 & $\mathrm{Te}$ & $4.0 \times 10^{2}$ & $7.5 \times 10^{1}$ \\
\hline $\mathrm{F}$ & $1.0 \times 10^{1}$ & $1.0 \times 10^{2}$ & I & $4.0 \times 10^{1}$ & 5.0 \\
\hline $\mathrm{Na}$ & $2.0 \times 10^{1}$ & $2.0 \times 10^{2}$ & $\mathrm{Xe}$ & 0 & 0 \\
\hline $\mathrm{Al}$ & $5.0 \times 10^{2}$ & $1.0 \times 10^{3}$ & $\mathrm{Cs}$ & $2.0 \times 10^{3}$ & $1.0 \times 10^{2}$ \\
\hline $\mathbf{P}$ & $5.0 \times 10^{4}$ & $2.0 \times 10^{4}$ & $\mathrm{Ba}$ & 4.0 & $2.0 \times 10^{2}$ \\
\hline S & $1.0 \times 10^{3}$ & $2.4 \times 10^{2}$ & $\mathrm{La}$ & $3.0 \times 10^{1}$ & $1.0 \times 10^{3}$ \\
\hline $\mathrm{Cl}$ & $1.0 \times 10^{3}$ & $1.9 \times 10^{2}$ & $\mathrm{Ce}$ & $3.0 \times 10^{1}$ & $1.0 \times 10^{3}$ \\
\hline $\mathrm{Ar}$ & 0 & 0 & $\mathrm{Pr}$ & $1.0 \times 10^{2}$ & $1.0 \times 10^{3}$ \\
\hline $\mathbf{K}$ & $1.0 \times 10^{3}$ & $2.0 \times 10^{3}$ & $\mathrm{Nd}$ & $1.0 \times 10^{2}$ & $1.0 \times 10^{3}$ \\
\hline $\mathrm{Ca}$ & $1.0 \times 10^{3}$ & $3.3 \times 10^{2}$ & $\mathrm{Pm}$ & $3.0 \times 10^{1}$ & $1.0 \times 10^{3}$ \\
\hline $\mathrm{Sc}$ & $1.0 \times 10^{2}$ & $1.0 \times 10^{3}$ & $\mathrm{Sm}$ & $2.5 \times 10^{1}$ & $1.0 \times 10^{3}$ \\
\hline $\mathrm{Cr}$ & $2.0 \times 10^{2}$ & $2.0 \times 10^{3}$ & $\mathrm{Eu}$ & $5.0 \times 10^{1}$ & $1.0 \times 10^{3}$ \\
\hline $\mathrm{Mn}$ & $4.0 \times 10^{2}$ & $9.0 \times 10^{4}$ & Gd & $2.5 \times 10^{1}$ & $1.0 \times 10^{3}$ \\
\hline $\mathrm{Fe}$ & $2.0 \times 10^{2}$ & $3.2 \times 10^{3}$ & $\mathrm{~Tb}$ & $2.5 \times 10^{1}$ & $1.0 \times 10^{3}$ \\
\hline Co & $3.0 \times 10^{2}$ & $2.0 \times 10^{2}$ & Ho & $2.5 \times 10^{1}$ & $1.0 \times 10^{3}$ \\
\hline $\mathrm{Ni}$ & $1.0 \times 10^{2}$ & $1.0 \times 10^{2}$ & $\mathrm{Ta}$ & $1.0 \times 10^{2}$ & $3.0 \times 10^{1}$ \\
\hline $\mathrm{Cu}$ & $2.0 \times 10^{2}$ & $4.0 \times 10^{2}$ & W & $1.2 \times 10^{3}$ & $1.0 \times 10^{1}$ \\
\hline $\mathrm{Zn}$ & $1.0 \times 10^{3}$ & $1.0 \times 10^{4}$ & Ir & $1.0 \times 10^{1}$ & $2.0 \times 10^{2}$ \\
\hline $\mathrm{Ge}$ & $4.0 \times 10^{3}$ & $2.0 \times 10^{4}$ & $\mathrm{Au}$ & $3.5 \times 10^{1}$ & $1.0 \times 10^{3}$ \\
\hline As & $3.0 \times 10^{2}$ & $3.0 \times 10^{2}$ & $\mathrm{Hg}$ & $1.0 \times 10^{3}$ & $2.0 \times 10^{4}$ \\
\hline $\mathrm{Se}$ & $2.0 \times 10^{2}$ & $2.0 \times 10^{2}$ & $\mathrm{TI}$ & $1.0 \times 10^{4}$ & $1.5 \times 10^{4}$ \\
\hline $\mathrm{Br}$ & $4.2 \times 10^{2}$ & $3.3 \times 10^{2}$ & $\mathrm{~Pb}$ & $3.0 \times 10^{2}$ & $1.0 \times 10^{2}$ \\
\hline $\mathbf{K r}$ & 0 & 0 & $\mathbf{B i}$ & $1.5 \times 10^{1}$ & $1.0 \times 10^{1}$ \\
\hline $\mathrm{Rb}$ & $2.0 \times 10^{3}$ & $1.0 \times 10^{3}$ & Po & $1.0 \times 10^{2}$ & $2.0 \times 10^{4}$ \\
\hline $\mathbf{S r}$ & $6.0 \times 10^{1}$ & $1.0 \times 10^{2}$ & $\mathrm{Rn}$ & 0 & 0 \\
\hline$Y$ & $3.0 \times 10^{1}$ & $1.0 \times 10^{3}$ & $\mathbf{R a}$ & $5.0 \times 10^{1}$ & $2.5 \times 10^{2}$ \\
\hline $\mathbf{Z r}$ & $3.0 \times 10^{2}$ & 6.7 & Ac & $1.5 \times 10^{1}$ & $1.0 \times 10^{3}$ \\
\hline $\mathrm{Nb}$ & $3.0 \times 10^{2}$ & $1.0 \times 10^{2}$ & $\mathrm{Th}$ & $1.0 \times 10^{2}$ & $5.0 \times 10^{2}$ \\
\hline Mo & $1.0 \times 10^{1}$ & $1.0 \times 10^{1}$ & $\mathrm{~Pa}$ & $1.0 \times 10^{1}$ & $1.1 \times 10^{2}$ \\
\hline $\mathrm{Tc}$ & $2.0 \times 10^{1}$ & 5.0 & $\mathrm{U}$ & $1.0 \times 10^{1}$ & $6.0 \times 10^{1}$ \\
\hline $\mathbf{R u}$ & $1.0 \times 10^{1}$ & $3.0 \times 10^{2}$ & $\mathrm{~Np}$ & $3.0 \times 10^{1}$ & $4.0 \times 10^{2}$ \\
\hline $\mathrm{Rh}$ & $1.0 \times 10^{1}$ & $3.0 \times 10^{2}$ & $\mathrm{Pu}$ & $3.0 \times 10^{1}$ & $1.0 \times 10^{2}$ \\
\hline $\mathrm{Pd}$ & $1.0 \times 10^{1}$ & $3.0 \times 10^{2}$ & Am & $3.0 \times 10^{1}$ & $1.0 \times 10^{3}$ \\
\hline $\mathrm{Ag}$ & 5.0 & $7.7 \times 10^{2}$ & $\mathrm{Cm}$ & $3.0 \times 10^{1}$ & $1.0 \times 10^{3}$ \\
\hline $\mathrm{Cd}$ & $2.0 \times 10^{2}$ & $2.0 \times 10^{3}$ & $\mathrm{Cf}$ & $2.5 \times 10^{1}$ & $1.0 \times 10^{3}$ \\
\hline
\end{tabular}




$$
\begin{aligned}
F D W= & \text { fraction of drinking water from site }(1.0, \text { dimensionless), } \\
W S R_{i j, 1}(t)= & \text { ratio of well water concentration of radionuclide } j \text { at time } t \text { to } \\
& \text { the initial soil concentration of radionuclide } i(\mathrm{~g} / \mathrm{L}), \\
W S R_{i j, 2}(t)= & \text { ratio of surface water concentration of radionuclide } j \text { at time } \\
& t \text { to the initial soil concentration of radionuclide } i(\mathrm{~g} / \mathrm{L}), \text { and } \\
F D 1= & \text { fraction of well water used for drinking (balanced from } \\
& \text { surface water; } 1.0, \text { dimensionless). }
\end{aligned}
$$

The annual intake of drinking water is a dietary factor from Table D.2. The water/soil concentration ratios are calculated by using the models described in Appendixes $\mathrm{E}$ and $\mathrm{I}$.

\section{D.3 REFERENCES}

Eckerman, K.F., et al., 1988, Limiting Values of Radionuclide Intake and Air Concentration and Dose Conversion Factors for Inhalation, Submersion, and Ingestion, EPA-520/1-88-020, Federal Guidance Report No. 11, prepared by Oak Ridge National Laboratory, Oak Ridge, Tenn., for U.S. Environmental Protection Agency, Office of Radiation Programs, Washington, D.C.

International Commission on Radiological Protection, 1979-1982, Limits for Intakes of Radionuclides by Workers, a report of Committee 2 of the International Commission on Radiological Protection, adopted by the Commission in July 1978, ICRP Publication 30, Part 1 (and Supplement), Part 2 (and Supplement), Part 3 (and Supplements A and B), and Index, Annals of the ICRP, Pergamon Press, New York, N.Y.

U.S. Department of Energy, 1988, Internal Dose Conversion Factors for Calculation of Dose to the Public, DOE/EH-0071, Assistant Secretary for Environment, Safety and Health, Washington, D.C. 
APPENDIX E:

WATER PATHWAY FACTORS 


\section{APPENDIX E:}

\section{WATER PATHWAY FACTORS}

Water pathway factors are components of the environmental transport factors for water-dependent ingestion pathways. A water-dependent ingestion pathway can be divided into two segments: (1) a water pathway segment that extends from the contaminated zone to a point where transport through the food chain begins (a well or surface water body) and (2) a food chain pathway segment that extends from the point of entry of a radionuclide from water into the food chain to a point of human exposure. Transport through the water pathway segment is characterized by a water/soil concentration ratio, which is defined as the ratio of the concentration of the principal radionuclide (either parent or progeny) in water used for drinking, irrigation, or livestock water to the initial parent radionuclide concentration in the contaminated zone. Transport through the food chain pathway segment is characterized by a water exposure factor, which is defined as the ratio of the quantity of a radionuclide ingested annually to the concentration in water used for drinking, irrigation, or livestock water. The environmental transport factor for the water-dependent ingestion pathway can be expressed as a product of a water exposure factor and a water/soil concentration ratio:

$$
E T F_{i j, p q r}(t)=W E F_{i j, p q r}(t) \times W S R_{i j, r}(t)
$$

where

$$
\begin{aligned}
E T F_{i j, p q r}(t)= & E_{i j, p q r}(t) / S_{i}(0)=\text { environmental transport factor at time } t \text { for } \\
& \text { the } j \text { th principal radionuclide transported through the pqrth } \\
& \text { ingestion pathway }(\mathrm{g} / \mathrm{yr})-E_{i j, p q r}(t) \text { is the rate of ingestion } \\
& (\mathrm{pCi} / \mathrm{yr}) \text { at time } t \text { of the } j \text { th principal radionuclide } \\
& \text { transported through the pqrth pathway from the contami- } \\
& \text { nated zone to a point of human exposure, and } S_{i}(0) \text { is the } \\
& \text { initial concentration (pCi/g) of the } i \text { th principal radionuclide } \\
& \text { in the contaminated zone; } \\
i j= & \text { subscript index of a variable whose quantity is assigned to } \\
& \text { the principal radionuclide } j \text { as the result of the decay of the }
\end{aligned}
$$


principal radionuclide $i$, which initially exists in the contaminated zone. ${ }^{1}$

$p, q, r=$ pathway indices - the indices $p$ and $q$ identify the food chain segment of the pathway (see Table 2.1 and Equation D.1), and the index $r$ identifies the water pathway segment, that is, the segment from the contaminated zone to well water $(r=1)$ or surface water $(r=2)$;

$$
\begin{aligned}
W E F_{i j, p q r}(t)= & E_{i j, p q r}(t) / W_{i j, r}(t)=\text { water exposure factor at time } t \text { for the } j \text { th } \\
& \text { principal radionuclide transported through the pqrth } \\
& \text { pathway from the point of water use to the point of exposure } \\
& (\mathrm{L} / \mathrm{yr})-E_{i j, p q r}(t) \text { is the rate of ingestion (pCi/yr) at time } t \text { of } \\
& \text { the } j \text { th principal radionuclide transported through the } p q r \text { th } \\
& \text { pathway, and } W_{i j, r}(t) \text { is the concentration in water }(\mathrm{pCi} / \mathrm{L}) \text { at } \\
& \text { time } t \text { of the } j \text { th principal radionuclide transported through } \\
& \text { the } r \text { th water pathway segment at the point of entry into the } \\
& p q \text { th food chain; and }
\end{aligned}
$$

$W S R_{i j, r}(t)=W_{i j, r}(t) / S_{i}(0)=$ water/soil concentration ratio at time $t$ for the $r$ th water pathway segment $(\mathrm{g} / \mathrm{L})-W_{i j, r}(t)$ is the concentration in water $(\mathrm{pCi} / \mathrm{L})$ at time $t$ of the $j$ th principal radionuclide transported through the $r$ th water pathway segment at the point of entry into a food chain pathway segment, and $S_{i}(0)$ is the average concentration ( $\mathrm{pCi} / \mathrm{g}$ ) at time 0 of the $i$ th principal radionuclide in the contaminated zone.

The water exposure factor $W E F_{i j, p q r}(t)$ is discussed in Appendix D. The water/soil concentration ratio $W S R_{i j, r}(t)$ is discussed in this appendix and Appendix $\mathrm{I}$.

A water soil concentration ratio, $W S R_{i j, r}$ is determined by the rate at which the parent radionuclide $i$ is leached from the contaminated zone, the amount of ingrowth of radionuclide $j$ from radionuclide $i$ along the water pathway from the boundary of the

1 In the remainder of this appendix, a variable with a subscript index of $i j$ refers directly to radionuclide $j$. However, the quantity of the variable is actually associated with the ingrowth and decay relationship between radionuclides $j$ and $i$. 
contaminated zone to the point of water use, the time for radionuclide $j$ and its precursors of the same decay chain to be transported along the water pathway, and the dilution that occurs along this pathway. The model for estimating radionuclide leaching and formulas for calculating the leach rate are given in Section E.1. Formulas that relate the radionuclide concentration in water at the point of use to parameters that characterize the leaching and transport processes are derived in Section E.2. Formulas of dilution factors and other transport parameters used to calculate $W S R_{i j, r}$ are given in Section E.3. The transfer functions of radioactive decay products transporting through the unsaturated zone and saturated zone are discussed in Appendix $\mathbf{I}$.

\section{E.1 RADIONUCLIDE LEACHING FROM THE CONTAMINATED ZONE}

Radionuclides adsorbed in soil are subject to leaching by infiltrating water. Radionuclides leaching from the contaminated zone are the sources of groundwater contamination. Therefore, the first step in calculating radionuclide concentrations in groundwater is to estimate the leaching of radionuclides from the contaminated zone.

A sorption-desorption, ion-exchange leaching model is used in the RESRAD code. This model is characterized by a nuclide-dependent, first-order leach rate constant, $L_{i}$, which is defined as the fraction of available radionuclide $i$ leached out per unit time. The radionuclide release rate (source strength, in pCi/yr), $\dot{R}_{i}(t)$, can be written as (Yu 1987)

$$
\dot{R}_{i}(t)=L_{i} \rho_{b}^{(c z)} A T(t) S_{i}(t)
$$

where

$$
\begin{aligned}
L_{i}= & \text { leach rate for radionuclide } i\left(\mathrm{yr}^{-1}\right), \\
\rho_{b}{ }^{(c z)}= & \text { bulk density of the contaminated zone }\left(\mathrm{kg} / \mathrm{m}^{3}\right), \\
A= & \text { area of the contaminated zone }\left(\mathrm{m}^{2}\right), \\
T(t)= & \text { thickness of the contaminated zone at time } t(\mathrm{~m}), \text { and } \\
S_{i}(t)= & \text { average concentration of the } i \text { th principal radionuclide in the } \\
& \text { contaminated zone available for leaching at time } t(\mathrm{pCi} / \mathrm{kg}) .
\end{aligned}
$$

The first-order leach rate constant used in the current version of RESRAD is a timeindependent radionuclide leach rate constant that is estimated on the basis of the soil 
residence time for the initial thickness of the contaminated zone. A time-independent radionuclide leach rate constant for radionuclide $i, L_{i}$, may be written as

$$
L_{i}=\frac{I}{\theta^{(c z)} T_{0} R_{d_{i}}^{(c z)}}
$$

where

$$
\begin{aligned}
I= & \text { infiltration rate }(\mathrm{m} / \mathrm{yr}) \\
\theta^{(c z)}= & \text { volumetric water content of the contaminated zone, and } \\
T_{0}= & \text { initial thickness of the contaminated zone }(\mathrm{m}), \text { and } \\
R_{d_{i}}^{(c z)}= & \text { retardation factor in the contaminated zone for radionuclide } i \\
& \text { (dimensionless). }
\end{aligned}
$$

The infiltration rate is given by

$$
I=\left(1-C_{e}\right)\left[\left(1-C_{r}\right) P_{r}+I_{r r}\right]
$$

where

$C_{e}=$ evapotranspiration coefficient $(0.5$, dimensionless),

$C_{r}=$ runoff coefficient $(0.2$, dimensionless),

$P_{r}=$ precipitation rate (annual rainfall, $1.0 \mathrm{~m} / \mathrm{yr}$ ), and

$I_{r r}=$ irrigation rate $(0.2 \mathrm{~m} / \mathrm{yr})$.

To calculate the infiltration rate, the average evapotranspiration coefficient is used rather than the average evapotranspiration rate, $E_{t}$ (see Geraghty et al. [1973] for U.S. average). Using the average evapotranspiration rate does not take into account the correlation between precipitation and evapotranspiration and, for arid regions, can give a spurious negative infiltration rate. The evapotranspiration coefficient is related to the evapotranspiration rate by the formula $C_{e}=E_{t} /\left[\left(1-C_{r}\right) P_{r}+I_{r r}\right]$. Runoff coefficients for a specific site may be obtained from Table E.1. It is assumed that irrigation water is controlled by ditching or by the duration of application so that none will be lost by runoff. The default irrigation rate is for humid regions where irrigation is unnecessary; an appropriate generic 
TABLE E.1 Runoff Coefficient Values

\begin{tabular}{lcc}
\hline \multicolumn{1}{c}{ Type of Area } & Coefficient & Value \\
\hline Agricultural environment & & \\
& & \\
Flat land with average slopes of 0.3 to $0.9 \mathrm{~m} / \mathrm{mi}$ & & \\
Rolling land with average slopes of 4.6 to $6.1 \mathrm{~m} / \mathrm{mi}$ & $\mathrm{c}_{1}$ & 0.3 \\
Hilly land with average slopes of 46 to $76 \mathrm{~m} / \mathrm{mi}$ & $\mathrm{c}_{1}$ & 0.1 \\
Open sandy loam & $\mathrm{c}_{1}$ & 0.4 \\
Intermediate combinations of clay and loam & $\mathrm{c}_{2}$ & 0.2 \\
Tight, impervious clay & $\mathrm{c}_{2}$ & 0.1 \\
& $\mathrm{c}_{2}$ & \\
Woodlands & & 0.2 \\
Cultivated lands & $\mathrm{c}_{3}$ & 0.1 \\
& $\mathrm{c}_{3}$ & \\
Urban environment & & \\
Flat, residential area - about $30 \%$ impervious & & 0.4 \\
Moderately steep, residential area - about 50\% impervious & $\mathrm{C}_{\mathrm{r}}$ & 0.65 \\
Moderately steep, built-up area - about 70\% impervious & $\mathrm{C}_{\mathrm{r}}$ & 0.8 \\
\hline
\end{tabular}

a The runoff coefficient for an agricultural environment is given by $C_{r}=1-c_{1}-c_{2}-c_{3}$. Source: Data from Gray (1970).

value for arid regions would be $I_{r r}=1 \mathrm{~m} / \mathrm{yr}$. Site-specific values for the precipitation and irrigation rates should be used whenever possible.

The volumetric water content of the contaminated zone, $\theta^{(c z)}$, is the product of the saturated water content of the contaminated zone, $\theta_{\text {sat }}^{(c z)}$, and the saturation ratio of the contaminated zone, $R_{s}^{(c z)}$. The saturated water content is the water content when the soil material is saturated. Hence, $\theta_{s a t}$ equals $p_{t}$, where $p_{t}$ is the total porosity of the soil material, that is, ${ }^{2}$

$$
\theta_{\text {sat }}=p_{t}
$$

The saturation ratio, $R_{s}$, is defined as the ratio of $\theta$ over $\theta_{\text {sat }}$, that is,

2. The superscript is omitted for a general definition. The definition applies for all zones. 


$$
R_{s}=\theta / \theta_{\text {sat }}=\theta / p_{t} .
$$

When the medium is saturated, $R_{s}$ equals unity. Under unsaturated infiltration conditions, the saturation ratio is a function of the infiltration rate, the saturated hydraulic conductivity, and the texture of the soil. The saturation ratio can be estimated by using the following equation (Clapp and Hornberger 1978):

$$
R_{s}=\left(\frac{I}{K_{\text {sat }}}\right) \frac{1}{2 b+3},
$$

where

$$
\begin{aligned}
I & =\text { infiltration rate }(\mathrm{m} / \mathrm{yr}), \\
K_{\text {sat }} & =\text { saturated hydraulic conductivity (m/yr), and } \\
b & =\text { soil-specific exponential parameter (dimensionless). }
\end{aligned}
$$

Representative values of $K_{\text {sat }}, \theta_{\text {sat }}$, and $b$ for various soil textures are listed in Table E.2.

TABLE E.2 Representative Values of Saturated Hydraulic Conductivity, Saturated Water Content, and the Soil-Specific Exponential Parameter

\begin{tabular}{lccc}
\hline \multicolumn{1}{c}{ Texture } & $\begin{array}{c}\text { Hydraulic } \\
\text { Conductivity, } \\
\mathrm{K}_{\text {sat }}(\mathrm{m} / \mathrm{yr})\end{array}$ & $\begin{array}{c}\text { Saturated } \\
\text { Water } \\
\text { Content, } \theta_{\text {sat }}\end{array}$ & $\begin{array}{c}\text { Soil-Specific } \\
\text { Exponential } \\
\text { Parameter, b }\end{array}$ \\
\hline Sand & $5.55 \times 10^{3}$ & 0.395 & 4.05 \\
Loamy sand & $4.93 \times 10^{3}$ & 0.410 & 4.38 \\
Sandy loam & $1.09 \times 10^{3}$ & 0.435 & 4.90 \\
Silty loam & $2.27 \times 10^{2}$ & 0.485 & 5.30 \\
Loam & $2.19 \times 10^{2}$ & 0.451 & 5.39 \\
Sandy clay loam & $1.99 \times 10^{2}$ & 0.420 & 7.12 \\
Silty clay loam & $5.36 \times 10^{1}$ & 0.477 & 7.75 \\
Clay loam & $7.73 \times 10^{1}$ & 0.476 & 8.52 \\
Sandy clay & $6.84 \times 10^{1}$ & 0.426 & 10.40 \\
Silty clay & $3.26 \times 10^{1}$ & 0.492 & 10.40 \\
Clay & $4.05 \times 10^{1}$ & 0.482 & 11.40 \\
\hline
\end{tabular}

Source: Data from Clapp and Hornberger (1978). 
The retardation factor for radionuclide $i, R_{d_{i}}$, is the ratio of the average pore water velocity to the radionuclide transport velocity. Assuming that the adsorption-desorption process can be represented with a linear Freundlich isotherm, the retardation factor can be calculated with the following formula (Yu 1987):

$$
R_{d_{i}}=1+\frac{\rho_{b} K_{d_{i}}}{\theta}=1+\frac{\rho_{b} K_{d_{i}}}{p_{t} R_{s}}
$$

where

$$
\begin{aligned}
\rho_{b} & =\text { bulk soil density }\left(\mathrm{g} / \mathrm{cm}^{3}\right), \\
K_{d_{i}} & =\text { distribution coefficient for the } i \text { th principal radionuclide }\left(\mathrm{cm}^{3} / \mathrm{g}\right), \text { and } \\
\theta & =\text { volumetric water content (dimensionless). }
\end{aligned}
$$

The distribution coefficient is the radionuclide equilibrium concentration ratio of the adsorbed radionuclide (in soil) to the desorbed radionuclide (in water). Representative distribution coefficients are given in Tables E.3 through E.6.

\section{E.2 WATER/SOIL CONCENTRATION RATIOS IN TERMS OF NUCLIDE WATER-TRANSPORT PARAMETERS}

A water/soil concentration ratio can be expressed in terms of functions that characterize the source terms and transport processes and that are applicable for both simple and complex hydrogeological strata. By introducing simplifying approximations for the functional form of the breakthrough curve that are generally applicable, even for complex hydrogeological structures, the transport and source functions can be specified by a small number of nuclide water-transport parameters. Various models can be used to derive the relationships between these parameters and measurable quantities. The analysis is applicable to either the groundwater or surface water pathway; hence, in the following derivation, the subscript $r$ that is used to identify different water pathways has been omitted in order to simplify the expressions.

According to the definitions presented in Equation E.1, the water/soil concentration ratio, $W S R_{i j}(t)$, can be expressed as

$$
W S R_{i j}(t)=\frac{W_{i j}(t)}{S_{i}(0)},
$$


TABLE E.3 Typical Average Distribution Coefficients for Various Elements in Sand, Soils, and Clays

\begin{tabular}{|c|c|c|c|c|c|c|c|}
\hline \multirow[b]{2}{*}{ Element } & \multicolumn{2}{|c|}{$\begin{array}{c}\text { Average } \\
\text { Distribution } \\
\text { Coefficient, } \\
\mathrm{K}_{\mathrm{d}}\left(\mathrm{cm}^{3} / \mathrm{g}\right) \\
\end{array}$} & \multirow[b]{2}{*}{$\begin{array}{l}\text { Geometric } \\
\text { Standard } \\
\text { Deviation }\end{array}$} & \multirow[b]{2}{*}{ Element } & \multicolumn{2}{|c|}{$\begin{array}{c}\text { Average } \\
\text { Distribution } \\
\text { Coefficient, } \\
\mathrm{K}_{\mathrm{d}}\left(\mathrm{cm}^{3} / \mathrm{g}\right) \\
\end{array}$} & \multirow[b]{2}{*}{$\begin{array}{l}\text { Geometric } \\
\text { Standard } \\
\text { Deviation }\end{array}$} \\
\hline & $\begin{array}{l}\text { Soils } \\
\text { and } \\
\text { Clays }\end{array}$ & Sand $^{b}$ & & & $\begin{array}{l}\text { Soils } \\
\text { and } \\
\text { Clays }\end{array}$ & Sand & \\
\hline As & 3 & 0.3 & 1.8 & $\mathrm{Hg}$ & 100 & 10 & - \\
\hline $\mathrm{Ba}$ & 50 & 5 & - & $\mathrm{Ni}$ & 1,000 & 100 & - \\
\hline $\mathrm{Cd}$ & 7 & 0.7 & 2.4 & $\mathrm{Pu}$ & 2,000 & 200 & 10 \\
\hline $\mathrm{Ce}$ & 1,000 & 100 & 3.7 & $\mathrm{Ra}^{\mathrm{d}}$ & 70 & 7 & 7 \\
\hline $\mathrm{Cs}^{\mathrm{c}}$ & 500 & 80 & 2.4 & $\mathrm{Se}$ & 3 & 0.3 & 1.9 \\
\hline $\mathrm{Cr}(+6)$ & 4 & 0.4 & 9.0 & $\mathrm{Ag}$ & 100 & 10 & 3.7 \\
\hline $\mathrm{Co}$ & 1,000 & 100 & - & $\mathrm{Sr}^{\mathrm{d}, \mathrm{e}}$ & 30 & 3 & 7.4 \\
\hline $\mathrm{Cu}$ & 20 & 2 & 3.0 & $\mathrm{Th}$ & 60,000 & 6,000 & 4.5 \\
\hline $\mathbf{F}$ & 0 & 0 & - & Tritium & 0 & 0 & - \\
\hline $\mathrm{Fe}$ & 1,000 & 100 & - & $\mathbf{U}$ & 50 & 5 & 3.6 \\
\hline $\mathrm{La}$ & 1,000 & 100 & - & $\mathrm{Va}$ & 1,000 & 100 & - \\
\hline $\mathrm{Pb}$ & 100 & 10 & 5.5 & $\mathrm{Zn}$ & 20 & 2 & 6.7 \\
\hline $\mathbf{L i}$ & 500 & 50 & - & $\mathrm{Zr}$ & 1,000 & 100 & - \\
\hline $\mathrm{Mn}$ & 200 & 20 & 15 & & & & \\
\hline
\end{tabular}

a Data for $\mathrm{As}, \mathrm{Cd}, \mathrm{Ce}, \mathrm{Cr}, \mathrm{Co}, \mathrm{Cu}, \mathrm{Pb}, \mathrm{Mn}, \mathrm{Pu}, \mathrm{Se}, \mathrm{Ag}, \mathrm{Sr}$, Th, U, and $\mathrm{Zn}$ from Baes and Sharp (1983); the values of $K_{d}$ are the geometric means of the literature data (see also Gilbert et al. 1983, pp. 3-57 to 3-60). Data for other elements from Nuclear Safety Associates (1980), except as noted. All values have been rounded to one significant figure.

b The values are taken to be $10 \%$ of the values of soils and clays (Nuclear Safety Associates 1980), except for Cs.

c From Isherwood (1981), using soil and quartz sand data, rounded to one significant digit.

d Sources: U.S. Nuclear Regulatory Commission (1980); Gee et al. (1980).

e The corresponding values from Isherwood (1981) for $\mathrm{Sr}$ are 73 for soils, 2.5 for quartz sand, and 24 for other sands. 
TABLE E.4 Distribution Coefficients for Strontium and Cesium ${ }^{\text {a }}$

\begin{tabular}{lll}
\hline & \multicolumn{2}{c}{$\mathrm{K}_{\mathrm{d}}\left(\mathrm{cm}^{3} / \mathrm{g}\right)$} \\
\cline { 2 - 3 } \multicolumn{1}{c}{ Conditions } & \multicolumn{1}{c}{ Strontium } & \multicolumn{1}{c}{ Cesium } \\
\hline & & \\
Basalt, 32-80 mesh & $16-140$ & $790-9,500$ \\
Basalt, 0.5-4 mm, 300 ppm TDS & $220-1,200$ & $39-280$ \\
Basalt, 0.5-4 mm, sea water & 1.1 & 6.5 \\
Basalt-fractured in-situ measurement & 3 & \\
Sand, quartz - pH 7.7 & $1.7-3.8$ & $22-310$ \\
Sands & $13-43$ & 100 \\
Carbonate, greater than 4 mm & 0.19 & 14 \\
Dolomite, 4,000 ppm TDS & $5-14$ & \\
Granite, greater than 4 mm & 1.7 & 34 \\
Granodiorite, 100-200 mesh & $4-9$ & $8-9$ \\
Granodiorite, 0.5-1 mm & $11-23$ & $1,000-1,800$ \\
Hanford sediments & 50 & 300 \\
Tuff & $45-4,000$ & $800-18,000$ \\
Soils & $19-280$ & $190-1,100$ \\
Shaley siltstone, greater than 4 mm & 8 & 310 \\
Sandstone, greater than 4 mm & 1.4 & 100 \\
Alluvium, 0.5-4 mm & $48-2,500$ & $120-3,200$ \\
Salt, greater than 4 mm, saturated brine & 0.19 & 0.027 \\
\hline
\end{tabular}

a All values have been rounded to two significant figures.

Source: Data from Isherwood (1981).

where $W S R_{i j}(t)$ is given in units of $(\mathrm{g} / \mathrm{L})$, and $W_{i j}(t)$ is the average concentration at time $t$ of the $j$ th principal radionuclide in water at the point of use in units of $\mathrm{pCi} / \mathrm{L}$ due to the original concentration at $t=0$ of the $i$ th principal radionuclide, $S_{i}(0)$, in units of $\mathrm{pCi} / \mathrm{g}$.

The concentration of the $j$ th principal radionuclide at the point of use at time $t$ as a result of the original contamination of the $i$ th principal radionuclide, $W_{i j}(t)$, is the sum of all contributions from the decay products of the original contamination, $S_{i}(0)$, which was leached from the contaminated zone at a time earlier than $t$. If $k$ is a radionuclide in the decay chain from $i$ to $j$ ( $i$ and $j$ inclusive), the concentration of radionuclide $k$ in the contaminated zone resulting from an original contamination of the $i$ th radionuclide $S_{i}(0)$ can be expressed as follows by using the source factor $S F_{i k}$ as defined in Section 3.2.3 and Appendix G:

$$
S_{k}(t)=S_{i}(0) S F_{i k}(t)
$$


TABLE E.5 Distribution Coefficients for Thorium and Uranium

Conditions

Thorium

Silt loam, Ca-saturated clay, $\mathrm{pH} 6.5$

Montmorillonite, Ca-saturated clay, $\mathrm{pH} 6.5$

Clay soil, $5 \mathrm{mM} \mathrm{Ca}\left(\mathrm{NO}_{3}\right)_{2}$, pH 6.5

Medium sand; $\mathrm{pH} 8.15$

Very fine sand, $\mathrm{pH} 8.15$

Silt/clay, $\mathrm{pH} 8.15$

Schist soil, 1 g/L Th, pH 3.2

Schist soil, $0.1 \mathrm{~g} / \mathrm{L}$ Th

Mlite, $1 \mathrm{~g} / \mathrm{L}$ Th, $\mathrm{pH} 3.2$

nlite, $0.1 \mathrm{~g} / \mathrm{L}$ Th, $\mathrm{pH} 3.2$

nlite, $0.1 \mathrm{~g} / \mathrm{L} \mathrm{Th}, \mathrm{pH}>6$

Uranium

Silt loam, U(VI), Ca-saturated, $\mathrm{pH} 6.5$

Clay soil, U(VI), $5 \mathrm{mM} \mathrm{Ca}\left(\mathrm{NO}_{3}\right)_{2}, \mathrm{pH} 6.5$

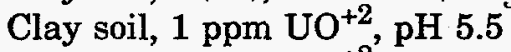

Clay soil, $1 \mathrm{ppm} \mathrm{\textrm {UO } ^ { + 2 }}, \mathrm{pH} 10$

Clay soil, 1 ppm UO ${ }^{+2}$, pH 12

Dolomite, 100-325 mesh, brine, $\mathrm{pH} 6.9$

Limestone, 100-170 mesh, brine, $\mathrm{pH} 6.9$
$\mathrm{K}_{\mathrm{d}}\left(\mathrm{cm}^{3} / \mathrm{g}\right)$
160,000

400,000

160,000

$40-130$

$310-470$

270-10,000

8

603.2

120

1,000

$<100,000$

Source: Data from Isherwood (1981).

The release rate (atoms/yr) of $k$ atoms from the contaminated zone into the unsaturated zone can be written by substituting the following in Equation E.2:

$$
\dot{R}_{i k}(t)=\frac{S F_{i k}(t) S_{i}(0)}{\lambda_{k}} L_{k} \rho_{b}^{(c z)} T(t) A \times \text { constant }
$$

where $\lambda_{k}$ is the decay constant of radionuclide $k$ and constant is a unit conversion factor. Use of the leach rate as defined in Equation E.3 yields

$$
\dot{R}_{i k}(t)=\frac{S F_{i k}(t) S_{i}(0)}{\lambda_{k}} \frac{I}{\theta^{c z} T_{0} R_{d_{k}}^{(c z)}} \rho_{b}^{(c z)} T(t) A \times \text { constant }
$$


TABLE E.6 Distribution Coefficients for Several Radionuclides from Various Sources

\begin{tabular}{|c|c|c|c|c|c|c|c|}
\hline Element & $\mathrm{pH}$ & $\begin{array}{c}\mathrm{K}_{\mathrm{d}} \\
\left(\mathrm{cm}^{3} / \mathrm{g}\right)\end{array}$ & Source & Element & $\mathrm{pH}$ & $\begin{array}{c}\mathrm{K}_{\mathrm{d}} \\
\left(\mathrm{cm}^{3} / \mathrm{g}\right)\end{array}$ & Source \\
\hline \multirow[t]{8}{*}{ Uranium } & 2 & 0 & Rancon (1973) & Lead & 2.2 & 1,850 & Gee et al. (1980) \\
\hline & 8 & 100 & & & 7.7 & 10,000 & \\
\hline & 10 & 600 & & & & & \\
\hline & 13 & 50 & & & $4-9$ & $99^{\mathrm{c}}$ & Baes and Sharp (1983) \\
\hline & 2.2 & 1.3 & Gee et al. (1980) & Strontium & 2 & 0.1 & Staley et al. (1979) \\
\hline & 7.7 & 23,000 & & & 3 & 6 & \\
\hline & & & & & 7 & 500 & \\
\hline & $4-9$ & $45^{\mathrm{a}}$ & Baes and Sharp (1983) & & 10 & 170 & \\
\hline \multirow[t]{10}{*}{ Thorium } & 2 & 500 & Rancon (1973) & & $4-9$ & $27^{\mathrm{d}}$ & Baes and Sharp (1983) \\
\hline & 5 & 3,000 & & & & & \\
\hline & 7 & 50,000 & & Cesium & 3 & 45 & Staley et al. (1979) \\
\hline & 13 & 50 & & & 5 & 150 & \\
\hline & & & & & 8 & 905 & \\
\hline & 2.2 & 1.2 & Gee et al. (1980) & & 10 & 650 & \\
\hline & 7.7 & 80,000 & & & & & \\
\hline & & & & & $4-9$ & $1,100^{\mathrm{e}}$ & Baes and Sharp (1983) \\
\hline & $4-9$ & $60,000^{\mathrm{b}}$ & Baes and Sharp (1983) & & & & \\
\hline & & & & Plutonium & 2 & 150 & Staley et al. (1979) \\
\hline \multirow[t]{7}{*}{ Radium } & 2 & $\mathbf{0}$ & U.S. Nuclear & & 5 & 250 & \\
\hline & 4 & 12 & Regulatory & & 7 & 8,500 & \\
\hline & 6 & 60 & Commission (1980) & & 11 & 1,000 & \\
\hline & 7 & 100 & & & & & \\
\hline & & & & & $4-9$ & $1,800^{f}$ & Baes and Sharp (1983) \\
\hline & 2.2 & 13 & Gee et al. (1980) & & & & \\
\hline & 7.7 & 2,400 & & Deuterium & All & 0 & \\
\hline
\end{tabular}

a Geometric mean (GM) of values ranging from 11 to 4,400, with a geometric standard deviation (GSD) of 3.7 .

b GM of values ranging from 2,000 to 510,000 , with a GSD of 4.5 .

c GM of values ranging from 4.5 to 7,600 , with a GSD of 5.5.

d GM of values ranging from 0.15 to 3,300 , with a GSD of 7.4 .

e GM of values ranging from 10 to 52,000 , with a GSD of 6.7 .

f GM of values ranging from 11 to 300,000 , with a GSD of 10 .

The transfer function, $G_{k j}(t)$ is defined as follows: if $N_{k}$ atoms of radionuclide $k$ are released at time $t_{0}$ from the contaminated zone into the unsaturated zone, $n_{j}(t) d t=N_{k} G_{k j}\left(t-t_{0}\right) d t$ atoms of radionuclide $j$ will arrive at the point of use between $t$ and $t+d t$. This function is developed in Appendix $\mathrm{I}$.

The release rate, $\dot{R}_{i k}(t)$ of $k$ atoms into the unsaturated zone will result in a release of $j$ atoms from the saturated zone to the point of use of 


$$
r_{k j}(t)=\dot{R}_{i k}(t) * G_{k j}(t)=\int_{0}^{t} \dot{R}_{i k}\left(t^{\prime}\right) G_{k j}\left(t-t^{\prime}\right) d t^{\prime},
$$

where the asterisk is used to denote the time convolution of the functions, and $r_{k j}(t)$ is the release amount of radionuclide $j$ at the point of use at time $t$ due to the release of radionuclide $k$ from the contaminated zone.

The concentration of $j$ at the point of use resulting from $k$ atoms being released from the contaminated zone can be estimated by dividing the activity by the water flow rate, $I A$, and multiplying by the dilution factor, $f$ :

$$
W_{k j}(t)=\frac{\lambda_{j} r_{k j}(t) f}{I A \times \text { constant }}
$$

The water/soil concentration, $W S R_{i j}$, can be derived by summing the concentrations of nuclide $j$ from all the decay products of nuclide $i$, that is, nuclide $k$ (which then will decay to nuclide $j$ ) and then dividing by the original contamination of $i, S_{i}(0)$ :

$$
\begin{gathered}
W S R_{i j}(t)=\frac{\sum_{k} \frac{\lambda_{j} r_{k j}(t) f}{I A \text { constant }}}{S_{i}(0)} \\
=\frac{\lambda_{j} \rho_{b}^{c z} f}{\theta^{c z} T_{0}} \sum_{k}\left(\frac{S F_{i k}(t) T(t)}{\lambda_{k} R_{d_{k}}{ }^{(c z)}}\right) * G_{k j}(t) .
\end{gathered}
$$

By defining the source-term leaching ratio, $Q_{j}(t)$, as

$$
Q_{j}(t)=\frac{T(t)}{T_{0} R_{d_{j}}^{(c z)}}
$$

$W S R_{i j}(t)$ can be explicitly written as

$$
W S R_{i j}(t)=\frac{\lambda_{j} \rho_{b}^{c z} f}{\theta^{c z}} \sum_{k} \int_{0}^{t} \frac{S F_{i k}\left(t^{\prime}\right) Q_{k}\left(t^{\prime}\right)}{\lambda_{k}} G_{k j}\left(t-t^{\prime}\right) d t^{\prime}
$$




\section{E.3 WATER TRANSPORT PARAMETERS}

\section{E.3.1 On-Site Groundwater Pathway $(r=1)$}

The water transport parameters for radionuclide $i$ are the breakthrough time $\Delta t_{i}$ (the time following the radiological survey at which radionuclides first appear in the water at the point of use), the rise time $\delta t_{i r}$ (the time following the breakthrough time for the radionuclide concentration in the water to attain a maximum value), and the dilution factor $f_{i r}$ (the ratio between the concentration in the water at the point of use to the concentration in the infiltrating water as it leaves the unsaturated zone). Two models are used for calculating these parameters: a mass-balance (MB) model and a nondispersion (ND) model. In the MB model, it is assumed that all of the radionuclides released from the contaminated zone are withdrawn through the well. In the ND model, it is assumed that the dispersivity is nil, the unsaturated zone consists of one or more horizontal homogeneous strata, the saturated zone is a single homogeneous stratum, and water withdrawal introduces only a minor perturbation in the water flow. These assumptions lead to a pattern of flow lines from which the dilution factor can be estimated by geometric considerations.

The user has the option of selecting which model to use. Usually, the MB model is used for smaller contaminated areas (e.g., $1,000 \mathrm{~m}^{2}$ or less) and the ND model is used for larger areas. The breakthrough times are the same for both models; the rise times and dilution factors are different.

\section{E.3.1.1 Breakthrough Time}

The well from which water is withdrawn for domestic use or irrigation is conservatively assumed to be located either in the center of the contaminated zone (in the MB model) or at the downgradient edge of the contaminated zone (in the ND model). For either location, radionuclides are assumed to enter the well as soon as they reach the water table; hence, the transport time through the aquifer is nil and the breakthrough time is equal to the transport time through the unsaturated zone, which is the sum of the times that a radionuclide is transported through those strata of the unsaturated zone that lie below the contaminated zone. 


$$
\Delta t_{i}=\sum_{m=1}^{n+1} \Delta t_{i m}^{(u z)}
$$

where

$$
n=\text { number of distinct horizontal strata in the unsaturated zone below }
$$
the contaminated zone at time 0 , and

$$
\begin{aligned}
\Delta t_{i m}^{(u z)}= & \text { transport time for the } i \text { th principal radionuclide through the } m \text { th } \\
& \text { stratum }(\mathrm{yr}) .
\end{aligned}
$$

The upper bound, $n+1$, of the summation in Equation E.19 is for the $(n+1)$ th unsaturated stratum created by the decrease in the water table. The thickness of this $(n+1)$ th stratum, $\Delta z_{n+1}$, is equal to the product of the water table drop rate, $v_{w t}$, and time. That is,

$$
\Delta z_{n+1}=v_{w t} \times t
$$

where $v_{w t}=$ water table drop rate $(0.001 \mathrm{~m} / \mathrm{yr})$.

The hydrogeological and hydrogeochemical properties of the $(n+1)$ th unsaturated stratum are assumed to be the same as those of the saturated stratum. The RESRAD code allows up to five horizontal strata below the contaminated zone, that is, $n \leq 5$. If $n=0$, the contaminated zone extends down to the aquifer.

The formula for the transport time is

$$
\Delta t_{i m}^{(u z)}=\frac{\Delta z_{m} R_{d_{i m}^{(u z)}}^{(u z)} R_{e_{m}}^{(u z)} s_{m}}{I}
$$

where

$$
\begin{aligned}
\Delta z_{m}= & \text { thickness of the } m \text { th stratum }\left(\Delta z_{1}=4 \mathrm{~m}, \Delta z_{2}, \ldots, \Delta z_{5}=0\right), \\
R_{d i m}^{(u z)}= & \text { retardation factor of the ith principal radionuclide in the } m \text { th } \\
& \text { stratum of the unsaturated zone, }
\end{aligned}
$$


$p_{e_{m}}^{(u z)}=$ effective porosity of the $m$ th stratum of the unsaturated zone $(0.2$, dimensionless), and

$R_{s_{m}}^{(u z)}=$ saturation ratio of the $m$ th stratum (dimensionless).

The unsaturated zone retardation factors, $R_{d_{i m}}^{(u z)}$, are calculated by the formula

$$
R_{d_{i m}}^{(u z)}=1+\frac{\rho_{b_{m}}^{(u z)} K_{d_{i m}}^{(u z)}}{\left(p_{t_{m}}^{(u z)} R_{s_{m}}^{(u z)}\right)}
$$

where

$$
\begin{aligned}
\rho_{b}^{(u z)}= & \text { bulk soil density in the } m \text { th stratum }\left(1.5 \mathrm{~g} / \mathrm{cm}^{3}\right) \\
K_{d_{i m}}^{(u z)}= & \text { distribution coefficient for the } i \text { th principal radionuclide in the } \\
& m \text { th stratum }\left(\mathrm{cm}^{3} / \mathrm{g}\right) \text {, and } \\
p_{t_{m}}^{(u z)}= & \text { total porosity of the } m \text { th stratum }(0.4, \text { dimensionless). }
\end{aligned}
$$

The saturation ratio, $R_{s_{m}}^{(u z)}$, can be determined by using Equation E.7.

Data that may be used to estimate breakthrough times when site-specific measurements are not available are given in Tables E.2 through E.7, as follows: saturated water contents (porosities), soil-specific exponential parameters, and saturated hydraulic conductivities are given in Table E.2; distribution coefficients are given in Tables E.3 through E.6; and total porosities and effective porosities are given in Table E.7.

Hydraulic conductivity is a critical hydrological parameter that can differ by several orders of magnitude from one site to another. Site-specific values should be used for deriving soil guidelines. Default values of the distribution coefficient values used in the RESRAD code are the values for soils and clays given in Table E.3. The default distribution coefficient for technetium is zero. For other elements not listed in Table E.3, the default value is the value of the nearest listed element in the same column of the periodic table. If there are no other elements in the same column, a zero value is assumed. 
TABLE E.7 Representative Porosity Values

\begin{tabular}{|c|c|c|c|c|}
\hline \multirow[b]{2}{*}{ Material } & \multicolumn{2}{|c|}{ Total Porosity, $p_{t}$} & \multicolumn{2}{|c|}{ Effective Porosity, $\mathrm{p}_{\mathrm{e}}$} \\
\hline & Range & $\begin{array}{l}\text { Arithmetic } \\
\text { Mean }\end{array}$ & Range & $\begin{array}{c}\text { Arithmetic } \\
\text { Mean }\end{array}$ \\
\hline \multicolumn{5}{|l|}{ Sedimentary material } \\
\hline Sandstone (fine) & - & - & $0.02-0.40$ & 0.21 \\
\hline Sandstone (medium) & $0.14-0.49$ & 0.34 & $0.12-0.41$ & 0.27 \\
\hline Siltstone & $0.21-0.41$ & 0.35 & $0.01-0.33$ & 0.12 \\
\hline Sand (fine) & $0.25-0.53$ & 0.43 & $0.01-0.46$ & 0.33 \\
\hline Sand (medium) & - & - & $0.16-0.46$ & 0.32 \\
\hline Sand (coarse) & $0.31-0.46$ & 0.39 & $0.18-0.43$ & 0.30 \\
\hline Gravel (fine) & $0.25-0.38$ & 0.34 & $0.13-0.40$ & 0.28 \\
\hline Gravel (medium) & - & - & $0.17-0.44$ & 0.24 \\
\hline Gravel (coarse) & $0.24-0.36$ & 0.28 & $0.13-0.25$ & 0.21 \\
\hline Silt & $0.34-0.51$ & 0.45 & $0.01-0.39$ & 0.20 \\
\hline Clay & $0.34-0.57$ & 0.42 & $0.01-0.18$ & 0.06 \\
\hline Limestone & $0.07-0.56$ & 0.30 & $\sim 0-0.36$ & 0.14 \\
\hline \multicolumn{5}{|l|}{ Wind-laid material } \\
\hline Loess & - & - & $0.14-0.22$ & 0.18 \\
\hline Eolian sand & - & - & $0.32-0.47$ & 0.38 \\
\hline Tuff & - & - & $0.02-0.47$ & 0.21 \\
\hline \multicolumn{5}{|l|}{ Igneous rock } \\
\hline Weathered granite & $0.34-0.57$ & 0.45 & - & - \\
\hline Weathered gabbro & $0.42-0.45$ & 0.43 & - & - \\
\hline Basalt & $0.03-0.35$ & 0.17 & - & - \\
\hline \multicolumn{5}{|l|}{ Metamorphic rock } \\
\hline Schist & $0.04-0.49$ & 0.38 & $0.22-0.33$ & 0.26 \\
\hline
\end{tabular}

Source: Data from McWorter and Sunada (1977).

Distribution coefficients depend strongly on soil type, the $\mathrm{pH}$ and Eh of the soil, and the presence of other ions (see Tables E.4 through E.6). Thus, considerable uncertainty can be introduced by using default values for the distribution coefficients. This uncertainty is a critical matter, particularly in cases in which the water-dependent pathways are the dominant contributors to the total dose/source concentration ratios. Default values for the distribution coefficients are provided only for the purpose of obtaining preliminary estimates; site-specific values should be used for deriving soil guidelines whenever possible. 
In addition to the option of using default values, four other options have been implemented in RESRAD to derive distribution coefficients when site-specific data are not available. The first option is to derive the distribution coefficients from the soil-to-plant transfer coefficients by using the correlation proposed by Sheppard and Sheppard (1989). The second option uses the groundwater concentration data, if available, to derive the distribution coefficients. The third option uses leach rate data, if available, to derive the distribution coefficients. The fourth option uses solubility limit data to derive an equivalent distribution coefficient. The first three options are discussed in detail in Appendix $H$; the fourth option is presented in Appendix $\mathrm{J}$.

The distance from the ground surface to the water table, $D_{w t}(t)$, at time $t$ is

$$
D_{w t}(t)=C_{d}(t)+T(t)+\sum_{m=1}^{n+1} \Delta z_{m}
$$

where

$$
\begin{aligned}
C_{d}(t) & =\text { cover depth at time } t(\mathrm{~m}), \\
T(t) & =\text { thickness of contaminated zone at time } t(\mathrm{~m}), \text { and } \\
\Delta z_{m} & \text { is as defined in Equations E.20 and E.21. }
\end{aligned}
$$

In the current version of RESRAD, the water table is assumed to be below the contaminated zone, that is, $D_{w t}(0) \geq C_{d}(0)+T(0)$.

\section{E.3.1.2 Rise Time}

When the well is located at the downgradient edge of the contaminated zone, as shown in Figure E.1 (i.e., if the ND model is used), the rise time is given by the formula

$$
\begin{aligned}
\delta t_{i 1} & =\tau_{\ell_{i}}, & & \zeta \leq d_{w}, D_{w t}(t) \geq C_{d}(t)+T(t) \\
& =\tau_{\ell_{i}} /\left(\zeta / d_{w}\right), & & \zeta>d_{w}, D_{w t}(t) \geq C_{d}(t)+T(t) \\
& =0, & & \zeta>d_{w}, D_{w t}(t)<C_{d}(t)+T(t),
\end{aligned}
$$

where

$$
\begin{aligned}
\zeta= & \left(I / V_{w f r}\right) l=\text { distance from the water table to the } \\
& \text { lower boundary of contamination in the aquifer at }
\end{aligned}
$$




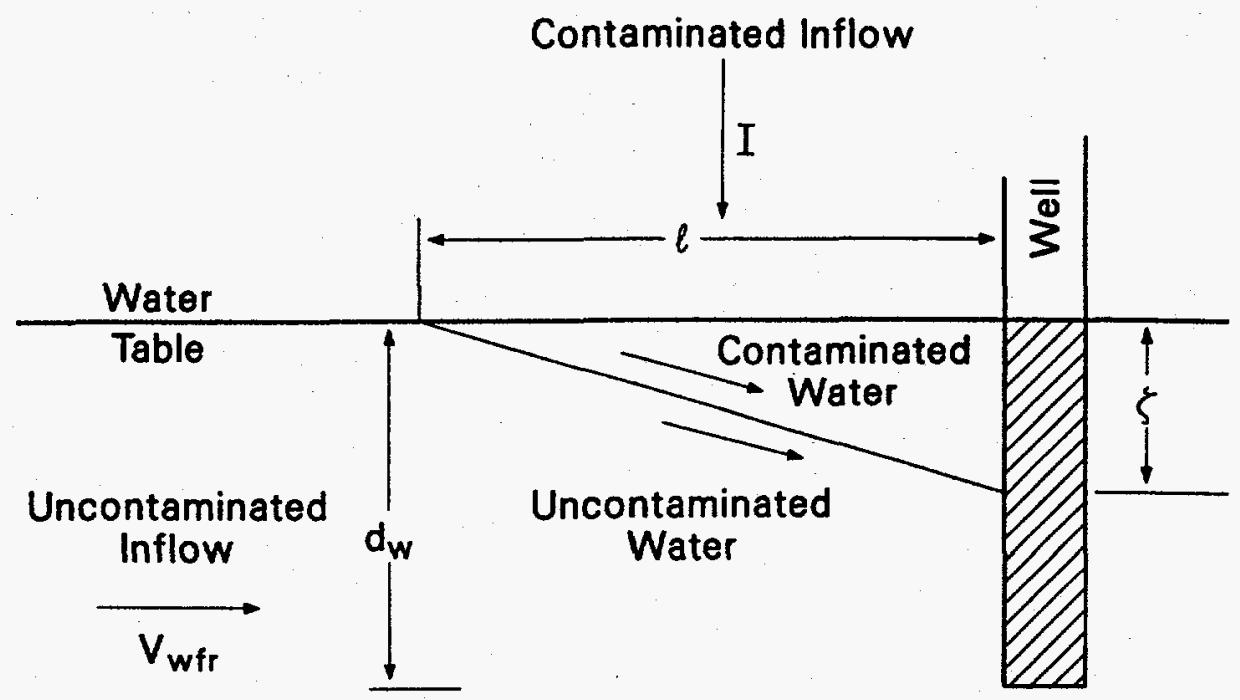

FIGURE E.1 Dilution of Contaminated Inflow by Uncontaminated Inflow in the Nondispersion Model for a Well Adjacent to the Contaminated Area

the downgradient edge of the contaminated zone (m),

$$
I=\text { infiltration rate }(\mathrm{m} / \mathrm{yr}) \text {, }
$$

$$
\begin{aligned}
V_{w f r}=K_{s}^{(s z)} J_{x}= & \text { water flow rate in the saturated zone (m/yr), } \\
K_{s}^{(s z)}= & \text { saturated hydraulic conductivity of the saturated } \\
& \text { zone }(100 \mathrm{~m} / \mathrm{yr}), \\
J_{x}= & \text { hydraulic gradient in the flow }(\mathrm{x}) \text { direction }(0.02, \\
& \text { dimensionless), } \\
\ell= & \text { length of the contaminated zone parallel to the } \\
& \text { hydraulic gradient (maximum distance from the } \\
& \text { upgradient edge to downgradient edge parallel to } \\
& \text { the hydraulic gradient, } 100 \mathrm{~m}), \\
d_{w}= & \text { distance of the well intake below the water table } \\
& (10 \mathrm{~m}),
\end{aligned}
$$




$$
\begin{aligned}
\tau_{\ell_{i}}=p_{e}^{(s z)} R_{d_{i}}^{(s z)} Q V_{w f r}= & \text { time for the } i \text { th principal radionuclide to be trans- } \\
& \text { ported from the upgradient edge to the } \\
& \text { downgradient edge of the saturated zone }(\mathrm{yr}), \\
p_{e}^{(s z)}= & \text { effective porosity of the aquifer }(0.2, \\
& \text { dimensionless); and } \\
R_{d_{i}}^{(s z)}= & \text { retardation factor for the } i \text { th principal radio- } \\
& \text { nuclide in the saturated zone (dimensionless). }
\end{aligned}
$$

Equation E.24 is used only in the ND model. Representative porosities are given in Table E.7. The infiltration rate is given by Equation E.4. Representative saturated hydraulic conductivities are given in Table E.2. Representative distribution coefficients are given in Tables E.3 through E.6.

If the well is in the center of the contaminated zone (i.e., if the MB model is used), the rise time is assumed to be zero. That is, for the MB model,

$$
\delta t_{i 1}=0
$$

\section{E.3.1.3 Dilution Factor}

It is assumed that the water flow is vertically downward from the bottom of the contaminated zone to the water table; hence, no dilution will occur in the unsaturated zone. The dilution in the saturated zone is estimated by using the conservative assumption that the dispersivity is zero.

The dilution factor for the MB model is a radionuclide-independent factor given by the formula

$$
\begin{aligned}
f_{1} & =\frac{A I}{U_{w}}, & & A I<U_{w} \\
& =1, & & A I \geq U_{w},
\end{aligned}
$$

where

$$
A=\text { area of the contaminated zone }\left(\mathrm{m}^{2}\right)
$$


$I=$ infiltration rate $(\mathrm{m} / \mathrm{yr})$, and

$U_{w}=$ well pumping rate $=$ annual volume of water withdrawn from well $\left(250 \mathrm{~m}^{3} / \mathrm{yr}\right)$.

The infiltration rate is calculated by Equation E.4.

For a well located at the downgradient edge of the contaminated zone, the dilution factor for the ND model is given by the formula

$$
\begin{array}{rlr}
f_{i 1}=\frac{\zeta}{d_{w}} & d_{r} \leq \frac{A}{\ell}, \zeta<d_{w} \\
& =\frac{A I}{U_{w}} & d_{r}>\frac{A}{\ell}, \zeta<d_{w} \\
& =1.0 & d_{r} \leq \frac{A}{\ell}, \zeta \geq d_{w} \\
=\frac{A I d_{w}}{U_{w} \zeta} & d_{r}>\frac{A}{\ell}, \zeta \geq d_{w},
\end{array}
$$

where $d_{r}$ is the effective pumping diameter $(\mathrm{m})$ and is calculated as

$$
d_{r}=\frac{U_{w}}{V_{w f r} d_{w}}
$$

Parameters used in Equations E.27 and E.28 are defined the same as those in Equations E.24 and E.26.

The dilution factors for a well that is located away from the edge of the contaminated zone can be significantly smaller than the dilution factors estimated by Equations E.26 and E.27 for a well located in or immediately adjacent to the contaminated zone. The onedimensional model used to obtain Equations E.26 and E.27 cannot be used to estimate this reduction; a two- or three-dimensional model must be used to estimate a more realistic dilution factor. In addition, the assumption of no dispersivity may be unnecessarily 
conservative if the well is not located very close to the contaminated zone. The estimation of off-site well concentrations is discussed further in Appendix $\mathrm{K}$.

\section{E.3.2 Surface Water Pathway $(r=2)$}

The surface water pathway will consist of an on-site groundwater pathway segment that extends to the edge of the contaminated zone, an off-site groundwater pathway segment that extends from the edge of the contaminated zone to a location where surface seepage occurs, and a surface water segment in which the contaminated groundwater is mixed with uncontaminated surface water. Contamination of the surface water can also occur as a consequence of erosion as contaminated soil is deposited in a nearby streambed or pond. This pathway is not included in the current version of RESRAD.

\section{E.3.2.1 Breakthrough Time}

The breakthrough time for the surface water pathway is assumed to be the same as that for the groundwater pathway, that is,

$$
\Delta t_{i 2}=\Delta t_{i 1}=\Delta t_{i},
$$

where $\Delta t_{i}$ is calculated by using Equations E.19 through E.22.

\section{E.3.2.2 Rise Time}

The rise time for the surface water pathway is assumed to be the same as that used in the ND model for the groundwater pathway, that is,

$$
\delta t_{i 2}=\delta t_{i 1},
$$

where $\delta t_{i 1}$ is given by Equation E.24.

\section{E.3.2.3 Dilution Factor}

The dilution factor for the surface water pathway is based on the assumption that the surface water is a pond and (1) the inflow and outflow for the pond are in steady-state equilibrium and (2) the annual inflow of radioactivity into the pond is equal to the annual quantity of radioactivity leached from the contaminated zone. With these assumptions, the 
dilution factor is the ratio of the annual volume of water that percolates through the contaminated zone to the annual total inflow of water into the pond. If, in addition, it is assumed that the infiltrating water flow is vertically downward, the dilution factor is given by the formula

$$
f_{2}=A / A_{w}
$$

where

$$
\begin{aligned}
A & =\text { area of contaminated zone }\left(10^{4} \mathrm{~m}^{2}\right), \text { and } \\
A_{w} & =\text { area of watershed }\left(10^{6} \mathrm{~m}^{2}\right)
\end{aligned}
$$

\section{E.4 REFERENCES}

Baes, C.F., and R.D. Sharp, 1983, "A Proposal for Estimation of Soil Leaching and Leaching Constants for Use in Assessment Models," Journal of Environmental Quality 12(1):17-28.

Clapp, R.B., and G.M. Hornberger, 1978, "Empirical Equations for Some Soil Hydraulic Properties," Water Resources Research 14:601-604.

Gee, G.W., et al., 1980, Interaction of Uranium Mill Tailings Leachate with Soils and Clay Liners, NUREG/CR-1494, PNL-3381, prepared by Pacific Northwest Laboratory, Richland, Wash., for Office of Nuclear Regulatory Research, U.S. Nuclear Regulatory Commission, Washington, D.C.

Geraghty, J.J., et al., 1973, Water Atlas of the United States, Water Information Center, Port Washington, N.Y.

Gilbert, T.L., et al., 1983, Pathways Analysis and Radiation Dose Estimates for Radioactive Residues at Formerly Utilized MED/AEC Sites, ORO-832 (Rev.), prepared by Division of Environmental Impact Studies, Argonne National Laboratory, Argonne, Ill., for U.S. Department of Energy, Oak Ridge Operations, Oak Ridge, Tenn., March (reprinted with corrections January 1984).

Gray, D.M. (editor), 1970, Handbook on the Principles of Hydrology, Water Information Center, Inc., Port Washington, N.Y.

Isherwood, D., 1981, Geoscience Data Base Handbook for Modeling a Nuclear Waste Repository, NUREG/CR-0912, Vols. 1 and 2, U.S. Nuclear Regulatory Commission, Washington, D.C.

McWorter, D.B., and D.K. Sunada, 1977, Ground-Water Hydrology and Hydraulics, Water Resources Publications, Fort Collins, Colo. 
Nuclear Safety Associates, Inc., 1980, Comparison of Alternatives for Long-Term Management of High-Level Radioactive Waste at the Western New York Nuclear Service Center, Appendix IIIC, prepared by Nuclear Safety Associates, Inc., Bethesda, Md., for Argonne National Laboratory, Argonne, Ill.

Rancon, D., 1973, "The Behavior in Underground Environments of Uranium and Thorium Discharge by the Nuclear Industry," in Proceedings of Environmental Behavior of Radionuclides Released in the Nuclear Industry, International Atomic Energy Agency, Vienna, Austria, pp. 333-346.

Sheppard, S.C., and M.I. Sheppard, 1989, "Impact of Correlations on Stochastic Estimates of Soil Contamination and Plant Uptake," Health Physics 57:653-657.

Staley, G.B., et al., 1979, "Radionuclide Migration from Low-Level Waste: A Generic Overview," in Management of Low-Level Radioactive Waste, M.W. Carter et al. (editors), Pergamon Press, New York, N.Y., pp. 1041-1072.

U.S. Nuclear Regulatory Commission, 1980, Final Generic Environmental Impact Statement on Uranium Milling - Vol. 1, Summary and Text; Vol. 2, Appendices A-F; Vol. 3, Appendices G-V, NUREG-0706, Office of Nuclear Material Safety and Safeguards, Washington, D.C.

Yu, C., 1987, "Modeling of Low-Level-Waste Disposal for Environmental Impact Analysis," in Waste Management '87: Waste Isolation in the U.S. Technical Programs and Public Education, proceedings of the Symposium on Waste Management, Tucson, Ariz., March 1-5, pp. 385-390. 
APPENDIX F:

SOIL INGESTION PATHWAY FACTORS 


\section{APPENDIX F: \\ SOIL INGESTION PATHWAY FACTORS}

Soil ingestion occurs very often among young children, especially those under 18 months old. Children incidentally ingest soil when playing on the ground indoors or outdoors. Adults ingest soil (or dust) while working in attics or other uncleaned areas of a house or doing yard work.

The dose/source ratio $D S R_{i}(t)$ for radionuclide $i$ caused by ingestion of contaminated soil (or dust) is calculated as the sum of the product of the dose conversion factor $D C F_{j 8}$, the environmental transport factor $E T F_{j 8}$, and the source factor $S F_{i j}$, in which both leaching and ingrowth and decay processes are considered, and radionuclide $j$ is the decay product of radionuclide $i$ (see Equation 3.9).

The dose conversion factor (DCF) for soil ingestion is the dose/exposure ratio, $D C F_{j 8}=H_{E_{j} 8} / E_{j}$, where $H_{E_{j} 8}$ is the committed effective dose equivalent incurred by an individual from intake by soil ingestion of a quantity $E_{j}$ of the $j$ th principal radionuclide. Dose conversion factor values for soil ingestion are the same as those for the food ingestion pathway in Table D.1.

The environmental transport factor (ETF) for soil ingestion is the ratio of the annual intake $E_{j 8}(t)$ of the $j$ th principal radionuclide by soil ingestion to the concentration $S_{j}(t)$ of the radionuclide in the soil, that is, $E T F_{j 8}(t)=E_{j 8}(t) / S_{j}(t)$. The environmental transport factor can be calculated by the following equation:

$$
E T F_{j 8}(t)=F S I \times F A_{8} \times F C D_{8}(t) \times F O_{8}
$$

where

$$
\begin{aligned}
E T F_{j 8}(t)= & \text { environmental transport factor at time } t \text { for soil ingestion for } \\
& \text { the } j \text { th principal radionuclide (g/yr) } \\
F S I= & \text { annual intake of soil }(36.5 \mathrm{~g} / \mathrm{yr}), \\
F A_{8}= & \text { area factor (dimensionless) }
\end{aligned}
$$




$$
\begin{aligned}
F C D_{8}(t) & =\text { cover and depth factor (dimensionless), and } \\
F O_{8} & =\text { occupancy factor (dimensionless) }
\end{aligned}
$$

The annual intake of soil depends on the age group. The U.S. Environmental Protection Agency (1990) suggests that a value of $70 \mathrm{~g} / \mathrm{yr}(200 \mathrm{mg} / \mathrm{d}, 350 \mathrm{~d} / \mathrm{yr})$ can be used as an average value for young children (under the age of 7); for other age groups (older than 7 years old), the average value is $35 \mathrm{~g} / \mathrm{yr}(100 \mathrm{mg} / \mathrm{d}, 350 \mathrm{~d} / \mathrm{yr})$, which accounts for intakes of both outdoor soil and indoor dust. In RESRAD, a soil ingestion rate of $36.5 \mathrm{~g} / \mathrm{yr}$ is used as the default, which is based on a daily intake rate of $100 \mathrm{mg}, 365 \mathrm{~d} / \mathrm{yr}$.

The area factor represents the fraction of the play or work area that might be contaminated. It is assumed that

$$
\begin{aligned}
F A_{8} & =A / 1,000, & & 0<A<1,000 \mathrm{~m}^{2} \\
& =1 & & A>1,000 \mathrm{~m}^{2},
\end{aligned}
$$

where $A$ is the area of the contaminated zone, and $1,000 \mathrm{~m}^{2}$ is the assumed play or work area, which is approximately the size of a single house lot.

The cover and depth factor is the same as for the inhalation pathway (see Equation B.5) and represents the fraction of soil particles at the ground surface that are contaminated. On the ground surface, a default soil mixing depth of $15 \mathrm{~cm}$ is assumed, within which soils are disturbed and mixed by human activities or wind. As with the inhalation pathway, a default occupancy factor of 0.45 is used in the RESRAD code and is based on the assumption that $50 \%$ of a person's time is spent indoors (where $40 \%$ of the dust or soil level on the floor or household surfaces is of outdoor origin), $25 \%$ is spent outdoors in the contaminated area, and $25 \%$ is spent off-site in an uncontaminated area. The assumption that $40 \%$ of indoor dust is of outdoor origin is based on the conclusions in two studies in which the fractions of indoor dust originating outdoors are reported as $31.3 \%$ (Calabrese and Stanek 1992) and 50\% (Stanek and Calabrese 1992).

The source factor is a correction factor for leaching and ingrowth and decay. The derivation of source factors is presented in Appendix G. 


\section{REFERENCES}

Calabrese, E.J., and Stanek, E.J., 1992, "What Proportion of Household Dust is Derived from Outdoor Soil?" Journal of Soil Contamination 1:253-263.

Stanek, E.J., and Calabrese, E.J., 1992, "Soil Ingestion in Children: Outdoor Soil or Indoor Dust?" Journal of Soil Contamination 1(1):1-28.

U.S. Environmental Protection Agency, 1990, Exposure Factors Handbook, EPA/600/8-89/043, Office of Health and Environmental Assessment, Washington, D.C. 
APPENDIX G:

SOURCE FACTORS 


\section{APPENDIX G:}

\section{SOURCE FACTORS}

The time dependence of dose/source ratios resulting from radioactive ingrowth and decay and infiltration leaching is taken into account by introducing source factors (SFs), $S F_{i j}(t)$. Radionuclides are divided into two groups: those with half-lives longer than six months (principal radionuclides) and those with half-lives of six months or less (associated radionuclides) (see Table 3.1). It is assumed that the associated radionuclides are in secular equilibrium with their principal radionuclide and that the leach rate of the associated radionuclides is the same as the leach rate of their principal radionuclides. Hence, only the SFs for the principal radionuclides need to be calculated.

Let $S_{i i}(0)$ be the initial concentration of the $i$ th principal radionuclide and let $S_{i j}(t)$ be the concentration at time $t$ of the $j$ th principal radionuclide that results from ingrowth from the initial inventory of the $i$ th principal radionuclide. The parameter $S_{i i}(t)$ is the concentration of the undecayed portion that is available for leaching of the $i$ th principal radionuclide $\left[S_{i i}(t)\right.$, which does not include any contribution from ingrowth, should be distinguished from

$$
S_{i}(t)=\sum_{j} S_{j i}(t)
$$

which does include the contribution from ingrowth]. The parameter $S_{i j}(0)=0$ unless $i=j$, and $S_{i j}(t)=0$ unless $i=j$ or the $j t h$ principal radionuclide is a decay product of the $i$ th principal radionuclide. The $\mathrm{SF}$ is defined as

$$
S F_{i j}(t)=S_{i j}(t) / S_{i}(0)
$$

The initial values of the SFs are $S F_{i j}(0)=0$ unless $i=j$, and $S F_{i i}(0)=1$. For $t>0, S F_{i j}(t)=0$ unless $i=j$ or the $j$ th principal radionuclide is a decay product of the ith principal radionuclide. When $i=j$, the inequality $1>S F_{i i}(t)>0$ will be satisfied for $t>0$.

The SF is a correction factor for the source term (i.e., radionuclide concentration in the contaminated zone), which includes the contribution from ingrowth and the removal due to radioactive decay and leaching. If leaching is negligible, the SF reduces to a factor that 
accounts for ingrowth and decay only. This factor is called the ingrowth-and-decay factor, $I D_{i j}(t)$.

Equations for calculating SFs and ingrowth-and-decay factors are given below. Branching radionuclides are a common complication and there are four possible cases to consider: (1) only associated radionuclides are involved; (2) a principal radionuclide parent branches to associated radionuclide progeny; (3) an associated radionuclide parent branches to at least one principal radionuclide decay product; and (4) a principal radionuclide parent branches to at least one principal radionuclide decay product. Cases 1 and 2 cover most of the radionuclides (case 3 does not occur at all) in Table 3.1, and with the assumption that all associated radionuclides are in equilibrium with their principal radionuclide parents, branching need not be considered in deriving SFs and ingrowth-and-decay factors. The longest associated radionuclide half-life in Table 3.1 is $\mathbf{1 8 . 7}$ days.

There are three case 4 branching principal radionuclides in Table 3.1: europium-152, plutonium-241, and curium-243. Case 4 is handled by treating all such principal radionuclides as double entries with the initial concentration of each part multiplied by the appropriate branching fraction. The ingrowth and decay of each portion is then calculated independently by ignoring (temporarily) the other portion. For example, curium-243 (part 1) follows the decay path curium-243 $\rightarrow$ plutonium-239 $\rightarrow$...lead-207, and the curium-243 (part 2) decay is curium-243 $\rightarrow$ americium-243 $\rightarrow$ neptunium-239 $\rightarrow$ plutonium-239 $\rightarrow$ lead-207. The final dose is obtained by simple addition of the part 1 and part 2 components. The advantage of this approach is that all other branching is of the case 1 or case 2 type and can be ignored, as described in the previous paragraph. The Source Factors Table of the DETAILED.REP file (Section 4) lists the individual contributions of each case 4 portion, and all Dose/Source Ratios in the DETAILED.REP file show the overall value for each case 4 portion.

The equation for ingrowth and decay and leaching of a nonbranching chain, assuming a first-order ion-exchange leaching (see Appendix E), is

$$
d A_{k} / d t=\lambda_{k} A_{k-1}-\left(\lambda_{k}+L_{k}\right) A_{k}
$$

where

$$
\lambda_{k}=\ln (2) / T_{1 / 2, k}=\text { radioactive decay constant of the } k \text { th radionuclide }\left(\mathrm{yr}^{-1}\right)
$$


$T_{4 / 2, k}=$ radioactive decay half-life of the $k$ th radionuclide (yr),

$A_{k}=$ activity concentration of the $k$ th radionuclide in the chain (pCi/g), and $L_{k}=$ leach rate of the $k$ th radionuclide $\left(\mathrm{yr}^{-1}\right)$; the radionuclide leach rate is defined in Appendix E (Equation E.3).

The solution to Equation G.3 is

$$
A_{k}(t)=\sum_{\ell=0}^{k} a_{k \ell} \times \exp \left[-\left(\lambda_{\ell}+L_{\ell}\right) t\right]
$$

where

$$
\begin{aligned}
& a_{00}=A_{o}(0) \\
& a_{k \ell}=\left[\lambda_{k} /\left(\lambda_{k}+L_{k}-\lambda_{\ell}-L_{\ell}\right)\right] a_{k-1, \ell}, \quad \ell=0,1, \ldots, k-1 ; \text { and } \\
& a_{k k}=A_{k}(0)-\sum_{\ell=0}^{k-1} a_{k \ell} .
\end{aligned}
$$

The source factor $S F_{i j}(t)$ can be calculated as

$$
S F_{i j}(t)=A_{\ell(i j)}(t) / A_{0}(0)
$$

where $\ell(i j)$ is the sequence number for the $j$ th principal decay product of the $i$ th principal radionuclide, numbered from the $i$ th principal radionuclide $[\ell(\mathrm{ii}) \equiv 0]$. The ingrowth-anddecay factor $I D_{i j}(t)$ is the source factor $S F_{i j}(t)$ when the leach rates $L_{\ell}$ and $L_{k}$ are set to zero. 


\section{APPENDIX H:}

DISTRIBUTION COEFFICIENTS 


\section{APPENDIX H:}

\section{DISTRIBUTION COEFFICIENTS}

The distribution coefficient, $K_{d}$, is a partitioning coefficient under equilibrium conditions that assumes a linear relationship between the concentration of a solute in the solid $(S)$ and the liquid $(C)$ phases; that is, $S=K_{d} C$. This model implicitly assumes that the dissolution of radionuclides is not limited by the solubility limit. The solubility limit model is discussed in Appendix J. The value of $K_{d}$ for different radionuclides is quite variable, depending strongly on soil type, the $\mathrm{pH}$ and $\mathrm{Eh}$ of the soil, and the presence of other ions. Thus, considerable uncertainty can be caused by using default values for the distribution coefficient, especially when the water-dependent pathways are the dominant factors in the dose/source concentration ratios. Therefore, site-specific values should always be used whenever they are available. The measurement methodology for the distribution coefficients is discussed in the RESRAD Data Collection Handbook (Yu et al. 1993).

In addition to the direct input of $K_{d}$ values from the screen, RESRAD provides four optional methods for deriving the distribution coefficient. The first method requires inputting a greater than zero value for the elapsed time since material placement (TI) and providing the groundwater concentration of the radionuclide, which is measured at the same time as the radionuclide soil concentration. The second method uses the input solubility limit to derive an effective $K_{d}$. The third method uses the nonzero input leach rate (default is 0 ) to derive $K_{d}$. The last method is based on the correlation between the plant/soil concentration ratio and the water/soil distribution coefficient, which can be invoked by setting the $K_{d}$ value to -1 on the input screen. Only one of the four methods can be used in each RESRAD execution. If more than one of the requirements is satisfied, RESRAD will always choose according to the following order - the groundwater concentration method first, the solubility limit method second, the leach rate method third, and the plant/soil concentration ratio method last. The solubility limit method is described in Appendix $\mathrm{J}$; the other three methods are discussed below. 


\section{H.1 GROUNDWATER CONCENTRATION METHOD}

The groundwater concentration method requires inputting two parameters - the elapsed time since material placement $(T I)$ and the groundwater concentration $\left(W_{i}\right)$ for radionuclide $i$. The values for both parameters should be greater than zero. The radionuclide groundwater concentration is measured in an on-site well at the same time as the soil concentration is measured. The well is assumed to be located at the downgradient side of the site boundary for the nondispersion (ND) model (see Appendix E). For the mass balance (MB) model, in which the well is located at the center of the site, the groundwater input method is not applicable.

On the basis of measured soil and groundwater concentrations and the elapsed time between the disposal of radioactive wastes and the performance of a radiological survey, it is possible to determine the distribution coefficient of a radionuclide with the provided geological and geometrical information. To simplify the derivation procedures, it is assumed that the distribution coefficients of radionuclide $i$ in the contaminated zone, $K_{d_{i}}{ }^{(c z)}$, in the unsaturated zone, $K_{d_{i m}}{ }^{(u z)}$, and in the saturated zone, $K_{d_{i}}{ }^{(s z)}$, are equal. The geological and geometrical structure of the contaminated site is assumed to be unchanged between the time of the waste placement and the performance of the radiological survey; that is, the thickness of the cover material and the contaminated zone and the distance from the ground surface to the water table remain the same. Iterative calculations to find a best fit value for the distribution coefficient can then be carried out following the leaching model presented in Appendix E.

During the calculational iteration, input data are checked constantly for the possibility of finding a reasonable $K_{d}$ to match the specified environmental setting. If RESRAD finds that the input data violate the leaching model, thus making the derivation of a reasonable $K_{d}$ impossible, warning messages will appear on the screen, and the input groundwater concentration will be reset to zero. The default value of $K_{d}$ can then be used to continue the remaining calculations. This case leads to the same result as if no groundwater contamination had been observed during the radiological survey. The user is advised to check the input value of $T I$ or the groundwater concentration in the next RESRAD execution so that a reasonable $\dot{K}_{d}$ can be derived. Since the $K_{d}$ value of only one radionuclide is solved in each iteration, when a decay chain of more than one principal radionuclide is 
involved, the $K_{d}$ values for the other radionuclides will be used in the iteration. For example, if uranium-238 and uranium-234 exist in the contaminated soil, and the groundwater concentrations for both radionuclides are detected, then the $K_{d}$ value of uranium-238 will be used in the iteration procedure for uranium-234. Because of the listing order of principal radionuclides in the RESRAD code, the iteration procedure for uranium-234 will be performed first, so that the $K_{d}$ value for uranium-238 used in the iteration is obtained from the user input file rather than from an iteration procedure. This may result in an inaccurate $K_{d}$ for uranium-234, if the input $K_{d}$ for uranium-238 is inaccurate. To reduce such inaccuracy, it may be necessary to run RESRAD several times to obtain the correct values. In the repeated runs, the groundwater concentrations of each radionuclide are assigned sequentially according to the decay order. In the first run, only the first radionuclide in the decay chain is input with a nonzero groundwater concentration. For the second run, the derived $K_{d}$ for the first radionuclide is used, and the groundwater concentration of the second radionuclide in the decay chain is added (the groundwater concentration of the first radionuclide can either be set to zero or carried over from the first run). This procedure can be used for the remainder of the radionuclides in the decay chain. Sections H.1.1 and H.1.2 detail the iteration procedure used in RESRAD for deriving distribution coefficients by the groundwater concentration method.

\section{H.1.1 Breakthrough Time and Rise Time as Functions of $\mathbf{K}_{d_{1}}$}

According to Equations E.19 and E.21, the breakthrough time for radionuclide $i$ can be written as

$$
\Delta t_{i}=\frac{\sum_{m=1}^{n+1} \Delta Z_{m} R_{d_{i m}{ }^{(u z)} P_{e_{m}}{ }^{(u z)} R_{s_{m}}{ }^{(u z)}}^{I}}{I}
$$

Substituting $R_{d_{i m}}{ }^{(u z)}$ with Equation E.22, $\Delta t_{i}$ then becomes 


$$
\Delta t_{i}=\frac{\sum_{m=1}^{n+1} \Delta Z_{m} P_{e_{m}}{ }^{(u z)} R_{s_{m}}{ }^{(u z)}\left[1+\frac{\rho_{b_{m}}{ }^{(u z)} K_{d_{i}}{ }^{(u z)}}{P_{t_{m}}{ }^{(u z)} R_{s_{m}}{ }^{(u z)}}\right]}{I} .
$$

Equation H.2, after rearrangement, can be expressed as a linear function of $K_{d_{i}}$;

$$
\Delta t_{i}=X_{1}+X_{2} K_{d_{i}}
$$

where

$$
X_{1}=\frac{\sum_{m=1}^{n+1} \Delta Z_{m} P_{e_{m}}{ }^{(u z)} R_{s_{m}}{ }^{(u z)}}{I}
$$

and

$$
X_{2}=\sum_{m=1}^{n+1} \frac{\Delta Z_{m} P_{e_{m}}{ }^{(u z)} \rho_{b_{m}}{ }^{(u z)}}{P_{t_{m}}{ }^{(u z)} I}
$$

The rise time, $\delta t_{i}$, depends on the geometrical characteristics of the site. For the MB model, the value of $\delta t_{i}$ equals 0 because the well is located at the center of the contaminated zone. For the nondispersion model, $\delta t_{i}$ is a function of $\tau_{\ell_{i}}$ and can be expressed as

$$
\delta t_{i}=\left(\tau_{\ell_{i}}\right) \chi
$$

The value of $\chi$, according to Equation E.24, is

$$
\begin{array}{cll}
1 & \zeta \leq d_{w}, & D_{w t}(t) \geq C_{d}(t)+T(t) \\
\chi=1 /\left(\zeta / d_{w}\right) & \zeta>d_{w}, & D_{w t}(t) \geq C_{d}(t)+T(t) \\
0 & \zeta>d_{w}, & D_{w t}(t)<C_{d}(t)+T(t)
\end{array}
$$

By incorporating the definition of $\tau_{\ell_{i}}$ into Equation H.6, $\delta_{t_{i}}$ then can be written as 


$$
\begin{aligned}
\delta t_{i} & =\left[\frac{P_{e}^{(s z)} R_{d_{i}}^{(s z)} l}{V_{w f r}}\right] \chi \\
& =\left[\frac{P_{e}^{(s z)} l}{V_{w f r}}\right] \chi+\left[\frac{P_{e}^{(s z)} l \rho_{b}^{(s z)}}{V_{w f r} P_{t}^{(s z)} R_{s}^{(s z)}}\right] \chi K_{d_{i}} \\
& =X_{3}+X_{4} K_{d_{i}},
\end{aligned}
$$

where

$$
X_{3}=\left[\frac{P_{e}^{(s z)} l}{V_{w f r}}\right] \chi
$$

and

$$
X_{4}=\left[\frac{P_{e}^{(s z)} l \rho_{b}^{(s z)}}{V_{w f r} P_{t}^{(s z)}}\right] \chi
$$

because

$$
R_{s}^{(s z)}=1
$$

The leach rate constant $L_{i}$ for radionuclide $i$ is also a function of $K_{d_{i}}$. From Equation E.3, $L_{i}$ can be expressed as

$$
L_{i}=\frac{1}{X_{5}+X_{6} K_{d_{i}}}
$$

with

$$
X_{5}=\frac{P_{t}^{(c z)} R_{s}^{(c z)} T(t)}{I}
$$

and

$$
X_{6}=\frac{T(t) \rho_{b}^{(c z)}}{I}
$$




\section{H.1.2 Solution for the Distribution Coefficient}

\section{H.1.2.1 Relationship between the Water/Soil Concentration Ratio and the Distribution Coefficient}

For a principal radionuclide that is not a decay product of any other radionuclide, the relationship between the water/soil concentration ratio [the ratio of water concentration at time $t$ to the input soil concentration, $\left.W_{i}(t) / S_{i}(0)\right]$ and the distribution coefficient $\left(K_{d_{i}}\right)$ is such that for a contaminated site with a specific geological structure, an increase in $K_{d_{i}}$ will result in an increase in the breakthrough time $\Delta t_{i}$ and the rise time $\delta t_{i}$, but will result in a decrease in the leach rate constant $\left(L_{i}\right)$; therefore, the maximum value of $W_{i}(t) / S_{i}(0)$ will decrease. When the principal radionuclide $i$ is a decay product of other radionuclides, the times to observe the beginning of a nonzero water/soil concentration ratio $W_{i}(t) / S_{i}(0)$ and its maximum are not necessarily $\Delta t_{i}$ and $\Delta t_{i}+\delta t_{i}$, respectively. These times depend not only on the $K_{d}$ of nuclide $i$ but also on the $K_{d}$ 's of its parent radionuclides. It is difficult to define precisely the magnitude range of the distribution coefficient $K_{d_{i}}$ based on a measured groundwater concentration $W_{i}$. To make it simpler, the range of $K_{d_{i}}$ is defined by considering radionuclide $i$ independently; that is, the range is the same as that for a radionuclide that does not originate from the decay of other radionuclides. Although this assumption is inaccurate, for the possible values of $T I$ (which are always less than 100) in most cases, the defined range is a good approximation and includes the final solution. After the range is defined, an iteration procedure is used to find a best fit value for $K_{d_{i}}$. An internal absolute time frame is used in the calculation of the water/soil concentration ratio; that is, the origin $t=0$ corresponds to the time the contaminants were placed on-site. Whenever the input $T I$ is greater than 0, RESRAD will calculate the soil concentrations of all principal radionuclides at the placement time. The ratios of water concentrations at different times with the measured soil concentrations are then calculated. The iteration procedure will find a best fit value of $K_{d_{i}}$, so that at time $T I$, the water/soil concentration ratio is the measured value of $\left(W_{i} / S_{i}\right)_{m}$. The derived $K_{d_{i}}$ will result in a $T I$ that is greater than the corresponding breakthrough time $\left(\Delta t_{i}\right)$ and smaller than the corresponding rise time $\left(\Delta t_{i}+\delta t_{i}\right)$. 


\section{H.1.2.2 Definition and Magnitude Range of the Distribution Coefficients}

The various distribution coefficients used in the iterative calculation based on the groundwater concentration method are defined as follows:

$\left(K_{d_{i}}\right)_{r}$ - The distribution coefficient of radionuclide $i$ that causes the maximum water/soil concentration ratio to occur at time $T I$ (absolute time frame).

$\left(K_{d_{i}}\right)_{b t}$ - The distribution coefficient of radionuclide $i$ that causes the breakthrough time to equal TI (absolute time frame).

The solution of the distribution coefficient for radionuclide $i, K_{d_{i}}$, can be found only for the ND model. The distribution coefficient $\left(K_{d_{i}}\right)_{r}$ that has its peak at $T I$ should be the lower limit for $K_{d_{i}}$. The preliminary requirement for observing any concentration in groundwater is that the absolute time $T I$ must be equal to or greater than the breakthrough time $\Delta t_{i}$. Thus $\left(K_{d_{i}}\right)_{b t}$ is the upper limit for $K_{d_{i}}$.

\section{H.1.2.3 Calculation of $\left(K_{d_{i}}\right)$ and $\left(K_{d_{i}}\right)_{b t}$}

From the above discussion, if the distribution coefficient for radionuclide $i$ is $\left(K_{d_{i}}\right)_{r}$, then the peak of the water/soil concentration ratio will occur at time TI (absolute time). On the basis of this information, $\left(K_{d_{i}}\right)_{r}$ can then be solved by

$$
\begin{aligned}
T I & =\Delta t_{i}+\delta t_{i} \\
& =\left(X_{1}+X_{3}\right)+\left(X_{2}+X_{4}\right)\left(K_{d_{i}}\right)_{r} .
\end{aligned}
$$

Therefore,

$$
\left(K_{d_{i}}\right)_{r}=\frac{T I-\left(X_{1}+X_{3}\right)}{X_{2}+X_{4}}
$$

The water/soil concentration ratio, $\left(W_{i}(t) / S_{i}(0)\right)_{r}$, calculated at time $T I$ (absolute time) with $\left(K_{d_{i}}\right)_{r}$ will be greater than (or equal to) the measured value $\left(W_{i} / S_{i}\right)_{m}$. The value of $\left(K_{d_{i}}\right)_{b t}$ can easily be solved by setting $T I$ to $\Delta t_{i}$ : 


$$
\begin{gathered}
T I=\Delta t_{i} \\
=X_{1}+X_{2}\left(K_{d_{i}}\right)_{b t} \\
\left(K_{d_{i}}\right)_{b t}=\frac{\left(T I-X_{1}\right)}{X_{2}} .
\end{gathered}
$$

Because, by definition, the distribution coefficient $K_{d_{i}}$ should always be greater than or equal to zero, any negative solution for $\left(K_{d_{i}}\right)_{b t}$ is not allowed. The occurrence of a negative $\left(K_{d_{i}}\right)_{b t}$ indicates that no contamination of radionuclide $i$ should be observed at time TI (absolute time) in accordance with the specified geological structure of the site, because the elapsed time $T I$ is shorter than the breakthrough time $\Delta t_{i}$; this is obviously contrary to the observation. Therefore, if the calculated $\left(K_{d_{i}}\right)_{b t}$ is less than zero, a warning message will appear on the screen, and the water concentration will be neglected.

\section{H.1.2.4 Flow Diagram for the Iteration Procedure}

Once the magnitude range for $K_{d_{i}}$ is solved, an iterative calculation is performed to solve for $K_{d_{i}}$. A preliminary check must be made, however, to make sure that it is possible to find a solution. When $\left(K_{d_{i}}\right)_{b t}$ is less than 0 , a warning message will appear on the screen and the iteration will be aborted. The normal calculation will be continued by setting $W_{i}$ to 0 and using the default or input distribution coefficient. Figure H.1 illustrates the flow diagram for the iteration procedure.

\section{H.2 THE LEACH RATE METHOD}

When a nonzero leach rate for radionuclide $j^{1}$ is input, RESRAD will calculate the distribution coefficient on the basis of this value. Equation E.3 is used first to derive the retardation factor for the contaminated zone, then Equation E.8 is used to obtain $K_{d}$, the distribution coefficient of the contaminated zone. After $K_{d}$ is obtained, its magnitude will be checked for validity ( $K_{d}$ must be greater than or equal to 0 ). If $K_{d}$ is negative, indicating that

1 The index $j$ corresponds to principal radionuclides present originally as well as any principal radionuclides resulting from ingrowth. 


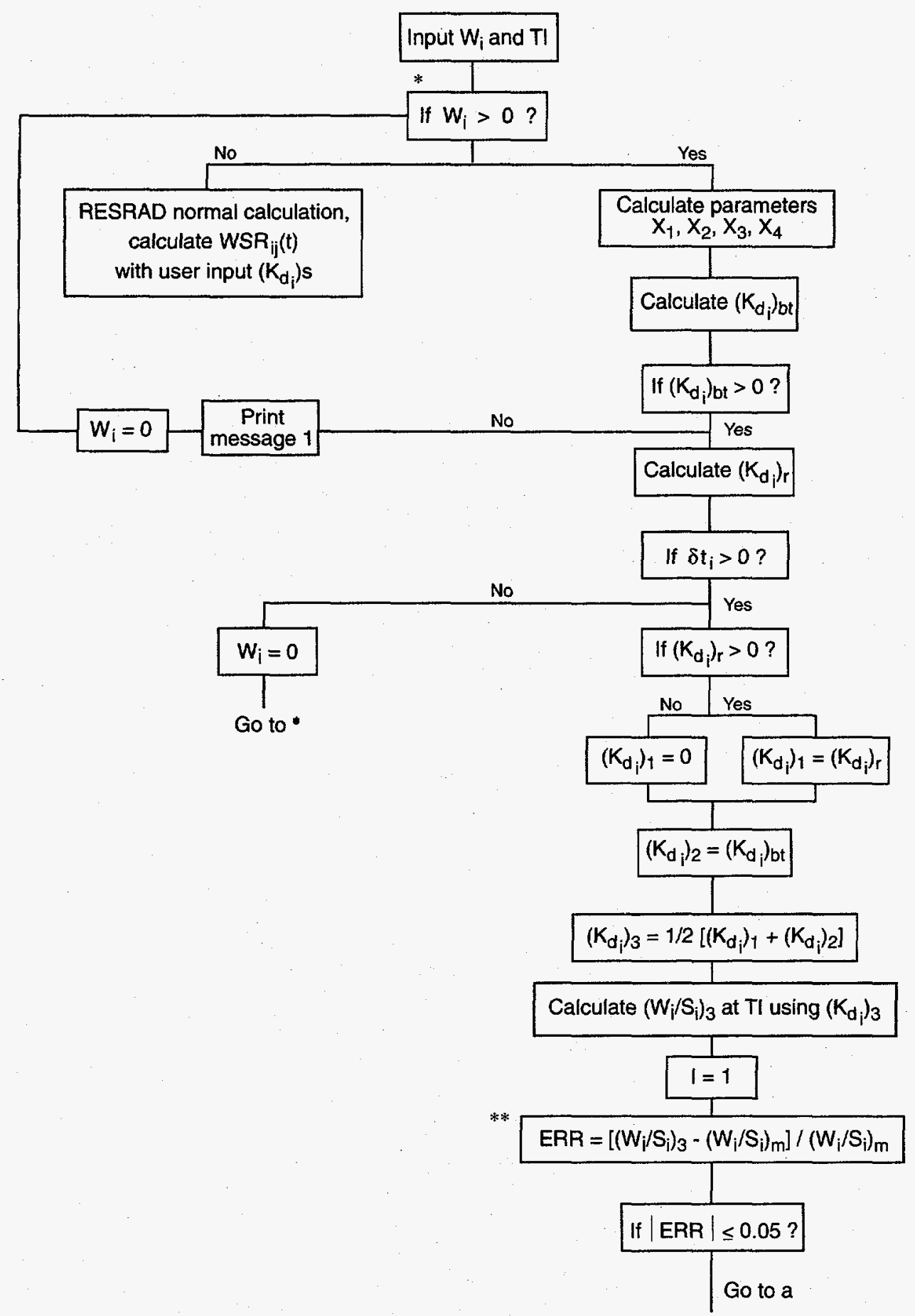

Message 1: With the input information, it is impossible to observe a nonzero concentration of "radionuclide $i$ " in groundwater. Calculation resumes by setting the groundwater concentration of "radionuclide $i$ " to 0 .

FIGURE H.1 Flow Diagram for the Iterative Calculations 


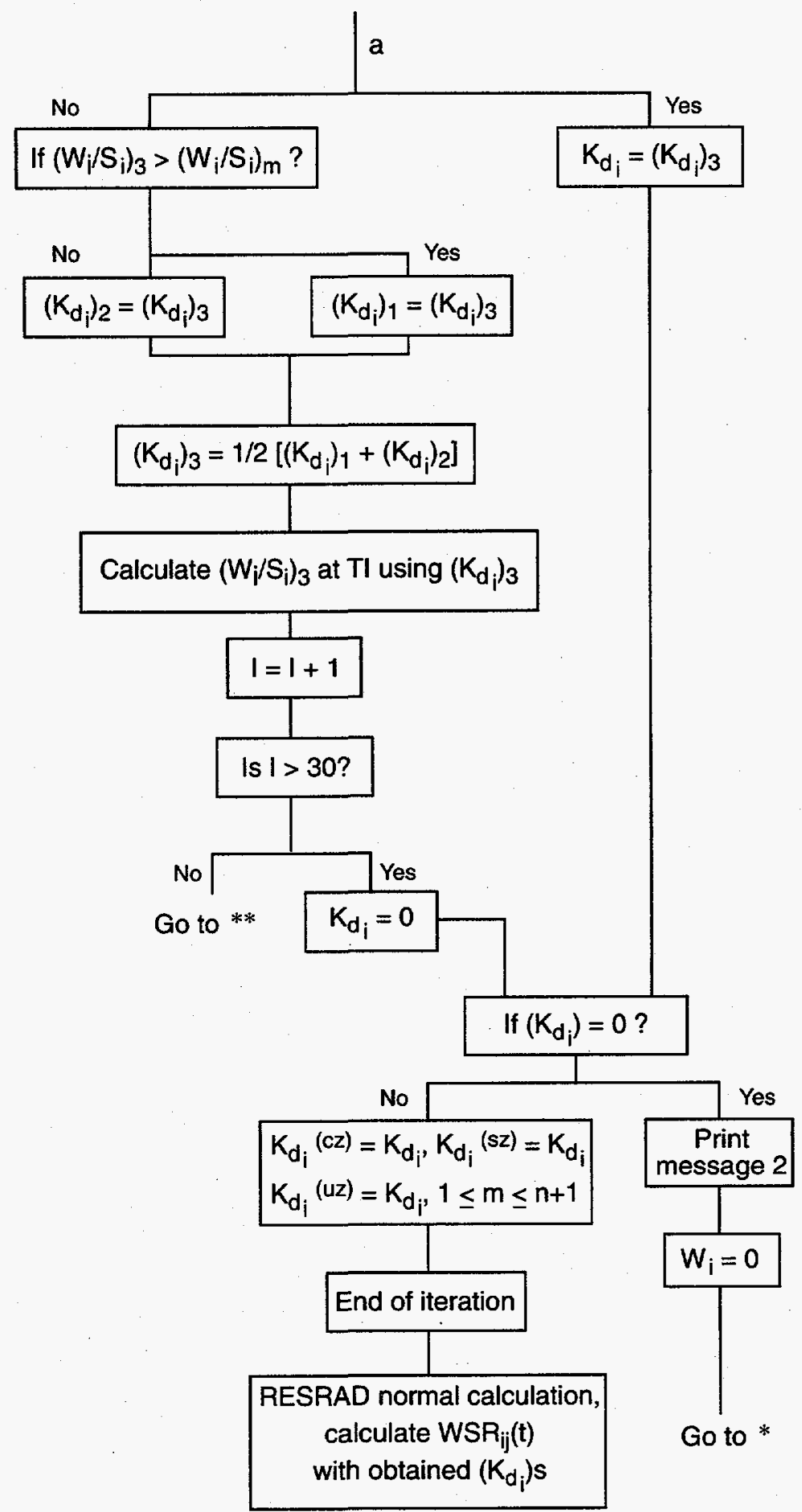

Message 2:

Iteration without convergence. Calculation resumes by setting the groundwater concentration of "radionuclide $i$ " to 0 .

\section{FIGURE H.1 (Cont.)}


the input leach rate is unreasonably large according to the leaching model in Appendix $\mathbf{E}$, then the input leach rate will be neglected, and its value will be recalculated with the input or default distribution coefficient. This process results in the same situation as if the input leach rate of radionuclide $j$ were zero. Otherwise, the derived $K_{d}$ will replace the screen input value and RESRAD will continue the remaining calculations.

When an acceptable $K_{d}$ is derived, this value will be used not only for the contaminated zone but for the unsaturated zone(s) and the saturated zone. If the user does not want to have the input distribution coefficients of the unsaturated zone(s) and the saturated zone substituted, another execution of RESRAD with the derived $K_{d}$ for the contaminated zone and a zero input leach rate is required.

\section{H.3 THE PLANT/SOIL CONCENTRATION METHOD}

Another method for deriving the distribution coefficient is the plant/soil concentration method. According to Baes et al. (1984) and Sheppard and Sheppard (1989), a strong correlation exists between the plant/soil concentration ratio $(C R)$ and the contaminated zone distribution coefficient $K_{d}$. Sheppard and Thibault (1990) proposed the following correlation equation:

$$
\ln K_{d}=a+b(\ln C R)
$$

where $a$ and $b$ are constants. From experimental data, the value for the coefficient $b$ is found to be -0.5. The value of $a$ depends on soil type: for sandy soil, $a=2.11$; for loamy soil, $a=3.36$; for clayey soil, $a=3.78$; and for organic soil, $a=4.62$. Baes et al. (1984) also proposed the above correlation equation (Equation H.23), however, with different coefficient values. Equation H.23 provides a method to estimate the distribution coefficient from the plant/soil concentration ratio, especially when experimental or literature data are not available.

The plant/soil concentration ratio $(C R)$ used is based on the weights of dry soil and wet plants. The soil-to-plant transfer coefficient, $B_{j v}$, used in RESRAD is also based on the weights of dry soil and wet plants. Therefore, the RESRAD default $B_{j v}$ values or the userspecified values of the soil-to-plant transfer factors can be used in Equation H.23 for the calculation of $K_{d}$; 


$$
\ln K_{d_{j}}=a+b\left(\ln B_{j v}\right),
$$

where $j$ is the radionuclide index. When the input distribution coefficient $K_{d}$ value (whether it is for the contaminated, unsaturated, or saturated zone) is -1 , the soil-to-plant transfer factor $B_{j v}$ will be used for calculating the distribution coefficients by using Equation H.24. In the current version of RESRAD, loamy soil is assumed, that is, $a=3.36$ in Equation H.24. It is also assumed that the derived $K_{d}$ applies to all zones. If the user would like to use different $K_{d}$ values for different soil layers, then RESRAD must be executed once again with the derived value assigned to the proper soil layer.

\section{H.4 REFERENCES}

Baes, C.F., III, et al., 1984, A Review and Analysis of Parameters for Assessing Transport of Environmentally Released Radionuclides through Agriculture, prepared by Oak Ridge National Laboratory, Oak Ridge, Tenn. for Martin Marietta Energy Systems, Oak Ridge, Tenn.

Sheppard, S.C., and Sheppard, M.I., 1989, "Impact of Correlations on Stochastic Estimates of Soil Contaminations and Plant Update," Health Physics 57:653:657.

Sheppard, M.I., and Thibault, D.H., 1990, "Default Soil Solid/Liquid Partition Coefficients, $\mathrm{K}_{d} \mathrm{~s}$, for Four Major Soil Types: a Compendium," Health Physics 59:471-482.

Yu, C., et al., 1993, Data Collection Handbook to Support Modeling the Impacts of Radioactive Material in Soil, ANL/EAIS-8, prepared by Argonne National Laboratory, Argonne, Ill., for U.S. Department of Energy, Washington, D.C. 


\section{APPENDIX I:}

RADIOACTIVE DECAY PRODUCT TRANSFER FUNCTION 


\section{APPENDIX I:}

\section{RADIOACTIVE DECAY PRODUCT TRANSFER FUNCTION}

\section{I.1 INTRODUCTION}

The transfer function, $G_{k j}$, used in Appendix $\mathrm{E}$ was derived in two steps. In the first, the transfer function, $g_{k j}$, through a soil layer of fixed thickness was derived. The function, $g_{k j}$, relates the release of radionuclide $k$ from the contaminated zone to the observation of radionuclide $j$ at the point of water use as a result of the decay of radionuclide $k$. When there is more than one soil layer (i.e., unsaturated zone), this function can be derived analytically if it is assumed that the radionuclides in the decay chain travel through the zones such that their relative velocities remain fixed. Under these conditions, the unsaturated zones can be treated as a single, thick, soil layer. In the second step, consideration was given to the transport through the saturated zone where the distance the released radionuclides travel is not fixed but related to where they were released, that is, the distance from the point of release to the downgradient edge of the contaminated zone. The transfer function $G_{k j}$ is the average of $g_{k j}$ 's, which vary in travel distance.

\section{I.2 MATHEMATICAL MODEL OF TRANSFER FUNCTIONS}

Consider the simplest case of radionuclide $i$ being released from the contaminated zone and observed at the point of use. The transfer function through a fixed distance is simply a delta $(\delta)$ function in time multiplied by the decay factor for the amount of time it takes to travel this distance $\left(T_{i}\right)$,

$$
g_{i i}(t)=g_{i i}\left(t, T_{i}\right)=e^{-\lambda_{i} T_{i}} \delta\left(t-T_{i}\right)
$$

The transfer function $G_{i i}(t)$ through the unsaturated and saturated zones is the average over all paths of the fixed distance transfer function, $g_{i i}(t)$. This is because $g$ is a function not only of $t$ but also of the breakthrough times and decay constant of radionuclide $i$; 


$$
\begin{aligned}
G_{i i}(t) & =\int_{0}^{1} g_{i i}\left(t, T_{u i}+x T_{s i}\right) d x \\
& =\frac{e^{-\lambda_{i} t}}{T_{s i}}
\end{aligned}
$$

where $G_{i i}(t)$ is nonzero when $t$ is between $T_{u i}$ and $T_{u i}+T_{s i}$. The transit time for radionuclide $i$ to travel through all of the unsaturated zones (the breakthrough time) is represented by $T_{u i}$, and $T_{s i}$ is the transit time for radionuclide $i$ through the saturated zone (the rise time).

In general, if $g_{i j}(t,\{T\})$ is the transfer function through a given depth of a soil layer where $\{T\}$ is the set of breakthrough times, then the transfer function $G_{i j}(t)$ through the unsaturated zones of fixed depths and the saturated zone of variable length is the average of $g_{i j}(t)$ through the different travel distances:

$$
G_{i j}(t)=\int_{0}^{1} g_{i j}\left(t,\left\{T_{u}+x T_{s}\right\}\right) d x
$$

where $\left\{T_{u}\right\}$ is the set of unsaturated breakthrough times, and $\left\{T_{s}\right\}$ is the set of saturated rise times (i.e., maximum time to travel the saturated zone).

For the case in which $i$ decays directly into $j$, the transfer function, $g_{2, i j}$ (i.e., the fixed length transfer function for a decay chain length of 2), will be nonzero for all times between the transit times of the two radionuclides, $T_{i}$ and $T_{j}$ (let $T_{1}$ be the smaller and $T_{2}$ be the larger; that is, subscript 1 refers to the faster moving radionuclide and subscript 2 refers to the slower one). These two extremes correspond to the decay of radionuclide $i$ to $j$ at the beginning and at the end of the travel but not necessarily in that order. For radionuclide $j$ to arrive at a time between $T_{1}$ and $T_{2}$, the location of the decay of $i$ to $j$ can be calculated by letting $x$ represent the fraction of distance that $i$ travels before decay:

$$
t=x T_{i}+(1-x) T_{j}
$$


Consider a small layer around $x$ with a thickness of $d x$. As the result of the decay of nuclide $i$, which is released at the top, only a fraction of the $i$ nuclides will arrive at this small layer. Another fraction will arrive as decay products (nuclide $j$ ). Some of the $i$ nuclides will decay to $j$ nuclides in the small layer. In turn, some of the $j$ nuclides will reach the bottom between times $t$ and $t+d t$, where $d t$ is the difference in arrival times from the case in which $i$ decays at the top of the small layer and $i$ decays at the bottom of the small layer. The unsaturated transfer function can then be expressed as

$$
g_{2, i j}(t) d t=\lambda_{i}\left(d x T_{i}\right) e^{-\lambda_{i} T_{i} x} e^{-\lambda_{j} T_{j}(1-x)}
$$

and

$$
g_{2, i j}(t)=\frac{\lambda_{i} T_{i}}{T_{2}-T_{1}} e^{\frac{\left(\lambda_{1}-\lambda_{2}\right) T_{1} T_{2}}{T_{1}-T_{2}}} e^{\left(\frac{\lambda_{1} T_{1}-\lambda_{2} T_{2}}{T_{2}-T_{1}}\right) t}
$$

If it is assumed that the relative transport speed (i.e., the speed ratio) between nuclide $i$ and nuclide $j$ in the saturated zone is the same as that in the unsaturated zone, then the saturated zone can be treated as another unsaturated zone (with a saturation ratio of one), and the distance that the radionuclides travel through this zone will depend on the horizontal location of the release point to the point of water use. The transfer function, $G_{2, i j}(t)$, through the unsaturated and saturated zones can be derived by integrating the unsaturated transfer function, $g_{2, i j}(t)$, as described in Equation I.6.

$$
\begin{gathered}
G_{2, i j}(t)=\int_{x_{L}}^{x_{H}} g_{2, i j}\left(t,\left\{T_{u}+x T_{s}\right\}\right) d x \\
=\frac{\lambda_{i} T_{i}}{T_{1}(F-1)} e^{\frac{\lambda_{1} \lambda_{2} F}{F-1} t} \int_{x_{L}}^{x_{H}} e^{\frac{\left(\lambda_{1}-\lambda_{2}\right) F\left(T_{u i}+x T_{s i}\right)}{1-F} d x}
\end{gathered}
$$


The transfer function is nonzero in the region $T_{u 1}<t<T_{u 2}+T_{s 2}$, and the limits of the integral can be defined as

$$
x_{L}=\max \left[\frac{t-T_{u 2}}{T_{s 2}}, 0\right] ; \quad x_{H}=\min \left[\frac{t-T_{u 1}}{T_{s 1}}, 1\right]
$$

and

$$
\begin{aligned}
& F=\text { ratio of the transport speeds } \\
& F=\frac{T_{u 2}+T_{s 2}}{T_{u 1}+T_{s 1}}
\end{aligned}
$$

In the region where this integral is nonzero, $G_{2, i j}(t)$ can be expressed as

$$
G_{2, i j}(t)=\frac{\lambda_{i}}{\lambda_{1}-\lambda_{2}} \frac{T_{u i}}{T_{u 1} T_{s 2}} e^{\frac{\lambda_{1}-F \lambda_{2}}{F-1} t}\left(e^{-\alpha T_{L}}-e^{-\alpha T_{H}}\right)
$$

where

$$
\begin{gathered}
\alpha=\frac{\left(\lambda_{1}-\lambda_{2}\right) F}{F-1} \text { and } \\
T_{L}=T_{u 1}+x_{L} T_{s 1} ; T_{H}=T_{u 1}+x_{H} T_{s 1}
\end{gathered}
$$

If both $i$ and $j$ have the same breakthrough time, then this expression for $G_{2}$ is invalid. A similar derivation can be used in this case, with $g_{2}$ in Equation I.5 replaced by Bateman's equation for two radionuclides and a $\delta$ function.

For the case in which $i$ decays to $k$, which in turn decays to $j$, the transfer function $g_{3, i j}$ is nonzero between the smallest and the largest transit times. For $g_{2, i j}$, for a given time $t$ between the larger and the smaller transit times, there is only one solution for the location in the transport path for radioactive decay from $i$ to $j$. For $g_{3, i j}$, there is more than 
one solution for the locations in the transport path where the two decays occur at a given time $t$. Therefore, the transfer function $g_{3, i j}(t)$ is the sum of all the solutions:

$$
g_{3, i j}(t) d t=\sum \lambda_{i} T_{i} \lambda_{k} T_{k} e^{-\lambda_{i} x_{i} T_{i}} e^{-\lambda_{j} x_{j} T_{j}} e^{-\lambda_{k} x_{k} T_{k}} d x_{i} d x_{k}
$$

Let the smallest transit time among $T_{i}, T_{j}$, and $T_{k}$ be $T_{1}$, the largest transit time be $T_{3}$, and the intermediate transit time be $T_{2}$. Then, $x_{1}, x_{2}$, and $x_{3}$ are governed by the following equations:

$$
\begin{gathered}
t=x_{1} T_{1}+x_{2} T_{2}+x_{3} T_{3} \text { and } \\
x_{1}+x_{2}+x_{3}=1
\end{gathered}
$$

where $x_{1}$ is the path fraction traveled by the fastest moving radionuclide, $x_{3}$ is the path fraction traveled by the slowest moving radionuclide, and $x_{2}=1-x_{1}-x_{3}$ is the path fraction traveled by the other radionuclide. The transfer function $g_{3, i j}(t)$ in Equation $\mathrm{I} .12$ can be expressed in terms of subscript indexes 1,2 , and 3 by correlating radionuclides $i, j$, and $k$ to the transit times $T_{1}, T_{2}$, and $T_{3}$ (not necessarily in that order). The two differentials in the right side of the equation can then be converted to the differential of time $t, d t$, and the selected differential of one of the path fractions, $d x_{1}, d x_{2}$, or $d x_{3}$, by multiplying by the Jacobian of the conversion. The differential of time, $d t$, in both sides of the equation then cancels out, and the summation in the right side of the equation can be replaced by integrating over the selected path fraction for the appropriate limits. It is easier to determine the limits for the path fraction $x_{2}$ than for $x_{1}$ and $x_{3}$. In the paragraph below, an example is given to illustrate the derivation of the transfer function $g_{3, i j}$.

Assuming $T_{i}, T_{k}$, and $T_{j}$ can be designated as $T_{1}, T_{3}$, and $T_{2}$, respectively, then $g_{3, i j}$ in Equation 1.12 can be written as

$$
g_{3, i j}(t) d t=\sum \lambda_{i} T_{i} \lambda_{k} T_{k} e^{-\lambda_{1} x_{1} T_{1}} e^{-\lambda_{2} x_{2} T_{2}} e^{-\lambda_{3} x_{3} T_{3}} d x_{1} d x_{3}
$$

If $t$ is between $T_{1}$ and $T_{2}$, then the smallest value of $x_{2}$ is 0 ; the largest value is $\left(t-T_{1}\right) /\left(T_{2}-T_{1}\right)$. Otherwise, if $t$ is between $T_{2}$ and $T_{3}$, the smallest value of $x_{2}$ is 0 ; the largest value is 
$\left(T_{3}-t\right) /\left(T_{3}-T_{2}\right)$. From Equations $I .13$ and I.14, the path fractions $x_{1}$ and $x_{3}$ can be written as functions of time $t$ and path fraction $x_{2}$ by

$$
\begin{gathered}
x_{1}=\frac{t-T_{3}}{T_{1}-T_{3}}-\left(\frac{T_{2}-T_{3}}{T_{1}-T_{3}}\right) x_{2} \text { and } \\
x_{3}=\frac{t-T_{1}}{T_{3}-T_{1}}-\left(\frac{T_{2}-T_{1}}{T_{3}-T_{1}}\right) x_{2} .
\end{gathered}
$$

The value of the Jacobian to convert $\left(x_{1}, x_{3}\right)$ to $\left(x_{2}, t\right)$ is $1 /\left(T_{3}-T_{1}\right)$. Then the transfer function, $g_{3, i j}(t)$, in Equation I.15 can be obtained by integrating over the path fraction $x_{2}$ :

$$
g_{3, i j}(t)=\frac{\lambda_{i} T_{i} \lambda_{k} T_{k}}{T_{3}-T_{1}} \int_{0}^{x_{2}^{\max }} e^{-\lambda_{2} T_{2} x_{2}} e^{-\lambda_{1} T_{1} x_{1}} e^{-\lambda_{3} T_{3} x_{3}} d x_{2}
$$

Performing this integral yields

$$
g_{3, i j}=\frac{\lambda_{i} T_{i} \lambda_{k} T_{k}}{T_{3}-T_{1}} e^{-\lambda_{1} T_{1}\left(\frac{t-T_{3}}{T_{1}-T_{3}}\right)} e^{-\lambda_{3} T_{3}\left(\frac{t-T_{1}}{T_{3}-T_{1}}\right)}\left[\frac{1-e^{-\alpha^{\prime}\left(\frac{t-T_{M}}{T_{2}-T_{M}}\right)}}{\alpha^{\prime}}\right]
$$

where

$$
\alpha^{\prime}=\lambda_{2} T_{2}-\frac{T_{2}-T_{3}}{T_{1}-T_{3}} \lambda_{1} T_{1}-\frac{T_{2}-T_{1}}{T_{3}-T_{1}} \lambda_{3} T_{3}
$$

and

$$
T_{M}= \begin{cases}T_{1} & T_{1} \leq t<T_{2} \\ T_{3} & T_{2} \leq t \leq T_{3}\end{cases}
$$


As with $g_{1}$ and $g_{2}$, this unsaturated transfer function $g_{3, i j}$ can be similarly integrated to obtain the transfer function $G_{3, i j}$ through the unsaturated and saturated zones.

In general, if there are $n$ radionuclides in the decay chain from $i$ to $j$, the transfer function, $g_{n, i j}$, can be expressed as

$$
g_{n, i j}=\frac{\prod_{1}^{n} \lambda_{k} T_{k}}{\lambda_{j} T_{j}\left(T_{n}-T_{1}\right)} \int_{0}^{x_{2}^{M} x_{3}^{M}} \int_{0}^{M} \ldots \int_{0}^{x_{n-1}^{M}} e^{-\sum_{1}^{n} \lambda_{i} T_{i} x_{i}} d x_{n-1} \ldots d x_{3} d x_{2}
$$

where

$$
\begin{gathered}
x_{1}=\frac{t-T_{n}-\sum_{i=2}^{n-2}\left(T_{i}-T_{n}\right) x_{i}}{T_{1}-T_{n}} \\
x_{n}=\frac{t-T_{1}-\sum_{i=2}^{n-2}\left(T_{i}-T_{1}\right) x_{i}}{T_{n}-T_{1} x}
\end{gathered}
$$

and the limits of the integration are given as

$$
x_{i}^{M}=\frac{t-T_{M}-\sum_{j=2}^{i-1} x_{j}\left(T_{j}-T_{M}\right)}{T_{i}-T_{M}}
$$

where $T_{M}$ is $T_{n}$ if $t>T_{i}$, or $T_{1}$ if $t<T_{i}$.

However, the analytical expression for the transfer functions $g_{4, i j}, g_{5, i j}, g_{6, i j}$, etc., are too complicated to be implemented in the computer program. Another methodology was developed to find the time integral of the transfer functions, $\int g_{n, i j}(t) d t$ and $\int G_{n, i j}(t) d t$, when $n$ is greater than 3. This methodology is described in Section I.3. 


\section{I.3 TRANSFER FUNCTION TIME INTEGRALS}

For the general case in which there are $n$ nuclides involved in the decay chain, the transport path of fixed length can be broken into $n$ segments whose fractional length can be denoted by $x_{i}$, along which the $i$ th nuclide in the decay chain travels. The summation of the fractional length $x_{i}$ is then $1, \sum_{i=1}^{n} x_{i}=1$. Considering the decay of nuclide $i$ into $i+1$ at the depth given by $\sum_{j=i}^{i} x_{j}$ and width $\Delta x_{i}$, the contribution to nuclide $n$ at the bottom is given by

$$
\begin{aligned}
& c_{n}=\left(\prod_{i=1}^{n-1} \lambda_{i} T_{i} e^{-\lambda_{i} T_{i} X_{i}} \Delta x_{i}\right) e^{-\lambda_{n} T_{n} X_{n}} \\
& =e^{-\lambda_{n} T_{n}} \prod_{i=1}^{n-1} \lambda_{i} T_{i} e^{-\left(\lambda_{i} T_{i}-\lambda_{n} T_{n}\right) x_{i}} \Delta x_{i}
\end{aligned}
$$

To obtain the time integral, this function must be integrated over all the $x$ 's. This is an $n-1$ dimensional integral. If $x_{n-1}$ is integrated first, the limits of $x_{n-1}$ are from 0 to $1-\sum_{j=1}^{n-2} x_{j}$. Next, the limits of $x_{n-2}$ are from 0 to $1-\sum_{j=1}^{n-3} x_{j}$. This continues until the last integral over $x_{1}$ is completed from 0 to 1 . The integral is equivalent to the time-integrated value of the transfer function $g_{n}(t)$ and can be written as

$$
\Omega_{n}=\int g_{n}(t) d t=e^{-\lambda_{n} T_{n}} I_{n-1}\left(\alpha_{1}, \ldots, \alpha_{n-1}\right) \prod_{i=1}^{n-1} \lambda_{i} T_{i}
$$

where the integral function is defined as

$$
I_{n}\left(\alpha_{1}, \ldots \alpha_{n}\right)=\int_{0}^{1} \int_{0}^{1-x_{1}} \int_{0}^{1-x_{2}} \ldots \int_{0}^{1-\sum_{i=1}^{n-1}} e^{-\sum_{j=1}^{n} \alpha_{j} x_{j}} d x_{n} \ldots d x_{3} d x_{2} d x_{1}
$$

and the parameter $\alpha_{i}$ is defined as 


$$
\alpha_{i}=\lambda_{i} T_{i}-\lambda_{n+1} T_{n+1}
$$

Performing the first (innermost) integral will show the recursive relation (with initialization, i.e., $I_{0}=1$ ):

$$
I_{n}\left(\alpha_{1}, \ldots, \alpha_{n-1}\right)=\frac{I_{n-1}\left(\alpha_{1}, \ldots, \alpha_{n-1}\right)-e^{-\alpha_{n}} I_{n-1}\left(\alpha_{1}-\alpha_{n}, \ldots, \alpha_{n-1}-\alpha_{n}\right)}{\alpha_{n}}
$$

When the radionuclides travel with the same velocity, the above expression for the integral of the transfer function (Equation I.27) reduces to Bateman's equation.

This methodology can be extended to obtain the time integral of the transfer function $G_{n}(t)$ through both the unsaturated and saturated zones by assuming that the relative transport speeds (speed ratios) of radionuclides in the saturated zone are the same as those in the unsaturated zone. Equation 1.27 can be expressed as

$$
\Omega_{n}=\sum_{i=1}^{n} a_{i} e^{-\lambda_{i} T_{i}}
$$

where

$$
a_{i}=\sum_{i=1}^{n} \frac{\prod_{j=1}^{n-1} \lambda_{j} T_{j}}{\prod_{j=1, j \neq i}^{n}\left(\lambda_{j} T_{j}-\lambda_{i} T_{i}\right)}
$$

Assuming the ratio of transit times between radionuclide $j$ and radionuclide 1 is $F_{j}$, then $a_{i}$ can be written as

$$
a_{i}=\sum_{i=1}^{n} \frac{\prod_{j=1}^{n-1} \lambda_{j} F_{j}}{\prod_{j=1, j \neq i}^{n}\left(\lambda_{j} F_{j}-\lambda_{i} F_{i}\right)} .
$$

The time-integrated value of $G_{n}(t)$ can be obtained by replacing $T_{i}$ with $T_{u i}+x T_{s i}$, then integrating the right side of the equation over $x$ within the limits of 0 and 1. 


$$
\begin{aligned}
\Omega_{n}^{s+u} & =\int G_{n}(t) d t=\int_{0}^{1} \sum_{i=1}^{n} a_{i} e^{-\lambda_{i}\left(T_{u i}+x T_{s i}\right)} d x \\
& =\sum_{i=1}^{n} \frac{a_{i}}{\lambda_{i} T_{s i}}\left[e^{-\lambda_{i} T_{u i}}-e^{\lambda_{i}\left(T_{u i}+T_{s i}\right)}\right]
\end{aligned}
$$

where $T_{u i}$ is the transit time through the unsaturated zone for radionuclide $i$, and $T_{s i}$ is the transit time through the saturated zone for radionuclide $i$. Parameters $T_{u i}$ and $T_{s i}$ can be calculated by using Equations E.23 and E.26 or E.27, respectively.

The time-integrated value $\Omega_{n}{ }^{s+u}$ can be used in conjunction with the time distribution of the transfer function, $G_{n}(t)$, to estimate the water/soil concentration ratios. The shape of the time distribution depends on the relative transport speed of the radionuclide of interest at the point of water use compared to all the radionuclides that appeared earlier in the decay chain. For a decay chain with a length greater than three, the following approximation is used in RESRAD. If the slowest moving radionuclide is one of the first three in the decay chain, then the time distribution of the third radionuclide in the chain, $G_{3}(t)$, is used for the rest of the radionuclides. Otherwise, a constant time distribution between the breakthrough time of the fastest moving radionuclide through the unsaturated zone and a cutoff time is assumed. The cutoff time is the largest of the individual cutoff times of radionuclides in the decay chain. The individual cutoff time of radionuclide $i$ is defined as the smaller of $T_{u i}+T_{s i}$ and the relative decay time $\tau_{i}$. The relative decay time $\tau_{i}$ is the sum of the smallest unsaturated zone breakthrough time for all of the radionuclides in the decay chain plus twice the inverse of the decay constant of radionuclide $i$. Once the time distribution of the transfer function, $G_{n}$, is determined, the time-integrated value $\Omega_{n}{ }^{s+u}$ is used to normalize the transfer function.

In general, the derivation of the transfer function through the unsaturated and saturated zone, $G(t)$, used in RESRAD can be illustrated by the following example. Consider a decay chain of $n$ radionuclides: 
nuclide $1 \rightarrow$ nuclide $2 \rightarrow$ nuclide $3 \ldots \rightarrow$ nuclide $n$,

where the numbers are used to indicate the decay order, $n$ is used for the radionuclide of interest at the point of water use and 1 is used for the initial parent radionuclide leaching out from the contaminated zone. If $n \leq 3$, the transfer function $G_{n}(t)$ is derived by using the mathematical model described in Section I.2. For $n>3$, the transfer function $G_{n}(t)$ is

$$
G_{n}(t)=G_{3}(t) \times \frac{\Omega_{n}^{u+s}}{\Omega_{3}^{u+s}},
$$

if the maximum of $\left(T_{u 1}+T_{s 1}, T_{u 2}+T_{s 2}, \ldots T_{u n}+T_{s n}\right)$ is $\left(T_{u 1}+T_{s 1}\right),\left(T_{u 2}+T_{s 2}\right)$, or $\left(T_{u 3}+\right.$ $\left.T_{s 3}\right)$, or

$$
G_{n}(t)=\frac{\Omega_{n}^{u+\mathrm{s}}}{T_{c u t}-T_{u, \min }},
$$

where $T_{u, \min }=$ the minimum of $\left(T_{u 1}, T_{u 3}, \ldots T_{u n}\right), T_{c u t}=$ the maximum of $\left(T_{c u t, 1}, T_{c u t, 2}\right.$, $\left.T_{c u t, 3 \cdots} \ldots T_{c u t, n}\right)$, and $T_{c u t, i}$ is defined as the minimum of $\left(\left(T_{u i}+T_{s i}\right), T_{u, \min }+2 / \lambda_{i}\right)$. 
APPENDIX J:

ESTIMATION OF THE DISTRIBUTION COEFFICIENT

ON THE BASIS OF THE SOLUBILITY CONSTANT 


\section{APPENDIX J:}

\section{ESTIMATION OF THE DISTRIBUTION COEFFICIENT ON THE BASIS OF THE SOLUBILITY CONSTANT}

\section{J.1 INTRODUCTION}

The present leaching model in the RESRAD code requires the input of an empirical distribution coefficient, $K_{d}$, which represents the ratio of the solute concentration in soil to that in solution under equilibrium conditions. Parameters that affect the measurement of the distribution coefficient are the physical and chemical characteristics of the soil and the chemical species present in the contact solution. For soils contaminated with radionuclides, the radionuclides may be associated with particulate matter in several chemical forms, resulting in differences in leachability and solubility. When in contact with chemical species in solution, these radionuclides might be desorbed into the solution phase, possibly by dissolution or complexation. Therefore, the investigation of radionuclide distribution among different geochemical species and the solubility of these geochemical species will be useful for predicting the possible maximum concentration of a radionuclide in soil solution. The estimated maximum radionuclide concentration in soil solution can be further utilized to calculate the distribution coefficient under more conservative conditions for modeling purposes. The methodology for estimating the distribution coefficient on the basis of radionuclide solubility is presented, and sample calculations are provided to demonstrate the use of this methodology.

\section{J.2 METHODOLOGY}

In the earliest stage of the leaching process, geochemical species with high solubilities are first released into the solution phase by dissolution. As leaching proceeds, geochemical species with low solubilities will start to dissolve. The investigation of radionuclide distribution among different geochemical species can provide useful information for predicting the radionuclide concentration under equilibrium desorption conditions. Unfortunately, laboratory information on possible solid phase and solution species of radionuclides is usually unavailable, and the little information that is available is mostly very site- and solution-specific, making extrapolation of data very difficult. To estimate the possible solids present in a given soil solution system, available thermodynamic data were 
used to construct Eh-pH diagrams (Garrels and Christ 1965; Pourbaix 1966) and activity-pH diagrams (Rai and Lindsay 1975; Rai and Serne 1977). Ames and Rai (1978) reported that although Eh-pH diagrams can be used to predict the presence of different solids for elements that exist in more than one oxidation state, these diagrams do not indicate the total amount of the element and the relative amount of various species in solution under specific conditions. Therefore, activity-pH diagrams were used.

\section{J.2.1 Activity-pH Diagrams for Radionuclides}

The methodology used for developing a stability and/or activity-pH diagram utilizes thermodynamic standard free energies of formation $\left(\Delta \mathrm{G}_{f}^{\circ}\right)$ to calculate equilibrium constants for various minerals/solids under standard conditions, that is, $1 \mathrm{~atm}$ and $25^{\circ} \mathrm{C}$. On the basis of the estimated equilibrium reaction constant, the equation for the activities of related chemical species in a specific mineral or solid can be formulated. For any given soil solution system for which there is insufficient chemical information, activities of common cations (such as $\mathrm{Ca}^{+2}, \mathrm{Mg}^{+2}, \mathrm{~K}^{+}, \mathrm{Na}^{+}$, etc.) and anions (such as $\mathrm{Cl}^{-}, \mathrm{HCO}_{3}^{-}, \mathrm{CO}_{3}^{-2}, \mathrm{NO}_{3}^{-}, \mathrm{F}^{-}, \mathrm{SO}_{4}^{-2}$, and $\mathrm{PO}_{4}^{-3}$ ) are usually selected to represent expected environmental conditions. The equation for the activity of the radionuclide being investigated can then be simplified to be a function of the solution $\mathrm{pH}$. For each radionuclide investigated, the stability diagram can be constructed so as to provide information on the saturate solubility of the radionuclide under given environmental conditions with different solution $\mathrm{pHs}$. Typical soil concentrations of selected elements and inorganic anions have been reported by Rai and Lindsay (1975), Dvorak et al. (1978), and Nieto and Frankenberger (1985). For sites with specific soil solution properties, the stability diagram can be modified to correspond to the actual ionic activities. This modification would result in an upward or downward shift of the isotherms. Rai and Lindsay (1975) used Al-minerals, such as analcime $\left(\mathrm{NaAlSi}_{2} \mathrm{O}_{6} \cdot \mathrm{H}_{2} \mathrm{O}\right.$ ), to present the details of the method for constructing stability diagrams.

Chemical information on contaminated soils and contact solutions is needed for an accurate prediction of possible reactions in the soil-solution system. To aid in estimating the possible solution composition under typical environmental conditions, stability diagrams available in the literature are reviewed. Stability diagrams are summarized in Section J.4 for some of the radionuclides considered in the current version of RESRAD. 


\section{J.2.2 Estimation of the Saturate Solubility for a Specific Radionuclide}

Consider a soil-solution system in which the initial concentration of radionuclide $i$ in soil is $S_{i}(0)$ (pCi/g soil). Assume that all of radionuclide $i$ can be released into the soil solution under the worst desorption conditions; the total activity of radionuclide $i$ in this soil solution system $C_{\text {tot, } i}(\mathrm{~mol} / \mathrm{L})$ is

$$
C_{\text {tot }, i}=S_{i}(0) \times M_{s} \times \frac{1}{S A_{i}} \times \frac{1}{A W_{i}} \times \frac{1}{V_{\text {solution }}} \times \frac{1,000 \mathrm{~mL}}{\mathrm{~L}},
$$

where

$$
\begin{aligned}
M s & =\text { total mass of contaminated soil }(\mathrm{g}), \\
& =\text { volume of contaminated soil } \times \text { bulk density of soil, } \\
& =V \times \rho_{b}, \\
V_{\text {solution }} & =\text { volume of soil solution }(\mathrm{mL}), \\
& =\text { volume of contaminated soil } \times \text { total porosity } \times \text { saturation } \\
& \text { ratio, } \\
& =V \times p_{t} \times R_{s}, \\
S A_{i} & =\text { specific activity of radionuclide } i(\mathrm{pCi} / \mathrm{g}), \\
A W_{i} & =\text { atomic weight of radionuclide } i(\mathrm{~g} / \mathrm{mole}),
\end{aligned}
$$

and

$$
C_{t o t, i}=S_{i}(0) \times \frac{\rho_{b}}{p_{t} R_{s}} \times \frac{1}{S A_{i}} \times \frac{1}{A W_{i}} \times 1,000\left(\frac{\mathrm{mol}}{\mathrm{L}}\right)
$$

In the stability diagram of radionuclide $i$, any solid with its stability line below another solid's stability line is more stable than another solid. Thus, the maximum activity of radionuclide $i$ in soil solution $\left(C_{t o t, i}\right)$ in the stability diagram can be used as the starting point to search for the saturate solubility by the following process:

1. In the stability diagram for radionuclide $i$, draw a horizontal line $\mathrm{L}_{1}$ with $\log A_{\mathrm{i}}=\log C_{t o t, i}$ to represent the total activity of radionuclide $i$ in 
the soil solution system. Any solids with a solubility line below $\mathrm{L}_{1}$ might be present as stable solids in the given soil solution system.

2. At a specific solution $\mathrm{pH}$, draw a vertical line $\mathrm{L}_{2}$. The intercept between $\mathrm{L}_{2}$ and the solubility line right below $L_{1}$ indicates the activity of radionuclide $i$ in a saturate soil solution, that is, saturate solubility $S_{\max }$, at the specific solution $\mathrm{pH}$.

\section{J.2.3 Estimation of the Distribution Coefficient for Input into RESRAD}

For the soil solution system discussed above, assume that, at equilibrium conditions, $\mathrm{X}$ fraction (\%) of total activity of radionuclide $i$ will be released into the solution phase. On the basis of the radioactivity measurement, the empirical distribution coefficient $K_{d}$ for radionuclide $i$ is defined as

$$
K_{d_{i}}=\frac{\text { Radioactivity Remaining in Soil Phase, } p \text { Ci/g soil }}{\text { Radioactivity Released into Solution Phase, } p \text { Ci/mL solution }} .
$$

Then

$$
\begin{gathered}
K_{d_{i}}=\frac{S_{i}(0) \times(1-X) \times M_{s}}{M_{s}} \times \frac{V_{\text {solution }}}{S_{i}(0) \times X \times M_{s}} \\
=\frac{1-X}{X} \times \frac{p_{t} R_{s}}{\rho_{b}}
\end{gathered}
$$

Calculation of the distribution coefficient can be performed by using the following procedure:

1. Calculate the maximum mass concentration of radionuclide $i, C_{\max }$, on the basis of the estimated saturate solubility.

$$
\begin{gathered}
C_{\max }=S_{\max }\left(\frac{\mathrm{mol}}{\mathrm{L}}\right) \times \frac{\mathrm{L}}{1,000 \mathrm{~mL}} \times A W_{i}\left(\frac{\mathrm{g}}{\mathrm{mol}}\right),\left(\frac{\mathrm{g}}{\mathrm{mL} \mathrm{solution}}\right) \\
S_{\max }=\text { Saturate Solubility }
\end{gathered}
$$


2. Calculate the fraction of the total radionuclide mass released into the solution phase, $X_{\max }$

$$
\begin{gathered}
X_{\max }=\frac{C_{\max } \times S A_{i} \times V_{\text {solution }}}{S_{i}(0) \times M_{s}} \\
=C_{\max } \times \frac{S A_{i}}{S_{i}(0)} \times \frac{p_{t} R_{s}}{\rho_{b}}
\end{gathered}
$$

3. Calculate the distribution coefficient.

$$
K_{d_{i}}=\frac{1-X_{\max }}{X_{\max }} \times \frac{p_{t} R_{s}}{\rho_{b}}
$$

\section{J.3 SAMPLE CALCULATION}

A sample calculation is summarized in this section by using uranium-238 as the principle contaminant. Consider a contaminated site with the following properties:

Contaminated soil

Area

$1,000 \mathrm{~m}^{2}$

Depth

$1 \mathrm{~m}$

Bulk density, $\rho_{b}$

$1.6 \mathrm{~g} / \mathrm{cm}^{3}$

Total porosity, $\mathbf{p}_{\mathrm{t}}$

0.4

Saturation ratio, $R_{s}$

0.5

Contaminant (uranium-238)

Initial concentration

$1,000 \mathrm{pCi} / \mathrm{g}$ soil

Specific activity

$3.36 \times 10^{5} \mathrm{pCi} / \mathrm{g}$

1. Calculate the total activity of uranium-238 in the soil solution system. 


$$
\begin{gathered}
C_{t o t, i}=S_{i}(0) \times \frac{\rho_{b}}{p_{t} R_{s}} \times \frac{1}{S A_{i}} \times \frac{1}{A W_{i}} \times 1,000 \\
=1,000 \times \frac{1.6}{0.4 \times 0.5} \times \frac{1}{\left(3.36 \times 10^{5}\right)} \times \frac{1}{238.1} \times 1,000=0.1\left(\frac{\mathrm{mol}}{\mathrm{L}}\right)
\end{gathered}
$$

2. Estimate the saturate solubility of uranium-238 in solution. On the basis of the stability diagram for $\mathrm{UO}_{2}{ }^{+2}$ shown in Figure J.11, draw a horizontal line $\mathrm{L}_{1}$ with $\log A_{i}=-1$ to represent the total activity of uranium-238. Draw a vertical line $\mathrm{L}_{2}$ to represent the solution $\mathrm{pH}$. The $\mathrm{UO}_{2}{ }^{+2}$ solid with a stability line right below $\mathrm{L}_{1}$ at each solution $\mathrm{pH}$ is used as the reference solid. The intercept between $\mathrm{L}_{2}$ and the stability line of the reference solid indicates the saturate solubility of $\mathrm{UO}_{2}{ }^{+2}$ in the soil solution system investigated. For soil solution systems with a solution $\mathrm{pH}$ varying from 4 to 9 , the estimated saturate solubilities are listed below.

\begin{tabular}{clc}
\hline Solution pH & Reference Solid & $\begin{array}{c}\text { Saturate Solubility } \\
\mathrm{S}_{\text {max }}, \text { mol/L }\end{array}$ \\
\hline 4 & $\mathrm{UO}_{2}(\mathrm{OH})_{2} \cdot \mathrm{H}_{2} \mathrm{O}$ & $4 \times 10^{-3}$ \\
5 & $\mathrm{UO}_{2}(\mathrm{OH})_{2} \cdot \mathrm{H}_{2} \mathrm{O}$ & $5 \times 10^{-5}$ \\
6 & $\mathrm{UO}_{2}(\mathrm{OH})_{2} \cdot \mathrm{H}_{2} \mathrm{O}$ & $3 \times 10^{-7}$ \\
7 & $\mathrm{UO}_{2} \mathrm{CO}_{3}$ & $1 \times 10^{-3}$ \\
8 & $\mathrm{UO}_{2} \mathrm{CO}_{3}$ & $1 \times 10^{-5}$ \\
9 & $\mathrm{UO}_{2} \mathrm{CO}_{3}$ & $1 \times 10^{-7}$ \\
\hline
\end{tabular}

3. Calculate the distribution coefficient for each soil solution system with a different solution $\mathrm{pH}$.

For solution $\mathrm{pH}=5$, the distribution coefficient is calculated on the basis of the following process.

a. Calculate the maximum mass concentration of uranium-238 in the soil solution as 


$$
\begin{aligned}
C_{\max }= & 5 \times 10^{-5} \frac{\mathrm{mol}}{\mathrm{L}} \times \frac{\mathrm{L}}{1,000 \mathrm{~mL}} \times \frac{238.1 \mathrm{~g}}{\mathrm{~mol}} \\
= & 1.19 \times 10^{-5} \frac{\mathrm{g} U-238}{\mathrm{~mL} \text { solution }} .
\end{aligned}
$$

b. Calculate the maximum fraction of total uranium-238 released into the solution phase as

$$
\begin{gathered}
X_{\max }=C_{\max } \times \frac{S A_{i}}{S_{i}(0)} \times \frac{p_{t} R_{s}}{\rho_{b}} \\
=1.19 \times 10^{-5} \times \frac{3.36 \times 10^{5}}{1,000} \times \frac{0.4 \times 0.5}{1.6}=5 \times 10^{-4} .
\end{gathered}
$$

c. Calculate the distribution coefficient as

$$
\begin{gathered}
K_{d_{i}}=\frac{1-X_{\max }}{X_{\max }} \times \frac{p_{t} R_{s}}{\rho_{b}} \\
K_{d_{i}}=\frac{1-\left(5 \times 10^{-4}\right)}{5 \times 10^{-4}} \times \frac{0.4 \times 0.5}{1.6}=250\left(\frac{\mathrm{ml}}{\mathrm{g}}\right) .
\end{gathered}
$$

4. The distribution coefficients, $K_{d}$, for uranium-238 in soil solution systems with different solution $\mathrm{pHs}$ are calculated and summarized below.

\begin{tabular}{cc}
\hline Solution $\mathrm{pH}$ & Calculated $\mathrm{K}_{\mathrm{d}}(\mathrm{mL} / \mathrm{g})$ \\
\hline 4 & 3 \\
5 & 250 \\
6 & $4.17 \times 10^{4}$ \\
7 & 12.38 \\
8 & $1.25 \times 10^{4}$ \\
9 & $1.25 \times 10^{5}$ \\
\hline
\end{tabular}




\section{J.4 RESRAD INPUT REQUIREMENTS}

The RESRAD code provides an additional option for calculating the distribution coefficient by using the solubility constant. To use this option, users need to input the solubility constant. With the input solubility constant, RESRAD will estimate a distribution coefficient corresponding to the solubility limit desorption conditions.

Constants of specific activity and atomic weight for radionuclides considered in the current RESRAD database are summarized in Table J.1.

\section{J.5 COMPILATION OF STABILITY DIAGRAMS}

The chemistry and geochemistry of radionuclides in RESRAD will be briefly reviewed in this section from the standpoint of the relative solubilities of possible solid phases. The work summarized below is based on the assumption that radiochemical properties of all isotopes of an element are identical in respect to their reaction with geological materials.

TABLE J.1 Constants of Atomic Weight and Specific Activity for Radionuclides in the Current RESRAD Database

\begin{tabular}{lcclcc}
\hline Radionuclide & $\begin{array}{c}\text { Atomic Weight } \\
\text { (g/mol) }\end{array}$ & $\begin{array}{c}\text { Specific Activity } \\
(\mathrm{pCi} / \mathrm{g})\end{array}$ & Radionuclide & $\begin{array}{c}\text { Atomic Weight } \\
\text { (g/mol) }\end{array}$ & $\begin{array}{c}\text { Specific Activity } \\
(\mathrm{pCi} / \mathrm{g})\end{array}$ \\
\hline Ac-227 & 227.1 & $7.24 \times 10^{13}$ & Ni-63 & 62.9 & $5.68 \times 10^{13}$ \\
Am-241 & 241.1 & $3.42 \times 10^{12}$ & Np-237 & 237.1 & $7.04 \times 10^{8}$ \\
Am-243 & 243.1 & $2.00 \times 10^{11}$ & Pa-231 & 231.1 & $4.72 \times 10^{10}$ \\
C-14 & 14.0 & $4.45 \times 10^{12}$ & Pb-210 & 210.0 & $7.63 \times 10^{13}$ \\
Ca-41 & 41.0 & $8.47 \times 10^{10}$ & Pu-238 & 238.1 & $1.71 \times 10^{13}$ \\
Ce-144 & 144.0 & $3.19 \times 10^{15}$ & Pu-239 & 239.1 & $6.20 \times 10^{10}$ \\
Cl-36 & 36.0 & $3.30 \times 10^{10}$ & Pu-240 & 240.1 & $2.27 \times 10^{11}$ \\
Cm-243 & 243.1 & $5.16 \times 10^{13}$ & Pu-241 & 241.1 & $1.03 \times 10^{14}$ \\
Cm-244 & 244.1 & $8.09 \times 10^{13}$ & Pu-242 & 242.1 & $3.93 \times 10^{9}$ \\
Co-57 & 56.9 & $8.46 \times 10^{15}$ & Ra-226 & 226.1 & $9.88 \times 10^{11}$ \\
Co-60 & 59.9 & $1.13 \times 10^{15}$ & Ra-228 & 228.1 & $2.72 \times 10^{14}$ \\
Cs-134 & 134.0 & $1.29 \times 10^{15}$ & Ru-106 & 106.0 & $3.26 \times 10^{15}$ \\
Cs-135 & 135.9 & $8.83 \times 10^{8}$ & Sb-125 & 125.0 & $1.03 \times 10^{15}$ \\
Cs-137 & 137.0 & $8.65 \times 10^{13}$ & Sm-151 & 151.0 & $2.63 \times 10^{13}$ \\
Eu-152 & 152.0 & $1.81 \times 10^{14}$ & Sr-90 & 89.9 & $1.38 \times 10^{14}$ \\
Eu-154 & 154.0 & $2.73 \times 10^{14}$ & Tc-99 & 98.9 & $1.69 \times 10^{10}$ \\
Eu-155 & 155.0 & $4.65 \times 10^{14}$ & Th-228 & 228.1 & $8.19 \times 10^{14}$ \\
Fe-55 & 55.0 & $2.42 \times 10^{15}$ & Th-229 & 229.1 & $2.14 \times 10^{11}$ \\
Gd-152 & 152.0 & $2.18 \times 10^{1}$ & Th-230 & 230.1 & $2.02 \times 10^{10}$ \\
H-3 & 3.0 & $9.61 \times 10^{15}$ & Th-232 & 232.1 & $1.09 \times 10^{5}$ \\
I-129 & 129.0 & $1.73 \times 10^{8}$ & U-232 & 232.1 & $2.14 \times 10^{13}$ \\
K-40 & 40.0 & $6.99 \times 10^{6}$ & U-233 & 233.1 & $9.63 \times 10^{11}$ \\
Mn-54 & 54.0 & $7.74 \times 10^{15}$ & U-234 & 234.1 & $6.23 \times 10^{9}$ \\
Na-22 & 22.0 & $6.24 \times 10^{15}$ & U-235 & 235.1 & $2.16 \times 10^{6}$ \\
Nb-94 & 93.9 & $1.90 \times 10^{11}$ & U-236 & 236.1 & $6.46 \times 10^{8}$ \\
Ni-59 & 59.0 & $8.08 \times 10^{10}$ & U-238 & 238.1 & $3.36 \times 10^{5}$ \\
\hline & & & & &
\end{tabular}




\section{J.5.1 Americium}

Scant information is available on the possible solid phases of americium in soil and rock environments (Ames and Rai 1978). The relative solubility of several americium solids in an oxidizing environment $\left(\mathrm{pO}_{2}=0.68 \mathrm{~atm}\right.$ ) have been estimated by Latimer (1952) and Keller (1971) as a function of solution $\mathrm{pH}$. On the basis of this information, the stability diagram for americium solids has been constructed by Ames and Rai (1978) as shown in Figure J.1. It can be seen that the solubility of the americium solids decreases rapidly as the solution $\mathrm{pH}$ increases. Except for $\mathrm{Am}(\mathrm{OH})_{3}$, changing the soil solution system from oxidized to reduced conditions will increase the activities of $\mathrm{Am}^{+3}$ associated with each possible solid phase.

\section{J.5.2 Antimony}

Thermodynamic data for $\mathrm{Sb}_{2} \mathrm{O}_{3}$ (Sillen and Martell 1964), $\mathrm{Sb}_{4} \mathrm{O}_{6}, \mathrm{Sb}(\mathrm{OH})_{3}, \mathrm{Sb}_{2} \mathrm{O}_{4}$, $\mathrm{Sb}_{2} \mathrm{O}_{5}, \mathrm{Sb}_{2} \mathrm{~S}_{3}, \mathrm{SbCl}_{3}$, and $\mathrm{SbF}_{3}$ (Wagman et al., 1968) compounds were selected by Ames and Rai (1978) to construct the stability diagram shown in Figure J.2 for antimony solids in an oxidizing soil environment with $\mathrm{pO}_{2}=0.68$ atm. The $\mathrm{SbCl}_{3}, \mathrm{SbF}_{3}$, and $\mathrm{Sb}_{2} \mathrm{~S}_{3}$ solids are very soluble in the oxidizing environment and therefore are not shown in this figure.

\section{J.5.3 Cerium}

The stability diagram for cerium solids is shown in Figure J.3. The thermodynamic data for the compounds used for developing this figure were obtained from Schumm et al. (1973) for $\mathrm{Ce}_{2} \mathrm{O}_{3}$ and $\mathrm{CeO}_{2}$, Baes and Mesmer (1976) for $\mathrm{Ce}(\mathrm{OH})$, and Sillen and Martell (1964) for $\mathrm{CePO}_{4}$.

\section{J.5.4 Cesium}

Thermodynamic data are available for the following cesium solids: $\mathrm{Cs}(\mathrm{OH}), \mathrm{Cs}_{2} \mathrm{O}$, $\mathrm{CsCl}, \mathrm{CsClO}_{4}, \mathrm{Cs}_{2} \mathrm{SO}_{4}, \mathrm{Cs}_{2} \mathrm{CO}_{3}, \mathrm{CsHCO}_{3}, \mathrm{CsNO}_{3}$, and CsF. As reported by Ames and Rai (1978), all these solids are highly soluble and, therefore, stability diagrams for cesium are not presented. 


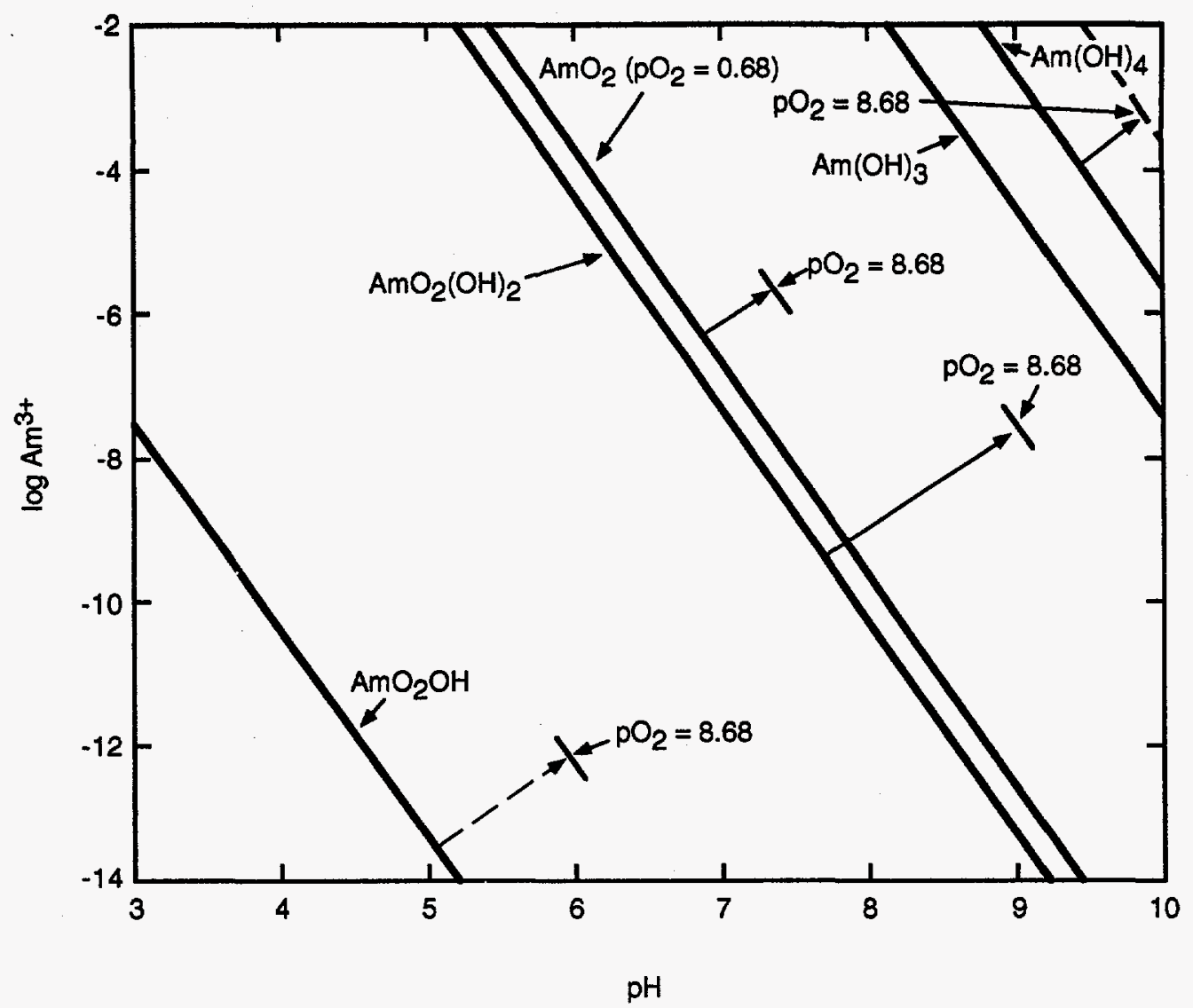

FIGURE J.1 Relative Stability of Various Americium Solids in an Oxidizing Soil Environment $\left[\mathrm{pO}_{2(\mathrm{~g})}=0.68 \mathrm{~atm}\right]$ (Source: Adapted from Ames and Rai 1978)

\section{J.5.5 Cobalt}

The stability of cobalt solids depends on the $\mathrm{pH}$ and the oxidation-reduction environment. Sources from which thermodynamic data for various cobalt solids were obtained were Sillen and Martell (1964) for $\mathrm{Co}(\mathrm{OH})_{3}, \mathrm{CoCO}_{3}$, and $\mathrm{CoOOH}$; Wagman et al. (1969) for $\mathrm{CoO}$ and $\mathrm{CoHPO}_{4}$; Robie and Waldbaum (1968) for $\mathrm{Co}_{3}\left(\mathrm{PO}_{4}\right)_{2}$; and Chase et al. (1975) for $\mathrm{Co}_{3} \mathrm{O}_{4}$. The stability diagram for cobalt is shown in Figure J.4.

\section{J.5.6 Curium}

Curium can form hydrides, hydroxides, halides, oxides, and organometallic compounds (Keller 1971). A search for thermodynamic data for these compounds was 


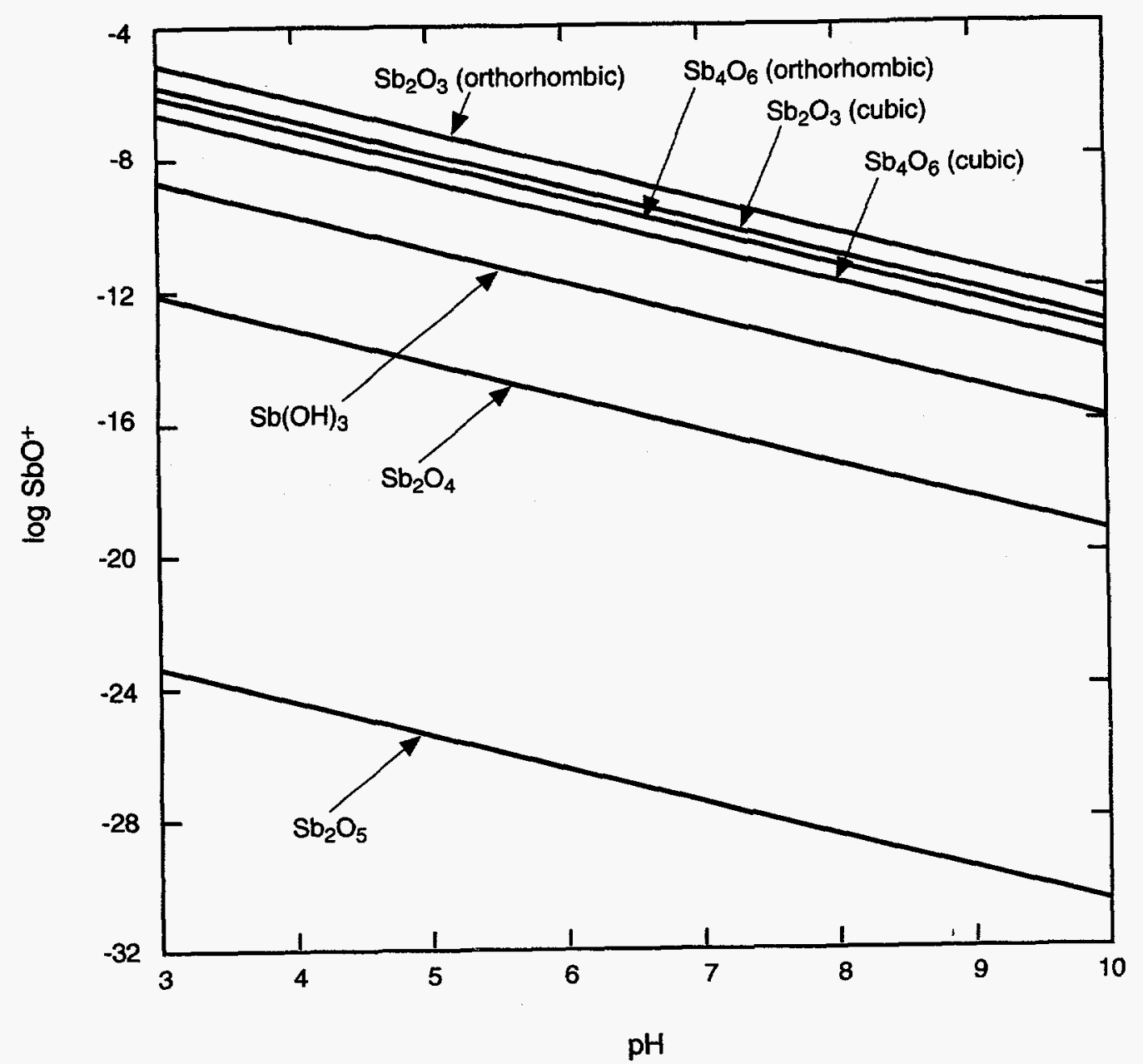

FIGURE J.2 Activity of $\mathrm{SbO}^{+}$in Equilibrium with Various Antimony Solids in an Oxidizing Soil Environment $\left[\mathrm{pO}_{2(\mathrm{~g})}=0.68 \mathrm{~atm}\right]$ (Source: Adapted from Ames and Rai 1978)

unsuccessful except for $\mathrm{CmF}_{3}$. Therefore, a stability diagram for curium cannot be developed at this time.

\section{J.5.7 Europium}

Europium forms oxides, hydroxides, and salts with chlorides and sulfates. The stability diagram for europium is shown in Figure J.5. The thermodynamic data for the compounds used for developing this figure were obtained from Shumm et al. (1973) for $\mathrm{Eu}_{2} \mathrm{O}_{3}$ and $\mathrm{Eu}(\mathrm{OH})_{3}$ and Latimer (1952) for $\mathrm{Eu}_{2}\left(\mathrm{SO}_{4}\right)_{3} \cdot 8 \mathrm{H}_{2} \mathrm{O}$. The other solids were too soluble and fall beyond the graph boundaries. 


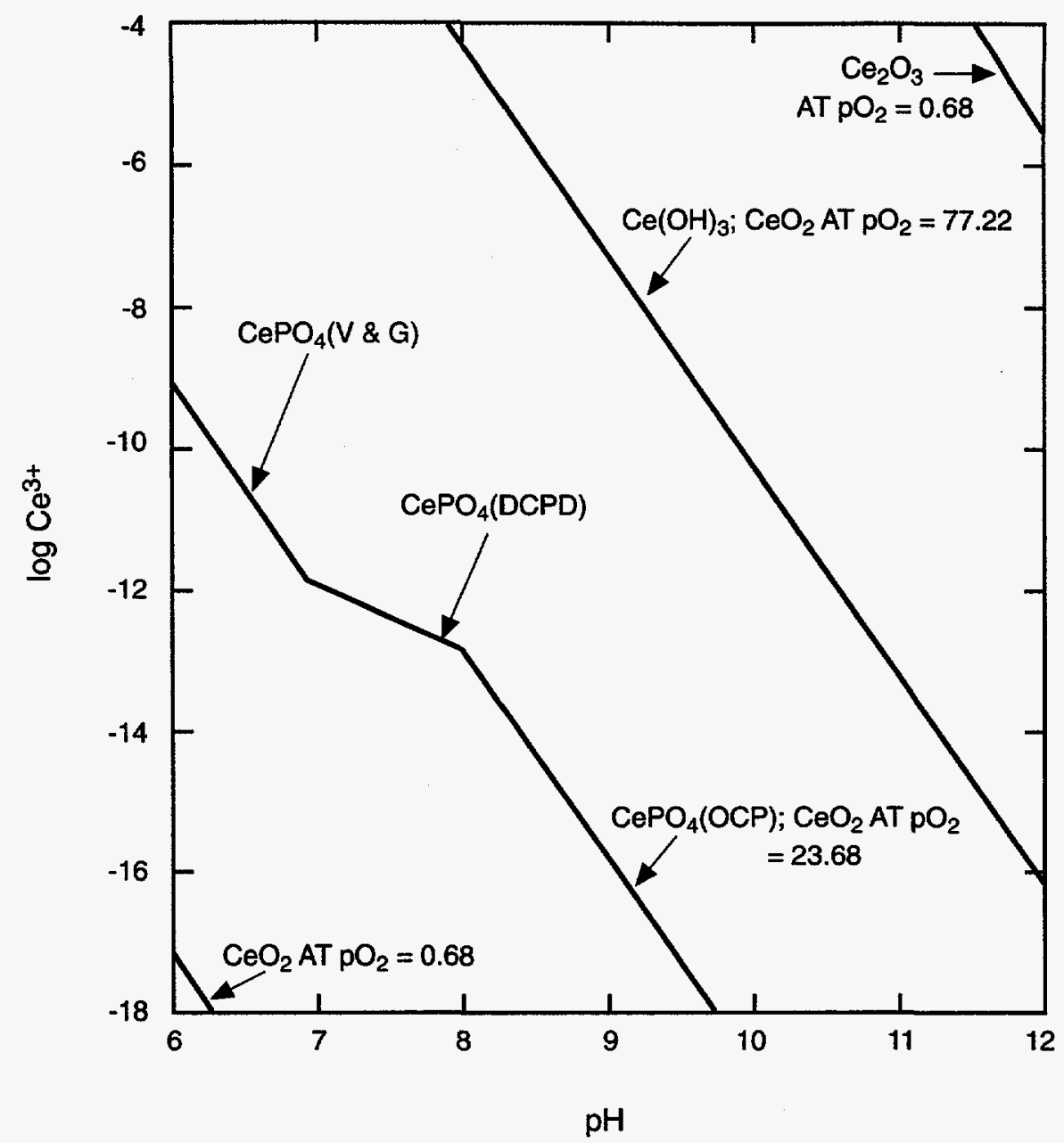

FIGURE J.3 Activity of $\mathrm{Ce}^{3+}$ in Equilibrium with Phosphate Levels from Variscite and Gibbsite (V \& G), Dicalcium Phosphate Dihydrate (DCPD), and Octacalcium Phosphate (OCP) (Source: Adapted from Ames and Rai 1978)

\section{J.5.8 Iodine}

Most iodine compounds are very soluble. Some of the insoluble or sparingly soluble compounds include the iodites of lead and palladium, the hypoiodites of silver and mercury, and barium periodates (Pourbaix 1966). In normal soils, the concentration of most of these elements (lead, palladium, arsenic, mercury, and barium) that form compounds with iodine is very low (Ames and Rai 1978). Therefore, they would not be present in soils. 


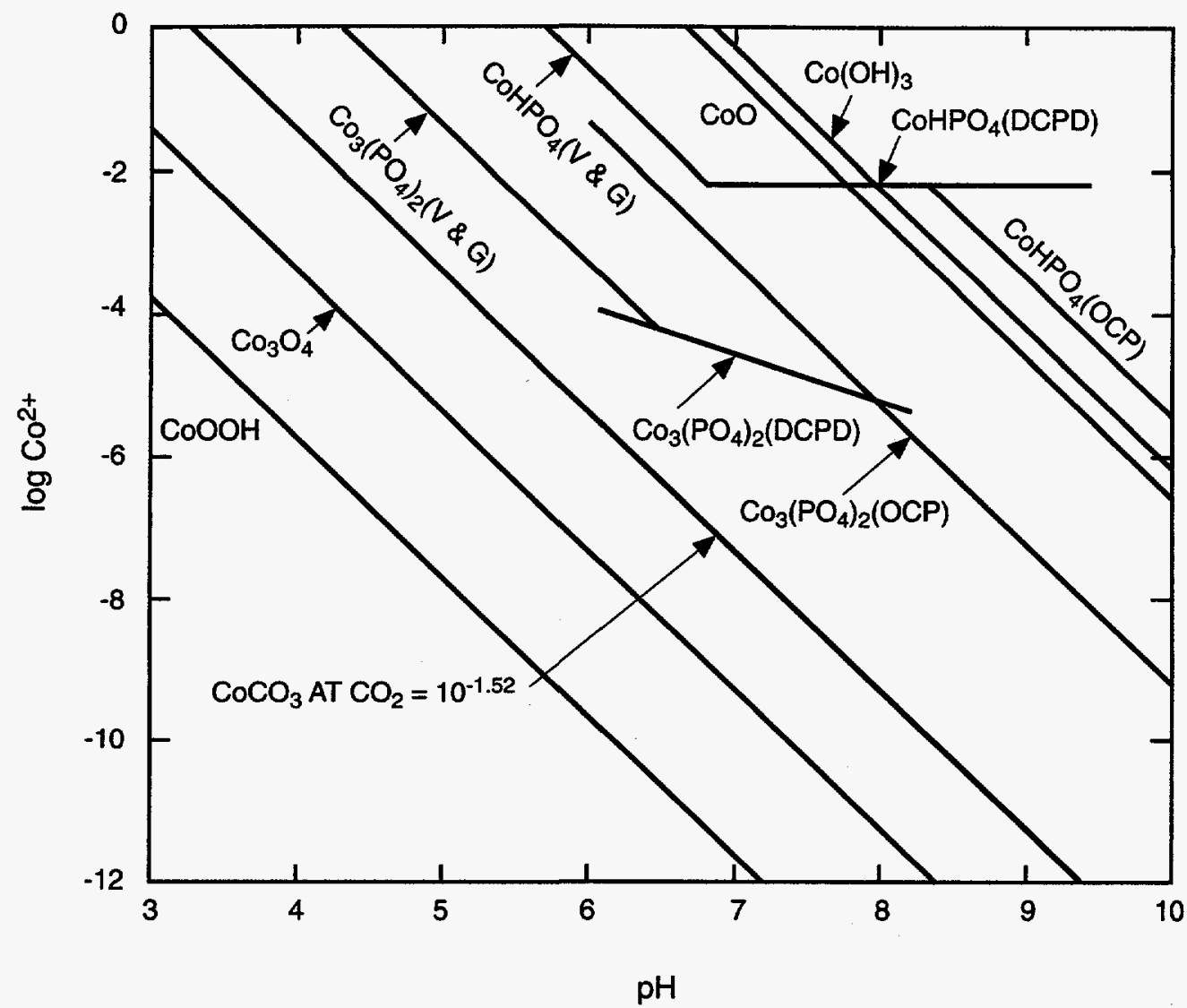

FIGURE J.4 Relative Stability of Cobalt Solids in an Oxidizing Soil Environment $\left[\mathrm{pO}_{2(\mathrm{~g})}=0.68 \mathrm{~atm}, \mathrm{pCa}^{2+}=2.5\right]$ with Phosphate Levels in Equilibrium with Variscite and Gibbsite (V \& G), Dicalcium Phosphate Dihydrate (DCPD), and Octacalcium Phosphate (OCP) (Source: Adapted from Ames and Rai 1978)

\section{J.5.9 Neptunium}

Thermodynamic data are unavailable for neptunium compounds other than oxides and hydroxides (Burney and Hartour 1974). The stability diagram for neptunium shown in Figure J.6 relates the activity of $\mathrm{NpO}_{2}{ }^{+}$to $\mathrm{pH}$ in an oxidizing environment $\left(\mathrm{pO}_{2}=0.68\right.$ atm $)$ in equilibrium with neptunium oxides and hydroxides.

\section{J.5.10 Plutonium}

The stability diagram for plutonium shown in Figure J.7 relates the activity of $\mathrm{Pu}^{+4}$ to $\mathrm{pH}$ in an oxidizing environment $\left(\mathrm{pO}_{2}=0.68 \mathrm{~atm}\right)$ in equilibrium with various plutonium solids. The parameters indicated in parentheses after the mineral formulas refer to the 


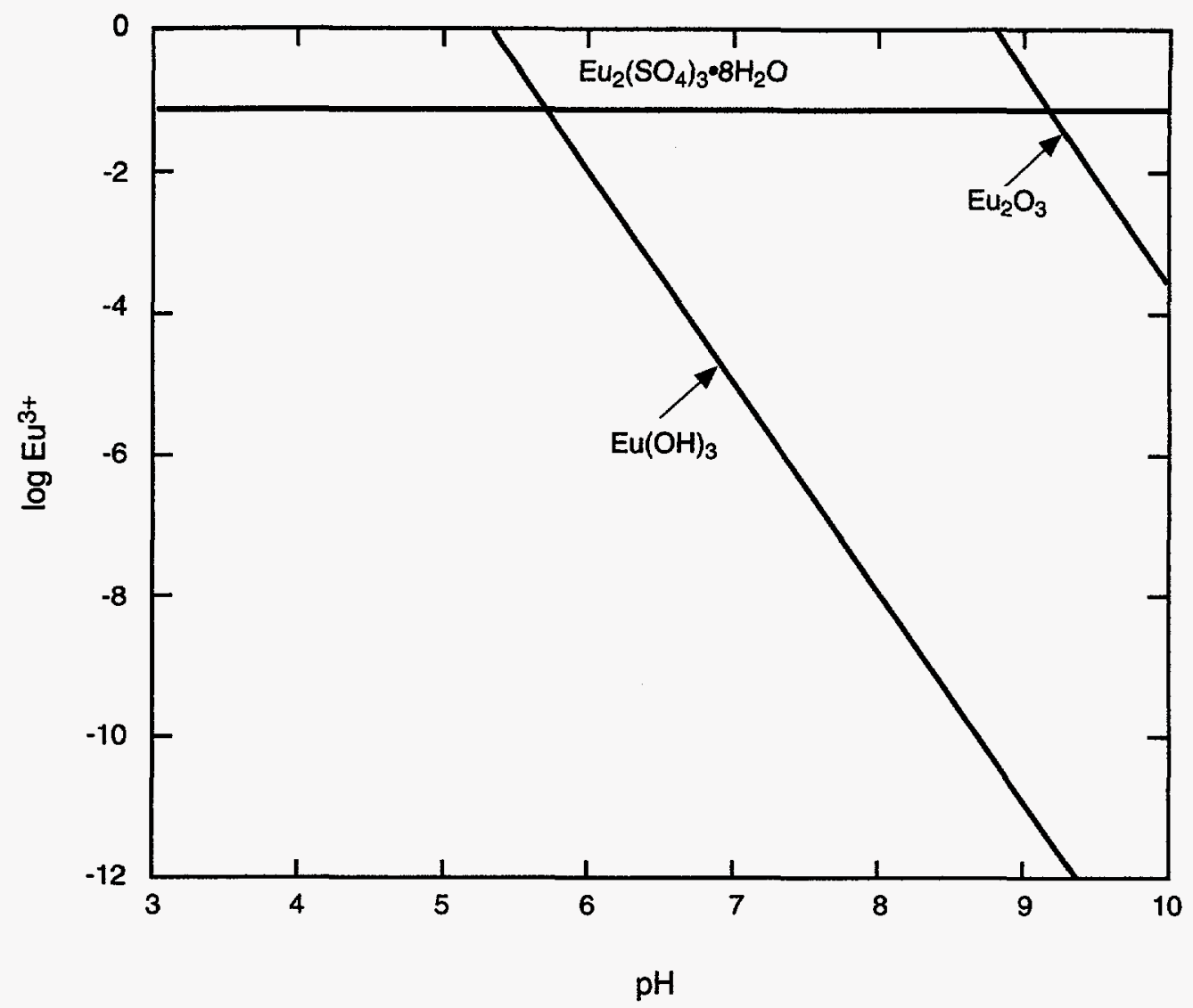

FIGURE J.5 Relative Stability of Various Europium Solids at $\mathrm{pSO}_{4}^{2-}=2.5$ (Source: Adapted from Ames and Rai 1978)

additional condition of equilibrium. For example, (V\&G) indicated after the $\mathrm{Pu}\left(\mathrm{HPO}_{4}\right)_{2}$ mineral formula denotes that the mineral is considered to be in equilibrium with variscite and gibbsite.

\section{J.5.11 Radium}

The compounds formed by radium and their solubilities are similar to barium. Thermodynamic data for radium compounds are available only for radium nitrate, chloride, iodate, and sulfate (Parker et al. 1971). All of the compounds except radium sulfate are very soluble. Therefore, the stability diagram for radium compounds is not presented. The solubility product for radium sulfate is $4.25 \times 10^{-11}$ at $25^{\circ} \mathrm{C}$ and $1 \mathrm{~atm}$. 


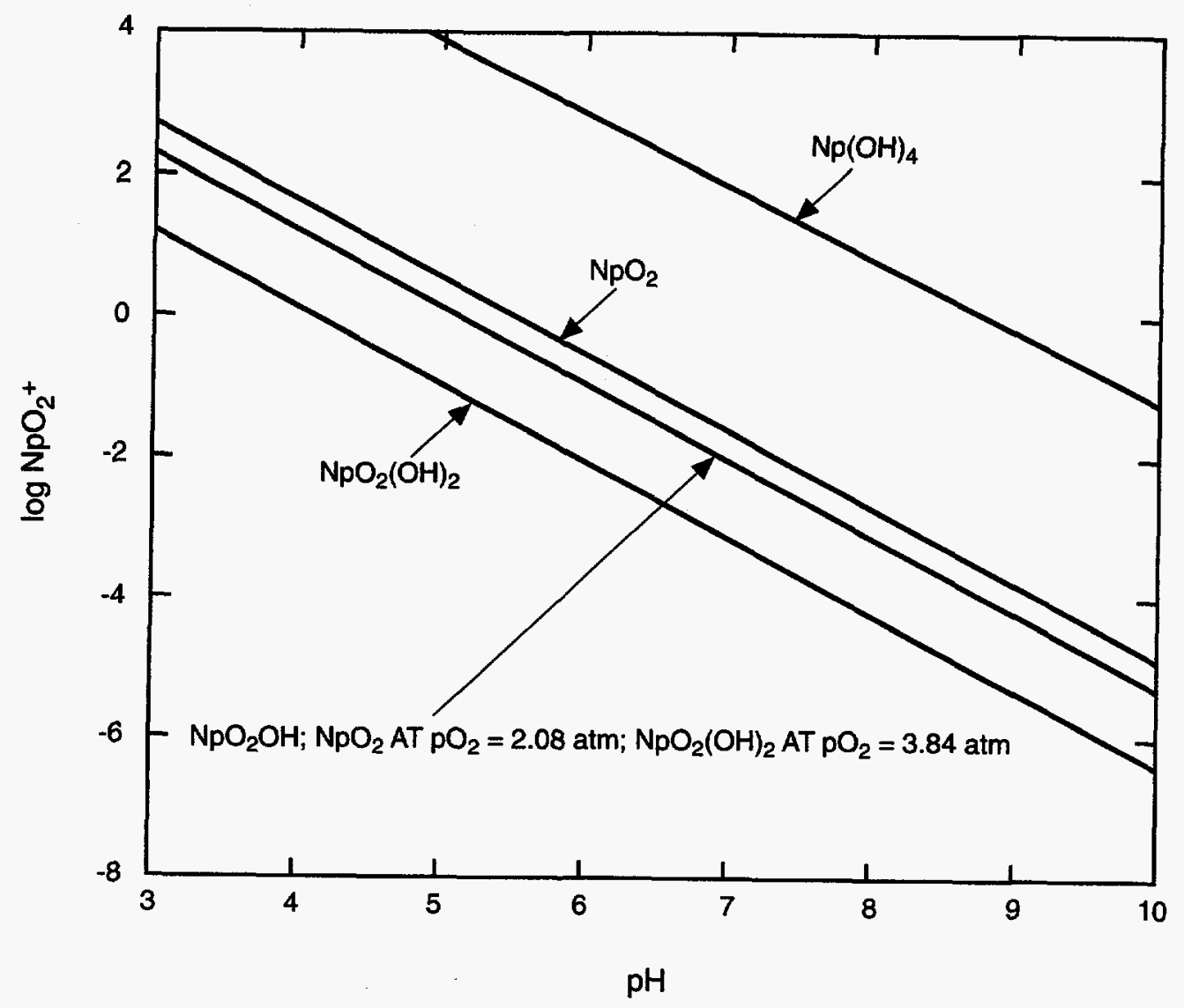

FIGURE J.6 Relative Stability of Various Neptunium Solids in an Oxidizing Soil Environment $\left[\mathrm{pO}_{2(\mathrm{~g})}=0.68 \mathrm{~atm}\right]$ (Source: Adapted from Ames and Rai 1978)

\section{J.5.12 Ruthenium}

Ruthenium is generally present in association with platinum group metals. Ruthenium can also form discrete solid compounds such as $\mathrm{RuO}_{2}, \mathrm{RuO}_{4}, \mathrm{Ru}(\mathrm{OH}), \mathrm{Ru}(\mathrm{OH})_{4}$, $\mathrm{RuCl}_{3}$, and $\mathrm{RuS}_{2}$. The stability diagram for ruthenium solids under oxidizing conditions $\left(\mathrm{pO}_{2}=0.68 \mathrm{~atm}\right)$ is shown in Figure J.8. The thermodynamic data for $\mathrm{Ru}(\mathrm{OH})_{3}$ and $\mathrm{Ru}(\mathrm{OH})_{4}$ were obtained from Sillen and Martell (1964); the data for $\mathrm{RuO}_{2}$ and $\mathrm{RuO}_{4}$ were obtained from Wagman (1969).

\section{J.5.13 Strontium}

Strontium is an alkaline-earth and forms several salts. Thermodynamic data for various strontium compounds are available in the literature. Except for $\mathrm{SrSiO}_{3}, \mathrm{SrHPO}_{4}$, 


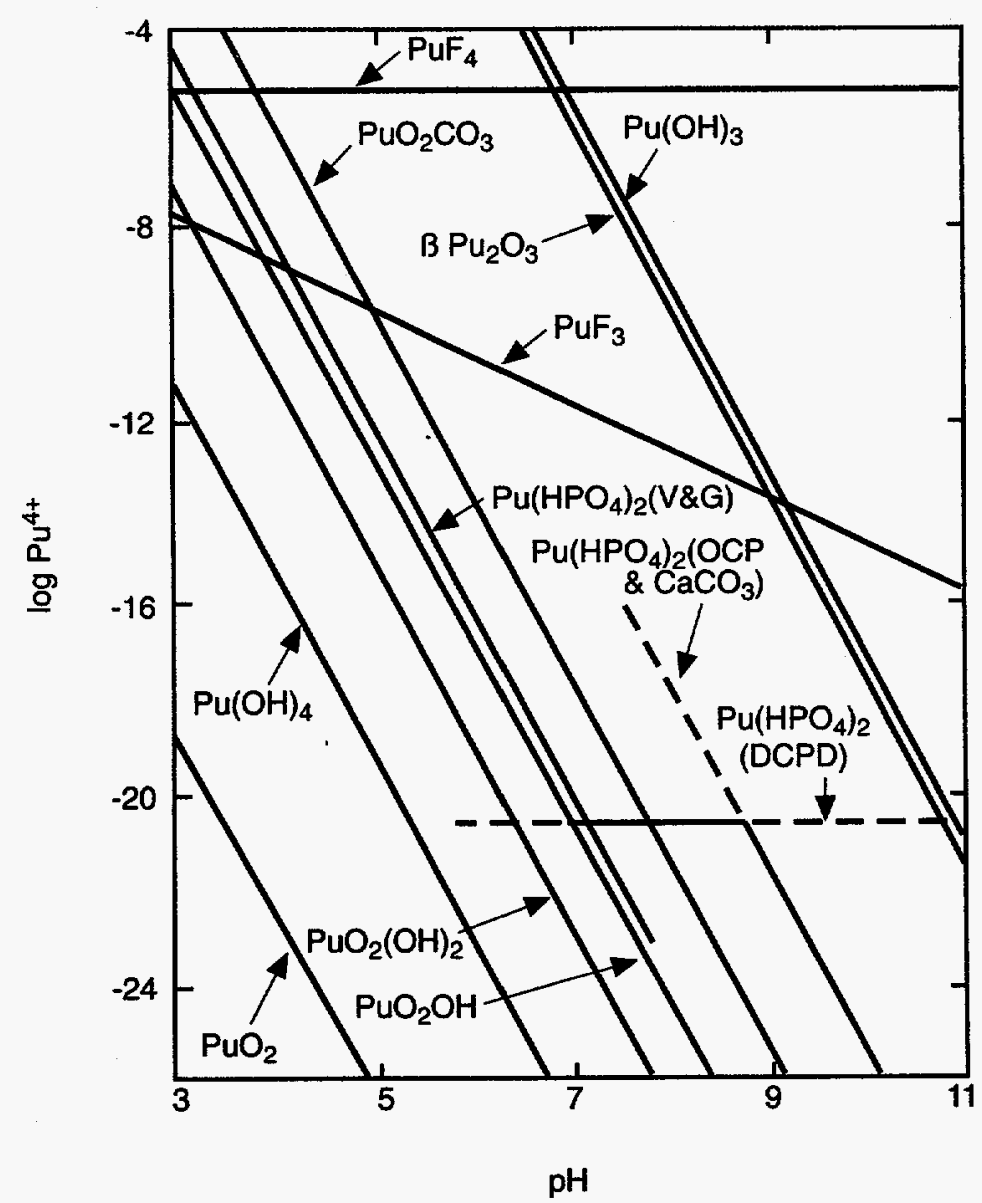

FIGURE J.7 Relative Stability of Various Plutonium Solids in an Oxidizing Soil Environment $\left[\mathrm{pO}_{2(\mathrm{~g})}=\right.$ 0.68 atm, $\mathrm{pCO}_{2(\mathrm{~g})}=3.52$ atm], $\mathrm{pF}^{-}=3.5$, with $\mathrm{Phosphate}$ Levels in Equilibrium with Variscite and Gibbsite (V \& G), Dicalcium Phosphate Dihydrate (DCPD), and Octacalcium Phosphate (OCP) (Source: Adapted from Ames and Rai 1978)

$\mathrm{Sr}_{3}\left(\mathrm{PO}_{4}\right)_{2}, \mathrm{SrCO}_{3}$, and $\mathrm{SrSO}_{4}$, the strontium solids are too soluble to construct a stability diagram. As shown in Figure J.9, the stability diagram for strontium was developed on the basis of thermodynamic data from Sillen and Martell (1964) for $\mathrm{Sr}_{3}\left(\mathrm{PO}_{4}\right)_{2}$ and Parker et al. (1971) for $\mathrm{SrSiO}_{3}, \mathrm{SrHPO}_{4}, \mathrm{SrCO}_{3}$, and $\mathrm{SrSO}_{4}$. 


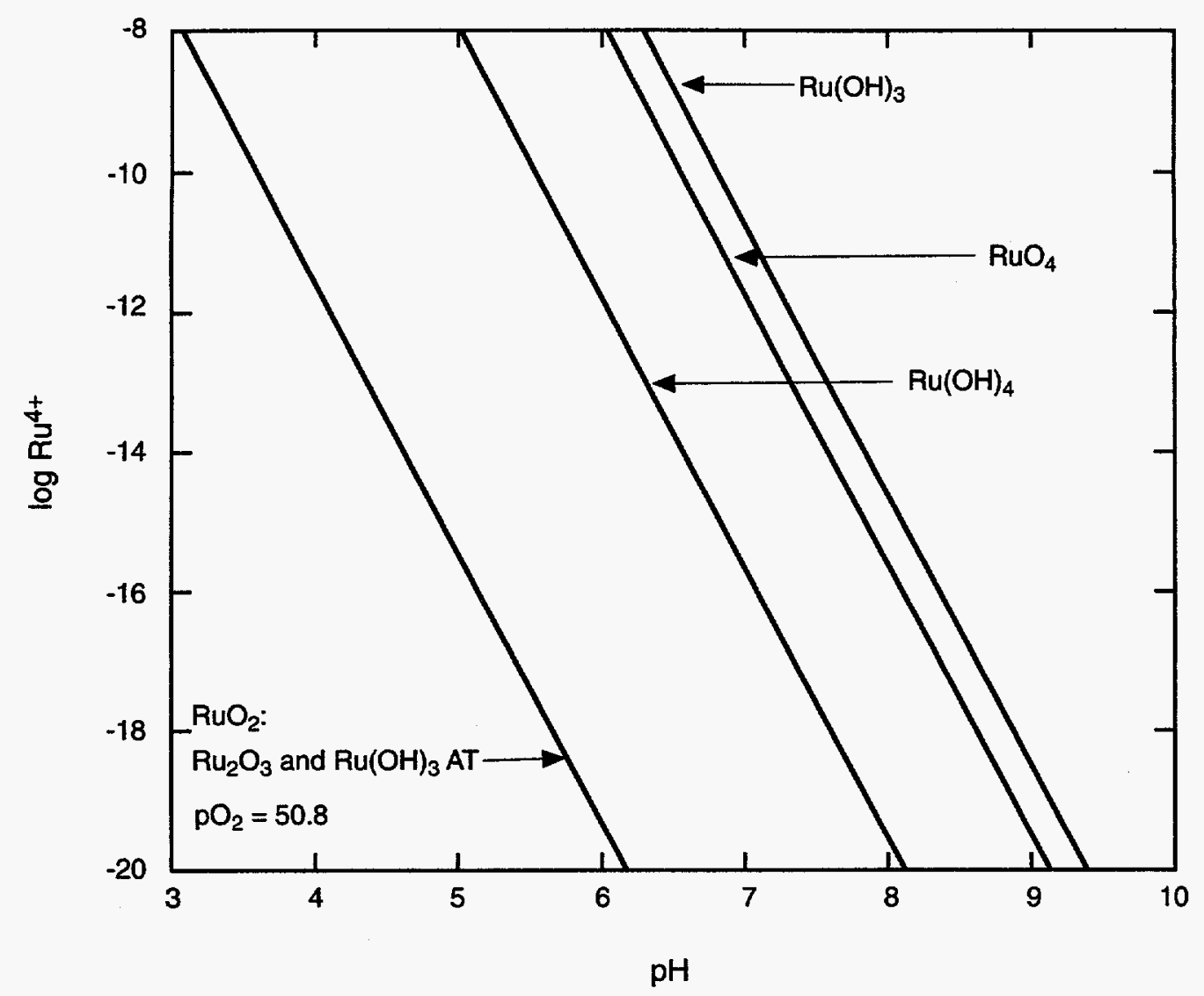

FIGURE J.8 Relative Stability of Various Ruthenium Solids in an Oxidizing Soil Environment $\left[\mathrm{pO}_{2(\mathrm{~g})}=0.68 \mathrm{~atm}, \mathrm{pCl}^{-}=\mathrm{pSO}_{4}{ }^{2-}=2.5\right]$ (Source: Adapted from Ames and Rai 1978)

\section{J.5.14 Technetium}

Baes and Mesmer (1976) reported that technetium (VII) forms strong peracids $\left(\mathrm{HMO}_{4}\right)$ and that its oxides are very soluble. Scant information is available concerning the solubility of its salts (Ames and Rai 1978).

\section{J.5.15 Thorium}

Common insoluble thorium compounds include hydroxides, fluorides, and phosphates. Soluble compounds include chlorides, nitrates, and sulfates. The stability diagram for thorium, as shown in Figure J.10, is constructed for an assumed environmental condition of equilibrium with various thorium solid phases. The thermodynamic data for $\mathrm{ThO}_{2}(\mathrm{~s})$ were selected from Baes and Mesmer (1976). The data for the other compounds were selected from Sillen and Martell (1964). 


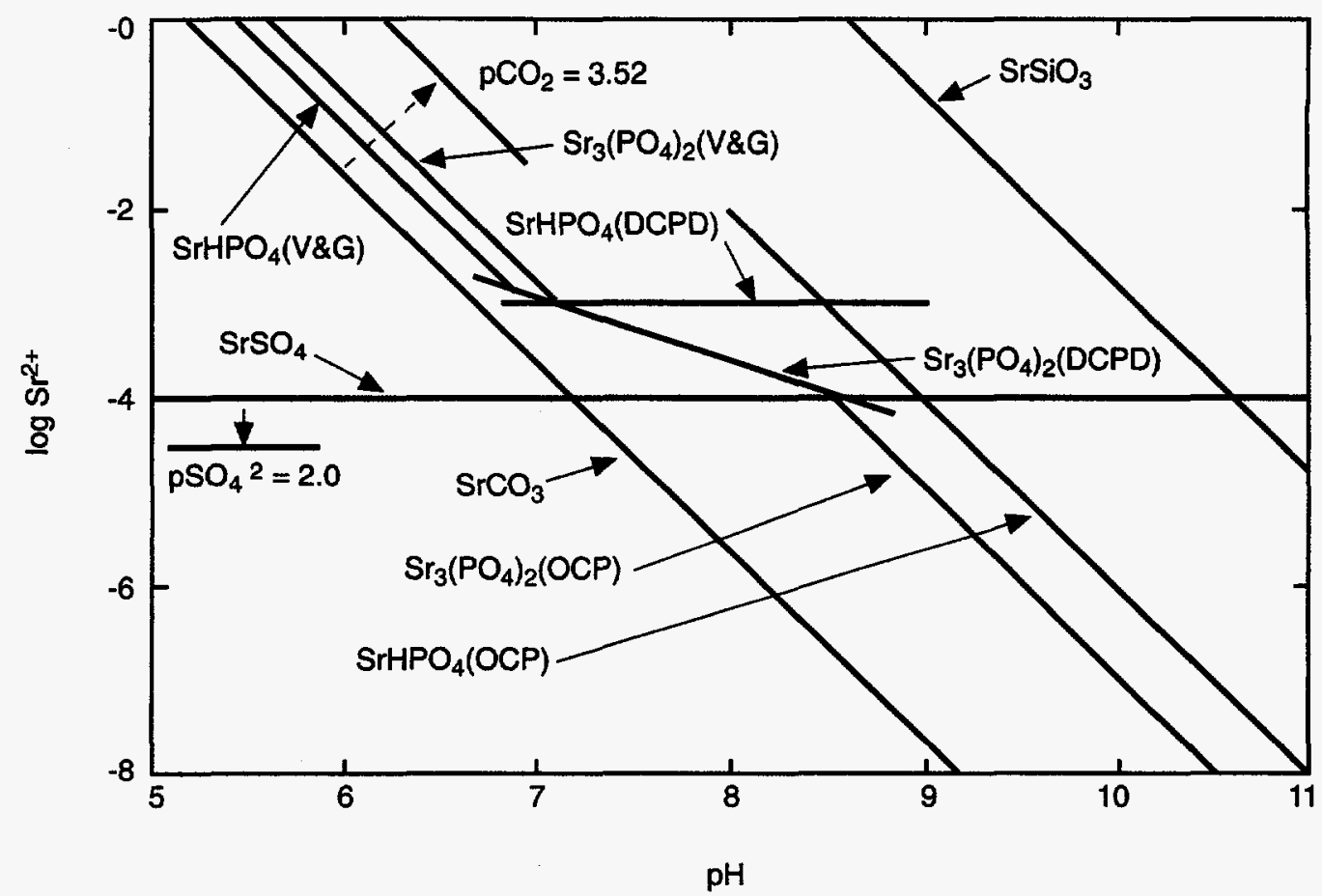

FIGURE J.9 Relative Stability of Various Strontium Solids at $\mathrm{pCa}^{2+}=$ $\mathrm{pSO}_{4}^{2-}=2.5, \mathrm{pH}_{4} \mathrm{SiO}_{4}=3.1$, and $\mathrm{pCO}_{2(\mathrm{~g})}=1.52$ atm in Equilibrium with Variscite and Gibbsite (V \& G), Dicalcium Phosphate Dihydrate (DCPD), and Octacalcium Phosphate (OCP) (Redox potential has little effect on strontium solubilization.) (Source: Adapted from Ames and Rai 1978)

\section{J.5.16 Tritium}

Tritium is a radioactive isotope of hydrogen. Thus, the behavior of tritium in soils would be expected either to be similar to hydrogen or to exist as an ion, gas, or liquid (tritiated water). In the soil solution system investigated, tritium can travel rapidly at about the same velocity as the soil water or groundwater.

\section{J.5.17 Uranium}

The stability diagram of uranium solids presented by Ames and Rai (1978) in terms of the uranyl ion activity is shown in Figure J.11. Thermodynamic data for (1) $\mathrm{Na}_{2} \mathrm{UO}_{4}$ and $\mathrm{UO}_{2} \mathrm{CO}_{3}$ were obtained from Garrels and Christ (1965); (2) data for $\mathrm{UO}_{2}(\mathrm{OH})_{2}$, $\mathrm{UO}_{2}(\mathrm{OH})_{2} \cdot \mathrm{H}_{2} \mathrm{O}$, and $\mathrm{Na}_{4} \mathrm{UO}_{2}\left(\mathrm{CO}_{3}\right)_{3}$ were obtained from Sillen and Martell (1964); and (3) the remaining species were obtained from Palei (1963). 


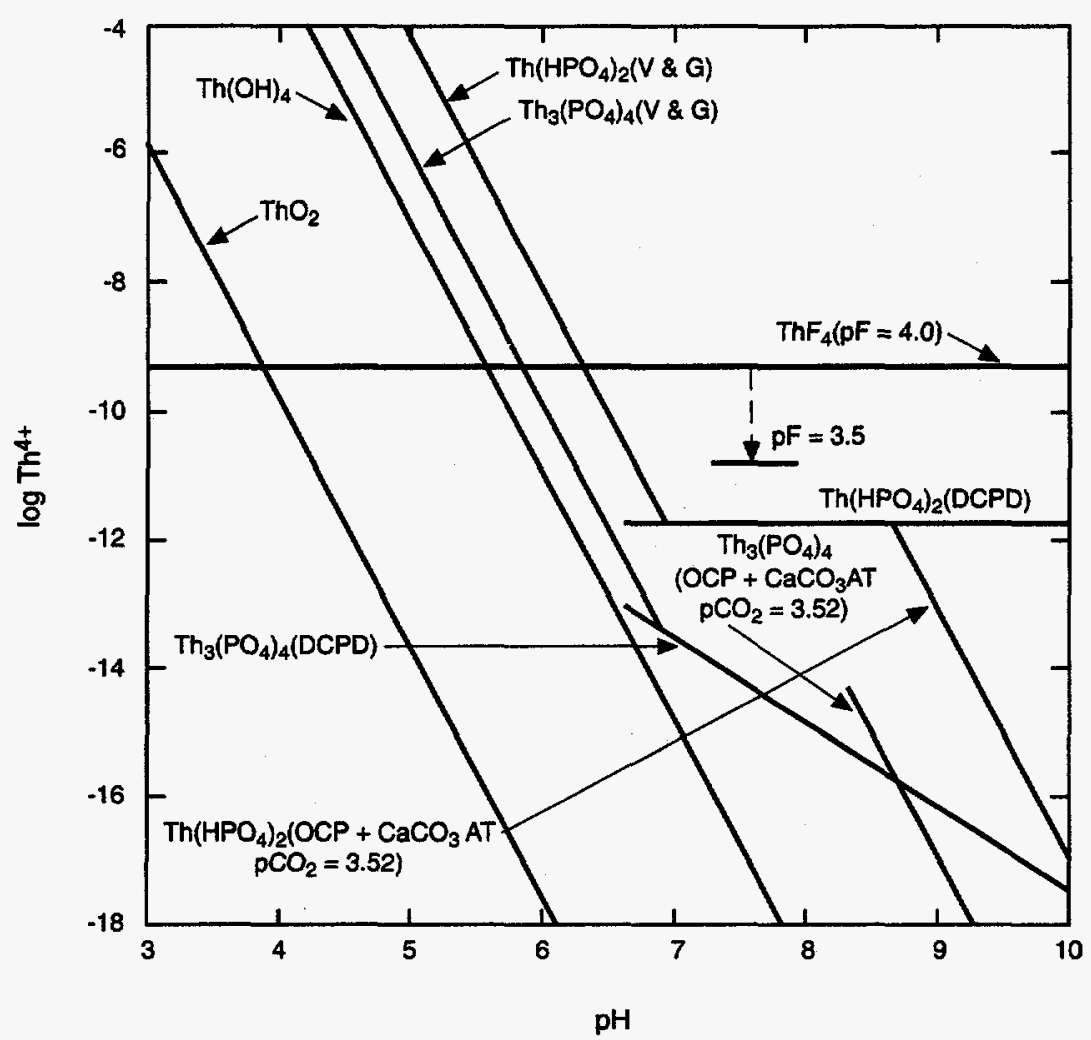

FIGURE J.10 Relative Stability of Various Thorium Solids in Equilibrium with Variscite and Gibbsite (V \& G), Dicalcium Phosphate Dihydrate (DCPD), and Octacalcium Phosphate (OCP) (Source: Adapted from Ames and Rai 1978) 


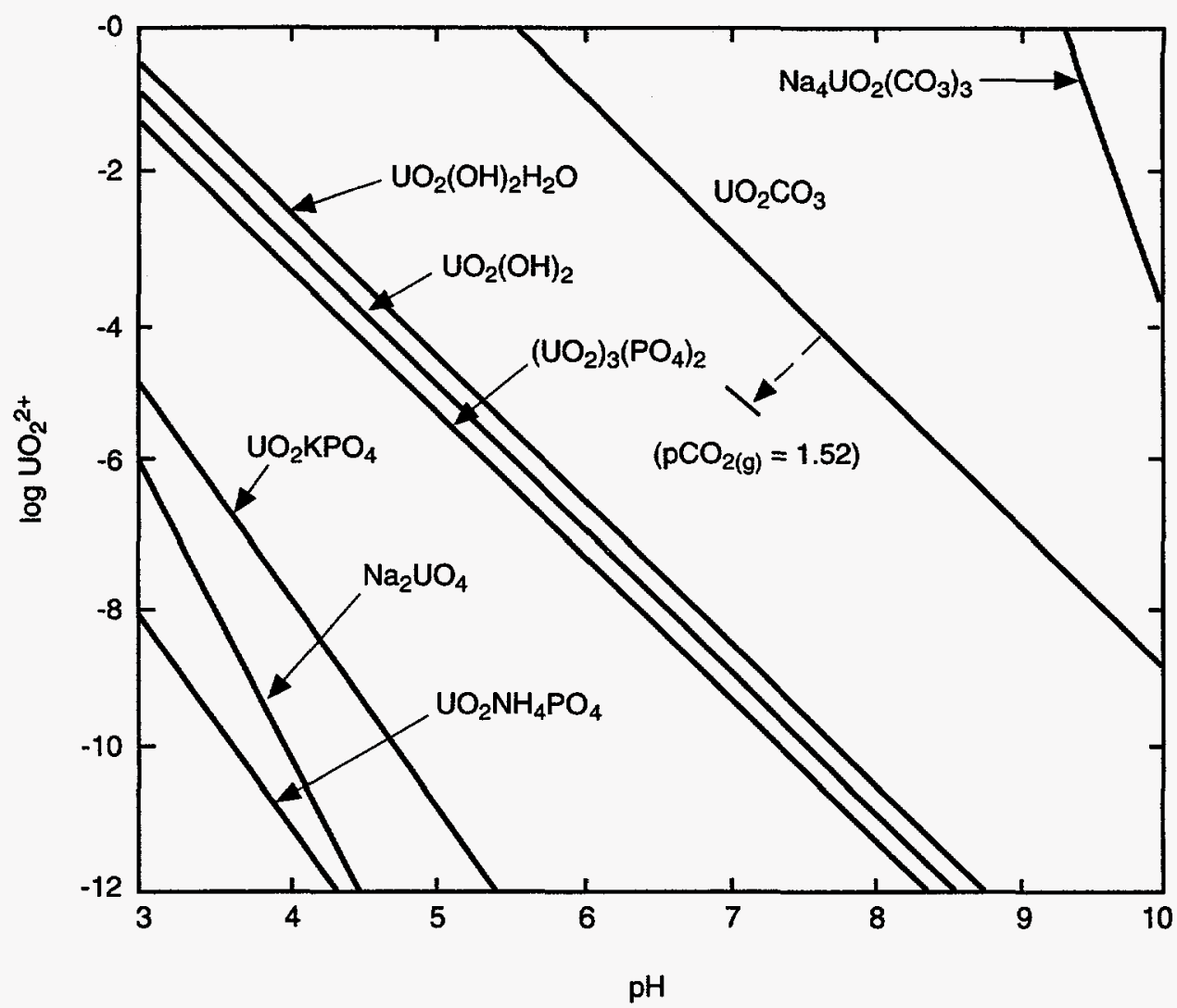

FIGURE J.11 Relative Stability of Various Uranium Solids in an Oxidizing Soil Environment $\left[\mathrm{pO}_{2(\mathrm{~g})}=0.68 \mathrm{~atm}, \mathrm{PCO}_{2(\mathrm{~g})}=3.52\right.$, and $\mathrm{pNa}^{+}=\mathrm{pNH}_{4}^{+}=3.0$ ] with Phosphate Levels in Equilibrium with Variscite and Gibbsite (V \& G) (Source: Adapted from Ames and Rai 1978) 


\section{J.6 REFERENCES}

Ames, L.L., and Rai, D., 1978, Radionuclide Interaction with Rock and Soil Media, Volume I: Processes Influencing Radionuclide Mobility and Retention, Element Chemistry and Geochemistry; Conclusions and Evaluation, EPA520/6-78-007-a, U.S. Environmental Protection Agency, Office of Radiation Programs, Las Vegas, N.V.

Baes, C.F., Jr., and R.E. Mesmer, 1976, The Hydrolysis of Cations, John Wiley \& Sons, New York, N.Y.

Burney, G.A., and R.M. Harbour, 1974, Radiochemistry of Neptunium, NAS-NS-3600, NAS-NRC Nuclear Scientific Series, Technical Information Center, Office of Information Services, U.S. Atomic Energy Commission, Oak Ridge, Tenn.

Chase, M.W., et al., 1975, "JANAF Thermodynamical Tables, 1975 Supplement," Journal of Physical and Chemical Reference Data 4:1-175.

Dvorak, A.J., et al., 1978, Impacts of Coal-Fired Power Plants on Fish, Wildlife, and Their Habitats, FWS/OBS-78/29, prepared for the U.S. Fish and Wildlife Service, Ann Arbor, Mi., by the Division of Environmental Impact Studies, Argonne National Laboratory, Argonne, Ill.

Garrels, R.M., and C.L. Christ, 1965, Solutions, Minerals, and Equilibria, Harper and Row, New York, N.Y., pp. 213-216.

Keller, C., 1971, The Chemistry of the Transuranium Elements, Vol. 3, Kernchemie in Einzeldarstellungen, Verlag Chemie/GmbH, Weinheim/Gergstr, Germany.

Latimer, W.M., 1952, Oxidation Potentials, 2nd ed., Prentice-Hall, Englewood Cliffs, N.J.

Nieto, K.F., and W.T. Frankenberger, Jr., 1985, "Single Column Ion Chromatography, I. Analysis of Inorganic Anions in Soils," Soil Science Society of America Journal 49:587-592.

Palei, P.N., 1963, Analytical Chemistry of Uranium, translated by N. Kaner, Israel Program for Scientific Translations Ltd., Jerusalem, Israel, Daniel Davey \& Co., Inc., Analytical Chemistry of Elements Series, New York, N.Y.

Parker, V.B., et al., 1971, Selected Values of Chemical Thermodynamic Properties, Tables for the Alkaline Earth Elements (Elements 92 through 97) in the Standard Order of Arrangement, NBS Technical Note 270-6, National Bureau of Standards, Government Printing Office, Washington, D.C.

Pourbaix, M., 1966, Atlas of Electrochemical Equilibria in Aqueous Solution, Pergamon Press, Oxford, United Kingdom.

Rai, D., and W.L. Lindsay, 1975, "A Thermodynamic Model for Predicting the Formation, Stability, and Weathering of Common Soil Minerals," Soil Science Society of America Proceedings 39:991-996. 
Rai, D., and R.J. Serne, 1977, "Plutonium Activities in Soil Solutions and the Stability and Formation of Selected Plutonium Minerals," Journal of Environmental Quality 6:89-95.

Robie, R.A., and D.R. Waldbaum, 1968, "Thermodynamic Properties of Minerals and Related Substances at $298.15^{\circ} \mathrm{K}\left(25^{\circ} \mathrm{C}\right)$ and One Atmosphere (1.013 bars) Pressure and at Higher Temperatures," Geological Survey Bulletin 1259:238-248.

Schumm, R.H., et al., 1973, Selected Values of Chemical Thermodynamic Properties, Tables for the Lanthanide (Rare Earth) Elements (Elements 62 through 76) in the Standard Order of Arrangement, NBS Technical Note 270-7, National Bureau of Standards, Government Printing Office, Washington, D.C.

Sillen, I.G., and A.E. Martell, 1964, Stability Constants of Metal-Ion Complexes, Special Publication No. 17, The Chemical Society, London, United Kingdom.

Wagman, D.D., et al., 1968, Selected Values of Chemical Thermodynamic Properties, Tables for the First Thirty-Four Elements in the Standard Order of Arrangement, NBS Technical Note 270-3, National Bureau of Standards, Government Printing Office, Washington, D.C.

Wagman, D.D., et al., 1969, Selected Values of Chemical Thermodynamic Properties, Tables for the Elements 35 through 53 in the Standard Order of Arrangement, NBS Technical Note 270-4, National Bureau of Standards, Government Printing Office, Washington, D.C. 
APPENDIX K:

ESTIMATION OF INDIVIDUAL AND COLLECTIVE OFF-SITE DOSES 


\section{APPENDIX K:}

\section{ESTIMATION OF INDIVIDUAL AND COLLECTIVE OFF-SITE DOSES}

The current version of the RESRAD code is designed for calculating on-site individual doses; it does not include models for calculating individual and collective doses at off-site locations. RESRAD modules for calculating off-site doses may be available in the future. However, in the interim, off-site doses can be estimated by using the methods presented in this appendix.

The principal environmental pathways for estimating off-site individual and collective doses are airborne and waterborne pathways; the latter include surface water and subsurface or groundwater.

The methodology for estimating off-site individual and collective doses from airborne pathways is presented in Section K.1. The methodologies for estimating off-site doses from waterborne pathways are presented in Sections K.2 and K.3.

\section{K.1 AIRBORNE PATHWAY}

To calculate site-specific estimates of collective (population) doses, the user must obtain the population distribution by radius and sector and the geographical and meteorological parameters characterizing the population in the vicinity of the contaminated area. Using these data, an external computer code, such as CAP88-PC (U.S. Environmental Protection Agency 1992) or, for the uranium series nuclides, the MILDOS-AREA code (Yuan et al. 1989) can be used to calculate the individual and collective off-site doses if the airborne release rates can be determined. Depending on the user's application, dose calculations derived by using these codes can be made for a single year (e.g., the year of maximum airborne release) or for multiple years. For single-year dose calculations, the radionuclide release rates for the particular year need to be estimated first and used as input to run the selected computer code. For multiple-year dose calculations, the estimated cumulative releases need to be adjusted for radioactive decay for the time period of concern and input into the code.

Except for sites involving gaseous radionuclides (e.g., tritium, carbon, radon, etc.), the airborne releases from a contaminated area usually consist of fugitive dusts generated 
by wind erosion and/or by the mechanical disturbance caused by human activities. The MILDOS-AREA code includes a method for calculating the airborne dust release generated from wind erosion processes. However, the airborne release generated by mechanical disturbance is site specific and must be estimated by the user. The rates of gaseous radionuclide releases are also site specific and can usually be related to the concentrations of radionuclides in the contaminated zone (see Appendix $C$ for radon and Appendix $L$ for tritium and carbon-14).

The RESRAD code does not include a model for estimating the airborne fugitive dust emission from the contaminated zone. The code uses an empirically determined mass loading factor from the air immediately above the contaminated area at a height of 1 meter, or $\mathrm{Cm}(0)$, to calculate the radionuclide concentrations in the on-site air. Fields et al. (1986) used the following equation to approximate the relationship between the mass loading factor and the airborne dust release rate:

$$
Q=C m(0) \quad V d A
$$

where

$$
\begin{aligned}
Q & =\text { airborne release rate }(\mathrm{g} / \mathrm{s}), \\
C m(0) & =\text { mass loading factor }\left(\mathrm{g} / \mathrm{m}^{3}\right) \\
V d & =\text { deposition velocity }(\mathrm{m} / \mathrm{s}), \text { and } \\
A & =\text { area of contamination }\left(\mathrm{m}^{2}\right) .
\end{aligned}
$$

By multiplying the value of $Q$ by the radionuclide concentrations, $S$ (pCi/g), in the contaminated zone, the radionuclide release rates can be obtained. These release rates can then be input into the CAP88-PC or MILDOS-AREA code to calculate doses to off-site individuals or the collective population.

Both the CAP88-PC and MULDOS-AREA codes were designed to calculate doses to individuals and the collective population from airborne releases of radionuclides. The radionuclide transport models in both codes use the Gaussian plume dispersion equation to compute radionuclide concentrations in air, deposition rates on ground surfaces, concentrations in foods, and human intake rates resulting from inhalation and ingestion of contaminated air and food products. The MILDOS-AREA code only calculates doses for 
uranium series nuclides. The CAP88 code does not model the resuspension of deposited material into the atmosphere.

For off-site individual receptor locations at distances shorter than 1.3 times the largest extent of the contaminated area, the MILDOS-AREA dispersion model is recommended because the area source dispersion model in the MLLOS-AREA code is more accurate at these distances. However, because the MILDOS-AREA code was designed only for uranium series nuclides, the CAP88-PC code can be used for radionuclides other than the uranium series. To obtain the best results for near-source receptors, individual doses calculated by the CAP88-PC code may be rescaled by the ratios of the relative concentration $(X / Q)$ values calculated by MILDOS-AREA to those calculated by CAP88-PC.

For a site for which site-specific meteorological data, such as windrose data, are not available, meteorological data from the nearest airport should be used. The following simplified approach can also be used to estimate off-site doses when CAP88-PC or MILDOSAREA are not available. Usually, the primary concern is the potential dose to off-site individuals living closest to the contaminated area or at the site boundaries. These locations are usually within a few kilometers from the contaminated area, and the dispersion of released material to these locations can be described adequately by a sector-averaged Gaussian plume model. The air concentration at $x$ meters from the center of the contaminated site can be calculated by using the following equation:

$$
C m(x)=\frac{8 \sqrt{2} Q f}{\sqrt{\pi} \pi x \sigma_{z} u} \exp \left[-\frac{1}{2}\left(\frac{H}{\sigma_{z}}\right)^{2}\right]
$$

where

$$
\begin{aligned}
C m(x)= & \text { air concentration at off-site location } x \text { meters from the } \\
& \text { center of the contaminated area }\left(\mathrm{g} / \mathrm{m}^{3}\right), \\
Q= & \text { average emission rate from the contaminated area }(\mathrm{g} / \mathrm{s}), \\
f= & \text { fraction of the year assumed wind blows toward the } \\
& 22.5^{\circ} \text { sector where the receptor is located, }
\end{aligned}
$$




$$
\begin{aligned}
H= & \text { relative height between the contaminated area and the } \\
& \text { receptor }(\mathrm{m}), \\
x= & \text { downwind distance of the receptor location }(\mathrm{m}), \\
\sigma_{z}= & \text { annual average vertical dispersion coefficient }(\mathrm{m}), \text { and } \\
u= & \text { annual mean wind speed }(\mathrm{m} / \mathrm{s})
\end{aligned}
$$

The annual average vertical dispersion coefficient, $\sigma_{z}$, may be estimated from the PasquillGifford dispersion curves (Pasquill 1974; Gifford 1968) or their fitted empirical equations (Eimutis and Konicek 1972) at the distances of interest. The value of $\mathrm{Cm}(x)$ calculated by using Equation K.2 can then be input into RESRAD as the mass loading factors for the inhalation and the foliar deposition pathways to calculate the inhalation and waterindependent ingestion doses. Collective doses can be estimated by computing average off-site doses for various sectors of interest and multiplying by the estimated population in that sector.

The calculation of off-site doses requires two steps to account for the following two processes. Dust resuspended from the contaminated site and transported to the off-site location may be inhaled or deposited onto vegetation. Also, deposition of dust originating from the contaminated site over the previously uncontaminated off-site area will occur, and off-site radionuclide soil concentrations may build up over time.

To calculate off-site doses using RESRAD, the user must run the code twice. With the exceptions noted below, most input parameters will correspond to the off-site location. The radionuclide concentrations should correspond to the contaminated site. The size of the off-site area should be set to $20,000 \mathrm{~m}^{2}$, and the dilution length for airborne dust should be set to zero (this will set all area factors calculated by RESRAD to unity). To suppress the water-dependent pathways, all fractions of water (drinking, household, livestock, irrigation) obtained from the site should be set to zero.

The first step (transport of resuspended dust from contaminated site to off-site location) requires the user to suppress all pathways except the inhalation and plant, meat, and milk ingestion pathways. If the plant, meat, and/or milk pathways do not apply, these should also be suppressed. The depth of roots should be set to zero to suppress the root uptake subpathway. The airborne concentrations calculated with Equation $\mathrm{K} .2$ should be 
entered as the mass loading for both inhalation and foliar deposition. To correctly account for losses due to leaching and erosion, the user must enter values for parameters at the contaminated site that affect these processes (i.e., thickness of contaminated zone, mixing layer thickness, precipitation, irrigation, runoff, evapotranspiration, soil properties and distribution coefficients for the contaminated zone). RESRAD will then calculate off-site doses as a function of time resulting from inhalation and foliar deposition of contaminated dust.

In the second step, time-dependent scaling factors must be derived to account for the deposition and accumulation in soil of contaminated dust at the off-site location. The amount of contaminated soil deposited after one year is given by the following equation:

$$
M_{d e p}(x)=3.2 \times 10^{7} \mathrm{Cm}(x) \mathrm{Vd}
$$

where

$$
\begin{aligned}
M_{d e p}(x) & =\text { dust deposition rate }\left(\mathrm{g} / \mathrm{m}^{2}-\mathrm{yr}\right), \text { and } \\
3.2 \times 10^{7} & =\text { conversion factor }(\mathrm{s} / \mathrm{yr})
\end{aligned}
$$

It is then assumed that this dust is mixed with previously uncontaminated soil by plowing it into the top layer of soil. The fraction of off-site surface soil that originated from the contaminated site can be obtained by the following equation:

$$
F_{\text {cont }}(x, t)=\frac{M_{d e p}(x) t}{10^{6} \rho_{b} d_{m}}
$$

where

$$
\begin{aligned}
F_{c o n t}(x, t)= & \text { fraction of soil within the surface mixing layer that originated } \\
& \text { from the contaminated site (dimensionless), } \\
t= & \text { elapsed time }(\mathrm{yr}), \\
10^{6}= & \text { unit conversion factor }\left(\mathrm{cm}^{3} / \mathrm{m}^{3}\right), \\
\rho_{b}= & \text { bulk density of off-site soil }\left(1.5 \mathrm{~g} / \mathrm{cm}^{3}\right), \text { and } \\
d_{m}= & \text { depth of mixing layer }(0.15 \mathrm{~m}) .
\end{aligned}
$$


The input file generated from the first run may now be modified by activating all applicable pathways, except drinking water and aquatic food pathways. In addition, the mass loading factors for inhalation and foliar deposition should be reset to the site-specific values [actual dust loading, not $\mathrm{Cm}(\mathrm{x})$ ]. To avoid potential conflicts when the thickness of the original contaminated area is not equal to the newly contaminated off-site area, the depth to roots should be set equal to the mixing depth (i.e., $0.15 \mathrm{~m}$ ). The doses calculated by the code at the user specified times should be scaled by the contaminated soil fractions using Equation K.4. The inhalation and ingestion doses calculated from the first run should then be added to the scaled doses calculated for these pathways in the second run at each user specified time to obtain the total dose.

Equation K.2 provides an adequate estimation for off-site locations at distances greater than five times the largest extent (dimension) of the contaminated area. For receptors at distances less than five times the largest extent of the contaminated area, this point source dispersion approximation will usually overestimate the air concentrations.

\section{K.2 GROUNDWATER PATHWAY}

The groundwater transport model implemented in the RESRAD code for estimating on-site well and surface water concentrations does not include the dispersion process. If the dispersion process is not considered, the breakthrough times for the off-site well and surface water sources will be overestimated, and the off-site well water concentrations may also be overestimated. Depending on the complexity of the hydrogeological conditions of the site, it may be possible to use one of the following computer codes to estimate off-site well and surface water concentrations: MASCOT (Gureghian 1987), FEMA (Yeh and Huff 1985), TRIPM (Gureghian 1983), LAYFLO (Gureghian and Jansen 1983, 1985), and FECWASTE (Yeh and Strand 1982).

The FEMA, TRIPM, and FECWASTE codes use the finite-element (numerical) method to solve the transport equation. Both the FEMA and TRIPM codes consider advection, longitudinal and transverse dispersion, and (radioactive) decay of the solute; TRIPM also considers the ingrowth of a radionuclide. Prescribing the boundary condition for the time-dependent areal source entering the saturated zone would be difficult with either of these two codes. The FECWASTE code models the movement of a radionuclide in the $x-z$ 
plane, thus accounting for advective and dispersive transport in both the vertical and longitudinal directions and for radioactive decay. Neither ingrowth nor lateral dispersion (in the $y$ direction) is considered.

MASCOT is an analytical code that models the movement of the nuclides in a fourmember decay chain through a homogeneous aquifer and accounts for the effects of advection, dispersion in the three principal directions, and the decay and ingrowth of nuclides. Areal sources and a first order leach rate can be specified as the boundary condition; MASCOT can thus model the transport through a single unsaturated layer under the conditions idealized in RESRAD. If the saturated and unsaturated zones possess the same geochemical properties, the vertical transport through the unsaturated zone and the horizontal transport through the saturated zone can be modeled by summing the results of a number of simulations of MASCOT, each using a different path length to simulate the range of path lengths from the extremities of the contaminated zone to the source.

Burkholder and Rosinger (1980) present an analytical model that considers advection, longitudinal dispersion, and decay and ingrowth of the radionuclides for the onedimensional transport of a three-member decay chain through a single layer of porous media. The semianalytical model LAYFLO also considers the same processes for the one-dimensional transport of a three-member decay chain through multiple layers of porous media. Incorporating the boundary condition for the time-dependent areal concentration entering the saturated zone into these analytical models would be difficult. Disregarding the lateral dispersion will overpredict the solute concentration in the water. The LAYFLO code, however, can be used to estimate the shortest time (i.e., the breakthrough time) required for the radionuclide to be transported from the contaminated site to the off-site water source. If the breakthrough time is greater than the period of concern for the dose evaluation, the contribution of the groundwater pathway can be ignored.

Given the limitations and drawbacks associated with the use of the transport models discussed above, the user may prefer to use the approximate method that follows. This method makes use of the unsaturated zone transport model of RESRAD in which advective transport and the decay and ingrowth of the radionuclides are considered. The effects of transverse dispersion are included in an off-site dilution factor. 
The RESRAD code can be used to model the transport in the saturated zone by specifying that the saturated zone be an unsaturated zone with a unit saturation ratio. One of the five unsaturated zones permitted in RESRAD can be used for this purpose. Considering Equation E.7, it is apparent that setting the hydraulic conductivity of this unsaturated zone equal to the infiltration (percolation) rate, $I$, (Equation E.4) would ensure a saturation ratio of one.

$$
\hat{K}_{s}^{u z}=I=\left(1-C_{e}\right)\left[\left(1-C_{r}\right) P_{r}+I_{r r}\right],
$$

where

$$
\begin{aligned}
\hat{K}_{s}^{(u z)}= & \text { hydraulic conductivity of the unsaturated stratum } \\
& \text { simulating the saturated zone }(\mathrm{m} / \mathrm{yr}) \\
I= & \text { infiltration rate }(\mathrm{m} / \mathrm{yr}) \\
C_{e}= & \text { evapotranspiration coefficient, } \\
C_{r}= & \text { runoff coefficient, } \\
P_{r}= & \text { precipitation rate }(\mathrm{m} / \mathrm{yr}), \text { and } \\
I_{r r}= & \text { irrigation rate }(\mathrm{m} / \mathrm{yr}) .
\end{aligned}
$$

The depth of this "unsaturated" zone, $\Delta z_{s z}$, is given by

$$
\Delta z_{s z}=\frac{L^{(s z)} \times I}{K_{s}^{(s z)} \times J_{x}},
$$

where

$$
\begin{aligned}
\Delta z_{s z}= & \text { thickness of the unsaturated stratum simulating the } \\
& \text { saturated zone }(\mathrm{m}), \\
L^{(s z)}= & \text { distance from the downgradient edge of the contaminated } \\
& \text { zone to the well or the surface water source }(\mathrm{m}), \\
I= & \text { infiltration rate }(\mathrm{m} / \mathrm{yr}), \\
K_{s}^{(s z)}= & \text { hydraulic conductivity of the saturated zone }(\mathrm{m} / \mathrm{yr}) \text {, and } \\
J_{x}= & \text { hydraulic gradient in the direction of flow (dimensionless). }
\end{aligned}
$$


It is assumed that the vertical dispersion is negligible and that the aquifer is "deep." A deep aquifer is one in which the recharge over the length under consideration is small compared to the flow of the aquifer, so as not to have an effect on the flow rate. The path of the contamination is shown in Figure K.1. Under these assumptions, the vertical dilution factor - dilution occurring in the well due to the mixing of contaminated and uncontaminated waters entering along the depth of the well - is given by Equation K.7. The rise time, which is the additional travel time (above the breakthrough time) of the most upgradient contamination reaching the well, is also given in Equation K.7.

$$
\begin{array}{lll}
D F_{v}=0, & \text { and } \delta_{t}=0 & \text { if } \xi \geq d_{w} \\
D F_{v}=\frac{d_{w}-\xi}{d_{w}}, & \text { and } \delta_{t}=\tau_{l} \frac{d_{w}-\xi}{\zeta} & \text { if } \xi<d_{w}<\xi+\zeta \\
D F_{v}=\frac{\zeta}{d_{w}} & \text { and } \delta_{t}=\tau_{l} & \text { if } d_{w} \geq \xi+\zeta,
\end{array}
$$

where

$$
\begin{aligned}
D F_{v} & =\text { vertical dilution factor, } \\
\delta_{t} & =\text { rise time }(\mathrm{yr}), \\
d_{w} & =\text { depth of the well below the water table }(\mathrm{m}),
\end{aligned}
$$

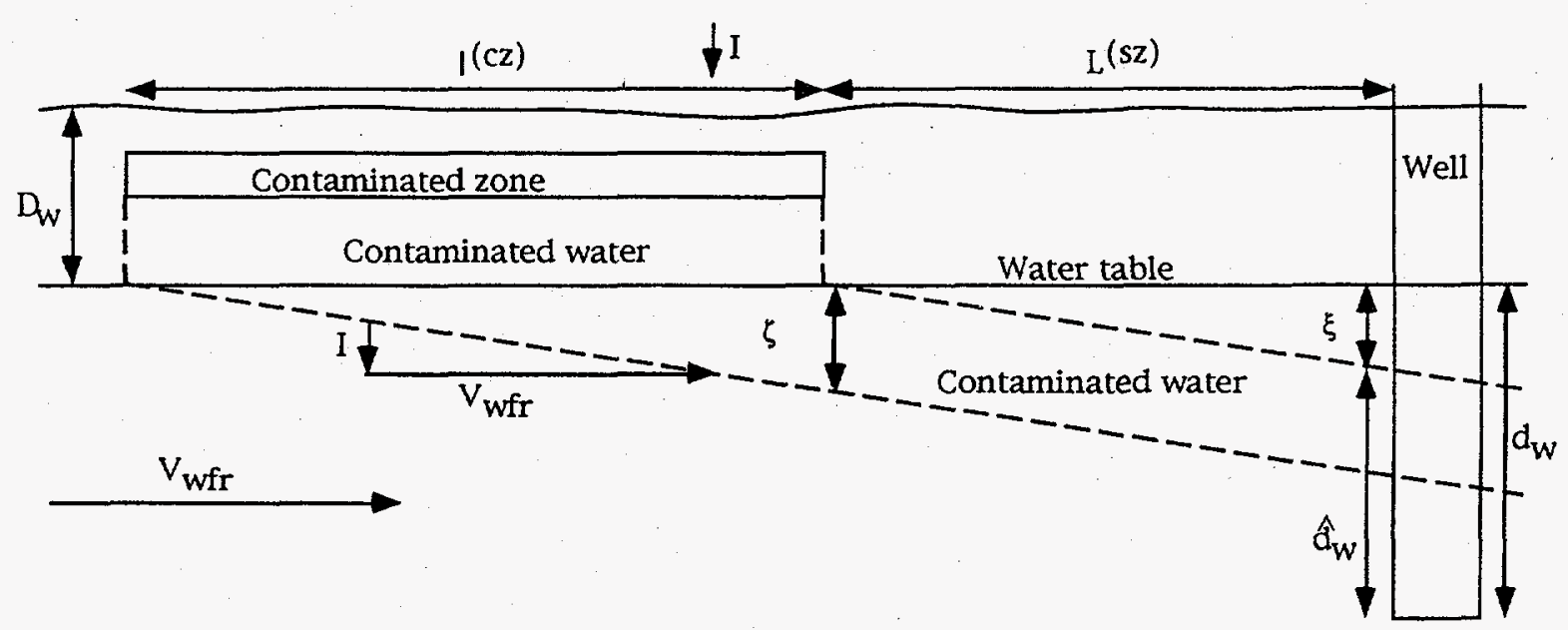

FIGURE K.1 Movement of Contaminated Water in the Horizontal Plane 


$$
\begin{aligned}
\xi=L^{(s z)} \times I / V_{w f r}= & \text { depth from the water table to the upper boundary of } \\
& \text { contamination at the well (m), } \\
L^{(s z)}= & \text { distance from the downgradient edge of the } \\
& \text { contaminated zone to the well (m), } \\
I= & \text { infiltration rate (m/yr), } \\
V_{w f r}=K_{s}^{(s z)} \times J_{x}= & \text { Darcy flow velocity in the saturated zone (m/yr), } \\
K_{s}^{(s z)}= & \text { hydraulic conductivity of the saturated zone (m/yr), } \\
J_{x}= & \text { hydraulic gradient in the direction of flow (m/m), } \\
\tau_{l}=\frac{P_{e}^{(s z)} \times R_{d}^{(s z)} \times l}{V_{w f r}} & \text { time taken by the nuclide to traverse the length of the } \\
& \text { contaminated zone (yr), } \\
l= & \text { length of the contaminated zone parallel to the } \\
& \text { aquifer flow (m), } \\
P_{e}^{(s z)}= & \text { effective porosity of the saturated zone, } \\
R_{d}^{(s z)}= & \text { retardation factor of the nuclide in the saturated zone, } \\
& \text { and } \\
\zeta=l \times I / V_{w f r}= & \text { depth from the water table to the lower boundary of } \\
& \text { contamination at the downgradient edge of the } \\
& \text { contaminated zone (m). }
\end{aligned}
$$

Considering Equations E.24 and K.7, the value for depth of the well below the water table to be input to RESRAD, $\hat{d}_{w}$, in order to achieve the desired rise time is given by

$$
\hat{d}_{w}=d_{w}-\xi=d_{w}-\frac{L^{(s z)} \times I}{K_{s}^{(s z)} \times J_{x}},
$$

where

$\hat{d}_{w}=$ value of depth of well below the water table to be used in RESRAD for the off-site well (m),

$d_{w}=$ actual depth of well below the water table (m), 
$\xi$ = depth from the water table to the upper boundary of contamination at the well $(\mathrm{m})$,

$L^{(s z)}=$ distance from the downgradient edge of the contaminated zone to the well (m),

$I=$ infiltration rate $(\mathrm{m} / \mathrm{yr})$,

$K_{s}^{(s z)}=$ hydraulic conductivity of the saturated zone $(\mathrm{m} / \mathrm{yr})$, and

$J_{x}=$ hydraulic gradient in the direction of flow (dimensionless).

If Equation K.8 yields a negative value for $\hat{d}_{w}$, the contamination will pass under the offsite well. In this case, the water pathway can be suppressed since it will not contribute to the dose.

The dilution due to lateral dispersion is obtained by solving the lateral dispersion equation under the appropriate initial condition,

$$
\begin{aligned}
& R_{d} \frac{\partial c}{\partial t}=D_{y} \frac{\partial^{2} c}{\partial y^{2}} \\
& c(y, t)=1, \quad \text { for }|y| \leq w / 2, \quad t=0 \quad \text { and } \\
& c(y, t)=0, \quad \text { for }|y|>w / 2, \quad t=0 \text {, }
\end{aligned}
$$

where

$$
\begin{aligned}
R_{d}= & \text { retardation factor of the nuclide in the saturated zone, } \\
D_{y}= & \text { lateral dispersion coefficient of the nuclide in the } \\
& \text { saturated zone }\left(\mathrm{m}^{2} / \mathrm{yr}\right), \\
y= & \text { lateral distance from the center line of the contaminated } \\
& \text { zone }(\mathrm{m}), \\
c(y, t)= & \text { normalized concentration at } y \text { after dispersing for time } t, \\
w=A / l= & \text { width of the contaminated zone }(\mathrm{m}), \\
A= & \text { area of the contaminated zone }\left(\mathrm{m}^{2}\right), \text { and } \\
l= & \text { length of the contaminated zone parallel to the aquifer } \\
& \text { flow }(\mathrm{m}) .
\end{aligned}
$$


The solution to Equation K.9 is

$$
\begin{aligned}
c(y, t) & =\sqrt{\frac{R_{d}}{4 \pi D_{y} t}} \int_{y-w / 2}^{y+w / 2} e^{\frac{-\Psi^{2} R_{d}}{4 D_{y} t}} d \Psi \\
& =\frac{1}{2}\left[\operatorname{erf}\left(\frac{w}{2}+y\right) \sqrt{\frac{R_{d}}{4 D_{y} t}}+\operatorname{erf}\left(\frac{w}{2}-y\right) \sqrt{\frac{R_{d}}{4 D_{y} t}}\right]
\end{aligned}
$$

where

$$
\text { erf }()=\text { error function tabulated in mathematical handbooks. }
$$

The lateral dispersion coefficient is related to the lateral dispersivity by an empirical relationship:

$$
D_{y}=\alpha_{T} V_{x}
$$

where

$$
\begin{aligned}
D_{y}= & \text { lateral dispersion coefficient of the nuclide in the } \\
& \text { saturated zone }\left(\mathrm{m}^{2} / \mathrm{yr}\right) \\
\alpha_{T}= & \text { lateral dispersivity }(\mathrm{m}), \text { and } \\
V_{x}= & \text { absolute flow velocity in the saturated zone }(\mathrm{m} / \mathrm{yr}) .
\end{aligned}
$$

The mean transport time of the nuclide for a distance, $L$, is given by

$$
t=\frac{L R_{d}}{V_{x}}
$$

where

$$
\begin{aligned}
t= & \text { mean travel time of radionuclide }(\mathrm{yr}), \\
L=L^{(s z)}+l / 2= & \text { distance from the center of the contaminated zone to the } \\
& \text { well }(\mathrm{m}), \\
L^{(s z)}= & \text { distance from the downgradient edge of the } \\
& \text { contaminated zone to the well }(\mathrm{m}),
\end{aligned}
$$




$$
\begin{aligned}
l= & \text { length of the contaminated zone parallel to the aquifer } \\
& \text { flow }(\mathrm{m}), \\
R_{d}= & \text { retardation factor of the nuclide in the saturated zone, } \\
& \text { and } \\
V_{x}= & \text { absolute flow velocity in the saturated zone }(\mathrm{m} / \mathrm{yr}) .
\end{aligned}
$$

Introducing Equations K.11 and K.12 in the solution of Equation K.9 yields

$$
c\left(y, L^{(s z)}\right)=\frac{1}{2}\left[\operatorname{erf}\left(\frac{w+2 y}{4 \sqrt{\alpha_{T} L}}\right)+\operatorname{erf}\left(\frac{w-2 y}{4 \sqrt{\alpha_{T} L}}\right)\right] .
$$

The horizontal dilution factor - dilution due to lateral dispersion and the convergence of streamlines in the horizontal plane due to the withdrawal of water - for a well located on the downgradient centerline is given by

$$
\begin{aligned}
D F_{h} \quad & =\frac{1}{d_{r}} \int_{-d_{r} / 2}^{d_{r} / 2} c\left(y, L^{(s z)}\right) d y \\
& =\frac{w}{d_{r}} \int_{0}^{d_{r} / 2 w}\left[\operatorname{erf}\left(\frac{1+2 y / w}{4 \sqrt{\alpha_{T} L} / w}\right)+\operatorname{erf}\left(\frac{1-2 y / w}{4 \sqrt{\alpha_{T} L} / w}\right)\right] d\left(\frac{y}{w}\right) \\
& =\frac{4}{d_{r}} \sqrt{\frac{\alpha_{T} L}{\pi}} \sum_{n=0}^{\infty}(-1)^{n} \frac{\left(w+d_{r}\right)^{2 n+2}-\left(w-d_{r}\right)^{2 n+2}}{\left(16 \alpha_{T} L\right)^{n+1}(2 n+2)(2 n+1) n !},
\end{aligned}
$$

where

$$
\begin{aligned}
D F_{h}= & \text { horizontal dilution factor, } \\
d_{r}= & \text { effective diameter of the well (Equation E.39) (m), } \\
L^{(s z)}= & \text { distance from the downgradient edge of the } \\
& \text { contaminated zone to the well }(\mathrm{m}), \\
w=A / l= & \text { width of the contaminated zone }(\mathrm{m}), \\
A= & \text { area of the contaminated zone }\left(\mathrm{m}^{2}\right),
\end{aligned}
$$




$$
\begin{aligned}
l= & \text { length of the contaminated zone parallel to the aquifer } \\
& \text { flow }(\mathrm{m}), \\
\operatorname{erf}()= & \text { error function tabulated in mathematical handbooks, } \\
\alpha_{T}= & \text { lateral dispersivity (m), and } \\
L=L^{(s z)}+l / 2= & \text { distance from the center of the contaminated zone to the } \\
& \text { well (m). }
\end{aligned}
$$

If the effective diameter of the well is small in comparison to the width of the contaminated zone, $D F_{h}$ can be approximated by

$$
D F_{h}=\operatorname{erf}\left(\frac{w}{4 \sqrt{\alpha_{T} L}}\right)
$$

If the off-site saturated zone is simulated as an unsaturated zone by using the input values obtained from Equations K.5, K.6, and K.8, RESRAD will compute the proper breakthrough time, rise time, and the decay and transfer functions (Appendix I). However, RESRAD will not compute the proper dilution factor for the well water concentration. The user will have to multiply those output quantities (and only those quantities) that are directly affected by the well water pathway by the $D F / f_{1}$ factors given in Equation K.16. This may necessitate a second run of RESRAD during which all but the well water pathways are suppressed. (Simply suppressing the other pathways in the "Set pathway" option may not be sufficient; for example, if well water is used for irrigation of crops, foliar deposition and direct root uptake from the contaminated zone will have to be suppressed in the second run of RESRAD by selecting a cover depth greater than the root and mixing zone depths.)

$$
\begin{array}{ll}
\frac{D F}{f_{1}}=\frac{d_{w}-\zeta}{d_{w}} D F_{h} & \text { if } w \geq d_{r} \\
\frac{D F}{f_{1}}=\frac{d_{w}-\zeta}{d_{w}} \frac{d_{r}}{w} D F_{h} & \text { if } w<d_{r},
\end{array}
$$

where

$$
\frac{D F}{f_{1}}=\text { multiplication factor to introduce off-site dilution, }
$$




$$
\begin{aligned}
d_{w}= & \text { depth of the well below the water table (m), } \\
\xi=L^{(s z)} \times I / V_{w f r}= & \text { depth from the water table to the upper boundary of } \\
& \text { contamination at the well (m), } \\
L^{(s z)}= & \text { distance from the downgradient edge of the } \\
& \text { contaminated zone to the well (m), } \\
I= & \text { infiltration rate (Equation E.4) (m/yr), } \\
V_{w f r}=K_{s}^{(s z)} \times J_{x}= & \text { Darcy flow velocity in the saturated zone(m/yr), } \\
K_{s}^{(s z)}= & \text { hydraulic conductivity of the saturated zone (m/yr), } \\
J_{x}= & \text { hydraulic gradient in the direction of flow (m/m), } \\
w=A / l= & \text { width of the contaminated zone (m), } \\
A= & \text { area of the contaminated zone (m } \left.{ }^{2}\right), \\
l= & \text { length of the contaminated zone parallel to the } \\
D F_{h}= & \text { horizontal dilution factor that can be obtained by } \\
& \text { summing the convergent series in Equation } \mathrm{K} 14 \text { or } \\
& \text { from Equation } \mathrm{K} .15 \text {. } \\
d_{r}= & \text { effective diameter of the well (Equation E.28) (m), } \\
L=L^{(s z)}+l / 2= & \text { distance from the center of the contaminated zone to } \\
& \text { the well (m), and }
\end{aligned}
$$

The steps involved in simulating the saturated zone in RESRAD as an unsaturated zone are as follows:

1. Use Equation K.6 to determine the thickness of the unsaturated zone that simulates the saturated zone.

2. Determine the hydraulic conductivity of the unsaturated zone that simulates the saturated zone by using Equation K.5.

3. Compute the value of the depth of the well below the water table to be used in this simulation by using Equation K.8. 
4. Input the values computed in steps 1,2 , and 3 in RESRAD. Input all the other relevant (unadjusted) parameters. Suppress all pathways other than the drinking water pathways.

5. Run RESRAD. Multiply the output by the factor in Equation K.16 to introduce the proper dilution factors.

This approximate method will account for advective transport, lateral dispersion, and the decay and ingrowth of the radionuclides in the saturated zone but will not consider the effects of longitudinal dispersion. By neglecting the longitudinal dispersion, this method overestimates the breakthrough times. If the travel time is much larger than the time to leach the solute out of the contaminated zone, longitudinal dispersion will dilute the peak solute concentration; in this case, the peak solute concentration will also be overestimated.

\section{K.3 SURFACE WATER EXPOSURE PATHWAYS}

The radiological impact from surface water exposure pathways can be estimated by using the LADTAP-II code (Simpson and McGill 1980), which implements the radiological exposure models of U.S. Nuclear Regulatory Commission Regulatory Guide 1.109. If the radiological source terms (releases) are known, radiological impacts from potable water, aquatic foods, shoreline deposits, swimming, boating, and irrigation can be calculated in terms of the dose to the maximally exposed individual and the collective population.

\section{K.4 REFERENCES}

Burkholder, H.C., and E.L.J. Rosinger, 1980, "A Model for the Transport of Radionuclides and Their Decay Products through Geologic Media," Nuclear Technology 49:150-158.

Eimutis, E.C., and M.G. Konicek, 1972, "Derivation of Continuous Functions for the Lateral and Vertical Dispersion Coefficients," Atmospheric Environment 6:859-863.

Fields, D.E., et al., 1986, PRESTO-II: A Low-Level Waste Environmental Transport and Risk Assessment Code, ORNL-5970, UC-11, UC-41, Oak Ridge National Laboratory, Oak Ridge, Tenn.

Gifford, F.A., 1968, "Turbulent Diffusion-Type Schemes: A Review," Nuclear Safety 17:68-86.

Gureghian A.B., 1983, TRIPM: A Two-Dimensional Finite-Element Model for the Simultaneous Transport of Water and Reacting Solutes through Saturated and Unsaturated 
Porous Media, ONWI-465, Office of Nuclear Waste Isolation, Battelle Memorial Institute, Columbus, Ohio.

Gureghian A.B., 1987, Analytical Solutions for Multidimensional Transport of a FourMember Radionuclide Decay Chain in Ground Water, BMIOCRD-25, Office of Crystalline Repository Development, Battelle Memorial Institute, Columbus, Ohio.

Gureghian A.B., and G. Jansen, 1983, LAYFLO: A One-Dimensional Semianalytical Model for the Migration of a Three-Member Decay Chain in a Multilayered Geologic Medium, ONWI-466, Office of Nuclear Waste Isolation, Battelle Memorial Institute, Columbus, Ohio.

Gureghian A.B., and G. Jansen, 1985, "One-Dimensional Analytical Solutions for the Migration of a Three-Member Radionuclide Decay Chain in a Multilayered Geologic Medium," Water Resources Research 21:733-742.

Pasquill, F., 1974, Atmospheric Diffusion, 2nd ed., John Wiley \& Sons, New York, N.Y.

Simpson, D.B., and B.L. McGill, 1980, User's Manual for LADTAP II: A Computer Program for Calculating Radiation Exposure to Man from Routine Release of Nuclear Reactor Liquid Effluents, NUREG/CR-1276, U.S. Nuclear Regulatory Commission, Washington, D.C.

U.S. Environmental Protection Agency, 1992, User's Guide for CAP-88-PC, Version 1.0, 4023-92-001, Office of Radiation Programs, Las Vegas, Nev.

Yeh G.T., and R.H. Strand, 1982, FECWASTE: User's Manual of a Finite-Element Code for Simulating WASTE Transport through Saturated-Unsaturated Porous Media, ORNL/TM8104, Oak Ridge National Laboratory, Oak Ridge, Tenn.

Yeh, G.T., and D.D. Huff, 1985, FEMA: A Finite Model of Material Transport through Aquifers, ORNL-6063, Oak Ridge National Laboratory, Oak Ridge, Tenn.

Yuan, Y.C., et al., 1989, MILDOS-AREA: An Enhanced Version of MILDOS for Large-Area Sources, ANL/ES-161, Argonne National Laboratory, Argonne, Ill. 
APPENDIX L:

TRITIUM AND CARBON-14 PATHWAY MODELS 


\section{APPENDIX L: \\ TRITIUM AND CARBON-14 PATHWAY MODELS}

Pathway analysis models for deriving soil concentration guidelines in the RESRAD code have been developed primarily for radionuclides in solid form. Tritium (H-3) and carbon-14 (C-14), which are ubiquitous, mobile, and have an abundance of stable isotopes in the environment, require special consideration in radiological analysis in order to obtain a more accurate assessment of their potential hazards. ${ }^{1}$ This appendix describes the pathway analysis models developed for tritium and carbon-14.

\section{L.1 GENERAL APPROACH}

The pathway analysis models in RESRAD are inherently complex. To avoid additional complexity, the basic strategy employed in developing the tritium and carbon-14 pathway models was to maintain the general RESRAD approach but to add pathwaydependent submodels in the code to take into account the special behavior of these hydrogen and carbon isotopes in the environment.

Numerous pathway analysis models have been developed for estimating radionuclide concentrations in food products and doses to humans from the environmental release oftritium (Evans 1969; Moore et al. 1979; National Council on Radiation Protection and Measurement 1979) and carbon-14 (Killough and Rohwer 1978; Moore et al. 1979; Zach and Sheppard 1991). Many of these models, however, have evolved from analyses of atmospheric sources and, therefore, are not appropriate for ground sources such as residual radioactive materials on or beneath the ground surface.

The specific activity methodology that has been used extensively for calculating doses from tritium and carbon-14 assumes, conservatively, that a state of equilibrium exists among their concentrations in all environmental media - air, water, food products, and body tissues. When this methodology is applied to contaminated soil, an infinitely large uniformly distributed source area is assumed. Consequently, the methodology is not specifically useful

1 In general, it is assumed that tritium is transferred in environmental media through its association with water as tritiated water (HTO); carbon-14 follows the conversion of $\mathrm{CO}_{2}$ and becomes fixed in vegetation and reaches man primarily through the ingestion pathway. 
for a radioactively contaminated site with a finite area. This simplistic, yet conservative, approach may, however, be applicable to some of the individual pathways such as soil-toplant and water-to-fish transfers. The advantage of using this method for individual pathways is that the uncertainty introduced into the pathway analysis can be reduced because of the minimal number of parameters required. The major goal in developing the tritium and carbon-14 models was to maintain sufficient flexibility in the code so that sitespecific data can be used for a conservative, yet realistic, analysis of the potential hazards associated with the presence of these hydrogen and carbon isotopes in the environment.

The following sections describe the submodels developed for calculating the effective dose equivalents from soils containing residual activities of tritium and carbon-14. These submodels are for estimating radionuclide concentrations in soil and soil water, in air, and in food products.

Estimations of radionuclide concentrations in groundwater or surface water for sites contaminated with tritium and carbon-14 can be derived by using the nuclide water pathway submodels described in Appendix E. Therefore, this appendix does not include a waterpathway-related submodel.

\section{L.2 TRITIUM SUBMODELS}

Tritium, with an atomic mass number of 3 and a decay half-life of 12.26 years, is a naturally occurring isotope of hydrogen produced by the interaction of cosmic ray protons and neutrons with nitrogen and oxygen atoms. Tritium is also a ternary fission product of nuclear reactors as a result of neutron interaction with coolant additives, deuterium (H-2), and other reactor components. Because tritium decays to helium-3 by emitting a low-energy beta particle $\left(E_{\max }=0.0186 \mathrm{MeV}\right.$ ), it does not pose an external hazard. However, it can pose an internal radiological hazard once inhaled or ingested. The ingestion dose conversion factor is about $1 / 800$ of that of cesium-137+D. Because tritium (H-3) has essentially the same chemical behavior as stable isotopes of hydrogen (i.e., $\mathrm{H}-1$ and $\mathrm{H}-2$ ), it will occur in organisms throughout ecosystems in concentrations that depend on the ratio of tritium to stable hydrogen in the environment. ${ }^{2}$

2 The specific activity of elemental tritium is approximately $9,700 \mathrm{Ci} / \mathrm{g}$. 
Tritium released to the environment is usually converted to the oxide form quite rapidly and is dispersed like ordinary water. The relatively large mass difference between ordinary water and HTO has some influence on HTO's properties. For example, the vapor pressure of HTO is about $10 \%$ lower, and the molecular diffusion coefficient in air is about 5\% lower than that of ordinary water (Murphy 1990). In general, the circulation of tritium would be expected to closely follow that of water. To estimate the tritium concentration in air (as vapor), water, and food, the tritium concentration in the soil water in the contaminated zone must be estimated first.

\section{L.2.1 Tritium in Soil Water}

The tritium concentration in soil water in the form of HTO in the contaminated zone can be expressed as follows:

$$
W_{H-3}^{(c z)}=\frac{\rho_{b}^{(c z)} \times S_{H-3}}{\theta^{(c z)} \times R_{d}^{(c z)}}
$$

where

$$
\begin{aligned}
W_{H-3}{ }^{(c z)}= & \text { concentration of tritium in soil water in the contaminated zone } \\
& \left(\mathrm{pCi} / \mathrm{cm}^{3}\right), \\
\rho_{b}{ }^{(c z)}= & \text { bulk density of the contaminated zone }\left(1.5 \mathrm{~g} / \mathrm{cm}^{3}\right), \\
S_{H-3}= & \text { concentration of tritium in contaminated soil }(\mathrm{pCi} / \mathrm{g}), \\
\theta^{(c z)}= & \text { volumetric water content of the contaminated zone (dimension- } \\
& \text { less), and } \\
R_{d}{ }^{(c z)}= & \text { retardation factor of tritium in the contaminated zone (dimen- } \\
& \text { sionless, see Appendix } \mathrm{E}) .
\end{aligned}
$$

The volumetric water content, $\theta^{(c z)}$, is expressed in Equation E. 6 of Appendix E as

$$
\theta^{(c z)}=p_{t}^{(c z)} \times R_{s}^{(c z)}
$$


where

$$
\begin{aligned}
& p_{t}^{(c z)}=\text { total soil porosity, and } \\
& R_{s}^{(c z)}=\text { saturation ratio. }
\end{aligned}
$$

The retardation factor, $R_{d}$, is expressed in Equation E.8 of Appendix E as

$$
R_{d}=1+\frac{\rho_{b} \times K_{d}}{\theta}
$$

Substituting Equations L.2 and L.3 into Equation L.1, $W_{H-3}^{(c z)}$ can be written as

$$
W_{H-3}{ }^{(c z)}=\frac{\rho_{b}{ }^{(c z)} \times S_{H-3}}{\left[\rho_{t}^{(c z)} R_{s}{ }^{(c z)}\right]+\left[\rho_{b}{ }^{(c s)} K_{d}{ }^{(c z)}\right]}
$$

where

$$
K_{d}^{(c z)}=\text { distribution coefficient for tritium }\left(0 \mathrm{~cm}^{3} / \mathrm{g}\right) \text {. }
$$

For the case of HTO, tritium transport generally follows that of stable hydrogen in water (i.e., $K_{d}=0$ ); therefore,

$$
W_{H-3}^{(c z)}=\frac{\rho_{b}{ }^{(c z)} \times S_{H-3}}{\rho_{t} \times R_{s}} .
$$

\section{L.2.2 Tritium in Air}

The tritium concentration in the air above an area of soil contaminated with tritium is influenced by the tritium flux from the ground surface, environmental and meteorological factors, location, and time. If tritium closely follows the transport of stable hydrogen in the environment, the relationship of tritium in air versus tritium in the ground will be analogous to that of stable hydrogen in these media. In the case of HTO, this relationship implies that tritium concentrations in air and soil will depend on the ratio of water vapor in air to the water content in soil.

For HTO in the contaminated zone, the tritium flux or the evaporation rate from a contaminated site can be estimated by using the water balance equation (Equation E.4) and other site-specific parameters. The primary environmental parameter that describes the concentration of water vapor in air is site-specific absolute humidity. Although other factors 
such as wind speed and temperature can temporarily affect the humidity in a localized area, the long-term average water concentration in air can be represented by the average humidity measured at the site.

The equilibrium tritium concentration in air resulting from an infinitely large contaminated area can be related to the stable hydrogen content in air and soil water in proportion to the tritium concentration in soil water as follows:

$$
C_{H-3, a}{ }^{e q m}=\frac{W_{H-3}{ }^{(c z)} \times C_{H, a}^{e q m}}{W_{H}}
$$

where

$$
\begin{aligned}
C_{H-3, a}{ }^{\text {eqm }}= & \text { average equilibrium concentration of tritium in air }\left(\mathrm{pCi} / \mathrm{m}^{3}\right), \\
C_{H, a}{ }^{\text {eqm }}= & \text { average equilibrium concentration of hydrogen in air }\left(\mathrm{g} / \mathrm{m}^{3}\right), \\
& \text { and } \\
W_{H}= & \text { mass fraction of hydrogen in water }\left(1 \mathrm{~g} \mathrm{H} \text { per } 9 \mathrm{~cm}^{3} \mathrm{H}_{2} \mathrm{O}\right) .
\end{aligned}
$$

The average equilibrium concentration of hydrogen in air can be expressed as

$$
C_{H, a}^{e q m}=\frac{1}{9} \times H_{a}
$$

where

$1 / 9=$ mass fraction of hydrogen in water (dimensionless, because $1 \mathrm{~g} \mathrm{H}_{2} \mathrm{O}$ $\left.=1 \mathrm{~cm}^{3} \mathrm{H}_{2} \mathrm{O}\right)$, and

$H_{a}=$ average absolute humidity in $\operatorname{air}\left(8 \mathrm{~g} / \mathrm{m}^{3}\right)$.

Equation L.6 can be rewritten as

$$
C_{H-3, a}^{e q m}=W_{H-3}^{(c z)} \times H_{a}
$$

Site-specific values of the absolute humidity are required as input to determine the equilibrium concentration of tritium in air. Because of the wide variability in atmospheric humidity from one location to another within the United States, a contour map of absolute humidity values is provided for user reference (Figure L.1). 


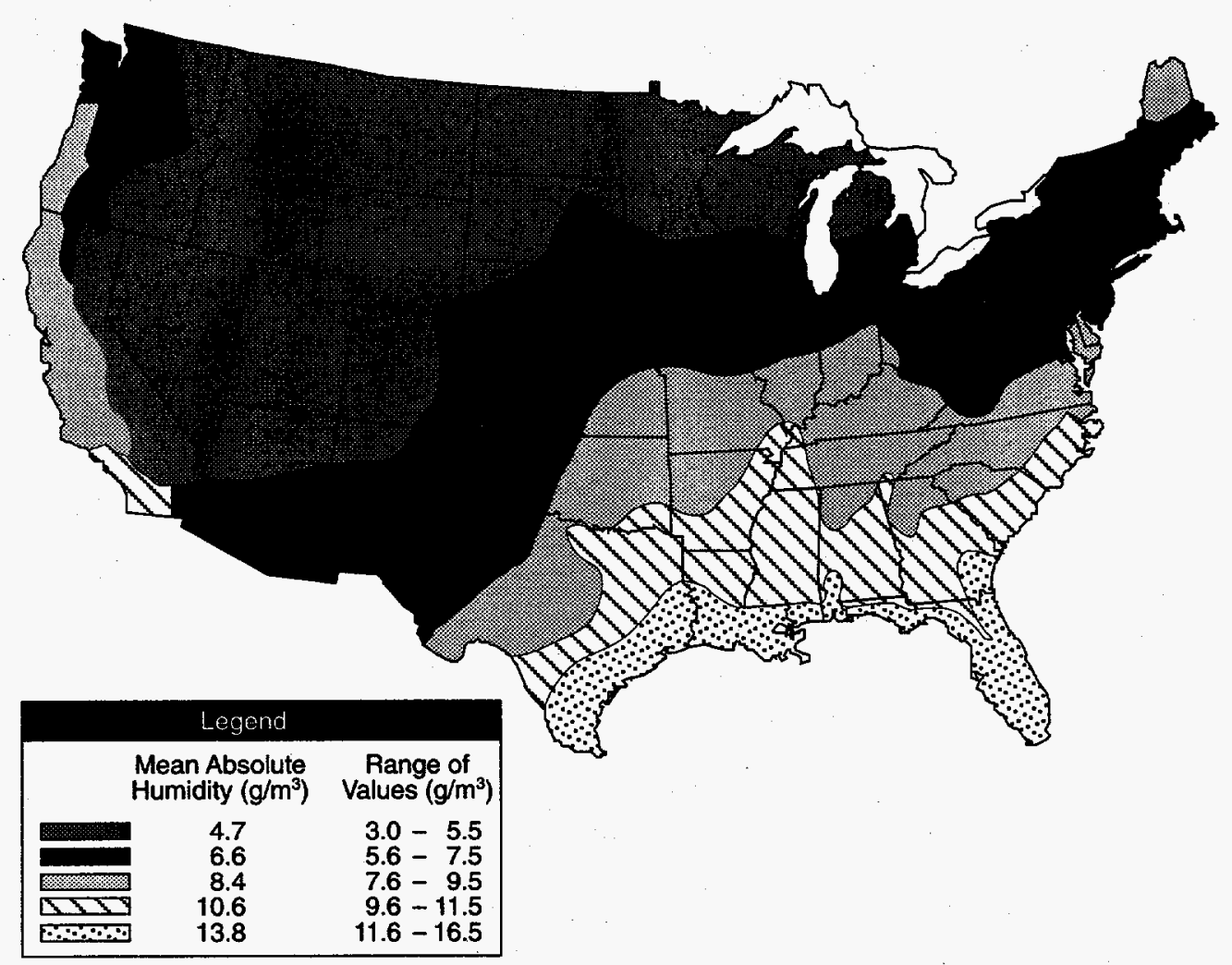

FIGURE L.1 Absolute Humidity by Geographical Region (Source: Etnier 1980)

For a local source with a finite area $A$, the tritium concentration in air can be estimated by using the following approximation, which neglects the small amount of water retained in plants relative to the amount released from the soil:

$$
C_{H-3, a}=\frac{3.17 \times 10^{-8} \times 0.5 \times E V S N \times \sqrt{A}}{H_{m i x} \times U},
$$

where

$$
\begin{aligned}
C_{H-3, a}= & \text { average concentration of tritium in air over a contaminated } \\
& \text { area of finite size }\left(\mathrm{pCi} / \mathrm{m}^{3}\right),
\end{aligned}
$$

$3.17 \times 10^{-8}=$ unit conversion factor $(\mathrm{yr} / \mathrm{s})$,

$$
\begin{aligned}
0.5= & \text { time fraction wind is blowing toward receptor } \\
& \text { (dimensionless), }
\end{aligned}
$$




$$
\begin{aligned}
E V S N= & \text { tritium flux (evasion rate) from the contaminated area } \\
& \left(\mathrm{pCi} / \mathrm{m}^{2}-\mathrm{yr}\right), \\
A= & \text { area of contaminated zone }\left(10,000 \mathrm{~m}^{2}\right), \\
H_{\text {mix }}= & \text { height at which the tritiated water vapor is uniformly mixed } \\
& (2 \mathrm{~m} \text { for the human inhalation pathway; } 1.0 \mathrm{~m} \text { for the plant, } \\
& \text { meat, and milk ingestion pathways), and } \\
U= & \text { annual average wind speed }(2 \mathrm{~m} / \mathrm{s}) .
\end{aligned}
$$

For HTO, the tritium flux can be calculated on the basis of the water balance equation for the contaminated site:

$$
\begin{aligned}
& E V S N=10^{6} \times W_{H-3}^{(c z)} \times E_{t}, \\
& E_{t}=C_{e} \times\left[\left(1-C_{r}\right) P_{r}+I_{r r}\right],
\end{aligned}
$$

where

$$
\begin{aligned}
10^{6}= & \text { unit conversion factor }\left(\mathrm{cm}^{3} / \mathrm{m}^{3}\right), \text { and } \\
E_{t}= & \text { evapotranspiration rate (see Equation E.4 in Appendix E for } \\
& \text { definitions of constituent parameters) }(\mathrm{m} / \mathrm{yr}) .
\end{aligned}
$$

The tritium concentration in air calculated by using Equation L.10 should always be less than the equilibrium value calculated by using Equation L. 8 for an infinitely large source area. For tritium in forms other than tritiated water vapor, the tritium flux, EVSN, may be obtained by using site-specific measurements.

The tritium flux to the atmosphere results in a fractional loss of tritium from soil water in addition to the loss of tritium because of radioactive decay and leaching.

To account for the tritium lost to air from the contaminated zone, an evasion source factor for tritium is included in addition to the source factor for radioactive decay and leaching. The evasion source factor, $S F_{E}(t)$, can be calculated as follows:

$$
S F_{E}(t)=e^{-\left(E_{c} \times t\right)},
$$


where

$$
\begin{aligned}
& E_{c}=0, d_{r e f}-C_{d}(t)<0 \\
& E_{c}=E_{H-3} \times \frac{d_{r e f}-C_{d}(t)}{T(t)}, 0 \leq d_{r e f}-C_{d}(t) \leq T(t) \\
& E_{c}=E_{H-3}, d_{r e f}-C_{d}(t)>T(t) \\
& E_{H-3}=\frac{10^{-6} E V S N}{\rho_{b}{ }^{(c z)} S_{H-3} d_{r e f}}
\end{aligned}
$$

and where

$$
\begin{aligned}
S F_{E}(t)= & \text { source factor for tritium due to evapotranspiration } \\
& \text { (dimensionless), } \\
E_{c}= & \text { evasion rate adjusted for cover thickness }\left(\mathrm{yr}^{-1}\right) \\
E_{H-3}= & \text { evasion rate constant }\left(\mathrm{yr}^{-1}\right) \\
d_{r e f}= & \text { reference evasion depth }(0.3 \mathrm{~m}), \\
C_{d}(t)= & \text { cover thickness }(\mathrm{m}), \\
T(t)= & \text { thickness of the contaminated zone }(\mathrm{m}), \\
t= & \text { elapsed time (yr), and } \\
10^{-6}= & \text { unit conversion factor }\left(\mathrm{m}^{3} / \mathrm{cm}^{3}\right) .
\end{aligned}
$$

\section{L.2.3 Tritium in Food}

Tritium concentrations in plants grown on contaminated soil are modeled by assuming that the transfer of tritium from soil to plants follows the path of stable hydrogen from soil to plants as

$$
C_{H-3, p k}=\frac{W_{H-3}^{(c z)} \times C_{H, p k}}{W_{H}}
$$

or

$$
C_{H-3, p k}=9 \times W_{H-3}^{(c z)} \times C_{H, p k},
$$


where

$C_{H-3, p k}=$ concentration of tritium in plants, pathway $p=3$ (produce ingestion), $p=4$ (meat ingestion), or $p=5$ (milk ingestion); for plant type $k=1$ (leafy vegetables), $k=2$ (other vegetables, fruits, and grains), or $k=3$ (fodder) (pCi/g); and

$C_{H, p k}=$ mass fraction of hydrogen in plants (dimensionless).

The mass fraction of hydrogen in plants is estimated to have a value of 0.10 (see Table L.1). Therefore, Equation L.14 can be rewritten as

$$
C_{H-3, p k}=0.9 \times W_{H-3}^{(c z)}
$$

By substituting the expression for tritium in soil water (Equation L.4) into Equation L.15, Equation L.13 can be rewritten as

$$
C_{H-3, p k}=\frac{0.9 \times \rho_{b}^{(c z)} \times S_{H-3}}{\left(p_{t}^{(c z)} \times R_{s}^{(c z)}\right)+\left(\rho_{b}^{(c z)} \times K_{d}^{(c z)}\right)}
$$

The transfer of tritium to foods such as produce, meat, milk, and fish is calculated by using the general equations derived in Appendix D. However, the food transfer coefficients for tritium must be derived by taking into account site-specific parameters such as humidity, animal diets, and intake rates.

TABLE L.1 Water Content of Food Types

\begin{tabular}{lc}
\hline \multicolumn{1}{c}{ Food Type } & $\begin{array}{c}\text { Water Fraction } \\
(\mathrm{kg} / \mathrm{kg})\end{array}$ \\
\hline Fresh fruits, vegetables, and fodder & 0.80 \\
Grain and stored feed & 0.12 \\
Meat & 0.60 \\
Milk & 0.88 \\
\hline
\end{tabular}

Source: Napier et al. (1988). 
The plant-food/soil concentration ratio for root uptake is calculated by combining Equations L.16 and D.8:

$$
F S R_{H-3,31 k}=\frac{0.9 \times \rho_{b}^{(c z)}}{\left(p_{t}^{(c z)} \times R_{s}^{(c z)}\right)+\left(\rho_{b}^{(c z)} \times K_{d}^{(c z)}\right)}
$$

The transfer of tritium to meat and milk can be calculated by correlation with the transfer of stable hydrogen to meat and milk, by calculating the intake rate of hydrogen in the animal diet as follows:

$$
\begin{gathered}
F Q R_{H-3, p} \equiv \frac{C_{H-3, p}}{F I_{H-3, p}}=\frac{C_{H, p}}{F I_{H, p}} \\
F I_{H, p}=\left(W_{H} \times F I_{p 5}\right)+\left(C_{H, p q 3} \times F I_{p q 3}\right) \\
F I_{H-3, p}=1,000 \times\left[\left(W_{H-3, r} \times F I_{p 5}\right)+\left(C_{H-3, p q 3} \times F I_{p q 3}\right)\right] \\
C_{H, p}=0.11 C_{H_{2} O, p}
\end{gathered}
$$

where

$$
\begin{aligned}
C_{H-3, p}= & \text { concentration of tritium in meat }(p=4) \text { or milk }(p=5)(\mathrm{pCi} / \mathrm{kg}), \\
F I_{H-3, p}= & \text { daily intake rate of tritium in livestock diet (pCi/d), } \\
C_{H, p}= & \text { mass fraction of stable hydrogen in meat or milk (dimension- } \\
& \text { less), } \\
F I_{H, p}= & \text { daily intake rate of stable hydrogen in livestock diet }(\mathrm{kg} / \mathrm{d}), \\
W_{H}= & \text { mass fraction of stable hydrogen in livestock water }(0.11, \\
& \text { dimensionless), } \\
F I_{p 5}= & \text { daily intake rate of livestock water }(p=4,50 \mathrm{~L} / \mathrm{d} ; p=5, \\
& 160 \mathrm{~L} / \mathrm{d}), \\
C_{H, p q 3}= & \text { mass fraction of stable hydrogen in livestock fodder }(0.10, \\
& \text { dimensionless), }
\end{aligned}
$$




$$
\begin{aligned}
F I_{p q 3}= & \text { daily intake rate of livestock fodder }(p=4,68 \mathrm{~kg} / \mathrm{d} ; p=5, \\
& 55 \mathrm{~kg} / \mathrm{d}), \\
1,000= & \text { unit conversion factor }\left(\mathrm{cm}^{3} / \mathrm{L}\right. \text { for water, g/kg for fodder). } \\
W_{H-3, r}= & \text { concentration of tritium in livestock water }(r=1 \text { for well water, } \\
& r=2 \text { for surface water) }\left(\mathrm{pCi} / \mathrm{cm}^{3}\right), \\
C_{H-3, p q 3}= & \text { concentration of tritium in livestock fodder (pCi/g), and } \\
C_{H_{2} O, p=} & \text { mass fraction of water in meat or milk (dimensionless, default } \\
& \text { values in Table L.1). }
\end{aligned}
$$

By using Equations L.18 through L.20 for each food type $p$, the tritium transfer factor, $F Q R_{H-3, p}$, can be derived as follows:

$$
F Q R_{H-3, p}=\frac{C_{H, p}}{\left(W_{H} \times F I_{p 5}\right)+\left(C_{H, p q 3} \times F I_{p q 3}\right)}
$$

The values of $C_{H, p}$ and $C_{H, p q 3}$ can be derived from the water contents in meat, milk, and fodder. Table L.1 shows the default values of these parameters used in the RESRAD code. These parameters are not expected to be very site specific.

The concentration of tritium in meat or milk can then be calculated as

$$
C_{H-3, p}=F I_{H-3, p} \times F Q R_{H-3, p} .
$$

Tritium transfer from contaminated water to fish or other aquatic foods is calculated by using aquatic bioaccumulation factors, $F W R_{i 6 k}$, as described in Section D.2.2.

$$
C_{H-3,6 k}=F W R_{H-3,6 k} \times W_{H-3,2},
$$

where

$$
\begin{aligned}
F W R_{H-3,6 k}= & \text { fish } / \text { water }(k=1) \text { and crustacea-mollusk } / \text { water }(k=2) \text { con- } \\
& \text { centration ratio (bioaccumulation factor) for tritium }(1.0 \mathrm{~L} / \mathrm{kg}), \\
C_{H-3,6 k}= & \text { concentration of tritium in fish or other aquatic foods }(\mathrm{pCi} / \mathrm{kg}), \\
& \text { and } \\
W_{H-3,2}= & \text { concentration of tritium in surface water }(\mathrm{pCi} / \mathrm{L}) .
\end{aligned}
$$


In the RESRAD calculations, the plant and food transfer factors are used to calculate the environmental transfer factors $\left(E T F_{H-3, p q}\right)$ of tritium from the contaminated zone for pathways $p$ and subpathways $q$ as a function of time. This approach maintains the general pathway analysis methodology in RESRAD so that site-specific variables, such as depth and area factors associated with the various pathways, can still be effective.

\section{L.2.4 Tritium Absorption Dose}

Water vapor is absorbed through the skin at approximately $50 \%$ of the inhalation rate of water vapor (International Commission on Radiological Protection 1979-1982; Hamby 1993). To account for dermal absorption of HTO vapor in air, RESRAD multiplies the dose from inhalation alone by a factor of 1.5 .

\section{L.3 CARBON-14 SUBMODELS}

Carbon-14, with a radiological half-life of 5,730 years, is a naturally occurring isotope of carbon produced in the stratosphere by the interaction of cosmic ray neutrons with nitrogen atoms. Carbon-14 is also produced in appreciable quantities in atmospheric and underground testing of nuclear and thermonuclear devices, as well as in nuclear power operations (primarily the result of fission and neutron activation). Carbon-14 decays to stable nitrogen by emitting a beta particle $\left(E_{\max }=0.156 \mathrm{MeV}\right)$ and does not pose an external hazard. Because carbon-14 emits a higher energy beta particle, it poses a more severe internal radiological hazard than tritium. The ingestion dose conversion factor is approximately 30 times that of tritium. One of the most important pathways involving the radiological dose to humans from soil contaminated with carbon-14 is the plant ingestion pathway. In addition to direct root uptake from soil and foliar deposition of dust particles contaminated with carbon-14, carbon in gases volatilized from the soil is directly incorporated into the plant by the process of photosynthesis.

Like models developed for tritium, much of the modeling for carbon-14 has evolved from analyses of atmospheric sources and is not appropriate for ground contamination sources (U.S. Nuclear Regulatory Commission 1977; Killough and Rohwer 1978; Zach and Sheppard 1991). 
The models used for calculating the dose from carbon-14 in air usually assume a steady-state relationship among carbon isotopes, from the point of photosynthetic fixation through the food chain to man. In the natural environment, the ratio of carbon-14 to carbon-12 (the common form of stable carbon) in the body, as well as in plants and animals, is the same as it is in the atmosphere. At a site contaminated with carbon-14, the amount of carbon-14 in air (and hence in all living organisms) would increase over the natural background.

\section{L.3.1 Carbon-14 in Air}

Inorganic and organic reactions convert most forms of soil carbon to $\mathrm{CO}_{2}$. Because of the volatile nature of $\mathrm{CO}_{2}$, soil carbon is usually lost to the air where it becomes absorbed in plants through photosynthesis. The concentration of carbon-14 in air above a contaminated zone depends on the volatilization (evasion) rate of carbon from the soil, the size and location of the source area, and meteorological dispersion conditions.

Sheppard et al. (1991) measured the rate of carbon-14 loss from soils in outdoor lysimeter experiments. They found that the evasion rate, $E$, was not a strong function of soil properties. For clay and loam soils, the evasion rate was estimated to be $3.8 \times 10^{-7} / \mathrm{s}$; for sand and organic soils, the evasion rate was estimated to be $6.7 \times 10^{-7} / \mathrm{s}$. However, evasion rates from as low as $1.0 \times 10^{-10} / \mathrm{s}$ from native carbonates and humified soils have been observed by Amiro et al. (1991).

For a local contaminated source of area $A$, the carbon-14 concentration in air can be estimated in a manner similar to that for Equation L.9 for tritium:

$$
C_{C-14, a}=\frac{3.17 \times 10^{-8} \times 0.5 \times E V S N \times \sqrt{A}}{H_{m i x} \times U}
$$

where

$$
\begin{aligned}
C_{C-14, a}= & \text { average concentration of carbon-14 in air over a contaminated } \\
& \text { area of finite size }\left(\mathrm{pCi} / \mathrm{m}^{3}\right),
\end{aligned}
$$

$3.17 \times 10^{-8}=$ unit conversion factor $(\mathrm{yr} / \mathrm{s})$,

$$
0.5 \text { = time fraction wind is blowing toward receptor (dimensionless), }
$$




$$
\begin{aligned}
E V S N= & \text { carbon-14 flux (evasion rate) from the contaminated area } \\
& \left(\mathrm{pCi} / \mathrm{m}^{2}-\mathrm{y}\right), \\
A= & \text { area of contaminated zone }\left(10,000 \mathrm{~m}^{2}\right), \\
H_{m i x}= & \text { height into which the tritiated water vapor is uniformly mixed } \\
& (2 \mathrm{~m} \text { for the human inhalation pathway; } 1.0 \mathrm{~m} \text { for the plant, } \\
& \text { meat, and milk ingestion pathways), and } \\
U= & \text { annual average wind speed }(2 \mathrm{~m} / \mathrm{s}) .
\end{aligned}
$$

The flux of gaseous carbon-14 to the atmosphere from soil was correlated to the evasion loss rate by Sheppard et al. (1991) for a reference depth of $30 \mathrm{~cm}$ as follows:

$$
E V S N=10^{6} \times S_{C-14} \times E_{C-14} \times \rho_{b}^{(c z)} \times d_{r e f}
$$

where

$$
\begin{aligned}
10^{6} & =\text { unit conversion factor }\left(\mathrm{cm}^{3} / \mathrm{m}^{3}\right) \\
S_{C-14} & =\text { concentration of carbon-14 in contaminated soil }(\mathrm{pCi} / \mathrm{g}), \\
E_{C-14} & =\text { evasion loss rate constant }\left(22 \mathrm{yr}^{-1}\right) \\
\rho_{b}{ }^{(c z)} & =\text { bulk density of the contaminated zone }\left(1.5 \mathrm{~g} / \mathrm{cm}^{3}\right), \text { and } \\
d_{r e f} & =\text { reference soil depth }(0.3 \mathrm{~m})
\end{aligned}
$$

The evasion rate of carbon-14 describes the fraction of the soil inventory of carbon-14 that is lost to the atmosphere per unit time. This rate can usually be obtained by measuring the carbon-14 inventory as a function of time in the contaminated zone. Table L.2 lists the measured evasion rates for carbon-14 from lysimeter experiments.

The transport of carbon-14 is assumed to follow that of stable carbon in the environment. For an infinitely large source
TABLE L.2 Carbon-14 Evasion Rate Factor

\begin{tabular}{lc}
\hline & $\begin{array}{c}\text { Evasion } \\
\text { Rate } \\
\left(\mathrm{yr}^{-1}\right)\end{array}$ \\
\hline Soil Type & 12 \\
Loamy soils & 12 \\
Sandy soils & 22 \\
Organic soils & 22 \\
Stable carbon in carbonate soils & 0.0032 \\
Water & 0.91 \\
\hline
\end{tabular}

Sources: Sheppard et al. (1991); Amiro and Davis (1991). 
area, the ratio of carbon-14 in air to carbon-14 in soil is analogous to that of stable carbon in air and soil:

$$
C_{C-14, a}^{e q m}=C_{C, a}^{e q m} \times \frac{E_{C-14} \times S_{C-14}}{E_{C-12} \times S_{C}}
$$

where:

$$
\begin{aligned}
C_{C-14, a}{ }^{\text {eqm }}= & \text { equilibrium concentration of carbon-14 in air }\left(\mathrm{pCi} / \mathrm{m}^{3}\right), \\
C_{c, a}{ }^{\text {eqm }}= & \text { equilibrium concentration of stable carbon in air }\left(0.16 \mathrm{~g} / \mathrm{m}^{3}\right), \\
E_{C-14}= & \text { evasion rate of carbon-14 in soil }(22 / \mathrm{yr}), \\
S_{C-14}= & \text { concentration of carbon }-14 \text { in soil }(\mathrm{pCi} / \mathrm{g}), \\
E_{C-12}= & \text { average evasion rate of stable carbon from soil }(0.0032 / \mathrm{yr}), \\
& \text { and } \\
S_{C}= & \text { fraction of soil which is stable carbon }(0.03, \text { dimensionless }) .
\end{aligned}
$$

For a finite source contaminated with carbon-14, the concentration of carbon-14 in air (calculated with Equation L.25) should always be less than the concentration of carbon-14 from an infinitely large contaminated source area (calculated with Equation L.27).

To account for the carbon-14 lost to air from the contaminated zone, an evasion source factor for carbon-14 is needed in addition to the source factor for radioactive decay and leaching. The evasion source factor, $S F_{E}(t)$ can be derived as follows:

$$
S F_{E}(t)=e^{-\left(E_{c} \times t\right)}
$$

where

$$
\begin{aligned}
& E_{c}=0, d_{r e f}-C_{d}(t)<0 \\
& E_{c}=E_{C-14}{ }^{\prime} \times \frac{d_{r e f}-C_{d}(t)}{T(t)}, 0 \leq d_{r e f}-C_{d}(t) \leq T(t) \\
& E_{c}=E_{C-14}, d_{r e f}-C_{d}(t)>T(t)
\end{aligned}
$$

and where

$$
\begin{aligned}
S F_{E}(t)= & \text { source factor for carbon-14 due to atmospheric evasion } \\
& \text { (dimensionless), }
\end{aligned}
$$




$$
\begin{aligned}
E_{c} & =\text { evasion rate adjusted for cover thickness }\left(\mathrm{yr}^{-1}\right), \\
d_{r e f} & =\text { reference evasion depth }(0.3 \mathrm{~m}) \\
C_{d}(t) & =\text { cover thickness }(\mathrm{m}) \\
T(t) & =\text { thickness of the contaminated zone }(\mathrm{m}), \text { and } \\
t & =\text { elapsed time (yr). }
\end{aligned}
$$

\section{L.3.2 Carbon-14 in Food}

The main pathway for carbon-14 intake by humans from a carbon-14-contaminated site is usually through food ingestion. Carbon-14 soil-to-plant transfer factors, $B_{C-14, v}$, are often used to estimate the radiological dose to humans from soil contaminated with carbon-14 (Coughtrey et al. 1985; Oztunali and Roles 1985; Zach and Sheppard 1991).

The transfer factors for carbon-14 currently used in most pathway analysis models are derived primarily from early data compiled by $\mathrm{Ng}$ et al. (1968). As stated by Sheppard et al. (1991), Ng's derivation treated carbon-14 in the same manner as trace elements by basing the soil-to-plant transfer factor, $B_{C-14, v}$, on the average stable carbon contents of vegetation and soil. This method of determining the transfer factor for carbon is overly conservative for the dose estimation because vegetation incorporates most of its carbon from the atmosphere during photosynthesis. Sheppard et al. (1991) describe the implications of this transfer coefficient for carbon-14 and indicate that two separate pathways exist: (1) direct root uptake and (2) uptake by foliage of gases volatilized from the soil and absorbed from the atmosphere.

Therefore, the quantification and comparison of these two pathways is important for deriving the meaningful transfer coefficient for a specific site contaminated with carbon-14. In general, the direct root uptake pathway should be dependent on the magnitude of the soil source; the atmospheric pathway should be dependent on the volatilization rate from the soil, the uptake rate of foliage, and the meteorological dispersion conditions. Also, the size of the source area is an important factor because it determines air concentrations from local sources. In RESRAD, carbon-14 in plants is assumed to be derived from these two separate pathways. For each pathway, the transport of carbon-14 is assumed to follow that of stable carbon as 


$$
\begin{gathered}
C_{\mathrm{C}-14, v s}=1,000 \times S_{\mathrm{C}-14} \times \frac{C_{C, v s}}{S_{C}} \\
C_{\mathrm{C}-14, v a}=C_{\mathrm{C}-14, a} \times \frac{C_{C, v a}}{C_{C, a}}
\end{gathered}
$$

where

$$
\begin{aligned}
C_{\mathrm{C}-14, v s}= & \text { concentration of carbon-14 in plants derived from carbon-14 in } \\
& \text { soil (pCi/kg), } \\
1,000= & \text { unit conversion factor }(\mathrm{g} / \mathrm{kg}), \\
S_{\mathrm{C}-14}= & \text { concentration of carbon-14 in soil (pCi/g), } \\
C_{C, v s}= & \text { fraction of plant mass that is carbon derived from soil } \\
& \text { (dimensionless), } \\
S_{C}= & \text { fraction of soil that is stable carbon }(0.03, \text { dimensionless), } \\
C_{\mathrm{C}-14, v a}= & \text { concentration of carbon-14 in plants derived from carbon-14 in } \\
& \text { air (pCi } / \mathrm{kg}), \\
C_{\mathrm{C}-14, a}= & \text { concentration of carbon-14 in air (pCi } \left./ \mathrm{m}^{3}\right), \\
C_{C, v a}= & \text { fraction of plant mass that is carbon derived from } \\
& \text { photosynthesis (dimensionless), and } \\
C_{C, a}= & \text { concentration of stable carbon in air }\left(1.6 \times 10^{-4} \mathrm{~kg} / \mathrm{m}^{3}\right) .
\end{aligned}
$$

Equations L.29 and L.30 can be merged into Equation L.31 for deriving the total concentration of carbon-14 in a plant from these two separate pathways as a function of sitespecific parameters:

$$
C_{\mathrm{C}-14, v}=C_{C, v} \times\left[\left(F_{a} \times \frac{C_{\mathrm{C}-14, a}}{C_{C, a}}\right)+\left(1,000 \times F_{s} \times \frac{S_{\mathrm{C}-14}}{S_{C}}\right)\right],
$$

where

$$
\begin{aligned}
C_{C-14, v} & =\text { concentration of carbon-14 in plants }(\mathrm{pCi} / \mathrm{kg}), \\
C_{C, v} & =\text { fraction of stable carbon in plants (dimensionless), }
\end{aligned}
$$




$$
\begin{aligned}
F_{a}= & C_{C, v a} / C_{C, v}=\text { fraction of carbon in plants derived from carbon in } \\
& \text { air (0.98, dimensionless), and } \\
F_{s}= & C_{C, v s} / C_{C, v}=\text { fraction of carbon in plants derived from carbon in } \\
& \text { soil }\left(0.02, \text { dimensionless; } F_{s}=0, \text { if } S_{c}=0\right) .
\end{aligned}
$$

Dividing both sides of Equation L.31 by $S_{\mathrm{C}-14}$, the soil-to-plant transfer factor, $B_{C-14, v}$, including all sources can be given as

$$
B_{C-14, v}=C_{C, v} \times\left[\left(F_{a} \times \frac{10^{-3} \times C_{\mathrm{C}-14, a}}{S_{\mathrm{C}-14} \times C_{C, a}}\right)+\left(\frac{F_{s}}{S_{C}}\right)\right]
$$

It has been shown that most of the plant carbon is assimilated from the atmosphere through photosynthesis. Only 1 to $2 \%$ is absorbed directly through the roots (Sheppard et al. 1991). In RESRAD, the default values of $F_{a}$ and $F_{s}$ are assumed to be 0.98 and 0.02 , respectively. Equation L.32 is used in RESRAD to calculate carbon-14 concentrations in various plant products consumed by either humans or livestock.

The carbon-14 concentrations in meat and milk are assumed to be derived totally from the livestock diets. The transfer of carbon-14 from the livestock diets to meat and milk is assumed to follow the same route as that of stable carbon. The carbon-14 contents in meat and milk are related to the intake rates of stable carbon and carbon-14 by livestock as

$$
\begin{gathered}
\frac{C_{\mathrm{C}-14, p}}{F I_{\mathrm{C}-14, p}}=\frac{C_{C, p}}{F I_{C, p}}, \\
F I_{C, p}=\left(W_{C} \times F I_{p 5}\right)+\sum_{k}\left(C_{C, p q k} \times F I_{p q k}\right),
\end{gathered}
$$

and

$$
F I_{\mathrm{C}-14, p}=\left(W_{\mathrm{C}-14, r} \times F I_{p 5}\right)+\sum_{k}\left(C_{\mathrm{C}-14, p q k} \times F I_{p q k}\right)
$$

where

$$
\begin{aligned}
C_{\mathrm{C}-14, p}= & \text { concentration of carbon-14 in meat }(p=4) \text { or milk }(p=5) \\
& (p C i / k g),
\end{aligned}
$$




$$
\begin{aligned}
F I_{C-14, p}= & \text { intake rate of carbon-14 in the diet of beef cattle }(p=4) \text { or milk } \\
& \text { cows }(p=5)(\mathrm{pCi} / \mathrm{d}), \\
C_{C, p}= & \text { fraction of stable carbon in meat or milk (beef }=0.24, \text { milk }= \\
& 0.070, \text { dimensionless), } \\
F I_{C, p}= & \text { intake rate of stable carbon in the diet of beef cattle or milk } \\
& \text { cows }(\mathrm{kg} / \mathrm{d}), \\
W_{C}= & \text { concentration of stable carbon in livestock water } \\
& \left(2.0 \times 10^{-5} \mathrm{~kg} / \mathrm{L}\right), \\
F I_{p 5}= & \text { livestock water intake rate }(160 \mathrm{~L} / \mathrm{d}, \text { milk cow; } 50 \mathrm{~L} / \mathrm{d}, \text { beef } \\
& \text { cattle), } \\
C_{C, p q k}= & \text { concentration of stable carbon in livestock fodder }(\mathrm{pCi} / \mathrm{kg}), \\
F I_{p q k}= & \text { livestock fodder intake rate }(\mathrm{p}=4,68 \mathrm{~kg} / \mathrm{d} ; \mathrm{p}=5,55 \mathrm{~kg} / \mathrm{d}), \\
W_{C-14, r}= & \text { concentration of carbon-14 in livestock water }(r=1 \text { for well } \\
& \text { water, } r=2 \text { for surface water) (pCi/L), and } \\
C_{C-14, p q k}= & \text { concentration of carbon-14 in livestock fodder (fresh pasture, } \\
& k=3, \text { or stored grain, } k=4)(\mathrm{pCi} / \mathrm{kg}) .
\end{aligned}
$$

Because various livestock feeds have significantly different carbon contents (see Table L.3), carbon-14 concentrations in livestock fodder are calculated in RESRAD by using the soil-to-plant transfer factors (see Equation L.32) for two different types of feed: fresh forage (carbon fraction $=0.090$ ) and stored grain (carbon fraction $=0.40$ ).

For food type $p$, the carbon-14 transfer factor, $F Q R_{C-14, p}$, can be derived by using Equations L.33 and L.34 as
TABLE L.3 Carbon Content of Food Types and Water

\begin{tabular}{lc}
\hline \multicolumn{1}{c}{ Food Type } & $\begin{array}{c}\text { Carbon } \\
\text { Fraction (kg/kg) }\end{array}$ \\
\hline Fresh fruits, vegetables, & 0.090 \\
and fodder & 0.40 \\
Grain and stored feed & 0.24 \\
Meat & 0.070 \\
Milk & $2 \times 10^{-5}$ \\
Water & \\
\hline
\end{tabular}

Source: Napier et al. (1988). 


$$
F Q R_{\mathrm{C}-14, p}=\frac{C_{C, p}}{\left(W_{C} \times F I_{p 5}\right)+\sum_{k}\left(C_{C, p q k} \times F I_{p q k}\right)}
$$

The values of $C_{C, p}$ and $C_{C, p q k}$ can be obtained by measuring the carbon contents in various local foods and feeds. However, these are not expected to be very site specific. Table L.3 shows the RESRAD default values for these parameters.

The concentrations of carbon-14 in meat and milk can then be calculated as

$$
C_{\mathrm{C}-14, p}=F Q R_{C-14, p} \times F I_{\mathrm{C}-14, p} .
$$

Carbon-14 in fish or other aquatic food is calculated by using aquatic bioaccumulation factors, $F W R_{\mathrm{C}-14,6 \mathrm{k}}$, as described in Section D.2.2.

$$
C_{\mathrm{C}-14,6 k}=F W R_{C-14,6 k} \times W_{\mathrm{C}-14,2},
$$

where

$$
\begin{aligned}
F W R_{\mathrm{C}-14,6 k}= & \mathrm{fish} / \text { water }(k=1) \text { and crustacea-mollusk/water }(k=2) \\
& \text { concentration ratio (bioaccumulation factor) for carbon-14 } \\
& \left(k=1,5 \times 10^{4} ; k=2,9.1 \times 10^{3} \mathrm{~L} / \mathrm{kg}\right), \text { and } \\
W_{\mathrm{C}-14,2}= & \text { concentration of carbon-14 in surface water }(\mathrm{pCi} / \mathrm{L}) .
\end{aligned}
$$

In the RESRAD calculations, these site-specific plant and food transfer factors, $B_{\mathrm{C}-14, v}, F Q R_{\mathrm{C}-14, p}$, and $F W R_{\mathrm{C}-14,6 k}$, are used in a similar manner to transfer factors assigned to other solid radionuclides. These transfer factors are used to calculate the time-dependent carbon-14 food/soil concentration ratios, $F S R_{\mathrm{C}-14, p q k}(t)$, in various foods due to residual carbon-14 contamination in soil. This approach assumes that the other site-specific variables, such as the depth and area factors associated with various pathways, are applicable to carbon-14.

\section{L.4 REFERENCES}

Amiro, B.D., and P.A. Davis, 1991, "A Pathway Model to Assess Transport of Radionuclides from Terrestrial and Aquatic Surfaces to the Atmosphere," Waste Management 11:41-57. 
Amiro, B.D., et al., 1991, "Relative Importance of Atmospheric and Root Uptake Pathways for ${ }^{14} \mathrm{CO}_{2}$, Transfer from Contaminated Soil to Plants," Health Physics 61:825-829.

Coughtrey, P.J., et al., 1985, The Assessment of Terrestrial Pathways of Radionuclides to Man: Experimental Studies on C-14 and H-3 Transfers in Soils and Plants, 555-1, Associated Nuclear Services, Epsom, United Kingdom.

Etnier, E.L., 1980, "Regional and Site-Specific Absolute Humidity Data for Use in Tritium Dose Calculations," Health Physics 39:318-320.

Evans, A.G., 1969, "New Dose Estimates from Chronic Tritium Exposures," Health Physics 16: $57-63$.

Hamby, D.M., 1992, "A Methodology for Estimating the Radiological Consequence of an Acute Aqueous Release," Health Physics 62:567-570.

International Commission on Radiological Protection, 1979-1982, Limits for Intakes of Radionuclides by Workers, a report of Committee 2 of the International Commission on Radiological Protection, adopted by the Commission in July 1978, ICRP Publication 30, Part 1 (and Supplement), Part 2 (and Supplement), Part 3 (and Supplements A and B), and Index, Annals of the ICRP, Pergamon Press, New York, N.Y.

Killough, G.G., and P.S. Rohwer, 1978, "A New Look at the Dosimetry of ${ }^{14} \mathrm{C}$ Released to the Atmosphere as Carbon Dioxide," Health Physics 34:141-159.

Moore, R.E., et al., 1979, AIRDOS-EPA: A Computerized Methodology for Estinating Environmental Concentrations and Dose to Man from Airborne Release of Radionuclides, ORNL-5532, Oak Ridge National Laboratory, Oak Ridge, Tenn., June.

Murphy, C.E., Jr., 1990, The Transport, Dispersion, and Cycling of Tritium in the Environment, WSRC-RP-90-462, Westinghouse Savannah River Company, Aiken, S.C.

Napier, B.A., et al., 1988, GENII-The Hanford Environmental Radiation Software Dosimetry System, PNL-6584/UC-600, Pacific Northwest Laboratory, Hanford, Wash., Dec.

National Council on Radiation Protection and Measurement, 1979, Tritium in the Environment, Washington, D.C.

Ng, Y.C., et al., 1968, Prediction of the Maximum Dosage to Man from the Fallout of Nuclear Devices, IV: Handbook for Estimating the Maximum Internal Dose from Radionuclides Released to the Biosphere, UCRL-50163, Lawrence Radiation Laboratory, University of California, Livermore, Calif.

Oztunali, O.I., and G.W. Roles, 1985, Update of Part 61, Impacts Analysis Methodology, NUREG/CR-4370, U.S. Nuclear Regulatory Commission, Washington, D.C. 
Sheppard, M.I., et al., 1991, "Mobility and Plant Uptake of Inorganic C-14 and C-14-Labelled PCB in Soils of High and Low Retention," Health Physics 61:481-492.

U.S. Nuclear Regulatory Commission, 1977, Calculation of Annual Dose to Man from Routine Releases of Reactor Effluents for the Purpose of Evaluating Compliance with 10 CFR Part 50, Appendix I, Rev. 1, Regulatory Guide 1.109, Office of Standards Developments, Washington, D.C.

Zach, R., and S.C. Sheppard, 1991, "Food-Chain and Dose Model, CALDOS, for Assessing Canada's Nuclear Fuel Waste Management Concept," Health Physics 60: 643-656. 
APPENDIX M:

OPTIMIZATION IN QUANTITATIVE ALARA ANALYSES 


\title{
APPENDIX M:
}

\section{OPTIMIZATION IN QUANTITATIVE ALARA ANALYSES}

\author{
by R. E. Baker
}

\section{M.1 OPTIMIZATION}

The International Commission on Radiological Protection considers the as low as reasonably achievable (ALARA) process and optimization to be synonymous. Optimization for radiological protection occurs when the total of the cost for radiological protection plus the cost of the detriment is minimal. (This is also the condition for the maximum benefit.) The following exercise demonstrates, mathematically, the relationship between costs and benefits, which is the basis for ALARA.

\section{M.1.1 Cost-Benefit Analysis}

The net benefit, $B$, of a practice (or activity) is equal to the gross benefit, $V$; minus the production cost, $P$; minus the protection cost, $X$; and minus the detriment cost, $Y$, for the selected level of protection.

$$
B=V-(P+X+Y)
$$

The total detriment cost, $Y$, includes two components: a health detriment cost, $Y_{H}$, and a nonhealth detriment cost, $Y_{N H}$. The health detriment essentially consists of radiationinduced fatal and nonfatal cancers and hereditary diseases. The risk is presumed to be linearly proportional to the collective dose, $S$, of the exposed population. The health detriment cost is also linearly proportional to the collective dose; for example, $Y_{H}=\alpha S$ where $\alpha$ is the assumed monetary worth of a unit of collective dose related to health issues. The nonhealth detriment consists of all other factors that contribute to the cost but are not necessarily related to health effects, for example, risk aversion attitudes, political or policy decisions, public relation considerations, aesthetic considerations, and management practices and policies. The nonhealth detriment cost is usually nonlinear with collective dose but is

* R.E. Baker is a consultant to the U.S. Department of Energy, Office of Environmental Guidance, Washington, D.C. 
frequently a complex function of dose, $H$. If the distribution of individual doses is described by $N_{j} f_{j}\left(H_{j}\right)$, where $N_{j}$ is the number of individuals receiving an average dose, $H_{j}$ and $f_{j}\left(H_{j}\right)$ is a function of the average dose to the $j$ th group, the nonhealth detriment cost is given by

$$
Y_{N H}=\beta \Sigma_{j} N_{j} f\left(H_{j}\right)
$$

where $\beta$ is the monetary worth of a unit of collective dose related to nonhealth issues. Thus, the total detriment cost can be derived as follows:

$$
Y=Y_{H}+Y_{N H}
$$

and

$$
Y=\alpha S+\beta \Sigma_{j} N_{j} f_{j}\left(H_{j}\right)
$$

To maximize the benefit (which is the same as minimizing the cost), Equation M.1 is differentiated with respect to $S$ and set equal to zero:

$$
\frac{d B}{d S}=\frac{d V}{d S}-\frac{d P}{d S}-\frac{d X}{d S}-\frac{d Y}{d S}=0
$$

Because $B, V$, and $P$ are generally constants; that is, they do not vary with collective dose,

$$
\frac{d B}{d S}=0, \quad \frac{d V}{d S}=0, \quad \frac{d P}{d S}=0
$$

The equation becomes

$$
\frac{d X}{d S}=-\frac{d Y}{d S}
$$

and

$$
\frac{d X}{d S}=-\left[\alpha+\frac{\beta \Sigma_{j} d\left(N_{j} f_{j}\left(H_{j}\right)\right)}{d S}\right]
$$




\section{M.1.1.1 The Alpha Term}

Considering only the health detriment part of the total detriment cost, the minimum cost (optimization) for continuous variables occurs when

$$
\frac{d X}{d S}=-\alpha
$$

In practice, the variables are usually incremental rather than continuous and the optimization occurs when

$$
\frac{X_{B}-X_{A}}{S_{B}-S_{A}}=-\alpha
$$

where the subscripts $A$ and $B$ indicate the cost of two (of several) candidate radiological protection systems and associated health detriments, and $\alpha$ is the selected monetary worth of a unit of collective dose.

In other words, where several candidate alternative actions have been identified to achieve a particular task, each alternative having a unique cost and exposure potential, the alternatives should be evaluated with respect to cost and potential individual and collective doses. The potential dose to the maximally exposed individual must be within the appropriate dose limit - whether the application is for workers, the public, or both - regardless of the cost for doing so. Arranging the alternatives in order of progressively higher system cost, the differences in cost and collective dose are determined and the cost per unit of collective dose is compared to the assumed worth of avoiding a unit of collective dose, for example, $\$$ per person-rem (or $\$$ per person-Sv).

\section{M.1.1.2 The Value of Alpha}

The worth of avoiding a unit of collective dose (the $\alpha$ coefficient) is difficult to quantify in an unambiguous manner. The collective dose can be related to presumed risk if the prudent assumption (for radiation protection purposes) is made that over the range of doses found in the environment or workplace, the probability of experiencing a radiationinduced health effect is linearly proportional to the dose. On the basis of data and extrapolations to date, the risk of experiencing a fatal cancer or severe genetic disease is about 500 per million person-rem. There would also be about 300 nonfatal cancers. Thus, 
the question arises, how much is it worth to avoid these potential health effects? Historically, many values have been proposed or postulated ranging from "a few pounds Sterling" to several thousands of dollars per person-rem. The range of reasons used to arrive at the estimates is as broad as the range of resulting values. The fact is there is no generally accepted value for $\alpha$. In applications dealing with potential doses to the public from reactor effluents, the NRC assumed a value of $\$ 1,000$ per person-rem. This could be viewed as a willingness to spend $\$ 1,000$ to avoid 0.0005 fatal cancers or about $\$ 2,000,000$ per fatal cancer. If the nonfatal cancers are considered, the commitment would be about $\$ 1.2$ million per cancer avoided. Other reasons would consider the loss of lifetime, loss of wages, insurance premiums, risks from prescription drugs, risk of highway travel, hospital costs, cost for training workers, and many other factors in deriving values for $\alpha$.

\section{M.1.1.3 The Beta Term}

Considering only the nonhealth part of the total detriment cost, the protection cost that exceeds the health detriment cost is the cost of the nonhealth detriment cost. This is the cost of the additional efforts and equipment that result in lower potential exposures because radiation sources are reduced by removal and disposal in another location, further processing of radwaste streams, additional shielding, and other alternative actions, beyond those justified by health considerations. The value of $\alpha$ is generally assumed to be constant for the entire range of individual doses contributing to the collective dose. In some instances, there have been attempts to combine the $\alpha$ and $\beta$ terms so that the optimization equation would take the following form:

$$
\frac{d X}{d S}=-(\alpha+\beta)
$$

However, the $\beta$ coefficient, in contrast to the $\alpha$ coefficient, is generally multiplied by a nonlinear function of collective dose. Further, the $B$ coefficient may be a variable that is related to specific ranges of individual doses.

\section{M.1.1.4 The Value of Beta}

The value of $B$ is not related to a particular impact, such as quantifiable specific health effects, in the case of determining a value for $\alpha$. Rather, it depends on such 
qualitative factors as perception, risk aversion, public opinion, fear of the unknown, managerial alternatives, esthetics, ethical issues, political issues, and public relations considerations.

The nonhealth detriment can sometimes be costed for some applications by assuming various values for $B$, for example, if it is desirable to avoid doses that are near the limit. The dose distribution can be influenced by using one or more values for $B$ applied to the collective dose for the exposed group receiving individual doses between $H_{M}$ and $H_{L(L I M I T)}$. A smaller value for $B$ could be applied to the collective dose for the group receiving individual doses in the next lower interval between $H_{N}$ and $H_{M}$, and $\beta=0$ could be applied to the collective dose fraction composed of the lowest individual doses. These $B$ values do not represent health considerations. Rather, they are dose-avoidance considerations. For continuous functions, the optimum condition is given by

$$
\frac{d X}{d S}=-\left\{\alpha+\beta \Sigma_{j} \frac{d\left[N_{j} f_{j}\left(H_{j}\right)\right]}{d S}\right\}
$$

And the optimization equation for incremental evaluations may be given by

$$
\frac{X_{B}-X_{A}}{S_{B}-S_{A}}=-\left(\alpha+\beta_{1} S_{N M}+\beta_{2} S_{M L}\right)
$$

In many instances, the nonhealth detriment cost can be evaluated by subtracting the health detriment cost from the protection cost, for example,

$$
\beta \Sigma_{j} N_{j} f_{j}\left(H_{j}\right)=X-\alpha S
$$

It is generally useful to be able to identify and quantify the costs of $Y_{H}$ and $Y_{N H}$. However, the value of $B$, generally cannot be determined retrospectively from the data, even if the nonhealth detriment cost is readily deduced.

\section{M.1.2 Degree of Effort}

The amount of effort that can be justified to analyze and remediate a property should be commensurate with the potential detriment of releasing the property for unconditioned use. This can be quantified by estimating (1) the potential dose to the maximally exposed 
individual (or critical population group) and (2) the collective dose to the exposed population with due consideration of spacial and temporal parameters. The individual dose must be within the appropriate limit regardless of cost. The health detriment cost is simply the product $\alpha Y$. Assuming a cost of $\$ 1,000$ per person-rem (a nominal value), the optional radiation protection cost should not exceed $\$ 1,000 \times S$, where $S$ is expressed in units of person-rem. Because this is an expression of cost-effectiveness rather than optimization, the optimum cost should be less than $\$ 1,000 \mathrm{~S}$.

\section{M.1.3 Other Analytical Methods}

Environmental and other factors, such as temperature, noise, odor, and esthetics, that are important to decision-making cannot be expressed in monetary terms and are not amenable to cost-benefit analyses. Other methods are available for analyzing the alternative actions to aid the decision-making process. One such method is multiattribute analysis in which weighting factors are assigned to each factor and used to multiply scaling values that range from 0 to 1 depending on the degree of acceptance for the attribute. The products for each alternative are then listed according to the products to provide an order of preference. The details of this and other systems, however, are beyond the scope of this report. 